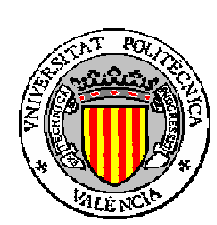

\title{
Modelado Cinemático y Control de Robots Móviles con Ruedas
}

\author{
Tesis Doctoral \\ Departamento de Ingeniería de Sistemas y Automática \\ Universidad Politécnica de Valencia
}

Autor: Luis Ignacio Gracia Calandín

Director: Dr. Josep Tornero i Montserrat 

En primer lugar, agradezco al director de la tesis Dr. Josep Tornero su valiosa ayuda, estímulo permanente y acertado asesoramiento a lo largo del trabajo.

En segundo lugar, vaya también mi agradecimiento a mis compañeros del Grupo de Investigación y del Departamento de Ingeniería de Sistemas y Automática que de un modo u otro han contribuido al desarrollo de la tesis.

A mi familia 

La presente tesis doctoral aborda el modelado cinemático y control de robots móviles con ruedas. En concreto se profundiza en los siguientes temas:

- Se plantea el modelado de una rueda genérica que incluye todos los tipos comunes: fija, orientable centrada, orientable descentrada (castor) y sueca (también denominada universal, Mecanum ó Ilon).

- Se describe un procedimiento eficiente para generar modelos cinemáticos, basado en el concepto de espacio nulo, el cual se aplica posteriormente a un gran número de tipos de robots móviles. Todos estos modelos son caracterizados en cuanto a su precisión o transmisión de errores (isotropía).

- Se deduce un novedoso planteamiento geométrico que establece la singularidad de cualquier modelo cinemático de cualquier robot con ruedas. Este planteamiento se aplica a todos los tipos de robots anteriores.

- Se desarrolla el modelado dinámico del robot para, a través de tres sucesivas aproximaciones y de la caracterización de las fricciones en las ruedas, llegar a un modelado cinemático con deslizamiento.

- Se plantea un esquema de control del robot con tres bucles de control anidados (dinámico, cinemático y de planificación) que es conceptualmente similar a los empleados en robots manipuladores. En particular se profundiza en el bucle cinemático de nivel medio e indirectamente en el de planificación, al caracterizar las referencias que puede seguir cada tipo de robot sin error.

- Se presentan experiencias de comprobación de los algoritmos de modelado con deslizamiento y de control del robot, realizadas sobre una plataforma eléctrica industrial (carretilla industrial).

- Finalmente se desarrollan dos soluciones para las aplicaciones de aparcamiento en paralelo, con pre-planificación y caracterización geométrica, y de seguimiento de línea por visión. 



\section{ABSTRACT}

This $\mathrm{PhD}$ thesis deals with the kinematic modeling and control of wheeled mobile robots. In particular it focuses on the following issues:

- It is modeled a generic wheel that includes all the common types: fixed, centered orientable, off-centered orientable (castor) and swedish (also referred to as universal, Mecanum or Ilon).

- It is developed an efficient procedure, based on the null space concept, to obtain kinematic models. This procedure is applied to many mobile robots and the accuracy of the obtained models is characterized through an isotropy analysis.

- It is deduced a new geometric approach that establishes the singularity of any kinematic model of any wheeled mobile robot. This geometric approach is applied to all the mobile robots previously mentioned.

- It is proposed a kinematic modeling with slip obtained from successive approximations of the robot dynamic model and the characterization of the friction on the wheels.

- It is suggested a kinematic control scheme with three nested loops (dynamic, kinematic and planning) that is similar to the approaches used for robotic manipulators. It is studied in depth the kinematic loop and indirectly the planning loop, through the characterization of the references that each mobile robot can track with no error.

- An industrial forklift has been used to test the algorithms of kinematic modeling with slip and robot control.

- Finally two solutions have been developed for the robotic applications of parallel parking and line tracking with a vision system. 



\section{RESUM}

La present tesi doctoral aborda el modelatge cinemàtic i control de robots mòbils amb rodes. En concret s'aprofundix en els temes següents:

- Es planteja el modelatge d'una roda genèrica que inclou tots els tipus comuns: fixa, orientable centrada, orientable descentrada (castor) i sueca (també nomenada universal, Mecanum o Ilon).

- Es descriu un procediment eficient per a generar models cinemàtics, basat en el concepte d'espai nul, el qual s'aplica posteriorment a un gran nombre de tipus de robots mòbils. Tots estos models són caracteritzats quant a la seua precisió o transmissió d'errors (isotropia).

- Es deduïx un nou plantejament geomètric que estableix la singularitat de qualsevol model cinemàtic de qualsevol robot amb rodes. Esta regla s'aplica a tots els tipus de robots anteriors.

- Es desenvolupa el modelatge dinàmic del robot per a, a través de tres successives aproximacions i de la caracterització de les friccions en les rodes, arribar a un modelatge cinemàtic amb lliscament.

- Es planteja un esquema de control del robot amb tres bucles de control niats (dinàmic, cinemàtic i de planificació) que es conceptualment similar als empleats per robots manipuladors. En particular s'aprofundeix en el bucle cinemàtic i indirectament en el de planificació, al caracteritzar les referències que pot seguir cada tipus de robot sense error.

- Es presenten experiències de comprovació dels algoritmes de modelatge amb lliscament i de control del robot, realitzades sobre una plataforma elèctrica industrial (carretó industrial).

- Finalment es desenvolupen dos solucions per a les aplicacions d'aparcament en paral-lel, amb pre-planificació i caracterització geomètrica, i de seguiment de línia per visió. 



\section{ÍNDICE GENERAL}

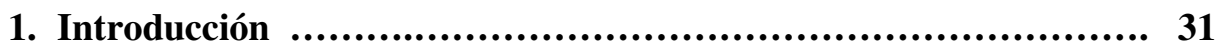

$1.1 \quad$ Introducción .................................................. 31

1.2 Objetivos de la tesis .................................. 32

$1.3 \quad$ Estructura de la tesis ........................................ 33

2. Estado del arte

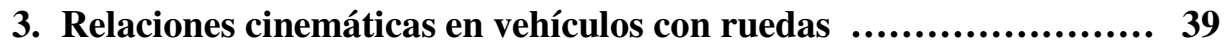

3.1 Introducción ...................................................... 39

3.2 Supuestos considerados .................................. 42

3.3 Relaciones cinemáticas ..................................... 44

3.3.1 Sistemas de coordenadas $\ldots \ldots \ldots \ldots \ldots \ldots \ldots \ldots \ldots \ldots \ldots . \ldots \ldots$

3.3.2 Obtención de la velocidad de deslizamiento de rueda ....... 46

3.3.3 Particularización de la ecuación de rueda ..................... 49

3.3.4 Matriz Jacobiana de rueda ................................ 50

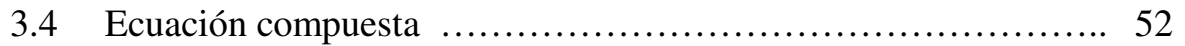

$3.5 \quad$ Ruedas especiales ........................................ 54

3.5.1 Rueda doble y rueda castor doble ........................ 54

3.5.2 Rueda tipo bola ....................................... 58

3.5.3 Rueda ortogonal ................................... 59

3.6 Resultados más relevantes y conclusiones del capítulo .......... 61 


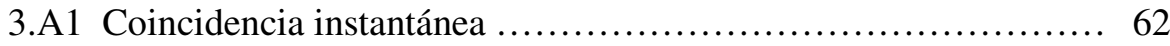

4. Modelado cinemático de vehículos con ruedas sin deslizamiento ..... 65

$4.1 \quad$ Introducción ................................................... 65

4.2 Modelado y caracterización con espacio nulo .................... 67

4.3 Modelado y caracterización con rangos ........................... 69

4.4 Modelado y caracterización con matrices Jacobianas de rueda ..... 70

4.4.1 Problema cinemático directo ............................ 72

4.4.2 Problema cinemático inverso .......................... 73

4.4.3 Solución inversa accionada ............................ 75

4.4.4 Árbol de accionamiento .................................. 77

4.4.5 Solución directa sensorizada ............................ 79

4.4.6 Árbol de sensorización .................................... 80

4.4.7 Discusión del método de matrices Jacobianas de rueda ..... 82

4.4.8 Demostraciones para el método de matrices Jacobianas de rueda ............................................. 84

4.5 Solución cinemática ampliada ............................... 95

4.6 Modelado y caracterización del vehículo triciclo .................. 96

4.7 Resultados más relevantes y conclusiones del capítulo ...........102

5. Modelos cinemáticos de vehículos y transmisión de errores ......... 103

5.1 Introducción ................................................ 103

5.2 Restricciones al movimiento ................................. 105

5.3 Obtención de las cinco clases de vehículos ..................... 107

5.3.1 Demostración de las implicaciones de (5.9) .............. 110

5.4 Transmisión de errores en los modelos cinemáticos: Isotropía .... 113

5.5 Caracterización de las cinco clases de vehículos ............... 117

5.5.1 Tipo $1(3,0)$ : Vehículo omnidireccional ................. 117 
5.5.2 Tipo 2 (2,0): Vehículo diferencial ..................... 122

5.5.3 Tipo 3 (2,1): Vehículo con una rueda orientable ............129

5.5.4 Tipo $4(1,1)$ : Vehículo triciclo y bicicleta ................ 135

5.5.5 Tipo 5 (1,2): Vehículo con dos ruedas orientables ......... 138

5.6 Resultados más relevantes y conclusiones del capítulo ........... 141

6. Singularidad de los modelos cinemáticos de vehículos ............... 143

$6.1 \quad$ Introducción ................................................. 143

6.2 Reformulación de ecuaciones ............................ 145

6.3 Problemática de la singularidad ............................. 147

6.4 Caracterización de la singularidad ........................... 149

6.5 Singularidad de los cinco tipos de vehículos .................... 154

6.5.1 Tipo 1: Vehículo omnidireccional ....................... 154

6.5.2 Tipo 2: Vehículo diferencial ............................ 156

6.5.3 Tipo 3: Vehículo con una rueda orientable ................. 156

6.5.4 Tipo 4: Vehículo triciclo y bicicleta .................... 156

6.5.5 Tipo 5: Vehículo con dos ruedas orientables ............... 160

6.6 Extensión de la caracterización de la singularidad ................. 161

6.7 Resultados más relevantes y conclusiones del capítulo ........... 162

7. Modelado cinemático de vehículos con ruedas con deslizamiento ... 163

7.1 Introducción ................................................. 163

7.2 Modelado dinámico de vehículos con ruedas ..................... 168

7.3 Modelos de tracción (fuerzas de fricción en las ruedas) ............ 173

7.4 Modelos con deslizamiento .................................... 179

7.4.1 Modelo del movimiento quasi-estático ................... 179

7.4.2 Modelo cinemático con deslizamiento .................... 181

7.4.3 Uso práctico del modelo cinemático con deslizamiento $\ldots 183$ 
7.4.4 Solución de Mínimos Cuadrados ponderada del modelo cinemático

7.5 Simulación y resultados experimentales

7.5.1 Carretilla industrial (triciclo)

7.5.2 Estimación del vector de velocidad del vehículo con el Filtro de Kalman

7.5.3 Resultados de simulación ................................... 192

7.5.4 Resultados experimentales ............................... 198

7.6 Resultados más relevantes y conclusiones del capítulo ........... 206

7.A1 Fórmulas de Pacejka utilizadas en la simulación ................... 208

8. Control cinemático de vehículos con ruedas ........................ 213

8.1 Introducción .............................................. 213

8.2 Esquema global del control del vehículo ...................... 217

8.3 Control de posición ............................................ 219

8.4 Modelo cinemático inverso de rueda ........................... 221

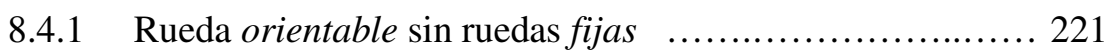

8.4.2 Rueda fija y particularización de rueda orientable ..........222

8.4.3 Rueda castor ........................................... 224

8.4.4 Rueda sueca .......................................... 224

8.5 Tipos de referencias posibles para cada tipo de vehículo ......... 226

8.5.1 Introducción ......................................... 226

8.5.2 Vehículo tipo 1: Omnidireccional ...................... 229

8.5.3 Vehículo tipo 2: Diferencial ............................... 229

8.5.4 Vehículo tipo 4: Triciclo y bicicleta ......................... 230

8.5.5 Vehículo tipo 3 (una rueda orientable) y tipo 5 (dos ruedas orientables) ........................................ 231

8.5.6 Resumen de tipos de referencias posibles .................. 232

8.6 Aplicación del control al caso del triciclo ........................ 234 
8.6.1 Particularización del control .............................. 234

8.6.2 Resultados de simulación .................................... 235

8.6.3 Resultados con carretilla industrial .........................2239

8.6 Resultados más relevantes y conclusiones del capítulo ........... 243

9. Aplicaciones para el control cinemático de vehículos con ruedas ... 245

9.1 Introducción ............................................... 245

9.2 Seguimiento de línea por vision ............................... 247

9.2.1 Posicionamiento por visión …....................... 247

9.2.2 Control cinemático para el seguimiento de línea por visión .262

9.2.3 Resultados del seguimiento de línea por visión .............. 263

9.3 Planificador de aparcamiento ................................... 268

9.3.1 Introducción ............................................... 268

9.3.2 Planificador de aparcamiento en línea .................... 269

9.3.3 Resultados del planificador en simulación ............... 278

9.4 Resultados más relevantes y conclusiones del capítulo ........... 283

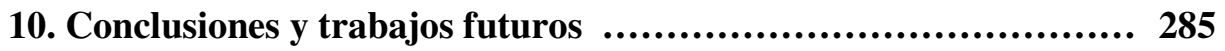

10.1 Conclusiones ................................................. 285

10.2 Trabajos futuros ........................................... 287

Bibliografía .............................................................. 289 



\section{ÍNDICE DE TABLAS Y ALGORITMOS}

Tabla 3.1- Asignación de sistemas de coordenadas utilizada ............... 45

Tabla 3.2- Nomenclatura de variables y parámetros ..................... 46

Tabla 5.1- Relación de vehículos no degenerados posibles ................. 109

Tabla 7.1- Nuevas variables y parámetros en (7.11) ....................... 169

Tabla 7.2- Nuevas variables y parámetros en (7.17) ....................... 170

Tabla 7.3- Comparativa de cómputo WLS-FK2 y modelo diferencial-FK1 .. 208

Tabla 7.4- Variables y parámetros de la fórmula mágica de Pacejka ........ 205

Tabla 8.1- Referencias posibles según el tipo de vehículo y marco considerados ................................................. 233

Tabla 9.1- Valores de parámetros para el ejemplo $1^{\circ}$ de aparcamiento ...... 279

Tabla 9.2- Valores de parámetros para el ejemplo $2^{\circ}$ de aparcamiento ....... 280

Algoritmo1- Detección de colisión frontal/trasera ........................ 275

Algoritmo2-Cálculo de $\mathrm{dx}_{1}$ de modo que se pase por $\left(x_{\mathrm{P} 2}, y_{\mathrm{P} 2}\right)=(0,0) \ldots \ldots \ldots 276$

Algoritmo3-Cálculo del punto de aproximación ............................ 276

Algoritmo4-Cálculo del punto previo al de aproximación .................... 278

Algoritmo5-Cálculo de maniobras en la fase 3 ........................ 278 



\section{ÍNDICE DE FIGURAS}

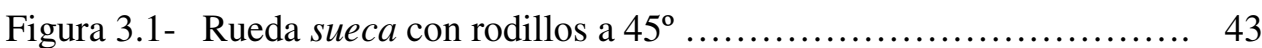

Figura 3.2- Sistemas de coordenadas utilizados en el vehículo ............... 45

Figura 3.3- Sistemas y variables de la formulación cinemática recursiva ..... 48

Figura 3.4- Vista de planta con variables y parámetros definidos en (3.8) ... 48

Figura 3.5- Rueda doble y rueda castor doble ............................ 54

Figura 3.6- Equivalencia entre la rueda doble y la orientable centrada ........ 55

Figura 3.7- Equivalencia entre la rueda castor doble y la castor ............. 56

Figura 3.8- Rueda tipo bola con tres rodillos formando un Rectángulo horizontal .................................... 58

Figura 3.9- Rueda ortogonal con ensamblaje longitudinal .................. 60

Figura 3.10- Rueda ortogonal con ensamblaje lateral ......................... 60

Figura 3.11- Ejemplo de bola en movimiento en un sistema unidimensional .... 62

Figura 4.1- Árbol de solución de la ecuación genérica “A $\cdot \mathrm{x}=\mathrm{B} \cdot \mathrm{y}$ ” ........ 71

Figura 4.2- Árbol para el vector de velocidad del vehículo .................. 72

Figura 4.3- Árbol para el vector compuesto de velocidades de rueda ......... 73

Figura 4.4- Árbol de caracterización del accionamiento del vehículo ......... 78

Figura 4.5- Árbol de caracterización de la sensoriazación del vehículo ....... 81

Figura 4.6- Asignación de sistemas de coordenadas en el vehículo triciclo .... 96

Figura 5.1- Vehículo omnidireccional isotrópico con tres ruedas suecas ..... 116

Figura 5.2- Vehículo omnidireccional quasi-isotrópico con tres ruedas castor 119 
Figura 5.3- Vehículo diferencial con rueda sueca ......................... 120

Figura 5.4- Condiciones de isotropía para el vehículo diferencial con

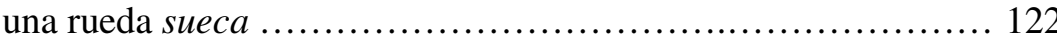

Figura 5.5- Vehículo diferencial con rueda castor ........................ 123

Figura 5.6- Condiciones de isotropía para el vehículo diferencial con una rueda castor ............................................... 125

Figura 5.7- Vehículo con una rueda orientable y dos ruedas suecas ......... 127

Figura 5.8- Configuraciones isotrópicas para el vehículo tipo 3 con ruedas suecas .................................................... 128

Figura 5.9- Vehículo con una rueda orientable y dos ruedas castor ........... 129

Figura 5.10- Configuraciones isotrópicas para el vehículo tipo 3 con ruedas castor

Figura 5.11- Vehículo triciclo (una rueda orientable y dos fijas dependientes). 133

Figura 5.12- Vehículo bicicleta con una rueda adicional sueca o castor ........ 134

Figura 5.13- Vehículo con dos ruedas orientables ......................... 136

Figura 6.1- Línea definida por el vector fila de tres elementos $\lambda=\left(\lambda_{x} \lambda_{y} d_{\lambda}\right) .150$

Figura 6.2- Singularidad del vehículo omnidireccional con ruedas suecas .... 155

Figura 6.3- Configuraciones singulares del vehículo omnidireccional con ruedas castor

Figura 6.4- Singularidad del vehículo tipo 2 con rueda suecalcastor adicional

Figura 6.5- Configuraciones singulares comunes para el vehículo tipo 3 ..... 157

Figura 6.6- Configuraciones singulares para el vehículo tipo 3 con ruedas suecas ............................................... 158

Figura 6.7- Configuraciones singulares para el vehículo tipo 3 con ruedas castor ............................................... 158

Figura 6.8- Configuraciones singulares para el vehículo triciclo

Figura 6.9- Configuraciones singulares para el vehículo bicicleta con una rueda suecalcastor adicional

Figura 6.10- Configuraciones singulares para el vehículo tipo 5 con una rueda suecalcastor adicional 
Figura 7.1- Fenómenos estáticos en la fricción ............................ 173

Figura 7.2- Rueda con fricción distribuida o puntual ….................... 174

Figura 7.3- Coeficiente de adhesión para fricción de Coulomb y

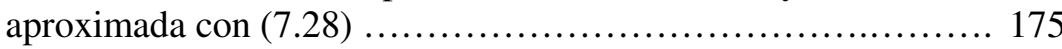

Figura 7.4- Coeficientes admisibles para el caso bidimensional de Coulomb 176

Figura 7.5- Curva propuesta en [Dugoff et al. 70] para el coeficiente de fricción en distintas condiciones de velocidad y adherencia ...... 177

Figura 7.6- Curva estática para el coeficiente de fricción lateral vs. ángulo de deslizamiento ....................................... 178

Figura 7.7- Carretilla industrial Nichiyu FBT15 serie 65

Figura 7.8- Vista de planta de la carretilla industrial con la representación esquemática tipo triciclo

Figura 7.9- Diferencia entre los elementos del vector de velocidad del vehículo al calcularlos con MCD y con WLS optimizado

Figura 7.10- Vector de velocidad calculado con MCD para los 1155 puntos ... 194

Figura 7.11- Error numérico medio cometido en (7.63) al calcular el vector de velocidad calculado con MCD

Figura 7.12- Caminos obtenidos con MD, MQE, MCD y WLS para pares nulos y condiciones iniciales no nulas

Figura 7.13-Caminos obtenidos con MD, MQE, MCD y WLS con pares de rotación no nulos y condiciones iniciales no nulas

Figura 7.14- Evolución de los elementos del vector de velocidad del vehículo según el MD para el segundo ejemplo de simulación

Figura 7.15-Camino obtenido con WLS optimizado y no optimizado al recorrer aproximadamente un rectángulo

Figura 7.16- Medidas de velocidades en las ruedas fijas y en la orientación de la rueda orientable en experimento del rectángulo

Figura 7.17- Camino obtenido con el FK1 optimizado y no optimizado para los datos de la Figura 7.16

Figura 7.18-Camino obtenido con el FK2 optimizado y no optimizado para los datos de la Figura 7.16 
Figura 7.19- Caminos obtenidos con WLS, el FK1, el FK2 y el modelo diferencial para distintos experimentos tipo rectángulo

Figura 7.20- Caminos obtenidos con WLS, el FK1, el FK2 y el modelo diferencial para el segundo tipo de camino en forma de tirabuzón 203

Figura 7.21-Caminos obtenidos con WLS, el FK1, el FK2 y el modelo diferencial para el tercer tipo de camino en forma de $\mathrm{D}$

Figura 7.22-Caminos obtenidos con WLS, el FK1, el FK2 y el modelo diferencial para el cuarto tipo de camino en forma de ocho

Figura 7.23-Caminos obtenidos con WLS, el FK1, el FK2 y el modelo diferencial para el quinto tipo de camino en forma de triple bucle 204

Figura 7.24- Interfaz del terminal táctil incorporado en la carretilla industrial . 205

Figura 7.25-Coeficiente longitudinal en deslizamiento longitudinal puro y velocidad longitudinal de rueda entre 1 y $18 \mathrm{~m} / \mathrm{s}$

Figura 7.26- Coeficiente lateral en deslizamiento lateral puro y velocidad longitudinal de rueda entre 1 y $9 \mathrm{~m} / \mathrm{s}$

Figura 7.27- Coeficiente de adhesión longitudinal en deslizamiento combinado

Figura 7.28- Proyección de las curvas de nivel del coeficiente de adhesión longitudinal en deslizamiento combinado respecto al ángulo de deslizamiento

Figura 7.29- Coeficiente de adhesión lateral en deslizamiento combinado

Figura 7.30- Proyección de las curvas de nivel del coeficiente de adhesión lateral en deslizamiento combinado respecto al ratio de deslizamiento

Figura 8.1- Esquema general de control del vehículo ..................... 218

Figura 8.2- Ejemplos de función: $\mathrm{f}_{1}$ no es $\mathrm{C}^{0} ; \mathrm{f}_{2}$ es $\mathrm{C}^{0}$ pero no $\mathrm{C}^{1} \ldots \ldots \ldots \ldots 227$

Figura 8.3- Variables del camino en un punto de tangente continua .......... 228

Figura 8.4- Variables y parámetros de la carretilla industrial

Figura 8.5- Ejemplo $1^{\circ}$ de seguimiento de referencia: camino seguido por la referencia, origen de $\mathrm{R}$ y punto medio de las rueda fijas ........ 236

Figura 8.6- Orientación $\beta_{3}$, error en $x$ y error en $y$ para ejemplo $1^{\circ} \ldots \ldots \ldots 236$ 
Figura 8.7- Ejemplo $2^{\circ}$ de seguimiento de referencia: camino seguido por la referencia, origen de $\mathrm{R}$ y punto medio de las rueda fijas ........ 237

Figura 8.8- Orientación $\beta_{3}$, error en $x$ y error en $y$ para ejemplo $2^{\circ}$

Figura 8.9- Experiencias de seguimiento de trayectoria rectilínea: camino seguido por referencia, origen de $\mathrm{R}$ y punto medio ruedas fijas ... 240

Figura 8.10- Orientación $\beta_{3}$, error en $x$ y error en $y$ en caso de recta 241

Figura 8.11-Experiencia de seguimiento de trayectoria circular: camino seguido por referencia, origen de $\mathrm{R}$ y punto medio ruedas fijas ... 241

Figura 8.12- Orientación $\beta_{3}$, error en $x$ y error en $y$ para el círculo .......... 242

Figura 9.1- Modelo básico de formación de imagen ..................... 250

Figura 9.2- Relación entre el sistema de coordenadas del suelo y el de la cámara

Figura 9.3- Vista de planta del vehículo, cámara, línea a seguir y sus sistemas de coordenadas

Figura 9.4- Vista de perfil de la cámara, línea a seguir y sus sistemas de coordenadas

Figura 9.5- Posibilidades de líneas de seguimiento .......................... 254

Figura 9.6- Situación de calibración ........................................ 256

Figura 9.7- Ejemplo de calibración 258

Figura 9.8- Líneas detectadas en ejemplo de posicionamiento con sistema calibrado 259

Figura 9.9- Posicionamientos obtenidos 260

Figura 9.10- Posicionamiento en campo de chufas 260

Figura 9.11- Transformada de Hough de la Figura 9.10 (b) 261

Figura 9.12- Posicionamiento obtenido con sistema de visión 262

Figura 9.13- Trazados en seguimiento de línea simulado: Referencia, $\mathrm{P}$ ó origen de $\mathrm{R}$ y punto medio de las ruedas fijas 264

Figura 9.14-Evolución de las velocidades de control de las ruedas fijas del vehículo diferencial en el seguimiento de línea simulado 
Figura 9.15- Vehículo de pruebas tipo diferencial ........................ 266

Figura 9.16- Interfaz desarrollada para el seguimiento de línea .............. 267

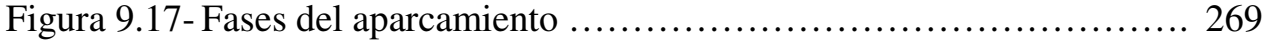

Figura 9.18- Parámetros geométricos del espacio de aparcamiento ........... 270

Figura 9.19- Parámetros geométricos del vehículo tipo coche .............. 271

Figura 9.20- Descripción del CIR y distancias $d_{1}, d_{2}, d_{3}$ y $d_{4} \ldots \ldots \ldots \ldots \ldots \ldots . \ldots 272$

Figura 9.21- Relación entre el ángulo $\theta$ y la posición de $\mathrm{P}_{i} \ldots \ldots \ldots \ldots \ldots \ldots . \ldots 273$

Figura 9.22- Desplazamiento entre dos arcos simétricos de valor $\xi$......... 273

Figura 9.23-Características de la colisión en la primera maniobra ............ 275

Figura 9.24- Maniobra previa para alcanza el punto de posicionamiento ...... 277

Figura 9.25- Interfaz de los parámetros del vehículo ..................... 279

Figura 9.26- Interfaz de los parámetros del espacio de aparcamiento ......... 279

Figura 9.27-Ejemplo $1^{\circ}$ de aparcamiento con el planificador desarrollado ..... 280

Figura 9.28-Ejemplo $2^{\circ}$ de aparcamiento con el planificador desarrollado ..... 280

Figura 9.29-Zoom del ejemplo $2^{\circ}$ de aparcamiento ....................... 281

Figura 9.30- Relación maniobras-ratio en el ejemplo $1^{\circ}$ de aparcamiento ...... 281

Figura 9.31- Vehículo eléctrico de pruebas sobre el que implementar el Planificador de aparcamiento desarrollado 282 
A continuación se indican los símbolos utilizados, agrupados según el capítulo donde aparecen por primera vez.

\section{En el Capítulo 3}

\begin{tabular}{ll}
$\mathbf{p}$ & Postura del vehículo \\
$\dot{\mathbf{p}}$ & Vector de velocidad del vehículo \\
$\mathrm{R}$ & Sistema de coordenadas del vehículo \\
$\overline{\mathrm{R}}$ & Sistema de coordenadas estacionario y coincidente con el $\mathrm{R}$ \\
$\mathrm{S}_{i}$ & Sistema de coordenadas del brazo de dirección de la rueda $i$ \\
$\mathrm{~L}_{i}$ & Sistema de coordenadas de la rueda $i$ \\
$\mathrm{M}_{i}$ & Sistema de coordenadas del rodillo de la rueda $i$ \\
$\mathrm{E}_{i}$ & Sistema de coordenadas entre rodillo de la rueda $i$ y el suelo \\
$\overline{\mathrm{E}}_{i}$ & Sistema de coordenadas estacionario y coincidente con el $\mathrm{E}_{i}$ \\
$\mathrm{G}$ & Sistema de coordenadas global estacionario \\
$\overline{\mathrm{R}}_{\mathrm{R}} \mathbf{v}_{\mathrm{E} i}$ & Velocidad de deslizamiento entre el rodillo y el suelo respecto al sistema $\mathrm{R}$ \\
$\overline{\mathrm{E}} \mathbf{v}_{\mathrm{E} i} \equiv \mathbf{v}_{\text {slip } i}$ & Velocidad de deslizamiento entre el rodillo y el suelo respecto al sistema $\mathrm{E}_{i}$ \\
${ }_{\overline{\mathrm{R}}} v_{\mathrm{R} x} \equiv v_{x}$ & Velocidad del vehículo respecto a la coordenada $X$ del sistema $\mathrm{R}$ \\
${ }_{\overline{\mathrm{R}}} v_{\mathrm{R} y} \equiv v_{y}$ & Velocidad del vehículo respecto a la coordenada $Y$ del sistema $\mathrm{R}$ \\
$\overline{\mathrm{R}}^{\mathrm{R}} \omega_{\mathrm{R}} \equiv \omega$ & Velocidad angular del vehículo \\
$\dot{\beta}_{i}$ & Velocidad angular del brazo de dirección de rueda respecto a la plataforma \\
$\dot{\varphi}_{i}$ & Velocidad de rotación de la rueda \\
$\dot{\varphi}_{\mathrm{r} i}$ & Velocidad de rotación del rodillo \\
$\mathrm{r}_{i}$ & Radio equivalente de la rueda \\
$\mathrm{r}_{\mathrm{r} i}$ & Radio del rodillo \\
\hline
\end{tabular}




\begin{tabular}{|c|c|}
\hline $\operatorname{Rot}(\cdot)$ & Matriz bidimensional de rotación \\
\hline $1_{i}$ & $\begin{array}{l}\text { Distancia del origen del sistema del vehículo al eje de articulación } \\
\text { de la dirección de la rueda }\end{array}$ \\
\hline $\mathrm{d}_{i}$ & Distancia del eje de articulación de dirección al centro de la rueda \\
\hline$\alpha_{i}$ & Ángulo entre el vector definido por $l_{i}$ y el sistema del vehículo $\mathrm{R}$ \\
\hline$\beta_{i}$ & Ángulo del brazo de dirección \\
\hline$\delta_{i}$ & Ángulo entre el brazo de dirección y la rueda \\
\hline$\gamma_{i}$ & Ángulo entre la rueda y el rodillo \\
\hline $\cos (\cdot) \equiv \mathrm{c}$. & Función coseno \\
\hline $\sin (\cdot) \equiv \mathrm{s}$ & Función seno \\
\hline $\operatorname{Rot}_{Z}(\cdot)$ & Matriz de rotación tridimensional en el eje $Z$ \\
\hline${ }^{\mathrm{G}} \theta_{\mathrm{R}}$ & Orientación del vehículo \\
\hline${ }^{\mathrm{R}} \mathbf{v}_{\mathrm{R}}$ & Velocidad lineal del vehículo respecto al sistema $\bar{R}$ \\
\hline$\dot{\mathbf{q}}_{\mathrm{w} i}$ & Vector de velocidad de rueda \\
\hline $\mathbf{J}_{i}$ & Matriz Jacobiana de rueda \\
\hline $\mathbf{J}$ & Matriz Jacobiana compuesta \\
\hline$\overline{\mathbf{I}}$ & Matriz compuesta de matrices identidad de dimensión 3 \\
\hline $\mathrm{f}$ & Subíndice de rueda fija \\
\hline o & Subíndice de rueda orientable \\
\hline $\mathrm{c}$ & Subíndice de rueda castor \\
\hline $\mathrm{s}$ & Subíndice de rueda sueca \\
\hline $\mathrm{N}$ & Número de ruedas del vehículo \\
\hline$\dot{\mathbf{q}}_{\mathrm{w}}$ & Vector de todas las velocidades de ruedas \\
\hline $\mathbf{v}_{\text {slip }}$ & Vector de todas las velocidades de deslizamiento de rueda \\
\hline $\mathbf{A}$ & Matriz compuesta del vehículo \\
\hline$\dot{\mathbf{q}}$ & Vector de todas las velocidades (de ruedas y del vehículo) \\
\hline $\mathbf{A}_{\mathrm{p}}$ & Parte de la matriz compuesta que multiplica al vector $\dot{\mathbf{p}}$ \\
\hline $\mathbf{A}_{\mathrm{w}}$ & Parte de la matriz compuesta que multiplica al vector $\dot{\mathbf{q}}_{\mathrm{w}}$ \\
\hline
\end{tabular}

\section{En el Capítulo 4}

$\begin{array}{ll}\mathcal{N}(\cdot) & \text { Espacio nulo } \\ \mathbf{B} & \text { Base del espacio nulo de la matriz compuesta del vehículo } \\ \boldsymbol{\eta} & \text { Vector de movilidad del vehículo } \\ m & \text { Grado de movilidad del vehículo } \\ k & \text { Número de velocidades (de ruedas y del vehículo) }\end{array}$




$\begin{array}{ll}g & \text { Rango de la matriz compuesta del vehículo } \\ \dot{\mathbf{q}}_{\mathrm{a}} & \text { Vector de velocidades asignadas } \\ \dot{\mathbf{q}}_{\text {na }} & \text { Vector de velocidades no asignadas } \\ \mathbf{B}_{\mathrm{a}} & \text { Submatriz de } \mathbf{B} \text { definida por las velocidades asignadas } \\ \mathbf{B}_{\text {na }} & \text { Submatriz de B definida por las velocidades no asignadas } \\ \boldsymbol{\beta}_{\mathrm{o}} & \text { Vector de todos los ángulos de las ruedas orientables } \\ \boldsymbol{\beta}_{\mathrm{c}} & \text { Vector de todos los ángulos de las ruedas castor } \\ \mathrm{l}_{12} & \text { Semidistancia entre las ruedas fijas para el vehículo tipo triciclo } \\ \mathrm{l}_{3} & \text { Distancia entre la rueda orientable y el origen del sistema R para el } \\ \mathrm{r} & \text { vehículo tipo triciclo } \\ \Delta(\cdot) & \text { Radio de las tres ruedas del vehículo tipo triciclo } \\ H & \text { Función auxiliar para el cálculo del error residual obtenido al aplicar el } \\ H & \text { algoritmo de Mínimos Cuadrados } \\ G & \text { Número de velocidades de rueda independientes } \\ \mathrm{w}_{i} & \text { Número de velocidades del vehículo independientes } \\ \mathbf{J}_{\mathrm{p}} & \text { Número de velocidades de la rueda } i \\ \dot{\mathbf{q}}_{\mathrm{p}} & \text { Reordenación de matriz Jacobiana compuesta según accionadas } \\ \mathrm{u} & \text { Reordenación del vector de velocidades de rueda según accionadas } \\ \mathrm{nu} & \text { Subíndice de accionadas } \\ \mathrm{s} & \text { Subíndice de no accionadas } \\ \mathrm{ns} & \text { Subíndice de sensorizadas } \\ & \text { Subíndice de no sensorizadas }\end{array}$

\section{En el Capítulo 5}

$\mathbf{K}_{\mathrm{r}} \quad$ Matriz de restricciones al movimiento del vehículo

$\mathbf{K}_{\mathrm{r}}^{*} \quad$ Matriz de restricciones al movimiento del vehículo debidas a las ruedas fijas y orientables

$\mathbf{K}_{\mathrm{rf}}^{*} \quad$ Matriz de restricciones al movimiento del vehículo debidas a las fijas

$\mathbf{K}_{\text {ro }}^{*} \quad$ Matriz de restricciones al movimiento del vehículo debidas a orientables

$\mathrm{N}_{\mathrm{f}} \quad$ Número de ruedas fijas en el vehículo

$\mathrm{N}_{\mathrm{o}} \quad$ Número de ruedas orientables en el vehículo

$m \quad$ Grado de movilidad del vehículo

d Grado de direccionabilidad del vehículo

H Matriz de relación entre vector de salida y vector de entrada

$\kappa \quad$ Número de condición de una matriz

$\|\cdot\|_{2} \quad$ Norma Euclídea 
${ }^{\dagger} \quad$ Pseudo inversa de una matriz

$\sigma_{\max } \quad$ Valor singular más grande de la matriz

$\sigma_{\min } \quad$ Valor singular más pequeño de la matriz

$\dot{\mathbf{q}}_{\text {na } \_ \text {S }} \quad$ Velocidades no asignadas utilizadas para isotropía

$\dot{\mathbf{q}}_{\text {na_ns }} \quad$ Velocidades no asignadas no utilizadas para isotropía

$\mathbf{B}_{\text {na_s }} \quad$ Submatriz definida por las velocidades no asignadas utilizadas en isotropía

$\mathbf{B}_{\text {na_ns }} \quad$ Submatriz definida por las velocidades no asignadas no utilizadas en isotropía

\section{En el Capítulo 6}

$\mathbf{E}_{i} \quad$ Vector de la ecuación de rueda que multiplica al vector de velocidad del vehículo $\dot{\mathbf{p}}$ y cuyo resultado es igual a cero

$\mathbf{F}_{i} \quad$ Vector de la ecuación de rueda que multiplica a $\mathbf{p}$ y cuyo resultado se iguala a la velocidad de rotación

$\mathbf{F}_{\beta i} \quad$ Vector de la ecuación de rueda que multiplica a $\dot{\mathbf{p}}$ y cuyo resultado se iguala a la velocidad de orientación

$\mathbf{F}_{\varphi i} \quad$ Vector de la ecuación de rueda que multiplica a $\dot{\mathbf{p}}$ y cuyo resultado se iguala a la velocidad de orientación y a la de rotación

E Agrupación de vectores $\mathbf{E}_{i}$

F Agrupación de vectores $\mathbf{F}_{i}$

r Matriz con los radios de las ruedas y distancias del brazo de dirección de las ruedas castor

a Subíndice de asignadas

na Subíndice de no asignadas

sg Subíndice de ruedas omnidireccionales singulares

nsg Subíndice de omnidireccionales no singulares

G Matriz que se multiplica por $\dot{\mathbf{p}}$ y cuyo resultado es igual a cero

$\lambda=\left(\begin{array}{lll}\lambda_{x} & \lambda_{y} & d_{\lambda}\end{array}\right)$ Vector fila que representa una recta en el espacio bidimensional

$\left(\lambda_{x} \lambda_{y}\right)^{\mathrm{T}} \quad$ Vector unitario bidimensional de una recta

$\mathrm{d}_{\lambda} \quad$ Distancia de una recta bidimensional al origen 


\section{En el Capítulo 7}

$\mathbf{F}_{\text {fric_c } i} \quad$ Fuerza de fricción debida a la ley de Coulomb

$\mu_{\mathrm{c} i} \quad$ Coeficiente de fricción o adhesión debido a la ley de Coulomb

$P_{\text {dis }} \quad$ Potencia disipada por las fuerzas de fricción

$v_{\mathrm{b}} \quad$ Velocidad de un bloque libre en una dimensión

$F_{\text {fric_b }} \quad$ Fuerza de fricción sobre bloque

$P_{\text {dis_b }} \quad$ Potencia disipada por fuerza de fricción sobre bloque

$T \quad$ Energía cinética del vehículo

Q Vector de fuerzas generalizadas sobre el vehículo

$\mathrm{N}_{\mathrm{o}} \quad$ Número de ruedas orientables en el vehículo

$\mathrm{N}_{\mathrm{c}} \quad$ Número de ruedas castor en el vehículo

$\mathrm{N}_{\mathrm{s}} \quad$ Número de ruedas suecas en el vehículo

$\tau_{\mathrm{r} i} \quad$ Par de rotación de la rueda $i$

$\tau_{\text {osi }} \quad$ Par de direccionamiento de la rueda orientable $i$

$\tau_{\mathrm{cs} i} \quad$ Par de direccionamiento de la rueda castor $i$

$\dot{\beta}_{\mathrm{o} i} \quad$ Velocidad de direccionamiento de la rueda orientable $i$

$\dot{\beta}_{\text {ci }} \quad$ Velocidad de direccionamiento de la rueda castor $i$

$\mathbf{F}_{\text {fric } i} \quad$ Fuerza de fricción en la rueda $i$ respecto al sistema $E_{i}$

${ }^{\mathrm{G}} \dot{\mathbf{p}} \quad$ Vector de velocidad del vehículo respecto al sistema global $\mathrm{G}$

${ }^{\overline{\mathrm{R}}} \dot{\mathbf{p}} \quad$ Vector de velocidad del vehículo respecto al sistema coincidente

$\tau \quad$ Vector de pares agrupados

$\mathbf{F}_{\text {fric }} \quad$ Agrupación de las fuerzas de fricción en las ruedas

$\mathrm{M}_{\mathrm{T}} \quad$ Masa del vehículo sin incluir las ruedas castor

$\mathrm{M}_{\mathrm{c} i} \quad$ Masa de la rueda castor $i$

$\mathrm{I}_{\mathrm{T}}$

Momento de inercia del vehículo sin incluir las ruedas castor

Momento de inercia de la ruedas $i$ respecto a su eje de rotación

Momento de inercia del rodillo de la rueda sueca $i$

$\mathrm{I}_{\mathrm{osi}}$

Momento de inercia de la rueda orientable $i$ respecto al eje $Z$

$\mathrm{I}_{\mathrm{cs} i}$

Momento de inercia de toda la rueda castor $i$ respecto al eje $Z$

${ }^{\mathrm{G}} \mathbf{v}_{\mathrm{CM}}$

Velocidad del CM del vehículo (sin incluir castor) respecto a $\mathrm{G}$

${ }^{\mathrm{G}} \mathbf{v}_{\mathrm{R}}$

Velocidad del origen del sistema $\mathrm{R}$ del vehículo respecto al sistema $\mathrm{G}$

${ }^{\mathrm{G}} \mathbf{v}_{\mathrm{cm} i}$

Velocidad de la rueda castor $i$ respecto al sistema global G

Funciones para rueda castor $i$ que dependen de $1_{i}, \mathrm{~d}_{\mathrm{cm} i}, \alpha_{i},{ }^{\mathrm{G}} \theta_{\mathrm{R}}, \mathrm{y} \beta_{i}$.

$F_{\mathrm{N}} \quad$ Fuerza de reacción en el punto de contacto entre rueda y suelo 
$F_{\text {fric } a} \quad$ Fuerza de fricción en la coordenada $a$

$\mu_{a} \quad$ Coeficiente de fricción o adhesión en la coordenada $a$

$\boldsymbol{\mu} \quad$ Coeficiente de fricción o adhesión vectorial

$\boldsymbol{\mu}^{*} \quad$ Coeficiente de fricción vectorial que maximiza el ratio de disipación

$\mu_{y} \quad$ Coeficiente de fricción longitudinal en la rueda

$\mu_{x} \quad$ Coeficiente de fricción lateral en la rueda

$s \quad$ Ratio de deslizamiento longitudinal

$v_{\text {wheel } y} \quad$ Velocidad longitudinal del centro de la rueda

$\alpha_{s} \quad$ Ángulo de deslizamiento

$\infty \quad$ Subíndice que indica el valor estacionario de una variable o vector

${ }^{\bar{R}} \mathbf{V}_{\mathrm{R}} \quad$ Velocidad del sistema $\mathrm{R}$ del vehículo respecto su coincidente

${ }^{\mathrm{G}} \dot{\mathbf{q}} \quad$ Vector de velocidades con vector de velocidad del vehículo respecto a $\mathrm{G}$

${ }^{\bar{R}} \dot{q} \quad$ Vector de velocidades con vector de velocidad del vehículo respecto a $\bar{R}$

$\tau_{\text {raj }} \quad$ Par de rotación no libre de la rueda $j$

$\tau_{\text {csaj }} \quad$ Par de dirección no libre de la rueda castor $j$

$\dot{\varphi}_{\text {raj }} \quad$ Velocidad de rotación no libre de rueda $j$

$\dot{\beta}_{\text {caj }} \quad$ Velocidad de dirección no libre de rueda castor $j$

$\mathbf{f}_{\text {fric } i}(\cdot) \quad$ Función genérica bidimensional para el cálculo de fuerza de fricción

$\sqrt{\boldsymbol{\mu}} \quad$ Matriz de ponderación de la ecuación cinemática sin deslizamiento

$\sqrt{\boldsymbol{\mu}_{i}} \quad$ Matriz de ponderación de las ecuaciones cinemáticas de la rueda $i$

$J_{\mathrm{LS}} \quad$ Índice minimizado por el algoritmo de mínimos cuadrados

$1_{\mathrm{CM}} \quad$ Distancia del CM de la carretilla al eje de rotación de las ruedas fijas

$\mathbf{A}_{\mu \mathrm{a}} \quad$ Matriz de velocidades asignadas con ponderación de ecuaciones

$\mathbf{A}_{\text {una }} \quad$ Matriz de velocidades no asignadas con ponderación de ecuaciones

$\left(\mu_{x 12}, \mu_{y 12}\right) \quad$ Coeficientes de fricción lateral y longitudinal de ruedas fijas de carretilla

$\mu_{x 3} \quad$ Coeficiente de fricción lateral de la rueda orientable de la carretilla

$\mathrm{k}_{11} \quad$ Constante de relación entre coeficientes de fricción $\mu_{x 12}$ y $\mu_{y 12}$

$\mathrm{k}_{12} \quad$ Constante de relación entre coeficientes de fricción $\mu_{x 12}$ y $\mu_{x 3}$

$\mathrm{T} \quad$ Periodo del sistema de adquisición de medidas

$\mathbf{y}_{1 k} \quad$ Vector de salida para el FK1 en el instante discreto $k$

$\mathbf{C}_{1 k} \quad$ Matriz de salida para el FK1 en el instante discreto $k$

$\mathbf{y}_{2 k} \quad$ Vector de salida para el FK2 en el instante discreto $k$

$\mathbf{C}_{2 k} \quad$ Matriz de salida para el FK2 en el instante discreto $k$

$\mathbf{Q}_{k} \quad$ Matriz de covarianza de ruido en el proceso en el instante $k$ 


\begin{tabular}{|c|c|}
\hline $\mathbf{R}_{k}$ & Matriz de covarianza de ruido en la medida en el instante $k$ \\
\hline $\mathbf{P}_{k}$ & Matriz de covarianza del error en el instante $k$ \\
\hline $\mathbf{K}_{k}$ & Matriz de corrección o de ganancia en el instante $k$ \\
\hline $\mathrm{k}_{13}$ & $\begin{array}{l}\text { Desviación típica del ruido en los elementos del vector } \dot{\mathbf{p}} \text { de la ecuación } \\
\text { de estado }\end{array}$ \\
\hline $\mathrm{k}_{14}$ & Desviación típica del ruido en la velocidad de las ruedas fijas para el FK1 \\
\hline $\mathrm{k}_{15}$ & $\begin{array}{l}\text { Desviación típica del error de la ecuación correspondiente a la coordenada } \\
\text { perpendicular de la rueda orientable para el FK1 }\end{array}$ \\
\hline $\mathrm{k}_{16}$ & $\begin{array}{l}\text { Desviación típica del error de la ecuación correspondiente a la coordenada } \\
\text { perpendicular de las ruedas fijas para el FK2 }\end{array}$ \\
\hline $\mathrm{k}_{17}$ & Desviación típica del ruido en la velocidad de las ruedas fijas para el FK2 \\
\hline $\mathrm{k}_{18}$ & $\begin{array}{l}\text { Desviación típica del error de la ecuación correspondiente a la coordenada } \\
\text { perpendicular de la rueda orientable para el FK2 }\end{array}$ \\
\hline$J_{\mathrm{MQE}}$ & Î́ndice de error del modelo de movimiento quasi-estático en simulación \\
\hline$J_{\mathrm{MCD}}$ & Índice de error del modelo cinemático con deslizamiento en simulación \\
\hline$J_{\mathrm{wLS}}$ & $\begin{array}{l}\text { Índice de error de la solución de mínimos cuadrados ponderada en } \\
\text { simulación }\end{array}$ \\
\hline$J_{\mathrm{k}}$ & $\begin{array}{l}\text { Índice de error para los caminos obtenidos con WLS, FK1, FK2 y el } \\
\text { modelo diferencial en los experimentos reales }\end{array}$ \\
\hline$k_{7}$ & Factor de pico de la fórmula mágica de $P a c e j k a$ \\
\hline$k_{8}$ & Factor de forma de la fórmula mágica de Pacejka \\
\hline$k_{9}$ & Factor de rigidez de la fórmula mágica de Pacejka \\
\hline$k_{10}$ & Factor de curvatura de la fórmula mágica de Pacejka \\
\hline
\end{tabular}

\section{En el Capítulo 8}

$\mathbf{p}_{\text {ref }} \quad$ Postura de referencia establecida por el planificador

$\dot{\mathbf{p}}_{\text {control }} \quad$ Vector de velocidad del vehículo a conseguir por control dinámico

$\dot{\varphi}_{i \text { ref }} \quad$ Velocidad de rotación a conseguir por el control dinámico

$\dot{\beta}_{i \text { ref }} \quad$ Velocidad de dirección a conseguir por control dinámico

$\beta_{i \text { ref }} \quad$ Orientación de rueda orientable a conseguir por control dinámico

$V_{i} \quad$ Tensiones aplicadas a los actuadores

$\tau_{i} \quad$ Pares aplicados por los actuadores

$\dot{\varphi}_{i \mathrm{~s}} \quad$ Velocidad de rotación sensorizada 
$\dot{\beta}_{i \text { s }} \quad$ Velocidad de dirección sensorizada

$\beta_{i \mathrm{~s}} \quad$ Orientación sensorizada de rueda

$\beta_{i \text { castor }} \quad$ Orientación de rueda castor con alguna velocidad de rueda accionada

$\mathbf{A}_{\mathrm{c}} \quad$ Matriz diagonal del control de posición

B $_{\mathrm{c}} \quad$ Matriz diagonal del control de posición

$a_{\mathrm{c}} \quad$ Polos asignados por el control de posición

$\mathrm{T} \quad$ Periodo del sistema de adquisición de medidas y control

$\theta \equiv{ }^{\mathrm{G}} \theta_{\mathrm{R}} \quad$ Orientación del vehículo

${ }^{\overline{\mathrm{R}}} \dot{\mathbf{p}} \quad$ Vector de velocidad del vehículo respecto al sistema coincidente $\overline{\mathrm{R}}$

${ }^{\mathrm{G}} \dot{\mathbf{p}} \quad$ Vector de velocidad del vehículo respecto al sistema global G

${ }^{\mathrm{G}} v_{x} \quad$ Velocidad del vehículo respecto a la coordenada $X$ del sistema global G

${ }^{\mathrm{G}} v_{y} \quad$ Velocidad del vehículo respecto a la coordenada $Y$ del sistema global $\mathrm{G}$

$f_{i}(\theta) \quad$ Función genérica que sólo depende de la variable $\theta$

$\mathrm{C}^{0} \quad$ Una función es $\mathrm{C}^{0}$ si es continua

$\mathrm{C}^{\mathrm{n}} \quad$ Una función es $\mathrm{C}^{\mathrm{n}}$ si su derivada enésima es continua

$\chi \quad$ Ángulo que forma el vector tangente al camino o curva con el eje $X$

$v_{\mathrm{a}} \quad$ Velocidad de avance de la trayectoria 2D

$\omega_{\mathrm{g}} \quad$ Velocidad de giro de la trayectoria 2D

$\kappa \quad$ Curvatura del camino

$\rho_{\mathrm{c}} \quad$ Radio de curvatura del camino

$\mathrm{C}_{\mathrm{c}} \quad$ Centro de curvatura del camino

e Distancia entre el punto que hace el seguimiento (origen de R) y el eje de rotación de las ruedas fijas de la carretilla

${ }^{\mathrm{G}} x_{\mathrm{R} \text { ref }} \quad$ Posición de referencia del vehículo respecto a la coordenada $X$ de $\mathrm{G}$

${ }^{\mathrm{G}} y_{\mathrm{R} \text { ref }} \quad$ Posición de referencia del vehículo respecto a la coordenada $Y$ de $\mathrm{G}$

${ }^{\mathrm{G}} v_{x \text { control }}$

${ }^{\mathrm{G}} v_{y \text { control }}$

Velocidad de control de la coordenada $X$ del vector $\dot{\mathbf{p}}$

Velocidad de control de la coordenada $Y$ del vector $\dot{\mathbf{p}}$

$a_{x}$

Polo asignado a la dinámica de la coordenada $X$ del vector $\dot{\mathbf{p}}$

$a_{y}$

Polo asignado a la dinámica de la coordenada $Y$ del vector $\dot{\mathbf{p}}$ 


\section{En el Capítulo 9}

$\left(X_{c}, Y_{c}, Z_{c}\right) \quad$ Sistema de coordenadas de la cámara

$\left(x_{c}, y_{c}, z_{c}\right) \quad$ Posición de un punto respecto al sistema de coordenadas de la cámara

$\left(X_{u}, Y_{u}\right) \quad$ Sistema de coordenadas del plano de la imagen

$\left(x_{u}, y_{u}\right) \quad$ Posición de un punto en el plano de la imagen

$f \quad$ Distancia focal efectiva de la cámara

$\left(x_{p}, y_{p}\right) \quad$ Posición de un punto en el plano de la imagen en coordenadas de píxel

$\left(C_{x}, C_{y}\right) \quad$ Coordenadas en píxeles del centro del plano imagen

$\left(\mathrm{d}_{x}, \mathrm{~d}_{y}\right) \quad$ Tamaño de los elementos sensores de la cámara en los dos ejes

$\left(X_{w}, Y_{w}, Z_{w}\right)$ Sistema de coordenadas del suelo

$\left(x_{w}, y_{w}, z_{w}\right)$ Posición de un punto respecto al sistema de coordenadas del suelo

$\left(t_{x}, t_{y}, t_{z}\right) \quad$ Vector de desplazamiento entre los sistemas de la cámara y el del suelo

$\phi \quad$ Ángulo de inclinación de la cámara respecto a la vertical

$\psi \quad$ Ángulo de giro entre el sistema de la cámara y el del suelo

v Altura del objetivo de la cámara respecto a la línea a seguir

P Punto solidario al vehículo perteneciente al eje del objetivo de la cámara que se quiere que haga el seguimiento

$\mathrm{Z}_{\mathrm{P}} \quad$ Distancia del punto $\mathrm{P}$ al objetivo de la cámara

$h \quad$ Distancia entre $\mathrm{P}$ y la línea a seguir

d Distancia real de separación entre líneas paralelas a seguir

$\mathrm{n} \quad$ Parámetro que indica la línea a seguir de entre las que son paralelas

$\left(x_{1}, x_{2}, \mathrm{~A}\right) \quad$ Valores de plano imagen para poder aplicar sistema de calibración

$\left(x_{w}, y_{w}\right) \quad$ Valores de plano real para poder aplicar sistema de calibración

J Anchura del espacio de aparcamiento

$\mathrm{H} \quad$ Longitud del espacio de aparcamiento

MS Margen de seguridad con los obstáculos

J' Ancho efectivo del espacio de aparcamiento

$\mathrm{H}^{\prime} \quad$ Longitud efectiva del espacio de aparcamiento

$\mathrm{a}_{1} \quad$ Distancia entre el eje trasero de ruedas y la parte delantera del vehículo

$\mathrm{b}_{1} \quad$ Distancia entre el eje trasero de ruedas y la parte de atrás del vehículo

$\mathrm{h}_{1} \quad$ Distancia entre el eje trasero de ruedas y el centro de la rueda orientable

$\mathrm{d}_{1} \quad$ Distancia entre los centros de las ruedas fijas

$\mathrm{c}_{1} \quad$ Ancho del vehículo

r Radio de las ruedas

$\phi_{\mathrm{d}} \quad$ Orientación de la rueda de dirección equivalente

$\phi_{\mathrm{d} \max } \quad$ Máxima orientación posible de la rueda de dirección equivalente

$\mathrm{P}_{\mathrm{f}} \quad$ Punto fijo entre las ruedas fijas que sigue la trayectoria 
CIR Centro instantáneo de rotación del vehículo

$\rho \quad$ Radio de giro, desde el CIR hasta el punto $\mathrm{P}_{\mathrm{f}}$

$\mathrm{P}_{i} \quad$ Esquina del vehículo

$d_{i} \quad$ Distancia del CIR a la esquina del vehículo $\mathrm{P}_{i}$

$\xi \quad$ Ángulo de cada uno de los arcos simétricos de una maniobra

$\mathrm{dx}_{1} \quad$ Distancia del punto $\mathrm{P}_{\mathrm{f}}$ al obstáculo delantero al iniciar primera maniobra

$\mathrm{dx}_{2} \quad$ Separación inicial entre el punto $\mathrm{P}_{\mathrm{f}} \mathrm{y}$ los obstáculos delantero y trasero

$\mathrm{dy}_{3} \quad$ Distancia recorrida en pasada de reconocimiento a partir de la detección del obstáculo delantero

$\mathrm{W}_{\max } \quad$ Distancia actual disponible al obstáculo lateral

ratio

Medida porcentual del espació longitudinal disponible para el aparcamiento 


\section{CAPÍTULO 1 \\ INTRODUCCIÓN}

\subsection{INTRODUCCIÓN}

Una de las temáticas clásicas del área de conocimiento de Ingeniería de Sistemas y Automática es la robótica.

El mundo de la robótica está experimentando un crecimiento explosivo impulsado por los avances en computación, sensores, electrónica, comunicaciones y software. Los robots están en la antesala de revolucionar los procedimientos que se emplean en la agricultura, minería, industria en general, etc. atrayendo los distintos mercados.

Dentro de la robótica se encuentra el campo de los robots manipuladores, que ha experimentado un alto desarrollo desde la década de los setenta, y la denominada robótica móvil, que ha cobrado una importancia creciente durante los años ochenta y noventa.

Tanto en los robots manipuladores como en la robótica móvil existen puntos de interés común: el modelado cinemático, el modelado dinámico, el control (arquitecturas, algoritmos...), la planificación, el reconocimiento del entorno, etc.

En el caso de robots manipuladores existe ya una abundante bibliografía que aborda los aspectos anteriores, mientras que para robots móviles todavía se están realizando un considerable número de investigaciones y desarrollos al respecto.

Así pues, la presente tesis surge ante la necesidad de dar respuesta a las cuestiones cinemáticas (modelado, control, ...) de los robots móviles con ruedas. 


\subsection{OBJETIVOS DE LA TESIS}

En el año 2000 se estableció como meta de la presente tesis doctoral el estudio de aquellas cuestiones relacionadas con la cinemática de robots móviles con ruedas.

Durante el desarrollo de la tesis se han ido planteando y resolviendo objetivos concretos. A continuación se describen los objetivos principales de la tesis:

- Desarrollar una metodología completa de modelado cinemático de vehículos sin deslizamiento, mejorando (y unificando en algunos casos) lo desarrollado por otros autores. (Capítulos 3 y 4 )

- Proporcionar una completa guía sobre los modelos cinemáticos de vehículos, su transmisión de errores (Isotropía) y condiciones de singularidad. (Capítulos 5 y 6 )

- Establecer un modelado cinemático con deslizamiento a partir, a diferencia de otros autores, de principios físicos. (Capítulo 7)

- Obtener un método de control cinemático que anule el error en el seguimiento de referencias para cualquier tipo de vehículo. (Capítulo 8)

- Plantear soluciones a dos aplicaciones concretas, el seguimiento de línea por visión y el aparcamiento en paralelo, sobre las que poder aplicar el control cinemático anterior. (Capítulo 9) 


\subsection{ESTRUCTURA DE LA TESIS}

La presente tesis doctoral está organizada en diez capítulos, incluyendo éste.

El Capítulo 2, estado del arte, es preliminar y representa una mirada de conjunto a las distintas temáticas de la tesis.

La revisión anterior se completa de forma pormenorizada en el resto de capítulos, fundamentalmente en su introducción particular, lo que permite encauzar el desarrollo de los mismos.

El último punto de cada capítulo presenta las aportaciones y conclusiones correspondientes al mismo.

En el Capítulo 3 se deducen las relaciones cinemáticas entre variables de rueda y vehículo para una rueda genérica, que incluye los tipos habituales (fija, orientable, castor, sueca), empleando una eficiente formulación cinemática recursiva.

En el Capítulo 4 se presentan tres métodos para generar modelos cinemáticos de vehículos con ruedas sin deslizamiento, los cuales son aplicados al vehículo tipo triciclo y comparados.

En el Capítulo 5 se deduce una clasificación genérica de vehículos para los que se obtiene su caracterización, modelado cinemático y transmisión de errores a través del concepto de isotropía.

En el Capítulo 6 se obtiene un planteamiento geométrico general que caracteriza la singularidad de cualquier modelo cinemático de cualquier vehículo con ruedas, este planteamiento se aplica a los cinco tipos de vehículos deducidos en el capítulo anterior.

En el Capítulo 7 se deducen tres tipos de modelos con deslizamiento a partir de sucesivas aproximaciones del modelo dinámico del vehículo. Estos modelos se comparan, para el vehículo triciclo, con el modelo dinámico en simulación y con el filtro de Kalman en una situación real.

En el Capítulo 8 se presenta un control cinemático de vehículos basado en tres bucles anidados y se caracterizan las referencias que puede seguir sin error cada tipo de vehículo. El control cinemático anterior se particulariza para el caso del vehículo triciclo, probándose tanto en simulación como en una situación real. 
En el Capítulo 9 se ha dado solución a dos aplicaciones robóticas concretas: el seguimiento de línea por visión y el aparcamiento en línea.

Finalmente en el Capítulo 10 se destacan las conclusiones más relevantes y se propone una serie de trabajos futuros para distintas líneas de la tesis. 


\section{CAPÍTULO 2 ESTADO DEL ARTE}

Con objeto de no descontextualizar la revisión bibliográfica, dada la disparidad de capítulos, aquí se plantea una visión de conjunto sobre las distintas temáticas de la tesis. Posteriormente, en la introducción particular de cada capítulo se realiza un estudio más detallado de las distintas referencias o fuentes que permite encauzar los desarrollos del capítulo en cuestión.

El modelado y el control son puntos de interés tanto en robots manipuladores como en robots móviles.

En el caso de los robots manipuladores existe una abundante bibliografía, ver por ejemplo [Fu et al. 88] y [Ollero 01], que aborda su modelado cinemático, dinámico y/o control.

La mayor parte de los robots manipuladores son brazos articulados y tradicionalmente se modelan, desde el punto de vista cinemático, con matrices de transformación homogénea entre sistemas de coordenadas. Para el modelado dinámico se manejan distintas formulaciones y métodos: Lagrange-Euler, Newton-Euler, ecuaciones generalizadas de d'Alambert, etc.

Para mejorar sus prestaciones se investiga en técnicas para identificar los modelos dinámicos eficientemente y en métodos de control de articulaciones que compensan no-linealidades y acoplamientos [An et al. 88], así como en optimización dinámica y control adaptativo para distintas condiciones de trabajo [Craig 88] [Ortega et al. 89]. 
Por otro lado, para la robótica móvil existe una reciente bibliografía, ver por ejemplo [Inoue et al. 97] [Lyshevski et al. 00] [O'Connor et al. 96] [Canudas et al. 97] [Samson 95], que aborda su modelado cinemático, dinámico y/o control.

Desde el punto de vista del modelado cinemático de vehículos con ruedas, las publicaciones que han causado un mayor impacto hasta ahora son [Muir et al. 87] [Campion et al. 96] [Alexander et al. 89], referidas en un gran número de publicaciones y libros.

Prácticamente sólo en [Muir et al. 87] se plantea un método sistemático basado en matrices de transformación homogénea, de forma análoga al caso de robots manipuladores, para obtener las relaciones cinemáticas entre las variables de rueda y del vehículo, lo cual permite un posterior modelado del vehículo en su conjunto. En el Capítulo 3 de la tesis se plantea una forma más genérica y eficiente de obtener las relaciones anteriores, utilizando una formulación cinemática recursiva. Posteriormente en [Muir et al. 87] se realiza un estudio del modelo del vehículo aplicando un tratamiento de ecuación algebraica matricial genérica y grafos en forma de árbol.

En [Campion et al. 96] fundamentalmente se realiza una clasificación de todos los vehículos en cinco tipos básicos y se obtienen modelos cinemáticos directos con variables de entrada sin sentido físico. Esta clasificación genérica se utiliza en varios capítulos de la tesis $(5,6$ y 8$)$.

En [Alexander et al. 89] se plantean modelos cinemáticos directos e inversos parciales para relacionar variables de rueda y del vehículo, no permitiendo una perspectiva global del vehículo.

Por otra parte la transmisión de errores en los modelos cinemáticos directos o inversos, cuando se considera un error de entrada, ha sido estudiada por distintos autores a través de la caracterización de matrices isotrópicas [Saha et al. 95] [Low et al. 05] [Kim2 et al. 04] [Kim et al. 05].

Otros autores la han estudiado desde un punto de vista empírico para la corrección de errores sistemáticos [Borenstein et al. 94] o estimación de distintos parámetros del error [Martinelli 02] [Kleeman 95]. 
Un aspecto importante es que los modelos cinemáticos dependen de los ángulos de las ruedas con articulación de dirección, por lo que hay valores especiales de estos ángulos para los que se produce la singularidad del modelo, que implica deslizamiento o pérdida de movilidad en el vehículo.

La singularidad de robots manipuladores ha sido ampliamente estudiada [Tourassis et al. 92] [Dinesh et al. 92] [Liu et al. 03] [Lipkin et al. 91] mientras que en vehículos sólo existe alguna pequeña aproximación [Yi et al. 02].

Por otro lado, la necesidad de considerar modelos cinemáticos con deslizamiento puede venir dada por una redundancia en la información de sensores, de forma similar al filtro de Kalman, o por una sobre-actuación en el vehículo.

En [Muir et al. 87] se considera una solución cinemática con información redundante que viola el modelo de sólido rígido del vehículo, mientras que la propuesta en [Kim et al. 04] minimiza una norma Euclídea sin un sentido físico definido. En [Tham et al. 98] se consideran varias relaciones cinemáticas con variables de deslizamiento sin una justificación rigurosa. Finalmente en [Alexander et al. 89] se propone minimizar una función de disipación que en general no produce una solución correcta y que no tiene en cuenta fuerzas externas.

Otros autores han abordado el modelado dinámico de vehículos con deslizamiento [Balakrishna et al. 95] [Williams et al. 02] [Lindgren et al. 02].

Para el control del vehículo o robot móvil algunos autores han planteado métodos geométricos [Ollero et al. 94] [Shin 90] y otros la utilización de herramientas de la teoría de control clásica: aproximación lineal [O'Connor et al. 96]; linealización exacta [d'Andréa-Novel et al. 95] [De Luca et al. 93] [Park et al. 99] [Tzafestas et al. 01]; controlabilidad [Samson 95] [Monaco et al. 91] [Murray et al. 93]; estabilidad por Lyapunov [Lyshevski et al. 00] [Canudas et al. 97] [Dixon et al. 00]; control adaptativo [Inoue et al. 97] [Dixon et al. 01] [Fukao et al. 00]; control predictivo [Ollero et al. 91]; etc.

Por otro lado, el seguimiento de línea es una aplicación habitual en robótica móvil, utilizándose normalmente cables o sensores ópticos. Con los últimos avances tecnológicos se ha ido incrementando el uso de sistemas de visión para 
esta aplicación. La visión tiene algunas ventajas respecto a los otros métodos aunque con el inconveniente del coste computacional asociado al tratamiento de imagen: umbralizado, segmentación, detección de contornos, etc. Para mejorar dicho tratamiento se utilizan herramientas como la transformada de Hough [Hough 59]. En [Marchant 95] se establece el posicionamiento a partir de un sistema de visión respecto a una línea, para lo cual se realiza una aproximación (innecesaria como demuestra el Capítulo 9) que da lugar a relaciones inexactas.

Por otra parte, para el aparcamiento en línea se pueden utilizar planificadores de propósito general [Latombe 91] [Laumond et al. 94], con alto coste computacional, o planificadores más específicos [Tilbury et al. 93] [Zhao et al. 05] [Paromtchik et al. 96] [Jiang et al. 99] [Holve et al. 96] [Miyata et al. 96] [Baturone et al. 04] [Cuesta et al. 04].

En concreto en [Paromtchik et al. 96] [Jiang et al. 99] se realiza el aparcamiento con una preplanificación de tres fases y acciones de control preestablecidas o caracterización geométrica. La caracterización geométrica de [Jiang et al. 99] es incompleta, además de no optimizarse determinados parámetros del aparcamiento. 


\section{CAPÍTULO 3 RELACIONES CINEMÁTICAS EN VEHÍCULOS CON RUEDAS}

\subsection{INTRODUCCIÓN}

El primer paso para obtener modelos cinemáticos para distintos tipos de vehículos, bien sean con o sin deslizamiento, es conseguir las relaciones cinemáticas entre los distintos tipos de variables que intervienen en el vehículo.

Dichas variables son ([Campion et al. 96] [Muir et al. 89]):

Asumiendo movimiento horizontal, la posición de la estructura del vehículo queda completamente definida con tres variables escalares, dos lineales y otra angular (ej. $x, y, \theta$ ), cuya forma vectorial (ej. p) se denomina postura del vehículo.

Su derivada de primer orden respecto al tiempo $(\dot{\mathbf{p}})$ se denomina vector de velocidad del vehículo, y separadamente $\left(v_{x}, v_{x}, \omega\right)$ velocidades del vehículo.

De igual modo, las articulaciones de dirección y rotación de la rueda dan lugar al vector de velocidad de rueda y a las velocidades de rueda.

Varias publicaciones han abordado el modelado de una rueda como paso previo al modelado de todo el vehículo. 
Quizás, [Muir et al. 89] sea la metodología de modelado más destacada, donde se emplean matrices de transformación homogénea para relacionar sistemas de coordenadas, de forma análoga al caso tradicional de robots manipuladores. El resultado es una relación (matriz Jacobiana de rueda) entre el vector de velocidad del vehículo y las velocidades de rueda. Sin embargo, lo planteado en [Muir et al. 89] tiene los siguientes inconvenientes:

- Se consideran tres ecuaciones por rueda, cuando realmente sólo hay dos restricciones (bajo el supuesto de no deslizamiento) por rueda. Esto se debe a introducir una variable de velocidad de rueda sin sentido práctico, ya que no puede ser sensorizada ni actuada. Esto produce un coste computacional innecesario, además de una inconsistencia cinemática al calcular la evolución del vehículo con información redundante, como se muestra en el capítulo de modelado con deslizamiento. Por todo ello, se debería haber sustituido la tercera ecuación en las otras dos.

- La rotación de rueda se incluye considerando un ficticio par planar entre la rueda y la superficie. Este innecesario y ad-hoc procedimiento contrasta con la sistemática empleada de matrices de transformación. En su lugar se deberían utilizar dos sistemas de coordenadas adicionales, como se propone en [Shin et al. 01].

- Se asume no deslizamiento innecesariamente pronto, por lo que se complica la identificación del mismo en etapas posteriores.

[Rajagopalan 97] continua el método de matrices de transformación de [Muir et al. 89] y lo extiende a un nuevo tipo de rueda, con la columna de dirección inclinada y desplazada.

Otros estudios cinemáticos relevantes son [Alexander et al. 89] y [Campion et al. 96]. En concreto, [Alexander et al. 89] utiliza un planteamiento vectorial para modelar la rueda, sólo valido para ruedas fijas y orientables (centradas). Mientras que [Campion et al. 96] no justifica las relaciones cinemáticas de rueda utilizadas, que son la clave para la clasificación y caracterización posterior.

Otro interesante estudio es [Kim et al. 04], donde la cinemática de rueda se obtiene por procedimientos vectoriales. La ecuación de rueda explicita las velocidades de deslizamiento en las dos direcciones, que son ocasionalmente utilizadas para conseguir una matriz Jacobiana cuadrada (añadiendo ecuaciones escalares triviales). Esta modificación facilita pasar de modelos directos a inversos y viceversa. No obstante, estas ecuaciones triviales deberían ser eliminadas en una etapa posterior para evitar innecesario coste computacional. 
Además, se realiza un estudio previo de movilidad basado en la fórmula de Grübler, clásica en sistemas mecánicos.

Finalmente, [Ollero 01] [Leow et al. 02] [Low et al. 05] son ejemplos de estudios donde se deducen de forma no sistemática fórmulas y relaciones cinemáticas, válidas sólo para casos particulares.

Así pues, el objetivo de este capítulo es obtener de forma sistemática y completa dichas relaciones cinemáticas. Para ello se empiezan estableciendo los supuestos considerados. Posteriormente, se obtienen las relaciones cinemáticas de rueda una formulación cinemática recursiva, descrita en [Fu et al. 88]. Dichas relaciones se particularizan para los distintos tipos de rueda. A continuación se plantea la ecuación cinemática completa del vehículo. Y por último se destacan los resultados más relevantes del capítulo. 


\subsection{SUPUESTOS CONSIDERADOS}

Se van a considerar los siguientes supuestos prácticos para el estudio:

- Supuestos de diseño:

1) Los vehículos no poseen en su estructura partes flexibles, es decir: toda la estructura es rígida.

2) Por cada rueda puede haber una o ninguna articulación de dirección.

3) Todos los ejes de dirección existentes son perpendiculares a la superficie por la que se desplaza el vehículo.

4) Las ruedas pueden estar directamente en contacto con el suelo o a través de unos rodillos.

- Supuesto operacional: La superficie de desplazamiento (suelo) es plana.

El primer supuesto de diseño permite aplicar la formulación de sólido rígido, mientras que los otros tres limitan los tipos de rueda a los que va dirigido esta metodología. En particular, los tipos de rueda que se consideran son:

- Rueda fija: No posee articulación de dirección, por lo que su posición respecto a la estructura es fija.

- Rueda orientable centrada (orientable): Tiene articulación de dirección, es decir es orientables respecto a la estructura del vehículo, pasando su eje de dirección por el centro de rotación de la rueda.

- Rueda orientable descentrada (castor): Posee articulación de dirección, es decir es orientable respecto a la estructura del vehículo, no pasando su eje de dirección por el centro de rotación de la rueda.

- Rueda fija con rodillos (sueca, universal, Mecanum ó Ilon): Es fija respecto a la estructura del vehículo y posee rodillos entre la rueda y el suelo con una determinada orientación fija respecto a la rueda.

En la Figura 3.1 se muestra este tipo de rueda con rodillos con la clásica orientación de $45^{\circ}$. Otra orientación típica es $90^{\circ}$.

En ocasiones se utilizan dos hileras de rodillos para garantizar una mejor continuidad del punto de contacto con el suelo. No obstante, esto último añade una complicación para el control y la odometría, ya que el punto de contacto con el suelo se mueve de la hilera interior a la exterior. No obstante, si la distancia entre los rodillos es pequeña en comparación con las dimensiones de la rueda y del vehículo dicho problema resulta menor. 
Los tipos de rueda fija, orientable y castor se engloban dentro del tipo convencional, y también el tipo castor y sueca dentro del omnidireccional. Como se indica en el capítulo posterior, las ruedas omnidireccionales no restringen el movimiento del vehículo mientras que las otras sí.

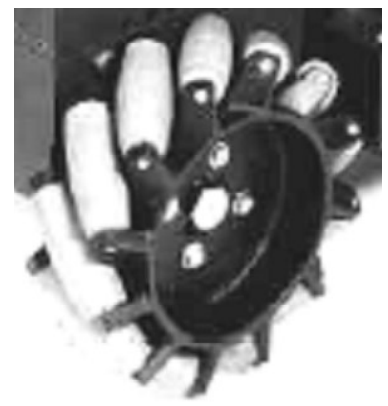

Foto de rueda real

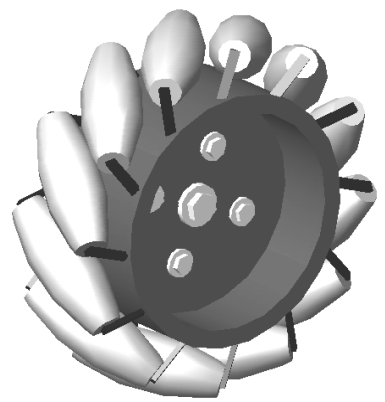

Representación 3D

Figura 3.1- Rueda sueca (también llamada Mecanum ó Ilon) con rodillos a $45^{\circ}$

Existen otro tipo de ruedas menos habituales (especiales) que se analizan en el punto 3.5 del capítulo. En concreto se presentan las ruedas doble y castor doble, que pueden modelarse como dos ruedas castor con mismo eje de rotación y ángulo de dirección, y las ruedas tipo bola y ortogonal, que pertenecen al grupo de omnidireccionales y que funcionalmente son equivalentes a las ruedas suecas.

También se han planteado variantes en los mecanismos de dirección: en [Rajagopalan 97] se considera una columna de dirección con cierta inclinación y descentrada respecto a la rueda (no contempla el supuesto de diseño tercero).

Por otra parte, el supuesto operacional restringe el campo de aplicaciones prácticas. Cabe destacar que [Muir et al. 89] asume además el de nodeslizamiento, no considerado aquí por ser innecesario en una primera instancia. De hecho el método de modelado planteado permite modelar ruedas con deslizamiento.

A pesar de que pueda parecer que los supuestos establecidos limitan en exceso la validez de los resultados, se cumplen en la mayoría de aplicaciones prácticas con vehículos autoguiados y por tanto serán validos. 


\subsection{RELACIONES CINEMÁTICAS}

En este apartado se van a obtener mediante un método sistemático las relaciones cinemáticas a que da lugar una rueda como parte de un vehículo.

El único método sistemático que hay en la bibliografía al respecto es [Muir et al. 89] que emplea, como se ha comentado en la introducción, las matrices de transformación homogénea. No obstante, para emplear este método con la Figura 3.2 habría que definir un total de 11 sistemas de coordenadas (por el método de asignación de Sheth - Uicker [Sheth et al. 71]), dando lugar a idéntico número de matrices de transformación entre sistemas adyacentes. De forma que, la aparatosidad de las matrices y sus productos, hasta llegar a la relación cinemática final de rueda genérica, es enorme. De hecho, es bien conocido en aplicaciones de tiempo real de robótica, el elevado coste computacional de utilizar matrices de transformación. Resultando este método poco adecuado (poco manejable) para indicar todo el proceso.

Así pues, se va a utilizar como alternativa una recursividad cinemática, desarrollada originalmente como parte de la formulación dinámica de NewtonEuler, para obtener las relaciones cinemáticas de una forma mucho más sencilla.

\subsubsection{Sistemas de coordenadas}

La asignación de los sistemas de coordenadas del vehículo es clave para la posterior formulación. Por tanto, merece la pena hacer una asignación de sistemas conveniente para que la formulación sea lo más sencilla posible.

Para los robots manipuladores (cadenas cinemáticas abiertas) se utiliza el método de asignación de coordenadas de Denavit - Hartenberg [Denavit et al. 55]. Pero en el caso de vehículos autoguiados, al existir múltiples cadenas cinemáticas cerradas, se produce una ambiguiedad a la hora de elegir el orden de las articulaciones. Para evitar este problema en [Muir et al. 89] se utiliza, como se ha comentado, el método de asignación Sheth-Uicker [Sheth et al. 71].

En nuestro caso, la elección de sistemas de coordenadas se indica en la Tabla 3.1 para un vehículo genérico de $\mathrm{N}$ ruedas. Dicha elección consiste en asignar un sistema de coordenadas por eslabón móvil $\left(\mathrm{R}, \mathrm{S}_{i}, \mathrm{~L}_{i}, \mathrm{M}_{i}\right)$, uno estacionario coincidente con el de la estructura robot $(\bar{R})$ y otros en el punto de contacto entre los rodillos y el suelo $\left(\mathrm{E}_{i}\right)$, con sus instantáneamente coincidentes asociados $\left(\overline{\mathrm{E}}_{i}\right)$. El sistema instantáneamente coincidente $\overline{\mathrm{R}}$ permite evitar la dependencia con un sistema de coordenadas estacionario global (el concepto de coincidencia instantánea se explica en el punto 3.A1). 
Tabla 3.1- Asignación de sistemas de coordenadas utilizada

\begin{tabular}{|c|c|}
\hline Nombre & Descripción \\
\hline R (Robot) & $\begin{array}{l}\text { Sistema de coordenadas solidario al vehículo, con el eje } Z \\
\text { ortogonal a la superficie de desplazamiento }\end{array}$ \\
\hline $\begin{array}{l}\mathrm{S}_{i}(\text { Sistema dirección }) \\
\quad \text { para } i=1 . . \mathrm{N}\end{array}$ & $\begin{array}{l}\text { Sistema de coordenadas que se mueve con la articulación de } \\
\text { dirección } i \text {, con el eje } Z \text { coincidente con el eje de articulación de } \\
\text { la dirección } i \text { y el eje } Y \text { en la dirección del brazo de dirección }\end{array}$ \\
\hline $\begin{array}{c}\mathrm{L}_{i}(\text { Rueda }) \\
\text { para } i=1 . . \mathrm{N}\end{array}$ & $\begin{array}{l}\text { Sistema que se mueve con la rueda } i \text {, con origen en el centro de } \\
\text { la rueda y el eje } X \text { en la dirección del eje de rotación de la rueda }\end{array}$ \\
\hline $\begin{array}{l}\mathrm{M}_{i}(\text { Rodillo }) \\
\text { para } i=1 . . \mathrm{N}\end{array}$ & $\begin{array}{l}\text { Sistema de coordenadas que se mueve con el rodillo (en } \\
\text { contacto con el suelo) de la rueda } i \text {, con origen en el centro del } \\
\text { rodillo y el eje } X \text { en la dirección del eje de rotación del rodillo }\end{array}$ \\
\hline $\begin{array}{l}\mathrm{E}_{i}(\text { Punto de contacto } \\
\text { del rodillo con suelo }) \\
\quad \text { para } i=1 . . \mathrm{N}\end{array}$ & $\begin{array}{l}\text { Sistema de coordenadas que se mueve con el rodillo (en } \\
\text { contacto con el suelo) de la rueda } i \text {, con origen en dicho punto } \\
\text { de contacto, el eje } X \text { con la misma dirección y sentido que el de } \\
\mathrm{M}_{i} \text { y el eje } Z \text { perpendicular a la superficie de desplazamiento }\end{array}$ \\
\hline $\begin{array}{c}\overline{\mathrm{R}} \text { (Coincidencia } \\
\text { instantánea del Robot) }\end{array}$ & $\begin{array}{l}\text { Sistema de coordenadas coincidente con el sistema de } \\
\text { coordenadas } \mathrm{R} \text { y estacionario respecto la superficie }\end{array}$ \\
\hline $\begin{array}{l}\overline{\mathrm{E}}_{i}(\text { Coincidencia } \\
\left.\text { instantánea } \mathrm{E}_{i}\right)\end{array}$ & $\begin{array}{l}\text { Sistema de coordenadas coincidente con el sistema } \mathrm{E}_{i} \mathrm{y} \\
\text { estacionario respecto a la superficie }\end{array}$ \\
\hline G (Global) & Sistemas de coordenadas global estacionario \\
\hline
\end{tabular}

En la Figura 3.1 se indica la representación gráfica de estos sistemas de coordenadas para un vehículo con rueda genérica $i$. Los eslabones móviles son: la estructura del robot, el brazo de dirección, la rueda y los rodillos. Las articulaciones entre los eslabones móviles son pares de revolución (tres).

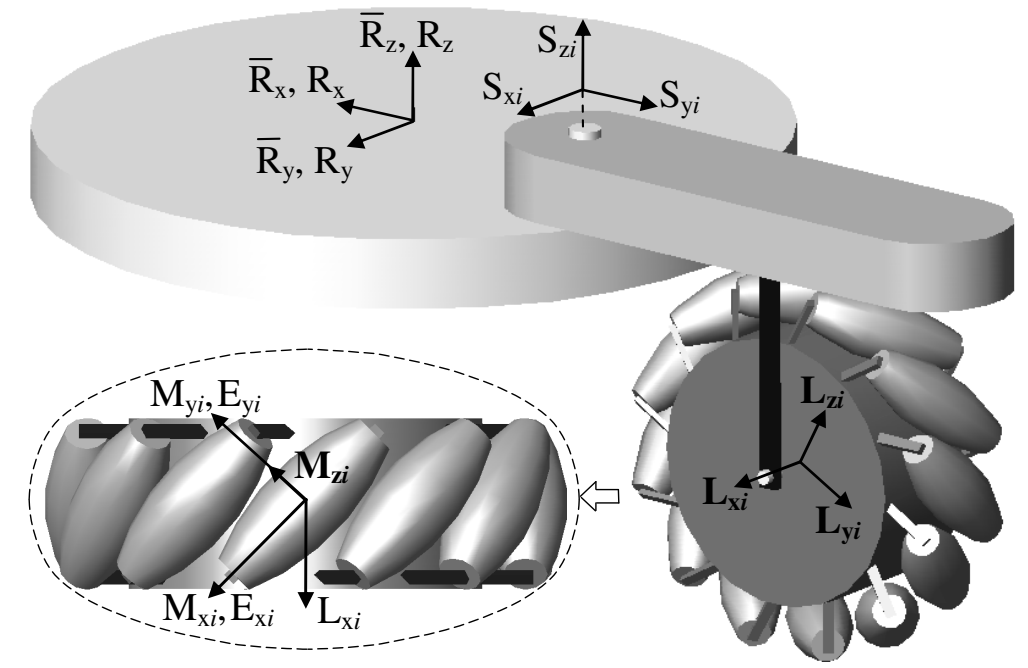

Figura 3.2- Sistemas de coordenadas utilizados en el vehículo 


\subsubsection{Obtención de la velocidad de deslizamiento de rueda}

Las ecuaciones recursivas (cinemáticas de velocidad) descritas en [Fu et al. 88]:

$$
\mathbf{v}_{i}=\frac{d^{*} \mathbf{p}_{i}^{*}}{d t}+\left(\omega_{i-1} \times \mathbf{p}_{i}^{*}\right)+\mathbf{v}_{i-1} \quad \boldsymbol{\omega}_{i}=\boldsymbol{\omega}_{i-1}+\boldsymbol{\omega}_{i}^{*}
$$

donde el significado de cada término es (ver Figura 3.3):

$\frac{d^{*}}{d t}$ Derivada respecto al sistema de coordenadas $i-1$

$\omega_{i}^{*}$ Velocidad angular del sistema de coordenadas $i$ respecto al $i-1$ en coordenadas del sistema 0

$\boldsymbol{\omega}_{i} \quad$ Velocidad angular del sistema de coordenadas $i$ respecto al 0

$\mathbf{p}_{i}^{*} \quad$ Vector del origen del sistema $i-1$ al de $i$ en coordenadas del sistema 0

$\mathbf{v}_{i}$ Velocidad del origen del sistema de coordenadas $i$ respecto al del 0 en coordenadas del sistema 0

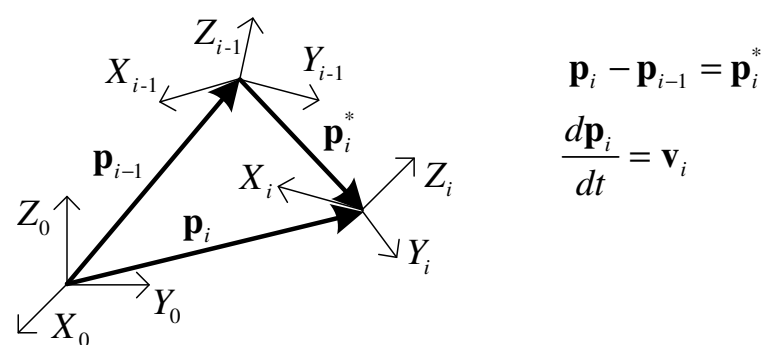

Figura 3.3- Sistemas y variables de la formulación cinemática recursiva

Los sistemas de coordenadas de la Tabla 3.1 numerados son: $\{0-\overline{\mathrm{R}}, 1-\mathrm{R}$, 2- $\left.\mathrm{S}_{i}, 3-\mathrm{L}_{i}, 4-\mathrm{M}_{i}, 5-\mathrm{E}_{i}\right\}$. Al trabajar con sistemas de coordenadas entre sólidos rígidos, el vector $\mathbf{p}_{i}^{*}$ es siempre constante. Por tanto:

$$
\frac{d^{*} \mathbf{p}_{i}^{*}}{d t}=0
$$

Aplicando recursivamente (3.1) y utilizando la notación de la Tabla 3.2:

$\mathbf{v}_{1}={ }^{\bar{R}} \mathbf{v}_{\mathrm{R}}$

$\mathbf{v}_{2}={ }^{\overline{\mathrm{R}}} \mathbf{v}_{\mathrm{R}}+{ }^{\overline{\mathrm{T}}} \boldsymbol{\omega}_{\mathrm{R}} \times{ }^{\mathrm{R}} \mathbf{d}_{S i}$

$\mathbf{v}_{3}={ }^{\bar{R}} \mathbf{v}_{\mathrm{R}}+{ }^{\overline{\mathrm{R}}} \boldsymbol{\omega}_{\mathrm{R}} \times{ }^{\mathrm{R}} \mathbf{d}_{\mathrm{L} i}+{ }^{\mathrm{R}} \boldsymbol{\omega}_{3 i} \times{ }^{\mathrm{R}, S i} \mathbf{d}_{\mathrm{L} i}$

$\mathbf{v}_{4}={ }^{\overline{\mathrm{R}}} \mathbf{v}_{\mathrm{R}}+{ }^{\overline{\mathrm{R}}} \boldsymbol{\omega}_{\mathrm{R}} \times{ }^{\mathrm{R}} \mathbf{d}_{\mathrm{M} i}+{ }^{\mathrm{R}} \boldsymbol{\omega}_{5 i} \times{ }^{\mathrm{R}, S i} \mathbf{d}_{\mathrm{M} i}+{ }^{\mathrm{R}, S i} \boldsymbol{\omega}_{\mathrm{Li}} \times{ }^{\mathrm{R}, \mathrm{Li}} \mathbf{d}_{\mathrm{M} i}$

$$
\omega_{1}={ }^{\overline{\mathrm{R}}} \omega_{\mathrm{R}}
$$

$\boldsymbol{\omega}_{2}={ }^{\overline{\mathrm{R}}} \boldsymbol{\omega}_{\mathrm{R}}+{ }^{\mathrm{R}} \boldsymbol{\omega}_{\mathrm{Si}}$

$\mathbf{v}_{5}={ }^{\bar{R}} \mathbf{v}_{\mathrm{R}}+{ }^{\bar{R}} \boldsymbol{\omega}_{\mathrm{R}} \times{ }^{\mathrm{R}} \mathbf{d}_{\mathrm{E} i}+{ }^{\mathrm{R}} \boldsymbol{\omega}_{S i} \times{ }^{\mathrm{R}, S i} \mathbf{d}_{\mathrm{E} i}+{ }^{\mathrm{R}, S i} \boldsymbol{\omega}_{\mathrm{Li}} \times{ }^{\mathrm{R}, L i} \mathbf{d}_{\mathrm{E} i}+{ }^{\mathrm{R}, L i} \boldsymbol{\omega}_{\mathrm{M} i} \times{ }^{\mathrm{R}, M i} \mathbf{d}_{\mathrm{E} i}$ 
siendo $\mathbf{v}_{5}$ la velocidad de deslizamiento ${ }^{\overline{\mathrm{R}}} \mathbf{v}_{\mathrm{E} i}$ entre el rodillo y la superficie respecto al sistema de coordenadas $\overline{\mathrm{R}}$.

Tabla 3.2- Nomenclatura de variables y parámetros

\begin{tabular}{|c|l|}
\hline Nombre & \multicolumn{1}{|c|}{ Descripción } \\
\hline${ }^{\mathrm{H}, \mathrm{A}} \mathbf{d}_{\mathrm{B}}$ & $\begin{array}{l}\text { Vector que va del origen del sistema A al origen del sistema B en } \\
\text { coordenadas del sistema } \mathrm{H}\end{array}$ \\
\hline${ }^{\mathrm{A}} \boldsymbol{\theta}_{\mathrm{B}}$ & $\begin{array}{l}\text { Desplazamiento de rotación entre el eje } X \text { del sistema B y el eje } X \text { del } \\
\text { sistema A respecto al eje } Z \text { de A (según la regla de Maxwell) }\end{array}$ \\
\hline${ }^{\mathrm{H}, \mathrm{A}} \mathbf{v}_{\mathrm{B}}$ & $\begin{array}{l}\text { Velocidad lineal del origen del sistema B respecto al de A en coordenadas } \\
\text { del sistema H }\end{array}$ \\
\hline${ }^{\mathrm{H}, \mathrm{A}} \boldsymbol{\omega}_{\mathrm{B}}$ & Velocidad angular del sistema B respecto al A en coordenadas del sistema H \\
\hline
\end{tabular}

Si H no se explicita, A toma su lugar.

Teniendo en cuenta los sistemas de coordenadas y articulaciones de la Figura 3.2, dicha velocidad resulta:

$$
\left(\begin{array}{c}
{ }^{\overline{\mathrm{R}}} v_{\mathrm{E} i x} \\
{ }^{\mathrm{R}} v_{\mathrm{E} i y} \\
0
\end{array}\right)=\left(\begin{array}{c}
v_{x} \\
v_{y} \\
0
\end{array}\right)+\left(\begin{array}{c}
0 \\
0 \\
\omega
\end{array}\right) \times{ }^{\mathrm{R}} \mathbf{d}_{\mathrm{E} i}+\left(\begin{array}{c}
0 \\
0 \\
\dot{\beta}_{i}
\end{array}\right) \times{ }^{\mathrm{R}, \mathrm{S} i} \mathbf{d}_{\mathrm{E} i}+\left(\begin{array}{c}
\dot{\varphi}_{i} \cdot \cos \left({ }^{\mathrm{R}} \theta_{\mathrm{L} i}\right) \\
\dot{\varphi}_{i} \cdot \sin \left({ }^{\mathrm{R}} \theta_{\mathrm{L} i}\right) \\
0
\end{array}\right) \times\left(\begin{array}{c}
0 \\
0 \\
-\mathrm{r}_{i}
\end{array}\right)+\left(\begin{array}{c}
\dot{\varphi}_{\mathrm{r} i} \cdot \cos \left({ }^{\mathrm{R}} \theta_{\mathrm{Ei}}\right) \\
\dot{\varphi}_{\mathrm{r} i} \cdot \sin \left({ }^{\mathrm{R}} \theta_{\mathrm{Ei}}\right) \\
0
\end{array}\right) \times\left(\begin{array}{c}
0 \\
0 \\
-\mathrm{r}_{\mathrm{r} i}
\end{array}\right)(3
$$

con el siguiente significado de las nuevas variables y constantes:

- $\dot{\mathbf{p}}=\left(v_{x} v_{y} \omega\right)^{\mathrm{T}} \equiv\left({ }^{\overline{\mathrm{R}}} v_{\mathrm{R} x}{ }^{\overline{\mathrm{R}}} v_{\mathrm{R} y}{ }^{\overline{\mathrm{R}}} \omega_{\mathrm{R}}\right)^{\mathrm{T}}$ vector de velocidad del vehículo respecto al sistema instantáneamente coincidente $\overline{\mathrm{R}}$;

- $\dot{\beta}_{i}$ velocidad angular del brazo de dirección respecto a la plataforma;

- $\left(\dot{\varphi}_{i}, \dot{\varphi}_{\mathrm{ri}}\right)$ velocidad de rotación de la rueda y del rodillo respecto a $\mathrm{L}_{x i} \mathrm{y}_{x i}$;

$-\left(\mathrm{r}_{i}, \mathrm{r}_{\mathrm{r} i}\right)$ radio equivalente de la rueda y del rodillo.

De modo que, de (3.4) cada rueda introduce dos ecuaciones escalares:

$$
{ }^{\overline{\mathrm{R}}} \mathbf{v}_{\mathrm{E} i}=\left(\begin{array}{cccccc}
1 & 0 & -{ }^{\mathrm{R}} d_{\mathrm{E} i y} & -{ }^{\mathrm{R}, S i} d_{\mathrm{E} i y} & -\mathrm{r}_{i} \cdot \sin \left({ }^{\mathrm{R}} \theta_{\mathrm{L} i}\right) & -\mathrm{r}_{\mathrm{ri}} \cdot \sin \left({ }^{\mathrm{R}} \theta_{\mathrm{Ei}}\right) \\
0 & 1 & { }^{\mathrm{R}} d_{\mathrm{E} i x} & { }^{\mathrm{R}, S i} d_{\mathrm{E} i x} & \mathrm{r}_{i} \cdot \cos \left({ }^{\mathrm{R}} \theta_{\mathrm{L} i}\right) & \mathrm{r}_{\mathrm{r} i} \cdot \cos \left({ }^{\mathrm{R}} \theta_{\mathrm{Ei}}\right)
\end{array}\right) \cdot\left(\begin{array}{c}
\dot{\mathbf{p}} \\
\dot{\beta}_{i} \\
\dot{\varphi}_{i} \\
\dot{\varphi}_{\mathrm{ri}}
\end{array}\right)
$$

donde la componente $Z$ de velocidad se ha obviado y el subíndice $x / y$ en los vectores de distancia indica la componente correspondiente. 
La velocidad de deslizamiento ${ }^{\overline{\mathrm{R}}} \mathbf{v}_{\mathrm{E} i}$ de (3.5) se puede expresar también respecto a la dirección de los rodillos ${ }^{\overline{\mathrm{E} i}} \mathbf{v}_{\mathrm{E} i} \equiv \mathbf{v}_{\mathrm{slip} i}$ aplicando una rotación de $-{ }^{\mathrm{R}} \theta_{\mathrm{E} i}$ :

$$
\begin{aligned}
& { }^{\overline{\mathrm{E}} i} \mathbf{v}_{\mathrm{E} i}=\boldsymbol{\operatorname { R o t }}\left(-{ }^{\mathrm{R}} \theta_{\mathrm{E} i}\right) \cdot{ }^{\overline{\mathrm{R}}} \mathbf{v}_{\mathrm{E} i} \\
& \mathbf{v}_{\text {Slip } i}=\left(\begin{array}{lllll}
\operatorname{Rot}\left(-{ }^{\mathrm{R}} \theta_{\mathrm{E} i}\right) & { }^{\mathrm{E} i} d_{\mathrm{R} y} & { }^{\mathrm{E} i} \mathrm{~d}_{\mathrm{S} i y} & \mathrm{r}_{i} \cdot \sin \left({ }^{\mathrm{L} i} \theta_{\mathrm{E} i}\right) & 0 \\
-{ }^{\mathrm{E} i} d_{\mathrm{R} x} & -{ }^{\mathrm{E} i} \mathrm{~d}_{\mathrm{S} i x} & \mathrm{r}_{i} \cdot \cos \left({ }^{(i} \theta_{\mathrm{E} i}\right) & \mathrm{r}_{\mathrm{ri}}
\end{array}\right) \cdot\left(\begin{array}{c}
\dot{\mathbf{p}} \\
\dot{\beta}_{i} \\
\dot{\varphi}_{i} \\
\dot{\varphi}_{\mathrm{r} i}
\end{array}\right)
\end{aligned}
$$

donde $\operatorname{Rot}(x)$ es una matriz de rotación en dos dimensiones:

$$
\boldsymbol{\operatorname { R o t }}(x)=\left(\begin{array}{cc}
\cos (x) & -\sin (x) \\
\sin (x) & \cos (x)
\end{array}\right)
$$

con las propiedades $\operatorname{Rot}^{-1}(x)=\operatorname{Rot}^{\mathrm{T}}(x)=\boldsymbol{\operatorname { R o t }}(-x)$.

Se utilizará la siguiente notación (representada en la Figura 3.4):

$$
\begin{aligned}
& { }^{\mathrm{R}} \mathrm{d}_{\mathrm{S} i x}=1_{i} \cdot \cos \left(\alpha_{i}\right) \quad{ }^{\mathrm{R}} \mathrm{d}_{\mathrm{Siy}}=1_{i} \cdot \sin \left(\alpha_{i}\right) \quad{ }^{\mathrm{S} i} \mathrm{~d}_{\mathrm{E} i y}=\mathrm{d}_{i} \\
& { }^{\mathrm{R}} \theta_{\mathrm{Si}}=\beta_{i} \quad{ }^{\mathrm{S} i} \theta_{\mathrm{Li}}=\delta_{i} \quad{ }^{\mathrm{L} i} \theta_{\mathrm{Ei}}=\gamma_{i} \text {. }
\end{aligned}
$$

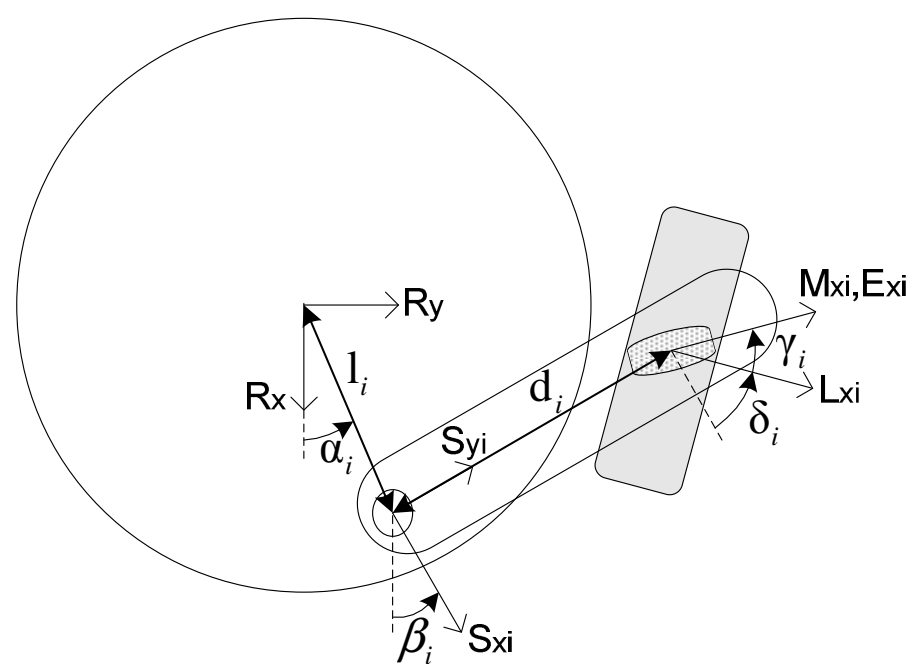

Figura 3.4- Vista de planta con variables y parámetros definidos en (3.8)

Esta notación se ha tomado de [Campion et al. 96], aunque la sustitución exacta para (3.8) sería: $\left\{{ }^{\mathrm{R}} \theta_{\mathrm{Si}} \rightarrow \alpha_{i}+\beta_{i}\right.$ para ruedas fijas, orientables y suecas y $\alpha_{i}+\beta_{i}+\pi$ para ruedas castor $\} ;\left\{{ }^{\mathrm{L} i} \theta_{\mathrm{Ei}} \rightarrow \gamma_{i}-\pi / 2\right\} ;\left\{{ }^{{ }^{i} i} \theta_{\mathrm{Li}} \rightarrow 0\right\}$. 
También, en lo que sigue se utilizará la forma compacta:

$$
\cos (x) \equiv \mathrm{c} x, \sin (x) \equiv \mathrm{s} x
$$

Las componentes de distancia de (3.6) con la notación de (3.8) resultan:

$$
\begin{aligned}
{ }^{\mathrm{E} i} d_{\mathrm{R} y} & =1_{i} \cdot \sin \left(\beta_{i}+\delta_{i}+\gamma_{i}-\alpha_{i}\right)-\mathrm{d}_{i} \cdot \cos \left(\delta_{i}+\gamma_{i}\right) \\
{ }^{\mathrm{E} i} d_{\mathrm{R} x} & =-1_{i} \cdot \cos \left(\beta_{i}+\delta_{i}+\gamma_{i}-\alpha_{i}\right)-\mathrm{d}_{i} \cdot \sin \left(\delta_{i}+\gamma_{i}\right) \\
{ }^{\mathrm{E} i} \mathrm{~d}_{\mathrm{S} i y} & =-\mathrm{d}_{i} \cdot \cos \left(\delta_{i}+\gamma_{i}\right) \quad{ }_{\mathrm{E} i} \mathrm{~d}_{\mathrm{S} i x}=-\mathrm{d}_{i} \cdot \sin \left(\delta_{i}+\gamma_{i}\right)
\end{aligned}
$$

\subsubsection{Particularización de la ecuación de rueda}

A continuación se particularizará (3.6) para cada tipo de rueda:

a) Rueda fija y orientable: Son nulos los parámetros $\left\{\mathrm{r}_{\mathrm{r} i}, \mathrm{~d}_{i}, \delta_{i}, \gamma_{i}\right\}$ (rueda alineada con el eje $\mathrm{S}_{i y}$ ), y $\beta_{i}$ es constante y variable respectivamente::

$$
\mathbf{v}_{\mathrm{slip} i}=\left(\begin{array}{cccc}
\mathrm{c} \beta_{i} & \mathrm{~s} \beta_{i} & 1_{i} \cdot \mathrm{s}\left(\beta_{i}-\alpha_{i}\right) & 0 \\
-\mathrm{s} \beta_{i} & \mathrm{c} \beta_{i} & 1_{i} \cdot \mathrm{c}\left(\beta_{i}-\alpha_{i}\right) & \mathrm{r}_{i}
\end{array}\right) \cdot\left(\begin{array}{c}
\dot{\mathbf{p}} \\
\dot{\varphi}_{i}
\end{array}\right)
$$

En la expresión anterior, para el caso de rueda orientable, la velocidad de direccionamiento afecta a la cinemática de rueda a través del ángulo pero no de forma instantánea.

b) Rueda castor: Son nulos los parámetros $\left\{\mathrm{r}_{\mathrm{r} i}, \gamma_{i}\right\}$, resultando:

$$
\mathbf{v}_{\text {slip } i}=\left(\begin{array}{ccccc}
\mathrm{c}\left(\beta_{i}+\delta_{i}\right) & \mathrm{s}\left(\beta_{i}+\delta_{i}\right) & \mathrm{l}_{i} \cdot \mathrm{s}\left(\beta_{i}+\delta_{i}-\alpha_{i}\right)-\mathrm{d}_{i} \cdot \mathrm{c} \delta_{i} & -\mathrm{d}_{i} \cdot \mathrm{c} \delta_{i} & 0 \\
-\mathrm{s}\left(\beta_{i}+\delta_{i}\right) & \mathrm{c}\left(\beta_{i}+\delta_{i}\right) & 1_{i} \cdot \mathrm{c}\left(\beta_{i}+\delta_{i}-\alpha_{i}\right)+\mathrm{d}_{i} \cdot \mathrm{s} \delta_{i} & \mathrm{~d}_{i} \cdot \mathrm{s} \delta_{i} & \mathrm{r}_{i}
\end{array}\right) \cdot\left(\begin{array}{c}
\dot{\mathbf{p}} \\
\dot{\beta}_{i} \\
\dot{\varphi}_{i}
\end{array}\right)
$$

Notar que la expresión anterior describe una rueda castor completamente genérica debido al ángulo $\delta_{i}$, considerado nulo en otros estudios.

c) Rueda sueca: Son nulos los parámetros $\left\{\mathrm{d}_{i}, \delta_{i}\right\}$ (rueda alineada con el eje $S_{i y}$ ) y el ángulo de dirección $\beta_{i}$ es constante, resultando: 
$\mathbf{v}_{\mathrm{slip} i}=\left(\begin{array}{ccccc}\mathrm{c}\left(\beta_{i}+\gamma_{i}\right) & \mathrm{s}\left(\beta_{i}+\gamma_{i}\right) & 1_{i} \cdot \mathrm{s}\left(\beta_{i}+\gamma_{i}-\alpha_{i}\right) & \mathrm{r}_{i} \cdot \mathrm{s} \gamma_{i} & 0 \\ -\mathrm{s}\left(\beta_{i}+\gamma_{i}\right) & \mathrm{c}\left(\beta_{i}+\gamma_{i}\right) & 1_{i} \cdot \mathrm{c}\left(\beta_{i}+\gamma_{i}-\alpha_{i}\right) & \mathrm{r}_{i} \cdot \mathrm{c} \gamma_{i} & \mathrm{r}_{\mathrm{r} i}\end{array}\right) \cdot\left(\begin{array}{c}\dot{\mathbf{p}} \\ \dot{\varphi}_{i} \\ \dot{\varphi}_{\mathrm{r} i}\end{array}\right)$

En la expresión anterior, bajo el supuesto de que la fuerza de fricción es siempre suficiente para suministrar la aceleración requerida, la (libre) rotación del rodillo garantiza no deslizamiento en la dirección de $\mathrm{E}_{i y}$. Sin embargo, dado que en la práctica dicha variable no es accesible (ni para sensorizar ni para actuar), sólo la primera componente ${ }^{\overline{\mathrm{E}} i} v_{\mathrm{E} i x}$ tiene utilidad práctica:

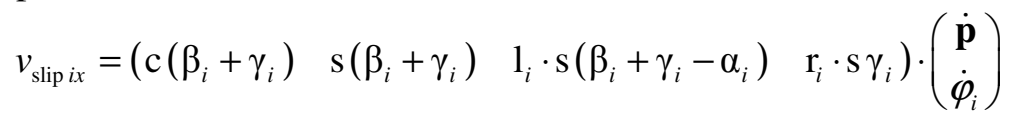

Para referir el vector de velocidad del vehículo respecto a un sistema de coordenadas global fijo G se premultiplica por una matriz de rotación:

$$
{ }^{\mathrm{G}} \dot{\mathbf{p}}=\left(\begin{array}{ccc}
\boldsymbol{\operatorname { R o t }}\left({ }^{\mathrm{G}} \boldsymbol{\theta}_{\mathrm{R}}\right) & 0 \\
0 & 0 & 1
\end{array}\right) \cdot{ }^{\overline{\mathrm{R}}} \dot{\mathbf{p}}=\operatorname{Rot}_{Z}\left({ }^{\mathrm{G}} \boldsymbol{\theta}_{\mathrm{R}}\right) \cdot \dot{\mathbf{p}},
$$

donde $\operatorname{Rot}_{z}(x)$ es una rotación $3 \mathrm{D}$ en el eje $Z$ de valor $x$ y $\operatorname{Rot}(x)$ ya se ha definido en (3.7).

\subsubsection{Matriz Jacobiana de rueda}

En este apartado se obtiene la relación entre el vector de velocidad del vehículo

$\dot{\mathbf{p}}$, respecto al sistema de coordenadas instantáneamente coincidente $\overline{\mathrm{R}}$, y el vector de velocidad de rueda para deslizamiento nulo. A dicha relación se le denomina matriz Jacobiana de rueda.

Despejando la velocidad lineal del vehículo en (3.6), aplicando una rotación en $2 \mathrm{D}$ de ${ }^{\mathrm{R}} \theta_{\mathrm{E} i}$ y anulando la velocidad de deslizamiento, se obtiene:

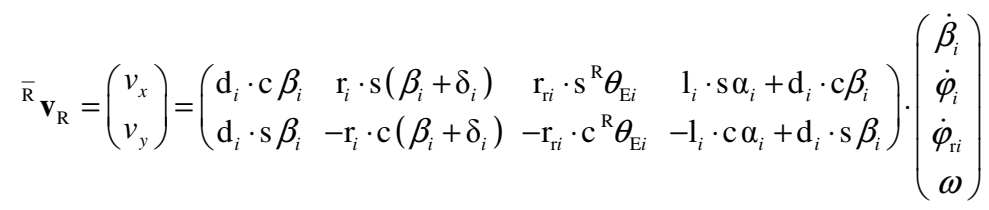


La expresión anterior relaciona la velocidad lineal del vehículo ${ }^{\overline{\mathrm{R}}} \mathbf{v}_{\mathrm{R}}$ con las velocidades de rueda $\left\{\dot{\beta}_{i}, \dot{\varphi}_{i}, \dot{\varphi}_{\mathrm{ri}}\right\}$ y la velocidad de rotación del vehículo $\omega$.

Alternativamente, se puede mantener en (3.16) un tercer elemento identidad para tener en el miembro de la izquierda el vector de velocidad del vehículo:

$$
\dot{\mathbf{p}}=\left(\begin{array}{cccc}
\mathrm{d}_{i} \cdot \mathrm{c} \beta_{i} & \mathrm{r}_{i} \cdot \mathrm{s}\left(\beta_{i}+\delta_{i}\right) & \mathrm{r}_{\mathrm{r} i} \cdot \mathrm{s}^{\mathrm{R}} \theta_{\mathrm{E} i} & 1_{i} \cdot \mathrm{s} \alpha_{i}+\mathrm{d}_{i} \cdot \mathrm{c} \beta_{i} \\
\mathrm{~d}_{i} \cdot \mathrm{s} \beta_{i} & -\mathrm{r}_{i} \cdot \mathrm{c}\left(\beta_{i}+\delta_{i}\right) & -\mathrm{r}_{\mathrm{r} i} \cdot \mathrm{c}^{\mathrm{R}} \theta_{\mathrm{E} i} & -1_{i} \cdot \mathrm{c} \alpha_{i}+\mathrm{d}_{i} \cdot \mathrm{s} \beta_{i} \\
0 & 0 & 0 & 1
\end{array}\right) \cdot\left(\begin{array}{c}
\dot{\beta}_{i} \\
\dot{\varphi}_{i} \\
\dot{\varphi}_{\mathrm{r} i} \\
\omega
\end{array}\right)
$$

Esto último permite mantener una homogeneidad que da lugar a un posterior análisis a través de matrices o pseudo matrices Jacobianas de ruedas:

$$
\dot{\mathbf{p}}=\mathbf{J}_{i} \cdot \dot{\mathbf{q}}_{\mathrm{w} i}
$$

donde $\dot{\mathbf{q}}_{\mathrm{wi}}$ es el vector de velocidad de rueda, que en este caso incluye además la velocidad angular del vehículo, y $\mathbf{J}$ la matriz Jacobiana de rueda.

En concreto, según el tipo de rueda considerada en (3.17) se tienen las siguientes pseudo matrices Jacobianas y vectores de velocidades de rueda:

$$
\begin{gathered}
\mathbf{J}_{\mathrm{f} / \mathrm{o} i}=\left(\begin{array}{cc}
\mathrm{r}_{i} \cdot \mathrm{s} \beta_{i} & 1_{i} \cdot \mathrm{s} \alpha_{i} \\
-\mathrm{r}_{i} \cdot \mathrm{c} \beta_{i} & -1_{i} \cdot \mathrm{c} \alpha_{i} \\
0 & 1
\end{array}\right) \quad \dot{\mathbf{q}}_{\mathrm{wf} / / i}=\left(\begin{array}{c}
\dot{\varphi}_{i} \\
\omega
\end{array}\right) \\
\mathbf{J}_{\mathrm{s} i}=\left(\begin{array}{ccc}
\mathrm{r}_{i} \cdot \mathrm{s} \beta_{i} & \mathrm{r}_{\mathrm{r} i} \cdot \mathrm{s}\left(\beta_{i}+\gamma_{i}\right) & 1_{i} \cdot \mathrm{s} \alpha_{i} \\
-\mathrm{r}_{i} \cdot \mathrm{c} \beta_{i} & -\mathrm{r}_{\mathrm{r} i} \cdot \mathrm{c}\left(\beta_{i}+\gamma_{i}\right) & -1_{i} \cdot \mathrm{c} \alpha_{i} \\
0 & 0 & 1
\end{array}\right) \quad \dot{\mathbf{q}}_{\mathrm{ws} i}=\left(\begin{array}{c}
\dot{\varphi}_{i} \\
\dot{\varphi}_{\mathrm{r} i} \\
\omega
\end{array}\right) \\
\mathbf{J}_{\mathrm{c} i}=\left(\begin{array}{ccc}
\mathrm{d}_{i} \cdot \mathrm{c} \beta_{i} & \mathrm{r}_{i} \cdot \mathrm{s}\left(\beta_{i}+\delta_{i}\right) & 1_{i} \cdot \mathrm{s} \alpha_{i}+\mathrm{d}_{i} \cdot \mathrm{c} \beta_{i} \\
\mathrm{~d}_{i} \cdot \mathrm{s} \beta_{i} & -\mathrm{r}_{i} \cdot \mathrm{c}\left(\beta_{i}+\delta_{i}\right) & -1_{i} \cdot \mathrm{c} \alpha_{i}+\mathrm{d}_{i} \cdot \mathrm{s} \beta_{i} \\
0 & 0 & 1
\end{array}\right) \quad \dot{\mathbf{q}}_{\mathrm{wc} i}=\left(\begin{array}{c}
\dot{\beta}_{i} \\
\dot{\varphi}_{i} \\
\omega
\end{array}\right)
\end{gathered}
$$

donde el subíndice $\{\mathrm{f}, \mathrm{o}, \mathrm{c}, \mathrm{s}\}$ es para rueda $\{$ fija, orientable, castor, sueca $\}$.

Todas las matrices Jacobianas anteriores siempre (pseudo) invertibles. 


\subsection{ECUACIÓN COMPUESTA}

Una vez calculadas las relaciones cinemáticas entre el vector de velocidad del vehículo y el de cada rueda en sus distintas formas $\{(3.5),(3.6),(3.19), \ldots\}$, cabe combinar todas ellas en una sola ecuación compuesta. Esto tiene gran relevancia para el tipo de análisis que posteriormente se podrá aplicar a dicha ecuación.

A continuación se detallan las dos formas de ecuación compuesta que son consideradas en este estudio de mayor utilidad.

La primera ecuación compuesta que se plantea es la obtenida juntando las matrices Jacobianas de rueda de (3.19):

$$
\left(\begin{array}{c}
\mathbf{I} \\
\mathbf{I} \\
\vdots \\
\mathbf{I}
\end{array}\right) \cdot \dot{\mathbf{p}}=\left(\begin{array}{cccc}
\mathbf{J}_{1} & 0 & \cdots & 0 \\
0 & \mathbf{J}_{2} & \ddots & \vdots \\
\vdots & \ddots & \ddots & 0 \\
0 & \cdots & 0 & \mathbf{J}_{\mathrm{N}}
\end{array}\right) \cdot\left(\begin{array}{c}
\dot{\mathbf{q}}_{\mathrm{w} 1} \\
\dot{\mathbf{q}}_{\mathrm{w} 2} \\
\vdots \\
\dot{\mathbf{q}}_{\mathrm{wN}}
\end{array}\right) \Rightarrow \overline{\mathbf{I}} \cdot \dot{\mathbf{p}}=\mathbf{J} \cdot \dot{\mathbf{q}}_{\mathrm{w}}
$$

siendo I la matriz identidad de dimensión 3 y $\dot{\mathbf{q}}_{\mathrm{w}}$ el vector de todas las velocidades de ruedas.

La ecuación compuesta anterior constituye un sistema de $3 \cdot \mathrm{N}$ ecuaciones.

El segundo tipo de ecuación compuesta consiste en agrupar las velocidades de deslizamiento de (3.6):

$$
\begin{aligned}
& \mathbf{v}_{\mathrm{slip} i}=\left(\begin{array}{ccccc}
\operatorname{Rot}\left(-{ }^{\mathrm{R}} \theta_{\mathrm{E} i}\right) & { }^{\mathrm{E} i} d_{\mathrm{R} y} & { }^{\mathrm{E} i} \mathrm{~d}_{\mathrm{S} i y} & \mathrm{r}_{i} \cdot \sin \left({ }^{\mathrm{L} i} \theta_{\mathrm{E} i}\right) & 0 \\
& -{ }^{\mathrm{E} i} d_{\mathrm{R} x} & -{ }^{\mathrm{E} i} \mathrm{~d}_{\mathrm{S} i x} & \mathrm{r}_{i} \cdot \cos \left({ }^{\mathrm{L} i} \theta_{\mathrm{E} i}\right) & \mathrm{r}_{\mathrm{ri} i}
\end{array}\right) \cdot\left(\begin{array}{c}
\dot{\mathbf{p}} \\
\dot{\beta}_{i} \\
\dot{\varphi}_{i} \\
\dot{\varphi}_{\mathrm{r} i}
\end{array}\right) \\
& \mathbf{v}_{\text {slip } i}=\left(\begin{array}{ll}
\mathbf{A}_{\mathrm{p} i} & \mathbf{A}_{\mathrm{w} i}
\end{array}\right) \cdot\left(\begin{array}{c}
\dot{\mathbf{p}} \\
\dot{\mathbf{q}}_{\mathrm{w} i}
\end{array}\right)=\mathbf{A}_{i} \cdot\left(\begin{array}{c}
\dot{\mathbf{p}} \\
\dot{\mathbf{q}}_{\mathrm{w} i}
\end{array}\right) \\
& \mathbf{v}_{\text {slip }}=\left(\begin{array}{c}
\mathbf{v}_{\text {slip } 1} \\
\vdots \\
\mathbf{v}_{\text {slip } 2} \\
\vdots \\
\mathbf{v}_{\text {slip N }}
\end{array}\right)=\left(\begin{array}{cccc}
\mathbf{A}_{\mathrm{p} 1} & \mathbf{A}_{\mathrm{w} 1} & \cdots & 0 \\
\vdots & \vdots & \ddots & \vdots \\
\mathbf{A}_{\mathrm{pN}} & 0 & \cdots & \mathbf{A}_{\mathrm{wN}}
\end{array}\right) \cdot\left(\begin{array}{c}
\dot{\mathbf{p}} \\
\dot{\mathbf{q}}_{\mathrm{w} 1} \\
\vdots \\
\dot{\mathbf{q}}_{\mathrm{wN}}
\end{array}\right) \\
& \mathbf{v}_{\text {slip }}=\left(\begin{array}{ll}
\mathbf{A}_{\mathrm{p}} & \mathbf{A}_{\mathrm{w}}
\end{array}\right) \cdot\left(\begin{array}{c}
\dot{\mathbf{p}} \\
\dot{\mathbf{q}}_{\mathrm{w}}
\end{array}\right)=\mathbf{A} \cdot\left(\begin{array}{c}
\dot{\mathbf{p}} \\
\dot{\mathbf{q}}_{\mathrm{w}}
\end{array}\right)=\mathbf{A} \cdot \dot{\mathbf{q}}
\end{aligned}
$$


donde $\mathbf{v}_{\text {slip }}$ es el vector de todas las velocidades de deslizamiento, A es la matriz compuesta del vehículo y $\dot{\mathbf{q}}$ el vector de todas las velocidades.

En caso de deslizamiento nulo se obtiene:

$$
\mathbf{A} \cdot \dot{\mathbf{q}}=\mathbf{0}
$$

La expresión anterior permite un análisis a través del concepto de espacio nulo, tal y como se aborda en el siguiente capítulo.

También puede plantearse con (3.23) qué variables pueden funcionar como incógnitas y cuáles pasar al otro miembro para que exista solución única, lo que implica un análisis de rangos de matrices y submatrices. Esto se aborda también en el capítulo siguiente. 


\subsection{RUEDAS ESPECIALES}

\subsubsection{Rueda doble y rueda castor doble}

La rueda doble [Leow et al. 00] está formada por dos ruedas descentradas simétricas, con el plano definido por ambas perpendicular al brazo de dirección, ver Figura 3.5 (a). Mientras que la rueda castor doble [Wada et al. 00] tiene la disposición equivalente de un vehículo remolque, ver Figura 3.5 (b). En cualquier caso, ambos tipos de rueda se pueden incluir en el proceso de modelado y análisis de los capítulos siguientes considerándolas como dos ruedas castor con eje de rotación $\mathrm{L}_{x i} \mathrm{y}$ ángulo de dirección $\beta_{i}$ común.

A pesar de la equivalencia cinemática entre ruedas dobles y simples, mostrada en los siguientes subapartados, las ruedas dobles tienen las siguientes ventajas:

- Las ruedas orientables y castor requieren, al reorientar, superar (cuando la rotación de rueda está bloqueada) un par de fricción seca. Mientras que las ruedas dobles únicamente deben superar la fricción por rodadura.

- La capacidad de carga de las ruedas dobles es el doble que la de una orientable/castor, bajo el supuesto de ruedas y actuadores idénticos.

- Los dos actuadores de las ruedas dobles pueden ser idénticos (menos coste), mientras que las ruedas orientables y castor requieren normalmente dos actuadores de características distintas, ya que con uno se hace un control de posición (orientación de la dirección) y con el otro de velocidad (rotación de la rueda).

El principal inconveniente que presentan las ruedas dobles es que tienen más elementos y son más grandes, para un mismo tamaño de rueda.

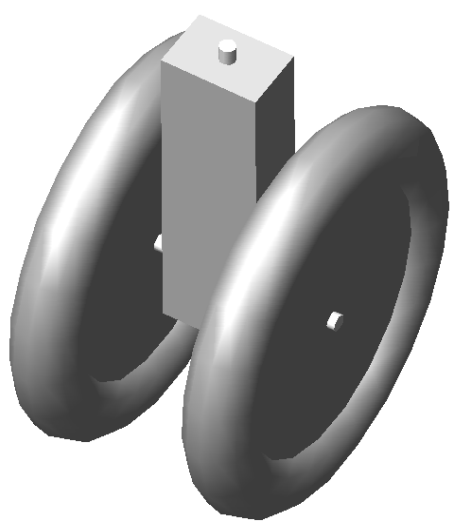

(a) Doble

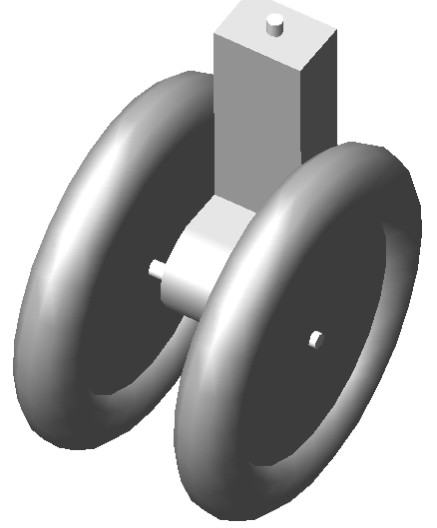

(b) Castor doble

Figura 3.5- Rueda doble y rueda castor doble 


\subsubsection{A) Equivalencia entre la rueda doble y la orientable (centrada)}

En la figura siguiente se aprecia la disposición de ambas opciones.

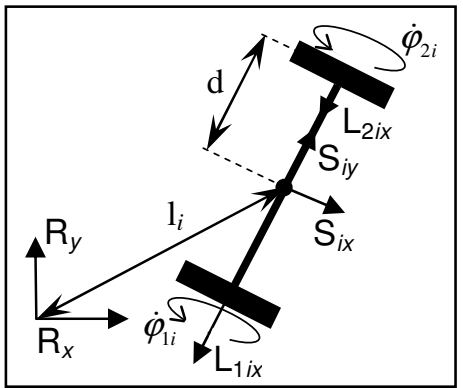

a) Rueda doble

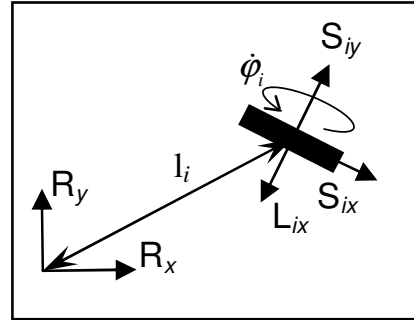

b) Rueda orientable

Figura 3.6- Equivalencia entre la rueda doble y la orientable (centrada)

Utilizando la Figura 3.6, existen las siguientes relaciones:

$$
\begin{array}{lll}
\alpha_{i}=\alpha_{i 1}=\alpha_{i 2} & \beta_{i}=\beta_{i 1}=\beta_{i 2} & \delta_{i}=\delta_{1 i}=\delta_{2 i}=-90^{\circ} \\
\mathrm{d}_{i}=0 & \mathrm{~d}_{1 i}=-\mathrm{d}_{2 i}=-\mathrm{d} &
\end{array}
$$

A continuación se particulariza (3.12), con deslizamiento nulo, para cada una de las ruedas:

$$
\begin{gathered}
\mathbf{0}=\left(\begin{array}{cccc}
\mathrm{s} \beta_{i} & -\mathrm{c} \beta_{i} & -1_{i} \cdot \mathrm{c}\left(\beta_{i}-\alpha_{i}\right) & 0 \\
\mathrm{c} \beta_{i} & \mathrm{~s} \beta_{i} & 1_{i} \cdot \mathrm{s}\left(\beta_{i}-\alpha_{i}\right) & \mathrm{r}_{i}
\end{array}\right) \cdot\left(\begin{array}{c}
\dot{\mathbf{p}} \\
\dot{\varphi}_{i}
\end{array}\right) \\
\mathbf{0}=\left(\begin{array}{ccccc}
\mathrm{s} \beta_{i} & -\mathrm{c} \beta_{i} & -1_{i} \cdot \mathrm{c}\left(\beta_{i}-\alpha_{i}\right) & 0 & 0 \\
\mathrm{c} \beta_{i} & \mathrm{~s} \beta_{i} & 1_{i} \cdot \mathrm{s}\left(\beta_{i}-\alpha_{i}\right)+\mathrm{d} & \mathrm{d} & \mathrm{r}_{1 i}
\end{array}\right) \cdot\left(\begin{array}{c}
\dot{\mathbf{p}} \\
\dot{\beta}_{i} \\
\dot{\varphi}_{1 i}
\end{array}\right) \\
\mathbf{0}=\left(\begin{array}{ccccc}
\mathrm{s} \beta_{i} & -\mathrm{c} \beta_{i} & -1_{i} \cdot \mathrm{c}\left(\beta_{i}-\alpha_{i}\right) & 0 & 0 \\
\mathrm{c} \beta_{i} & \mathrm{~s} \beta_{i} & 1_{i} \cdot \mathrm{s}\left(\beta_{i}-\alpha_{i}\right)-\mathrm{d} & -\mathrm{d} & \mathrm{r}_{2 i}
\end{array}\right) \cdot\left(\begin{array}{c}
\dot{\mathbf{p}} \\
\dot{\beta}_{i} \\
\dot{\varphi}_{2 i}
\end{array}\right)
\end{gathered}
$$

Notar que la primera ecuación de las tres expresiones anteriores coincide.

La semisuma de la segunda ecuación de (3.26) y (3.27) es igual a la segunda ecuación de (3.25) a través de la relación entre variables:

$$
\dot{\varphi}_{i} \cdot \mathrm{r}_{i}=\left(\dot{\varphi}_{1 i} \cdot \mathrm{r}_{1 i}+\dot{\varphi}_{2 i} \cdot \mathrm{r}_{2 i}\right) / 2
$$


La semiresta de las segundas ecuaciones de (3.26) y (3.27) da lugar a:

$$
\dot{\beta}_{i}=\left(\dot{\varphi}_{2 i} \cdot \mathrm{r}_{2 i}-\dot{\varphi}_{1 i} \cdot \mathrm{r}_{1 i}\right) /(2 \cdot \mathrm{d})-\omega
$$

La expresión anterior relaciona velocidades angulares, integrándola se obtiene la relación entre ángulos:

$$
\beta_{i}=\left(\varphi_{2 i} \cdot \mathrm{r}_{2 i}-\varphi_{1 i} \cdot \mathrm{r}_{1 i}\right) /(2 \cdot \mathrm{d})-{ }^{\bar{R}} \theta_{R}+\text { Constante }
$$

Apuntar que, la orientación del brazo de dirección en términos absolutos sólo depende de la rotación de las ruedas. Sin embargo, en términos relativos (ya que se mide respecto a la plataforma del vehículo) depende también de la evolución de la orientación del vehículo, como evidencia la anterior expresión.

Recapitulando, la rueda doble da lugar a 3 ecuaciones independientes: dos de ellas equivalentes (una de ellas exacta) a las aportadas por la rueda orientable centrada; mientras que la tercera establece la relación entre la orientación del brazo de dirección, la orientación del vehículo y la rotación de las dos ruedas.

En otras palabras, la rueda doble funciona como una orientable que se orienta por la diferencia en la rotación de las dos ruedas que la componen.

\subsubsection{B) Equivalencia entre rueda castor doble ó remolque y la castor}

En la figura siguiente se aprecia la disposición de una rueda castor doble.

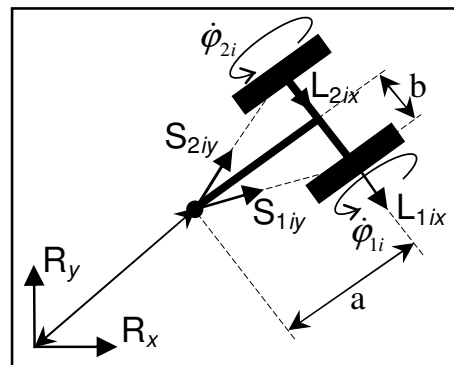

a) Rueda castor doble

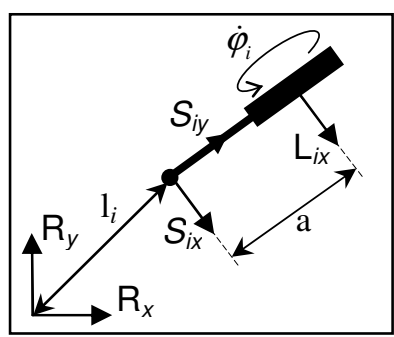

b) Rueda castor

Figura 3.7- Equivalencia entre la rueda castor doble y la castor

En primer lugar, notar en la Figura 3.7 a) que la disposición de la rueda castor doble es exactamente igual que la que tiene un vehículo remolque, por lo que son el mismo tipo de elemento cinemático a considerar. 
Utilizando la Figura 3.7, existen las siguientes relaciones:

$$
\begin{array}{llll}
\alpha_{i}=\alpha_{i 1}=\alpha_{i 2} & \beta_{i}=\beta_{i 1}+\delta_{1 i}=\beta_{i 2}+\delta_{2 i} & \dot{\beta}_{i}=\dot{\beta}_{i 1}=\dot{\beta}_{i 2} & \delta_{i}=0 \\
\mathrm{~d}_{i}=\mathrm{a} & \mathrm{d}_{1 i}=\mathrm{d}_{2 i}=\sqrt{\mathrm{a}^{2}+\mathrm{b}^{2}} & \delta_{i 1}=-\delta_{i 2}=\arctan (\mathrm{b} / \mathrm{a}) &
\end{array}
$$

A continuación se particulariza (3.12), con deslizamiento nulo, para cada una de las ruedas:

$$
\begin{aligned}
\mathbf{0} & =\left(\begin{array}{ccccc}
\mathrm{c} \beta_{i} & \mathrm{~s} \beta_{i} & 1_{i} \cdot \mathrm{s}\left(\beta_{i}-\alpha_{i}\right)-\mathrm{a} & -\mathrm{a} & 0 \\
-\mathrm{s} \beta_{i} & \mathrm{c} \beta_{i} & 1_{i} \cdot \mathrm{c}\left(\beta_{i}-\alpha_{i}\right) & 0 & \mathrm{r}_{i}
\end{array}\right) \cdot\left(\begin{array}{c}
\dot{\mathbf{p}} \\
\dot{\beta}_{i} \\
\dot{\varphi}_{i}
\end{array}\right) \\
\mathbf{0} & =\left(\begin{array}{ccccc}
\mathrm{c} \beta_{i} & \mathrm{~s} \beta_{i} & 1_{i} \cdot \mathrm{s}\left(\beta_{i}-\alpha_{i}\right)-\mathrm{a} & -\mathrm{a} & 0 \\
-\mathrm{s} \beta_{i} & \mathrm{c} \beta_{i} & 1_{i} \cdot \mathrm{c}\left(\beta_{i}-\alpha_{i}\right)+\mathrm{b} & \mathrm{b} & \mathrm{r}_{1 i}
\end{array}\right) \cdot\left(\begin{array}{c}
\dot{\mathbf{p}} \\
\dot{\beta}_{i} \\
\dot{\varphi}_{1 i}
\end{array}\right) \\
\mathbf{0} & =\left(\begin{array}{ccccc}
\mathrm{c} \beta_{i} & \mathrm{~s} \beta_{i} & 1_{i} \cdot \mathrm{s}\left(\beta_{i}-\alpha_{i}\right)-\mathrm{a} & -\mathrm{a} & 0 \\
-\mathrm{s} \beta_{i} & \mathrm{c} \beta_{i} & 1_{i} \cdot \mathrm{c}\left(\beta_{i}-\alpha_{i}\right)-\mathrm{b} & -\mathrm{b} & \mathrm{r}_{2 i}
\end{array}\right) \cdot\left(\begin{array}{c}
\dot{\mathbf{p}} \\
\dot{\beta}_{i} \\
\dot{\varphi}_{2 i}
\end{array}\right)
\end{aligned}
$$

Notar que la primera ecuación de las tres expresiones anteriores coincide.

La semisuma de la segunda ecuación de (3.33) y (3.34) es igual a la segunda ecuación de (3.32) a través de la relación entre variables:

$$
\dot{\varphi}_{i} \cdot \mathrm{r}_{i}=\left(\dot{\varphi}_{1 i} \cdot \mathrm{r}_{1 i}+\dot{\varphi}_{2 i} \cdot \mathrm{r}_{2 i}\right) / 2
$$

La semiresta de las segundas ecuaciones de (3.33) y (3.34) da lugar a:

$$
\dot{\beta}_{i}=\left(\dot{\varphi}_{2 i} \cdot \mathrm{r}_{2 i}-\dot{\varphi}_{1 i} \cdot \mathrm{r}_{1 i}\right) /(2 \cdot \mathrm{b})-\omega
$$

La expresión anterior relaciona velocidades angulares, integrándola se obtiene la relación entre ángulos:

$$
\beta_{i}=\left(\varphi_{2 i} \cdot \mathrm{r}_{2 i}-\varphi_{1 i} \cdot \mathrm{r}_{1 i}\right) /(2 \cdot \mathrm{b})-{ }^{\bar{R}} \theta_{R}+\text { Constante }
$$

Al igual que ocurría en el subapartado anterior, la orientación del brazo de dirección en términos absolutos sólo depende de la rotación de las ruedas. Sin embargo, en términos relativos (ya que se mide respecto a la plataforma del vehículo) depende también de la evolución de la orientación del vehículo. 
Recapitulando, la rueda castor doble da lugar a 3 ecuaciones independientes: dos de ellas equivalentes (una de ellas exacta) a las aportadas por la rueda castor; mientras que la tercera establece la relación entre la orientación del brazo de dirección, la orientación del vehículo y la rotación de las dos ruedas.

En otras palabras, la rueda castor doble, o un remolque (que a efectos funcionales es lo mismo), es como una castor que se orienta por la diferencia en la rotación de las dos ruedas que la componen.

\subsubsection{Rueda tipo bola}

Este tipo de rueda consiste en una rueda esférica con algún tipo de mecanismo de accionamiento. Para el mecanismo de accionamiento se suelen utilizar un conjunto de rodillos: en [West et al. 92] y [Luo et al. 98] se emplean dos rodillos horizontales y paralelos; en [Ostrovskaya et al. 00] se usan tres rodillos formando un rectángulo horizontal (Figura 3.8); mientras que en [West et al. 95] se utilizan rodillos con posiciones más complejas. Otro tipo de mecanismo de dirección se propone en [Ferrière et al. 98], el cual consiste en una rueda sueca que gira sobre la rueda esférica.

En general, el mecanismo de dirección de la rueda tipo bola puede restringir el movimiento de la rueda esférica en una o dos direcciones. Por ejemplo: el mecanismo de [West et al. 92] y [Luo et al. 98] restringe el movimiento en la dirección perpendicular a los rodillos paralelos cuando un de ellos (o los dos) se accionan; el mecanismo de [Ferrière et al. 98] restringe el movimiento en el eje de rotación del rodillo de la rueda sueca cuando ésta se acciona; y el mecanismo de [Ostrovskaya et al. 00] restringe el movimiento en las direcciones perpendiculares de los rodillos accionados (pueden ser uno ó dos).

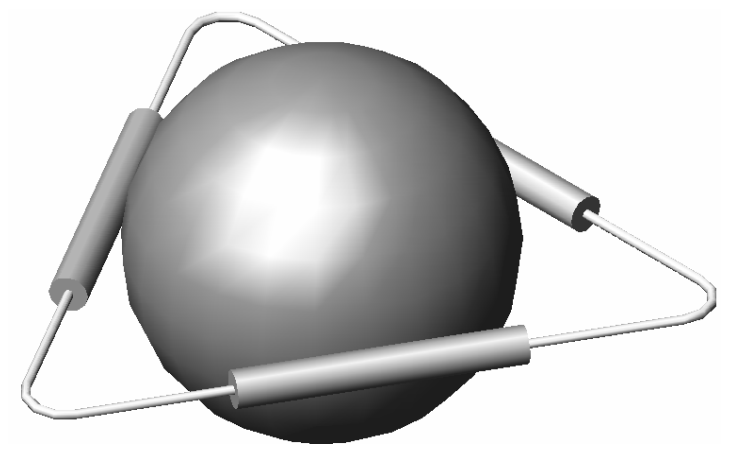

Figura 3.8- Rueda tipo bola con tres rodillos formando un rectángulo horizontal 
Desde el punto de vista de la funcionalidad, la rueda tipo bola es equivalente a la sueca. En esta última las restricciones al movimiento vienen dadas por la posible actuación sobre la rotación de la rueda y/o del rodillo en contacto con el suelo. Normalmente, tanto para la rueda tipo bola como para la sueca se suele restringir el movimiento sólo en una dirección, ó en ninguna cuando va libre.

\subsubsection{Rueda ortogonal}

Este tipo de rueda fue presentada en [Killough et al. 92] [Pin et al. 94] y consiste en dos esferas de igual diámetro que han sido rebanadas en dos extremos opuestos para asemejarse a ruedas redondeadas anchas. Las dos quasi-esferas se sitúan con sus ejes en direcciones perpendiculares u ortogonales. Las quasiesferas pueden rotar libremente entorno a sus ejes. Un soporte sostiene los extremos de los ejes de rotación de las quasi-esferas, el cuál les permite rotar en la parte esférica de su superficie, mientras que giran libremente en la dirección ortogonal. Esto produce el mismo efecto que las ruedas suecas. De modo que, con las dos ruedas quasi-esféricas correctamente alineadas y de movimiento sincronizado, el contacto con el suelo de una rueda esférica u otra está asegurado en todo momento. De forma que la rueda ortogonal se acciona en una dirección mientras que la dirección perpendicular permanece sin restricción.

Existen dos diseños: ensamblaje longitudinal (Figura 3.9) y ensamblaje lateral (Figura 3.10). Este ultimo caso, aunque es de construcción un poco más compleja porque requiere una transmisión por engranajes o correa, simplifica la cinemática y dinámica del vehículo ya que la línea recta que caracteriza el movimiento restringido se mantiene constante respecto a la estructura del vehículo. En concreto esta línea es la recta que pasa por los puntos de contacto (cuando los hay) entre el suelo y las dos quasi-esferas.

De hecho, el ensamblaje longitudinal no es recomendable a nivel práctico por dos motivos. En primer lugar porque, cuando se considera que sólo una de las dos quasi-esferas está en contacto con el suelo, la línea recta que caracteriza el movimiento restringido (aunque tenga la misma dirección) va cambiando del punto de contacto de una quasi-esfera a la otra. Esto hace que según qué quasiesfera esté en contacto con el suelo se tiene una cinemática/dinámica u otra.

Más aún, no es recomendable el ensamblaje longitudinal porque, si se considera que las dos quasi-esferas pueden estar en contacto con el suelo simultáneamente, se tienen dos líneas paralelas independientes de restricción al movimiento, supuestamente con la misma velocidad de movimiento, y por tanto 
incompatibles con una velocidad angular del vehículo no nula. Por lo que, en general una de las dos quasi-esferas deslizaría inevitablemente. Los dos problemas indicados (cambio de cinemática/dinámica y deslizamiento) serían menos importantes en la medida en que la distancia entre las dos quasi-esferas fuera despreciable respecto a las dimensiones del vehículo.

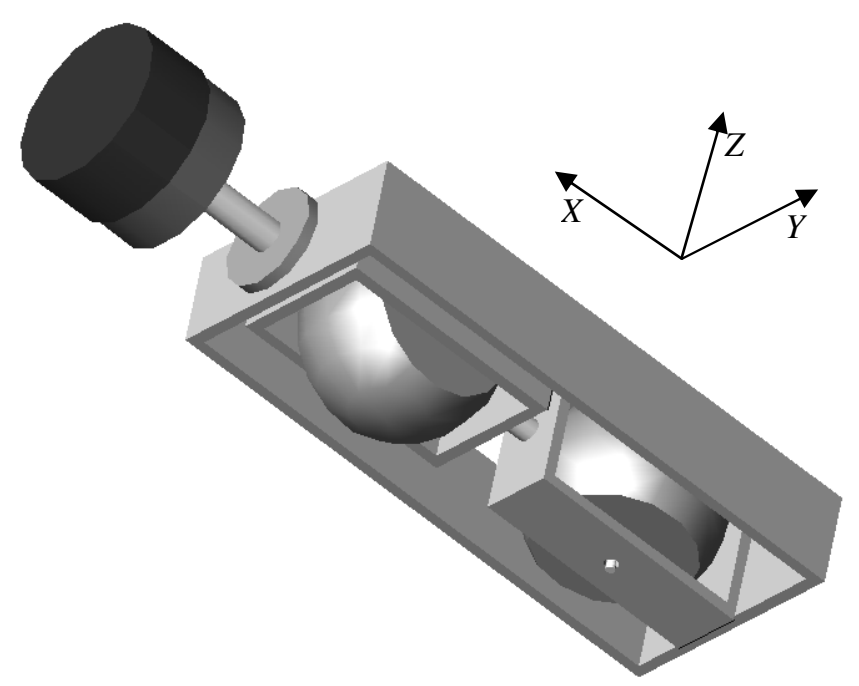

Figura 3.9- Rueda ortogonal con ensamblaje longitudinal

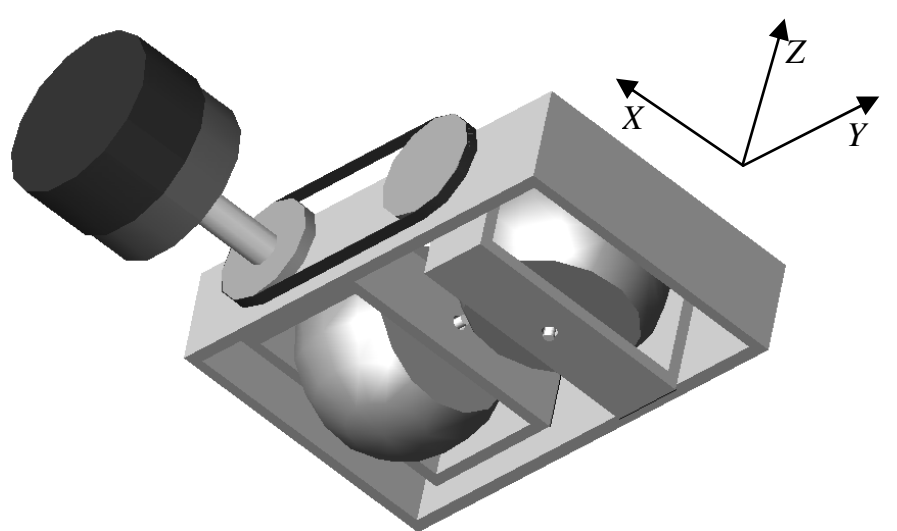

Figura 3.10- Rueda ortogonal con ensamblaje lateral

Al igual que ocurría con la rueda tipo bola, la rueda ortogonal es equivalente funcionalmente a la sueca, aunque presenta algunas ventajas de índole práctica: el contacto con el suelo es continuo, tiene menos elementos y el hueco de la rueda es más pequeño. 


\subsection{RESULTADOS MÁS RELEVANTES Y CONCLUSIONES DEL CAPÍTULO}

A continuación se indican las mejoras fundamentales que aporta el presente capítulo (de obtención de relaciones cinemáticas) respecto a los planteamientos de otros autores:

- El modelado cinemático se basa en una rueda genérica que incluye los tipos más comunes: fija, orientable, castor y sueca.

Cabe indicar que la rueda castor es completamente genérica, no como en otros estudios (los que sí la consideran) que obligan a que la rueda esté alineada con el brazo de dirección, es decir $\delta_{i}=0$.

- Se ha utilizado un método sistemático, sencillo, eficiente y novedoso, basado en una formulación cinemática recursiva, para obtener el modelo de rueda.

- El modelo de rueda consiste en dos ecuaciones escalares que explicitan las velocidades de deslizamiento de rueda, lo que resulta útil para posteriores etapas de análisis y/o modelado con deslizamiento.

- Se ha demostrado la equivalencia cinemática de la rueda doble con la orientable y de la castor doble (ó vehículo remolque) con la castor, a pesar de que las primeras presentan una serie de ventajas prácticas respecto a las segundas. 


\section{A1 COINCIDENCIA INSTANTÁNEA}

Los sistemas de coordenadas de coincidencia instantánea son una herramienta matemática conceptual que permite resolver con mayor facilidad el problema cinemático, tanto directo como inverso, del vehículo.

Consideramos el caso más simple de sistema unidimensional en el que una bola (punto) se desplaza en una línea recta. La posición de la bola está representada, respecto a un sistema de coordenadas fijo global por $r$ tal y como se observa en la Figura 3.11:

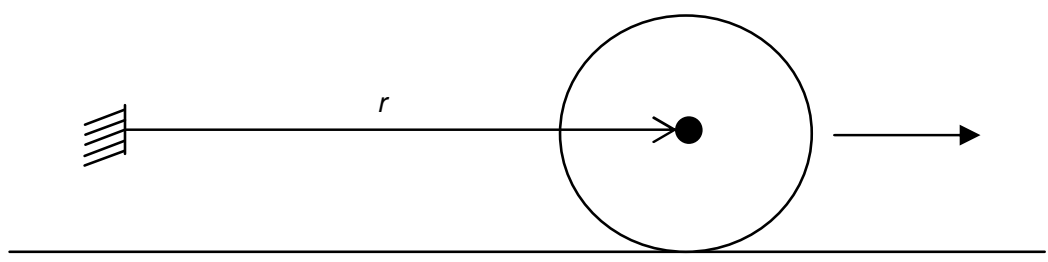

Figura 3.11- Ejemplo de bola en movimiento en un sistema unidimensional

La bola se mueve hacia la derecha con una velocidad $v_{r}$ y aceleración $a_{r}$.

Consideremos ahora el caso de un sistema de referencia estacionario que coincide con la posición actual de la bola. Se puede observar que en ese caso la posición de la bola $(\bar{r})$ respecto a ese sistema de referencia es nula, no así la velocidad y aceleración que tomarán el valor que corresponda.

Supongamos ahora que para cada instante evaluamos la posición, velocidad y aceleración de la bola respecto al sistema estacionario que coincida con la posición de la bola (concepto de sistema de coordenadas instantáneamente coincidente). En ese caso tendremos que la posición de la bola en cada instante respecto al sistema de coordenadas que le corresponda (el que la sigue) es nula, no siendo así para la velocidad y aceleración.

Esto es en esencia en lo que consiste un sistema de referencia instantáneamente coincidente (de coincidencia instantánea), un sistema de coordenadas que sigue al objeto, en este caso la bola, pero que es estacionario para cada instante evaluado. Realmente lo que ocurre es que en cada instante se utiliza un sistema de coordenadas estacionario distinto que cumple la coincidencia con el objeto. 
Es importante recalcar que, al utilizar como sistema de referencia un sistema de coordenadas de coincidencia instantánea, no se cumple que al derivar la ecuación de posición (velocidad) obtengamos la velocidad (aceleración). Esto queda de manifiesto en lo indicado dos párrafos más arriba.

Una forma de explicar lo anterior es la siguiente: en el caso de un sistema de coordenadas instantáneamente coincidente realmente está compuesto por muchos sistemas de coordenadas (que siguen) al objeto y de los cuales sólo se considera uno en cada instante. Por lo tanto a pesar de que conceptualmente es un único sistema de coordenadas (también es así en la ecuación) físicamente son muchos distintos. Esto último es lo que intuitivamente explica que no se cumplan las reglas tradicionales de derivación.

Por tanto, en general para ecuaciones en las que existan de forma implícita sistemas de coordenadas instantáneamente coincidentes hay que explicitar (a través de lema de velocidad en cascada) la derivación de cada matriz que relaciona los sistemas de coordenadas y sustituirlas por su valor.

En el ejemplo anteriormente descrito (una bola en un sistema unidimensional) el hecho de introducir sistemas de coincidencia instantánea no aporta ninguna ventaja, puesto que la velocidad y aceleración de la bola respecto al sistema global y al coincidente son iguales.

La necesidad práctica de esta herramienta conceptual surge en el caso multidimensional, en el que el sistema de coincidencia instantánea estacionario nos permite referir a él la velocidad y aceleración del objeto sin necesidad de conocer cuál es el sistema de referencia global o mejor dicho la posición relativa del objeto respecto a éste.

Además el hecho de referir la velocidad y aceleración respecto al sistema de coordenadas de coincidencia instantánea equivale a hacerlo respecto al propio móvil (posición del móvil), lo cual tiene más sentido físico y es más habitual en el trabajo con vehículos. 



\section{CAPÍTULO 4 \\ MODELADO CINEMÁTICO DE VEHÍCULOS CON RUEDAS SIN DESLIZAMIENTO}

\subsection{INTRODUCCIÓN}

El gran número de posibilidades en la configuración (ejes, ruedas,...) de los distintos vehículos, como demuestra la abundante bibliografía [Whittaker 62] [Everett 79] [Iijima et al. 88] [Helmers 85] [Rogers 84], hace necesario un método de modelado cinemático sistemático. La importancia de los modelos cinemáticos estriba en su utilización en una posterior planificación, control, etc., como se comprueba en [Fortune et al. 88] [Kanayama et al. 86] [Wilfong 88] [Samson et al. 91] [Murray et al. 93] entre otros.

Por ejemplo, [Alexander et al. 89] plantea, para deslizamiento nulo, modelos cinemáticos directos e inversos parciales que relacionan las variables de velocidad de rueda con las de la postura del vehículo. Lo anterior, si bien es de gran utilidad práctica en un control geométrico, no permite una perspectiva global del vehículo.

Por otra parte, en [Muir et al. 87] se presenta la metodología de modelado cinemático más destacada de la bibliografía, donde la ecuación Jacobiana de rueda se combina para obtener una ecuación compuesta global del vehículo. Dicha ecuación es tratada genéricamente a través de un diagrama en forma de árbol y del algoritmo de Mínimos Cuadrados, obteniéndose cuatro diagramas particularizados y las soluciones directa e inversa. 
En este sentido, el presente capítulo desarrolla dos metodologías para la generación de los modelos cinemáticos de vehículos, tanto directos como inversos, a partir de las relaciones cinemáticas aportadas por sus ruedas, bajo el supuesto de no-deslizamiento. Además, se valora la conveniencia de la metodología desarrollada en [Muir et al. 87].

Los supuestos considerados son los mismos que del segundo punto del capítulo anterior (al utilizarse las relaciones cinemáticas allí obtenidas) y adicionalmente el de deslizamiento nulo entre la rueda y el suelo.

Así pues, primeramente en el punto 4.2 del capítulo se presenta una metodología de generación de modelos cinemáticos basada en el concepto de espacio nulo.

Posteriormente en el punto 4.3 se describe, como metodología equivalente, un método basado en el análisis de rangos de matrices.

Mientras que en el punto 4.4 se indica la metodología de modelado propuesta en [Muir et al. 87], sobre la que se han realizado un par de mejoras, y se discute su conveniencia.

Adicionalmente, en el punto 4.5 se introduce el concepto de solución cinemática ampliada, pasada por alto en otros estudios.

Las tres alternativas metodológicas de modelado presentadas se comparan en el punto 4.6 en base al modelado del vehículo tipo triciclo, para el que también se calculan las soluciones cinemáticas ampliadas.

Finalmente se destacan los resultados más relevantes del capítulo. 


\subsection{MODELADO Y CARACTERIZACIÓN CON ESPACIO NULO}

En el presente apartado se presenta un método para el modelado y caracterización de vehículos con ruedas basado en el espacio nulo.

Las relaciones cinemáticas utilizadas como punto de partida para este método es la ecuación compuesta (3.22) con deslizamiento nulo:

$$
\begin{gathered}
\mathbf{v}_{\text {slip }}=\left(\begin{array}{c}
\mathbf{v}_{\text {slip } 1} \\
\vdots \\
\mathbf{v}_{\text {slip } 2} \\
\vdots \\
\mathbf{v}_{\text {slip N }}
\end{array}\right)=\left(\begin{array}{cccc}
\mathbf{A}_{\mathrm{p} 1} & \mathbf{A}_{\mathrm{w} 1} & \cdots & 0 \\
\vdots & \vdots & \ddots & \vdots \\
\mathbf{A}_{\mathrm{pN}} & 0 & \cdots & \mathbf{A}_{\mathrm{wN}}
\end{array}\right) \cdot\left(\begin{array}{c}
\dot{\mathbf{p}} \\
\dot{\mathbf{q}}_{\mathrm{w} 1} \\
\vdots \\
\dot{\mathbf{q}}_{\mathrm{wN}}
\end{array}\right)=\mathbf{0} \\
\mathbf{v}_{\text {slip }}=\left(\begin{array}{ll}
\mathbf{A}_{\mathrm{p}} & \mathbf{A}_{\mathrm{w}}
\end{array}\right) \cdot\left(\begin{array}{c}
\dot{\mathbf{p}} \\
\dot{\mathbf{q}}_{\mathrm{w}}
\end{array}\right)=\mathbf{A} \cdot\left(\begin{array}{c}
\dot{\mathbf{p}} \\
\dot{\mathbf{q}}_{\mathrm{w}}
\end{array}\right)=\mathbf{A} \cdot \dot{\mathbf{q}}=\mathbf{0}
\end{gathered}
$$

Dicha ecuación se caracteriza por tener el mínimo número de ecuaciones $2 \cdot \mathrm{N}$, aportadas por las $\mathrm{N}$ ruedas (sin tener en cuenta posibles dependencias entre ruedas), y por tener todas las velocidades de rueda y de postura en un miembro de la ecuación.

Las soluciones de $\dot{\mathbf{q}}$ en la ecuación anterior pertenecen al espacio nulo de $\mathbf{A}$ :

$$
\dot{\mathbf{q}} \in \mathcal{N}(\mathbf{A}) \rightarrow \dot{\mathbf{q}}=\mathbf{B} \cdot \boldsymbol{\eta}
$$

donde la matriz $\mathbf{B}$ forma una base de $\mathcal{N}(\mathbf{A}), \boldsymbol{\eta}$ es un vector de dimensión $m$ que representa la movilidad del vehículo.

Además $m$ es el grado de movilidad del vehículo, que coincide con los grados de libertad en el movimiento (GDLs) o con los accionamientos independientes descritos en el punto 4.4, y que viene dado por la nulidad de A (teorema sobre la relación entre el rango y la nulidad de una matriz):

$$
m=\operatorname{dim}(\boldsymbol{\eta})=\operatorname{dim}(\mathcal{N}(\mathbf{A}))=\operatorname{dim}(\dot{\mathbf{q}})-\operatorname{rango}(\mathbf{A})=k-g
$$

Con objeto de utilizar variables con significado físico, el vector de movilidad $\boldsymbol{\eta}$ debe sustituirse con un conjunto de velocidades, con la connotación de libremente asignadas. Dependiendo de si se eligen velocidades de rueda o del vehículo se obtendrían modelos cinemáticos directos o inversos. Incluso si se utiliza una combinación de los dos tipos de velocidades se obtendría un modelo cinemático mixto. 
Para verificar si un conjunto $\dot{\mathbf{q}}_{\mathrm{a}}$ de $m$ velocidades pueden ser asignadas, se ha de verificar que el determinante que definen en (4.2) sea no nulo:

$$
\begin{aligned}
&\left(\begin{array}{c}
\dot{\mathbf{q}}_{\text {na }} \\
\dot{\mathbf{q}}_{\mathrm{a}}
\end{array}\right)=\left(\begin{array}{c}
\mathbf{B}_{\text {na }} \\
\mathbf{B}_{\mathrm{a}}
\end{array}\right) \cdot \boldsymbol{\eta} \\
& \text { if } \quad\left|\mathbf{B}_{\mathrm{a}}\right| \neq 0 \rightarrow \quad \rightarrow \quad \dot{\mathbf{q}}_{\text {na }}=\mathbf{B}_{\text {na }} \cdot \mathbf{B}_{\mathrm{a}}^{-1} \cdot \dot{\mathbf{q}}_{\mathrm{a}}
\end{aligned}
$$

donde $\dot{\mathbf{q}}_{\mathrm{na}}$ son las restantes velocidades (no asignadas) del vector de velocidades $\dot{\mathbf{q}}$.

No obstante, existe un camino alternativo a lo anterior basado en la propia obtención del espacio nulo. El algoritmo utilizado normalmente para obtener la matriz $\mathbf{B}$ (que forma una base de $\mathcal{N}(\mathbf{A})$ ), que computacionalmente es el más eficiente, se basa en el método del pivote de Gauss-Jordan, haciendo nulos los elementos por debajo y por encima del pivote. Llegándose a:

$$
\left(\begin{array}{cc}
\mathbf{I} & -\mathbf{B}_{\mathrm{na}} \cdot \mathbf{B}_{\mathrm{a}}^{-1} \\
\mathbf{0} & \mathbf{0}
\end{array}\right) \cdot\left(\begin{array}{c}
\dot{\mathbf{q}}_{\mathrm{na}} \\
\dot{\mathbf{q}}_{\mathrm{a}}
\end{array}\right)=\mathbf{0} \rightarrow\left(\begin{array}{c}
\dot{\mathbf{q}}_{\mathrm{na}} \\
\dot{\mathbf{q}}_{\mathrm{a}}
\end{array}\right)=\left(\begin{array}{c}
\mathbf{B}_{\mathrm{na}} \cdot \mathbf{B}_{\mathrm{a}}^{-1} \\
\mathbf{I}
\end{array}\right) \cdot \boldsymbol{\eta}
$$

De modo que, las últimas variables funcionan como el vector de movilidad del vehículo. Esto será así siempre que sea posible, es decir siempre que las últimas variables puedan funcionar como asignadas.

En caso contrario el algoritmo hace una recolocación de columnas (variables), para que las últimas variables puedan funcionar como asignadas. El criterio de recolocación de columnas (ó variables) es encontrar el conjunto de columnas (ó variables) más próximo a la parte final que den lugar a (4.7).

Por tanto, para obtener la solución para unas variables, que se desea funcionen como asignadas, basta con pasar al algoritmo que calcula la matriz $\mathbf{B}$ con las columnas de dichas variables en último lugar.

Con lo anterior se pueden conseguir todas las soluciones cinemáticas. En general, haría falta calcular la matriz $\mathbf{B}$ un número de veces igual a:

$$
\mathrm{C}_{k}^{m}=\left(\begin{array}{l}
k \\
m
\end{array}\right)=\frac{k !}{m ! \cdot(k-m) !}
$$

Es decir, una combinación de $k$ elementos ( $\mathrm{n}^{\mathrm{o}}$ de velocidades) tomados de $m$ en $m$ (grado de movilidad). 


\subsection{MODELADO Y CARACTERIZACIÓN CON RANGOS}

A continuación se describe un método alternativo al de utilizar el espacio nulo, con gran paralelismo con lo descrito en el punto 4.4, para calcular: el grado de movilidad del vehículo; las variables que pueden ser asignadas; soluciones cinemáticas, etc.

Utilizando el teorema sobre la relación entre el rango y la nulidad de una matriz (4.3), se deduce que habrá un número de velocidades $\dot{\mathbf{q}}_{\text {nа }}$ (de rueda y/o del vehículo) igual al rango de $\mathbf{A}(g)$ utilizadas para cumplir (4.1), y otro grupo $\dot{\mathbf{q}}_{\mathrm{a}}$ igual a la nulidad de $\mathbf{A}(m)$ que podrán asignarse libremente, que funcionarán como el vector de movilidad de (4.2).

Por tanto, la ecuación (4.1) se puede rescribir como:

$$
\mathbf{A}_{\mathrm{na}} \cdot \dot{\mathbf{q}}_{\mathrm{na}}=-\mathbf{A}_{\mathrm{a}} \cdot \dot{\mathbf{q}}_{\mathrm{a}}
$$

en la que se han pasado las velocidades asignadas al miembro de la derecha, para calcular el resto de las velocidades (no asignadas) para deslizamiento nulo.

Para saber qué conjuntos de $m$ velocidades (de rueda, postura, o combinadas) pueden ser asignadas (4.9) ha de tener solución única, lo que implica:

$$
\operatorname{rango}\left(\mathbf{A}_{\mathrm{na}}\right)=\operatorname{rango}(\mathbf{A})
$$

Es decir, para que un conjunto de variables igual al grado de movilidad puedan ser asignadas se ha de cumplir que al eliminar sus columnas asociadas en la matriz original A no disminuya el rango en la matriz resultante.

Al despejar en (4.9) las variables asignadas, la solución cinemática resulta:

$$
\dot{\mathbf{q}}_{\text {na }}=-\left(\mathbf{A}_{\text {na }}^{\mathrm{T}} \cdot \mathbf{A}_{\text {na }}\right)^{-1} \cdot \mathbf{A}_{\text {na }}^{\mathrm{T}} \cdot \mathbf{A}_{\mathrm{a}} \cdot \dot{\mathbf{q}}_{\mathrm{a}}
$$

llegándose al mismo resultado que en (4.6).

Notar que en la expresión anterior se ha aplicado la pseudo-inversa por la izquierda (algoritmo de Mínimos Cuadrados), aunque evidentemente el error residual del algoritmo será siempre nulo. En caso de asignar alguna velocidad adicional al conjunto marcado por (4.10) la expresión seguiría valiendo, aunque con error residual no nulo, y serviría para detectar deslizamiento. 


\subsection{MODELADO Y CARACTERIZACIÓN CON MATRICES JACOBIANAS DE RUEDA}

El modelado con matrices Jacobianas de rueda tratado en este punto se presenta en [Muir et al. 87]. Sin embargo respecto a lo presentado en [Muir et al. 87] se realizan dos mejoras fundamentales:

1) Se trabaja con un tipo de rueda más, la rueda sueca.

2) Las matrices Jacobianas de rueda propuestas son las de (3.19), que no son exactamente las de [Muir et al. 87], ya que presentan la ventaja de haber eliminado una variable sin sentido físico (menos coste computacional) y ser siempre (excepto para ruedas omnidireccionales singulares, ver Capítulo 7) invertibles ó pseudo invertibles. Esto posibilita siempre el análisis desarrollado a continuación, mientras que para las matrices Jacobianas originales no es posible cuando el vehículo posee ruedas orientables, por otro lado bastante frecuente.

Así pues, el punto de partida para el modelado son: por un lado las matrices Jacobianas de rueda de (3.19); y por otro la ecuación compuesta de matrices Jacobianas de (3.20). Ambas del capítulo de relaciones cinemáticas:

$$
\begin{gathered}
\mathbf{J}_{\mathrm{f} / \mathrm{o} i}=\left(\begin{array}{cc}
\mathrm{r}_{i} \cdot \mathrm{s} \beta_{i} & 1_{i} \cdot \mathrm{s} \alpha_{i} \\
-\mathrm{r}_{i} \cdot \mathrm{c} \beta_{i} & -1_{i} \cdot \mathrm{c} \alpha_{i} \\
0 & 1
\end{array}\right) \quad \dot{\mathbf{q}}_{\mathrm{w} f / o i}=\left(\begin{array}{c}
\dot{\varphi}_{i} \\
\omega
\end{array}\right) \\
\mathbf{J}_{\mathrm{s} i}=\left(\begin{array}{ccc}
\mathrm{r}_{i} \cdot \mathrm{s} \beta_{i} & \mathrm{r}_{\mathrm{r} i} \cdot \mathrm{s}\left(\beta_{i}+\gamma_{i}\right) & 1_{i} \cdot \mathrm{s} \alpha_{i} \\
-\mathrm{r}_{i} \cdot \mathrm{c} \beta_{i} & -\mathrm{r}_{\mathrm{r} i} \cdot \mathrm{c}\left(\beta_{i}+\gamma_{i}\right) & -1_{i} \cdot \mathrm{c} \alpha_{i} \\
0 & 0 & 1
\end{array}\right) \quad \dot{\mathbf{q}}_{\mathrm{ws} i}=\left(\begin{array}{c}
\dot{\varphi}_{i} \\
\dot{\varphi}_{\mathrm{r} i} \\
\omega
\end{array}\right) \\
\mathbf{J}_{\mathrm{c} i}=\left(\begin{array}{ccc}
\mathrm{d}_{i} \cdot \mathrm{c} \beta_{i} & \mathrm{r}_{i} \cdot \mathrm{s}\left(\beta_{i}+\delta_{i}\right) & 1_{i} \cdot \mathrm{s} \alpha_{i}+\mathrm{d}_{i} \cdot \mathrm{c} \beta_{i} \\
\mathrm{~d}_{i} \cdot \mathrm{s} \beta_{i} & -\mathrm{r}_{i} \cdot \mathrm{c}\left(\beta_{i}+\delta_{i}\right) & -1_{i} \cdot \mathrm{c} \alpha_{i}+\mathrm{d}_{i} \cdot \mathrm{s} \beta_{i} \\
0 & 0 & \\
0 & 1
\end{array}\right) \quad \dot{\mathbf{q}}_{\mathrm{wc} i}=\left(\begin{array}{c}
\dot{\beta}_{i} \\
\dot{\varphi}_{i} \\
\omega
\end{array}\right) \\
\left(\begin{array}{c}
\mathbf{I} \\
\mathbf{I} \\
\vdots \\
\mathbf{I}
\end{array}\right) \cdot \dot{\mathbf{p}}=\left(\begin{array}{cccc}
\mathbf{J}_{1} & 0 & \cdots & 0 \\
0 & \mathbf{J}_{2} & \ddots & \vdots \\
\vdots & \ddots & \ddots & 0 \\
0 & \cdots & 0 & \mathbf{J}_{\mathrm{N}}
\end{array}\right) \cdot\left(\begin{array}{c}
\dot{\mathbf{q}}_{\mathrm{w} 1} \\
\dot{\mathbf{q}}_{\mathrm{w} 2} \\
\vdots \\
\dot{\mathbf{q}}_{\mathrm{wN}}
\end{array}\right) \Rightarrow \overline{\mathbf{I}} \cdot \dot{\mathbf{p}}=\mathbf{J} \cdot \dot{\mathbf{q}}_{\mathrm{w}}
\end{gathered}
$$

donde el subíndice $\{\mathrm{f}, \mathrm{o}, \mathrm{c}, \mathrm{s}\}$ es para rueda $\{$ fija, orientable, castor, sueca $\}$ e I es la matriz identidad de dimensión 3. 
Para analizar la ecuación compuesta (4.13) se utiliza la teoría relativa a la resolución de una ecuación genérica de la forma:

$$
\mathbf{A}_{\mathrm{m} x \mathrm{n}} \cdot \mathbf{x}_{\mathrm{n} x 1}=\mathbf{B}_{\mathrm{m} x \mathrm{p}} \cdot \mathbf{y}_{\mathrm{p} x 1}
$$

Para resolverla se utiliza el diagrama de la Figura 4.1. Dicho diagrama utiliza pruebas de bifurcación basadas en el análisis de rangos de las matrices o, en algunos casos, el error residual de Mínimos Cuadrados.

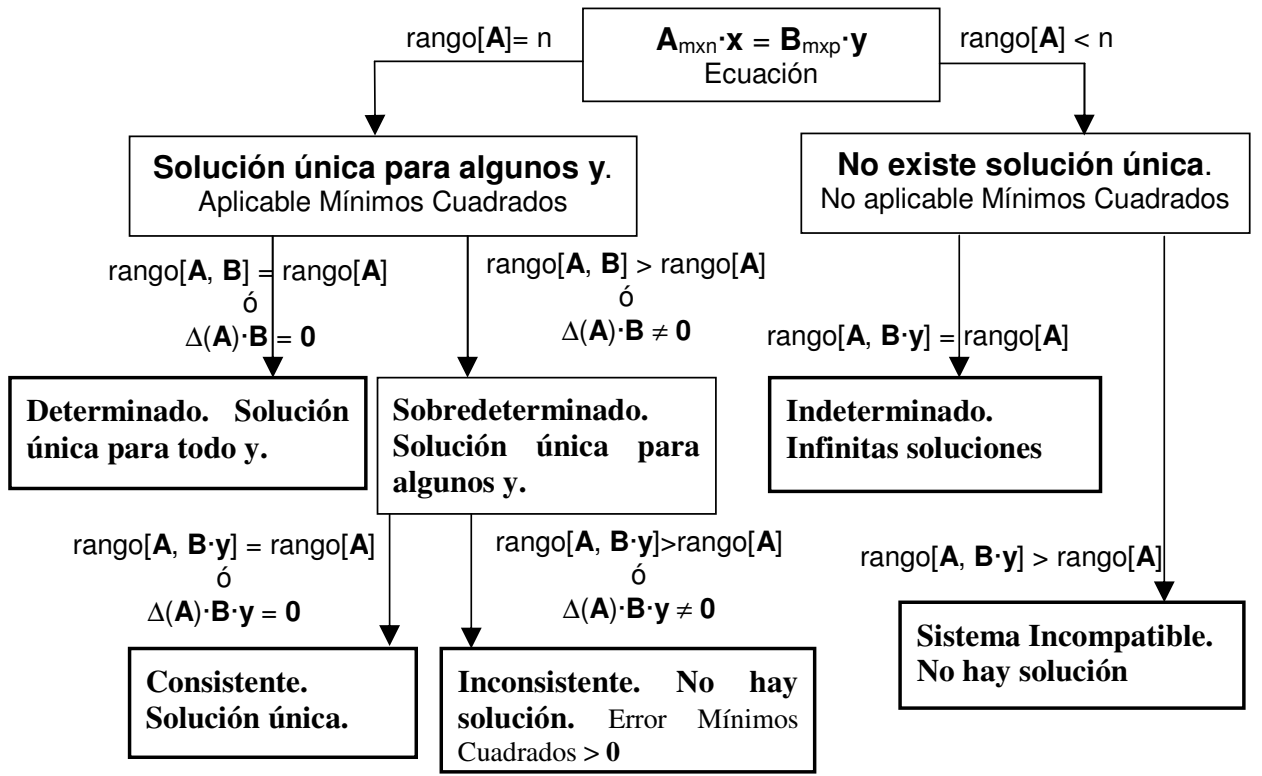

Figura 4.1- Árbol de solución de la ecuación genérica "A · x = B · y"

La solución de Mínimos Cuadrados de (4.14) y su error residual son:

$$
\begin{aligned}
& \mathbf{x}^{*}=\left(\mathbf{A}^{\mathrm{T}} \cdot \mathbf{A}\right)^{-1} \cdot \mathbf{A}^{\mathrm{T}} \cdot \mathbf{B} \cdot \mathbf{y} \\
& \text { Error }=\mathbf{A} \cdot \mathbf{x}^{*}-\mathbf{B} \cdot \mathbf{y}=\left(\mathbf{A} \cdot\left(\mathbf{A}^{\mathrm{T}} \cdot \mathbf{A}\right)^{-1} \cdot \mathbf{A}^{\mathrm{T}}-\mathbf{I}\right) \cdot \mathbf{B} \cdot \mathbf{y}=\Delta(\mathbf{A}) \cdot \mathbf{B} \cdot \mathbf{y} \\
& \text { donde: } \quad \Delta(\mathbf{A})=\left\{\begin{array}{ccc}
-\mathbf{I} & \text { para } & \mathbf{A}=\mathbf{0} \\
\mathbf{A} \cdot\left(\mathbf{A}^{\mathrm{T}} \cdot \mathbf{A}\right)^{-1} \cdot \mathbf{A}^{\mathrm{T}}-\mathbf{I} & \text { para } & \mathbf{A} \neq \mathbf{0}
\end{array}\right.
\end{aligned}
$$

Si se puede aplicar Mínimos Cuadrados, son equivalentes:

$$
\left.\begin{array}{c}
\Delta(\mathbf{A}) \cdot \mathbf{B} \cdot \mathbf{y}=0 \\
\operatorname{rango}[\mathbf{A} ; \mathbf{B} \cdot \mathbf{y}]=\operatorname{rango}[\mathbf{A}]
\end{array}\right\} \Rightarrow \text { Error nulo } \Rightarrow \text { Solución exacta }
$$




\subsubsection{Problema cinemático directo}

Para este caso, se considera como incógnita $\mathbf{x}$ el vector de velocidad de la postura del vehículo $\dot{\mathbf{p}}$. El árbol para este caso se particulariza en el de la Figura 4.2.

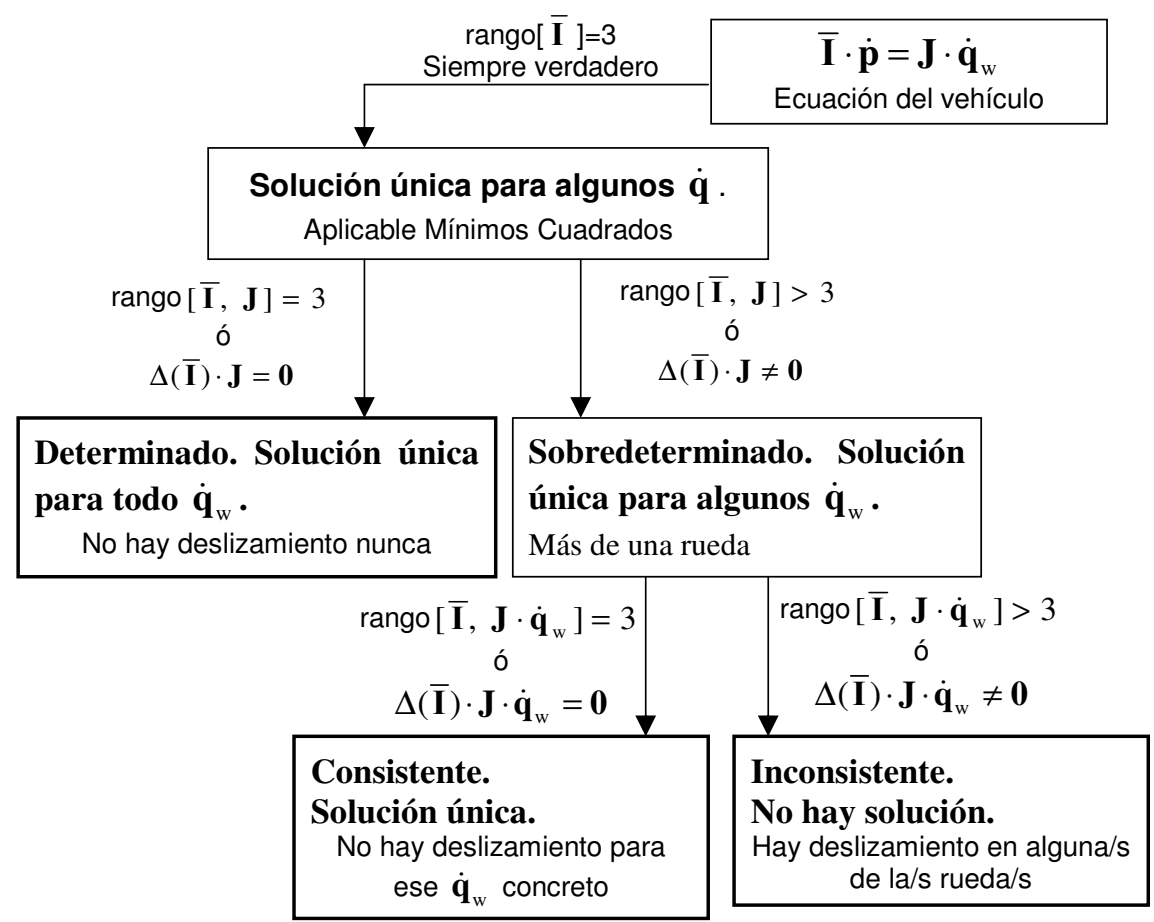

Figura 4.2- Árbol para el vector de velocidad del vehículo $\dot{\mathbf{p}}$

Como se puede apreciar en la Figura 4.2, en el caso sobredeterminado hay solución única (no se produce deslizamiento) cuando algunas velocidades de rueda están coordinadas (dependientes) con las asignadas libremente.

El número de velocidades de rueda sobre las que se puede actuar de forma independiente (asignadas libremente) es:

$$
H=\operatorname{dim}\left(\dot{\mathbf{q}}_{\mathrm{w}}\right)-(\operatorname{rango}[\overline{\mathbf{I}}, \mathbf{J}]-\operatorname{rango}[\overline{\mathbf{I}}])=\operatorname{dim}\left(\dot{\mathbf{q}}_{\mathrm{w}}\right)-\operatorname{rango}[\Delta(\overline{\mathbf{I}}) \cdot \mathbf{J}]
$$

La justificación de la ecuación anterior es: las velocidades de rueda independientes son todas menos el número de ecuaciones linealmente independientes que las ligan para no-deslizamiento. 


\subsubsection{Problema cinemático inverso}

En este caso la incógnita considerada es el vector compuesto de velocidades de rueda $\dot{\mathbf{q}}_{\mathrm{w}}$. La Figura 4.3 muestra el árbol correspondiente, siendo $\mathrm{w}_{i}$ el número de velocidades para la rueda $i$ (incluyendo la velocidad angular del vehículo).

Este árbol caracteriza la movilidad del vehículo, es decir establece las restricciones del vector velocidad del vehículo $\dot{\mathbf{p}}$.

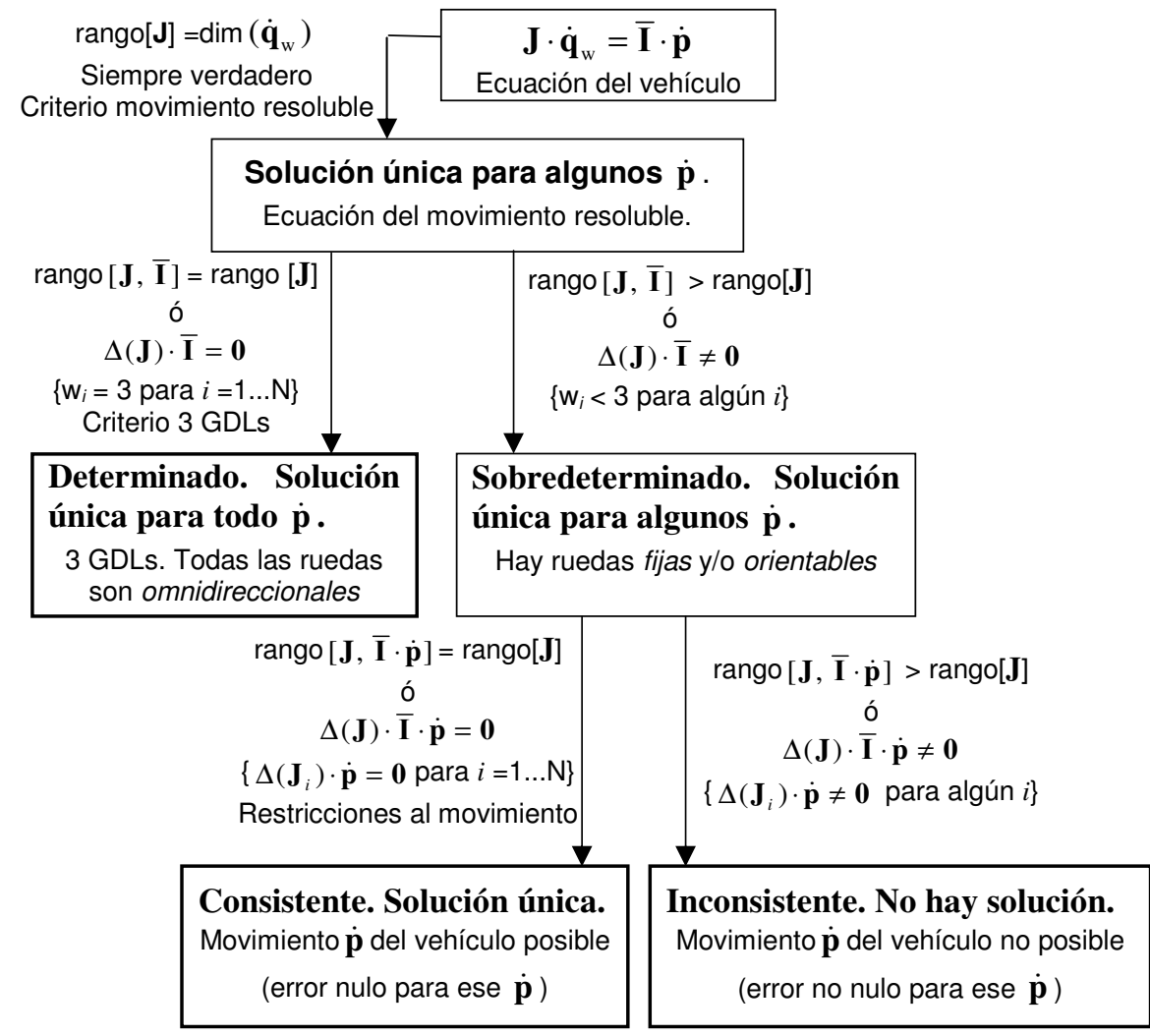

Figura 4.3- Árbol para el vector compuesto de velocidades de rueda $\dot{\mathbf{q}}_{\mathrm{w}}$

Para este árbol las pruebas generales de cada ramificación se derivan directamente del árbol de la Figura 4.1. Mientras que, las pruebas de simplificación, que afectan sólo a una misma rueda (van entre corchetes), se aplican cuando no hay acoplamientos entre ruedas. 
Entendiendo por acoplamiento entre ruedas la dependencia entre variables de velocidad de distintas ruedas, a parte de las fijadas por la ecuación compuesta del vehículo. Por ejemplo, el mecanismo de Ackerman acopla la orientación de las ruedas directrices en el vehículo tipo coche.

Para llegar a la prueba de simplificación que indica movilidad completa en el vehículo (3 GDLs), se realiza el siguiente análisis:

Como prueba la Demostración 4.1 (subapartado 4.4.8):

$$
\Delta(\mathbf{J}) \cdot \overline{\mathbf{I}}=\left(\begin{array}{c}
\Delta\left(\mathbf{J}_{1}\right) \\
\Delta\left(\mathbf{J}_{2}\right) \\
\vdots \\
\Delta\left(\mathbf{J}_{\mathrm{N}}\right)
\end{array}\right)
$$

por lo que la condición de 3 grados de libertad (GDLs) se puede expresar como:

$$
\Delta(\mathbf{J}) \cdot \overline{\mathbf{I}}=0 \quad \leftrightarrow \quad \Delta\left(\mathbf{J}_{i}\right)=0 \quad \forall i
$$

Lo anterior se cumple siempre que $\mathbf{J}_{\mathrm{i}}$ sea una matriz cuadrada (mismo número de filas que de columnas $\rightarrow$ mismo número de ecuaciones que de incógnitas) de rango completo (filas / columnas linealmente independientes). En cuyo caso el error residual al aplicar Mínimos Cuadrados resulta nulo.

Para que $\mathbf{J}_{\mathrm{i}}$ sea cuadrada ha de ser de $3 \times 3$, es decir la rueda ha de ser omnidireccional (castor o sueca) y tener tres variables de velocidad de rueda (incluyendo la velocidad angular del vehículo).

El resto de simplificaciones son inmediatas a partir la Demostración 4.1.

Para saber el número de velocidades del vehículo sobre las que se puede actuar independientemente (GDLs del vehículo) se utiliza:

$$
G=3-(\operatorname{rango}[\mathbf{J}, \overline{\mathbf{I}}]-\operatorname{rango}[\mathbf{J}])=3-\operatorname{rango}[\Delta(\mathbf{J}) \cdot \overline{\mathbf{I}}]
$$

El significado de la ecuación anterior es: se tienen todos los GDLs (3) menos el número de ecuaciones linealmente independientes que restringen el movimiento. 


\subsubsection{Solución inversa accionada}

En este punto se calculan los valores de las velocidades de rueda, separadas en accionadas y no accionadas, a partir del movimiento deseado en el vector de velocidad del vehículo, utilizando del algoritmo de Mínimos Cuadrados.

Como se ha comentado en el problema cinemático inverso, las velocidades del vehículo en general no se puede asignar de forma independiente, si no que hay que tener en cuenta los GDLs de los que se disponen. De lo contrario el error del algoritmo de Mínimos Cuadrados es no nulo y no se conseguiría el movimiento deseado.

Primeramente, se rescribe la matriz Jacobiana separando variables accionadas* (subíndice "u”) de no accionadas (subíndice "un”):

$$
\dot{\mathbf{p}}=\mathbf{J}_{i \mathrm{u}} \cdot \dot{\mathbf{q}}_{\mathrm{wiu}}+\mathbf{J}_{\text {inu }} \cdot \dot{\mathbf{q}}_{\text {winu }}
$$

*Nota: Evidentemente la velocidad angular del vehículo $\omega$ no debe elegirse como accionada, debido de la falta de sentido físico.

Con lo cual, la ecuación compuesta queda:

$$
\begin{aligned}
& \left(\begin{array}{c}
\mathbf{I} \\
\mathbf{I} \\
\vdots \\
\mathbf{I}
\end{array}\right) \cdot \dot{\mathbf{p}}=\left(\begin{array}{cccccccc}
\mathbf{J}_{1 \mathrm{u}} & 0 & \cdots & 0 & \mathbf{J}_{1 \mathrm{nu}} & 0 & \cdots & 0 \\
0 & \mathbf{J}_{2 \mathrm{u}} & \ddots & \vdots & 0 & \mathbf{J}_{2 \mathrm{nu}} & \ddots & \vdots \\
\vdots & \ddots & \ddots & 0 & \vdots & \ddots & \ddots & 0 \\
0 & \cdots & 0 & \mathbf{J}_{\mathrm{Nu}} & 0 & \cdots & 0 & \mathbf{J}_{\mathrm{Nnu}}
\end{array}\right) \cdot\left(\begin{array}{c}
\dot{\mathbf{q}}_{\mathrm{w} 1 \mathrm{u}} \\
\dot{\mathbf{q}}_{\mathrm{w} 2 \mathrm{u}} \\
\vdots \\
\dot{\mathbf{q}}_{\mathrm{wNu}} \\
\dot{\mathbf{q}}_{\mathrm{w} 1 \mathrm{nu}} \\
\dot{\mathbf{q}}_{\mathrm{w} 2 \mathrm{nu}} \\
\vdots \\
\dot{\mathbf{q}}_{\mathrm{wNnu}}
\end{array}\right) \\
& \overline{\mathbf{I}} \cdot \dot{\mathbf{p}}=\left(\begin{array}{ll}
\mathbf{J}_{\mathrm{u}} & \mathbf{J}_{\mathrm{nu}}
\end{array}\right) \cdot\left(\begin{array}{c}
\dot{\mathbf{q}}_{\text {wu }} \\
\dot{\mathbf{q}}_{\mathrm{wnu}}
\end{array}\right)=\mathbf{J}_{\mathrm{p}} \cdot \dot{\mathbf{q}}_{\mathrm{p}}
\end{aligned}
$$

Aplicando Mínimos Cuadrados a (4.24), para calcular $\dot{\mathbf{q}}_{\mathrm{p}}$, se obtiene:

$$
\dot{\mathbf{q}}_{\mathrm{p}}=\left(\mathbf{J}_{\mathrm{p}}^{\mathrm{T}} \cdot \mathbf{J}_{\mathrm{p}}\right)^{-1} \cdot \mathbf{J}_{\mathrm{p}}^{\mathrm{T}} \cdot \overline{\mathbf{I}} \cdot \dot{\mathbf{p}}
$$


La expresión anterior, utilizando (4.24), se rescribe (ver Demostración 4.2 del subapartado 4.4.8) en la denominada solución inversa accionada:

$$
\begin{gathered}
\dot{\mathbf{q}}_{\mathrm{wu}}=\left(\begin{array}{c}
\left(\mathbf{J}_{1 \mathrm{u}}^{\mathrm{T}} \cdot \Delta\left(\mathbf{J}_{1 \mathrm{nu}}\right) \cdot \mathbf{J}_{1 \mathrm{u}}\right)^{-1} \cdot \mathbf{J}_{1 \mathrm{u}}^{\mathrm{T}} \cdot \Delta\left(\mathbf{J}_{1 \mathrm{nu}}\right) \\
\left(\mathbf{J}_{2 \mathrm{u}}^{\mathrm{T}} \cdot \Delta\left(\mathbf{J}_{2 \mathrm{nu}}\right) \cdot \mathbf{J}_{2 \mathrm{u}}\right)^{-1} \cdot \mathbf{J}_{2 \mathrm{u}}^{\mathrm{T}} \cdot \Delta\left(\mathbf{J}_{2 \mathrm{nu}}\right) \\
\vdots \\
\left(\mathbf{J}_{\mathrm{Nu}}^{\mathrm{T}} \cdot \Delta\left(\mathbf{J}_{\mathrm{Nnu}}\right) \cdot \mathbf{J}_{\mathrm{Nu}}\right)^{-1} \cdot \mathbf{J}_{\mathrm{Nu}}^{\mathrm{T}} \cdot \Delta\left(\mathbf{J}_{\mathrm{Nnu}}\right)
\end{array}\right) \cdot \dot{\mathbf{p}} \\
\dot{\mathbf{q}}_{\mathrm{wnu}}=\left(\begin{array}{c}
\left(\mathbf{J}_{1 \mathrm{nu}}^{\mathrm{T}} \cdot \Delta\left(\mathbf{J}_{1 \mathrm{u}}\right) \cdot \mathbf{J}_{1 \mathrm{nu}}\right)^{-1} \cdot \mathbf{J}_{1 \mathrm{nu}}^{\mathrm{T}} \cdot \Delta\left(\mathbf{J}_{1 \mathrm{u}}\right) \\
\left(\mathbf{J}_{2 \mathrm{nu}}^{\mathrm{T}} \cdot \Delta\left(\mathbf{J}_{2 \mathrm{u}}\right) \cdot \mathbf{J}_{2 \mathrm{nu}}\right)^{-1} \cdot \mathbf{J}_{2 \mathrm{nu}}^{\mathrm{T}} \cdot \Delta\left(\mathbf{J}_{2 \mathrm{u}}\right) \\
\vdots \\
\left(\mathbf{J}_{\mathrm{Nnu}}^{\mathrm{T}} \cdot \Delta\left(\mathbf{J}_{\mathrm{Nu}}\right) \cdot \mathbf{J}_{\mathrm{Nnu}}\right)^{-1} \cdot \mathbf{J}_{\mathrm{Nnu}}^{\mathrm{T}} \cdot \Delta\left(\mathbf{J}_{\mathrm{Nu}}\right)
\end{array}\right) \cdot \dot{\mathbf{p}}
\end{gathered}
$$

En la expresión anterior el vector de velocidad de la rueda $i$ sólo depende de la matriz Jacobiana de la rueda $i$. Por tanto, la solución inversa para cada rueda es independiente de las ecuaciones cinemáticas del resto de ruedas, aunque tiene el inconveniente de que el vector $\dot{\mathbf{p}}$ no es, en general, independiente.

En particular, para una rueda $i$ tipo omnidireccional (castor o sueca) con las tres variables accionadas (o las tres no accionadas) la expresión se simplifica a (ver Demostración 4.4 del subaparado 4.4.8):

$$
\dot{\mathbf{q}}_{\mathrm{wiu}}=\mathbf{J}_{i \mathrm{u}}^{-1} \cdot \dot{\mathbf{p}}=\dot{\mathbf{q}}_{\mathrm{w} i}=\mathbf{J}_{i}^{-1} \cdot \dot{\mathbf{p}}
$$




\subsection{4 Árbol de accionamiento}

Este punto caracteriza el movimiento del vehículo, cuando las velocidades de rueda no accionadas alcanzan los valores dados por la solución inversa accionada, a partir de las accionadas. Es decir, las velocidades no accionadas se auto-ajustan para evitar el deslizamiento mientras se actúa sobre las accionadas.

Sustituyendo (4.26) en (4.13) y operando se obtiene:

$$
\begin{gathered}
\left(\begin{array}{c}
\mathbf{I}-\mathbf{J}_{1 \mathrm{nu}} \cdot\left(\mathbf{J}_{1 \mathrm{nu}}^{\mathrm{T}} \cdot \Delta\left(\mathbf{J}_{1 \mathrm{u}}\right) \cdot \mathbf{J}_{1 \mathrm{nu}}\right)^{-1} \cdot \mathbf{J}_{1 \mathrm{nu}}^{\mathrm{T}} \cdot \Delta\left(\mathbf{J}_{1 \mathrm{u}}\right) \\
\mathbf{I}-\mathbf{J}_{2 \mathrm{nu}} \cdot\left(\mathbf{J}_{2 \mathrm{nu}}^{\mathrm{T}} \cdot \Delta\left(\mathbf{J}_{2 \mathrm{u}}\right) \cdot \mathbf{J}_{2 \mathrm{nu}}\right)^{-1} \cdot \mathbf{J}_{2 \mathrm{nu}}^{\mathrm{T}} \cdot \Delta\left(\mathbf{J}_{2 \mathrm{u}}\right) \\
\vdots \\
\mathbf{I}-\mathbf{J}_{\mathrm{Nnu}} \cdot\left(\mathbf{J}_{\mathrm{Nnu}}^{\mathrm{T}} \cdot \Delta\left(\mathbf{J}_{\mathrm{Nu}}\right) \cdot \mathbf{J}_{\mathrm{Nnu}}\right)^{-1} \cdot \mathbf{J}_{\mathrm{Nnu}}^{\mathrm{T}} \cdot \Delta\left(\mathbf{J}_{\mathrm{Nu}}\right)
\end{array}\right) \cdot \dot{\mathbf{p}}=\left(\begin{array}{cccc}
\mathbf{J}_{1 \mathrm{u}} & 0 & \cdots & 0 \\
0 & \mathbf{J}_{2 \mathrm{u}} & \ddots & \vdots \\
\vdots & \ddots & \ddots & 0 \\
0 & \cdots & 0 & \mathbf{J}_{\mathrm{Nu}}
\end{array}\right) \cdot \dot{\mathbf{q}}_{\mathrm{wu}}(4.28) \\
\overline{\mathbf{I}}_{\mathrm{u}} \cdot \dot{\mathbf{p}}=\mathbf{J}_{\mathrm{u}} \cdot \dot{\mathbf{q}}_{\mathrm{wu}}
\end{gathered}
$$

La expresión anterior recibe el nombre de ecuación de accionamiento del vehículo, que al aplicarle el árbol de la Figura 4.1 da lugar al árbol de accionamiento de la Figura 4.4.

Algunas de las pruebas de cada ramificación del árbol anterior se derivan directamente de la Figura 4.1, siendo criterios con pleno sentido físico:

a) el criterio de actuadores suficientes permite actuar sobre todos los GDLs del vehículo (al margen de configuraciones singulares);

b) el criterio de actuación consistente garantiza que el conflicto entre actuadores es imposible;

c) el criterio de acoplamiento entre actuadores establece la forma de coordinar los actuadores redundantes para evitar el conflicto entre ellos. 


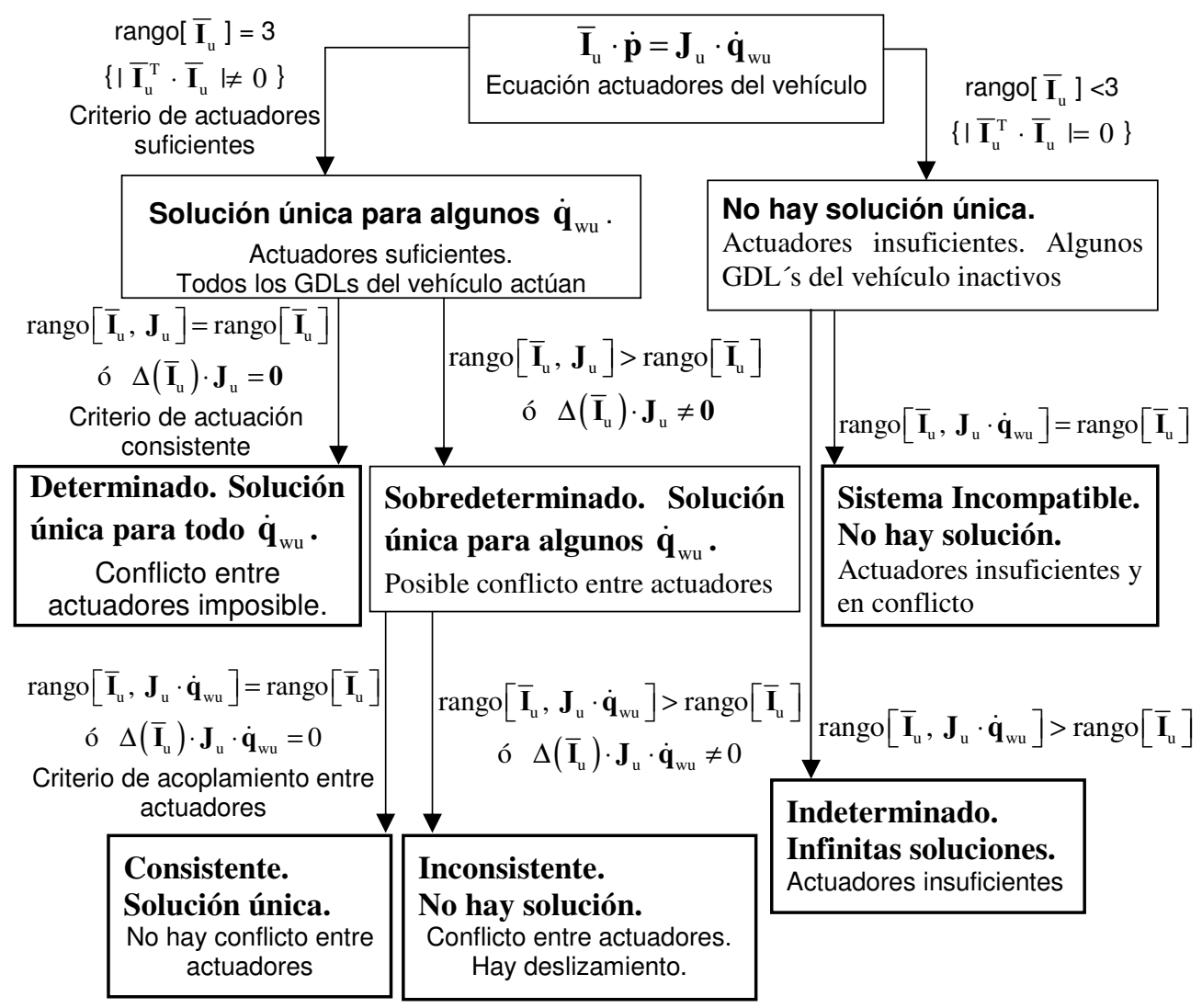

Figura 4.4- Árbol de caracterización del accionamiento del vehículo 


\subsubsection{Solución directa sensorizada}

En este punto se calcula la velocidad de la postura y las variables de velocidad de rueda no sensorizadas a partir de las variables de velocidad de rueda sensorizadas.

Primeramente, se separa la matriz Jacobiana en velocidades de rueda sensorizadas* (subíndice "s") y no sensorizadas (subíndice "ns"), obteniéndose:

$$
\dot{\mathbf{p}}=\mathbf{J}_{i \mathrm{~s}} \cdot \dot{\mathbf{q}}_{\mathrm{wis}}+\mathbf{J}_{\text {ins }} \cdot \dot{\mathbf{q}}_{\text {wins }}
$$

*Nota: Igual que antes no tiene sentido considerar sensorizada la velocidad $\omega$.

Reordenando la ecuación compuesta (4.13), utilizando (4.29), se obtiene:

$$
\begin{gathered}
\left(\begin{array}{ccccc}
\mathbf{I} & -\mathbf{J}_{\mathrm{nns}} & 0 & \cdots & 0 \\
\mathbf{I} & 0 & -\mathbf{J}_{2 \mathrm{~ns}} & \ddots & \vdots \\
\vdots & \vdots & \ddots & \ddots & 0 \\
\mathbf{I} & 0 & \cdots & 0 & -\mathbf{J}_{\mathrm{Nns}}
\end{array}\right) \cdot\left(\begin{array}{c}
\dot{\mathbf{p}} \\
\dot{\mathbf{q}}_{\mathrm{w} 1 \mathrm{~ns}} \\
\dot{\mathbf{q}}_{\mathrm{w} 2 \mathrm{~ns}} \\
\vdots \\
\dot{\mathbf{q}}_{\mathrm{wNns}}
\end{array}\right)=\left(\begin{array}{cccc}
\mathbf{J}_{1 \mathrm{~s}} & 0 & \cdots & 0 \\
0 & \mathbf{J}_{2 \mathrm{~s}} & \ddots & \vdots \\
\vdots & \ddots & \ddots & 0 \\
0 & \cdots & 0 & \mathbf{J}_{\mathrm{Ns}}
\end{array}\right) \cdot\left(\begin{array}{c}
\dot{\mathbf{q}}_{\mathrm{w} 1 \mathrm{~s}} \\
\dot{\mathbf{q}}_{\mathrm{w} 2 \mathrm{~s}} \\
\vdots \\
\dot{\mathbf{q}}_{\mathrm{wNs}}
\end{array}\right) \\
\left(\begin{array}{cc}
\dot{\mathbf{I}} & -\mathbf{J}_{\mathrm{ns}}
\end{array}\right) \cdot\left(\begin{array}{c}
\dot{\mathbf{p}} \\
\dot{\mathbf{q}}_{\mathrm{wns}}
\end{array}\right)=\mathbf{J}_{\mathrm{s}} \cdot \dot{\mathbf{q}}_{\mathrm{ws}} \rightarrow \overline{\mathbf{I}}_{\mathrm{ns}} \cdot\left(\begin{array}{c}
\dot{\mathbf{p}} \\
\dot{\mathbf{q}}_{\mathrm{wns}}
\end{array}\right)=\mathbf{J}_{\mathrm{s}} \cdot \dot{\mathbf{q}}_{\mathrm{ws}} \\
\text { Aplicando Mínimos Cuadrados en }(4.30): \\
\left(\begin{array}{c}
\dot{\mathbf{p}} \\
\dot{\mathbf{q}}_{\mathrm{wns}}
\end{array}\right)=\left(\overline{\mathbf{I}}_{\mathrm{ns}} \cdot \overline{\mathbf{I}}_{\mathrm{ns}}^{\mathrm{T}}\right)^{-1} \cdot \overline{\mathbf{I}}_{\mathrm{ns}}^{\mathrm{T}} \cdot \mathbf{J}_{\mathrm{s}} \cdot \dot{\mathbf{q}}_{\mathrm{ws}}
\end{gathered}
$$

Para poder aplicar (4.31) $\overline{\mathbf{I}}_{\mathrm{ns}}$ ha de ser pseudo invertible, lo cual se cumple siempre puesto que las matrices $\mathbf{J}$ e $\overline{\mathbf{I}}$ son siempre pseudo invertibles.

En particular, quedándose con las tres primeras componentes (velocidades del vehículo) en (4.31), y teniendo en cuenta (4.30), se llega a la siguiente solución directa sensorizada reducida (Demostración 4.5 del subaparado 4.4.8):

$$
\begin{aligned}
\dot{\mathbf{p}}= & \left(\Delta\left(\mathbf{J}_{1 \mathrm{~ns}}\right)+\Delta\left(\mathbf{J}_{2 \mathrm{~ns}}\right)+\cdots+\Delta\left(\mathbf{J}_{\mathrm{Nns}}\right)\right)^{-1} \cdot \\
& \cdot\left(\Delta\left(\mathbf{J}_{1 \mathrm{~ns}}\right) \cdot \mathbf{J}_{1 \mathrm{~s}} \quad \Delta\left(\mathbf{J}_{2 \mathrm{~ns}}\right) \cdot \mathbf{J}_{2 \mathrm{~s}} \quad \cdots \quad \Delta\left(\mathbf{J}_{\mathrm{Nns}}\right) \cdot \mathbf{J}_{\mathrm{Ns}}\right) \cdot \dot{\mathbf{q}}_{\mathrm{ws}}
\end{aligned}
$$


De la ecuación anterior se deduce que, si una rueda tiene tres variables de velocidad independientes (rueda omnidireccional) no sensorizadas, entonces se puede eliminar de la ecuación de solución directa sensorizada, puesto que no contribuye a dicha ecuación. Para demostrarlo, basta tener en cuenta que $\mathbf{J}_{i \text { ins }}$ será una matriz cuadrada de $3 \times 3$ de rango 3 (completo) y que al aplicarle la función $\Delta$ resulta una matriz nula.

En (4.32) se pueden obviar aquellas ruedas no sensorizadas que no aporten nuevas restricciones al movimiento del vehículo $\left(\Delta\left(\mathbf{J}_{i}\right) \cdot \mathbf{p}=0\right.$, ver Figura 4.3).

Hay que tener en cuenta que, para que las matrices Jacobianas (sensorizada y no sensorizada) de las ruedas con dirección (castor y orientable) sean calculables, hay que sensorizar el ángulo de la dirección. Otra alternativa sería realizar la integración numérica del ángulo a partir de la velocidad de giro (calculada en (4.31) en caso de no sensorizarla) y el valor inicial del ángulo:

$$
\beta_{i}(t)=\int \dot{\beta}_{i} \mathrm{~d} t+\beta_{i}(0)
$$

\subsection{6 Árbol de sensorización}

En este punto se calculan las velocidades del vehículo y las de rueda no sensorizadas, suponiendo que alcanzan los valores de no-deslizamiento de la solución inversa accionada, a partir de las velocidades de rueda sensorizadas.

Procediendo análogamente al árbol de accionamiento, se llega a la expresión (4.28) sin más que cambiar los subíndices u por s (accionadas por sensorizadas) y nu por ns (no accionadas por no sensorizadas):

$$
\begin{gathered}
\left(\begin{array}{c}
\mathbf{I}-\mathbf{J}_{1 \mathrm{~ns}} \cdot\left(\mathbf{J}_{1 \mathrm{~ns}}^{\mathrm{T}} \cdot \Delta\left(\mathbf{J}_{1 \mathrm{~s}}\right) \cdot \mathbf{J}_{1 \mathrm{~ns}}\right)^{-1} \cdot \mathbf{J}_{1 \mathrm{~ns}}^{\mathrm{T}} \cdot \Delta\left(\mathbf{J}_{1 \mathrm{~s}}\right) \\
\mathbf{I}-\mathbf{J}_{2 \mathrm{~ns}} \cdot\left(\mathbf{J}_{2 \mathrm{~ns}}^{\mathrm{T}} \cdot \Delta\left(\mathbf{J}_{2 \mathrm{~s}}\right) \cdot \mathbf{J}_{2 \mathrm{~ns}}\right)^{-1} \cdot \mathbf{J}_{2 \mathrm{~ns}}^{\mathrm{T}} \cdot \Delta\left(\mathbf{J}_{2 \mathrm{~s}}\right) \\
\vdots \\
\mathbf{I}-\mathbf{J}_{\mathrm{Nns}} \cdot\left(\mathbf{J}_{\mathrm{Nns}}^{\mathrm{T}} \cdot \Delta\left(\mathbf{J}_{\mathrm{Ns}}\right) \cdot \mathbf{J}_{\mathrm{Nns}}\right)^{-1} \cdot \mathbf{J}_{\mathrm{Nns}}^{\mathrm{T}} \cdot \Delta\left(\mathbf{J}_{\mathrm{Ns}}\right)
\end{array}\right) \cdot \dot{\mathbf{p}}=\left(\begin{array}{cccc}
\mathbf{J}_{1 \mathrm{~s}} & 0 & \cdots & 0 \\
0 & \mathbf{J}_{2 \mathrm{~s}} & \ddots & \vdots \\
\vdots & \ddots & \ddots & 0 \\
0 & \cdots & 0 & \mathbf{J}_{\mathrm{Ns}}
\end{array}\right) \cdot \dot{\mathbf{q}}_{\mathrm{ws}} \\
\overline{\mathbf{I}}_{\mathrm{s}} \cdot \dot{\mathbf{p}}=\mathbf{J}_{\mathrm{s}} \cdot \dot{\mathbf{q}}_{\mathrm{ws}}
\end{gathered}
$$


Esta expresión recibe el nombre de ecuación de sensores del vehículo y al aplicarle el árbol de la Figura 4.1 se obtiene el árbol de sensorización de la Figura 4.5 .

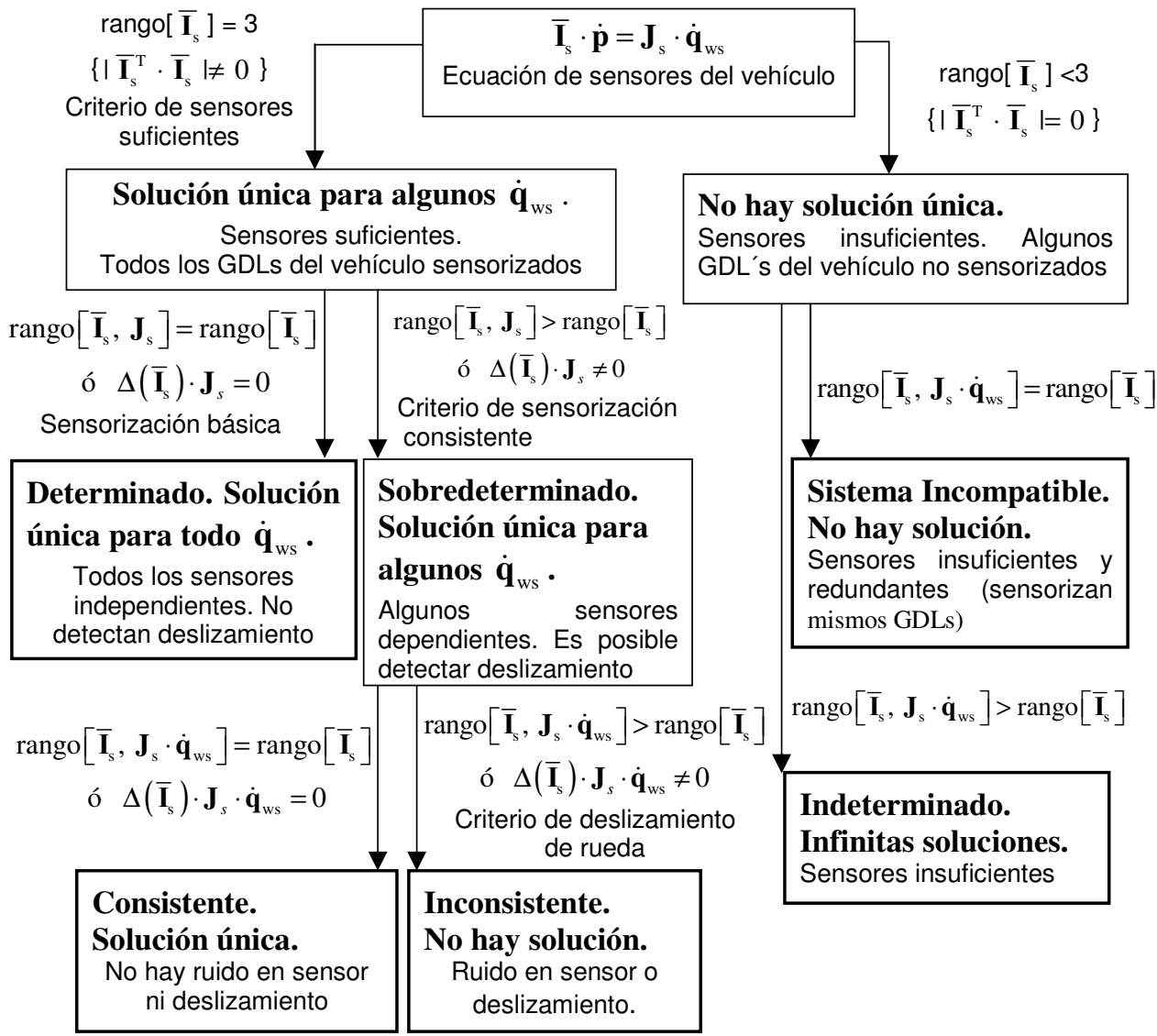

Figura 4.5- Árbol de caracterización de la sensorización del vehículo 


\subsubsection{Discusión del método de matrices Jacobianas de rueda}

Aquí se abordara de forma conceptual lo presentado en los seis subapartados anteriores, discutiendo la conveniencia de la forma de proceder según el caso.

En los subapartados de problema cinemático directo y problema cinemático inverso se utilizan diagramas en forma de árbol para evaluar: en un caso el número de velocidades de rueda que se pueden asignar independientemente (4.19); y en el otro el número máximo de velocidades del vehículo que se pueden asignar independientemente (4.22). Cabe destacar que:

- No se enfatiza que ambos valores, máximo número de velocidades de rueda y del vehiculo independientes, coinciden (Demostración 4.6 del subaparado 4.4.8), lo que pone de manifiesto una redundancia en el análisis. Además dichos valores coinciden con el grado de movilidad (Demostración 4.7 del subaparado 4.4.8).

+ Es de gran utilidad la simplificación que establece las restricciones al movimiento, ya que indica las restricciones que aporta cada rueda, lo que además implica una mayor simplicidad computacional. Esta simplificación es utilizada en el Capítulo 5 para clasificar los vehículos.

La solución inversa accionada calcula las velocidades de rueda, separando en accionadas y no accionadas, a partir de las de velocidad del vehículo (4.26). Cabe apuntar que:

- Las velocidades del vehículo en general no son independientes, ya que tienen las restricciones al movimiento indicadas en el problema cinemático inverso. Por tanto es una ecuación carente de sentido físico y de utilidad relativa.

+ La utilidad que se le da en [Muir et al. 87] a dicha ecuación es para expresar, en el apartado de árbol de accionamiento/sensorización, la ecuación de accionamiento/sensores del vehículo en función de las velocidades del vehículo y de las de rueda accionadas/sensorizadas.

- Lo anterior da elegancia en los árboles de actuación/sensorización, pero se puede realizar exactamente el mismo análisis en el diagrama manteniendo las velocidades de rueda no accionadas/sensorizadas junto con las del vehículo en un mismo miembro.

El árbol de accionamiento analiza la conveniencia (conflictos, insuficiencia) de una elección de velocidades de rueda como accionadas. Cabe destacar: 
- Como se ha comentado antes, no es necesario poner las velocidades de rueda no accionadas en función de las de postura para realizar el análisis.

+ Dicha sustitución, además de dar elegancia, hace que las matrices a invertir sean como máximo de $3 \times 3$, lo que es conveniente computacionalmente.

- No se explicita que la actuación consistente se consigue para un número de velocidades accionadas igual al número de velocidades del vehículo independientes $G$. Aunque a priori no se sabe cuáles pueden ser, acotaría bastante la búsqueda de una actuación consistente. Además eliminaría tres bloques, sin utilidad práctica, en el árbol de actuación.

La solución directa sensorizada establece los valores de las velocidades de rueda no sensorizadas y del vehículo a partir de las de rueda sensorizadas (4.31).

Mientras que el árbol de sensorización caracteriza la elección de sensores.

Como crítica a estos dos puntos:

- No se explicita que el número de sensores imprescindibles para calcular la solución directa sensorizada (reducida y/o completa) y para sensorizar todos los GDLs del vehículo coinciden con los GDLs del vehículo. Esto eliminaría tres bloques, sin utilidad práctica, en el árbol de actuación.

+ La solución directa reducida (4.32) presenta la ventaja computacional de invertir matrices de dimensión máxima $3 \times 3$.

- No se explicita que la única diferencia entre el árbol de actuación y de sensorización es el sentido conceptual atribuido a las velocidades de rueda elegidas, siendo incompatibles una actuación consistente y una sensorización consistente con las misma elección de velocidades.

+ El criterio de deslizamiento de rueda deja entrever la posibilidad, no sólo de cuantificar el deslizamiento por medio de la inecuación, sino realizar una estimación a través de la solución directa sensorizada de las velocidades del vehículo.

- El problema de lo anterior es la falta de sentido físico en la estimación.

Cabe indicar que la igualdad (4.10) es una generalización del criterio de actuación consistente y sensorización básica de los árboles de accionamiento y sensorización. 


\subsubsection{Demostraciones para el método de matrices Jacobianas de rueda}

Demostración 4.1: de la igualdad (4.20) teniendo en cuenta (4.13)

Sustituyendo en $\Delta(\mathbf{J}) \cdot \overline{\mathbf{I}}$ los valores indicados en (4.13), y además teniendo en cuenta (4.17), se obtiene:

$$
\begin{aligned}
& \Delta(\mathbf{J}) \cdot \overline{\mathbf{I}}=\left(\mathbf{J} \cdot\left(\mathbf{J}^{\mathrm{T}} \cdot \mathbf{J}\right)^{-1} \cdot \mathbf{J}^{\mathrm{T}}-\mathbf{I}\right) \cdot \overline{\mathbf{I}}= \\
& \left(\left(\begin{array}{cccc}
\mathbf{J}_{1} & 0 & \cdots & 0 \\
0 & \mathbf{J}_{2} & \ddots & \vdots \\
\vdots & \ddots & \ddots & 0 \\
0 & \cdots & 0 & \mathbf{J}_{\mathrm{N}}
\end{array}\right) \cdot\left(\left(\begin{array}{cccc}
\mathbf{J}_{1}^{\mathrm{T}} & 0 & \cdots & 0 \\
0 & \mathbf{J}_{2}^{\mathrm{T}} & \ddots & \vdots \\
\vdots & \ddots & \ddots & 0 \\
0 & \cdots & 0 & \mathbf{J}_{\mathrm{N}}^{\mathrm{T}}
\end{array}\right) \cdot\left(\begin{array}{cccc}
\mathbf{J}_{1} & 0 & \cdots & 0 \\
0 & \mathbf{J}_{2} & \ddots & \vdots \\
\vdots & \ddots & \ddots & 0 \\
0 & \cdots & 0 & \mathbf{J}_{\mathrm{N}}
\end{array}\right)\right)^{-1}\left(\begin{array}{cccc}
\mathbf{J}_{1}^{\mathrm{T}} & 0 & \cdots & 0 \\
0 & \mathbf{J}_{2}^{\mathrm{T}} & \ddots & \vdots \\
\vdots & \ddots & \ddots & 0 \\
0 & \cdots & 0 & \mathbf{J}_{\mathrm{N}}^{\mathrm{T}}
\end{array}\right)-\mathbf{I}\right) \cdot\left(\begin{array}{l}
\mathbf{I} \\
\mathbf{I} \\
\vdots \\
\mathbf{I}
\end{array}\right)
\end{aligned}
$$

Operando en la expresión anterior:

$$
\begin{aligned}
& \left(\left(\begin{array}{cccc}
\mathbf{J}_{1} & 0 & \cdots & 0 \\
0 & \mathbf{J}_{2} & \ddots & \vdots \\
\vdots & \ddots & \ddots & 0 \\
0 & \cdots & 0 & \mathbf{J}_{\mathrm{N}}
\end{array}\right) \cdot\left(\left(\left(\begin{array}{cccc}
\left.\mathbf{J}_{1}^{\mathrm{T}} \cdot \mathbf{J}_{1}\right) & 0 & \cdots & 0 \\
0 & \left(\mathbf{J}_{2}^{\mathrm{T}} \cdot \mathbf{J}_{2}\right) & \ddots & \vdots \\
\vdots & \ddots & \ddots & 0 \\
0 & \cdots & 0 & \left(\mathbf{J}_{\mathrm{N}}^{\mathrm{T}} \cdot \mathbf{J}_{\mathrm{N}}\right)
\end{array}\right)\right)^{-1}\left(\begin{array}{cccc}
\mathbf{J}_{1}^{\mathrm{T}} & 0 & \cdots & 0 \\
0 & \mathbf{J}_{2}^{\mathrm{T}} & \ddots & \vdots \\
\vdots & \ddots & \ddots & 0 \\
0 & \cdots & 0 & \mathbf{J}_{\mathrm{N}}^{\mathrm{T}}
\end{array}\right)-\mathbf{I}\right) \cdot\left(\begin{array}{c}
\mathbf{I} \\
\mathbf{I} \\
\vdots \\
\mathbf{I}
\end{array}\right)=\right. \\
& \left(\left(\begin{array}{cccc}
\mathbf{J}_{1} & 0 & \cdots & 0 \\
0 & \mathbf{J}_{2} & \ddots & \vdots \\
\vdots & \ddots & \ddots & 0 \\
0 & \cdots & 0 & \mathbf{J}_{N}
\end{array}\right) \cdot\left(\begin{array}{cccc}
\left(\mathbf{J}_{1}^{\mathrm{T}} \cdot \mathbf{J}_{\mathbf{1}}\right)^{-1} & 0 & \cdots & 0 \\
0 & \left(\mathbf{J}_{2}^{\mathrm{T}} \cdot \mathbf{J}_{2}\right)^{-1} & \ddots & \vdots \\
\vdots & \ddots & \ddots & 0 \\
0 & \cdots & 0 & \left(\mathbf{J}_{\mathrm{N}}^{\mathrm{T}} \cdot \mathbf{J}_{\mathrm{N}}\right)^{-1}
\end{array}\right) \cdot\left(\begin{array}{cccc}
\mathbf{J}_{1}^{\mathrm{T}} & 0 & \cdots & 0 \\
0 & \mathbf{J}_{2}^{\mathrm{T}} & \ddots & \vdots \\
\vdots & \ddots & \ddots & 0 \\
0 & \cdots & 0 & \mathbf{J}_{\mathrm{N}}^{\mathrm{T}}
\end{array}\right)-\left(\begin{array}{cccc}
\mathbf{I} & 0 & \cdots & 0 \\
0 & \mathbf{I} & \ddots & \vdots \\
\vdots & \ddots & \ddots & 0 \\
0 & \cdots & 0 & \mathbf{I}
\end{array}\right)\right) \cdot\left(\begin{array}{c}
\mathbf{I} \\
\mathbf{I} \\
\vdots \\
\mathbf{I}
\end{array}\right) \\
& =\left(\begin{array}{cccc}
\mathbf{J}_{1} \cdot\left(\mathbf{J}_{1}^{\mathrm{T}} \cdot \mathbf{J}_{1}\right)^{-1} \cdot \mathbf{J}_{1}^{\mathrm{T}}-\mathbf{I} & 0 & \cdots & 0 \\
0 & \mathbf{J}_{2} \cdot\left(\mathbf{J}_{2}^{\mathrm{T}} \cdot \mathbf{J}_{2}\right)^{-1} \cdot \mathbf{J}_{2}^{\mathrm{T}}-\mathbf{I} & \ddots & \vdots \\
\vdots & \ddots & \ddots & 0 \\
0 & \cdots & 0 & \mathbf{J}_{\mathrm{N}} \cdot\left(\mathbf{J}_{\mathrm{N}}^{\mathrm{T}} \cdot \mathbf{J}_{\mathrm{N}}\right)^{-1} \cdot \mathbf{J}_{\mathrm{N}}^{\mathrm{T}}-\mathbf{I}
\end{array}\right) \cdot\left(\begin{array}{c}
\mathbf{I} \\
\mathbf{I} \\
\vdots \\
\mathbf{I}
\end{array}\right)=\left(\begin{array}{c}
\Delta\left(\mathbf{J}_{1}\right) \\
\Delta\left(\mathbf{J}_{2}\right) \\
\vdots \\
\Delta\left(\mathbf{J}_{\mathrm{N}}\right)
\end{array}\right)
\end{aligned}
$$

Quedando así demostrada la igualdad (4.20). 
Demostración 4.2: obtención de (4.26) a partir de (4.25) y (4.24)

Se parte de (4.25) y se sustituyen $\mathbf{J}_{\mathrm{p}}$ y $\dot{\mathbf{q}}_{\mathrm{p}}$ según la notación de (4.24):

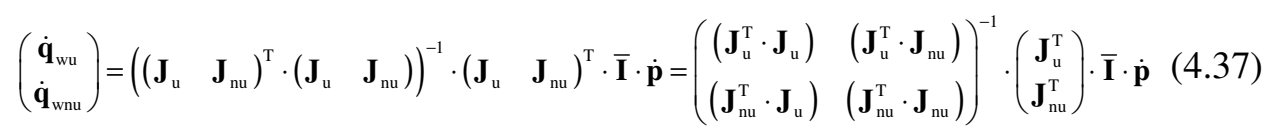

Para invertir la matriz de (4.37) se recurre al lema de inversión de matrices (ver Demostración 4.3):

$$
\left(\begin{array}{ll}
\mathbf{A}_{\mathrm{nxn}} & \mathbf{B}_{\mathrm{nxm}} \\
\mathbf{C}_{\mathrm{mxn}} & \mathbf{D}_{\mathrm{mxm}}
\end{array}\right)^{-1}=\left(\begin{array}{cc}
\left(\mathbf{A}-\mathbf{B} \cdot \mathbf{D}^{-1} \cdot \mathbf{C}\right)^{-1} & -\left(\mathbf{A}-\mathbf{B} \cdot \mathbf{D}^{-1} \cdot \mathbf{C}\right)^{-1} \cdot \mathbf{B} \cdot \mathbf{D}^{-1} \\
-\left(\mathbf{D}-\mathbf{C} \cdot \mathbf{A}^{-1} \cdot \mathbf{B}\right)^{-1} \cdot \mathbf{C} \cdot \mathbf{A}^{-1} & \left(\mathbf{D}-\mathbf{C} \cdot \mathbf{A}^{-1} \cdot \mathbf{B}\right)^{-1}
\end{array}\right)
$$

En primer lugar, se calculan por separado los elementos $(1,1)$ y $(2,2)$ de (4.38) para la matriz a invertir en (4.37):

$$
\begin{aligned}
& \left(\mathbf{A}-\mathbf{B} \cdot \mathbf{D}^{-1} \cdot \mathbf{C}\right)^{-1}=\left(\mathbf{J}_{\mathrm{u}}^{\mathrm{T}} \cdot \mathbf{J}_{\mathrm{u}}-\mathbf{J}_{\mathrm{u}}^{\mathrm{T}} \cdot \mathbf{J}_{\mathrm{nu}} \cdot\left(\mathbf{J}_{\mathrm{nu}}^{\mathrm{T}} \cdot \mathbf{J}_{\mathrm{nu}}\right)^{-1} \cdot \mathbf{J}_{\mathrm{nu}}^{\mathrm{T}} \cdot \mathbf{J}_{\mathrm{u}}\right)^{-1}= \\
& \left(\mathbf{J}_{\mathrm{u}}^{\mathrm{T}} \cdot\left(\mathbf{I}-\mathbf{J}_{\mathrm{nu}} \cdot\left(\mathbf{J}_{\mathrm{uu}}^{\mathrm{T}} \cdot \mathbf{J}_{\mathrm{nu}}\right)^{-1} \cdot \mathbf{J}_{\mathrm{nu}}^{\mathrm{T}}\right) \cdot \mathbf{J}_{\mathrm{u}}\right)^{-1}=-\left(\mathbf{J}_{\mathrm{u}}^{\mathrm{T}} \cdot \Delta\left(\mathbf{J}_{\mathrm{nu}}\right) \cdot \mathbf{J}_{\mathrm{u}}\right)^{-1} \\
& \left(\mathbf{D}-\mathbf{C} \cdot \mathbf{A}^{-1} \cdot \mathbf{B}\right)^{-1}=\left(\mathbf{J}_{\mathrm{nu}}^{\mathrm{T}} \cdot \mathbf{J}_{\mathrm{nu}}-\mathbf{J}_{\mathrm{nu}}^{\mathrm{T}} \cdot \mathbf{J}_{\mathrm{u}} \cdot\left(\mathbf{J}_{\mathrm{u}}^{\mathrm{T}} \cdot \mathbf{J}_{\mathrm{u}}\right)^{-1} \cdot \mathbf{J}_{\mathrm{u}}^{\mathrm{T}} \cdot \mathbf{J}_{\mathrm{nu}}\right)^{-1}= \\
& \left(\mathbf{J}_{\mathrm{uu}}^{\mathrm{T}} \cdot\left(\mathbf{I}-\mathbf{J}_{\mathrm{u}} \cdot\left(\mathbf{J}_{\mathrm{u}}^{\mathrm{T}} \cdot \mathbf{J}_{\mathrm{u}}\right)^{-1} \cdot \mathbf{J}_{\mathrm{u}}^{\mathrm{T}}\right) \cdot \mathbf{J}_{\mathrm{nu}}\right)^{-1}=-\left(\mathbf{J}_{\mathrm{nu}}^{\mathrm{T}} \cdot \Delta\left(\mathbf{J}_{\mathrm{u}}\right) \cdot \mathbf{J}_{\mathrm{nu}}\right)^{-1}
\end{aligned}
$$

Sustituyendo en (4.37) el lema de inversión (4.38), con los resultados de (4.39) y (4.40), se obtiene:

$$
\left(\begin{array}{c}
\dot{\mathbf{q}}_{\mathrm{wu}} \\
\dot{\mathbf{q}}_{\mathrm{wnu}}
\end{array}\right)=\left(\begin{array}{cc}
-\left(\mathbf{J}_{\mathrm{u}}^{\mathrm{T}} \cdot \Delta\left(\mathbf{J}_{\mathrm{nu}}\right) \cdot \mathbf{J}_{\mathrm{u}}\right)^{-1} & \left(\mathbf{J}_{\mathrm{u}}^{\mathrm{T}} \cdot \Delta\left(\mathbf{J}_{\mathrm{nu}}\right) \cdot \mathbf{J}_{\mathrm{u}}\right)^{-1} \cdot \mathbf{J}_{\mathrm{u}}^{\mathrm{T}} \cdot \mathbf{J}_{\mathrm{nu}} \cdot\left(\mathbf{J}_{\mathrm{nu}}^{\mathrm{T}} \cdot \mathbf{J}_{\mathrm{nu}}\right)^{-1} \\
\left(\mathbf{J}_{\mathrm{nu}}^{\mathrm{T}} \cdot \Delta\left(\mathbf{J}_{\mathrm{u}}\right) \cdot \mathbf{J}_{\mathrm{nu}}\right)^{-1} \cdot \mathbf{J}_{\mathrm{nu}}^{\mathrm{T}} \cdot \mathbf{J}_{\mathrm{u}} \cdot\left(\mathbf{J}_{\mathrm{u}}^{\mathrm{T}} \cdot \mathbf{J}_{\mathrm{u}}\right)^{-1} & -\left(\mathbf{J}_{\mathrm{nu}}^{\mathrm{T}} \cdot \Delta\left(\mathbf{J}_{\mathrm{u}}\right) \cdot \mathbf{J}_{\mathrm{nu}}\right)^{-1}
\end{array}\right) \cdot\left(\begin{array}{c}
\mathbf{J}_{\mathrm{u}}^{\mathrm{T}} \\
\mathbf{J}_{\mathrm{nu}}^{\mathrm{T}}
\end{array}\right) \cdot \overline{\mathbf{I}} \cdot \dot{\mathbf{p}}
$$

Operando en la expresión anterior:

$$
\left(\begin{array}{c}
\dot{\mathbf{q}}_{\mathrm{wu}} \\
\dot{\mathbf{q}}_{\mathrm{wu}}
\end{array}\right)=\left(\begin{array}{c}
\left(\mathbf{J}_{\mathrm{u}}^{\mathrm{T}} \cdot \Delta\left(\mathbf{J}_{\mathrm{nu}}\right) \cdot \mathbf{J}_{\mathrm{u}}\right)^{-1} \cdot \mathbf{J}_{\mathrm{u}}^{\mathrm{T}} \cdot\left(\mathbf{J}_{\mathrm{nu}} \cdot\left(\mathbf{J}_{\mathrm{nu}}^{\mathrm{T}} \cdot \mathbf{J}_{\mathrm{nu}}\right)^{-1} \cdot \mathbf{J}_{\mathrm{nu}}^{\mathrm{T}}-\mathbf{I}\right) \\
\left(\mathbf{J}_{\mathrm{nu}}^{\mathrm{T}} \cdot \Delta\left(\mathbf{J}_{\mathrm{u}}\right) \cdot \mathbf{J}_{\mathrm{nu}}\right)^{-1} \cdot \mathbf{J}_{\mathrm{nu}}^{\mathrm{T}} \cdot\left(\mathbf{J}_{\mathrm{u}} \cdot\left(\mathbf{J}_{\mathrm{u}}^{\mathrm{T}} \cdot \mathbf{J}_{\mathrm{u}}\right)^{-1} \cdot \mathbf{J}_{\mathrm{u}}^{\mathrm{T}}-\mathbf{I}\right)
\end{array}\right) \cdot \overline{\mathbf{I}} \cdot \dot{\mathbf{p}}=\left(\begin{array}{c}
\left(\mathbf{J}_{\mathrm{u}}^{\mathrm{T}} \cdot \Delta\left(\mathbf{J}_{\mathrm{nu}}\right) \cdot \mathbf{J}_{\mathrm{u}}\right)^{-1} \cdot \mathbf{J}_{\mathrm{u}}^{\mathrm{T}} \cdot \Delta\left(\mathbf{J}_{\mathrm{nu}}\right) \\
\left(\mathbf{J}_{\mathrm{nu}}^{\mathrm{T}} \cdot \Delta\left(\mathbf{J}_{\mathrm{u}}\right) \cdot \mathbf{J}_{\mathrm{nu}}\right)^{-1} \cdot \mathbf{J}_{\mathrm{nu}}^{\mathrm{T}} \cdot \Delta\left(\mathbf{J}_{\mathrm{u}}\right)
\end{array}\right) \cdot \overline{\mathbf{I}} \cdot \dot{\mathbf{p}}
$$

Por otro lado, teniendo en cuenta la Demostración 4.1: 


$$
\Delta(\mathbf{J})=\left(\begin{array}{cccc}
\Delta\left(\mathbf{J}_{1}\right) & 0 & \cdots & 0 \\
0 & \Delta\left(\mathbf{J}_{2}\right) & \ddots & \vdots \\
\vdots & \ddots & \ddots & 0 \\
0 & \cdots & 0 & \Delta\left(\mathbf{J}_{\mathrm{N}}\right)
\end{array}\right)
$$

Según la definición de matrices de (4.24) y la igualdad (4.43), (4.42) es:

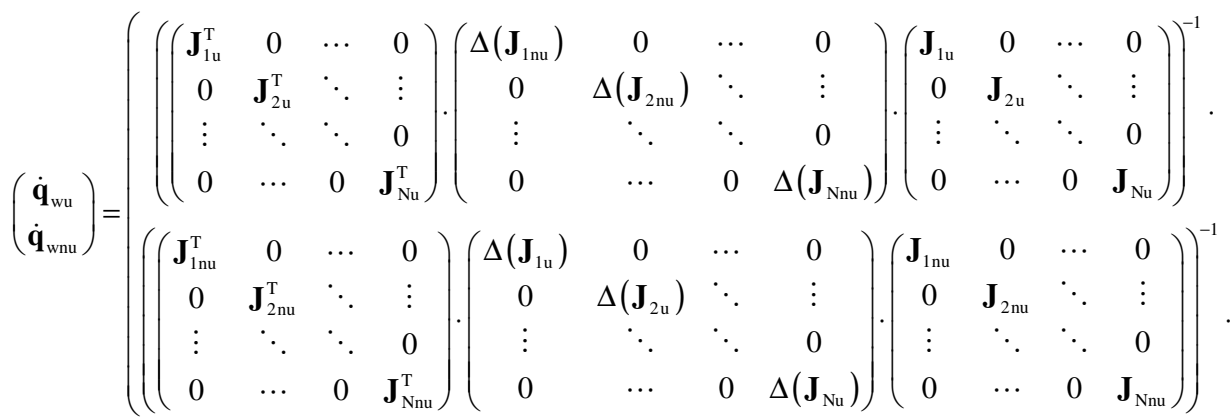

$$
\begin{aligned}
& \begin{array}{l}
\left.\left(\begin{array}{cccc}
\mathbf{J}_{1 \mathrm{u}}^{\mathrm{T}} & 0 & \cdots & 0 \\
0 & \mathbf{J}_{2 \mathrm{u}}^{\mathrm{T}} & \ddots & \vdots \\
\vdots & \ddots & \ddots & 0 \\
0 & \cdots & 0 & \mathbf{J}_{\mathrm{Nu}}^{\mathrm{T}}
\end{array}\right) \cdot\left(\begin{array}{cccc}
\Delta\left(\mathbf{J}_{\mathrm{1nu}}\right) & 0 & \cdots & 0 \\
0 & \Delta\left(\mathbf{J}_{2 \mathrm{nu}}\right) & \ddots & \vdots \\
\vdots & \ddots & \ddots & 0 \\
0 & \cdots & 0 & \Delta\left(\mathbf{J}_{\mathrm{Nuu}}\right.
\end{array}\right)\right) \cdot\left(\begin{array}{c}
\Delta \\
\mathbf{I} \\
\mathbf{I} \\
\vdots \\
\mathbf{I}
\end{array}\right) \cdot \dot{\mathbf{p}} \\
\left.\left(\begin{array}{cccc}
\mathbf{J}_{\text {1nu }}^{\mathrm{T}} & 0 & \cdots & 0 \\
0 & \mathbf{J}_{2 \mathrm{uu}}^{\mathrm{T}} & \ddots & \vdots \\
\vdots & \ddots & \ddots & 0 \\
0 & \cdots & 0 & \mathbf{J}_{\mathrm{Nnu}}^{\mathrm{T}}
\end{array}\right) \cdot\left(\begin{array}{cccc}
\Delta\left(\mathbf{J}_{1 \mathrm{u}}\right) & 0 & \cdots & 0 \\
0 & \Delta\left(\mathbf{J}_{2 \mathrm{u}}\right) & \ddots & \vdots \\
\vdots & \ddots & \ddots & 0 \\
0 & \cdots & 0 & \Delta\left(\mathbf{J}_{\mathrm{Nu}}\right.
\end{array}\right)\right)
\end{array} \\
& \text { (4.44) }
\end{aligned}
$$

Operando en la expresión anterior: 


$$
\begin{aligned}
& \left(\begin{array}{c}
\dot{\mathbf{q}}_{\mathrm{wu}} \\
\dot{\mathbf{q}}_{\mathrm{wnu}}
\end{array}\right)=\left(\begin{array}{cccc}
\left(\mathbf{J}_{1 \mathrm{u}}^{\mathrm{T}} \cdot \Delta\left(\mathbf{J}_{1 \mathrm{uu}}\right) \cdot \mathbf{J}_{1 \mathrm{u}}\right. & 0 & \cdots & 0 \\
0 & \mathbf{J}_{2 \mathrm{u}}^{\mathrm{T}} \cdot \Delta\left(\mathbf{J}_{2 \mathrm{uu}}\right) \cdot \mathbf{J}_{2 \mathrm{u}} & \ddots & \vdots \\
\vdots & \ddots & \ddots & 0 \\
0 & \cdots & 0 & \mathbf{J}_{\mathrm{Nu}}^{\mathrm{T}} \cdot \Delta\left(\mathbf{J}_{\mathrm{Nnu}}\right) \cdot \mathbf{J}_{\mathrm{Nu}}
\end{array}\right)^{-1} \cdot\left(\begin{array}{cccc}
\mathbf{J}_{1 \mathrm{u}}^{\mathrm{T}} & 0 & \cdots & 0 \\
0 & \mathbf{J}_{2 \mathrm{u}}^{\mathrm{T}} & \ddots & \vdots \\
\vdots & \ddots & \ddots & 0 \\
0 & \cdots & 0 & \mathbf{J}_{\mathrm{Nu}}^{\mathrm{T}}
\end{array}\right) .
\end{aligned}
$$

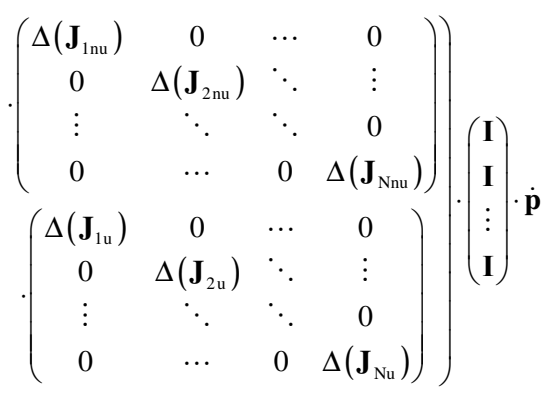

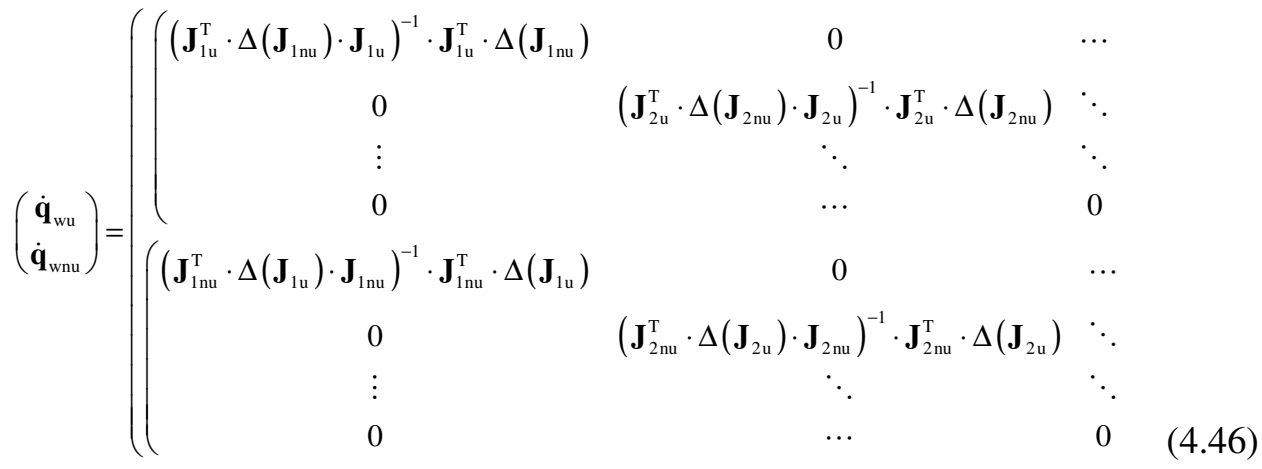

$$
\begin{aligned}
& \left.\begin{array}{c}
0 \\
\left(\mathbf{J}_{\mathrm{Nu}}^{\mathrm{T}} \cdot \Delta\left(\mathbf{J}_{\mathrm{Nnu}}\right) \cdot \mathbf{J}_{\mathrm{Nu}}\right)^{-1} \cdot \mathbf{J}_{\mathrm{Nu}}^{\mathrm{T}} \cdot \Delta\left(\mathbf{J}_{\mathrm{Nnu}}\right) \\
0 \\
\vdots \\
0 \\
\left(\mathbf{J}_{\mathrm{Nnu}}^{\mathrm{T}} \cdot \Delta\left(\mathbf{J}_{\mathrm{Nu}}\right) \cdot \mathbf{J}_{\mathrm{Nuu}}\right)^{-1} \cdot \mathbf{J}_{\mathrm{Nnu}}^{\mathrm{T}} \cdot \Delta\left(\mathbf{J}_{\mathrm{Nu}}\right)
\end{array}\right) \cdot\left(\begin{array}{c}
\mathbf{I} \\
\mathbf{I} \\
\vdots \\
\mathbf{I}
\end{array}\right) \cdot \dot{\mathbf{p}}
\end{aligned}
$$




$$
\left(\begin{array}{c}
\dot{\mathbf{q}}_{\mathrm{wu}} \\
\dot{\mathbf{q}}_{\mathrm{wnu}}
\end{array}\right)=\left(\begin{array}{c}
\left(\mathbf{J}_{1 \mathrm{u}}^{\mathrm{T}} \cdot \Delta\left(\mathbf{J}_{1 \mathrm{nu}}\right) \cdot \mathbf{J}_{1 \mathrm{u}}\right)^{-1} \cdot \mathbf{J}_{1 \mathrm{u}}^{\mathrm{T}} \cdot \Delta\left(\mathbf{J}_{1 \mathrm{nu}}\right) \\
\left(\mathbf{J}_{2 \mathrm{u}}^{\mathrm{T}} \cdot \Delta\left(\mathbf{J}_{2 \mathrm{nu}}\right) \cdot \mathbf{J}_{2 \mathrm{u}}\right)^{-1} \cdot \mathbf{J}_{2 \mathrm{u}}^{\mathrm{T}} \cdot \Delta\left(\mathbf{J}_{2 \mathrm{nu}}\right) \\
\vdots \\
\left(\mathbf{J}_{\mathrm{Nu}}^{\mathrm{T}} \cdot \Delta\left(\mathbf{J}_{\mathrm{Nnu}}\right) \cdot \mathbf{J}_{\mathrm{Nu}}\right)^{-1} \cdot \mathbf{J}_{\mathrm{Nu}}^{\mathrm{T}} \cdot \Delta\left(\mathbf{J}_{\mathrm{Nnu}}\right) \\
\left(\mathbf{J}_{1 \mathrm{nu}}^{\mathrm{T}} \cdot \Delta\left(\mathbf{J}_{1 \mathrm{u}}\right) \cdot \mathbf{J}_{1 \mathrm{nu}}\right)^{-1} \cdot \mathbf{J}_{1 \mathrm{nu}}^{\mathrm{T}} \cdot \Delta\left(\mathbf{J}_{1 \mathrm{u}}\right) \\
\left(\mathbf{J}_{2 \mathrm{nu}}^{\mathrm{T}} \cdot \Delta\left(\mathbf{J}_{2 \mathrm{u}}\right) \cdot \mathbf{J}_{2 \mathrm{nu}}\right)^{-1} \cdot \mathbf{J}_{2 \mathrm{uu}}^{\mathrm{T}} \cdot \Delta\left(\mathbf{J}_{2 \mathrm{u}}\right) \\
\vdots \\
\left(\mathbf{J}_{\mathrm{Nnu}}^{\mathrm{T}} \cdot \Delta\left(\mathbf{J}_{\mathrm{Nu}}\right) \cdot \mathbf{J}_{\mathrm{Nnu}}\right)^{-1} \cdot \mathbf{J}_{\mathrm{Nnu}}^{\mathrm{T}} \cdot \Delta\left(\mathbf{J}_{\mathrm{Nu}}\right)
\end{array}\right) \cdot \dot{\mathbf{p}}
$$

Llegándose a la expresión (4.26) C. Q. D.

\section{Demostración 4.3: del lema de inversión de matrices (4.38)}

Esta demostración va desglosarse en cuatro partes:

- En primer lugar se demostrará:

$$
\left(\begin{array}{cc}
\mathbf{A}_{\mathrm{nxn}} & \mathbf{B}_{\mathrm{nxm}} \\
\mathbf{0} & \mathbf{D}_{\mathrm{mxm}}
\end{array}\right)^{-1}=\left(\begin{array}{cc}
\mathbf{A}^{-1} & -\mathbf{A}^{-1} \cdot \mathbf{B} \cdot \mathbf{D}^{-1} \\
\mathbf{0} & \mathbf{D}^{-1}
\end{array}\right)
$$

Para ello se multiplica la matriz (sin invertir) del primer miembro de (4.48) por la del segundo miembro y se comprueba que el resultado es la identidad, como se muestra en (4.49), por tanto una es inversa de la otra.

$$
\left(\begin{array}{ll}
\mathbf{A} & \mathbf{B} \\
\mathbf{0} & \mathbf{D}
\end{array}\right) \cdot\left(\begin{array}{cc}
\mathbf{A}^{-1} & -\mathbf{A}^{-1} \cdot \mathbf{B} \cdot \mathbf{D}^{-1} \\
\mathbf{0} & \mathbf{D}^{-1}
\end{array}\right)=\left(\begin{array}{cc}
\mathbf{I}_{\mathrm{n}} & \mathbf{B} \cdot \mathbf{D}^{-1}-\mathbf{B} \cdot \mathbf{D}^{-1} \\
\mathbf{0} & \mathbf{I}_{\mathrm{m}}
\end{array}\right)=\left(\begin{array}{cc}
\mathbf{I}_{\mathrm{n}} & \mathbf{0} \\
\mathbf{0} & \mathbf{I}_{\mathrm{m}}
\end{array}\right)
$$

- A continuación se demostrará:

$$
\left(\begin{array}{cc}
\mathbf{A}_{\mathrm{nxn}} & \mathbf{0} \\
\mathbf{C}_{\mathrm{mxn}} & \mathbf{D}_{\mathrm{m} \times \mathrm{m}}
\end{array}\right)^{-1}=\left(\begin{array}{cc}
\mathbf{A}^{-1} & \mathbf{0} \\
-\mathbf{D}^{-1} \cdot \mathbf{C} \cdot \mathbf{A}^{-1} & \mathbf{D}^{-1}
\end{array}\right)
$$

Para ello se multiplica la matriz (sin invertir) del primer miembro de (4.50) por la del segundo miembro y se comprueba que el resultado es la identidad, como se muestra en (4.51), por tanto una es inversa de la otra. 


$$
\left(\begin{array}{ll}
\mathbf{A} & \mathbf{0} \\
\mathbf{C} & \mathbf{D}
\end{array}\right) \cdot\left(\begin{array}{cc}
\mathbf{A}^{-1} & \mathbf{0} \\
-\mathbf{D}^{-1} \cdot \mathbf{C} \cdot \mathbf{A}^{-1} & \mathbf{D}^{-1}
\end{array}\right)=\left(\begin{array}{cc}
\mathbf{I}_{\mathrm{n}} & \mathbf{0} \\
\mathbf{C} \cdot \mathbf{A}^{-1}-\mathbf{C} \cdot \mathbf{A}^{-1} & \mathbf{I}_{\mathrm{m}}
\end{array}\right)=\left(\begin{array}{cc}
\mathbf{I}_{\mathrm{n}} & \mathbf{0} \\
\mathbf{0} & \mathbf{I}_{\mathrm{m}}
\end{array}\right)
$$

- A continuación se demostrará:

$\left(\begin{array}{ll}\mathbf{A}_{\mathrm{nxn}} & \mathbf{B}_{\mathrm{nxm}} \\ \mathbf{C}_{\mathrm{m} \times n} & \mathbf{D}_{\mathrm{mxm}}\end{array}\right)^{-1}=\left(\begin{array}{cc}\mathbf{A}^{-1}+\mathbf{A}^{-1} \cdot \mathbf{B} \cdot\left(\mathbf{D}-\mathbf{C} \cdot \mathbf{A}^{-1} \cdot \mathbf{B}\right)^{-1} \cdot \mathbf{C} \cdot \mathbf{A}^{-1} & -\mathbf{A}^{-1} \cdot \mathbf{B} \cdot\left(\mathbf{D}-\mathbf{C} \cdot \mathbf{A}^{-1} \cdot \mathbf{B}\right)^{-1} \\ -\left(\mathbf{D}-\mathbf{C} \cdot \mathbf{A}^{-1} \cdot \mathbf{B}\right)^{-1} \cdot \mathbf{C} \cdot \mathbf{A}^{-1} & \left(\mathbf{D}-\mathbf{C} \cdot \mathbf{A}^{-1} \cdot \mathbf{B}\right)^{-1}\end{array}\right)$ (4.52)

Primero, observar que:

$$
\left(\begin{array}{ll}
\mathbf{A} & \mathbf{B} \\
\mathbf{C} & \mathbf{D}
\end{array}\right)=\left(\begin{array}{cc}
\mathbf{A} & \mathbf{0} \\
\mathbf{C} & \mathbf{I}_{\mathrm{m}}
\end{array}\right) \cdot\left(\begin{array}{cc}
\mathbf{I}_{\mathrm{n}} & \mathbf{A}^{-1} \cdot \mathbf{B} \\
\mathbf{0} & \left(\mathbf{D}-\mathbf{C} \cdot \mathbf{A}^{-1} \cdot \mathbf{B}\right)
\end{array}\right)
$$

Tomando la inversa a ambos lados de (4.53), utilizando (4.48) y (4.50) y operando resulta:

$$
\begin{aligned}
& \left(\begin{array}{ll}
\mathbf{A} & \mathbf{B} \\
\mathbf{C} & \mathbf{D}
\end{array}\right)^{-1}=\left(\begin{array}{cc}
\mathbf{I}_{\mathrm{n}} & \mathbf{A}^{-1} \cdot \mathbf{B} \\
\mathbf{0} & \left(\mathbf{D}-\mathbf{C} \cdot \mathbf{A}^{-1} \cdot \mathbf{B}\right)
\end{array}\right)^{-1} \cdot\left(\begin{array}{cc}
\mathbf{A} & \mathbf{0} \\
\mathbf{C} & \mathrm{I}_{\mathrm{m}}
\end{array}\right)^{-1}= \\
& \left(\begin{array}{cc}
\mathbf{I}_{\mathrm{n}} & -\mathbf{A}^{-1} \cdot \mathbf{B} \cdot\left(\mathbf{D}-\mathbf{C} \cdot \mathbf{A}^{-1} \cdot \mathbf{B}\right)^{-1} \\
\mathbf{0} & \left(\mathbf{D}-\mathbf{C} \cdot \mathbf{A}^{-1} \cdot \mathbf{B}\right)^{-1}
\end{array}\right) \cdot\left(\begin{array}{cc}
\mathbf{A}^{-1} & \mathbf{0} \\
-\mathbf{C} \cdot \mathbf{A}^{-1} & \mathbf{I}_{\mathrm{m}}
\end{array}\right)= \\
& \left(\begin{array}{cc}
\mathbf{A}^{-1}+\mathbf{A}^{-1} \cdot \mathbf{B} \cdot\left(\mathbf{D}-\mathbf{C} \cdot \mathbf{A}^{-1} \cdot \mathbf{B}\right)^{-1} \cdot \mathbf{C} \cdot \mathbf{A}^{-1} & -\mathbf{A}^{-1} \cdot \mathbf{B} \cdot\left(\mathbf{D}-\mathbf{C} \cdot \mathbf{A}^{-1} \cdot \mathbf{B}\right)^{-1} \\
-\left(\mathbf{D}-\mathbf{C} \cdot \mathbf{A}^{-1} \cdot \mathbf{B}\right)^{-1} \cdot \mathbf{C} \cdot \mathbf{A}^{-1} & \left(\mathbf{D}-\mathbf{C} \cdot \mathbf{A}^{-1} \cdot \mathbf{B}\right)^{-1}
\end{array}\right)
\end{aligned}
$$

Con lo cual queda demostrado (4.52).

- A continuación se demostrará:

$$
\left(\begin{array}{ll}
\mathbf{A}_{\mathrm{nxn}} & \mathbf{B}_{\mathrm{nxm}} \\
\mathbf{C}_{\mathrm{m} \times \mathrm{n}} & \mathbf{D}_{\mathrm{mxx}}
\end{array}\right)^{-1}=\left(\begin{array}{cc}
\left(\mathbf{A}-\mathbf{B} \cdot \mathbf{D}^{-1} \cdot \mathbf{C}\right)^{-1} & -\left(\mathbf{A}-\mathbf{B} \cdot \mathbf{D}^{-1} \cdot \mathbf{C}\right)^{-1} \cdot \mathbf{B} \cdot \mathbf{D}^{-1} \\
-\mathbf{D}^{-1} \cdot \mathbf{C} \cdot\left(\mathbf{A}-\mathbf{B} \cdot \mathbf{D}^{-1} \cdot \mathbf{C}\right)^{-1} & \mathbf{D}^{-1}+\mathbf{D}^{-1} \cdot \mathbf{C} \cdot\left(\mathbf{A}-\mathbf{B} \cdot \mathbf{D}^{-1} \cdot \mathbf{C}\right)^{-1} \cdot \mathbf{B} \cdot \mathbf{D}^{-1}
\end{array}\right)
$$

Primero, notar que:

$$
\left(\begin{array}{ll}
\mathbf{A} & \mathbf{B} \\
\mathbf{C} & \mathbf{D}
\end{array}\right)=\left(\begin{array}{cc}
\mathbf{I}_{\mathrm{n}} & \mathbf{B} \\
\mathbf{0} & \mathbf{D}
\end{array}\right) \cdot\left(\begin{array}{cc}
\mathbf{A}-\mathbf{B} \cdot \mathbf{D}^{-1} \cdot \mathbf{C} & \mathbf{0} \\
\mathbf{D}^{-1} \cdot \mathbf{C} & \mathbf{I}_{\mathrm{m}}
\end{array}\right)
$$


Tomando la inversa a ambos lados de (4.56), utilizando (4.48) y (4.50), y operando resulta:

$$
\begin{aligned}
& \left(\begin{array}{ll}
\mathbf{A} & \mathbf{B} \\
\mathbf{C} & \mathbf{D}
\end{array}\right)^{-1}=\left(\begin{array}{cc}
\left(\mathbf{A}-\mathbf{B} \cdot \mathbf{D}^{-1} \cdot \mathbf{C}\right) & \mathbf{0} \\
\mathbf{D}^{-1} \cdot \mathbf{C} & \mathbf{I}_{m}
\end{array}\right)^{-1} \cdot\left(\begin{array}{cc}
\mathbf{I}_{n} & \mathbf{B} \\
\mathbf{0} & \mathbf{D}
\end{array}\right)^{-1}= \\
& \left(\begin{array}{cc}
\left(\mathbf{A}-\mathbf{B} \cdot \mathbf{D}^{-1} \cdot \mathbf{C}\right)^{-1} & \mathbf{0} \\
-\mathbf{D}^{-1} \cdot \mathbf{C} \cdot\left(\mathbf{A}-\mathbf{B} \cdot \mathbf{D}^{-1} \cdot \mathbf{C}\right)^{-1} & \mathbf{I}_{m}
\end{array}\right) \cdot\left(\begin{array}{cc}
\mathbf{I}_{n} & -\mathbf{B} \cdot \mathbf{D}^{-1} \\
\mathbf{0} & \mathbf{D}^{-1}
\end{array}\right)= \\
& \left(\begin{array}{cc}
\left(\mathbf{A}-\mathbf{B} \cdot \mathbf{D}^{-1} \cdot \mathbf{C}\right)^{-1} & -\left(\mathbf{A}-\mathbf{B} \cdot \mathbf{D}^{-1} \cdot \mathbf{C}\right)^{-1} \cdot \mathbf{B} \cdot \mathbf{D}^{-1} \\
-\mathbf{D}^{-1} \cdot \mathbf{C} \cdot\left(\mathbf{A}-\mathbf{B} \cdot \mathbf{D}^{-1} \cdot \mathbf{C}\right)^{-1} & \mathbf{D}^{-1}+\mathbf{D}^{-1} \cdot \mathbf{C} \cdot\left(\mathbf{A}-\mathbf{B} \cdot \mathbf{D}^{-1} \cdot \mathbf{C}\right)^{-1} \cdot \mathbf{B} \cdot \mathbf{D}^{-1}
\end{array}\right)
\end{aligned}
$$

Con lo cual queda demostrado (4.55).

Por tanto, teniendo en cuenta (4.52) y (4.55):

$$
\begin{aligned}
& \left(\begin{array}{ll}
\mathbf{A} & \mathbf{B} \\
\mathbf{C} & \mathbf{D}
\end{array}\right)^{-1}=\left(\begin{array}{cc}
\mathbf{X} & \mathbf{Y} \\
\mathbf{W} & \mathbf{Z}
\end{array}\right) \\
& \left(\begin{array}{ll}
\mathbf{X} & \mathbf{Y} \\
\mathbf{W} & \mathbf{Z}
\end{array}\right)=\left(\begin{array}{cc}
\mathbf{A}^{-1}+\mathbf{A}^{-1} \cdot \mathbf{B} \cdot\left(\mathbf{D}-\mathbf{C} \cdot \mathbf{A}^{-1} \cdot \mathbf{B}\right)^{-1} \cdot \mathbf{C} \cdot \mathbf{A}^{-1} & -\mathbf{A}^{-1} \cdot \mathbf{B} \cdot\left(\mathbf{D}-\mathbf{C} \cdot \mathbf{A}^{-1} \cdot \mathbf{B}\right)^{-1} \\
-\left(\mathbf{D}-\mathbf{C} \cdot \mathbf{A}^{-1} \cdot \mathbf{B}\right)^{-1} \cdot \mathbf{C} \cdot \mathbf{A}^{-1} & \left(\mathbf{D}-\mathbf{C} \cdot \mathbf{A}^{-1} \cdot \mathbf{B}\right)^{-1}
\end{array}\right) \\
& \left(\begin{array}{cc}
\mathbf{X} & \mathbf{Y} \\
\mathbf{W} & \mathbf{Z}
\end{array}\right)=\left(\begin{array}{cc}
\left(\mathbf{A}-\mathbf{B} \cdot \mathbf{D}^{-1} \cdot \mathbf{C}\right)^{-1} & -\left(\mathbf{A}-\mathbf{B} \cdot \mathbf{D}^{-1} \cdot \mathbf{C}\right)^{-1} \cdot \mathbf{B} \cdot \mathbf{D}^{-1} \\
-\mathbf{D}^{-1} \cdot \mathbf{C} \cdot\left(\mathbf{A}-\mathbf{B} \cdot \mathbf{D}^{-1} \cdot \mathbf{C}\right)^{-1} & \mathbf{D}^{-1}+\mathbf{D}^{-1} \cdot \mathbf{C} \cdot\left(\mathbf{A}-\mathbf{B} \cdot \mathbf{D}^{-1} \cdot \mathbf{C}\right)^{-1} \cdot \mathbf{B} \cdot \mathbf{D}^{-1}
\end{array}\right)
\end{aligned}
$$

Así pues, tomando $\mathbf{W}$ y $\mathbf{Z}$ de (4.58) b $\mathbf{X}$ e $\mathbf{Y}$ de (4.58) :

$$
\left(\begin{array}{ll}
\mathbf{A} & \mathbf{B} \\
\mathbf{C} & \mathbf{D}
\end{array}\right)^{-1}=\left(\begin{array}{cc}
\mathbf{X} & \mathbf{Y} \\
\mathbf{W} & \mathbf{Z}
\end{array}\right)=\left(\begin{array}{cc}
\left(\mathbf{A}-\mathbf{B} \cdot \mathbf{D}^{-1} \cdot \mathbf{C}\right)^{-1} & -\left(\mathbf{A}-\mathbf{B} \cdot \mathbf{D}^{-1} \cdot \mathbf{C}\right)^{-1} \cdot \mathbf{B} \cdot \mathbf{D}^{-1} \\
-\left(\mathbf{D}-\mathbf{C} \cdot \mathbf{A}^{-1} \cdot \mathbf{B}\right)^{-1} \cdot \mathbf{C} \cdot \mathbf{A}^{-1} & \left(\mathbf{D}-\mathbf{C} \cdot \mathbf{A}^{-1} \cdot \mathbf{B}\right)^{-1}
\end{array}\right)
$$

Como se observa la igualdad anterior coincide con el lema (4.38) C. Q. D.

Demostración 4.4: de la ecuación (4.27) a partir de la (4.26) bajo el supuesto de rueda tipo omnidireccional (castor o sueca) y las tres variables accionadas (o las tres no accionadas)

En primer lugar $\mathbf{J}_{\text {inu }}$ es una matriz nula, para el caso de las tres variables accionadas, por lo que según (4.17):

$$
\Delta\left(\mathbf{J}_{\text {inu }}\right)=-\mathbf{I}
$$


A partir de (4.26), (4.60) y de que $\mathbf{J}_{\text {iu }}$ es una matriz cuadrada de $3 \times 3$, operando se obtiene:

$$
\begin{aligned}
& \dot{\mathbf{q}}_{\text {wiu }}=\left(\mathbf{J}_{i \mathrm{u}}^{\mathrm{T}} \cdot \Delta\left(\mathbf{J}_{i \mathrm{uu}}\right) \cdot \mathbf{J}_{i \mathrm{u}}\right)^{-1} \cdot \mathbf{J}_{i \mathrm{u}}^{\mathrm{T}} \cdot \Delta\left(\mathbf{J}_{i \mathrm{nu}}\right) \cdot \dot{\mathbf{p}}=-\left(-\mathbf{J}_{i \mathrm{u}}^{\mathrm{T}} \cdot \mathbf{J}_{i \mathrm{u}}\right)^{-1} \cdot \mathbf{J}_{i \mathrm{u}}^{\mathrm{T}} \cdot \dot{\mathbf{p}} \\
& \dot{\mathbf{q}}_{\mathrm{wiu}}=\mathbf{J}_{i \mathrm{u}}^{-1} \cdot\left(\mathbf{J}_{i \mathrm{u}}^{\mathrm{T}}\right)^{-1} \cdot \mathbf{J}_{i \mathrm{u}}^{\mathrm{T}} \cdot \dot{\mathbf{p}}=\mathbf{J}_{i \mathrm{u}}^{-1} \cdot \dot{\mathbf{p}}=\mathbf{J}_{i}^{-1} \cdot \dot{\mathbf{p}}
\end{aligned}
$$

que coincide con (4.27) C. Q. D.

Para el caso de las tres variables no accionadas la demostración anterior es válida sin más que cambiar los subíndices "u" por "nu" y "nu" por "u".

Demostración 4.5: de la ecuación (4.32) a partir de la (4.31) y las definiciones de (4.30)

Partiendo de la ecuación (4.31) y según la notación de (4.30):

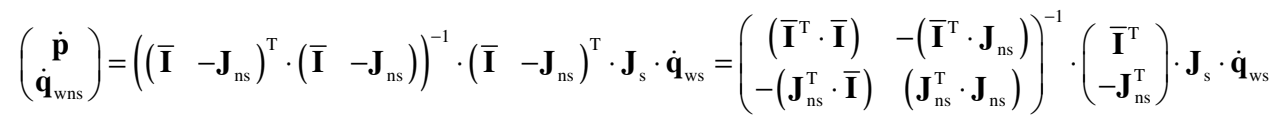

Para invertir la matriz que aparece en (4.62) se recurre al lema de inversión de matrices indicado en (4.59), probado en la Demostración 4.3.

Primero se calculan los elementos $(1,1)$ y $(2,2)$ de (4.59) para la matriz a invertir en (4.62):

$$
\begin{aligned}
& \left(\mathbf{A}-\mathbf{B} \cdot \mathbf{D}^{-1} \cdot \mathbf{C}\right)^{-1}=\left(\overline{\mathbf{I}}^{\mathrm{T}} \cdot \overline{\mathbf{I}}-\overline{\mathbf{I}}^{\mathrm{T}} \cdot \mathbf{J}_{\mathrm{ns}} \cdot\left(\mathbf{J}_{\mathrm{ns}}^{\mathrm{T}} \cdot \mathbf{J}_{\mathrm{ns}}\right)^{-1} \cdot \mathbf{J}_{\mathrm{ns}}^{\mathrm{T}} \cdot \overline{\mathbf{I}}\right)^{-1}= \\
& \left(\overline{\mathbf{I}}^{\mathrm{T}} \cdot\left(\overline{\mathbf{I}}-\mathbf{J}_{\mathrm{ns}} \cdot\left(\mathbf{J}_{\mathrm{ns}}^{\mathrm{T}} \cdot \mathbf{J}_{\mathrm{ns}}\right)^{-1} \cdot \mathbf{J}_{\mathrm{ns}}^{\mathrm{T}}\right) \cdot \overline{\mathbf{I}}\right)^{-1}=-\left(\overline{\mathbf{I}}^{\mathrm{T}} \cdot \Delta\left(\mathbf{J}_{\mathrm{ns}}\right) \cdot \overline{\mathbf{I}}\right)^{-1} \\
& \left(\mathbf{D}-\mathbf{C} \cdot \mathbf{A}^{-1} \cdot \mathbf{B}\right)^{-1}=\left(\mathbf{J}_{\mathrm{ns}}^{\mathrm{T}} \cdot \mathbf{J}_{\mathrm{ns}}-\mathbf{J}_{\mathrm{ns}}^{\mathrm{T}} \cdot \overline{\mathbf{I}} \cdot\left(\overline{\mathbf{I}}^{\mathrm{T}} \cdot \overline{\mathbf{I}}\right)^{-1} \cdot \overline{\mathbf{I}}^{\mathrm{T}} \cdot \mathbf{J}_{\mathrm{ns}}\right)^{-1}= \\
& \left(\mathbf{J}_{\mathrm{ns}}^{\mathrm{T}} \cdot\left(\overline{\mathbf{I}}-\overline{\mathbf{I}} \cdot\left(\overline{\mathbf{I}}^{\mathrm{T}} \cdot \overline{\mathbf{I}}\right)^{-1} \cdot \overline{\mathbf{I}}^{\mathrm{T}}\right) \cdot \mathbf{J}_{\mathrm{ns}}\right)^{-1}=-\left(\mathbf{J}_{\mathrm{ns}}^{\mathrm{T}} \cdot \Delta(\overline{\mathbf{I}}) \cdot \mathbf{J}_{\mathrm{ns}}\right)^{-1}
\end{aligned}
$$

Por tanto, teniendo en cuenta los resultados anteriores, (4.62) resulta: 
$\left(\begin{array}{c}\dot{\mathbf{p}} \\ \dot{\mathbf{q}}_{\mathrm{wns}}\end{array}\right)=\left(\begin{array}{cc}-\left(\overline{\mathbf{I}}^{\mathrm{T}} \cdot \Delta\left(\mathbf{J}_{\mathrm{ns}}\right) \cdot \overline{\mathbf{I}}\right)^{-1} & -\left(\overline{\mathbf{I}}^{\mathrm{T}} \cdot \Delta\left(\mathbf{J}_{\mathrm{ns}}\right) \cdot \overline{\mathbf{I}}\right)^{-1} \cdot \overline{\mathbf{I}}^{\mathrm{T}} \cdot \mathbf{J}_{\mathrm{ns}} \cdot\left(\mathbf{J}_{\mathrm{ns}}^{\mathrm{T}} \cdot \mathbf{J}_{\mathrm{ns}}\right)^{-1} \\ -\left(\mathbf{J}_{\mathrm{ns}}^{\mathrm{T}} \cdot \Delta(\overline{\mathbf{I}}) \cdot \mathbf{J}_{\mathrm{ns}}\right)^{-1} \cdot \mathbf{J}_{\mathrm{ns}}^{\mathrm{T}} \cdot \overline{\mathbf{I}} \cdot\left(\overline{\mathbf{I}}^{\mathrm{T}} \cdot \overline{\mathbf{I}}\right)^{-1} & -\left(\mathbf{J}_{\mathrm{ns}}^{\mathrm{T}} \cdot \Delta(\overline{\mathbf{I}}) \cdot \mathbf{J}_{\mathrm{ns}}\right)^{-1}\end{array}\right) \cdot\left(\begin{array}{c}\overline{\mathbf{I}}^{\mathrm{T}} \\ -\mathbf{J}_{\mathrm{ns}}^{\mathrm{T}}\end{array}\right) \cdot \mathbf{J}_{\mathrm{s}} \cdot \dot{\mathbf{q}}_{\mathrm{ws}}$

Operando en la expresión anterior:

$$
\left(\begin{array}{c}
\dot{\mathbf{p}} \\
\dot{\mathbf{q}}_{\mathrm{wns}}
\end{array}\right)=\left(\begin{array}{c}
\left(\overline{\mathbf{I}}^{\mathrm{T}} \cdot \Delta\left(\mathbf{J}_{\mathrm{ns}}\right) \cdot \overline{\mathbf{I}}\right)^{-1} \cdot \overline{\mathbf{I}}^{\mathrm{T}} \cdot\left(\mathbf{J}_{\mathrm{ns}} \cdot\left(\mathbf{J}_{\mathrm{ns}}^{\mathrm{T}} \cdot \mathbf{J}_{\mathrm{ns}}\right)^{-1} \cdot \mathbf{J}_{\mathrm{ns}}^{\mathrm{T}}-\mathbf{I}\right) \\
-\left(\mathbf{J}_{\mathrm{ns}}^{\mathrm{T}} \cdot \Delta(\overline{\mathbf{I}}) \cdot \mathbf{J}_{\mathrm{ns}}\right)^{-1} \cdot \mathbf{J}_{\mathrm{ns}}^{\mathrm{T}} \cdot\left(\overline{\mathbf{I}} \cdot\left(\overline{\mathbf{I}}^{\mathrm{T}} \cdot \overline{\mathbf{I}}\right)^{-1} \cdot \overline{\mathbf{I}}^{\mathrm{T}}-\mathbf{I}\right)
\end{array}\right) \cdot \mathbf{J}_{\mathrm{s}} \cdot \dot{\mathbf{q}}_{\mathrm{ws}}=\left(\begin{array}{c}
\left(\overline{\mathbf{I}}_{\mathrm{T}}^{\mathrm{T}} \cdot \Delta\left(\mathbf{J}_{\mathrm{ns}}\right) \cdot \overline{\mathbf{I}}\right)^{-1} \cdot \overline{\mathbf{I}}^{\mathrm{T}} \cdot \Delta\left(\mathbf{J}_{\mathrm{ns}}\right) \\
-\left(\mathbf{J}_{\mathrm{ns}}^{\mathrm{T}} \cdot \Delta(\overline{\mathbf{I}}) \cdot \mathbf{J}_{\mathrm{ns}}\right)^{-1} \cdot \mathbf{J}_{\mathrm{ns}}^{\mathrm{T}} \cdot \Delta(\overline{\mathbf{I}})
\end{array}\right) \cdot \mathbf{J}_{\mathrm{s}} \cdot \dot{\mathbf{q}}_{\mathrm{ws}}
$$

Analizando el elemento $\Delta(\overline{\mathbf{I}})$ de (4.65) se comprueba no simplificable:

$$
\begin{aligned}
& \left.\Delta(\overline{\mathbf{I}})=\overline{\mathbf{I}} \cdot\left(\overline{\mathbf{I}}^{\mathrm{T}} \cdot \overline{\mathbf{I}}\right)^{-1} \cdot \overline{\mathbf{I}}^{\mathrm{T}}-\mathbf{I}=\left(\begin{array}{l}
\mathbf{I} \\
\mathbf{I} \\
\vdots \\
\mathbf{I}
\end{array}\right) \cdot\left(\begin{array}{llll}
\mathbf{I} & \mathbf{I} & \ldots & \mathbf{I}
\end{array}\right) \cdot\left(\begin{array}{l}
\mathbf{I} \\
\mathbf{I} \\
\vdots \\
\mathbf{I}
\end{array}\right)\right)^{-1} \quad\left(\begin{array}{llll}
\mathbf{I} & \mathbf{I} & \ldots & \mathbf{I}
\end{array}\right)-\left(\begin{array}{cccc}
\mathbf{I} & 0 & 0 & 0 \\
0 & \mathbf{I} & 0 & 0 \\
0 & 0 & \ddots & 0 \\
0 & 0 & 0 & \mathbf{I}
\end{array}\right)
\end{aligned}
$$

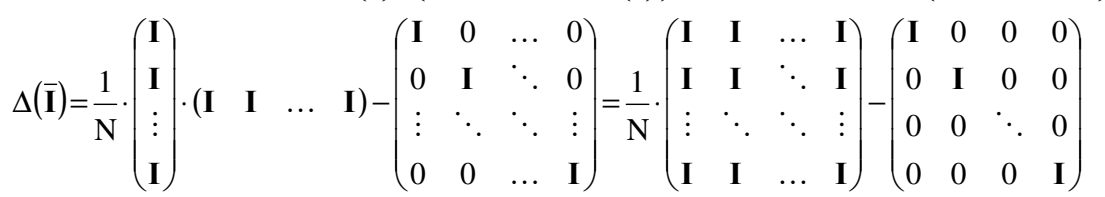

Así pues, sustituyendo en la primera fila de (4.65) las matrices por sus correspondientes valores, teniendo en cuenta (4.30), se tiene:

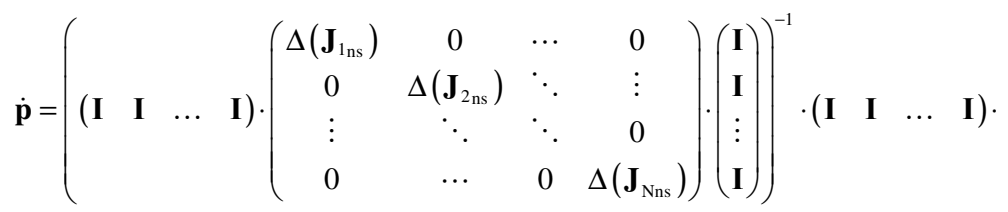

$$
\begin{aligned}
& \left(\begin{array}{cccc}
\Delta\left(\mathbf{J}_{1 \mathrm{~ns}}\right) & 0 & \cdots & 0 \\
0 & \Delta\left(\mathbf{J}_{2 \mathrm{~ns}}\right) & \ddots & \vdots \\
\vdots & \ddots & \ddots & 0 \\
0 & \cdots & 0 & \Delta\left(\mathbf{J}_{\mathrm{Nns}}\right)
\end{array}\right) \cdot\left(\begin{array}{cccc}
\mathbf{J}_{1 \mathrm{~s}} & 0 & \cdots & 0 \\
0 & \mathbf{J}_{2 \mathrm{~s}} & \ddots & \vdots \\
\vdots & \ddots & \ddots & 0 \\
0 & \cdots & 0 & \mathbf{J}_{\mathrm{Ns}}
\end{array}\right) \cdot \dot{\mathbf{q}}_{\mathrm{ws}} \\
& \dot{\mathbf{p}}=\left(\Delta\left(\mathbf{J}_{\text {lns }}\right)+\Delta\left(\mathbf{J}_{2 \mathrm{~ns}}\right)+\cdots+\Delta\left(\mathbf{J}_{\mathrm{Nns}}\right)\right)^{-1} \cdot\left(\Delta\left(\mathbf{J}_{\text {lns }}\right) \cdot \mathbf{J}_{1 \mathrm{~s}} \quad \Delta\left(\mathbf{J}_{2 \mathrm{~ns}}\right) \cdot \mathbf{J}_{2 \mathrm{~s}} \cdots \quad \Delta\left(\mathbf{J}_{\mathrm{Nns}}\right) \cdot \mathbf{J}_{\mathrm{Ns}}\right) \cdot \dot{\mathbf{q}}_{\mathrm{ws}}
\end{aligned}
$$

Expresión, esta última, que coincide con (4.32) C. Q. D. 
Demostración 4.6: de la coincidencia de las expresiones (4.19) y (4.22)

Se parte de (4.19):

$$
H=\operatorname{dim}\left(\dot{\mathbf{q}}_{\mathrm{w}}\right)-(\operatorname{rango}[\overline{\mathbf{I}}, \mathbf{J}]-\operatorname{rango}[\overline{\mathbf{I}}])
$$

Teniendo en cuenta que, $\mathbf{J}$ es de dimensión $(\mathrm{w}+\mathrm{h} \mathrm{x} w)$, con $\mathrm{h} \geq 0 \mathrm{y}$ $\mathrm{w}=\operatorname{dim}\left(\dot{\mathbf{q}}_{\mathrm{w}}\right)$, y que $\mathbf{J}$ es de rango completo, al estar formada por submatrices de rueda de rango completo (invertibles o pseudo invertibles), se cumple:

$$
\operatorname{rango}[\mathbf{J}]=\operatorname{dim}\left(\dot{\mathbf{q}}_{\mathrm{w}}\right)=\mathrm{w}
$$

donde $\dot{\mathbf{q}}_{\mathrm{w}}$ son las velocidades de rueda (incluyendo la velocidad angular del vehículo $\omega$ como velocidad en cada rueda).

Además, según la definición de $\overline{\mathbf{I}}$, se cumple:

$$
\operatorname{rango}[\overline{\mathbf{I}}]=3
$$

Así pues, sustituyendo (4.70) y (4.69) en (4.68) resulta:

$$
H=3-(\operatorname{rango}[\overline{\mathbf{I}}, \mathbf{J}]-\operatorname{rango}[\mathbf{J}])=3-(\operatorname{rango}[\mathbf{J}, \overline{\mathbf{I}}]-\operatorname{rango}[\mathbf{J}])=G
$$

Coincidiendo esta última expresión con (4.22) C. Q. D.

Demostración 4.7: de la coincidencia de la expresión (4.3) con (4.19) y (4.22)

La expresión (4.19) combinada con (4.69) es:

$$
\begin{aligned}
H=G & =\operatorname{rango}[\mathbf{J}]-(\operatorname{rango}[\mathbf{J}, \overline{\mathbf{I}}]-\operatorname{rango}[\overline{\mathbf{I}}]) \\
& =\operatorname{rango}[\mathbf{J}]+\operatorname{rango}[\overline{\mathbf{I}}]-\operatorname{rango}[\mathbf{J}, \overline{\mathbf{I}}]
\end{aligned}
$$

Según la definición de A en (4.1), se tiene:

$$
\operatorname{rango}[\overline{\mathbf{I}}, \mathbf{J}]=\operatorname{rango}[\mathbf{A}]+\mathrm{N}
$$

La $\mathrm{N}$ en la expresión anterior se debe a la ecuación identidad que, para cada rueda, relaciona la velocidad angular del vehículo $\omega$ en la matriz Jacobiana. 
Teniendo en cuenta (4.69) y (4.70) y la definición de $\dot{\mathbf{q}}$ en (4.1):

$$
\operatorname{rango}[\mathbf{J}]+\operatorname{rango}[\overline{\mathbf{I}}]=\operatorname{dim}\left(\dot{\mathbf{q}}_{\mathrm{w}}\right)+3=\operatorname{dim}(\dot{\mathbf{q}})+\mathrm{N}
$$

donde la $\mathrm{N}$ se debe a que el vector de velocidades de rueda $\dot{\mathbf{q}}_{\mathrm{w}}$ incluye la velocidad angular del vehículo $\omega$ como velocidad para cada rueda.

Sustituyendo (4.73) y (4.74) en (4.72), resulta:

$$
H=G=\operatorname{dim}(\dot{\mathbf{q}})-\operatorname{rango}(\mathbf{A})=m
$$

Coincidiendo esta última expresión con (4.3) C. Q. D. 


\subsection{SOLUCIÓN CINEMÁTICA AMPLIADA}

Además de lo expuesto en los tres métodos de modelado presentados, se va a dar una nueva perspectiva al número de velocidades, de rueda o del vehículo, que se pueden asignar libremente, manteniendo el no-deslizamiento.

La expresión (4.1) se puede rescribir como

$$
\mathbf{A}\left(\boldsymbol{\beta}_{\mathrm{o}}, \boldsymbol{\beta}_{\mathrm{c}}\right) \cdot \dot{\mathbf{q}}=\mathbf{0}
$$

donde $\boldsymbol{\beta}_{\mathrm{o}} / \boldsymbol{\beta}_{\mathrm{c}}$ son vectores con los ángulos de todas las ruedas orientables/castor.

Como se indico en el capítulo anterior, las velocidades de direccionamiento de las ruedas orientables $\dot{\beta}_{\text {oi }}$ no intervienen directamente en (4.76), al no incluirse en $\dot{\mathbf{q}}$, pero sí indirectamente al considerar la relación:

$$
\frac{\partial \boldsymbol{\beta}_{\mathrm{o}}}{\partial t}=\dot{\boldsymbol{\beta}}_{\mathrm{o}}
$$

Así pues, partiendo del supuesto de que se puede conseguir en cada instante el valor deseado en los ángulos $\boldsymbol{\beta}_{0}$, utilizando las velocidades, surge una variable más a asignar (GDL adicional) por cada rueda orientable.

Así, por cada rueda orientable en el vehículo asigna una velocidad adicional, de rueda o del vehículo, resultando la solución cinemática ampliada.

Para saber si un conjunto de velocidades (de rueda, de postura ó combinadas) puede ser asignado adicionalmente hay que comprobar que se puede cumplir su solución cinemática original a través de los ángulos correspondientes de las ruedas orientables.

Desde el punto de vista del control cinemático una velocidad puede cambiar de instantáneamente, pero para cumplir el supuesto anterior y conseguir los grados de libertad ampliados, surge la limitación (al no poderse dar valores infinitos a $\dot{\boldsymbol{\beta}}_{\mathrm{o}}$ ) de que las variables de velocidad $\dot{\mathbf{q}}$ cambien de forma continua.

Es decir, que las velocidades $\dot{\boldsymbol{\beta}}_{\text {o }}$ cambiarían de forma instantánea y el resto de velocidades de $\dot{\mathbf{q}}$ iy los ángulos $\boldsymbol{\beta}_{\mathrm{o}}$ ! de forma de forma continua.

No obstante, el control cinemático propuesto en un capítulo posterior presenta una estrategia más sencilla, conceptualmente y a nivel práctico, que utilizar soluciones cinemáticas ampliadas. 


\subsection{MODELADO Y CARACTERIZACIÓN DEL VEHÍCULO TRICICLO}

En la Figura 4.6 se puede apreciar el vehículo triciclo con la asignación de sistemas de coordenadas correspondiente.

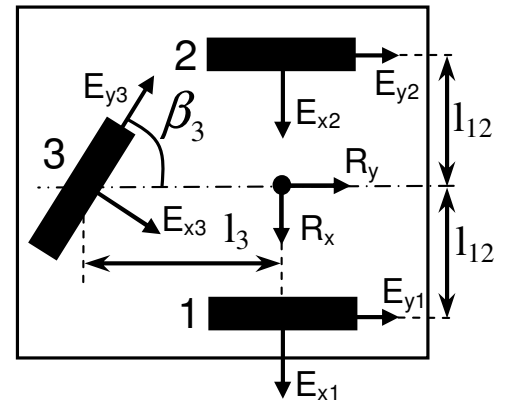

Figura 4.6- Asignación de sistemas de coordenadas en el vehículo triciclo

$\mathrm{Al}$ ser las ruedas 1 y 2 fijas: $\dot{\beta}_{1}=\dot{\beta}_{2}=0$.

La rueda 3 es orientable, por lo que su velocidad de direccionamiento no interviene en la cinemática instantánea.

Al no existir deslizamiento: $\mathbf{v}_{\text {slip 1 }}=\mathbf{v}_{\text {slip 2 }}=\mathbf{v}_{\text {slip 3 }}=\mathbf{0}$.

Las ecuaciones Jacobianas para cada rueda (utilizadas en el método desarrollado en el punto 4.4), utilizando (4.12), son:

$$
\begin{gathered}
\left(\begin{array}{c}
v_{x} \\
v_{y} \\
\omega
\end{array}\right)=\left(\begin{array}{cc}
0 & 0 \\
-\mathrm{r} & -1_{12} \\
0 & 1
\end{array}\right) \cdot\left(\begin{array}{c}
\dot{\varphi}_{1} \\
\omega
\end{array}\right)=\mathbf{J}_{1} \cdot \dot{\mathbf{q}}_{\mathrm{w} 1} \\
\left(\begin{array}{c}
v_{x} \\
v_{y} \\
\omega
\end{array}\right)=\left(\begin{array}{cc}
0 & 0 \\
-\mathrm{r} & 1_{12} \\
0 & 1
\end{array}\right) \cdot\left(\begin{array}{c}
\dot{\varphi}_{2} \\
\omega
\end{array}\right)=\mathbf{J}_{2} \cdot \dot{\mathbf{q}}_{\mathrm{w} 2} \\
\left(\begin{array}{c}
v_{x} \\
v_{y} \\
\omega
\end{array}\right)=\left(\begin{array}{cc}
\mathrm{r} \cdot \mathrm{s} \beta_{3} & -1_{3} \\
-\mathrm{r} \cdot \mathrm{c} \beta_{3} & 0 \\
0 & 1
\end{array}\right) \cdot\left(\begin{array}{c}
\dot{\varphi}_{3} \\
\omega
\end{array}\right)=\mathbf{J}_{3} \cdot \dot{\mathbf{q}}_{\mathrm{w} 3}
\end{gathered}
$$

La ecuación compuesta en la forma de (4.1), utilizada en los métodos de espacio nulo y rangos de matrices de los puntos 4.2 y 4.3 , es: 


$$
\left(\begin{array}{cccccc}
1 & 0 & 0 & 0 & 0 & 0 \\
0 & 1 & 1_{12} & \mathrm{r} & 0 & 0 \\
1 & 0 & 0 & 0 & 0 & 0 \\
0 & 1 & -1_{12} & 0 & \mathrm{r} & 0 \\
1 & 0 & 1_{3} & 0 & 0 & -\mathrm{r} \cdot \mathrm{s} \beta_{3} \\
0 & 1 & 0 & 0 & 0 & \mathrm{r} \cdot \mathrm{c} \beta_{3}
\end{array}\right) \cdot\left(\begin{array}{c}
v_{x} \\
v_{y} \\
\omega \\
\dot{\varphi}_{1} \\
\dot{\varphi}_{2} \\
\dot{\varphi}_{3}
\end{array}\right)=\left(\begin{array}{l}
0 \\
0 \\
0 \\
0 \\
0 \\
0
\end{array}\right) \rightarrow \mathbf{A} \cdot \dot{\mathbf{q}}=\mathbf{0}
$$

Calculando el grado de movilidad del vehículo con (4.3):

$$
m=\operatorname{dim}(\boldsymbol{\eta})=\operatorname{dim}(\mathcal{N}(\mathbf{A}))=\operatorname{dim}(\dot{\mathbf{q}})-\operatorname{rango}(\mathbf{A})=6-5=1
$$

Así, se dispone de 1 grado de movilidad para ser asignado a alguna de las velocidades, bien del vehículo (solución directa) o de rueda (solución inversa). Este resultado se obtiene también con los árboles de las Figuras 4.2 y 4.3.

Para establecer que variables pueden funcionar como asignadas se comprueba si al eliminar sus columnas de la matriz A, la nueva matriz tiene el mismo rango que la original (4.10). Procediendo se llega así se llega a que sólo la velocidad $v_{x}$ no puede funcionar como asignada.

Con el árbol de accionamiento de la Figura 4.4 se obtiene un accionamiento consistente actuando sobre cualquiera de las tres velocidades de rotación de rueda. Análogamente, aplicando el árbol de sensorización de la Figura 4.5, resulta una sensorización consistente para cualquier elección de velocidades de rotación de rueda que incluya al menos dos de ellas.

Ambos resultados concuerdan con lo obtenido en el análisis de rangos: todas las velocidades de rotación de rueda pueden funcionar como asignadas para que haya solución única (actuación consistente) y para detectar deslizamiento (sensorización consistente) basta con tomar al menos dos de ellas.

Para obtener los modelos cinemáticos directos e inversos se utilizará el algoritmo de cálculo del espacio nulo de A repetidamente, dejando cada vez una columna distinta, correspondiente a cada variable, al final de dicha matriz. El resultado obtenido es (4.80), donde se ratifica que la variable $v_{x}$ no puede funcionar como asignada. 


\begin{tabular}{|c|c|c|c|c|c|c|c|c|}
\hline$\left(\begin{array}{c}v_{y} \\
\omega \\
\dot{\varphi}_{1} \\
\dot{\varphi}_{2} \\
\dot{\varphi}_{3} \\
v_{x}\end{array}\right)=$ & $\left(\begin{array}{c}-\mathrm{r} \cdot \mathrm{c} \beta_{3} \\
\mathrm{r} \cdot \mathrm{s} \beta_{3} / 1_{3} \\
\frac{l_{3} \cdot \mathrm{c} \beta_{3}-1_{12} \cdot \mathrm{s} \beta_{3}}{M} \\
\frac{1_{3} \cdot \mathrm{c} \beta_{3}+1_{12} \cdot \mathrm{s} \beta_{3}}{1_{3}} \\
1 \\
0\end{array}\right.$ & $\cdot \eta$ & $\left(\begin{array}{c}v_{x} \\
\omega \\
\dot{\varphi}_{1} \\
\dot{\varphi}_{2} \\
\dot{\varphi}_{3} \\
v_{y}\end{array}\right)=$ & $\begin{array}{c}0 \\
-\tan \left(\beta_{3}\right) / 1_{3} \\
-1_{3}+1_{12} \cdot \tan \left(\beta_{3}\right) \\
1_{3} \cdot r \\
-1_{3}-1_{12} \cdot \tan \left(\beta_{3}\right) \\
1_{3} \cdot r \\
-\sec \left(\beta_{3}\right) / \mathrm{r} \\
1\end{array}$ & $\eta \eta$ & $\left(\begin{array}{c}v_{x} \\
v_{y} \\
\dot{\varphi}_{1} \\
\dot{\varphi}_{2} \\
\dot{\varphi}_{3} \\
\omega\end{array}\right)=$ & $\left(\begin{array}{c}0 \\
-1_{3} \cdot \cot \left(\beta_{3}\right) \\
\frac{-1_{12}+1_{3} \cdot \cot \left(\beta_{3}\right)}{\mathrm{r}} \\
\frac{1_{12}+1_{3} \cdot \cot \left(\beta_{3}\right)}{\mathrm{r}} \\
\frac{1_{3}}{\mathrm{r} \cdot \mathrm{s} \beta_{3}} \\
1\end{array}\right)$ & $\eta \eta$ \\
\hline$\left(\begin{array}{c}v_{x} \\
v_{y} \\
\omega \\
\dot{\varphi}_{2} \\
\dot{\varphi}_{3} \\
\dot{\varphi}_{1}\end{array}\right)=$ & $\left(\begin{array}{c}0 \\
\frac{\mathrm{r} \cdot 1_{3} \cdot \mathrm{c} \beta_{3}}{-1_{3} \cdot \mathrm{c} \beta_{3}+L \cdot \mathrm{s} \beta_{3}} \\
\frac{\mathrm{r} \cdot \mathrm{s} \beta_{3}}{1_{3} \cdot \mathrm{c} \beta_{3}-1_{12} \cdot \mathrm{s} \beta_{3}} \\
\frac{1_{3} \cdot \mathrm{c} \beta_{3}+1_{12} \cdot \mathrm{s} \beta_{3}}{1_{3} \cdot \mathrm{c} \beta_{3}-1_{12} \cdot \mathrm{s} \beta_{3}} \\
\frac{1_{3}}{1_{3} \cdot \mathrm{c} \beta_{3}-1_{12} \cdot \mathrm{s} \beta_{3}} \\
1\end{array}\right.$ & $\cdot \eta$ & $\left(\begin{array}{c}v_{x} \\
v_{y} \\
\omega \\
\dot{\varphi}_{1} \\
\dot{\varphi}_{3} \\
\dot{\varphi}_{2}\end{array}\right)=$ & $\left(\begin{array}{c}0 \\
\frac{-\mathrm{r} \cdot 1_{3} \cdot \mathrm{c} \beta_{3}}{1_{3} \cdot \mathrm{c} \beta_{3}+1_{12} \cdot \mathrm{s} \beta_{3}} \\
\frac{\mathrm{r} \cdot \mathrm{s} \beta_{3}}{\mathrm{l}_{3} \cdot \mathrm{c} \beta_{3}+1_{12} \cdot \mathrm{s} \beta_{3}} \\
\frac{1_{3} \cdot \mathrm{c} \beta_{3}-1_{12} \cdot \mathrm{s} \beta_{3}}{1_{3} \cdot \mathrm{c} \beta_{3}+1_{12} \cdot \mathrm{s} \beta_{3}} \\
\frac{1_{3}}{1_{3} \cdot \mathrm{c} \beta_{3}+1_{12} \cdot \mathrm{s} \beta_{3}} \\
1\end{array}\right.$ & $\cdot \eta$ & $\left(\begin{array}{c}v_{x} \\
v_{y} \\
\omega \\
\dot{\varphi}_{1} \\
\dot{\varphi}_{2} \\
\dot{\varphi}_{3}\end{array}\right)=$ & $\left(\begin{array}{c}0 \\
-\mathrm{r} \cdot \mathrm{c} \beta_{3} \\
\frac{\mathrm{r} \cdot \mathrm{s} \beta_{3}}{1_{3}} \\
\frac{\mathrm{l}_{3} \cdot \mathrm{c} \beta_{3}-\mathrm{l}_{12} \cdot \mathrm{s} \beta_{3}}{1_{3}} \\
\frac{\mathrm{l}_{3} \cdot \mathrm{c} \beta_{3}+1_{12} \cdot \mathrm{s} \beta_{3}}{1_{3}} \\
1\end{array}\right)$ & $\eta$ \\
\hline
\end{tabular}

Sustituyendo el grado de movilidad del vehículo por las distintas velocidades de rueda o del vehículo se obtienen los modelos cinemáticos directos o inversos de (4.81), teniendo estas velocidades la connotación de accionamiento independiente o GDL en el movimiento del vehículo.

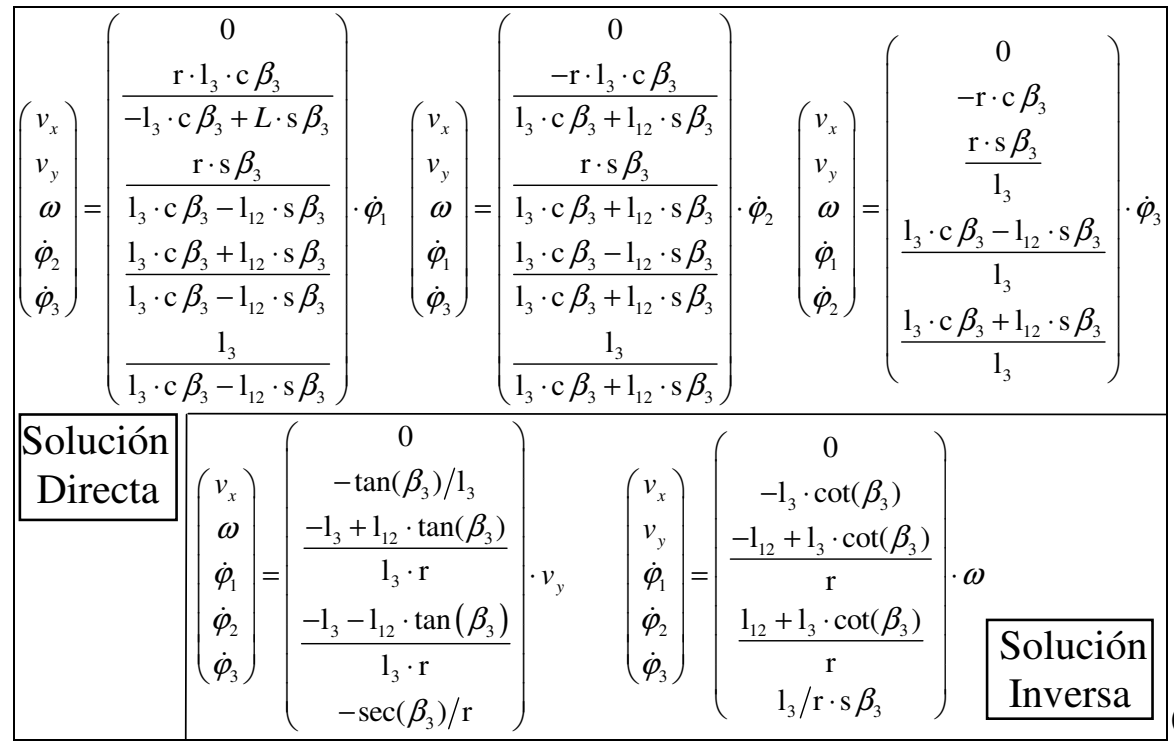


A las soluciones directas e inversas de (4.81) se llega igualmente con la ecuación (4.11) del método de rango de matrices, y a las directas reducidas (sólo velocidades del vehículo) con la ecuación (4.32) del método de matrices Jacobianas de rueda.

Desde el punto de vista de la solución cinemática ampliada, al tener una rueda orientable, se puede asignar una velocidad más, y según (4.81) todos las variables menos $v_{x}$ pueden funcionar como asignada adicional. En particular, haciendo pertenecer las dos variables a asignar al grupo de velocidades de ruedas (solución directa ampliada) o del vehículo (solución inversa ampliada), para un mayor sentido físico, y aplicando (4.11), se obtienen las soluciones de (4.82).

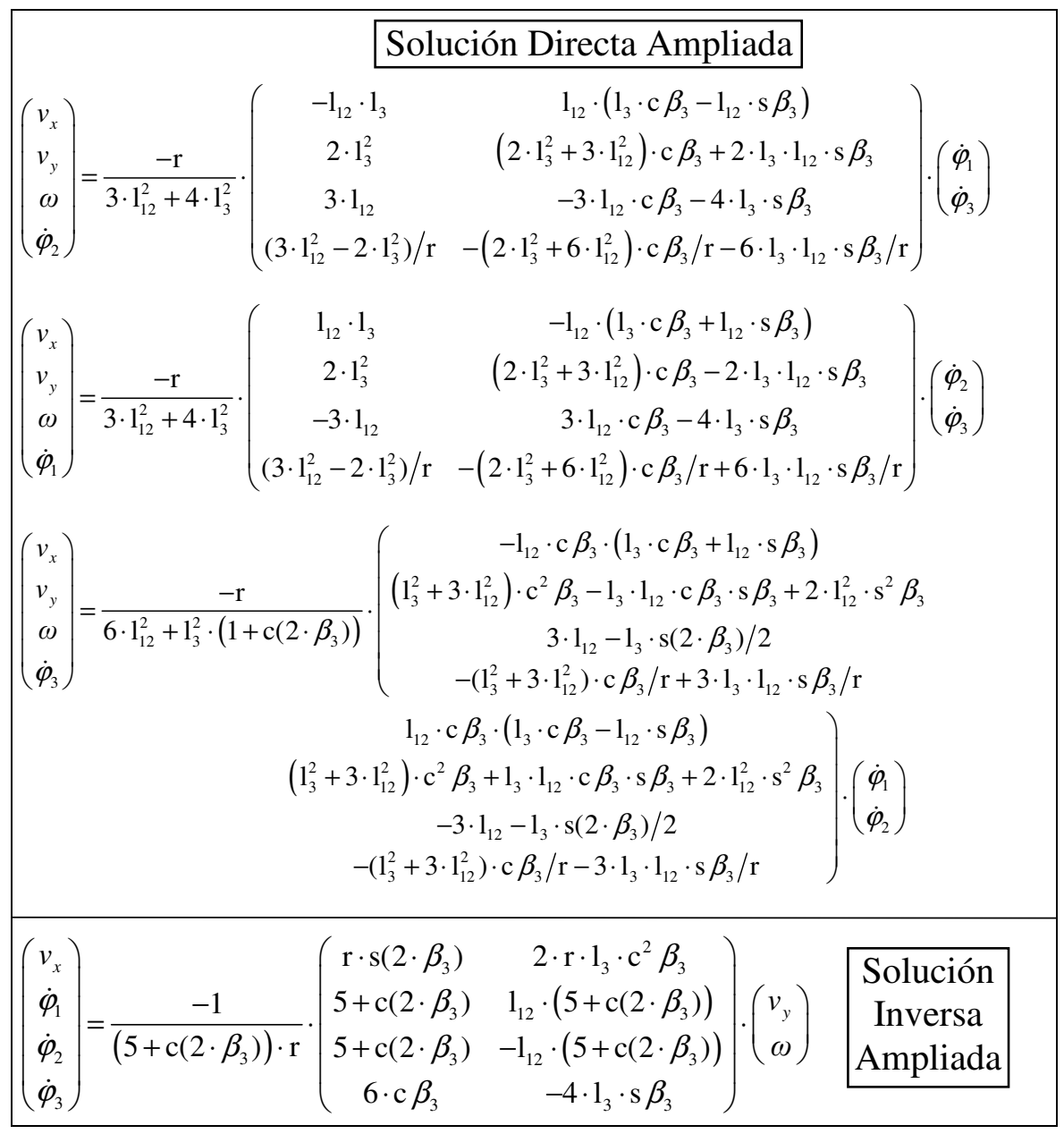


Las condiciones a cumplir por el ángulo de la rueda orientable en las soluciones ampliadas, obtenidas a partir la relación en (4.81) entre las dos variables que asignemos en (4.82), se muestran en (4.83).

\section{Condiciones para las Soluciones Ampliadas}

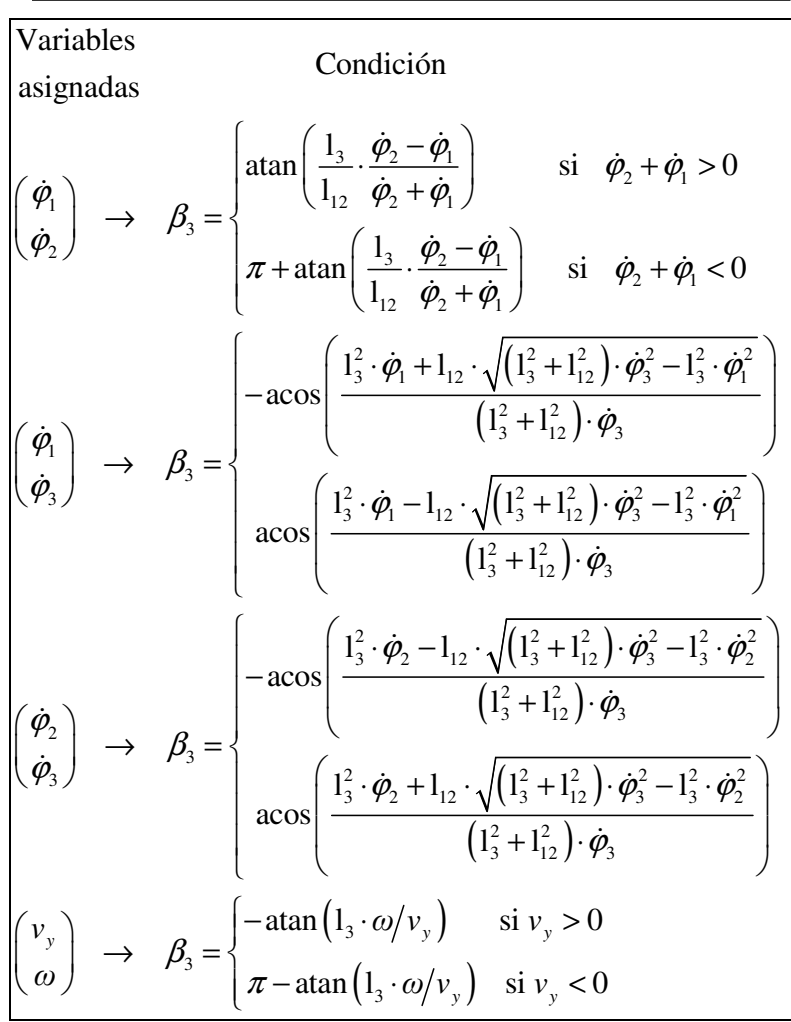

En (4.84) se indica como queda (4.82) al sustituir (en caso de que se consiga cierta simplificación) el valor de $\beta_{3}$ de (4.83). 


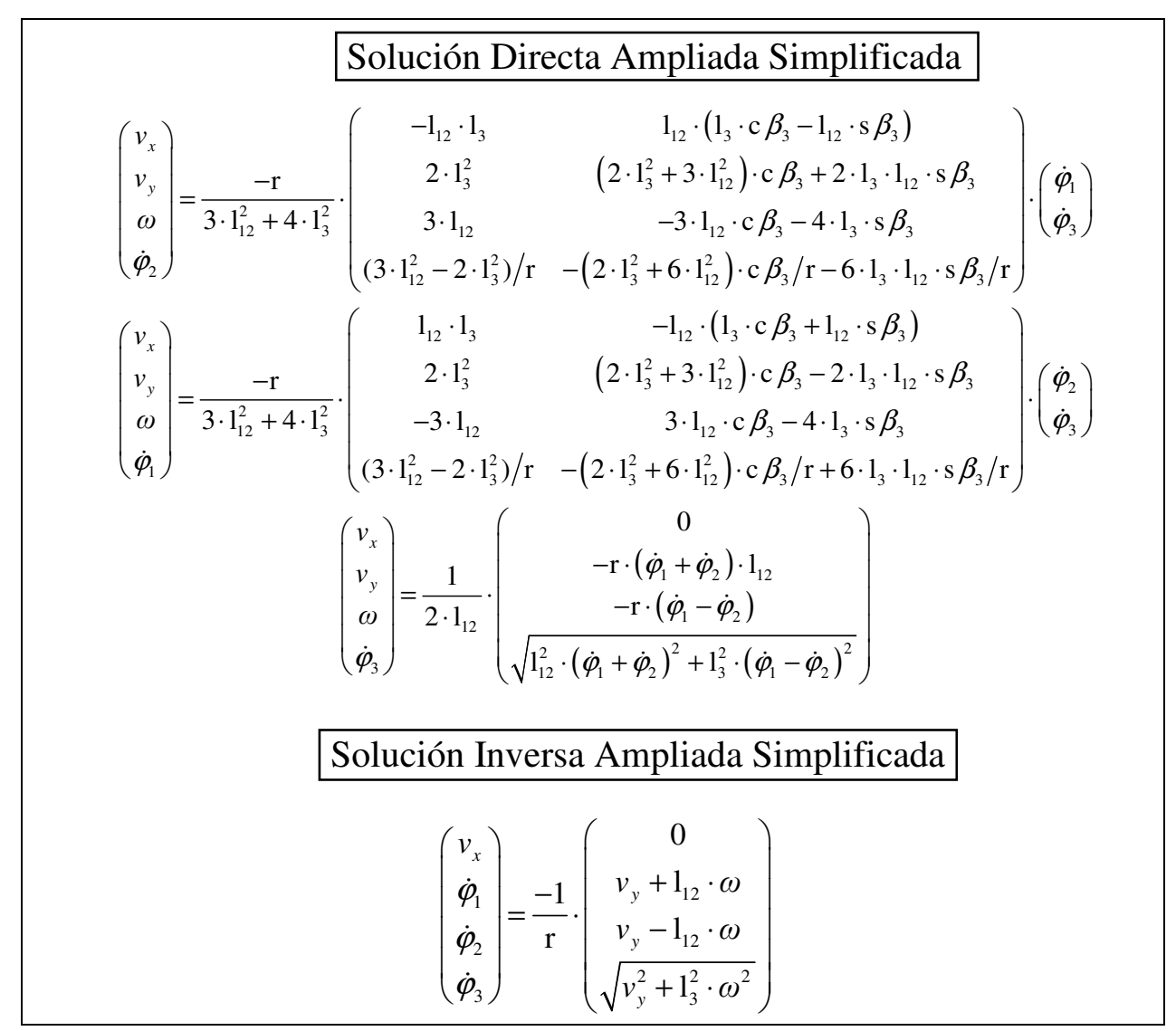

(4.84) 


\subsection{RESULTADOS MÁS RELEVANTES Y CONCLUSIONES DEL CAPÍTULO}

A continuación, se indican los aspectos más relevantes que aporta el capítulo.

- El método de modelado de espacio nulo es el más rápido, puesto que calcula los grados de libertad del vehículo a la vez que realiza la asignación de variables.

- El método de modelado basado en el espacio análisis de rangos simplifica conceptualmente el de matrices Jacobianas de rueda, aunque éste mantiene su interés computacional.

- Respecto al método de modelado desarrollado en [Muir et al. 87] se realizan las siguientes mejoras:

1) Las matrices Jacobianas de rueda utilizadas permiten siempre el análisis correspondiente, a diferencia de las propuestas en [Muir et al. 87] que se imposibilita en presencia de ruedas orientables.

2) Se contempla el análisis con un tipo de rueda más: la sueca.

3) Las pruebas de ramificación en los árboles se han duplicado, utilizando para ello el método de análisis de rangos de matrices.

- Se pone de manifiesto, tanto en el método de modelado de espacio nulo como en el de análisis de rangos, que no sólo hay soluciones cinemáticas directas e inversas (aunque puedan tener mayor utilidad) sino que también hay mixtas (con variables de velocidad de postura y de rueda conjuntamente como GDLs). Esto difiere del planteamiento tradicional.

- La solución cinemática ampliada propuesta evidencia una importante realidad práctica al trabajar con ruedas orientables, pasada por alto en otros estudios.

- El modelado del vehículo tipo triciclo ilustra los métodos descritos en los apartados anteriores y verifica los paralelismos y conexiones entre ellos.

- Todas las demostraciones del subapartado 4.4.8 son originales, a excepción del lema de inversión de matrices (Demostración 4.3). 


\section{CAPÍTULO 5 \\ MODELOS CINEMÁTICOS DE VEHÍCULOS Y TRASMISIÓN DE ERRORES (ISOTROPÍA)}

\subsection{INTRODUCCIÓN}

Existe un gran número de posibilidades en la configuración (ejes, ruedas..) de los posibles vehículos, como demuestra la abundante bibliografía ([Whittaker 62] [Everett 79] [Iijima et al. 88] [Helmers 85] [Rogers 84]).

No obstante es posible clasificar todos ellos, tal como se hace en [Campion et al. 96], en cinco tipos genéricos a partir de las ecuaciones aportadas por las ruedas y los conceptos de movilidad, direccionabilidad, maniobrabilidad y vehículo no degenerado.

En dicho estudio se obtienen los modelos cinemáticos de los cinco tipos de vehículos. El problema de estos modelos es que utilizan variables sin sentido físico como entradas, debido a emplear sólo parte del modelo (primer elemento de la ecuación (3.11)), además de no contemplar modelos inversos.

Por otra parte la transmisión de errores en los modelos cinemáticos directos o inversos, cuando se considera un error de entrada, ha sido estudiada por distintos autores a través de la caracterización de matrices isotrópicas. 
Por ejemplo, [Saha et al. 95] establece las condiciones de isotropía para un vehículo genérico con ruedas suecas, y las particulariza para el caso de tres y cuatro ruedas. Mientras que en [Low et al. 05] [Kim2 et al. 04] [Kim et al. 05] se estudian las condiciones de isotropía para un vehículo omnidireccional con tres ruedas castor.

Otros autores han estudiado la transmisión de errores desde un punto de vista empírico. Por ejemplo, en [Borenstein et al. 94] se presenta un método para medir, comparar y corregir errores sistemáticos en la cinemática de vehículos. Este mismo autor presenta en [Borenstein 95] el concepto compliant linkage (enlace de consistencia) para el control y diseño cinemático de robots. Este concepto se basa en dar consistencia a la actuación ajustando los errores del control para que no se produzca deslizamiento.

También, en [Kleeman 95] se realiza una estimación de la covarianza del error de odometría (modelo cinemático) para el vehículo diferencial (apartado 5.5.2). De forma similar en [Martinelli 02] se evalúa el error odométrico para el vehículo diferencial y síncrono (apartado 5.5.3) con objeto de establecer los parámetros del modelo de error tanto sistemático como no sistemático.

Así pues, el objetivo de este capítulo es proporcionar una completa guía sobre los modelos cinemáticos de vehículos y su transmisión de errores (Isotropía). Está guía se completa con el estudio de singularidad del Capítulo 6.

El contenido de cada punto de este capítulo se describe a continuación.

Alternativamente al método empleado en [Campion et al. 96] para obtener la clasificación de vehículos, en los puntos 5.2 y 5.3 se obtiene dicha clasificación en base a las restricciones al movimiento aportadas por cada rueda según el método descrito en el punto 4.4 del capítulo anterior.

La precisión o transmisión de errores en los modelos cinemáticos (isotropía), que proporciona un criterio de planificación/control, se discute y caracteriza en el punto 5.4.

En el punto 5.5 se caracterizan las posibilidades de actuación y sensorización en los cinco tipos de vehículos y se genera el modelado cinemático de cada uno con sus condiciones de isotropía.

Finalmente se destacan los resultados más relevantes del capítulo. 


\subsection{RESTRICCIONES AL MOVIMIENTO}

Las restricciones al movimiento aportadas por cada rueda decrementan el grado de movilidad del vehículo, que como se ha visto en el punto 4.4 del capítulo anterior tiene el sentido de grados de libertad en el movimiento del vehículo (GDLs) y/o de accionamientos independientes. El análisis de dichos GDLs son utilizados en [Campion et al. 96] para obtener la clasificación de vehículos.

En dicho estudio las restricciones al movimiento se obtienen con el primer elemento de (3.11). Como alternativa, aquí se utilizará lo desarrollado en el punto 4.4 del capítulo anterior.

Según la Figura 4.3, la expresión (4.19) y la Figura 4.2, las restricciones a los 3 GDLs del vehículo (máxima movilidad posible) vienen marcadas por:

$$
\begin{gathered}
\mathrm{N}^{\mathrm{o}} \text { de restricciones } \\
\text { al movmiento } \\
\text { (a los } 3 \text { GDLs) }
\end{gathered} \rightarrow\left\{\begin{array}{l}
\text { a) } \operatorname{rango}[\Delta(\mathbf{J}) \cdot \overline{\mathbf{I}}] \\
\text { b) } \operatorname{rango}[\mathbf{J}, \overline{\mathbf{I}}]-\operatorname{rango}[\mathbf{J}] \\
\text { c) } \operatorname{rango}[\Delta(\overline{\mathbf{I}}) \cdot \mathbf{J}]-\operatorname{rango}[\mathbf{J}]+3
\end{array}\right.
$$

De todas las opciones anteriores la primera es la más interesante ya que, al cumplirse (5.2) (Demostración 4.1), permite trabajar con cada rueda de forma independiente. Así se calcularían las restricciones que aportan las ruedas, y posteriormente se evaluaría su dependencia.

$$
\Delta(\mathbf{J}) \cdot \overline{\mathbf{I}}=\left(\begin{array}{c}
\Delta\left(\mathbf{J}_{1}\right) \\
\Delta\left(\mathbf{J}_{2}\right) \\
\vdots \\
\Delta\left(\mathbf{J}_{\mathrm{N}}\right)
\end{array}\right)=\mathbf{K}_{\mathrm{r}}
$$

Además $\mathbf{K}_{\mathrm{r}} \cdot \dot{\mathbf{p}}=\mathbf{0}$ son las ecuaciones de restricción al movimiento, como se desprende del árbol de la Figura 4.3.

Las restricciones que aporta cada rueda vienen dadas por $\Delta\left(\mathbf{J}_{i}\right)$ y las aportadas por todas por $\mathbf{K}_{\mathrm{r}}$.

A continuación se analizan las restricciones $\Delta\left(\mathbf{J}_{i}\right)$ para cada tipo de rueda. 
- Para las ruedas fijas y orientables, utilizando (4.12), se tiene:

$$
\begin{gathered}
\Delta\left(\mathbf{J}_{\mathrm{f} / \mathrm{i} i}\right)=\Delta\left(\begin{array}{cc}
\mathrm{r}_{i} \cdot \mathrm{s} \beta_{i} & 1_{i} \cdot \mathrm{s} \alpha_{i} \\
-\mathrm{r}_{i} \cdot \mathrm{c} \beta_{i} & -1_{i} \cdot \mathrm{c} \alpha_{i} \\
0 & 1
\end{array}\right)=\frac{-2}{2+1_{i}^{2} \cdot\left(1-\mathrm{c}\left(2 \cdot\left(\beta_{i}-\alpha_{i}\right)\right)\right)} . \\
\left(\begin{array}{ccc}
\mathrm{c}^{2} \beta_{i} & \mathrm{c} \beta_{i} \cdot \mathrm{s} \beta_{i} & 1_{i} \cdot \mathrm{c} \beta_{i} \cdot \mathrm{s}\left(\beta_{i}-\alpha_{i}\right) \\
\mathrm{c} \beta_{i} \cdot \mathrm{s} \beta_{i} & \mathrm{~s}^{2} \beta_{i} & 1_{i} \cdot \mathrm{s} \beta_{i} \cdot \mathrm{s}\left(\beta_{i}-\alpha_{i}\right) \\
1_{i} \cdot \mathrm{c} \beta_{i} \cdot \mathrm{s}\left(\beta_{i}-\alpha_{i}\right) & 1_{i} \cdot \mathrm{s} \beta_{i} \cdot \mathrm{s}\left(\beta_{i}-\alpha_{i}\right) & 1_{i}^{2} \cdot \mathrm{s}^{2}\left(\beta_{i}-\alpha_{i}\right)
\end{array}\right)
\end{gathered}
$$

En la matriz anterior sólo hay una fila/columna linealmente independiente, por lo que cada rueda orientable ó fija aporta una fila (restricción) a $\mathbf{K}_{\mathrm{r}}$.

- Para las rueda castor y suecas, utilizando (4.12), se tiene:

$$
\begin{gathered}
\Delta\left(\mathbf{J}_{\mathrm{c} i}\right)=\Delta\left(\begin{array}{ccc}
\mathrm{d}_{i} \cdot \mathrm{c} \beta_{i} & \mathrm{r}_{i} \cdot \mathrm{s}\left(\beta_{i}+\delta_{i}\right) & 1_{i} \cdot \mathrm{s} \alpha_{i}+\mathrm{d}_{i} \cdot \mathrm{c} \beta_{i} \\
\mathrm{~d}_{i} \cdot \mathrm{s} \beta_{i} & -\mathrm{r}_{i} \cdot \mathrm{c}\left(\beta_{i}+\delta_{i}\right) & -1_{i} \cdot \mathrm{c} \alpha_{i}+\mathrm{d}_{i} \cdot \mathrm{s} \beta_{i} \\
0 & 0 & 1
\end{array}\right)=\left(\begin{array}{lll}
0 & 0 & 0 \\
0 & 0 & 0 \\
0 & 0 & 0
\end{array}\right) \forall \delta_{i} \neq \pm \frac{\pi}{2} \\
\Delta\left(\mathbf{J}_{\mathrm{s} i}\right)=\Delta\left(\begin{array}{ccc}
\mathrm{r}_{i} \cdot \mathrm{s} \beta_{i} & \mathrm{r}_{\mathrm{ri}} \cdot \mathrm{s}\left(\beta_{i}+\gamma_{i}\right) & 1_{i} \cdot \mathrm{s} \alpha_{i} \\
-\mathrm{r}_{i} \cdot \mathrm{c} \beta_{i} & -\mathrm{r}_{\mathrm{r} i} \cdot \mathrm{c}\left(\beta_{i}+\gamma_{i}\right) & -\mathrm{l}_{i} \cdot \mathrm{c} \alpha_{i} \\
0 & 0 & 1
\end{array}\right)=\left(\begin{array}{ccc}
0 & 0 & 0 \\
0 & 0 & 0 \\
0 & 0 & 0
\end{array}\right) \forall \gamma_{i} \neq 0, \pi
\end{gathered}
$$

Por tanto las ruedas castor y suecas, siempre y cuando no sean singulares (ver Capítulo 6), no aportan filas linealmente independientes a $\mathbf{K}_{\mathrm{r}}$. 


\subsection{OBTENCIÓN DE LAS CINCO CLASES DE VEHÍCULOS}

Teniendo en cuenta (5.3), (5.4) y (5.5), la matriz $\mathbf{K}_{\mathrm{r}}$ se puede reducir sin perder generalidad a una matriz $\mathbf{K}_{\mathrm{r}}^{*}$ de dimensión $\left(\left(\mathrm{N}_{\mathrm{f}}+\mathrm{N}_{\mathrm{o}}\right)\right.$ x 3$)$, donde $\mathrm{N}_{\mathrm{f}}$ y $\mathrm{N}_{\mathrm{o}}$ son el número de ruedas fijas y orientables respectivamente, cumpliéndose:

$$
\operatorname{rango}\left[\mathbf{K}_{\mathrm{r}}^{*}\right] \leq 3
$$

Si el rango de $\mathbf{K}_{\mathrm{r}}^{*}$ fuera 3 habría 3 ecuaciones independientes de restricción al movimiento en (5.7), y su solución única sería $\dot{\mathbf{p}}=0 \rightarrow$ no movimiento posible.

$$
\mathbf{K}_{\mathrm{r}}^{*} \cdot \dot{\mathbf{p}}=\mathbf{0}
$$

Para que exista movimiento, y respetar (5.7), se debe cumplir:

$$
\operatorname{rango}\left[\mathbf{K}_{\mathrm{r}}^{*}\right] \leq 2
$$

La inecuación anterior caracteriza el Espacio definido por los cortes de los ejes de las ruedas fijas y orientables (E.c.f.), pudiendo ser (ver demostraciones del subapartado 5.3.1):

$$
\begin{aligned}
& \operatorname{rango}\left[\mathbf{K}_{\mathrm{r}}^{*}\right]=2 \rightarrow \text { E.c.f. es un punto } \\
& \operatorname{rango}\left[\mathbf{K}_{\mathrm{r}}^{*}\right]=1 \rightarrow \text { E.c.f. es } \underline{\text { recta }} \\
& \operatorname{rango}\left[\mathbf{K}_{\mathrm{r}}^{*}\right]=0 \rightarrow\left\{\begin{array}{l}
\text { No existen ruedas fijas ni orientables } \\
\text { E.c.f. puede considerarse todo el espacio 2D }
\end{array}\right.
\end{aligned}
$$

De hecho, este espacio (E.c.f.) establece las posibles ubicaciones del Centro Instantáneo de Rotación (CIR) del vehículo, pudiendo ser:

$$
\begin{aligned}
& \operatorname{rango}\left[\mathbf{K}_{\mathrm{r}}^{*}\right]=2 \rightarrow \text { CIR sólo se puede situar en un punto } \\
& \operatorname{rango}\left[\mathbf{K}_{\mathrm{r}}^{*}\right]=1 \rightarrow \text { CIR se puede situar en una recta } \\
& \operatorname{rango}\left[\mathbf{K}_{\mathrm{r}}^{*}\right]=0 \rightarrow \text { CIR se puede situar en todo el espacio 2D }
\end{aligned}
$$

El caso $\left\{\operatorname{rango}\left[\mathbf{K}_{\mathrm{rf}}{ }^{*}\right]=2\right\}$, donde $\mathbf{K}_{\mathrm{rf}}{ }^{*}$ es la parte de $\mathbf{K}_{\mathrm{r}}{ }^{*}$ aportada por las ruedas fijas, implica que hay dos ruedas fijas con sus ejes en distintas direcciones (aunque sean paralelas), lo que haría girar al vehículo permanente entorno a un CIR fijo. Evidentemente esto no es aceptable en la práctica, por lo que se asume:

$$
\operatorname{rango}\left[\mathbf{K}_{\mathrm{rf}}^{*}\right] \leq 1
$$


Como conclusión de (5.8) y (5.11), se define el vehículo no degenerado:

$$
\text { Ve degenerado }\left\{\begin{array}{l}
\text { a) } \operatorname{rango}\left[\mathbf{K}_{\mathrm{rf}}^{*}\right] \leq 1 \\
\text { b) } \operatorname{rango}\left[\mathbf{K}_{\mathrm{r}}^{*}\right]=\operatorname{rango}\left[\mathbf{K}_{\mathrm{rf}}^{*}\right]+\operatorname{rango}\left[\mathbf{K}_{\mathrm{ro}}^{*}\right] \leq 2
\end{array}\right.
$$

donde $\mathbf{K}_{\mathrm{ro}}{ }^{*}$ es la parte de $\mathbf{K}^{*}$ aportada por las ruedas orientables.

El grado de movilidad, ya introducido en el capítulo anterior, es:

$$
m=\operatorname{dim}(\boldsymbol{\eta})=3-\operatorname{rango}\left[\mathbf{K}_{\mathrm{r}}^{*}\right]
$$

La expresión (5.12) también se puede expresar en los siguientes tres puntos:

- Si el vehículo tiene más de una rueda fija todas ellas tienen un mismo eje común. Esto se deriva directamente de la condición a).

- Los centros de las ruedas orientables no pertenecen al eje común de las ruedas fijas.

Esto se deriva de haber igualado, en b), el rango de toda la matriz a la suma de los rangos de las matrices que la componen, es decir que ambas matrices han de ser linealmente independientes. Esto implica que el eje común de las posibles ruedas fijas no puede coincidir nunca con el eje de las ruedas orientables para alguna orientación de éstas.

La justificación de esta condición viene marcada por el hecho de que una rueda orientable en el eje común de las ruedas fijas implica* que el CIR, de existir, estaría de manera permanente en el centro de la rueda orientable sobre el eje común, dando lugar a una situación parecida a la comentado anteriormente de ruedas fijas con distintos ejes de rotación.

*Nota: Excepto en el caso de hacer coincidir permanentemente el eje de la rueda orientable y el eje común de las ruedas fijas, lo cual convertiría dicha rueda en fija a efectos prácticos y no tendría sentido definirla como orientable.

- El máximo número de ruedas orientables que pueden ser orientadas independientemente, para dirigir el vehículo, es de dos. Esto se deriva de la condición b). 
Se define el grado de direccionabilidad como:

$$
d=\operatorname{rango}\left[\mathbf{K}_{\mathrm{ro}}^{*}\right]
$$

El parámetro anterior indica el número de ruedas orientables que se pueden direccionar independientemente. El resto de ellas deberán direccionarse coordinadamente para garantizar la existencia de un CIR en cada instante.

Para un vehículo no degenerado, condiciones (5.12), se cumple:

$$
1 \leq m \leq 3, \quad 0 \leq d \leq 2 \text { y } 2 \leq m+d \leq 3
$$

De las inecuaciones de (5.15) se derivan las posibilidades de la Tabla 5.1.

Tabla 5.1- Relación de vehículos no degenerados posibles

\begin{tabular}{|c|c|c|c|c|c|}
\hline $\begin{array}{c}\text { Tipo X } \\
(m, d)\end{array}$ & Tipo 1 (3,0) & Tipo 2 (2,0) & Tipo 3 (2,1) & Tipo 4 (1,1) & Tipo 5 (1,2) \\
\hline$m$ & 3 & 2 & 2 & 1 & 1 \\
\hline$d$ & 0 & 0 & 1 & 1 & 2 \\
\hline
\end{tabular}

El grado de movilidad tiene el sentido físico de grados de libertad instantáneos ó velocidades del vehículo que independientemente se pueden asignar sin redireccionar ruedas.

Como se discute en el punto 4.5 del capítulo anterior, se consiguen grados de libertad adicionales modificando la orientación de las ruedas orientables, a través de sus velocidades de direccionamiento. Dichos GDLs adicionales coinciden el grado de direccionabilidad comentado.

Estas ruedas orientables son direccionables indirectamente a través de la velocidad de direccionamiento: $\beta_{i}=\int \dot{\beta}_{i} \mathrm{~d} t$, pero no instantáneamente.

Por tanto, el grado de direccionabilidad indica los grados de libertad no instantáneos para el movimiento del vehículo.

Así pues, la maniobrabilidad sería desde un punto de vista estricto el grado de movilidad, pero desde un punto de vista más amplio el grado de movilidad más el grado de direccionabilidad.

Por ejemplo, los vehículos de tipo $1(3,0)$ y tipo $5(1,2)$ tienen la misma maniobrabilidad desde un punto de vista amplio, pero el primero de forma instantánea y el segundo tras la reorientación de sus ruedas orientables. 


\subsubsection{Demostración de las implicaciones de (5.9)}

\section{Demostración 5.1: de la primera expresión de (5.9)}

La demostración se va realizar para tres ruedas del tipo fijo u orientables (misma matriz Jacobiana de rueda), que son las que restringen el movimiento. En caso de tener más ruedas la demostración se repetiría para cada terna de ruedas.

En la primera expresión de (5.9) se parte de:

$$
\operatorname{rango}\left[\mathbf{K}_{\mathrm{r}}^{*}\right]=2
$$

Puesto que cada rueda aporta una ecuación linealmente independiente a $\mathbf{K}_{\mathrm{r}}{ }^{*}$, se coge (por ejemplo) la primera fila de (5.3), resultando $\mathbf{K}_{\mathrm{r}}^{*}$ :

$$
\mathbf{K}_{\mathrm{r}}^{*}=-2 \cdot\left(\begin{array}{ccc}
\frac{\mathrm{c}^{2} \beta_{1}}{2+l_{1}^{2} \cdot\left(1-\mathrm{c}\left(2 \cdot\left(\beta_{1}-\alpha_{1}\right)\right)\right)} & \frac{\mathrm{c} \beta_{1} \cdot \mathrm{s} \beta_{1}}{2+l_{1}^{2} \cdot\left(1-\mathrm{c}\left(2 \cdot\left(\beta_{1}-\alpha_{1}\right)\right)\right)} & \frac{1_{1} \cdot \mathrm{c} \beta_{1} \cdot \mathrm{s}\left(\beta_{1}-\alpha_{1}\right)}{2+l_{1}^{2} \cdot\left(1-\mathrm{c}\left(2 \cdot\left(\beta_{1}-\alpha_{1}\right)\right)\right)} \\
\frac{\mathrm{c}^{2} \beta_{2}}{2+l_{2}^{2} \cdot\left(1-\mathrm{c}\left(2 \cdot\left(\beta_{2}-\alpha_{2}\right)\right)\right)} & \frac{\mathrm{c} \beta_{2} \cdot \mathrm{s} \beta_{2}}{2+l_{2}^{2} \cdot\left(1-\mathrm{c}\left(2 \cdot\left(\beta_{2}-\alpha_{2}\right)\right)\right)} & \frac{1_{2} \cdot \mathrm{c} \beta_{2} \cdot \mathrm{s}\left(\beta_{2}-\alpha_{2}\right)}{2+l_{2}^{2} \cdot\left(1-\mathrm{c}\left(2 \cdot\left(\beta_{2}-\alpha_{2}\right)\right)\right)} \\
\frac{\mathrm{c}^{2} \beta_{3}}{2+l_{3}^{2} \cdot\left(1-\mathrm{c}\left(2 \cdot\left(\beta_{3}-\alpha_{3}\right)\right)\right)} & \frac{\mathrm{c} \beta_{3} \cdot s \beta_{3}}{2+l_{3}^{2} \cdot\left(1-\mathrm{c}\left(2 \cdot\left(\beta_{3}-\alpha_{3}\right)\right)\right)} & \frac{1_{3} \cdot \mathrm{c} \beta_{3} \cdot \mathrm{s}\left(\beta_{3}-\alpha_{3}\right)}{2+l_{3}^{2} \cdot\left(1-\mathrm{c}\left(2 \cdot\left(\beta_{3}-\alpha_{3}\right)\right)\right)}
\end{array}\right)
$$

La expresión (5.16) implica que el determinante de $\mathbf{K}_{\mathrm{r}}^{*}$ en (5.17) es cero y que las tres filas aportadas a $\mathbf{K}_{\mathrm{r}}^{*}$ por las tres ruedas no son coincidentes (existe algún menor de orden 2 cuyo determinante es distinto de 0 ):

$$
\operatorname{rango}\left[\mathbf{K}_{\mathrm{r}}^{*}\right]=2 \rightarrow\left\{\begin{array}{l}
\text { a) }\left|\mathbf{K}_{\mathrm{r}}^{*}\right|=0 \rightarrow\left|\begin{array}{lll}
\mathrm{c} \beta_{1} & \mathrm{~s} \beta_{1} & 1_{1} \cdot \mathrm{s}\left(\beta_{1}-\alpha_{1}\right) \\
\mathrm{c} \beta_{2} & \mathrm{~s} \beta_{2} & 1_{2} \cdot \mathrm{s}\left(\beta_{2}-\alpha_{2}\right) \\
\mathrm{c} \beta_{3} & \mathrm{~s} \beta_{3} & 1_{3} \cdot \mathrm{s}\left(\beta_{3}-\alpha_{3}\right)
\end{array}\right| \\
\text { b) Restricciones de las } 3 \text { ruedas NO coincidentes }
\end{array}\right.
$$

El eje de rotación de una rueda fija u orientable $i$ viene dado por:

$$
\left(\begin{array}{ll}
\mathrm{c} \beta_{i} & \mathrm{~s} \beta_{i}
\end{array}\right) \cdot\left(\begin{array}{c}
x \\
-y
\end{array}\right)=-1_{i} \cdot \mathrm{s}\left(\beta_{i}-\alpha_{i}\right)
$$

donde $x$ e $y$ son respecto al sistema de coordenadas $\mathrm{R}$. 
El corte de los ejes de las tres ruedas viene dado por la ecuación matricial:

$$
\left(\begin{array}{cc}
\mathrm{c} \beta_{1} & \mathrm{~s} \beta_{1} \\
\mathrm{c} \beta_{2} & \mathrm{~s} \beta_{2} \\
\mathrm{c} \beta_{3} & \mathrm{~s} \beta_{3}
\end{array}\right) \cdot\left(\begin{array}{c}
x \\
-y
\end{array}\right)=-\left(\begin{array}{c}
1_{1} \cdot \mathrm{s}\left(\beta_{1}-\alpha_{1}\right) \\
1_{2} \cdot \mathrm{s}\left(\beta_{2}-\alpha_{2}\right) \\
1_{3} \cdot \mathrm{s}\left(\beta_{3}-\alpha_{3}\right)
\end{array}\right)
$$

Por tanto, si se cumple (5.18) los ejes de las tres ruedas se cortan en un punto (solución única en (5.20)) ó son paralelos si $\beta_{1}=\beta_{2}=\beta_{3}$ (pero no los tres coincidentes).

Para ejes paralelos se puede decir que el punto de corte se encuentra en el infinito (geometría proyectiva).

Así pues, si rango el rango de la matriz $\mathbf{K}_{\mathrm{r}}{ }^{*}$ es 2 los ejes de tres ruedas fijas $\mathbf{u}$ orientables se cortan en un único punto (incluyendo el infinito).

Dicho punto de corte viene dado por:

$$
\left(\begin{array}{l}
x_{\mathrm{c}} \\
y_{\mathrm{c}}
\end{array}\right)=\left\{\begin{array}{l}
\frac{1}{\mathrm{~s}\left(\beta_{1}-\beta_{2}\right)} \cdot\left(\begin{array}{l}
1_{1} \cdot \mathrm{s}\left(\beta_{1}-\alpha_{1}\right) \cdot \mathrm{s} \beta_{2}-1_{2} \cdot \mathrm{s}\left(\beta_{2}-\alpha_{2}\right) \cdot \mathrm{s} \beta_{1} \\
1_{1} \cdot \mathrm{s}\left(\beta_{1}-\alpha_{1}\right) \cdot \mathrm{c} \beta_{2}-1_{2} \cdot \mathrm{s}\left(\beta_{2}-\alpha_{2}\right) \cdot \mathrm{c} \beta_{1}
\end{array}\right) \text { si } \beta_{1} \neq \beta_{2} \\
\frac{1}{\mathrm{~s}\left(\beta_{1}-\beta_{3}\right)} \cdot\left(\begin{array}{l}
1_{1} \cdot \mathrm{s}\left(\beta_{1}-\alpha_{1}\right) \cdot \mathrm{s} \beta_{3}-1_{3} \cdot \mathrm{s}\left(\beta_{3}-\alpha_{3}\right) \cdot \mathrm{s} \beta_{1} \\
1_{1} \cdot \mathrm{s}\left(\beta_{1}-\alpha_{1}\right) \cdot \mathrm{c} \beta_{3}-1_{3} \cdot \mathrm{s}\left(\beta_{3}-\alpha_{3}\right) \cdot \mathrm{c} \beta_{1}
\end{array}\right) \text { si } \beta_{1}=\beta_{2}
\end{array}\right.
$$

\section{Demostración 5.2: de la segunda expresión de (5.9)}

La demostración se va realizar para dos ruedas del tipo fijo u orientables (misma matriz Jacobiana de rueda), que son las que restringen el movimiento. En caso de tener más ruedas la demostración se repetiría para cada par de ruedas.

En la segunda expresión de (5.9) se parte de:

$$
\operatorname{rango}\left[\mathbf{K}_{\mathrm{r}}^{*}\right]=1
$$

Puesto que cada rueda aporta una ecuación linealmente independiente a $\mathbf{K}_{\mathrm{r}}{ }^{*}$, se coge (por ejemplo) la primera fila de (5.3), resultando $\mathbf{K}_{\mathrm{r}}^{*}$ : 
$\mathbf{K}_{\mathrm{r}}^{*}=-2 \cdot\left(\begin{array}{ccc}\frac{\mathrm{c}^{2} \beta_{1}}{2+1_{1}^{2} \cdot\left(1-\mathrm{c}\left(2 \cdot\left(\beta_{1}-\alpha_{1}\right)\right)\right)} & \frac{\mathrm{c} \beta_{1} \cdot \mathrm{s} \beta_{1}}{2+1_{1}^{2} \cdot\left(1-\mathrm{c}\left(2 \cdot\left(\beta_{1}-\alpha_{1}\right)\right)\right)} & \frac{1_{1} \cdot \mathrm{c} \beta_{1} \cdot \mathrm{s}\left(\beta_{1}-\alpha_{1}\right)}{2+1_{1}^{2} \cdot\left(1-\mathrm{c}\left(2 \cdot\left(\beta_{1}-\alpha_{1}\right)\right)\right)} \\ \frac{\mathrm{c}^{2} \beta_{2}}{2+1_{2}^{2} \cdot\left(1-\mathrm{c}\left(2 \cdot\left(\beta_{2}-\alpha_{2}\right)\right)\right)} & \frac{\mathrm{c} \beta_{2} \cdot \mathrm{s} \beta_{2}}{2+1_{2}^{2} \cdot\left(1-\mathrm{c}\left(2 \cdot\left(\beta_{2}-\alpha_{2}\right)\right)\right)} & \frac{1_{2} \cdot \mathrm{c} \beta_{2} \cdot \mathrm{s}\left(\beta_{2}-\alpha_{2}\right)}{2+1_{2}^{2} \cdot\left(1-\mathrm{c}\left(2 \cdot\left(\beta_{2}-\alpha_{2}\right)\right)\right)}\end{array}\right)$

La condición (5.22) implica que los determinantes de los menores de orden 2 en $\mathbf{K}_{\mathrm{r}}^{*}$ nulos:

$$
\begin{aligned}
& \left|\mathbf{K}_{\mathrm{r} 12}^{*}\right|=\frac{-2 \cdot \mathrm{c} \beta_{1}}{2+l_{1}^{2} \cdot\left(1-\mathrm{c}\left(2 \cdot\left(\beta_{1}-\alpha_{1}\right)\right)\right)} \cdot \frac{\mathrm{c} \beta_{2}}{2+l_{2}^{2} \cdot\left(1-\mathrm{c}\left(2 \cdot\left(\beta_{2}-\alpha_{2}\right)\right)\right)} \cdot \begin{array}{ll}
\mathrm{c} \beta_{1} & \mathrm{~s} \beta_{1} \\
\mathrm{c} \beta_{2} & \mathrm{~s} \beta_{2}
\end{array} \mid=0 \\
& \left|\mathbf{K}_{\mathrm{r} 13}^{*}\right|=\frac{-2 \cdot \mathrm{c} \beta_{1}}{2+l_{1}^{2} \cdot\left(1-\mathrm{c}\left(2 \cdot\left(\beta_{1}-\alpha_{1}\right)\right)\right)} \cdot \frac{\mathrm{c} \beta_{2}}{2+l_{2}^{2} \cdot\left(1-\mathrm{c}\left(2 \cdot\left(\beta_{2}-\alpha_{2}\right)\right)\right)} \cdot \begin{array}{ll}
\mathrm{c} \beta_{1} & 1_{1} \cdot \mathrm{s}\left(\beta_{1}-\alpha_{1}\right) \\
\mathrm{c} \beta_{2} & 1_{2} \cdot \mathrm{s}\left(\beta_{2}-\alpha_{2}\right)
\end{array} \mid=0 \\
& \left|\mathbf{K}_{\mathrm{r} 23}^{*}\right|=\frac{-2 \cdot \mathrm{c} \beta_{1}}{2+l_{1}^{2} \cdot\left(1-\mathrm{c}\left(2 \cdot\left(\beta_{1}-\alpha_{1}\right)\right)\right)} \cdot \frac{\mathrm{c} \beta_{2}}{2+l_{2}^{2} \cdot\left(1-\mathrm{c}\left(2 \cdot\left(\beta_{2}-\alpha_{2}\right)\right)\right)} \cdot \begin{array}{ll}
\mathrm{s} \beta_{1} & 1_{1} \cdot \mathrm{s}\left(\beta_{1}-\alpha_{1}\right) \\
\mathrm{s} \beta_{2} & 1_{2} \cdot \mathrm{s}\left(\beta_{2}-\alpha_{2}\right)
\end{array} \mid=0
\end{aligned}
$$

Utilizando el mismo procedimiento que en la Demostración 5.1, el corte de los ejes de las dos ruedas viene dado por la ecuación matricial:

$$
\left(\begin{array}{cc}
\mathrm{c} \beta_{1} & \mathrm{~s} \beta_{1} \\
\mathrm{c} \beta_{2} & \mathrm{~s} \beta_{2}
\end{array}\right) \cdot\left(\begin{array}{c}
x \\
-y
\end{array}\right)=-\left(\begin{array}{c}
1_{1} \cdot \mathrm{s}\left(\beta_{1}-\alpha_{1}\right) \\
1_{2} \cdot \mathrm{s}\left(\beta_{2}-\alpha_{2}\right)
\end{array}\right)
$$

Por tanto, si se cumple (5.24) los ejes de las dos ruedas son coincidentes ó linealmente dependientes.

Así pues si el rango de la matriz $\mathbf{K}_{\mathrm{r}}^{*}$ es 1 , los ejes de dos ruedas fijas $\mathrm{u}$ orientables coinciden.

Demostración 5.3: de la tercera expresión de (5.9)

Se parte de:

$$
\operatorname{rango}\left[\mathbf{K}_{\mathrm{r}}^{*}\right]=0
$$

Lo anterior es posible si no hay ruedas fijas ni orientables, ya que cualquiera de ellas aportaría al menos una ecuación linealmente independiente.

En este caso propiamente no se puede hablar del corte de los ejes de las ruedas fijas y orientables, pero sí se puede decir que el CIR puede situarse en cualquier parte del espacio bidimensional, al no haber restricciones al movimiento. 


\subsection{TRANSMISIÓN DE ERRORES EN LOS MODELOS CINEMÁTICOS: ISOTROPÍA}

La precisión/transmisión de errores/isotropía de los modelos cinemáticos se va a estudiar en base a la siguiente ecuación algebraica genérica:

$$
\mathbf{H} \cdot \mathbf{x}=\mathbf{y}
$$

donde $\mathbf{x}$ es el vector de salida (incógnita) de dimensión $f^{\prime}, \mathbf{y}$ es el vector de entrada (conocido) de dimensión $f$ y $\mathbf{H}$ es la matriz de relación de dimensión $f \times x f^{\prime}$.

Se dice que el número de condición de la matriz $\mathbf{H}$ es una medida (de proporcionalidad si la matriz es cuadrada) de la amplificación del error relativo al calcular $\mathbf{x}$ en (5.27) (con la pseudoinversa por la izquierda si $f>f^{\prime}$ y con la pseudoinversa por la derecha si $f<f^{\prime}$ ) respecto al error relativo de los datos de entrada (y, H) [Golub et al. 83] [Higham 96].

Por ejemplo, un número de condición pequeño produce resultados precisos, mientras que un número grande produce gran amplificación del error. Un número de condición igual a la unidad ( $\mathbf{H}$ isotrópica) no produce error de amplificación y es la mejor situación posible.

Además, el número de condición de una matriz se puede definir basándose en cualquier norma. La norma Euclídea da lugar a una definición invariante con el número de condición:

$$
\kappa=\|\mathbf{H}\|_{2} \cdot\left\|\mathbf{H}^{\dagger}\right\|_{2}=\sigma_{\max } / \sigma_{\min } \rightarrow \kappa \in[1, \infty[
$$

donde $\sigma_{\max }$ y $\sigma_{\min }$ son los valores singulares más grande y más pequeño de la matriz rectangular $\mathbf{H}$.

De modo que, matrices singulares $\left(\sigma_{\min }=0\right)$ tienen un número de condición infinito, mientras que matrices isotrópicas tienen todos sus valores singulares iguales y distintos de cero. Para $\mathbf{H}$ isotrópica se cumple

$$
\mathrm{k} \cdot \mathbf{I}=\left\{\begin{array}{lll}
\mathbf{H}^{\mathrm{T}} \cdot \mathbf{H} & \text { si } f>f^{\prime} \\
\mathbf{H} \cdot \mathbf{H}^{\mathrm{T}} & \text { si } f<f^{\prime}
\end{array}\right.
$$

donde $\mathrm{k}$ es una constante de proporcionalidad. 
Otro aspecto importante es que las variables de $\mathbf{x}$, ej. velocidades lineales y angular del vehículo, pueden ser de diferentes unidades, ej. $\mathrm{m} / \mathrm{s} \mathrm{y} \mathrm{s}^{-1}$. Como consecuencia, los valores singulares asociados tienen diferentes unidades, ej. $\mathrm{m}^{-1}$ y sin unidades, resultando imposible ordenarlos [Lipkin et al. 88].

Para resolver este problema, se tiene que introducir la distancia característica D [Tandirci et al. 92], ej. la velocidad angular se multiplicaría por D y su columna asociada en $\mathbf{H}$ dividida por D. Este parámetro se ha optimizado tradicionalmente en robots manipuladores para alcanzar la mejor precisión (isotropía), denominándose ese valor distancia natural [Ma et al. 91]. Por ejemplo, en el vehículo diferencial la distancia natural es la mitad de la distancia entre las dos ruedas fijas (5.51).

Una interpretación de la distancia característica para velocidades lineales y angulares (ó fuerzas/pares) se muestra en [Stocco et al. 98] como la capacidad relativa del vehículo para el movimiento de traslación y rotación (con mismos valores en las velocidades de rueda). Por lo que [Stocco et al. 98] sugiere que la distancia característica debería ser elegida para satisfacer las demandas del movimiento y no ser un parámetro de diseño arbitrario.

En este sentido, en [Stocco et al. 99] se introducen dos matrices diagonales que escalan complemente los vectores $\mathbf{x}$ e $\mathbf{y}$ de (5.27). Esto representa una generalización del concepto de distancia característica como un factor de escalado. Estos factores pueden ser utilizados para alcanzar las demandas en el movimiento del vehículo o para alcanzar la isotropía. Por ejemplo si (5.27) es un modelo cinemático inverso y la matriz $\mathbf{H}$ está convenientemente escalada, las velocidades de rueda se utiliza completamente (es decir están equilibradas) para satisfacer los requerimientos definidos para el vector de velocidad del vehículo sin amplificación del error.

Sin embargo, si no está demasiado definido el rango para el movimiento del vehículo (ej. no está claro que velocidades relativas se requieren en dirección del vehículo) es razonable simplemente ajustar la distancia característica para alcanzar la isotropía, a través de la distancia natural, como habitualmente se hace. Esta opción será la que se utilice en el punto 5.5 del capítulo.

En nuestro caso, el vector de entrada $\mathbf{y}$ son las velocidades asignadas $\dot{\mathbf{q}}_{\mathrm{a}}$, por lo que sólo $m(f=m)$ ecuaciones escalares de (4.5) pueden ser consideradas para el análisis de isotropía. Por tanto, las condiciones para isotropía en un 
modelo cinemático se obtendrán para un conjunto $m$ de velocidades no asignadas $\dot{\mathbf{q}}_{\text {n__s}}$. En otras palabras, las condiciones de isotropía se establecerán para noamplificación del error entre el vector de entrada $\dot{\mathbf{q}}_{\mathrm{a}}$ y el vector de salida $\dot{\mathbf{q}}_{\text {na_s }}$, los dos con $m$ elementos escalares. Esta isotropía es bidireccional entre $\dot{\mathbf{q}}_{\mathrm{a}}$ y $\dot{\mathbf{q}}_{\text {na_s }}$. El procedimiento partiendo de (4.4) es:

$$
\begin{aligned}
& \left(\begin{array}{c}
\dot{\mathbf{q}}_{\text {na_ns }} \\
\dot{\mathbf{q}}_{\text {na_s }} \\
\dot{\mathbf{q}}_{\mathrm{a}}
\end{array}\right)=\left(\begin{array}{c}
\mathbf{B}_{\text {na_ns }} \\
\mathbf{B}_{\text {na_s }} \\
\mathbf{B}_{\mathrm{a}}
\end{array}\right) \cdot \boldsymbol{\eta} \\
& \dot{\mathbf{q}}_{\text {na_s }}=\left(\mathbf{B}_{\text {na_s }} \cdot \mathbf{B}_{\mathrm{a}}^{-1}\right) \cdot \dot{\mathbf{q}}_{\mathrm{a}} \\
& \left(\mathbf{B}_{\mathrm{a}} \cdot \mathbf{B}_{\text {na_s }}^{-1}\right) \cdot \dot{\mathbf{q}}_{\text {na_s }}=\dot{\mathbf{q}}_{\mathrm{a}},
\end{aligned}
$$

donde $\mathbf{B}_{\mathrm{a}}$ y $\mathbf{B}_{\text {na_s }}$ deben ser en general matrices no singulares, es decir no cualquier vector $\dot{\mathbf{q}}_{\mathrm{n} \_\_}$y $\dot{\mathbf{q}}_{\mathrm{a}}$ son posibles para un análisis de isotropía, el cual depende de la matriz $\mathbf{H}=\left(\mathbf{B}_{\mathrm{a}} \cdot \mathbf{B}_{\text {na_s }}^{-1}\right)$.

Las consideraciones anteriores proporcionan el siguiente criterio: la isotropía (no-amplificación del error) debe alcanzarse. Cuando el vehículo no tiene ruedas orientables ni castor éste es un criterio de diseño, ya que las condiciones de isotropía son permanentes.

En cambio si hay ruedas orientables y/o castor las condiciones se cumplen para determinadas configuraciones, denominadas configuraciones isotrópicas, por lo que dicho criterio es de planificación y/o control. Es decir, el planificador (generador de caminos) del nivel superior y/o el controlador tienen que desarrollar caminos y/o acciones de control que produzcan configuraciones próximas a las de isotropía. En este caso un vehículo sobre actuado sería útil, ya que permite cambiar de un modelo cinemático a otro, beneficiándose de la isotropía del segundo.

Un posible criterio de diseño, para el segundo caso, podría ser calcular los parámetros del vehículo (radios de ruedas, distancias entre ruedas, etc.) para minimizar el error medio de las condiciones de isotropía (o la media del número de condición) a lo largo del error de configuración. Esto conduce a un concepto de isotropía global. 
Por ejemplo en [Stocco2 et al. 98] se define la isotropía global como:

$$
\text { Isotropía Global }=\min _{x_{0}, x_{1} \in W} \frac{\sigma_{\min }\left(\mathbf{H}\left(\text { parámetros }, x_{0}\right)\right)}{\sigma_{\max }\left(\mathbf{H}\left(\text { parámetros }, x_{1}\right)\right)}
$$

donde $\sigma_{\min } / \sigma_{\max }$ es el valor singular más pequeño/grande de la matriz de relación $\mathbf{H}$ a lo largo de todo el espacio de configuración $W$ de las variables $x$ del sistema.

El índice anterior también depende de los parámetros del sistema. En [Stocco2 et al. 98] se calculan dichos parámetros para maximizar el índice.

De forma parecida en [Kim et al. 05] se define la isotropía global como la media de la isotropía local (inversa del número de condición $\kappa$ de (5.28)) a lo largo de todo el espacio de configuración, y posteriormente se maximiza a través de la distancia característica.

El criterio genérico anteriormente formulado, "la isotropía debe alcanzarse", se complementa perfectamente con el criterio de singularidad indicado en el capítulo posterior: "la singularidad debe evitarse". 


\subsection{CARACTERIZACIÓN DE LAS CINCO CLASES DE VEHÍCULOS}

En este punto se van a caracterizar las cinco clases de vehículos deducidas en el punto 5.3 para el caso de tres ruedas. El hecho de considerar tres ruedas es por cuestiones de estabilidad y no implica perdida de generalidad.

En primer lugar dicha caracterización consiste en establecer las opciones de sensorización y actuación, descritas en el punto 4.4 del capítulo anterior (Figura 4.4 y Figura 4.5), e indicar sus ventajas e inconvenientes prácticos.

A continuación se calcula el modelado cinemático del vehículo, que a diferencia de presentado en [Campion et al. 96] incluye las velocidades de rueda. En ése estudio aparecen entradas sin sentido físico debido a utilizar sólo parte de las ecuaciones de rueda, en concreto el primer elemento de (3.11).

Posteriormente se establecen las condiciones de isotropía de los modelos cinemáticos.

Toda la caracterización presentada en este punto se completa con la de singularidad del capítulo posterior.

Para los siguientes apartados se asumirá ruedas con mismos valores de parámetros $\mathrm{r}_{i}, \mathrm{r}_{\mathrm{ri}}, \mathrm{d}_{i}, \delta_{i}$, and $\gamma_{i}$ (cuando sea aplicable). También se considerará la habitual orientación de $90^{\circ}$ para los rodillos de las ruedas suecas $\left(\gamma=90^{\circ}\right)$ y la usual orientación de $0^{\circ}$ para la rueda castor respecto a su brazo de direccionamiento $(\delta=0)$.

\subsubsection{Tipo $1(3,0)$ : Vehículo omnidireccional}

Este vehículo tiene movilidad completa ruedas $(m=3)$, por lo que puede moverse en cada instante en cualquier dirección sin necesitar reorientación, de ahí que se le llame vehículo omnidireccional.

Está construido sin ruedas fijas ni orientables, es decir que utilizaría ruedas omnidireccionales (castor o suecas). En este estudio se considerarán las dos opciones más habituales: tres ruedas suecas y tres ruedas castor.

\subsubsection{A) Vehículo omnidireccional con tres ruedas suecas}

Dado que hay tres GDLs instantáneos se necesitan al menos tres actuadores 
/sensores para poder actuar/sensorizar sobre todos ellos. Por tanto no hay más opción que actuar/sensorizar las tres variables de rotación de las ruedas suecas.

Para modelar cinemáticamente este vehículo la ecuación (3.14) hay que considerarla tres veces, una por cada rueda sueca, por lo que (4.1) resulta:

$$
\mathbf{A}_{\mathrm{p}} \cdot \dot{\mathbf{p}}+\mathbf{A}_{\mathrm{w}} \cdot \dot{\boldsymbol{\varphi}}=\mathbf{0}
$$

con

$$
\begin{aligned}
& \dot{\boldsymbol{\varphi}}=\left(\begin{array}{lll}
\dot{\varphi}_{1} & \dot{\varphi}_{2} & \dot{\varphi}_{3}
\end{array}\right)^{\mathrm{T}} \quad \mathbf{A}_{\mathrm{w}}=\mathrm{r} \cdot \mathrm{s} \boldsymbol{\gamma} \cdot \mathbf{I} \\
& \mathbf{A}_{\mathrm{p}}=\left(\begin{array}{lll}
\mathrm{c}\left(\beta_{1}+\gamma\right) & \mathrm{s}\left(\beta_{1}+\gamma\right) & 1_{1} \cdot \mathrm{s}\left(\beta_{1}+\gamma-\alpha_{1}\right) \\
\mathrm{c}\left(\beta_{2}+\gamma\right) & \mathrm{s}\left(\beta_{2}+\gamma\right) & 1_{2} \cdot \mathrm{s}\left(\beta_{2}+\gamma-\alpha_{2}\right) \\
\mathrm{c}\left(\beta_{3}+\gamma\right) & \mathrm{s}\left(\beta_{3}+\gamma\right) & 1_{3} \cdot \mathrm{s}\left(\beta_{3}+\gamma-\alpha_{3}\right)
\end{array}\right)
\end{aligned}
$$

Una solución cinemática (4.2) para (5.33) es:

$$
\left(\begin{array}{c}
\dot{\mathbf{p}} \\
\dot{\boldsymbol{\varphi}}
\end{array}\right)=\left(\begin{array}{c}
-\mathbf{A}_{\mathrm{w}} \\
\mathbf{A}_{\mathrm{p}}
\end{array}\right) \cdot \boldsymbol{\eta}
$$

Resultando los modelos cinemáticos directo e inverso:

$$
\begin{aligned}
& \dot{\mathbf{p}}=-\mathrm{r} \cdot \mathrm{s} \gamma \cdot \mathbf{A}_{\mathrm{p}}^{-1} \cdot \dot{\boldsymbol{\varphi}} \\
& \dot{\boldsymbol{\varphi}}=-(\mathrm{r} \cdot \mathrm{s} \gamma)^{-1} \cdot \mathbf{A}_{\mathrm{p}} \cdot \dot{\mathbf{p}}
\end{aligned}
$$

La isotropía de los dos modelos cinemáticos anteriores depende de $\mathbf{A}_{\mathrm{p}}$.

Como se ha mencionado en la introducción de este capítulo, el análisis completo de isotropía para este vehículo se puede encontrar en [Saha et al. 95], por lo que no se va a duplicar aquí. El resultado es que los centros de las tres ruedas representan los vértices de un triángulo regular (equilátero) y el plano de cada rueda es perpendicular a la recta que une el centroide del triángulo y el centro de la rueda.

Si el sistema de coordenadas $\mathrm{R}$ se coloca en dicho centroide y su eje $\mathrm{X}$ cruza el centro de la primera rueda (ver Figura 5.1), los parámetros son $\alpha_{1}=0, \alpha_{2}=120^{\circ}, \alpha_{3}=240^{\circ}, \beta_{i}=\alpha_{i}, 1_{i}=\mathrm{L}, \mathrm{y}(5.36)$ resulta: 


$$
\begin{aligned}
& \dot{\mathbf{p}}=\frac{\mathrm{r}}{3} \cdot\left(\begin{array}{ccc}
0 & \sqrt{3} & -\sqrt{3} \\
-2 & 1 & 1 \\
-1 / \mathrm{L} & -1 / \mathrm{L} & -1 / \mathrm{L}
\end{array}\right) \cdot\left(\begin{array}{c}
\dot{\varphi}_{1} \\
\dot{\varphi}_{2} \\
\dot{\varphi}_{3}
\end{array}\right) \\
& \left(\begin{array}{c}
\dot{\varphi}_{1} \\
\dot{\varphi}_{2} \\
\dot{\varphi}_{3}
\end{array}\right)=\frac{1}{\mathrm{r}} \cdot\left(\begin{array}{ccc}
0 & -1 & -\mathrm{L} \\
\sqrt{3} / 2 & 0.5 & -\mathrm{L} \\
-\sqrt{3} / 2 & 0.5 & -\mathrm{L}
\end{array}\right) \cdot \dot{\mathbf{p}}
\end{aligned}
$$

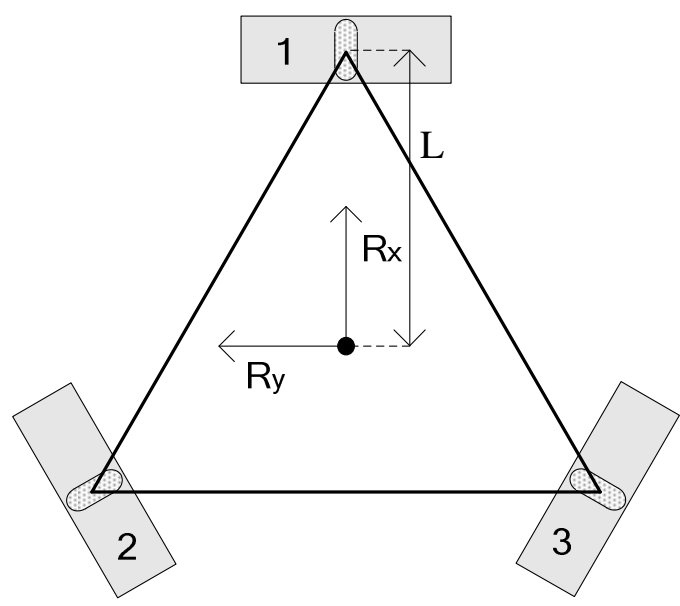

Figura 5.1- Vehículo omnidireccional (tipo 1) isotrópico con tres ruedas suecas

\subsubsection{B) Vehículo omnidireccional con tres ruedas castor}

Puesto que hay tres GDLs instantáneos, se necesitan al menos tres actuadores/sensores para poder actuar/sensorizar sobre todos ellos.

Las posibles actuaciones consistentes (al margen de las configuraciones singulares que se puedan producir de forma instantánea, ver Capítulo 6) son:

- Accionar las tres velocidades de rotación de las tres ruedas castor.

- Accionar las tres velocidades de dirección de las tres ruedas castor.

- Accionar dos velocidades de rotación y una de dirección.

- Accionar dos velocidades de dirección y una de rotación.

Sensorizando las mismas velocidades que en la actuación consistente se tendría una sensorización básica y al sensorizar alguna velocidades más resulta una sensorización consistente. 
Desde un punto de vista práctico, suele ser preferible actuar sobre las velocidades de rotación de las ruedas castor puesto que normalmente necesitan menores pares motor que los requeridos por las velocidades de dirección. Además, la tracción del vehículo es mejor sobre la superficie de trabajo al actuar sobre la rotación de las ruedas.

Al sensorizar también las velocidades de rotación dan un valor angular mayor para un mismo desplazamiento que en el caso de las velocidades de dirección. Esto permite un menor error para la misma resolución del encoder que sensoriza la velocidad de rotación/dirección. Aunque, dado que los ángulos de dirección están presentes implícitamente en la cinemática, es imprescindible sensorizar dichos ángulos para poder calcular la solución dada por los modelos.

En cuanto al modelado, la ecuación (3.12) hay que considerarla tres veces, una por cada rueda castor, por lo que (4.1) resulta:

$$
\mathbf{A}_{\mathrm{p}} \cdot \dot{\mathbf{p}}+\mathbf{A}_{\mathrm{w}} \cdot\left(\begin{array}{c}
\dot{\boldsymbol{\beta}} \\
\dot{\boldsymbol{\varphi}}
\end{array}\right)=\mathbf{0}
$$

con

$$
\begin{aligned}
\dot{\boldsymbol{\beta}}=\left(\begin{array}{lll}
\dot{\beta}_{1} & \dot{\beta}_{2} & \dot{\beta}_{3}
\end{array}\right)^{\mathrm{T}} & \dot{\boldsymbol{\varphi}}=\left(\begin{array}{lll}
\dot{\varphi}_{1} & \dot{\varphi}_{2} & \dot{\varphi}_{3}
\end{array}\right)^{\mathrm{T}} \\
\mathbf{A}_{\mathrm{w}}=\left(\begin{array}{cc}
-\mathrm{d} \cdot \mathbf{I} & \mathbf{0} \\
\mathbf{0} & \mathrm{r} \cdot \mathbf{I}
\end{array}\right) & \mathbf{A}_{\mathrm{p} 1}=\left(\begin{array}{ccc}
\mathrm{c} \beta_{1} & \mathrm{~s} \beta_{1} & 1_{1} \cdot \mathrm{s}\left(\beta_{1}-\alpha_{1}\right)-\mathrm{d} \\
\mathrm{c} \beta_{2} & \mathrm{~s} \beta_{2} & 1_{2} \cdot \mathrm{s}\left(\beta_{2}-\alpha_{2}\right)-\mathrm{d} \\
\mathrm{c} \beta_{3} & \mathrm{~s} \beta_{3} & 1_{3} \cdot \mathrm{s}\left(\beta_{3}-\alpha_{3}\right)-\mathrm{d}
\end{array}\right) \\
\mathbf{A}_{\mathrm{p}}=\left(\begin{array}{c}
\mathbf{A}_{\mathrm{p} 1} \\
\mathbf{A}_{\mathrm{p} 2}
\end{array}\right) & \mathbf{A}_{\mathrm{p} 2}=\left(\begin{array}{lll}
-\mathrm{s} \beta_{1} & \mathrm{c} \beta_{1} & 1_{1} \cdot \mathrm{c}\left(\beta_{1}-\alpha_{1}\right) \\
-\mathrm{s} \beta_{2} & \mathrm{c} \beta_{2} & 1_{2} \cdot \mathrm{c}\left(\beta_{2}-\alpha_{2}\right) \\
-\mathrm{s} \beta_{3} & \mathrm{c} \beta_{3} & 1_{3} \cdot \mathrm{c}\left(\beta_{3}-\alpha_{3}\right)
\end{array}\right)
\end{aligned}
$$

En este caso, una solución cinemática (4.2) para (5.38) es:

$$
\left(\begin{array}{c}
\dot{\mathbf{p}} \\
\dot{\boldsymbol{\beta}} \\
\dot{\boldsymbol{\varphi}}
\end{array}\right)=\left(\begin{array}{c}
\mathbf{I} \\
(1 / \mathrm{d}) \cdot \mathbf{A}_{\mathrm{p} 1} \\
-(1 / \mathrm{r}) \cdot \mathbf{A}_{\mathrm{p} 2}
\end{array}\right) \cdot \boldsymbol{\eta}
$$

Los modelos cinemáticos directos, con $\dot{\boldsymbol{\beta}}$ o $\dot{\boldsymbol{\varphi}}$ como velocidades asignadas 
(el resto de posibilidades se omiten), e inversos son:

$$
\begin{gathered}
\left(\begin{array}{c}
\dot{\mathbf{p}} \\
\dot{\boldsymbol{\varphi}}
\end{array}\right)=\mathrm{d} \cdot\left(\begin{array}{c}
\mathbf{A}_{\mathrm{p} 1}^{-1} \\
-\frac{\mathbf{A}_{\mathrm{p} 2}}{\mathrm{r}} \cdot \mathbf{A}_{\mathrm{p} 1}^{-1}
\end{array}\right) \cdot \dot{\boldsymbol{\beta}}\left(\begin{array}{c}
\dot{\mathbf{p}} \\
\dot{\boldsymbol{\beta}}
\end{array}\right)=-\mathrm{r} \cdot\left(\begin{array}{c}
\mathbf{A}_{\mathrm{p} 2}^{-1} \\
\frac{\mathbf{A}_{\mathrm{p} 1}}{\mathrm{~d}} \cdot \mathbf{A}_{\mathrm{p} 2}^{-1}
\end{array}\right) \cdot \dot{\boldsymbol{\varphi}} \\
\left(\begin{array}{c}
\dot{\boldsymbol{\beta}} \\
\dot{\boldsymbol{\varphi}}
\end{array}\right)=\left(\begin{array}{c}
1 / \mathrm{d} \cdot \mathbf{A}_{\mathrm{p} 1} \\
-1 / \mathrm{r} \cdot \mathbf{A}_{\mathrm{p} 2}
\end{array}\right) \cdot \dot{\mathbf{p}}
\end{gathered}
$$

En [Low et al. 05] se indica que la condición de isotropía para los modelos directo e inverso anteriores se alcanza con los ejes de direccionamiento en los vértices de un triángulo equilátero y con $\mathrm{d}=\mathrm{r}$. Sin embargo, este resultado de isotropía sólo es válido si se desprecia la distancia d del brazo de dirección respecto a la distancia entre el centroide del triángulo y el eje de dirección. En caso contrario las condiciones de isotropía dependen de los ángulos de dirección.

De hecho, el análisis isotrópico complete (para $\dot{\mathbf{q}}_{\text {na_s }}=\dot{\mathbf{p}}$ ) se puede encontrar [Kim2 et al. 04], donde las configuraciones isotrópicas (valores de $\beta_{1}, \beta_{2}, \beta_{3}$ ) se caracterizan en el modelo cinemático directo dependiendo del conjunto de velocidades asignadas. Además, estos autores desarrollan en [Kim et al. 05] una optimización de la isotropía global a través de la distancia característica. La isotropía global la definen, para un conjunto de velocidades asignadas, como la media de la inversa del número de condición (isotropía local) a lo largo del espacio de configuración dado por $\beta_{1}, \beta_{2}, \mathrm{y} \beta_{3}$.

Tanto [Low et al. 05] como [Kim2 et al. 04]-[Kim et al. 05] consideran ocasionalmente más de tres velocidades asignadas, ej. articulaciones accionadas, lo que no es lógico dentro del marco de modelos sin deslizamiento de (4.2).

Con las condiciones de isotropía de [Low et al. 05] y situando el sistema de coordenadas $\mathrm{R}$ en el centroide de este triángulo, con su eje $X$ cruzando el eje de dirección de la primera rueda (ver la Figura 5.2), los parámetros resultan: $\alpha_{1}=0, \alpha_{2}=120^{\circ}, \alpha_{3}=240^{\circ}, 1_{i}=\mathrm{L}, \mathrm{r}=\mathrm{d}$. Su sustitución en (5.41) se omite puesto que los modelos no se simplifican significativamente. 


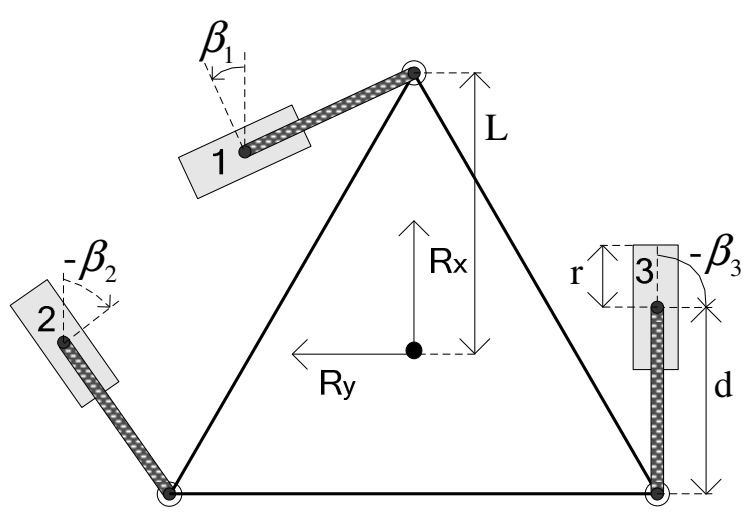

Figura 5.2- Vehículo omnidireccional quasi-isotrópico con tres ruedas castor

\subsubsection{Tipo 2 (2,0): Vehículo diferencial}

Este vehículo tiene una rueda fija independiente y otras posibles ruedas omnidireccionales, resultando una movilidad $m=2$. Este estudio considera dos ruedas fijas, numeradas 1 y 2 , con un eje de rotación común (el clásico mecanismo diferencial) más una rueda omnidireccional, numerada 3 , bien sueca o castor. Por lo que surgen dos posibilidades.

Para actuar/sensorizar sobre los dos GDLs instantáneos del vehículo se necesitan al menos dos actuadores/sensores. Al estar caracterizado este vehículo por las ruedas fijas dicha actuación/sensorización se centra normalmente en ellas, concretamente en su velocidad de rotación. Más aún cuando dichas ruedas suelen tener mayor tamaño e importancia que las adicionales del tipo omnidireccional, normalmente con la única finalidad de estabilizar el vehículo. La actuación sobre las dos ruedas fijas se realiza con un mecanismo denominado diferencial, que les transmite el movimiento y da nombre al vehículo.

Para este vehículo el origen del sistema de coordenadas $\mathrm{R}$ se ha situado (ver las Figuras 5.3 y 5.5) en el punto medio de la recta que une las dos ruedas fijas y con su eje $X$ coincidente con dicha recta, por lo que los parámetros resultan: $\beta_{1}=\beta_{2}=\alpha_{1}=0, \alpha_{2}=\pi, 1_{1}=l_{2}=1_{12}$.

Como excepción, para este vehículo no se asumirá el mismo radio para las tres ruedas en las ecuaciones del modelo. 


\subsubsection{A) Vehículo diferencial con rueda sueca}

Para este vehículo, mostrado en la Figura 5.3, hay que utilizar dos veces la ecuación (3.11) y una vez la (3.14), por lo que (4.1) resulta:

$$
\mathbf{A}_{\mathrm{p}} \cdot \dot{\mathbf{p}}+\mathbf{A}_{\mathrm{w}} \cdot \dot{\boldsymbol{\varphi}}=\mathbf{A}_{\mathrm{p}} \cdot \dot{\mathbf{p}}+\mathbf{A}_{\mathrm{w}} \cdot\left(\begin{array}{lll}
\dot{\varphi}_{1} & \dot{\varphi}_{2} & \dot{\varphi}_{3}
\end{array}\right)^{\mathrm{T}}=\mathbf{0}
$$

con

$$
\mathbf{A}_{\mathrm{w}}=\left(\begin{array}{ccc}
0 & 0 & 0 \\
\mathrm{r}_{1} & 0 & 0 \\
0 & 0 & 0 \\
0 & \mathrm{r}_{2} & 0 \\
0 & 0 & \mathrm{r}_{3}
\end{array}\right) \quad \mathbf{A}_{\mathrm{p}}=\left(\begin{array}{ccc}
1 & 0 & 0 \\
0 & 1 & 1_{12} \\
1 & 0 & 0 \\
0 & 1 & -1_{12} \\
-\mathrm{s} \beta_{3} & c \beta_{3} & 1_{3} \cdot c\left(\beta_{3}-\alpha_{3}\right)
\end{array}\right)
$$

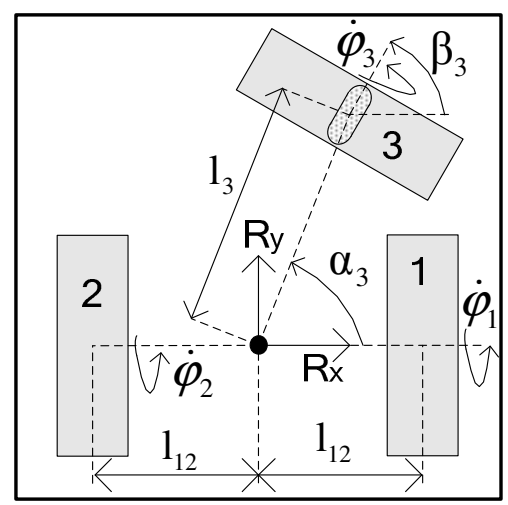

Figura 5.3- Vehículo diferencial (tipo 2) con rueda sueca

Una solución cinemática (4.2) para (5.42) es:

$$
\left(\begin{array}{l}
\dot{\mathbf{p}} \\
\dot{\boldsymbol{\varphi}}
\end{array}\right)=\left(\begin{array}{cc}
0 & 0 \\
-1_{12} & -1_{12} \\
-1 & 1 \\
2 \cdot 1_{12} / \mathrm{r}_{1} & 0 \\
0 & 2 \cdot 1_{12} / \mathrm{r}_{2} \\
\frac{1_{12} \cdot c \beta_{3}+1_{3} \cdot c\left(\beta_{3}-\alpha_{3}\right)}{\mathrm{r}_{3}} & \frac{1_{12} \cdot c \beta_{3}-1_{3} \cdot c\left(\beta_{3}-\alpha_{3}\right)}{\mathrm{r}_{3}}
\end{array}\right) \cdot \boldsymbol{\eta}
$$

Los modelos cinemáticos directos e inversos se pueden obtener de forma inmediata de (5.44) aplicando (4.5) para cualquier par de velocidades que no incluya $v_{x}$. Por ejemplo, para $\dot{\mathbf{q}}_{\mathrm{a}}=\left(\dot{\varphi}_{1} \dot{\varphi}_{2}\right)^{\mathrm{T}} \mathrm{y} \quad \dot{\mathbf{q}}_{\mathrm{a}}=\left(v_{y} \omega\right)^{\mathrm{T}}$ se obtienen los 
clásicos modelos diferenciales directo e inverso:

$$
\begin{gathered}
\dot{\mathbf{p}}=\left(\begin{array}{cc}
0 & 0 \\
-\mathrm{r}_{1} / 2 & -\mathrm{r}_{2} / 2 \\
-\mathrm{r}_{1} /\left(2 \cdot \mathrm{l}_{12}\right) & \mathrm{r}_{2} /\left(2 \cdot \mathrm{l}_{12}\right)
\end{array}\right) \cdot\left(\begin{array}{l}
\dot{\varphi}_{1} \\
\dot{\varphi}_{2}
\end{array}\right) \\
\left(\begin{array}{c}
v_{x} \\
\dot{\varphi}_{1} \\
\dot{\varphi}_{2}
\end{array}\right)=\left(\begin{array}{cc}
0 & 0 \\
-1 / \mathrm{r}_{1} & -1_{12} / \mathrm{r}_{1} \\
-1 / \mathrm{r}_{2} & 1_{12} / \mathrm{r}_{2}
\end{array}\right) \cdot\left(\begin{array}{l}
v_{y} \\
\omega
\end{array}\right)
\end{gathered}
$$

donde $\dot{\varphi}_{3}$ se omite para reconocer mejor los modelos.

Para el análisis de isotropía se considerarán las siguientes relaciones, obtenidas de (5.44), entre las velocidades no nulas del vehículo y las de rueda:

$$
\begin{gathered}
\left(\begin{array}{cc}
-1 / \mathrm{r}_{1} & -\mathrm{l}_{12} / \mathrm{r}_{1} \\
-1 / \mathrm{r}_{2} & \mathrm{l}_{12} / \mathrm{r}_{2}
\end{array}\right) \cdot\left(\begin{array}{c}
v_{y} \\
\omega
\end{array}\right)=\left(\begin{array}{c}
\dot{\varphi}_{1} \\
\dot{\varphi}_{2}
\end{array}\right) \\
\left(\begin{array}{cc}
-1 / \mathrm{r}_{1} & -1_{12} / \mathrm{r}_{1} \\
-\mathrm{c} \beta_{3} / \mathrm{r}_{3} & -\mathrm{l}_{3} \cdot \mathrm{c}\left(\beta_{3}-\alpha_{3}\right) / \mathrm{r}_{3}
\end{array}\right) \cdot\left(\begin{array}{c}
v_{y} \\
\omega
\end{array}\right)=\left(\begin{array}{c}
\dot{\varphi}_{1} \\
\dot{\varphi}_{3}
\end{array}\right)
\end{gathered}
$$

Como se ha comentado en el punto 5.4, es necesario introducir en (5.47) las distancias características $\mathrm{D}_{1}$ y $\mathrm{D}_{2}$ :

$$
\begin{gathered}
\left(\begin{array}{cc}
-1 / \mathrm{r}_{1} & -\mathrm{l}_{12} /\left(\mathrm{r}_{1} \cdot \mathrm{D}_{1}\right) \\
-1 / \mathrm{r}_{2} & 1_{12} /\left(\mathrm{r}_{2} \cdot \mathrm{D}_{1}\right)
\end{array}\right) \cdot\left(\begin{array}{c}
v_{y} \\
\mathrm{D}_{1} \cdot \omega
\end{array}\right)=\left(\begin{array}{c}
\dot{\varphi}_{1} \\
\dot{\varphi}_{2}
\end{array}\right) \\
\left(\begin{array}{cc}
-1 / \mathrm{r}_{1} & -\mathrm{l}_{12} /\left(\mathrm{r}_{1} \cdot \mathrm{D}_{2}\right) \\
-\mathrm{c} \beta_{3} / \mathrm{r}_{3} & -\mathrm{l}_{3} \cdot \mathrm{c}\left(\beta_{3}-\alpha_{3}\right) /\left(\mathrm{r}_{3} \cdot \mathrm{D}_{2}\right)
\end{array}\right) \cdot\left(\begin{array}{c}
v_{y} \\
\mathrm{D}_{2} \cdot \omega
\end{array}\right)=\left(\begin{array}{c}
\dot{\varphi}_{1} \\
\dot{\varphi}_{3}
\end{array}\right)
\end{gathered}
$$

Para establecer las condiciones de isotropía se aplica (5.29):

$$
\begin{aligned}
& \left(\begin{array}{cc}
1 / r_{1}^{2}+1 / r_{2}^{2} & \frac{l_{12}}{D_{1}} \cdot\left(1 / r_{1}^{2}-1 / r_{2}^{2}\right) \\
\frac{1_{12}}{D_{1}} \cdot\left(1 / r_{1}^{2}-1 / r_{2}^{2}\right) & \frac{l_{12}^{2}}{D_{1}^{2}} \cdot\left(1 / r_{1}^{2}+1 / r_{2}^{2}\right)
\end{array}\right)=k_{1} \cdot \mathbf{I} \\
& \left(\begin{array}{cc}
\frac{1}{\mathrm{r}_{1}^{2}}+\left(\frac{\mathrm{c} \beta_{3}}{\mathrm{r}_{3}}\right)^{2} & \frac{\mathrm{l}_{12}}{\mathrm{D}_{2} \cdot \mathrm{r}_{1}^{2}}+\frac{\mathrm{l}_{3} \cdot c \beta_{3} \cdot \mathrm{c}\left(\beta_{3}-\alpha_{3}\right)}{\mathrm{r}_{3}^{2} \cdot \mathrm{D}_{2}} \\
\frac{\mathrm{l}_{12}}{\mathrm{D}_{2} \cdot \mathrm{r}_{1}^{2}}+\frac{\mathrm{l}_{3} \cdot c \beta_{3} \cdot c\left(\beta_{3}-\alpha_{3}\right)}{\mathrm{r}_{3}^{2} \cdot \mathrm{D}_{2}} & \left(\frac{\mathrm{l}_{12}^{2}}{\mathrm{D}_{2}^{2} \cdot \mathrm{r}_{1}^{2}}+\left(\frac{\mathrm{l}_{3} \cdot \mathrm{c}\left(\beta_{3}-\alpha_{3}\right)}{\mathrm{r}_{3} \cdot \mathrm{D}_{2}}\right)^{2}\right)
\end{array}\right)=\mathrm{k}_{2} \cdot \mathbf{I}
\end{aligned}
$$


De (5.49) se obtienen los siguientes valores de parámetros:

$$
\mathrm{r}_{1}=\mathrm{r}_{2} \quad \mathrm{D}_{1}=\mathrm{l}_{12}
$$

Por lo que la isotropía de la primera relación de (5.48), entre las velocidades del vehículo no nulas $\left(v_{y}, \omega\right)$ y las velocidades de rotación de las dos ruedas fijas $\left(\dot{\varphi}_{1}, \dot{\varphi}_{2}\right)$, se alcanza para el mismo radio en dichas ruedas (5.51).

De (5.50) se obtienen dos ecuaciones: la primera (equivalencia de los elementos $(1,1)$ y $(2,2)$ de la matriz del miembro de la izquierda de (5.50)) se utiliza para calcular la distancia característica $\mathrm{D}_{2}$, y la segunda proporciona la siguiente relación:

$$
\mathrm{c} \beta_{3} \cdot \mathrm{l}_{3} \cdot \mathrm{c}\left(\beta_{3}-\alpha_{3}\right)=-\mathrm{l}_{12} \cdot\left(\mathrm{r}_{3} / \mathrm{r}_{1}\right)^{2}=-\mathrm{C}_{1}
$$

Esta condición de isotropía significa, ver Figura 5.4 (a), que la componente $X$ (respecto al sistema $\mathrm{R}$ ) del vector de distancia entre el origen de $\mathrm{R}$ y la línea definida por el plano de la rueda sueca, es igual a la constante negativa $-\mathrm{C}_{1}$.

Si se considera un sistema de coordenadas R' (en lugar de R) situado en la primera rueda fija, la expresión (5.52) es válida pero con $1_{12}=0$. En ese caso la condición de isotropía (5.52) resulta $\beta_{3}= \pm 90^{\circ}$, Figura 5.4 (b), donde el movimiento de avance viene dado por la rotación de la rueda fija y el angular por la rotación de la rueda sueca. Notar que la solución de isotropía $\beta_{3}-\alpha_{3}= \pm 90^{\circ}$ y $1_{3}=0$ no son válidas porque producen singularidad cinemática (ver Capítulo 6).

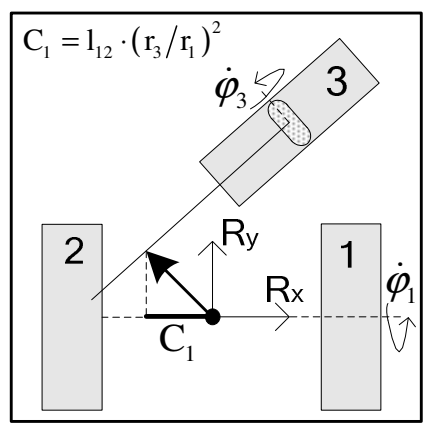

(a) $\mathrm{R}\left(\mathrm{l}_{12} \neq 0\right)$

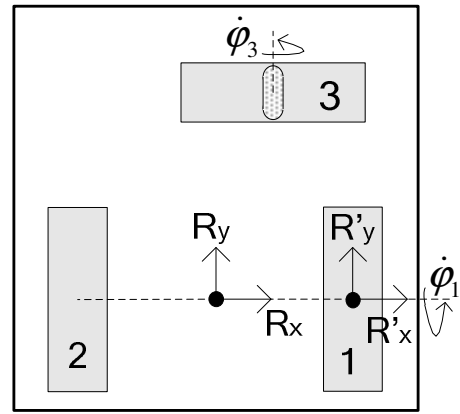

(b) R' $\left(1_{12}=0\right)$

Figura 5.4- Condiciones de isotropía para el vehículo diferencial (tipo 2) con una

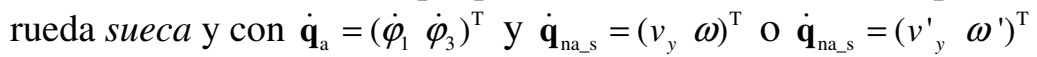




\subsubsection{B) Vehículo diferencial con rueda castor}

Para este vehículo, mostrado en la Figura 5.5, hay que utilizar dos veces la ecuación (3.11) y una vez la (3.12), por lo que (4.1) resulta:

$$
\mathbf{A}_{\mathrm{p}} \cdot \dot{\mathbf{p}}+\mathbf{A}_{\mathrm{w}} \cdot \dot{\mathbf{q}}_{\mathrm{w}}=\mathbf{A}_{\mathrm{p}} \cdot \dot{\mathbf{p}}+\mathbf{A}_{\mathrm{w}} \cdot\left(\begin{array}{llll}
\dot{\varphi}_{1} & \dot{\varphi}_{2} & \dot{\varphi}_{3} & \dot{\beta}_{3}
\end{array}\right)^{\mathrm{T}}=\mathbf{0}
$$

con

$$
\mathbf{A}_{\mathrm{w}}=\left(\begin{array}{cccc}
0 & 0 & 0 & 0 \\
\mathrm{r}_{1} & 0 & 0 & 0 \\
0 & 0 & 0 & 0 \\
0 & \mathrm{r}_{2} & 0 & 0 \\
0 & 0 & \mathrm{r}_{3} & 0 \\
0 & 0 & 0 & -\mathrm{d}_{3}
\end{array}\right) \mathbf{A}_{\mathrm{p}}=\left(\begin{array}{ccc}
1 & 0 & 0 \\
0 & 1 & 1_{12} \\
1 & 0 & 0 \\
0 & 1 & -1_{12} \\
-\mathrm{s} \beta_{3} & \mathrm{c} \beta_{3} & 1_{3} \cdot \mathrm{c}\left(\beta_{3}-\alpha_{3}\right) \\
\mathrm{c} \beta_{3} & \mathrm{~s} \beta_{3} & 1_{3} \cdot \mathrm{s}\left(\beta_{3}-\alpha_{3}\right)-\mathrm{d}_{3}
\end{array}\right)
$$

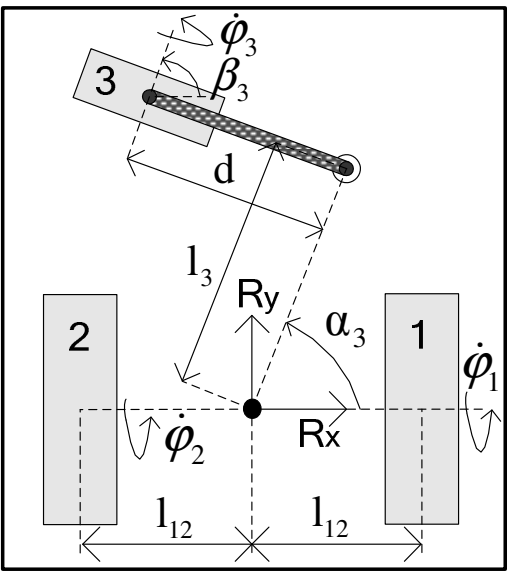

Figura 5.5- Vehículo diferencial (tipo 2) con rueda castor

Una solución cinemática (4.2) para (5.53) es:

$$
\left(\begin{array}{c}
\dot{\mathbf{p}} \\
\dot{\mathbf{q}}_{\mathrm{w}}
\end{array}\right)=\left(\begin{array}{ccccccc}
0 & -\mathrm{l}_{12} & -1 & 2 \cdot \mathrm{l}_{12} / \mathrm{r}_{1} & 0 & a_{1} / \mathrm{r}_{3} & -a_{3} / \mathrm{d}+1 \\
0 & -1_{12} & 1 & 0 & 2 \cdot 1_{12} / \mathrm{r}_{2} & a_{2} / \mathrm{r}_{3} & -a_{4} / \mathrm{d}-1
\end{array}\right)^{\mathrm{T}} \cdot \boldsymbol{\eta}
$$

con

$$
\begin{array}{ll}
a_{1}=1_{12} \cdot \mathrm{c} \beta_{3}+1_{3} \cdot \mathrm{c}\left(\beta_{3}-\alpha_{3}\right) & a_{2}=1_{12} \cdot \mathrm{c} \beta_{3}-1_{3} \cdot \mathrm{c}\left(\beta_{3}-\alpha_{3}\right) \\
a_{3}=1_{12} \cdot \mathrm{s} \beta_{3}+1_{3} \cdot \mathrm{s}\left(\beta_{3}-\alpha_{3}\right) & a_{4}=1_{12} \cdot \mathrm{s} \beta_{3}-1_{3} \cdot \mathrm{s}\left(\beta_{3}-\alpha_{3}\right)
\end{array}
$$

Como antes, los modelos cinemáticos directos e inversos se obtienen directamente de (5.55) aplicando (4.5) para cualquier par de velocidades 
asignadas que no incluya $v_{x}$. En particular para $\left(\dot{\varphi}_{1} \dot{\varphi}_{2}\right)^{\mathrm{T}}$ y $\left(v_{y} \omega\right)^{\mathrm{T}}$ como $\dot{\mathbf{q}}_{\mathrm{a}}$ resultan los mismos clásicos modelos directos e inversos de (5.45) y (5.46).

Para ese caso, las relaciones de (5.47) y las condiciones de isotropía (5.51) y (5.52) son válidas. Pero ahora, dado que el ángulo de dirección $\beta_{3}$ es variable, la isotropía de (5.52) se alcanza para una configuración particular, Figura 5.6 (a).

Además, las siguientes nuevas relaciones entre velocidades pueden considerarse para isotropía:

$$
\begin{aligned}
\left(\begin{array}{c}
\dot{\varphi}_{1} \\
\dot{\beta}_{3}
\end{array}\right) & =\left(\begin{array}{cc}
-1 / \mathrm{r}_{1} & -\mathrm{l}_{12} /\left(\mathrm{D}_{4} \cdot \mathrm{r}_{1}\right) \\
\frac{\mathrm{s} \beta_{3}}{\mathrm{~d}} & \frac{1_{3} \cdot \mathrm{s}\left(\beta_{3}-\alpha_{3}\right)-\mathrm{d}}{\mathrm{d} \cdot \mathrm{D}_{4}}
\end{array}\right) \cdot\left(\begin{array}{c}
v_{y} \\
\mathrm{D}_{4} \cdot \omega
\end{array}\right) \\
\left(\begin{array}{c}
\dot{\varphi}_{3} \\
\dot{\beta}_{3}
\end{array}\right) & =\left(\begin{array}{ll}
\frac{-\mathrm{c} \beta_{3}}{\mathrm{r}_{3}} & \frac{-\mathrm{l}_{3} \cdot \mathrm{c}\left(\beta_{3}-\alpha_{3}\right)}{\mathrm{r}_{3} \cdot \mathrm{D}_{5}} \\
\frac{\mathrm{s} \beta_{3}}{\mathrm{~d}} & \frac{1_{3} \cdot \mathrm{s}\left(\beta_{3}-\alpha_{3}\right)-\mathrm{d}}{\mathrm{d} \cdot \mathrm{D}_{5}}
\end{array}\right) \cdot\left(\begin{array}{c}
v_{y} \\
\mathrm{D}_{5} \cdot \omega
\end{array}\right) \\
\left(\begin{array}{c}
\dot{\varphi}_{1} \\
\dot{\varphi}_{2}
\end{array}\right) & =\left(\begin{array}{ll}
2 \cdot 1_{12} \cdot \mathrm{r}_{3} \cdot \frac{a_{4}+\mathrm{d}}{\mathrm{r}_{1} \cdot a_{5}} & 2 \cdot 1_{12} \cdot \frac{\mathrm{d} \cdot a_{2}}{\mathrm{r}_{1} \cdot a_{5}} \\
2 \cdot 1_{12} \cdot \mathrm{r}_{3} \cdot \frac{\mathrm{d}-a_{3}}{\mathrm{r}_{2} \cdot a_{5}} & -2 \cdot 1_{12} \cdot \frac{\mathrm{d} \cdot a_{1}}{\mathrm{r}_{2} \cdot a_{5}}
\end{array}\right) \cdot\left(\begin{array}{l}
\dot{\varphi}_{3} \\
\dot{\beta}_{3}
\end{array}\right) \\
\text { con } \quad a_{5} & =2 \cdot 1_{12} \cdot\left(1_{3} \cdot \mathrm{s} \beta_{3}+\mathrm{d} \cdot \mathrm{c} \beta_{3}\right)
\end{aligned}
$$

donde las distancias características necesarias $\mathrm{D}_{4}$ y $\mathrm{D}_{5}$ han sido introducidas.

Después de aplicar (5.29) a (5.57)a , se obtiene:

$$
\mathrm{s} \beta_{3} \cdot\left(1_{3} \cdot \mathrm{s}\left(\beta_{3}-\alpha_{3}\right)-\mathrm{d}\right)=-\left(\mathrm{d} / \mathrm{r}_{1}\right)^{2} \cdot \mathrm{l}_{12}=-\mathrm{C}_{2}
$$

La configuración isotrópica (valor de $\beta_{3}$ ) que cumple (5.58) es tal que la componente $X$ respecto al sistema $\mathrm{R}$ del vector de distancia entre el origen de $\mathrm{R}$ y la línea definida por el eje de rotación de la rueda castor, es igual a la constante negativa $-\mathrm{C}_{2}$ (ver Figura 9 (b)). Notar que esta configuración isotrópica no es possible seguro cuando $\left(\mathrm{l}_{3}+\mathrm{d}\right)<\mathrm{l}_{12} \cdot\left(\mathrm{d} / \mathrm{r}_{1}\right)^{2}=\mathrm{C}_{2}$, en caso contrario su existencia depende de $\alpha_{3}$.

Con el mismo procedimiento, para $(5.57)_{\mathrm{b}}$ se obtiene:

$$
\left(\mathrm{d} / \mathrm{r}_{3}\right)^{2} \cdot \mathrm{c} \beta_{3} \cdot 1_{3} \cdot \mathrm{c}\left(\beta_{3}-\alpha_{3}\right)=-\mathrm{s} \beta_{3} \cdot\left(1_{3} \cdot \mathrm{s}\left(\beta_{3}-\alpha_{3}\right)-\mathrm{d}\right)
$$


La Figura 9 (c) muestra de forma gráfica la correspondiente configuración isotrópica: la componente $X \quad\left(c_{1}\right)$ del primer vector de distancia (entre el origen de $\mathrm{R}$ y la línea que define el eje de rotación de la rueda castor) es igual al valor negativo de la misma componente $X\left(c_{2}\right)$ del segundo vector de distancia (entre el origen de $\mathrm{R}$ y la línea definida por el brazo de dirección de la rueda castor) multiplicado por el factor $\left(\mathrm{d} / \mathrm{r}_{3}\right)^{2}$. Esta configuración isotrópica siempre existe.

Para $(5.57)_{\mathrm{c}}$ se obtienen las siguientes relaciones:

$$
\begin{gathered}
\left(\mathrm{d}+a_{4}\right) \cdot a_{2} / \mathrm{r}_{1}^{2}=\left(\mathrm{d}-a_{3}\right) \cdot a_{1} / \mathrm{r}_{2}^{2} \\
\mathrm{r}_{3}^{2} \cdot\left(\left(\mathrm{d}+a_{4}\right)^{2} \cdot \mathrm{r}_{2}^{2}+\left(\mathrm{d}-a_{3}\right)^{2} \cdot \mathrm{r}_{1}^{2}\right)=\mathrm{d}^{2} \cdot\left(a_{1}^{2} \cdot \mathrm{r}_{2}^{2}+a_{2}^{2} \cdot \mathrm{r}_{1}^{2}\right),
\end{gathered}
$$

las cuales, asumiendo el mismo radio $\mathrm{r}$ par alas tres ruedas y sustituyendo los valores de $a_{1}, a_{2}, a_{3}$, y $a_{4}$, de (5.56), resultan:

$$
\begin{aligned}
\left(1_{3} \cdot \mathrm{c}\left(\beta_{3}-\alpha_{3}\right)\right) \cdot\left(\mathrm{d}-1_{3} \cdot \mathrm{s}\left(\beta_{3}-\alpha_{3}\right)\right) & =\left(1_{12} \cdot \mathrm{s} \beta_{3}\right) \cdot\left(1_{12} \cdot \mathrm{c} \beta_{3}\right) \\
& \rightarrow c_{5} \cdot c_{6}=c_{3} \cdot c_{4} \\
\left(1_{12} \cdot \mathrm{s} \beta_{3}\right)^{2}+\left(\mathrm{d}-1_{3} \cdot \mathrm{s}\left(\beta_{3}-\alpha_{3}\right)\right)^{2} & =\left(\mathrm{d}^{2} / \mathrm{r}^{2}\right) . \\
\left(\left(1_{12} \cdot \mathrm{c} \beta_{3}\right)^{2}+\left(1_{3} \cdot \mathrm{c}\left(\beta_{3}-\alpha_{3}\right)\right)^{2}\right) & \rightarrow c_{3}^{2}+c_{6}^{2}=\left(\mathrm{d}^{2} / \mathrm{r}^{2}\right) \cdot\left(c_{4}^{2}+c_{5}^{2}\right) .
\end{aligned}
$$

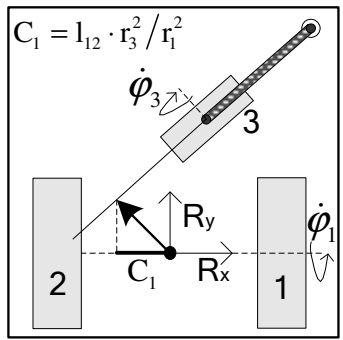

(a) $\dot{\mathbf{q}}_{\mathrm{a}}=\left(\dot{\varphi}_{1} \dot{\varphi}_{3}\right)^{\mathrm{T}} \quad \dot{\mathbf{q}}_{\mathrm{na} \_ \text {s }}=\left(v_{y} \omega\right)^{\mathrm{T}}$

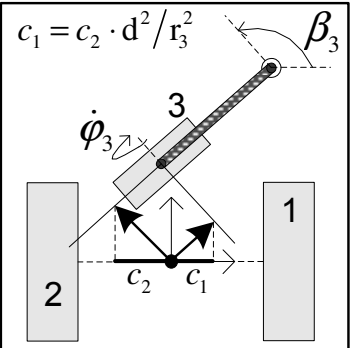

(c) $\dot{\mathbf{q}}_{\mathrm{a}}=\left(\dot{\varphi}_{3} \dot{\beta}_{3}\right)^{\mathrm{T}} \dot{\mathbf{q}}_{\mathrm{n} \_\mathrm{s}}=\left(v_{\mathrm{y}} \omega\right)^{\mathrm{T}}$

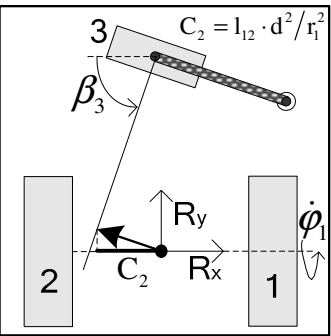

(b) $\dot{\mathbf{q}}_{\mathrm{a}}=\left(\dot{\varphi}_{1} \dot{\beta}_{3}\right)^{\mathrm{T}} \dot{\mathbf{q}}_{\mathrm{na} \_\mathrm{s}}=\left(v_{y} \omega\right)^{\mathrm{T}}$

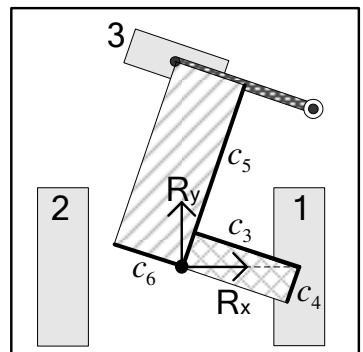

(d) $\dot{\mathbf{q}}_{\mathrm{a}}=\left(\dot{\varphi}_{1} \dot{\varphi}_{2}\right)^{\mathrm{T}} \quad \dot{\mathbf{q}}_{\mathrm{na} \_\mathrm{s}}=\left(\dot{\varphi}_{3} \dot{\beta}_{3}\right)^{\mathrm{T}}$

Figura 5.6- Condiciones de isotropía para el vehículo diferencial con rueda castor 
Las variables anteriores $c_{3}, c_{4}, c_{5}$, y $c_{6}$ se han representado en la Figura 9 (d). La primera condición de isotropía de (5.61) indica la equivalencia del área de dos rectángulos, mientras que la segunda no tiene un significado gráfico evidente. Notar que para alcanzar simultáneamente las dos condiciones de isotropía, una constante además de la variable de configuración $\beta_{3}\left(\right.$ ej. $\left.\alpha_{3}\right)$ se debe diseñar apropiadamente.

\subsubsection{Tipo 3 (2,1): Vehículo con una rueda orientable}

Este vehículo tiene una rueda orientable independiente y otras posibles ruedas omnidireccionales. El grado de movilidad, y por tanto el número de velocidades que se pueden asignar independientemente, es dos. Esto se debe a tener una ecuación de restricción. Además hay un GDL aparente aportado por la reorientación de la rueda orientable.

Un ejemplo de este tipo es el síncrono (Synchro Drive) donde múltiples ruedas orientables se sincronizan para tener siempre la misma orientación. Debido a esta sincronización todas equivalen conceptualmente a una única rueda y por tanto sólo se aprovecha uno de los dos GDLs instantáneos, más el aparente.

Al desaprovechar este GDL instantáneo se evita* actuar/sensorizar alguna rueda omnidireccional adicional, de menor tamaño e importancia. Manteniéndose un GDL instantáneo y otro aparente, suficiente en la mayoría de casos.

*Nota: Actuando/sensorizando sólo la rueda orientable, que caracteriza al vehículo, no se abarca toda la movilidad, ya que sólo se tendría su velocidad de rotación.

En este estudio se considera la rueda orientable, numerada $1, \mathrm{y}$ dos ruedas adicionales omnidireccionales del mismo tipo, numeradas 2 y 3 .

La actuación consistente vendría dada por actuar sobre la velocidad de rotación de la rueda orientable y la de una rueda omnidireccional adicional. Con esas mismas velocidades sensorizadas se consigue la sensorización básica, y sensorizando alguna más se convierte en consistente. Además, se debe accionar/sensorizar la dirección de la rueda orientable para disponer de su GDL aparente/computar los modelos cinemáticos.

Para este vehículo el origen del sistema de coordenadas $\mathrm{R}$ se ha situado en el centro de la rueda orientable (ver Figuras 5.7 y 5.9), por lo que $l_{1}=0$. 


\subsubsection{A) Vehículo con una rueda orientable y dos suecas}

Para el modelado de este vehículo, mostrado en la Figura 5.7, la expresión (3.11) se ha de utilizar una vez y la de (3.14) dos veces, por lo que (4.1) resulta:

$$
\mathbf{A}_{\mathrm{p}} \cdot \dot{\mathbf{p}}+\mathbf{A}_{\mathrm{w}} \cdot \dot{\boldsymbol{\varphi}}=\mathbf{A}_{\mathrm{p}} \cdot \dot{\mathbf{p}}+\mathbf{A}_{\mathrm{w}} \cdot\left(\begin{array}{lll}
\dot{\varphi}_{1} & \dot{\varphi}_{2} & \dot{\varphi}_{3}
\end{array}\right)^{\mathrm{T}}=\mathbf{0}
$$

con

$$
\mathbf{A}_{\mathrm{w}}=\left(\begin{array}{ccc}
0 & 0 & 0 \\
\mathrm{r}_{1} & 0 & 0 \\
0 & \mathrm{r}_{2} & 0 \\
0 & 0 & \mathrm{r}_{3}
\end{array}\right) \quad \mathbf{A}_{\mathrm{p}}=\left(\begin{array}{ccc}
\mathrm{c} \beta_{1} & \mathrm{~s} \beta_{1} & 0 \\
-\mathrm{s} \beta_{1} & \mathrm{c} \beta_{1} & 0 \\
-\mathrm{s} \beta_{2} & \mathrm{c} \beta_{2} & 1_{2} \cdot \mathrm{c}\left(\beta_{2}-\alpha_{2}\right) \\
-\mathrm{s} \beta_{3} & \mathrm{c} \beta_{3} & 1_{3} \cdot \mathrm{c}\left(\beta_{3}-\alpha_{3}\right)
\end{array}\right)
$$

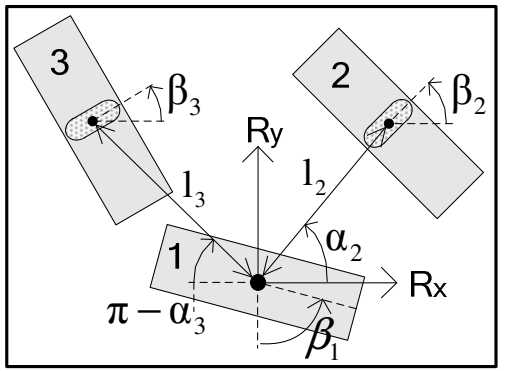

Figura 5.7- Vehículo con una rueda orientable (tipo 3) y dos ruedas suecas

A solución cinemática (4.2) para (5.62) es:

$$
\left(\begin{array}{l}
\dot{\mathbf{p}} \\
\dot{\boldsymbol{\varphi}}
\end{array}\right)=\left(\begin{array}{cc}
\mathrm{s} \beta_{1} & 0 \\
-\mathrm{c} \beta_{1} & 0 \\
0 & 1 \\
1 / \mathrm{r}_{1} & 0 \\
\mathrm{c}\left(\beta_{1}-\beta_{2}\right) / \mathrm{r}_{2} & -\mathrm{l}_{2} \cdot \mathrm{c}\left(\beta_{2}-\alpha_{2}\right) / \mathrm{r}_{2} \\
\mathrm{c}\left(\beta_{1}-\beta_{3}\right) / \mathrm{r}_{3} & -\mathrm{l}_{3} \cdot \mathrm{c}\left(\beta_{3}-\alpha_{3}\right) / \mathrm{r}_{3}
\end{array}\right) \cdot \boldsymbol{\eta}
$$

Los modelos cinemáticos directos e inversos se obtienen directamente de (5.64) aplicando (4.5) para cualquier par de velocidades excepto $\left(v_{x} v_{y}\right)^{\mathrm{T}}$. Notar que, (5.64) es directamente un modelo inverso donde las velocidades asignadas son el movimiento de avance del vehículo en la dirección del plano de la rueda orientable y el angular.

Para el análisis de isotropía existen seis relaciones entre pares de velocidades de ruedas y pares de velocidades del vehículo. Con el procedimiento 
mostrado para el vehículo de tipo 2, cada una de esas seis relaciones da lugar, aplicando (5.29), a una condición de isotropía.

En concreto, par alas velocidades asignadas $\dot{\mathbf{q}}_{\mathrm{a}}=\left(\dot{\varphi}_{1} \dot{\varphi}_{2}\right)^{\mathrm{T}}$ se obtiene $(5.65)_{\mathrm{a}}$, independientemente de las velocidades del vehículo consideradas. De igual modo, para las velocidades asignadas $\dot{\mathbf{q}}_{\mathrm{a}}=\left(\dot{\varphi}_{2} \dot{\varphi}_{3}\right)^{\mathrm{T}}$ resulta $(5.65)_{\mathrm{b}}$.

$$
\begin{gathered}
\mathrm{c}\left(\beta_{1}-\beta_{2}\right) \cdot \mathrm{c}\left(\beta_{2}-\alpha_{2}\right)=0 \\
\mathrm{c}\left(\beta_{1}-\beta_{3}\right) \cdot \mathrm{l}_{3} \cdot \mathrm{c}\left(\beta_{3}-\alpha_{3}\right)=-\left(\mathrm{r}_{3}^{2} / \mathrm{r}_{2}^{2}\right) \cdot \mathrm{c}\left(\beta_{1}-\beta_{2}\right) \cdot \mathrm{l}_{2} \cdot \mathrm{c}\left(\beta_{2}-\alpha_{2}\right)
\end{gathered}
$$

Las soluciones permanentes $\left\{\beta_{2}-\alpha_{2}= \pm 90^{\circ}\right\}$ para $(5.65)_{\mathrm{a}}$ y $\left\{\beta_{2}-\alpha_{2}=\right.$ $\left.\beta_{3}-\alpha_{3}= \pm 90^{\circ}\right\}$ para $\mathrm{b}$ no son válidas porque producen singuaridades cinemáticas (ver Capítulo 6).

La configuración isotrópica (valor de $\beta_{1}$ ) dado por (5.65)a, ver la Figura 5.8 (a), es tal que la rueda orientable es perpendicular a la primera rueda sueca. Mientras que la dada por (5.65) , ver la Figura 5.8 (b), es tal que la componente $X$ $\left(c_{7}\right)$ respecto al sistema $\mathrm{E}_{1}$ del primer vector de distancia entre el origen del sistema $\mathrm{E}_{1}$ y la línea definida por el plano de la rueda (sueca) 3 es igual al valor negativo de la misma componente $X\left(c_{8}\right)$ de la otra rueda sueca multiplicada por el factor $\left(\mathrm{r}_{3} / \mathrm{r}_{2}\right)^{2}$. Estas configuraciones isotrópicas siempre existen.

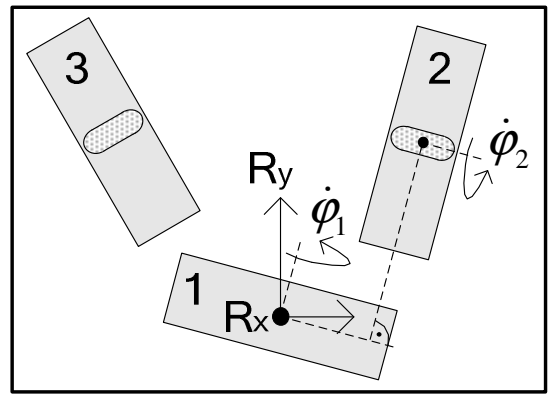

(a) $\dot{\mathbf{q}}_{\mathrm{a}}=\left(\dot{\varphi}_{1} \dot{\varphi}_{2}\right)^{\mathrm{T}}$

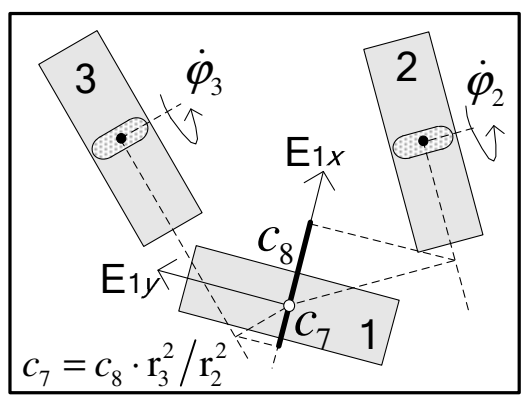

(b) $\dot{\mathbf{q}}_{\mathrm{a}}=\left(\dot{\varphi}_{2} \dot{\varphi}_{3}\right)^{\mathrm{T}} \rightarrow c_{7}=c_{8} \cdot\left(\mathrm{r}_{3} / \mathrm{r}_{2}\right)^{2}$

Figura 5.8- Configuraciones isotrópicas para el vehículo tipo 3 con ruedas suecas

Cabe señalar que la solución $\beta_{1}=\beta_{2}=\beta_{3}$, que cumple simultáneamente las dos condiciones de (5.65), no es válida por que produce una singularidad cinemática (ver Capítulo 6). 


\subsubsection{B) Vehículo con una rueda orientable y dos castor}

Para el modelado de este vehículo, mostrado en la Figura 5.9, la expresión (3.11) se utiliza una vez y la (3.12) dos, resultando (4.1):

$$
\mathbf{A}_{\mathrm{p}} \cdot \dot{\mathbf{p}}+\mathbf{A}_{\mathrm{w}} \cdot\left(\begin{array}{c}
\dot{\boldsymbol{\varphi}} \\
\dot{\boldsymbol{\beta}}
\end{array}\right)=\mathbf{A}_{\mathrm{p}} \cdot \dot{\mathbf{p}}+\mathbf{A}_{\mathrm{w}} \cdot\left(\begin{array}{lllll}
\dot{\varphi}_{1} & \dot{\varphi}_{2} & \dot{\varphi}_{3} & \dot{\beta}_{2} & \dot{\beta}_{3}
\end{array}\right)^{\mathrm{T}}=\mathbf{0}
$$

con

$$
\mathbf{A}_{\mathrm{w}}=\left(\begin{array}{cc}
0 & 0 \\
\mathrm{r} \cdot \mathbf{I} & \mathbf{0} \\
\mathbf{0} & -\mathrm{d} \cdot \mathbf{I}
\end{array}\right) \mathbf{A}_{\mathrm{p}}=\left(\begin{array}{ccc}
\mathrm{c} \beta_{1} & \mathrm{~s} \beta_{1} & 0 \\
-\mathrm{s} \beta_{1} & \mathrm{c} \beta_{1} & 0 \\
-\mathrm{s} \beta_{2} & \mathrm{c} \beta_{2} & 1_{2} \cdot \mathrm{c}\left(\beta_{2}-\alpha_{2}\right) \\
-\mathrm{s} \beta_{3} & \mathrm{c} \beta_{3} & 1_{3} \cdot \mathrm{c}\left(\beta_{3}-\alpha_{3}\right) \\
\mathrm{c} \beta_{2} & \mathrm{~s} \beta_{2} & 1_{2} \cdot \mathrm{s}\left(\beta_{2}-\alpha_{2}\right)-\mathrm{d} \\
\mathrm{c} \beta_{3} & \mathrm{~s} \beta_{3} & 1_{3} \cdot \mathrm{s}\left(\beta_{3}-\alpha_{3}\right)-\mathrm{d}
\end{array}\right)
$$

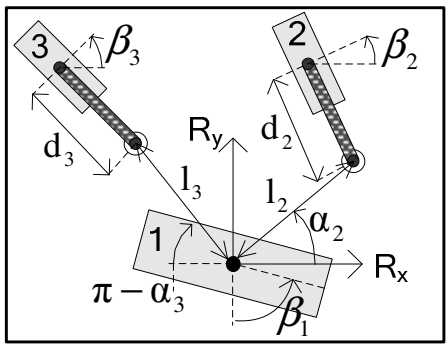

Figura 5.9- Vehículo con una rueda orientable (tipo 3) y dos ruedas castor

Una solución cinemática (4.2) para (5.66) es:

$$
\begin{aligned}
& \left(\begin{array}{c}
\dot{\mathbf{p}} \\
\dot{\boldsymbol{\varphi}} \\
\dot{\boldsymbol{\beta}}
\end{array}\right)=\left(\begin{array}{cc}
\mathrm{s} \beta_{1} & 0 \\
-\mathrm{c} \beta_{1} & 0 \\
0 & 1 \\
1 / \mathrm{r} & 0 \\
\mathrm{c} \beta_{12} / \mathrm{r} & -1_{2} \cdot \mathrm{c} \beta_{22} / \mathrm{r} \\
\mathrm{c} \beta_{13} / \mathrm{r} & -1_{3} \cdot \mathrm{c} \beta_{33} / \mathrm{r} \\
\mathrm{s} \beta_{12} / \mathrm{d} & 1_{2} \cdot \mathrm{s} \beta_{22} / \mathrm{d}-1 \\
\mathrm{~s} \beta_{13} / \mathrm{d} & 1_{3} \cdot \mathrm{s} \beta_{33} / \mathrm{d}-1
\end{array}\right) \cdot \boldsymbol{\eta} \\
& \text { con: } \beta_{12}=\beta_{1}-\beta_{2}, \beta_{13}=\beta_{1}-\beta_{3}, \beta_{22}=\beta_{2}-\alpha_{2}, \beta_{33}=\beta_{3}-\alpha_{3}
\end{aligned}
$$

Nuevamente (5.68) se puede considerar un modelo cinemático inverso con las mismas velocidades asignadas de antes. 
Los modelos cinemáticos directos e inversos se obitenen directamente de (5.68) para cualquier par de velocidades asignadas excepto $\left(v_{x} v_{y}\right)^{\mathrm{T}}$, surgiendo ocho posibilidades:

$$
\begin{aligned}
& \dot{\mathbf{q}}_{\mathrm{a} 1}=\left(\dot{\varphi}_{1} \dot{\varphi}_{2}\right)^{\mathrm{T}} \quad \dot{\mathbf{q}}_{\mathrm{a} 2}=\left(\dot{\varphi}_{1} \dot{\beta}_{2}\right)^{\mathrm{T}} \quad \dot{\mathbf{q}}_{\mathrm{a} 3}=\left(\dot{\varphi}_{2} \dot{\varphi}_{3}\right)^{\mathrm{T}} \quad \dot{\mathbf{q}}_{\mathrm{a} 4}=\left(\dot{\beta}_{2} \dot{\beta}_{3}\right)^{\mathrm{T}} \\
& \dot{\mathbf{q}}_{\mathrm{a} 5}=\left(\dot{\varphi}_{2} \dot{\beta}_{2}\right)^{\mathrm{T}} \quad \dot{\mathbf{q}}_{\mathrm{a} 6}=\left(\dot{\varphi}_{2} \dot{\beta}_{3}\right)^{\mathrm{T}} \quad \dot{\mathbf{q}}_{\mathrm{a} 7}=\left(v_{x} \omega\right)^{\mathrm{T}} \quad \dot{\mathbf{q}}_{\mathrm{a} 8}=\left(v_{y} \omega\right)^{\mathrm{T}}
\end{aligned}
$$

Por lo que quince relaciones pueden considerarse para el análisis de isotropía: seis entre un par de velocidades de rueda $\left(\dot{\mathbf{q}}_{\mathrm{a} 1}, \ldots, \dot{\mathbf{q}}_{\mathrm{a} 6}\right)$ y un par de velocidades del vehículo ( $\dot{\mathbf{q}}_{\mathrm{a} 7}, \dot{\mathbf{q}}_{\mathrm{a} 8}$ or $\boldsymbol{\eta}$, no importa cuál); y nueve entre dos pares de velocidades de rueda. Aquí se analizará la isotropía de las seis primeras relaciones ya que tienen más utilidad práctica que las otras.

Con el procedimiento utilizado para el vehículo de tipo 2 cada una de esas seis relaciones da lugar, al aplicar (5.29), a una condición de isotropía:

$$
\begin{aligned}
& \dot{\mathbf{q}}_{\mathrm{a} 1} \rightarrow \mathrm{c} \beta_{12} \cdot \mathrm{c} \beta_{22}=0 \\
& \dot{\mathbf{q}}_{\mathrm{a} 2} \rightarrow \mathrm{s} \beta_{12} \cdot\left(\mathrm{d}-1_{2} \cdot \mathrm{s} \beta_{22}\right)=0 \\
& \dot{\mathbf{q}}_{\mathrm{a3}} \rightarrow 1_{2} \cdot \mathrm{c} \beta_{22} \cdot \mathrm{c} \beta_{12}=-1_{3} \cdot \mathrm{c} \beta_{33} \cdot \mathrm{c} \beta_{13} \\
& \dot{\mathbf{q}}_{\mathrm{a} 4} \rightarrow \mathrm{s} \beta_{12} \cdot\left(\mathrm{d}-1_{2} \cdot \mathrm{s} \beta_{22}\right)=-\mathrm{s} \beta_{13} \cdot\left(\mathrm{d}-1_{3} \cdot \mathrm{s} \beta_{33}\right) \\
& \dot{\mathbf{q}}_{\mathrm{a} 5} \rightarrow 1_{2} \cdot \mathrm{c} \beta_{22} \cdot \mathrm{c} \beta_{12}=-(\mathrm{r} / \mathrm{d})^{2} \cdot \mathrm{s} \beta_{12} \cdot\left(\mathrm{d}-1_{2} \cdot \mathrm{s} \beta_{22}\right) \\
& \dot{\mathbf{q}}_{\mathrm{a} 6} \rightarrow 1_{2} \cdot \mathrm{c} \beta_{22} \cdot \mathrm{c} \beta_{12}=-(\mathrm{r} / \mathrm{d})^{2} \cdot \mathrm{s} \beta_{13} \cdot\left(\mathrm{d}-1_{3} \cdot \mathrm{s} \beta_{33}\right)
\end{aligned}
$$

El significado, ver la Figura 5.10, de las correspondientes configuraciones isotrópicas (sólo se consideran las no singulares, ej. $\beta_{22}=90^{\circ}$ para $(5.70)_{\mathrm{a}}$ es singular, ver Capítulo 6) es:

- (5.70) a el brazo de dirección de la primera rueda castor es perpendicular a la rueda orientable;

- (5.70) $)_{\mathrm{b}}$ el brazo de dirección de la primera rueda castor es paralelo a la rueda orientable:

- (5.70) c la componente $X\left(c_{10}\right)$ respecto al sistema $\mathrm{E}_{1}$ del vector de distancia entre el origen de $\mathrm{E}_{1}$ y la línea definida por el brazo de dirección de la primera rueda castor es igual al valor negativo de la misma componente $X\left(c_{11}\right)$ de la otra rueda castor;

- (5.70) da componente $X\left(c_{12}\right)$ respecto al sistema $\mathrm{E}_{1}$ del vector de distancia entre el origen de $\mathrm{E}_{1}$ y el eje de rotación de la primera rueda castor es igual al valor negativo de la misma componente $X\left(c_{13}\right)$ de la otra castor; 
- (5.70) $)_{\mathrm{e}}$ la anteriormente definida componente $c_{10}$ es igual al valor negativo de la componente $c_{12}$ multiplicada por $(\mathrm{r} / \mathrm{d})^{2}$;

- (5.70) $)_{\mathrm{f}}$ la anteriormente definida componente $c_{10}$ es igual al valor negativo de la componente $c_{13}$ multiplicada por el factor $(\mathrm{r} / \mathrm{d})^{2}$.

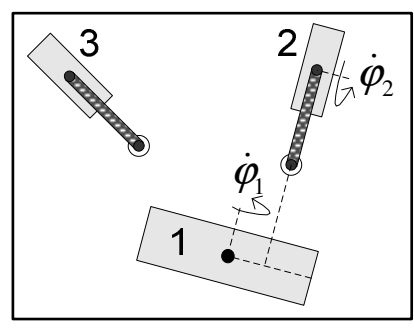

(a) $\dot{\mathbf{q}}_{\mathrm{a}}$

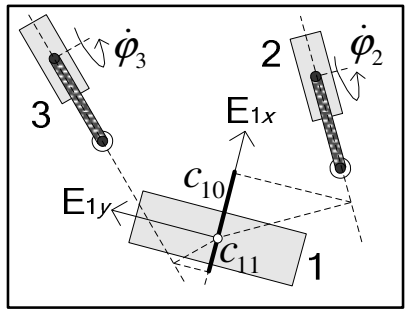

(c) $\dot{\mathbf{q}}_{\mathrm{a} 3} \rightarrow c_{10}=c_{11}$

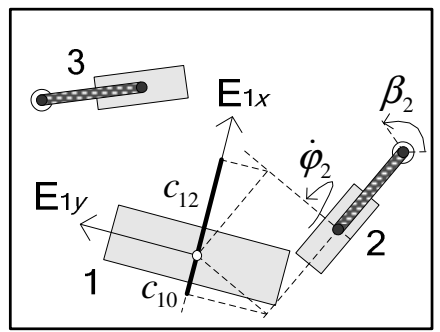

(e) $\dot{\mathbf{q}}_{\mathrm{a} 5} \rightarrow c_{10}=c_{12} \cdot(\mathrm{r} / \mathrm{d})^{2}$

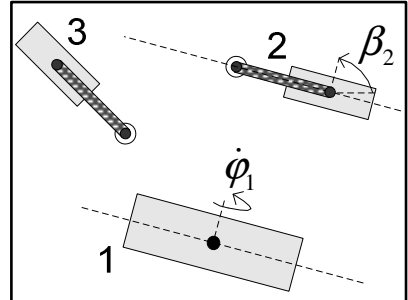

(b) $\dot{\mathbf{q}}_{\mathrm{a} 2}$

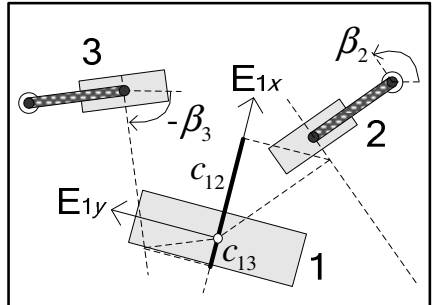

(d) $\dot{\mathbf{q}}_{\mathrm{a} 4} \rightarrow c_{12}=c_{13}$

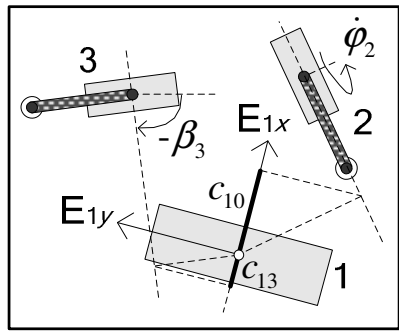

(f) $\dot{\mathbf{q}}_{\mathrm{a} 6} \rightarrow c_{10}=c_{13} \cdot(\mathrm{r} / \mathrm{d})^{2}$

Figura 5.10- Configuraciones isotrópicas para el vehículo tipo 3 con ruedas castor

Las anteriores configuraciones isotrópicas siempre existen. 


\subsubsection{Tipo 4 (1,1): Vehículo triciclo y bicicleta}

Este vehículo tiene una rueda orientable independiente y otra rueda fija independiente. En este estudio se considerará una rueda orientable (no. 1), una rueda fija (no. 2) y una tercera rueda (no. 3): fija dependiente, sueca o castor.

Este vehículo tiene un grado de movilidad $(m=1)$, por lo que no tiene sentido aplicarle el análisis de isotropía ya que, dentro del marco cinemático sin deslizamiento de (4.1), sólo una velocidad es libremente asignada, resultado cualquier relación entre velocidades escalar. Además hay otro GDL aparente aportado por la dirección de la rueda orientable.

Para disponer del GDL instantáneo se necesita un actuador/sensor. Además, se debe accionar/sensorizar la dirección de la rueda orientable para disponer del GDL aparente y poder computar los modelos cinemáticos.

Si la actuación se realiza sobre la rueda fija (trasera), se consigue una tracción trasera. En el caso de dos ruedas fijas dependientes se realiza a través del mecanismo diferencial ya comentado para el vehículo tipo 2 , que se coordina con la orientación de la rueda orientable. Este mecanismo también sirve para sensorizar las velocidades de rotación de las ruedas fijas.

Si se actúa sobre la velocidad de rotación de la rueda orientable la tracción es delantera. En ocasiones, se actúa simultáneamente sobre el mecanismo diferencial y sobre la rueda orientable (tracción completa) siendo necesaria la coordinación entre ambas actuaciones para evitar deslizamiento.

\subsubsection{A) Vehículo triciclo}

Este vehículo clásico consiste en una rueda orientable y dos ruedas fijas simétricas. Este vehículo se muestra en la Figura 5.11, donde el origen del sistema $\mathrm{R}$ se ha situado en el punto medio del eje de rotación de las ruedas fijas, y su eje $X$ coincidente con este eje de rotación.

Una variante del vehículo triciclo es el coche tradicional. Esta variante consiste en utilizar dos ruedas orientables acopladas a través del mecanismo de direccionamiento de Ackerman, resultando equivalentes a la única del triciclo. 
Para el modelado de este vehículo la ecuación (3.11) se utiliza tres veces, resultando (4.1) (con los parámetros de la Figura 5.11: $1_{1}=l_{2}=1_{12}, \beta_{1}=\beta_{2}=0^{\circ}$, $\alpha_{1}=0^{\circ}, \alpha_{2}=180^{\circ}$ y $\left.\alpha_{3}=90^{\circ}\right)$ :

$$
\mathbf{A}_{\mathrm{p}} \cdot \dot{\mathbf{p}}+\mathbf{A}_{\mathrm{w}} \cdot \dot{\boldsymbol{\varphi}}=\left(\begin{array}{ccc}
1 & 0 & 0 \\
1 & 0 & 0 \\
\mathrm{c} \beta_{3} & \mathrm{~s} \beta_{3} & -1_{3} \cdot \mathrm{c} \beta_{3} \\
0 & 1 & 1_{12} \\
0 & 1 & -1_{12} \\
-\mathrm{s} \beta_{3} & \mathrm{c} \beta_{3} & 1_{3} \cdot \mathrm{s} \beta_{3}
\end{array}\right) \cdot \dot{\mathbf{p}}+\mathrm{r} \cdot\left(\begin{array}{l}
\mathbf{0} \\
\mathbf{I}
\end{array}\right) \cdot\left(\begin{array}{l}
\dot{\varphi}_{1} \\
\dot{\varphi}_{2} \\
\dot{\varphi}_{3}
\end{array}\right)=\mathbf{0}
$$

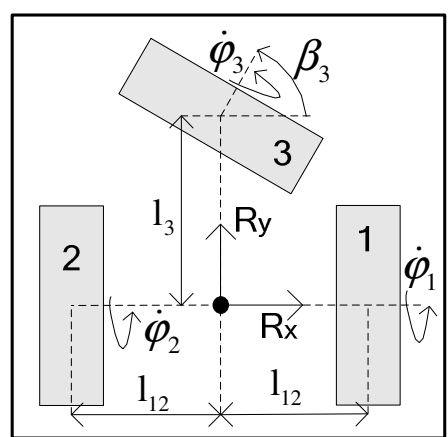

Figura 5.11- Vehículo triciclo (una rueda orientable y dos fijas dependientes)

Una solución cinemática (4.2) para (5.71) es:

$$
\left(\begin{array}{c}
\dot{p} \\
\dot{\boldsymbol{\varphi}}
\end{array}\right)=\left(\begin{array}{c}
0 \\
\mathrm{l}_{3} \cdot \mathrm{c} \beta_{3} \\
\mathrm{~s} \beta_{3} \\
-\left(\mathrm{L} \cdot \mathrm{s} \beta_{3}+1_{3} \cdot \mathrm{c} \beta_{3}\right) / \mathrm{r} \\
\left(\mathrm{L} \cdot \mathrm{s} \beta_{3}-1_{3} \cdot \mathrm{c} \beta_{3}\right) / \mathrm{r} \\
-\mathrm{l}_{3} / \mathrm{r}
\end{array}\right) \cdot \eta
$$

Los modelos directos e inversos se obtienen directamente de (5.72) aplicando (4.5) para cualquier velocidad asignada excepto $v_{x}$.

\subsubsection{B) Vehículo bicicleta con rueda sueca}

Este vehículo, mostrado en la Figura 5.12 (a), tiene una rueda fija alineada con una rueda orientable, y otra rueda sueca. El sistema de coordenadas $\mathrm{R}$ se ha situado en el centro de la rueda fija y con su eje $X$ concidente con el eje de rotación de dicha rueda. 
Para el modelado de este vehículo la ecuación (3.11) se utiliza dos veces y la (3.14) una, resultando (4.1) (con los parámetros obtenidos de la Figura 5.12 (a): $1_{1}$ $\left.=0, \beta_{1}=0^{\circ}, \alpha_{2}=90^{\circ}\right)$ :

$$
\mathbf{A}_{\mathrm{p}} \cdot \dot{\mathbf{p}}+\mathbf{A}_{\mathrm{w}} \cdot \dot{\boldsymbol{\varphi}}=\left(\begin{array}{ccc}
1 & 0 & 0 \\
\mathrm{c} \beta_{2} & \mathrm{~s} \beta_{2} & -\mathrm{l}_{2} \cdot \mathrm{c} \beta_{2} \\
0 & 1 & 0 \\
-\mathrm{s} \beta_{2} & \mathrm{c} \beta_{2} & 1_{2} \cdot \mathrm{s} \beta_{2} \\
-\mathrm{s} \beta_{3} & \mathrm{c} \beta_{3} & 1_{3} \cdot \mathrm{c}\left(\beta_{3}-\alpha_{3}\right)
\end{array}\right) \cdot \dot{\mathbf{p}}+\mathrm{r} \cdot\left(\begin{array}{l}
\mathbf{I} \\
\mathbf{I}
\end{array}\right) \cdot\left(\begin{array}{l}
\dot{\varphi}_{1} \\
\dot{\varphi}_{2} \\
\dot{\varphi}_{3}
\end{array}\right)=\mathbf{0}
$$

Una solución cinemática (4.2) para (5.73) es:

$$
\left(\begin{array}{c}
\dot{\mathbf{p}} \\
\dot{\boldsymbol{\varphi}}
\end{array}\right)=\left(\begin{array}{c}
0 \\
1_{2} \cdot \mathrm{c} \beta_{2} \\
\mathrm{~s} \beta_{2} \\
-1_{2} \cdot \mathrm{c} \beta_{2} / \mathrm{r} \\
-1_{2} / \mathrm{r} \\
-\frac{1}{\mathrm{r}} \cdot\left(\begin{array}{c}
1_{3} \cdot \mathrm{c}\left(\beta_{3}-\alpha_{3}\right) \cdot \mathrm{s} \beta_{2} \\
+1_{2} \cdot \mathrm{c} \beta_{2} \cdot \mathrm{c} \beta_{3}
\end{array}\right)
\end{array}\right) \cdot \eta
$$

Como antes, los modelos cinemáticos particulares se obtienen de (5.74) excepto para $v_{x}$ como velocidad asignada.

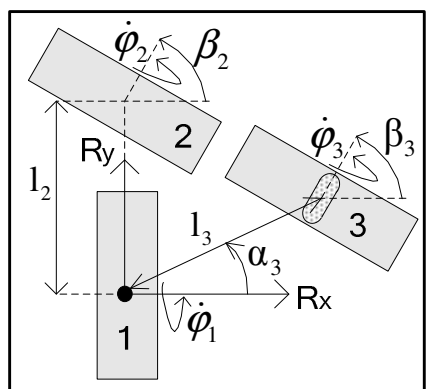

(a) con rueda sueca

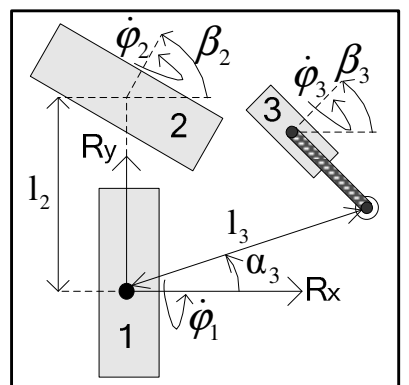

(b) con rueda castor

Figura 5.12- Vehículo bicicleta con rueda adicional sueca o castor

\subsubsection{B) Vehículo bicicleta con rueda castor}

Este vehículo, mostrado en la Figura 5.12 (b), tiene una rueda fija alineada con una rueda orientable, y otra rueda sueca. El sistema de coordenadas $\mathrm{R}$ se ha situado como en el subapartado 5.5.5.B). 
Para el modelado de este vehículo la ecuación (3.11) se utiliza dos veces y la (3.12) una, resultando (4.1) (con los parámetros obtenidos de la Figura 5.12 (b): $\left.1_{1}=0, \beta_{1}=0^{\circ}, \alpha_{2}=90^{\circ}\right)$ :

$$
\mathbf{A}_{\mathrm{p}} \cdot \dot{\mathbf{p}}+\mathbf{A}_{\mathrm{w}} \cdot \dot{\mathbf{q}}_{\mathrm{w}}=\left(\begin{array}{ccc}
1 & 0 & 0 \\
\mathrm{c} \beta_{2} & \mathrm{~s} \beta_{2} & -\mathrm{l}_{2} \cdot \mathrm{c} \beta_{2} \\
0 & 1 & 0 \\
-\mathrm{s} \beta_{2} & \mathrm{c} \beta_{2} & 1_{2} \cdot \mathrm{s} \beta_{2} \\
-\mathrm{s} \beta_{3} & \mathrm{c} \beta_{3} & 1_{3} \cdot \mathrm{c}\left(\beta_{3}-\alpha_{3}\right) \\
\mathrm{c} \beta_{3} & \mathrm{~s} \beta_{3} & 1_{3} \cdot \mathrm{s}\left(\beta_{3}-\alpha_{3}\right)-\mathrm{d}
\end{array}\right) \cdot \dot{\mathbf{p}}+\left(\begin{array}{c}
\mathbf{0} \\
\mathrm{r} \cdot \mathbf{I} \\
-\mathrm{d}
\end{array}\right) \cdot\left(\begin{array}{l}
\dot{\varphi}_{1} \\
\dot{\varphi}_{2} \\
\dot{\varphi}_{3} \\
\dot{\beta}_{3}
\end{array}\right)=\mathbf{0}
$$

Una solución cinemática (4.2) para (5.75) es:

$$
\left(\begin{array}{c}
\dot{\mathbf{p}} \\
\dot{\boldsymbol{\varphi}} \\
\dot{\beta}_{3}
\end{array}\right)=\left(\begin{array}{c}
0 \\
1_{2} \cdot \mathrm{c} \beta_{2} \\
\mathrm{~s} \beta_{2} \\
-1_{2} \cdot \mathrm{c} \beta_{2} / \mathrm{r} \\
-1_{2} / \mathrm{r} \\
\frac{1}{\mathrm{r}} \cdot\left(\begin{array}{l}
1_{3} \cdot \mathrm{c}\left(\beta_{3}-\alpha_{3}\right) \cdot \mathrm{s} \beta_{2} \\
+l_{2} \cdot \mathrm{c} \beta_{2} \cdot \mathrm{c} \beta_{3}
\end{array}\right) \\
\frac{1}{\mathrm{~d}} \cdot\left(\begin{array}{l}
\left.1_{3} \cdot \mathrm{s}\left(\beta_{3}-\alpha_{3}\right)-\mathrm{d}\right) \cdot \mathrm{s} \beta_{2} \\
+1_{2} \cdot \mathrm{c} \beta_{2} \cdot \mathrm{s} \beta_{3}
\end{array}\right)
\end{array}\right) \cdot \eta
$$

Como antes, los modelos cinemáticos particulares se obtienen de (5.74) excepto para $v_{x}$ como velocidad asignada.

\subsubsection{Tipo $5(1,2)$ : Vehículo con dos ruedas orientables}

Este vehículo está caracterizado por dos ruedas orientable independientes. En este estudio se considerarán estas dos ruedas, numeradas 1 y 2 , un una rueda omnidireccional, numerada 3, bien sueca o castor.

Como en el vehículo tipo 4, este vehículo tiene un grado de movilidad $(m=1)$ y por tanto el análisis de isotropía n es aplicable. Además del GDL instantáneo y hay dos aparentes debidos a la dirección de las dos ruedas orientables.

Por tanto se necesita un actuador/sensor para actuar/sensorizar sobre el GDL instantáneo y dos aguadores/sensores en las direcciones de las ruedas orientables para disponer de los 2 GDL aparentes/computar los modelos cinemáticos. 
Este tipo de vehículo se utiliza menos en la práctica debido a su falta de simetría y a la necesidad de utilizar tres actuadores y tres sensores, sin que además se consigan los 3 GDLs instantáneos como en el tipo 1.

El sistema de coordenadas R se ha situado, ver la Figura 5.13, en el punto medio de la recta que un las dos ruedas orientables, y con su eje $X$ coincidente con dicha recta. Los parámetros resultan: $1_{1}=1_{2}=1_{12}, \alpha_{1}=0^{\circ}, \alpha_{2}=180^{\circ}$.

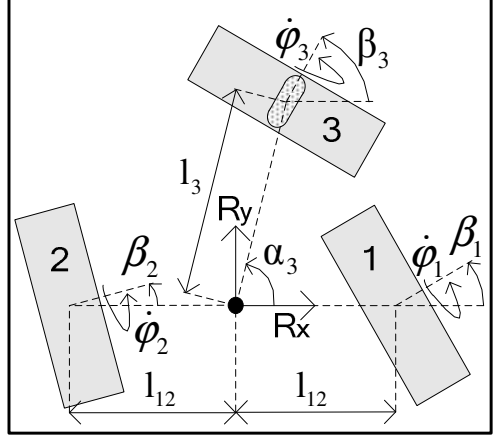

(a) con rueda sueca

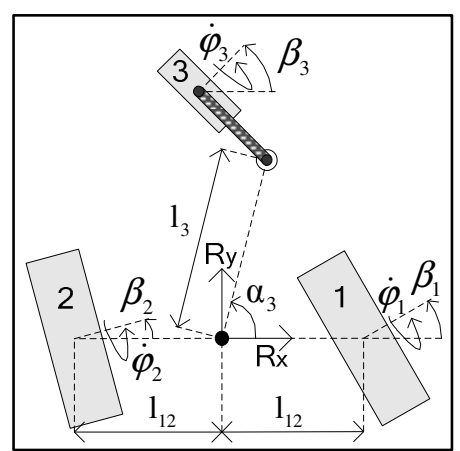

(b) con rueda castor

Figura 5.13- Vehículo con dos ruedas orientables (tipo 5)

Para el modelado de este vehículo con rueda sueca, ver Figura 5.13 (a), la ecuación (3.11) se ha de utilizar dos veces y la (3.14) una, resultando (4.1):

$$
\mathbf{A}_{\mathrm{p}} \cdot \dot{\mathbf{p}}+\mathbf{A}_{\mathrm{w}} \cdot \dot{\boldsymbol{\varphi}}=\left(\begin{array}{ccc}
\mathrm{c} \beta_{1} & \mathrm{~s} \beta_{1} & 1_{12} \cdot \mathrm{s} \beta_{1} \\
\mathrm{c} \beta_{2} & \mathrm{~s} \beta_{2} & -1_{12} \cdot \mathrm{s} \beta_{2} \\
-\mathrm{s} \beta_{1} & \mathrm{c} \beta_{1} & 1_{12} \cdot \mathrm{c} \beta_{1} \\
-\mathrm{s} \beta_{2} & \mathrm{c} \beta_{2} & -1_{12} \cdot \mathrm{c} \beta_{2} \\
-\mathrm{s} \beta_{3} & \mathrm{c} \beta_{3} & 1_{3} \cdot \mathrm{c}\left(\beta_{3}-\alpha_{3}\right)
\end{array}\right) \dot{\mathbf{p}}+\mathrm{r} \cdot\left(\begin{array}{l}
\mathbf{0} \\
\mathbf{I}
\end{array}\right) \cdot\left(\begin{array}{l}
\dot{\varphi}_{1} \\
\dot{\varphi}_{2} \\
\dot{\varphi}_{3}
\end{array}\right)=\mathbf{0}
$$

Mientras que para el modelado del vehículo con rueda castor, ver la Figura 5.13 (b), la ecuación (3.11) se ha de utilizar dos veces y (3.12) una, resultando:

$$
\mathbf{A}_{\mathrm{p}} \cdot \dot{\mathbf{p}}+\mathbf{A}_{\mathrm{w}} \cdot\left(\begin{array}{c}
\dot{\boldsymbol{\varphi}} \\
\dot{\beta}_{3}
\end{array}\right)=\left(\begin{array}{ccc}
\mathrm{c} \beta_{1} & \mathrm{~s} \beta_{1} & 1_{12} \cdot \mathrm{s} \beta_{1} \\
\mathrm{c} \beta_{2} & \mathrm{~s} \beta_{2} & -1_{12} \cdot \mathrm{s} \beta_{2} \\
-\mathrm{s} \beta_{1} & \mathrm{c} \beta_{1} & 1_{12} \cdot \mathrm{c} \beta_{1} \\
-\mathrm{s} \beta_{2} & \mathrm{c} \beta_{2} & -1_{12} \cdot \mathrm{c} \beta_{2} \\
-\mathrm{s} \beta_{3} & \mathrm{c} \beta_{3} & 1_{3} \cdot \mathrm{c}\left(\beta_{3}-\alpha_{3}\right) \\
\mathrm{c} \beta_{3} & \mathrm{~s} \beta_{3} & 1_{3} \cdot \mathrm{s}\left(\beta_{3}-\alpha_{3}\right)-\mathrm{d}
\end{array}\right) \cdot \dot{\mathbf{p}}+\left(\begin{array}{c}
\mathbf{0} \\
\mathrm{r} \cdot \mathbf{I} \\
-\mathrm{d}
\end{array}\right) \cdot\left(\begin{array}{l}
\dot{\varphi}_{1} \\
\dot{\varphi}_{2} \\
\dot{\varphi}_{3} \\
\dot{\beta}_{3}
\end{array}\right)=\mathbf{0}
$$


Una solución cinemática (4.2) para (5.77) y (5.78) es:

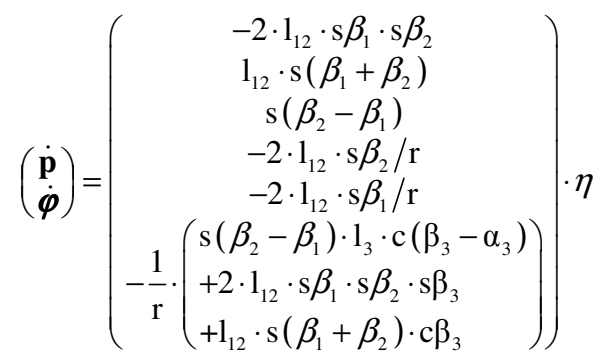

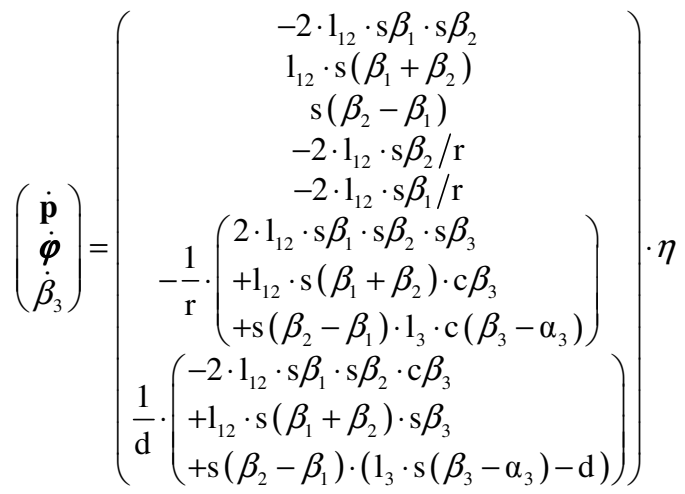

Los modelos directos e inversos se pueden obtener directamente de (5.79) y (5.80) aplicando (4.5) para cualquier velocidad como asignada. 


\subsection{RESULTADOS MÁS RELEVANTES Y CONCLUSIONES DEL CAPÍTULO}

A continuación se indican los aspectos más relevantes que aporta el capítulo:

- Las restricciones al movimiento aportadas por cada tipo de rueda son analizadas a partir de la matriz Jacobiana de rueda como alternativa al planteamiento de [Campion et al. 96]. Estas restricciones son las que dan lugar a la posterior clasificación de vehículos.

- Se han descrito y analizado las distintas opciones de actuación y sensorización para cada tipo de vehículo.

- Se han obtenido los modelos cinemáticos para cada tipo de vehículo, los cuales son genéricos e incluyen variables de rueda, a diferencia de [Campion et al. 96] que utiliza variables sin sentido físico.

- Un completo análisis de isotropía (transmisión de errores en el modelo) se ha desarrollado para los modelos cinemáticos.

- El modelado, análisis de actuación/sensorización y la caracterización de isotropía para todos los tipos de vehículos representa (junto con el estudio de singularidad del Capítulo 6) una guía de la cinemática de vehículos útil para el diseño mecánico, desarrollo de aplicaciones de control, la planificación de caminos, etc.

- Las demostraciones del subapartado 5.3.1 son originales. 

CAPÍTULO 6

\section{SINGULARIDAD DE LOS MODELOS CINEMÁTICOS DE VEHÍCULOS}

\subsection{INTRODUCCIÓN}

Un hecho importante es que, en general, los modelos cinemáticos correspondientes dependen de los parámetros de las ruedas y de los ángulos variables de las ruedas castor y orientables. De modo que hay valores especiales para los parámetros y ángulos variables en los que surge la singularidad del modelo cinemático. Esta singularidad implicará, según se describe en el punto 6.2 posterior, deslizamiento seguro o pérdida de movilidad en el vehículo.

La caracterización de la singularidad ha sido ampliamente estudiada para robots manipuladores, ver [Tourassis et al. 92] [Dinesh et al. 92] [Liu et al. 03] [Lipkin et al. 91] entre otras publicaciones relevantes. Por el contrario, en vehículos solo unos pocos autores han realizado alguna aproximación a este respecto. Por ejemplo, en [Yi et al. 02] se presenta un estudio parcial para un vehículo omnidireccional con ruedas castor, mientras que es no-singular con tres ruedas suecas [Loh et al. 03]. El estudio de [Leow et al. 02] se centra en el caso de tres ruedas orientables de forma poco acertada.

El objetivo de este capítulo es proporcionar un estudio completo sobre la singularidad de los modelos cinemáticos de vehículos. El contenido de cada punto del capítulo se describe a continuación. 
En primer lugar en el punto 6.2 se reformulan las ecuaciones de rueda y la ecuación compuesta del vehículo para que puedan ser utilizadas convenientemente en un punto posterior.

A continuación, en el punto 6.3 se introduce la problemática de la singularidad de los modelos cinemáticos de los vehículos, sus implicaciones y la utilidad práctica de su caracterización.

En el punto 6.4 analiza la singularidad de los modelos cinemáticos directos e inversos, o incluso mixtos. Como resultado se establece un planteamiento geométrico general, practico, y útil para caracterizar la singularidad de cualquier modelo cinemático de cualquier vehículo con ruedas comunes (fijas, orientables, castor y suecas).

Como aplicación del planteamiento geométrico anterior, en el punto 6.5 obtiene la singularidad del los modelos cinemáticos de los cinco tipos de vehículos clasificados en el punto 5.3 del capítulo anterior.

Posteriormente, en el punto 6.6 se consideran otro tipo de ruedas menos habituales (especiales) para las que se extiende la caracterización de la singularidad. Dos de estos tipos de rueda (dobles y castor dobles) ya fueron descritos en el segundo anexo del Capítulo 3, mientras que los otros dos tipos considerados (tipo bola y ortogonales) se presentan más detalladamente.

Finalmente en el punto de conclusiones se destacan los resultados más relevantes del capítulo. 


\subsection{REFORMULACIÓN DE ECUACIONES}

En este punto se van a reformular las ecuaciones de rueda y la compuesta del vehículo para que puedan ser utilizadas convenientemente en un punto posterior. Concretamente la reformulación va a consistir en añadir subíndices para cada una de las ruedas y en definir vectores/matrices que agrupen elementos.

Se reformulan las ecuaciones de rueda (3.11), (3.12) y (3.14) como sigue:

$$
\begin{aligned}
& \mathbf{v}_{\text {slip fi }}=\left(\begin{array}{cccc}
\mathrm{c} \beta_{\mathrm{fi}} & \mathrm{s} \beta_{\mathrm{fi}} & \mathrm{f}_{\mathrm{fi}} \cdot \mathrm{s}\left(\beta_{\mathrm{fi}}-\alpha_{\mathrm{fi}}\right) & 0 \\
-\mathrm{s} \beta_{\mathrm{fi}} & \mathrm{c} \beta_{\mathrm{fi}} & 1_{\mathrm{fi}} \cdot \mathrm{c}\left(\beta_{\mathrm{fi}}-\alpha_{\mathrm{fi}}\right) & \mathrm{r}_{\mathrm{fi}}
\end{array}\right) \cdot\left(\begin{array}{c}
\dot{\mathbf{p}} \\
\dot{\varphi}_{\mathrm{fi}}
\end{array}\right)=\left(\begin{array}{cc}
\mathbf{E}_{\mathrm{fi}} & 0 \\
\mathbf{F}_{\mathrm{fi}} & \mathrm{r}_{\mathrm{fi}}
\end{array}\right) \cdot\left(\begin{array}{c}
\dot{\mathbf{p}} \\
\dot{\varphi}_{\mathrm{fi}}
\end{array}\right) \\
& \mathbf{v}_{\text {slipoi }}=\left(\begin{array}{cccc}
\mathrm{c} \beta_{\mathrm{oi}} & \mathrm{s} \beta_{\mathrm{oi}} & 1_{\mathrm{o} i} \cdot \mathrm{s}\left(\beta_{\mathrm{oi}}-\alpha_{\mathrm{o}}\right) & 0 \\
-\mathrm{s} \beta_{\mathrm{oi}} & \mathrm{c} \beta_{\mathrm{oi}} & 1_{\mathrm{oi}} \cdot \mathrm{c}\left(\beta_{\mathrm{oi}}-\alpha_{\mathrm{oi}}\right) & \mathrm{r}_{\mathrm{oi}}
\end{array}\right) \cdot\left(\begin{array}{c}
\dot{\mathbf{p}} \\
\dot{\varphi}_{\mathrm{o} i}
\end{array}\right)=\left(\begin{array}{cc}
\mathbf{E}_{\mathrm{o} i} & 0 \\
\mathbf{F}_{\mathrm{oi}} & \mathrm{r}_{\mathrm{oi}}
\end{array}\right) \cdot\left(\begin{array}{c}
\dot{\mathbf{p}} \\
\dot{\varphi}_{\mathrm{o} i}
\end{array}\right)
\end{aligned}
$$

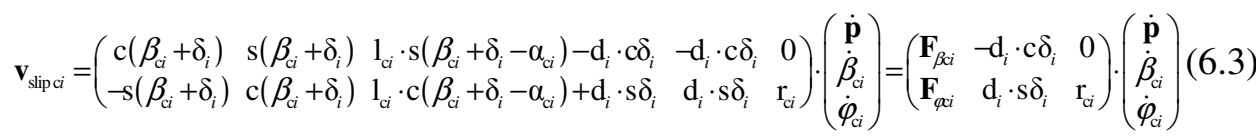

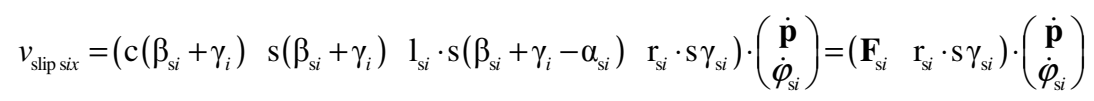

donde el subíndice $\{\mathrm{f}, \mathrm{o}, \mathrm{c}, \mathrm{s}\}$ es para rueda fija, orientable, castor y sueca.

Después de establecer las ecuaciones de rueda del vehículo (con sus correspondientes parámetros: $\left.\mathrm{r}_{i}, \mathrm{r}_{\mathrm{ri}}, 1_{i}, \mathrm{~d}_{i}, \alpha_{i}, \beta_{i}, \delta_{i}, \gamma_{i}\right)$, se define la ecuación compuesta del vehículo, por combinación de (6.1), (6.2), (6.3) y (6.4), como:

$$
\begin{gathered}
\mathbf{V}_{\text {slip }}=\left(\begin{array}{c|ccccc}
\mathbf{E}_{\mathrm{f}} & \mathbf{0} & \mathbf{0} & \mathbf{0} & \mathbf{0} & \mathbf{0} \\
\mathbf{E}_{\mathrm{o}} & \mathbf{0} & \mathbf{0} & \mathbf{0} & \mathbf{0} & \mathbf{0} \\
\hline \mathbf{F}_{\mathrm{f}} & \mathbf{r}_{\mathrm{f}} & \mathbf{0} & \mathbf{0} & \mathbf{0} & \mathbf{0} \\
\mathbf{F}_{\mathrm{o}} & \mathbf{0} & \mathbf{r}_{\mathrm{o}} & \mathbf{0} & \mathbf{0} & \mathbf{0} \\
\mathbf{F}_{\mathrm{s}} & \mathbf{0} & \mathbf{0} & \mathbf{r}_{\mathrm{s}} & \mathbf{0} & \mathbf{0} \\
\mathbf{F}_{\beta \mathrm{c}} & \mathbf{0} & \mathbf{0} & \mathbf{0} & \mathbf{d}_{\beta} & \mathbf{0} \\
\mathbf{F}_{\varphi \mathrm{c}} & \mathbf{0} & \mathbf{0} & \mathbf{0} & \mathbf{d}_{\varphi} & \mathbf{r}_{\mathrm{c}}
\end{array}\right) \cdot\left(\begin{array}{c}
\dot{\mathbf{p}} \\
\dot{\boldsymbol{\varphi}}_{\mathrm{f}} \\
\dot{\boldsymbol{\varphi}}_{\mathrm{o}} \\
\dot{\boldsymbol{\varphi}}_{\mathrm{s}} \\
\dot{\boldsymbol{\beta}}_{\mathrm{c}} \\
\dot{\boldsymbol{\varphi}}_{\mathrm{c}}
\end{array}\right) \\
\mathbf{V}_{\text {slip }}=\left(\begin{array}{ccc}
\mathbf{E} & \mathbf{0} \\
\mathbf{F} & \mathbf{r}
\end{array}\right) \cdot\left(\begin{array}{c}
\dot{\mathbf{p}} \\
\dot{\mathbf{q}}_{w}
\end{array}\right)=\mathbf{A} \cdot \dot{\mathbf{q}}
\end{gathered}
$$


donde $\left\{\mathbf{E}_{\mathrm{f}}, \mathbf{E}_{\mathrm{o}}, \mathbf{F}_{\mathrm{f}}, \mathbf{F}_{\mathrm{o}}, \mathbf{F}_{\mathrm{s}}, \mathbf{F}_{\beta \mathrm{c}}, \mathbf{F}_{\varphi \mathrm{c}}\right\}$ son matrices obtenidas por la combinación de los vectores fila $\left\{\mathbf{E}_{\mathrm{f} i}, \mathbf{E}_{\mathrm{o} i}, \mathbf{F}_{\mathrm{fi}}, \mathbf{F}_{\mathrm{o} i}, \mathbf{F}_{\mathrm{s} i}, \mathbf{F}_{\beta c i}, \mathbf{F}_{\varphi c i}\right\} ;\left\{\mathbf{r}_{\mathrm{f}}, \mathbf{r}_{\mathrm{o}}, \mathbf{r}_{\mathrm{s}}, \mathbf{r}_{\mathrm{c}}, \mathbf{d}_{\beta}, \mathbf{d}_{\varphi}\right\}$ son matrices diagonales con los elementos en la diagonal $\left\{\mathrm{r}_{\mathrm{f} i}, \mathrm{r}_{\mathrm{o} i}, \mathrm{r}_{\mathrm{s} i} \cdot \mathrm{s} \gamma_{\mathrm{s} i}, \mathrm{r}_{\mathrm{c} i},-\mathrm{d}_{i} \cdot \mathrm{c} \delta_{i}, \mathrm{~d}_{i} \cdot \mathrm{s}_{i}\right\}$; $\left\{\dot{\varphi}_{\mathrm{f}}, \dot{\boldsymbol{\varphi}}_{\mathrm{o}}, \dot{\boldsymbol{\varphi}}_{\mathrm{s}}, \dot{\boldsymbol{\beta}}_{\mathrm{c}}, \dot{\boldsymbol{\varphi}}_{\mathrm{c}}\right\}$ son vectores compuestos de los elementos $\left\{\dot{\varphi}_{\mathrm{fi}}, \dot{\varphi}_{\mathrm{oi}}, \dot{\varphi}_{\mathrm{si}}, \dot{\beta}_{\mathrm{c} i}, \dot{\varphi}_{\mathrm{c} i}\right\}$; $\dot{\mathbf{q}}_{w}$ es el vector de todas las velocidades de rueda; $\dot{\mathbf{q}}$ el vector de todas la velocidades; $y \mathbf{v}_{\text {slip }}$ es el vector de todas las velocidades de deslizamiento.

Como se ha indicado en el punto 4.2 del Capítulo 4, bajo el supuesto de no deslizamiento se tiene:

$$
\begin{gathered}
\mathbf{A} \cdot \dot{\mathbf{q}}=\mathbf{0} \\
\dot{\mathbf{q}} \in \mathcal{N}(\mathbf{A}) \rightarrow \dot{\mathbf{q}}=\mathbf{B} \cdot \boldsymbol{\eta} \\
m=\operatorname{dim}(\boldsymbol{\eta})=\operatorname{dim}(\mathcal{N}(\mathbf{A}))=\operatorname{dim}(\dot{\mathbf{q}})-\operatorname{rango}(\mathbf{A})=k-g \\
\left(\begin{array}{c}
\dot{\mathbf{q}}_{\text {na }} \\
\dot{\mathbf{q}}_{\mathrm{a}}
\end{array}\right)=\left(\begin{array}{c}
\mathbf{B}_{\text {na }} \\
\mathbf{B}_{\mathrm{a}}
\end{array}\right) \cdot \boldsymbol{\eta} \\
\text { if } \quad\left|\mathbf{B}_{\mathrm{a}}\right| \neq 0 \rightarrow \quad \rightarrow \quad \dot{\mathbf{q}}_{\text {na }}=\mathbf{B}_{\text {na }} \cdot \mathbf{B}_{\mathrm{a}}^{-1} \cdot \dot{\mathbf{q}}_{\mathrm{a}}
\end{gathered}
$$

donde la matriz $\mathbf{B}$ forma una base del espacio nulo de $\mathbf{A}, m$ es el grado de movilidad del vehículo, $\dot{\mathbf{q}}_{\mathrm{a}}$ son las velocidades asignadas y $\dot{\mathbf{q}}_{\mathrm{na}}$ las restantes velocidades (no asignadas).

Como se ha destrito en el punto 4.3 del Capítulo 4, (6.6) se puede reescribir:

$$
\mathbf{A}_{\mathrm{na}} \cdot \dot{\mathbf{q}}_{\mathrm{na}}=-\mathbf{A}_{\mathrm{a}} \cdot \dot{\mathbf{q}}_{\mathrm{a}}
$$

donde las $m$ velocidades asignadas se han pasado al miembro de la derecha.

Para que efectivamente dichas $m$ velocidades puedan ser asignadas se debe cumplir:

$$
\operatorname{rango}\left(\mathbf{A}_{\text {na }}\right)=\operatorname{rango}(\mathbf{A})
$$




\subsection{PROBLEMÁTICA DE LA SINGULARIDAD}

Un hecho importante es que, en general, la matriz $\mathbf{B}_{\mathbf{a}}$ (o equivalentemente la matriz $\mathbf{A}_{\mathrm{na}}$ ), y consecuentemente la correspondiente solución cinemática (6.10), depende de los parámetros de rueda $\left\{\mathrm{r}_{i}, \mathrm{r}_{\mathrm{ri}}, 1_{i}, \mathrm{~d}_{i}, \alpha_{i}, \delta_{i}, \gamma_{i}, \beta_{\mathrm{fi}}, \beta_{\mathrm{si}}\right\}$ y de los ángulos variables $\left\{\beta_{o i}, \beta_{c i}\right\}$ de las rueda castor y orientables. De modo que, para un conjunto $m$ de velocidades asignadas, hay valores especiales de los parámetros y ángulos variables para los que surge la singularidad, es decir $\mathbf{B}_{\mathbf{a}}$ resulta singular (o degenera), o equivalentemente $\mathbf{A}_{\text {na }}$ pierde rango (degenera).

Para estas situaciones hay dos planteamientos:

Pérdida de grados de movilidad:

Para evitar la incompatibilidad las velocidades asignadas se coordinan adecuadamente, lo que implica una perdida en el grado de movilidad $m$.

\section{Incompatibilidad cinemática:}

No hay ningún tipo de coordinación en las velocidades asignadas, por lo que la incompatibilidad cinemática queda sin resolver. Si las velocidades asignadas son velocidades de rueda (cinemática directa), el deslizamiento (debido a la incompatibilidad, no a las aceleraciones) es inevitable. Si se trata de velocidades del vehículo (cinemática inversa), se obtienen valores imposibles (infinitos) para las acciones de control.

Tener en cuenta que, el grado de movilidad $m$ también depende de los ángulos variables de las ruedas castor y orientable, ya que depende del rango de A. Por ejemplo un vehículo con dos ruedas orientables (tipo 5 en la clasificación del punto 5.3) en general tiene un grado de movilidad $m=1$, excepto cuando las dos ruedas están perpendiculares a su recta de unión donde la movilidad es $m=2$. $\mathrm{Si}$ en un momento dado se asignan menos de $m$ velocidades (siendo $m$ el grado de movilidad del momento) el modelo cinemático queda indefinido.

Las consideraciones anteriores proporcionan el siguiente criterio: la singularidad (pérdida de grados de movilidad, deslizamiento o acciones de control imposibles) debe evitarse. 
En concreto, cuando el vehículo no tiene ruedas orientables o castor, éste es un criterio de diseño, ya que las condiciones de singularidad son permanentes.

Por el contrario, si hay ruedas orientables o castor, las condiciones de singularidad se cumplen para configuraciones específicas: configuraciones singulares. En ese caso, el criterio comentado es un criterio de planificación y/o control, es decir el planificador de nivel superior (generador de caminos) y/o el controlador deben desarrollar caminos y/o acciones de control que impidan las singularidades.

Lo anterior se abordaría análogamente al caso de robots manipuladores, donde distintos planificadores y controladores de movimiento se han desarrollado considerando la singularidad (ver [Nenchev et al. 97] [Lloyd et al. 01] [Stanisic et al. 90] [Lai et al. 90] entre muchas otras publicaciones relevantes). Para este caso, un vehículo sobre-actuado sería útil en la medida que permite cambiar de un modelo cinemático a otro, evitando las singularidades.

El criterio genérico anteriormente formulado, "la singularidad debe evitarse", se complementa perfectamente con el criterio de isotropía presentado en el punto 5.4 del capítulo anterior: "la isotropía debe alcanzarse". 


\subsection{CARACTERIZACIÓN DE LA SINGULARIDAD}

Para caracterizar la singularidad de los modelos cinemáticos se va a utilizar la condición dada en (6.12).

La matriz $\mathbf{A}_{\text {na }}$ de (6.11), teniendo en cuenta (6.5), es:

$$
\mathbf{A}_{\text {na }}=\left(\begin{array}{c|c}
\mathbf{E} & \mathbf{0} \\
\mathbf{F}_{\mathrm{a}} & \mathbf{0} \\
\hline \mathbf{F}_{\text {na }} & \mathbf{r}_{\text {na }}
\end{array}\right)
$$

donde la segunda fila matricial es para las velocidades asignadas y la tercera fila matricial para las velocidades no asignadas.

En primer lugar la matriz $\mathbf{A}_{\text {na }}$ degenera cuando:

$$
\left|\mathbf{r}_{\mathrm{na}}\right|=0 \rightarrow \exists i:\left\{\mathrm{s} \gamma_{i}=0 \quad \text { ó } \quad \mathrm{c} \delta_{i}=0\right\}
$$

donde se ha asumido que $\mathrm{r}_{i} \neq 0$ y d $\mathrm{d}_{i} \neq 0$.

Las condiciones de (6.14) significan que hay alguna rueda sueca singular (rodillos con orientación paralela al plano de la rueda) o alguna rueda castor singular (plano de la rueda perpendicular al brazo de dirección).

Para la situación singular anterior las velocidades de ruedas correspondientes, $\dot{\varphi}_{\mathrm{si}}$ o $\dot{\beta}_{\mathrm{ci}}$, no afectan directamente al modelo cinemático (en el caso de $\dot{\beta}_{c i}$, afecta indirectamente a través de $\beta_{c i}$ ), por lo que su vector fila asociado se incluiría junto con la matriz $\left(\mathbf{E}^{\mathrm{T}} \mathbf{F}_{\mathrm{a}}^{\mathrm{T}}\right)^{\mathrm{T}}$ en (6.13), es decir:

$$
\begin{gathered}
\mathbf{G}=\left(\begin{array}{c}
\mathbf{E} \\
\mathbf{F}_{\text {a }} \\
\mathbf{F}_{\text {na_sg }}
\end{array}\right) \\
\mathbf{A}_{\text {na }}=\left(\begin{array}{c|c}
\mathbf{G} & \mathbf{0} \\
\hline \mathbf{F}_{\text {na_nsg }} & \mathbf{r}_{\text {na_nsg }}
\end{array}\right)
\end{gathered}
$$

donde $\mathbf{F}_{\text {n__sg }}$ es una matriz de tres columnas con los vectores fila $\mathbf{F}_{\mathrm{si}}$ y $\mathbf{F}_{\beta c i}$ de las 
ruedas omnidireccionales singulares, y $\left\{\mathbf{F}_{\text {na_nsg, }}, \mathbf{r}_{\text {na_nsg }}\right\}$ es igual que $\left\{\mathbf{F}_{\text {na }}, \mathbf{r}_{\text {na }}\right\}$ pero sin esos vectores fila.

Notar que, $\mathbf{F}_{\mathrm{s} i}$ y/o $\quad \mathbf{F}_{\beta c i}$ se incluyen en $\mathbf{G}$ tanto si la velocidad correspondiente es asignada como si la rueda $i$ es singular.

Las condiciones de (6.14) evidencian porque la clásica orientación de los rodillos a $90^{\circ}$ en las ruedas suecas $\left(\gamma=90^{\circ}\right)$ y la habitual orientación de las ruedas castor de $0^{\circ}\left(\delta=0^{\circ}\right)$ son deseables, ya que esos valores están lo más lejos posible de la singularidad de las ruedas omnidireccionales.

En segundo lugar la matriz $\mathbf{A}_{\text {na }}$ degenera cuando lo hace la matriz $\mathbf{G}$ :

$$
\operatorname{rango}(\mathbf{G})<3
$$

Para establecer las condiciones de (6.17) se considera el siguiente vector fila:

$$
\lambda=\left(\begin{array}{lll}
\lambda_{x} & \lambda_{y} & \mathrm{~d}_{\lambda}
\end{array}\right)
$$

donde $\left(\lambda_{x} \lambda_{y}\right)^{\mathrm{T}}$ es un vector unitario bidimensional de una recta (ver la Figura 6.1) y $\mathrm{d}_{\lambda}$ es la distancia de la recta al origen, con signo positive si el producto vectorial entre el vector distancia (del origen a la recta) y el vector de dirección es positivo respecto a la coordenada $Z$, y signo negativo en caso contrario.

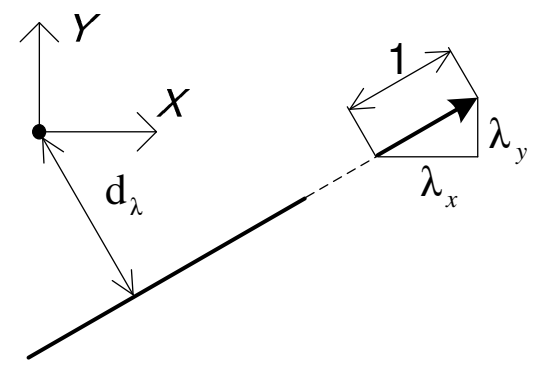

Figura 6.1- Línea definida por el vector fila de tres elementos $\lambda=\left(\begin{array}{lll}\lambda_{x} & \lambda_{y} & \mathrm{~d}_{\lambda}\end{array}\right)$

La ecuación de la recta definida por (6.18) es:

$$
\lambda_{x} \cdot y-\lambda_{y} \cdot x+\mathrm{d}_{\lambda}=\left(\begin{array}{lll}
\lambda_{x} & \lambda_{y} & \mathrm{~d}_{\lambda}
\end{array}\right) \cdot\left(\begin{array}{lll}
y & -x & 1
\end{array}\right)^{\mathrm{T}}=0
$$


La dependencia lineal de tres vectores $\lambda$, que representan tres rectas $\{a, b$, $c$ \} en el plano (6.19), viene dada por:

$$
\left|\begin{array}{lll}
\lambda_{x a} & \lambda_{y a} & \mathrm{~d}_{\lambda a} \\
\lambda_{x b} & \lambda_{y b} & \mathrm{~d}_{\lambda b} \\
\lambda_{x c} & \lambda_{y c} & \mathrm{~d}_{\lambda c}
\end{array}\right|=0
$$

La condición (6.20) implica que las tres líneas (6.19) son paralelas o intersectan en un punto común. Como extrapolación, cuando se consideran más de tres vectores/rectas y no hay ningún conjunto de tres vectores fila independientes, todas las rectas son paralelas o intersectan en un punto común.

En nuestro caso, los vectores $\left\{\mathbf{E}_{\mathrm{fi}}, \mathbf{E}_{\mathrm{o} i}, \mathbf{F}_{\mathrm{fi}}, \mathbf{F}_{\mathrm{o} i}, \mathbf{F}_{\mathrm{si}}, \mathbf{F}_{\beta c i}, \mathbf{F}_{\varphi c i}\right\}$ (definidos en (6.1),(6.2), (6.3) y (6.4)) representan con el significado de (6.18), las siguientes rectas respecto al sistema de coordenadas $\mathrm{R}$ :

- $\mathbf{E}_{\mathrm{f} i} / \mathbf{E}_{\mathrm{o} i}$ : eje de rotación de la rueda fija / orientable $i ;$

- $\mathbf{F}_{\mathrm{f} i} / \mathbf{F}_{\mathrm{o} i} / \mathbf{F}_{\varphi c i}$ : recta definida por el plano de la rueda fija / orientable/ castor $i$;

- $\mathbf{F}_{\mathrm{si}}$ : eje de rotación del rodillo de la rueda sueca $i$;

- $\mathbf{F}_{\beta c i}$ : eje de rotación de la rueda castor $i$;

De modo que la degeneración de $\mathbf{G}$, es decir la singularidad del modelo cinemático, se produce cuando todas las rectas asociadas a los vectores fila son paralelas o intersectan en un punto común.

Por tanto, el planteamiento geométrico que caracteriza la singularidad de modelos cinemáticos directos se enuncia como sigue:

Para un modelo cinemático con un conjunto $m$ de velocidades de rueda libremente asignadas, donde $m$ es el grado de movilidad actual (calculado con la ecuación (6.8)), se produce la singularidad cuando las siguientes rectas son paralelas o intersectan en un punto común:

1) los ejes de rotación de todas las ruedas fijas, orientables y omnidireccionales singulares;

2) las rectas definidas por los planos de todas las ruedas fijas, orientables y castor con la velocidad de rotación $\left(\dot{\varphi}_{\mathrm{fi}}, \dot{\varphi}_{\mathrm{oi}}, \dot{\varphi}_{\mathrm{ci}}\right)$ como asignada;

3) los ejes de rotación de los rodillos de todas las ruedas suecas con la velocidad de rotación $\dot{\varphi}_{\text {si }}$ como libremente asignada;

4) los ejes de rotación de todas las ruedas castor con la velocidad de direccionamiento $\dot{\beta}_{\mathrm{c} i}$ como libremente asignada. 
El planteamiento geométrico anterior sólo considera velocidades de rueda como asignadas. Para superar esta limitación y admitir velocidades del vehículo como asignadas (e un modelo cinemático inverso o incluso mixto), hay que reconsiderar la degeneración de la matriz $\mathbf{G}$.

En particular, si la velocidad del vehículo $v_{x}$ es libremente asignada la primera columna de la matriz $\mathbf{G}$ no debe considerarse para la degeneración. De modo que la degeneración se produce para:

$$
\left|\begin{array}{ll}
\lambda_{y a} & d_{\lambda a} \\
\lambda_{y b} & d_{\lambda b}
\end{array}\right|=\lambda_{y a} \cdot d_{\lambda b}-\lambda_{y b} \cdot d_{\lambda a}=0
$$

lo que significa que las rectas asociadas a los vectores fila de la matriz $\mathbf{G}$ son paralelas al eje $X$ del sistema de coordenadas $\mathrm{R}$ o intersectan en dicho eje.

De forma análoga, si la velocidad de vehículo $v_{y}$ es asignada todas las rectas asociadas son paralelas al eje $Y$ del sistema de coordenadas $\mathrm{R}$ o intersectan en dicho eje.

Si las dos velocidades lineales del vehículo son asignadas, sólo la tercera columna de $\mathbf{G}$ se considera para la degeneración:

$$
\left|d_{\lambda a}\right|=d_{\lambda a}=0
$$

lo que significa que las rectas asociadas con los vectores fila de la matriz $\mathbf{G}$ intersectan en el origen del sistema de coordenadas R.

Si la velocidad angular del vehículo es asignada la tercera columna de $\mathbf{G}$ no se debe considerar para la degeneración:

$$
\left|\begin{array}{ll}
\lambda_{x a} & \lambda_{y a} \\
\lambda_{x b} & \lambda_{y b}
\end{array}\right|=\lambda_{x a} \cdot \lambda_{y b}-\lambda_{x b} \cdot \lambda_{y a}=0
$$

lo que significa que las rectas asociadas los vectores fila de $\mathbf{G}$ son paralelas.

Si la velocidad angular y una de las lineales del vehículo $\left(\omega\right.$ y $v_{x}$ o $\left.v_{y}\right)$ son asignadas, la dependencia de los vectores fila es:

$$
\left|\lambda_{y a}\right|=\lambda_{y a}=0 \quad \text { ó } \quad\left|\lambda_{x a}\right|=\lambda_{x a}=0
$$

lo que implica que todas las rectas son paralelas al eje $X$ o $Y$ de sistema R. 
Finalmente, si las tres velocidades de rueda son asignadas (si es que es posible: $m=3$ ) el modelo cinemático inverso es no-singular:

Resumiendo, el planteamiento geométrico de singularidad se extiende para incluir cualquier modelo cinemático con los siguientes dos puntos:

5) si velocidades lineales del vehículo $\left(\begin{array}{lll}v_{x} & y / o & v_{y}\end{array}\right)$ son consideradas asignadas la recta (o rectas) definidas por el correspondiente eje $X$ y/o $Y$ del sistema de coordenadas $R$ se incorpora con el resto.

6) si la velocidad angular del vehículo $\omega$ es considerada asignada, todas las rectas indicadas deben ser necesariamente paralelas para que se produzca la singularidad.

Incluso, si más de $m$ velocidades son asignadas, es decir $\operatorname{rango}\left(\mathbf{A}_{\text {na }}\right)<g$, el planteamiento geométrico es válido para caracterizar la singularidad (degeneración de $\mathbf{A}_{\text {na }}$ ) de la solución al calcular $\dot{\mathbf{q}}_{\text {na }}$ aplicando la pseudo-inversa por la izquierda en (6.11).

El planteamiento geométrico establecido es completamente genérico y útil para establecer la singularidad de cualquier modelo cinemático de cualquier vehículo con ruedas comunes (fijas, orientables, castor y suecas). 


\subsection{SINGULARIDAD DE LOS CINCO TIPOS DE VEHÍCULOS}

Con objeto de mostrar aplicaciones prácticas del planteamiento geométrico de singularidad deducido en el punto anterior, se va a caracteriza la singularidad de los cinco tipos de vehículos (obtenidos en el punto 5.3 del capítulo anterior) para el caso de tres ruedas.

El hecho de considerar vehículos con tres ruedas es por motivos de estabilidad y no implica perdida de generalidad.

Así pues, en los siguientes apartados se representa de forma gráfica la singularidad, para cada conjunto de velocidades asignadas, en el caso de todas las rectas intersectando en un punto común.

El caso de rectas paralelas puede considerarse un caso límite donde las rectas intersectan en un punto en el infinito (geometría proyectiva).

Si no se muestra la singularidad de un vehículo para un conjunto de velocidades asignadas, y no es equivalente a otra mostrada, es porque es nosingular.

Para los parámetros de las ruedas castor y suecas se utilizarán los valores clásicos: $\left\{\gamma=90^{\circ}, \delta=0^{\circ}\right\}$.

\subsubsection{Tipo 1: Vehículo omnidireccional}

Este tipo de vehículo tiene movilidad completa $(m=3)$ y no tiene ruedas fijas ni orientables, es decir está construido con ruedas suecas o castor. Para este estudio se considera el mismo tipo de rueda, muy común en la práctica. Por lo que hay dos opciones: tres ruedas suecas o tres ruedas castor.

La singularidad de ambas opciones, dependiendo del conjunto de velocidades asignadas $\dot{\mathbf{q}}_{\mathrm{a}}$, se muestra en las Figuras 6.2 y 6.3. Las configuraciones singulares de la Figura 6.3 (e) y (f) fueron también apuntadas en [Yi et al. 02].

Notar que, asumiendo ruedas omnidireccionales no singulares, el modelo inverso cinemático de este vehículo es no-singular. 


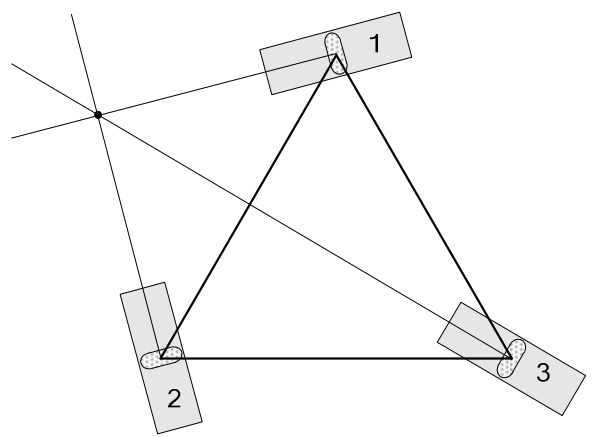

Figura 6.2- Singularidad del vehículo omnidireccional con ruedas suecas

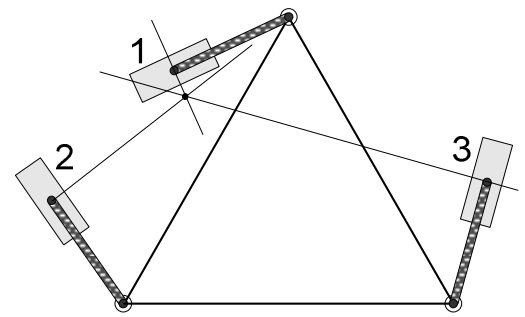

(a) $\dot{\mathbf{q}}_{\mathrm{a} 1}=\left(\dot{\beta}_{\mathrm{c} 1} \dot{\beta}_{\mathrm{c} 2} \dot{\beta}_{\mathrm{c} 3}\right)^{\mathrm{T}}$

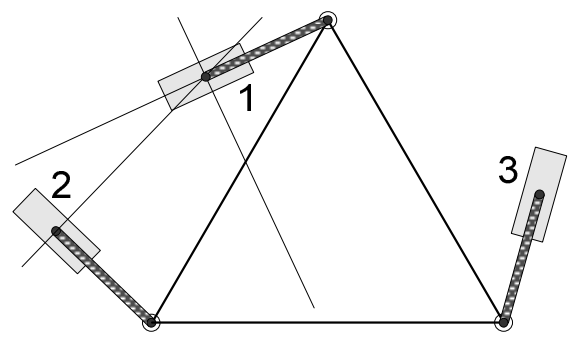

(c) $\dot{\mathbf{q}}_{\mathrm{a} 3}=\left(\dot{\varphi}_{\mathrm{c} 1} \dot{\beta}_{\mathrm{c} 1} \dot{\beta}_{\mathrm{c} 2}\right)^{\mathrm{T}}$

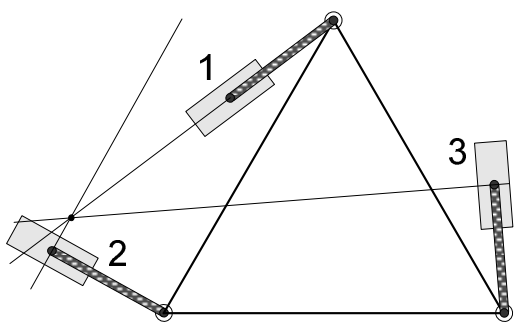

(e) $\dot{\mathbf{q}}_{\mathrm{a} 5}=\left(\dot{\varphi}_{\mathrm{c} 1} \dot{\beta}_{\mathrm{c} 2} \dot{\beta}_{\mathrm{c} 3}\right)^{\mathrm{T}}$

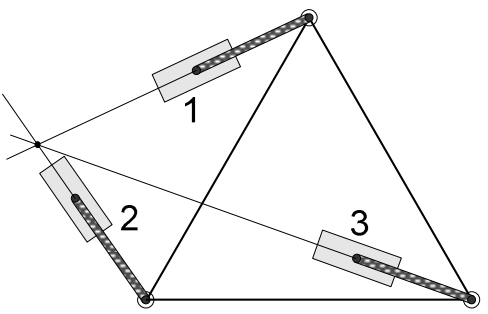

(b) $\dot{\mathbf{q}}_{\mathrm{a} 2}=\left(\dot{\varphi}_{\mathrm{c} 1} \dot{\varphi}_{\mathrm{c} 2} \dot{\varphi}_{\mathrm{c} 3}\right)^{\mathrm{T}}$

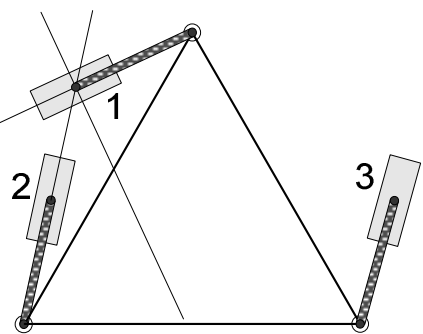

(d) $\dot{\mathbf{q}}_{\mathrm{a} 4}=\left(\dot{\varphi}_{\mathrm{c} 1} \dot{\beta}_{\mathrm{c} 1} \dot{\varphi}_{\mathrm{c} 2}\right)^{\mathrm{T}}$

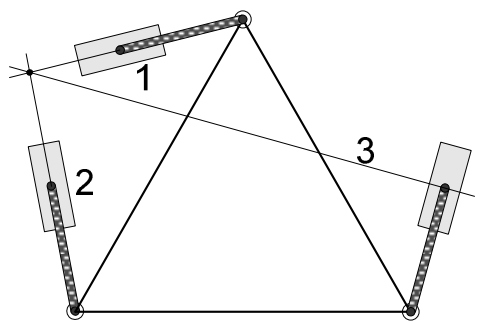

(f) $\dot{\mathbf{q}}_{\mathrm{a} 6}=\left(\begin{array}{lll}\dot{\varphi}_{\mathrm{c} 1} & \dot{\varphi}_{\mathrm{c} 2} & \left.\dot{\beta}_{\mathrm{c} 3}\right)^{\mathrm{T}}\end{array}\right.$

Figura 6.3-Configuraciones singulares del vehículo omnidireccional con ruedas castor 


\subsubsection{Tipo 2: Vehículo diferencial}

Este tipo de vehículo tiene una rueda fija y otras posibles ruedas omnidireccionales (suecas o castor), resultando una movilidad de dos $(m=2)$. Aquí se considerarán dos ruedas fijas con un eje de rotación común (el clásico mecanismo diferencial) y otra rueda omnidireccional, bien sueca o castor.

La singularidad de los dos vehículos se muestra en la Figura 6.4, excepto para los casos en que $v_{x}$ es considerada asignada, ya que resultan singulares para todo el espacio de configuración/de parámetros. Para este vehículo el origen del sistema $\mathrm{R}$ se ha situado en el punto medio del eje de rotación de las dos ruedas fijas y con su eje $X$ coincidente con dicho eje.

\subsubsection{Tipo 3: Vehículo con una rueda orientable}

Este vehículo tiene una rueda orientable independiente y otras posibles omnidireccionales, resultando una movilidad de dos $(m=2)$. En este estudio se considerará una rueda orientable y dos ruedas adicionales omnidireccionales del mismo tipo, bien suecas o castor. El origen del sistema de coordenadas $\mathrm{R}$ se ha situado en el centro de la rueda orientable.

La singularidad de este vehículo, con dos ruedas adicionales suecas o castor se representa en las Figuras 6.5, 6.6 y 6.7. Notar que las Figuras 6.6 (a), 6.7 (a) y 6.7 (b) son también válidas si $\dot{\varphi}_{\text {o1 }}$ se sustituye por $v_{x} \mathrm{o} v_{y}$.

\subsubsection{Tipo 4: Vehículo triciclo y bicicleta}

Este vehículo tiene una rueda orientable independiente y otra rueda fija independiente, resultando un grado de movilidad $(m=1)$. Aquí se considerará una rueda orientable, una rueda fija y otra rueda: fija dependiente, sueca o castor.

\subsubsection{A) Vehículo triciclo}

Este vehículo clásico consiste en una rueda orientable y otras dos ruedas fijas simétricas. El origen de sistema de coordenadas $\mathrm{R}$ se ha situado en el punto medio del eje de rotación de las ruedas fijas, y con su eje $X$ coincidente con dicho eje de rotación. La singularidad de este vehículo se muestra en la Figura 6.8 excepto para el caso $\dot{\mathbf{q}}_{\mathrm{a}}=v_{x}$, que es singular en todo el espacio de configuración. 


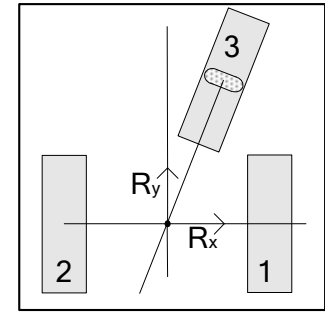

(a) $\dot{\mathbf{q}}_{\mathrm{a} 1}=\left(\dot{\varphi}_{\mathrm{s} 3} v_{\mathrm{y}}\right)^{\mathrm{T}}$

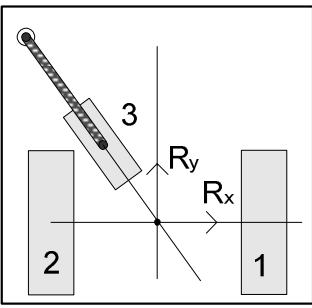

(c) $\dot{\mathbf{q}}_{\mathrm{a} 3}=\left(\dot{\varphi}_{\mathrm{c} 3} v_{\mathrm{y}}\right)^{\mathrm{T}}$

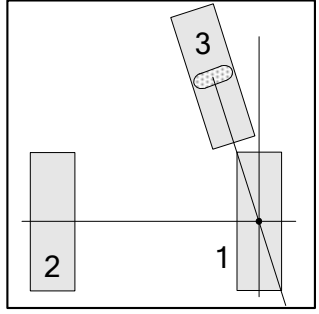

(b) $\dot{\mathbf{q}}_{\mathrm{a} 2}=\left(\dot{\varphi}_{\mathrm{f} 1} \dot{\varphi}_{\mathrm{s} 3}\right)^{\mathrm{T}}$

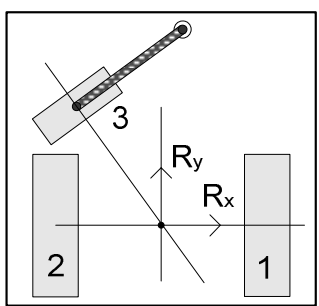

(d) $\dot{\mathbf{q}}_{\mathrm{a} 4}=\left(\dot{\beta}_{\mathrm{c} 3} v_{y}\right)^{\mathrm{T}}$

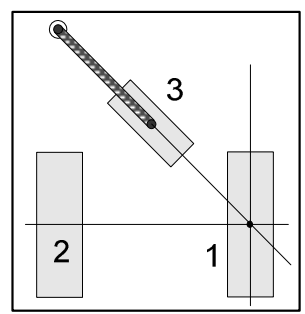

(e) $\dot{\mathbf{q}}_{\mathrm{a} 5}=\left(\dot{\varphi}_{\mathrm{f} 1} \dot{\varphi}_{\mathrm{c} 3}\right)^{\mathrm{T}}$

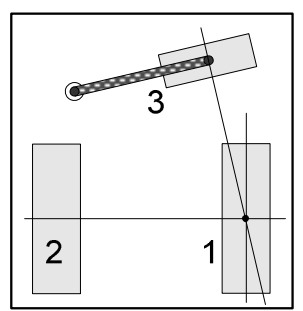

(f) $\dot{\mathbf{q}}_{\mathrm{a} 6}=\left(\dot{\varphi}_{\mathrm{f} 1} \dot{\beta}_{\mathrm{c} 3}\right)^{\mathrm{T}}$

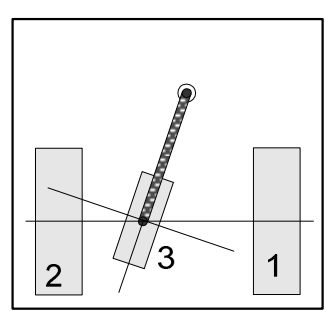

(g) $\dot{\mathbf{q}}_{\mathrm{a} 7}=\left(\dot{\varphi}_{\mathrm{c} 3} \dot{\beta}_{\mathrm{c} 3}\right)^{\mathrm{T}}$

Figura 6.4- Singularidad del vehículo tipo 2 con rueda suecalcastor adicional

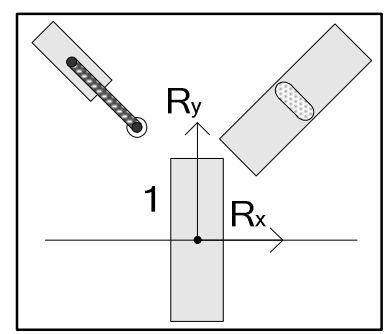

(a) $\dot{\mathbf{q}}_{\mathrm{a} 1}=\left(v_{x} \omega\right)^{\mathrm{T}}$

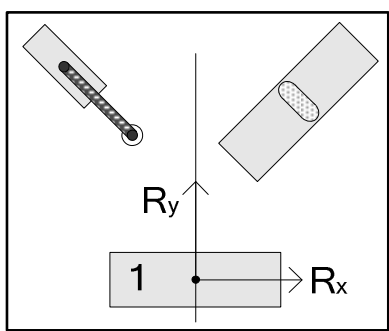

(b) $\dot{\mathbf{q}}_{\mathrm{a} 2}=\left(v_{y} \omega\right)^{\mathrm{T}}$

Figura 6.5- Configuraciones singulares comunes para el vehículo tipo 3 


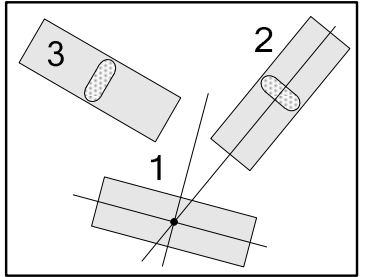

(a) $\dot{\mathbf{q}}_{\mathrm{a} 3}=\left(\dot{\varphi}_{\mathrm{o} 1} \dot{\varphi}_{\mathrm{s} 2}\right)^{\mathrm{T}}$

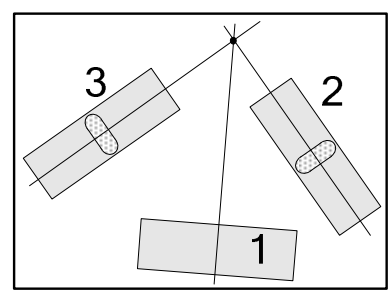

(b) $\dot{\mathbf{q}}_{\mathrm{a} 4}=\left(\dot{\varphi}_{\mathrm{s} 2} \dot{\varphi}_{\mathrm{s} 3}\right)^{\mathrm{T}}$

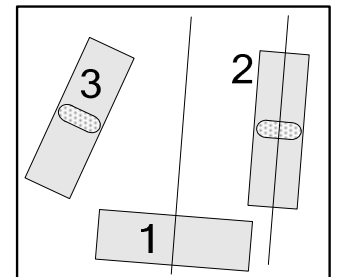

(c) $\dot{\mathbf{q}}_{\mathrm{a} 5}=\left(\dot{\varphi}_{\mathrm{s} 2} \omega\right)^{\mathrm{T}}$

Figura 6.6- Configuraciones singulares del vehículo tipo 3 con ruedas suecas

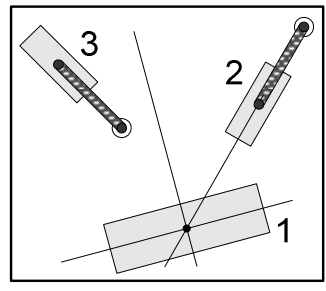

(a) $\dot{\mathbf{q}}_{\mathrm{a} 6}=\left(\dot{\varphi}_{\mathrm{o} 1} \dot{\varphi}_{\mathrm{c} 2}\right)^{\mathrm{T}}$

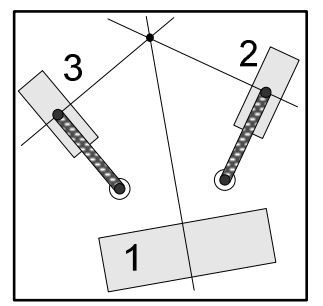

(d) $\dot{\mathbf{q}}_{\mathrm{a} 9}=\left(\dot{\beta}_{\mathrm{c} 2} \dot{\beta}_{\mathrm{c} 3}\right)^{\mathrm{T}}$

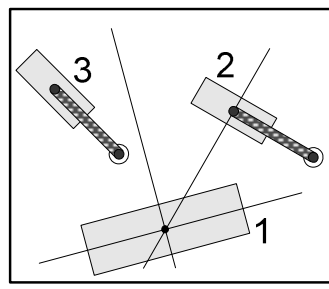

(b) $\dot{\mathbf{q}}_{\mathrm{a} 7}=\left(\dot{\varphi}_{\mathrm{o} 1} \dot{\beta}_{\mathrm{c} 2}\right)^{\mathrm{T}}$

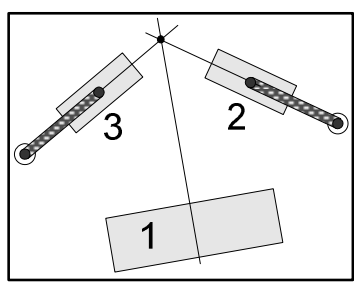

(c) $\dot{\mathbf{q}}_{\mathrm{a} 8}=\left(\dot{\varphi}_{\mathrm{c} 2} \dot{\varphi}_{\mathrm{c} 3}\right)^{\mathrm{T}}$

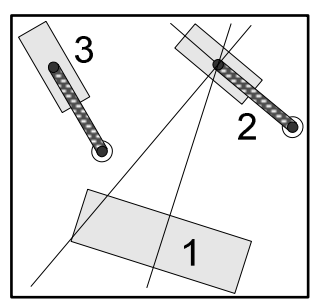

(e) $\dot{\mathbf{q}}_{\mathrm{a} 10}=\left(\dot{\varphi}_{\mathrm{c} 2} \dot{\beta}_{\mathrm{c} 2}\right)^{\mathrm{T}}$

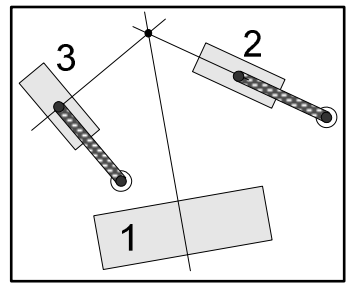

(f) $\dot{\mathbf{q}}_{\mathrm{a} 11}=\left(\dot{\varphi}_{\mathrm{c} 2} \dot{\beta}_{\mathrm{c} 3}\right)^{\mathrm{T}}$

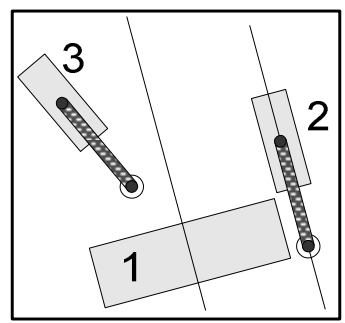

(e) $\dot{\mathbf{q}}_{\mathrm{a} 12}=\left(\dot{\varphi}_{\mathrm{c} 2} \omega\right)^{\mathrm{T}}$

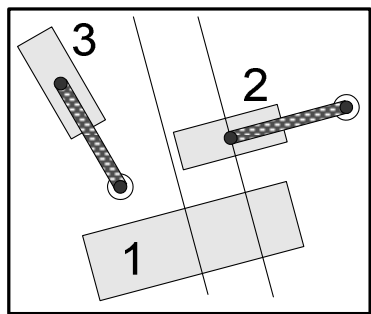

(f) $\dot{\mathbf{q}}_{\mathrm{a} 13}=\left(\dot{\beta}_{\mathrm{c} 2} \omega\right)^{\mathrm{T}}$

Figura 6.7- Configuraciones singulares del vehículo tipo 3 con ruedas castor 


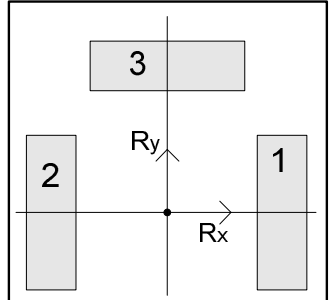

(a) $\dot{\mathbf{q}}_{\mathrm{a} 1}=v_{y}$

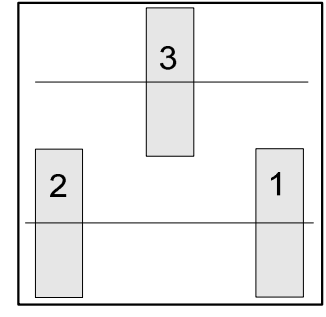

(b) $\dot{\mathbf{q}}_{\mathrm{a} 2}=\omega$

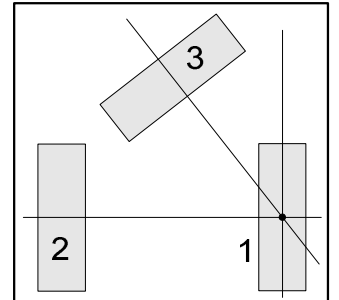

(c) $\dot{\mathbf{q}}_{\mathrm{a} 3}=\dot{\varphi}_{\mathrm{f}}$

Figura 6.8-Configuraciones singulares para el vehículo triciclo

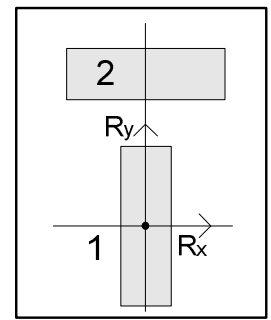

(a) $\dot{\mathbf{q}}_{\mathrm{a} 1}=v_{y}$

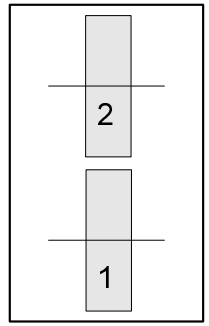

(b) $\dot{\mathbf{q}}_{\mathrm{a} 2}=\omega$

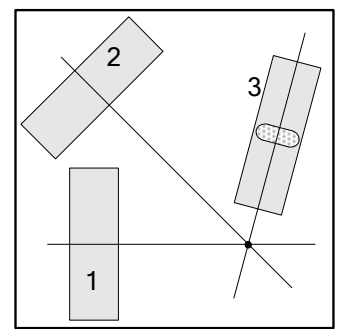

(c) $\dot{\mathbf{q}}_{\mathrm{a} 3}=\dot{\varphi}_{\mathrm{s} 3}$

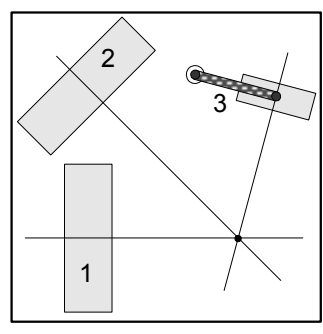

(d) $\dot{\mathbf{q}}_{\mathrm{a} 4}=\dot{\varphi}_{\mathrm{c} 3}$

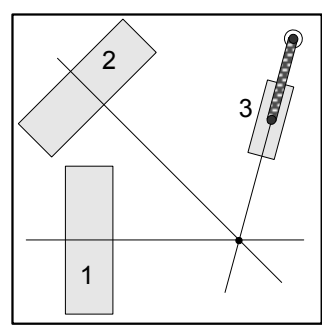

(e) $\dot{\mathbf{q}}_{\mathrm{a} 5}=\dot{\beta}_{\mathrm{c} 3}$

Figura 6.9- Configuraciones singulares para el vehículo bicicleta con una rueda suecalcastor adicional

\subsubsection{A) Vehículo bicicleta con rueda adicional sueca o castor}

Este vehículo tiene una rueda fija alineada con una rueda orientable, y otra rueda sueca o castor por motivos de estabilidad. El origen del sistema de coordenadas $\mathrm{R}$ se ha situado en el centro de la rueda fija, y con su eje $X$ coincidente con el eje de rotación de dicha rueda fija. La singularidad se muestra en la Figura 6.9. 


\subsubsection{Tipo 5: Vehículo con dos ruedas orientables}

Este vehículo está caracterizado por dos ruedas orientables independientes y tiene un grado de movilidad $(m=1)$. Para este trabajo se considerarán dos ruedas orientables y otra adicional del tipo sueca o castor. El origen del sistema de coordenadas $\mathrm{R}$ se ha situado en el punto medio de la recta que une las dos ruedas orientables, con su eje $X$ coincidente con dicha recta. Su singularidad se muestra en la Figura 6.10 excepto para el caso $\dot{\mathbf{q}}_{\mathrm{a}}=v_{x}$, que es singular en todo el espacio de configuración.

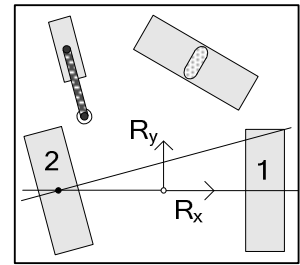

(a) $\dot{\mathbf{q}}_{\mathrm{a} 1}=v_{x}$

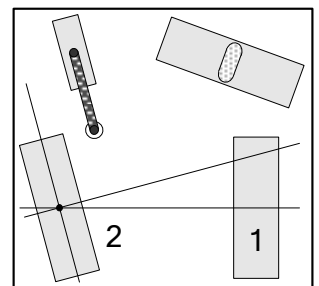

(e) $\dot{\mathbf{q}}_{\mathrm{a} 5}=\dot{\varphi}_{\mathrm{f} 2}$

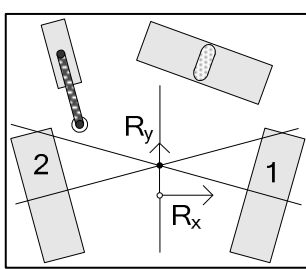

(b) $\dot{\mathbf{q}}_{\mathrm{a} 1}=v_{y}$

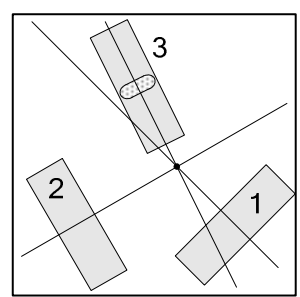

(f) $\dot{\mathbf{q}}_{\mathrm{a} 6}=\dot{\varphi}_{\mathrm{s} 3}$

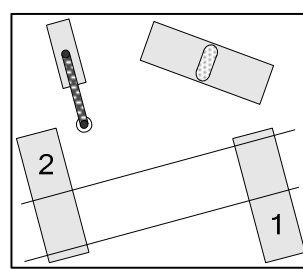

(c) $\dot{\mathbf{q}}_{\mathrm{a} 3}=\omega$

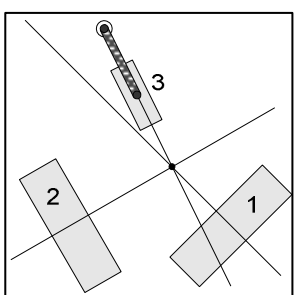

(g) $\dot{\mathbf{q}}_{\mathrm{a} 7}=\dot{\varphi}_{\mathrm{c} 3}$

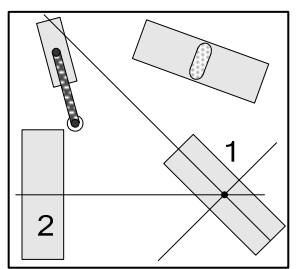

(d) $\dot{\mathbf{q}}_{\mathrm{a} 4}=\dot{\varphi}_{\mathrm{f} 1}$

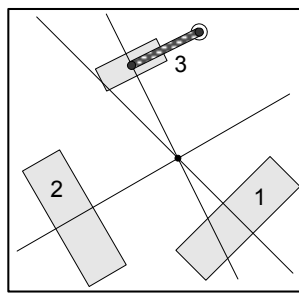

(h) $\dot{\mathbf{q}}_{\mathrm{a} 8}=\dot{\beta}_{\mathrm{c} 3}$

Figura 6.10- Configuraciones singulares para el vehículo tipo 5 con una rueda suecalcastor adicional 


\subsection{EXTENSIÓN DE LA CARACTERIZACIÓN DE LA SINGULARIDAD}

A continuación se consideran, para la caracterización de la singularidad, otro tipo de ruedas menos habituales (especiales), las cuáles ya fueron presentadas en el punto 3.5 del Capítulo 3.

La rueda doble y la castor doble (Figura 3.5) pueden considerarse como dos ruedas singulares castor y dos ruedas castor, respectivamente, con mismo eje de rotación $\mathrm{L}_{x i} \mathrm{y}$ ángulo de dirección $\beta_{i}$. Utilizando esta equivalencia, el modelado cinemático y la caracterización de la singularidad anteriores siguen siendo validos para este tipo de ruedas.

Por otro lado, las ruedas tipo bola (Figura 3.8) y ortogonal (Figuras 3.9 y 3.10) no han sido modeladas, pero dado que ambas son funcionalmente equivalentes a las ruedas suecas, se pueden incluir en la caracterización de la singularidad del punto 6.4 añadiendo la siguiente condición:

7) cada dirección restringida de las ruedas esféricas y ortogonales deben considerarse como velocidades libremente asignadas del conjunto $m$ y las lineas rectas definidas por dichas direcciones deben incorporarse con las otras líneas rectas del punto 6.4 . 


\subsection{RESULTADOS MÁS RELEVANTES Y CONCLUSIONES DEL CAPÍTULO}

A continuación se indican los aspectos más relevantes que aporta el capítulo:

- La principal aportación de este capítulo es el planteamiento geométrico formulado que caracteriza la singularidad de la cinemática de vehículos con ruedas comunes (fijas, orientables, castor y suecas) y/o especiales (dobles, castor dobles, tipo bola y ortogonales). Este planteamiento geométrico ha sido obtenido en base a principios sencillos y representa una forma novel, genérica, práctica y útil de caracterizar la singularidad de cualquier modelo cinemático de cualquier vehículo.

- Se han representado las configuraciones singulares de los cinco tipos de vehículos genéricos, clasificados en el capítulo anterior.

- La caracterización anterior representa (junto con los modelos y análisis de isotropía del capítulo anterior) una guía de la cinemática de vehículos útil para el diseño mecánico, desarrollo de aplicaciones de control, la planificación de caminos, etc. 


\section{CAPÍTULO 7 \\ MODELADO CINEMÁTICO DE VEHÍCULOS CON RUEDAS CON DESLIZAMIENTO}

\subsection{INTRODUCCIÓN}

Muchas de las publicaciones que tratan el tema del modelado cinemático asumen no-deslizamiento [Campion et al. 96] [Rajagopalan 97] [Low et al. 05] [Yi et al. 02] o acusan una falta de sentido físico en los modelos con deslizamiento [Muir et al. 87] [Alexander et al. 89] [Kim et al. 04] [Tham et al. 98].

En cualquier caso, a veces es necesario considerar modelos cinemáticos con deslizamiento, por ejemplo en una solución cinemática directa con información redundante (de modo similar al filtro de Kalman) o con accionamientos en las sobre-actuados de forma independiente.

En este sentido varias publicaciones han abordado el modelado cinemático de vehículos con deslizamiento.

En [Muir et al. 87] se considera una solución cinemática con información redundante $\left(\operatorname{dim}\left(\dot{\mathbf{q}}_{\mathrm{a}}\right)>m\right)$ aplicando la pseudo-inversa por la izquierda en (4.9), obteniéndose (4.11).

Sin embargo, esta solución de Mínimos Cuadrados (Least Square LS) propuesta en [Muir et al. 87] viola, como se apunta en [Kim et al. 04] con un ejemplo numérico, las restricciones cinemáticas (modelo de sólido rígido) del 
vehículo. Esta violación se produce, a diferencia de la explicación dada en [Kim et al. 04], porque se considera una ecuación de rueda con tres elementos escalares, en lugar de dos como (3.11) y (3.12) (la rueda sueca no la consideran). La tercera ecuación escalar relaciona la velocidad de direccionamiento con la velocidad angular del vehículo ${ }^{\overline{\mathrm{R}}} \omega_{\mathrm{R}}$ a través de la poco útil variable de rueda ${ }^{\overline{\mathrm{E}} i} \omega_{\mathrm{E} i}$ (no puede ser sensorizada ni actuada):

$$
{ }^{\overline{\mathrm{R}}} \omega_{\mathrm{R}}={ }^{\overline{\mathrm{E}} i} \omega_{\mathrm{E} i}-\dot{\beta}_{i}
$$

Como resultado, al aplicar el algoritmo de LS esta ecuación escalar no se garantiza, lo que es inaceptable ya que esta restricción debe cumplirse siempre, no como las otras dos que pueden permitir deslizamiento. Por ejemplo, una rueda fija o sueca tiene una velocidad de direccionamiento nula $\dot{\beta}_{i}$ y por tanto su velocidad angular ${ }^{\overline{\mathrm{E}} i} \omega_{\mathrm{E} i}$ debe ser siempre igual a la del vehículo ${ }^{\overline{\mathrm{R}}} \omega_{\mathrm{R}}$.

En [Kim et al. 04] se considera explícitamente las velocidades de deslizamiento de forma similar a (3.22), y se aplica la pseudo-inversa por la derecha ponderada para calcular las velocidades de rueda no-asignadas $\dot{\mathbf{q}}_{\mathrm{w} \_ \text {na }}$ y las velocidades de deslizamiento $\mathbf{v}_{\text {slip }}$. Por lo que el algoritmo minimiza la norma Euclídea ponderada de $\left(\dot{\mathbf{q}}_{\mathrm{w} \_n a} \mathbf{v}_{\text {slip }}\right)^{\mathrm{T}}$, lo que no tiene un sentido físico definido.

En [Tham et al. 98] se considera un vehículo con cuatro ruedas orientables con las velocidades de rotación y direccionamiento medidas. Se utilizan varias expresiones (que relacionan las velocidades de rueda y algunas variables de deslizamiento) junto con el filtro extendido de Kalman (FKE) para realizar una estimación adaptativa del estado. Sin embargo, las mencionadas relaciones con deslizamiento son completamente ad-hoc y no presentan una justificación rigurosa.

De forma similar, [Scheding et al. 99] desarrolla un modelo cinemático particular de un vehículo articulado, con eje trasero y delantero, que incluye el ángulo de deslizamiento de cada cuerpo. Estos ángulos y la postura del vehículo se estiman con un FKE.

La contribución [Alexander et al. 89] propone minimizar la función de disipación $P_{\mathrm{dis}}$ producida por las fuerzas de fricción de Coulomb $\mathbf{F}_{\text {fric_c } i}$ con los coeficientes de fricción dinámicos $\mu_{\mathrm{c} i}$ : 


$$
\begin{gathered}
\mathbf{F}_{\text {fric_c } i}=-\mu_{\mathrm{c} i} \cdot \mathbf{v}_{\text {slip } i} /\left|\mathbf{v}_{\text {slip } i}\right| \\
P_{\text {dis }}=-\sum_{i=1}^{\mathrm{N}}\left(\mathbf{F}_{\text {fric } \_c i} \cdot \mathbf{v}_{\text {slip } i}\right)=-\sum_{i=1}^{\mathrm{N}}\left(\mathbf{F}_{\text {fric_c } i}^{\mathrm{T}} \cdot \mathbf{v}_{\text {slip } i}\right)
\end{gathered}
$$

En este estudio se muestra que la minimización respecto al vector de velocidad del vehículo $\dot{\mathbf{p}}$ es equivalente a equilibrio de fuerzas y momentos y caracteriza un movimiento quasi-statico (aceleraciones nulas).

Sin embargo, este proceso de minimización no produce una solución correcta en el caso de una fuerza de fricción genérica. Esto se ilustrara con un ejemplo sencillo pero con los mismos principios de funcionamiento. Considérese un bloque libre en una dimensión sobre una superficie con una fuerza de fricción genérica:

$$
F_{\text {fric_b }}=-f\left(\left|v_{\mathrm{b}}\right|\right) \cdot v_{\mathrm{b}} /\left|v_{\mathrm{b}}\right|
$$

donde $v_{\mathrm{b}}$ es la velocidad (de deslizamiento) del bloque no nula y $f$ el módulo de la fuerza de fricción dependiente en el módulo de la velocidad de deslizamiento.

La función de disipación resulta:

$$
P_{\text {dis_b }}=-F_{\text {fric_b }} \cdot v_{\mathrm{b}}=\left|v_{\mathrm{b}}\right| \cdot f\left(\left|v_{\mathrm{b}}\right|\right)
$$

y su minimización respecto a la velocidad de deslizamiento $v_{\mathrm{b}}$ es:

$$
\frac{d P_{\text {dis_ }}}{d v_{\mathrm{b}}}=\frac{d P_{\text {dis_b }}}{d\left|v_{\mathrm{b}}\right|} \cdot \frac{d\left|v_{\mathrm{b}}\right|}{d v_{\mathrm{b}}}=\left(f\left(\left|v_{\mathrm{b}}\right|\right)+\left|v_{\mathrm{b}}\right| \cdot \frac{d f\left(\left|v_{\mathrm{b}}\right|\right)}{d\left|v_{\mathrm{b}}\right|}\right) \cdot \frac{v_{\mathrm{b}}}{\left|v_{\mathrm{b}}\right|}=0
$$

La solución de movimiento quasi-estático viene dada por:

$$
F_{\text {fric } \_ \text {b }}=-f\left(\left|v_{\mathrm{b}}\right|\right) \cdot v_{\mathrm{b}} /\left|v_{\mathrm{b}}\right|=0
$$

Comparando las dos expresiones anteriores:

$$
f\left(\left|v_{\mathrm{b}}\right|\right)+\left|v_{\mathrm{b}}\right| \cdot d f\left(\left|v_{\mathrm{b}}\right|\right) / d\left|v_{\mathrm{b}}\right|=\mathrm{k} \cdot f\left(\left|v_{\mathrm{b}}\right|\right)
$$

donde $\mathrm{k}$ es una constante genérica. 
La condición (7.8) se satisface, por ejemplo, para un módulo de la fuerza de fricción $f: 1,\left|v_{\mathrm{b}}\right|,\left|v_{\mathrm{b}}\right|^{2},\left|v_{\mathrm{b}}\right|^{3}, \ldots$, pero no para un módulo de la fuerza de fricción $f$ : $1+\left|v_{\mathrm{b}}\right|, 1+\left|v_{\mathrm{b}}\right|^{2},\left|v_{\mathrm{b}}\right|+\left|v_{\mathrm{b}}\right|^{2}, \ldots$

El procedimiento para un vehículo con varias ruedas deslizando es análogo al presentado, pero con fuerzas de fricción y velocidades de deslizamiento bidimensionales.

Por tanto se concluye que la minimización de la función de disipación no produce en general las ecuaciones de movimiento quasi-estático. Además, no tendría en cuenta otras posibles fuerzas externas, ej. el bloque sobre un plano inclinado con la componente tangencial del peso. (De hecho en el ejemplo presentado alguna fuerza externa debería considerarse en (7.7) para producir una velocidad $v_{\mathrm{b}}$ no nula.)

Por otra parte, algunas publicaciones han abordado el modelado dinámico de vehículos con deslizamiento.

Por ejemplo, [Balakrishna et al. 95] estudia en simulación el control de un vehículo omnidirectional con tres ruedas suecas basándose en modelos de tracción (relación entre velocidades de deslizamiento y fuerzas de fricción) específicos para las ruedas deslizantes.

De igual modo [Williams et al. 02] presenta un modelo dinámico de un robot omnidireccional con tres ruedas suecas y valida el modelo de las fricciones por deslizamiento con resultados experimentales.

También, [Lindgren et al. 02] utiliza un modelo de tracción para vehículos no urbanos junto con el FKE para estimar la postura de un vehículo agrícola en aplicaciones de navegación

Y por último, [Shin 90] estudia el desplazamiento lateral (deslizamiento) empleando el modelo bicicleta y un conjunto simplificado de ecuaciones cinemáticas y dinámicas.

El objetivo de este capítulo es proporcionar un modelado cinemático con deslizamiento que se base, a diferencia de los comentados anteriormente, en principios físicos. 
Con este objeto el punto 7.2 empieza con el modelado dinámico del vehículo, al que posteriormente se le aplicarán tres aproximaciones sucesivas (punto 7.4): el modelo de movimiento quasi-estático; el modelo cinemático con deslizamiento y la solución de Mínimos Cuadrados ponderada.

También, en el punto 7.3 se revisan y discuten distintos modelos de tracción, que relacionan la fuerza de fricción en la rueda con su velocidad de deslizamiento.

Como aplicación, práctica en el punto 7.5 se prueban los modelos con deslizamiento desarrollados a un vehículo tipo triciclo, primero en un entorno de simulación y posteriormente en una situación real, comparándolos con el filtro de Kalman.

Finalmente en el punto de conclusiones se destacan los resultados más relevantes del capítulo. 


\subsection{MODELADO DINÁMICO DE VEHÍCULO CON RUEDAS}

El mayor inconveniente de los métodos de modelado con deslizamiento descritos en la introducción previa el la falta de un sentido físico adecuado. Para superar esto, en este estudio se considera como punto de partida la dinámica del vehículo obtenida con la formulación de Lagrange. Posteriormente, este planteamiento dinámico se restringirá a un movimiento quasi-estático cinemático.

Las ecuaciones generales de Lagrange para un sistema dinámico de dimensión finita vienen dadas por:

$$
\frac{d}{d t}\left(\frac{\partial T}{\partial \dot{\mathbf{q}}}\right)-\frac{\partial T}{\partial \mathbf{q}}=\mathbf{Q}
$$

donde $\mathbf{q}$ es el vector de coordenadas generalizadas (en nuestro caso equivalente a ${ }^{\mathrm{G}} \mathbf{q}$ ), $\mathbf{Q}$ es el vector de fuerzas generalizadas (que incluye las fuerzas conservativas) y $T$ es la energía cinética del sistema.

El vector de fuerzas generalizadas es:

$$
\mathbf{Q}=\sum_{i} \mathbf{F}_{i} \cdot \frac{\partial \mathbf{r}_{i}}{\partial \mathbf{q}}=\sum_{i} \mathbf{F}_{i}^{\mathrm{T}} \cdot \frac{\partial \mathbf{v}_{i}}{\partial \dot{\mathbf{q}}}
$$

donde $\mathbf{F}_{i}$ son las fuerzas/pares que actúan sobre el sistema y $\mathbf{F}_{i} \bullet \delta \mathbf{r}_{i}$ su correspondiente trabajo virtual compatible con las restricciones del sistema.

Por ejemplo, el peso (fuerza conservativa) del vehículo no produce trabajo virtual para movimiento horizontal.

En nuestro caso el vector de fuerzas generalizadas es:

$$
\mathbf{Q}=\sum_{i=1}^{\mathrm{N}} \tau_{\mathrm{ri}} \cdot \frac{\partial \dot{\varphi}_{i}}{\partial \dot{\mathbf{q}}}+\sum_{i=1}^{\mathrm{No}} \tau_{\mathrm{osi}} \cdot \frac{\partial \dot{\beta}_{\mathrm{o} i}}{\partial \dot{\mathbf{q}}}+\sum_{i=1}^{\mathrm{Nc}} \tau_{\mathrm{csi}} \cdot \frac{\partial \dot{\beta}_{\text {ci }}}{\partial \dot{\mathbf{q}}}+\sum_{i=1}^{\mathrm{N}} \mathbf{F}_{\text {fric } i}^{\mathrm{T}} \cdot \frac{\partial \mathbf{v}_{\text {slip } i}}{\partial \dot{\mathbf{q}}}
$$

con el significado de variables y parámetros de la Tabla 7.1. 
Tabla 7.1- Nuevas variables y parámetros en (7.11)

\begin{tabular}{|c|l|}
\hline Símbolo & \multicolumn{1}{c|}{ Descripción } \\
\hline $\mathrm{N}_{\mathrm{o}} / \mathrm{N}_{\mathrm{c}}$ & Número de ruedas orientables / castor en el vehículo \\
\hline$\tau_{\mathrm{ri}}$ & Par de rotación de la rueda $i$ \\
\hline$\tau_{\mathrm{os} i} / \tau_{\mathrm{cs} i}$ & Par de direccionamiento de la rueda orientable / castor $i$ \\
\hline$\dot{\beta}_{\mathrm{o} i} / \dot{\beta}_{\mathrm{c} i}$ & Velocidad de direccionamiento de la rueda orientable / castor $i$ \\
\hline $\mathbf{F}_{\text {fric } i}$ & Fuerza de fricción en la rueda $i$ respecto al sistema $\mathrm{E}_{i}$ \\
\hline
\end{tabular}

Notar que una fuerza de fricción $\mathbf{F}_{\text {fric } i}$ en dirección contraria a la velocidad de deslizamiento $\mathbf{v}_{\text {slip } i}$ produce un signo negativo en el último término de (7.11), lo que indica un trabajo disipativo.

Considerando la velocidad de deslizamiento (3.6) y (4.1) respecto al sistema de coordenadas global:

$$
\begin{aligned}
& \mathbf{v}_{\text {slip }}=\left(\begin{array}{c}
\mathbf{v}_{\text {slip } 1} \\
\vdots \\
\mathbf{v}_{\text {slip N }}
\end{array}\right)=\left(\begin{array}{c}
{ }^{\overline{\mathrm{E}} 1} \mathbf{v}_{\mathrm{E} 1} \\
\vdots \\
\overline{{ }^{\mathrm{EN}}} \mathbf{v}_{\mathrm{EN}}
\end{array}\right)=\left(\begin{array}{cccc}
\mathbf{A}_{\mathrm{p} 1} & \mathbf{A}_{\mathrm{w} 1} & \cdots & 0 \\
\vdots & \vdots & \ddots & \vdots \\
\mathbf{A}_{\mathrm{pN}} & 0 & \cdots & \mathbf{A}_{\mathrm{wN}}
\end{array}\right) \cdot\left(\begin{array}{c}
{ }^{\overline{\mathrm{R}}} \dot{\mathbf{p}} \\
\mathbf{q}_{\mathrm{w} 1} \\
\vdots \\
\dot{\mathbf{q}}_{\mathrm{wN}}
\end{array}\right) \\
& \mathbf{v}_{\text {slip }}=\left(\begin{array}{ll}
\mathbf{A}_{\mathrm{p}} & \mathbf{A}_{\mathrm{w}}
\end{array}\right) \cdot\left(\begin{array}{c}
\dot{\mathbf{p}} \\
\dot{\mathbf{q}}_{\mathrm{w}}
\end{array}\right)={ }^{\overline{\mathrm{R}}} \mathbf{A} \cdot \overline{\mathrm{R}} \dot{\mathbf{q}} \\
& \mathbf{v}_{\text {slip }}=\left(\begin{array}{ll}
\mathbf{A}_{\mathrm{p}} \cdot \operatorname{Rot}_{Z}\left(-{ }^{\mathrm{G}} \boldsymbol{\theta}_{\mathrm{R}}\right) & \mathbf{A}_{\mathrm{w}}
\end{array}\right) \cdot\left(\begin{array}{c}
{ }^{\mathrm{G}} \dot{\mathbf{p}} \\
\dot{\mathbf{q}}_{\mathrm{w}}
\end{array}\right)={ }^{\mathrm{G}} \mathbf{A} \cdot{ }^{\mathrm{G}} \dot{\mathbf{q}} \\
& { }^{\mathrm{G}} \dot{\mathbf{p}}=\left(\begin{array}{ccc}
\boldsymbol{\operatorname { R o t }}\left({ }^{\mathrm{G}} \boldsymbol{\theta}_{\mathrm{R}}\right) & 0 \\
0 & 0 & 1
\end{array}\right) \cdot{ }^{\overline{\mathrm{R}}} \dot{\mathbf{p}}=\operatorname{Rot}_{Z}\left({ }^{\mathrm{G}} \boldsymbol{\theta}_{\mathrm{R}}\right) \cdot{ }^{\overline{\mathrm{R}}} \dot{\mathbf{p}}
\end{aligned}
$$

donde $\operatorname{Rot}_{\mathrm{Z}}(x)$ es una rotación en tres dimensiones en el eje $Z$ de $x, y \operatorname{Rot}(x)$ es una matriz de rotación en dos dimensiones:

$$
\boldsymbol{\operatorname { R o t }}(x)=\left(\begin{array}{cc}
\cos (x) & -\sin (x) \\
\sin (x) & \cos (x)
\end{array}\right)
$$

con las propiedades $\boldsymbol{R o t}^{-1}(x)=\operatorname{Rot}^{\mathrm{T}}(x)=\boldsymbol{\operatorname { R o t }}(-x)$. 
La expresión (7.11), utilizando (7.13), resulta:

$$
\mathbf{Q}=\boldsymbol{\tau}+\mathbf{F}_{\text {fric }}^{\mathrm{T}} \cdot \mathbf{A}=\boldsymbol{\tau}+\mathbf{A}^{\mathrm{T}} \cdot \mathbf{F}_{\text {fric }}
$$

donde $\boldsymbol{\tau}$ es el vector de pares agrupados y $\mathbf{F}_{\text {fric }}$ las fuerzas de fricción agrupadas.

Es interesante apuntar que el ultimo término de (7.16) también estaría presente en (7.9) si se considera no-deslizamiento debido a las restricciones dadas por (4.1), siendo en ese caso las fuerzas de fricción $\mathbf{F}_{\text {fric } i}$ igual a menos los multiplicadores de Lagrange $\lambda$ [Routh 1905].

La energía cinética del vehículo es:

$$
\begin{aligned}
T & =\frac{1}{2} \cdot\left(\mathrm{M}_{\mathrm{T}} \cdot\left({ }^{\mathrm{G}} v_{\mathrm{CMx}}^{2}+{ }^{\mathrm{G}} v_{\mathrm{CM} y}^{2}\right)+\mathrm{I}_{\mathrm{T}} \cdot \omega^{2}+\sum_{i=1}^{\mathrm{N}} \mathrm{I}_{\mathrm{ri}} \cdot \dot{\varphi}_{i}^{2}+\sum_{i=1}^{\mathrm{Ns}} \mathrm{I}_{\mathrm{rri}} \cdot \dot{\varphi}_{r i}^{2}\right) \\
& +\frac{1}{2} \cdot \sum_{i=1}^{\mathrm{Nc}}\left(\mathrm{M}_{\mathrm{ci}} \cdot\left({ }^{\mathrm{G}} v_{\mathrm{cmix}}^{2}+{ }^{\mathrm{G}} v_{\mathrm{cmiy}}^{2}\right)+\mathrm{I}_{\mathrm{csi}} \cdot\left(\dot{\beta}_{\mathrm{ci}}+\omega\right)^{2}\right) \\
& +\frac{1}{2} \cdot \sum_{i=1}^{\mathrm{No}}\left(\mathrm{I}_{\mathrm{osi}} \cdot\left(\dot{\beta}_{o i}^{2}+2 \cdot \dot{\beta}_{o i} \cdot \omega\right)\right)
\end{aligned}
$$

con el significado de variables y parámetros de la Tabla 7.2.

Tabla 7.2- Nuevas variables y parámetros en (7.17)

\begin{tabular}{|c|l|}
\hline Símbolo & \multicolumn{1}{|c|}{ Descripción } \\
\hline $\mathrm{N}_{\mathrm{s}}$ & Número de ruedas suecas en el vehículo \\
\hline $\mathrm{M}_{\mathrm{T}}$ & Masa del vehículo* (vehículo sin ruedas castor) \\
\hline $\mathrm{M}_{\mathrm{c} i}$ & Masa de la rueda castor $i$ \\
\hline $\mathrm{I}_{\mathrm{T}}$ & $\begin{array}{l}\text { Momento de inercia del vehículo* respecto al eje } Z \text { que cruza } \\
\text { su centro de masas (CM) }\end{array}$ \\
\hline $\mathrm{I}_{\mathrm{ri}}$ & Momento de inercia de la rueda $i$ respecto a su eje de rotación \\
\hline $\mathrm{I}_{\mathrm{rr} i}$ & Momento de inercia del rodillo de la rueda sueca $i$ \\
\hline $\mathrm{I}_{\mathrm{osi}} / \mathrm{I}_{\mathrm{cs} i}$ & $\begin{array}{l}\text { Momento de inercia de toda (brazo de dirección incluido) la } \\
\text { rueda orientable/ castor } i \text { con respecto al eje } Z \text { que cruza su CM }\end{array}$ \\
\hline${ }^{\mathrm{G}} \mathbf{v}_{\mathrm{CM}}$ & $\begin{array}{l}\text { Velocidad del centro de masas del vehículo* respecto al sistema } \\
\text { de coordenadas global G }\end{array}$ \\
\hline${ }^{\mathrm{G}} \mathbf{v}_{\mathrm{cm} i}$ & Velocidad del centro de masas de la rueda castor $i$ \\
\hline
\end{tabular}

Notar que (7.17) tiene en cuenta que el centro de masas (CM) de todas las ruedas centradas (fijas, orientables y castor) no cambia respecto a la estructura del vehículo. 
Aunque no se considera movimiento en el eje $Z$ en (7.17), la aceleración lineal del vehículo ${ }^{\mathrm{G}} \dot{\mathbf{v}}_{\mathrm{CM}}$ y los pares de reacción en la estructura debidos a $\tau_{\mathrm{ri}}$ redistribuyen las fuerzas normales en las ruedas.

Por conveniencia, el origen del sistema de coordenadas $\mathrm{R}$ de la estructura del vehículo se sitúa en el CM del vehículo", de modo que ${ }^{\mathrm{G}} \mathbf{v}_{\mathrm{CM}}={ }^{\mathrm{G}} \mathbf{v}_{\mathrm{R}}$.

Es fácil obtener de la Figura 3.4 la siguiente relación:

$$
{ }^{\mathrm{G}} \mathbf{d}_{\mathrm{cm} i}={ }^{\mathrm{G}} \mathbf{d}_{\mathrm{R}}+\left(\begin{array}{l}
1_{i} \cos \left({ }^{\mathrm{G}} \theta_{\mathrm{R}}+\alpha_{i}\right)-\mathrm{d}_{\mathrm{cm} i} \sin \left({ }^{\mathrm{G}} \theta_{\mathrm{R}}+\beta_{i}\right) \\
1_{i} \sin \left({ }^{\mathrm{G}} \theta_{\mathrm{R}}+\alpha_{i}\right)+\mathrm{d}_{\mathrm{cm} i} \cos \left({ }^{\mathrm{G}} \theta_{\mathrm{R}}+\beta_{i}\right)
\end{array}\right)
$$

donde $\mathrm{d}_{\mathrm{cm} i}$ es la distancia del eje $\mathrm{S}_{z i}$ al CM de la rueda castor $i$.

La primera y segunda derivada respecto al tiempo de ${ }^{\mathrm{G}} \mathbf{d}_{\mathrm{mci}}$ son:

$$
\begin{gathered}
{ }^{\mathrm{G}} \mathbf{v}_{\mathrm{cm} i}={ }^{\mathrm{G}} \mathbf{v}_{\mathrm{R}}+\mathbf{f}_{1 i} \cdot \boldsymbol{\omega}+\mathbf{f}_{2 i} \cdot \dot{\beta}_{i} \\
{ }^{\mathrm{G}} \dot{\mathbf{v}}_{\mathrm{cm} i}={ }^{\mathrm{G}} \dot{\mathbf{v}}_{\mathrm{R}}+\mathbf{f}_{3 i} \cdot \dot{\omega}+\mathbf{f}_{4 i} \cdot \ddot{\boldsymbol{\beta}}_{i}+\mathbf{f}_{5 i} \cdot \omega^{2}+\mathbf{f}_{6 i} \cdot \dot{\boldsymbol{\beta}}_{i}^{2}+\mathbf{f}_{7 i} \cdot 2 \cdot \omega \cdot \dot{\boldsymbol{\beta}}_{i}
\end{gathered}
$$

donde $\mathbf{f}_{j i}$ es función de $\mathrm{l}_{i}, \mathrm{~d}_{\mathrm{cmi}}, \alpha_{i},{ }^{\mathrm{G}} \theta_{\mathrm{R}}, \mathrm{y} \beta_{i}$.

La dinámica del vehículo (7.9), teniendo en cuenta \{(3.6), (7.11), (7.12), (7.13), (7.15), (7.16), (7.17), (7.19)\}, resulta:

$$
\begin{aligned}
& \left(\mathrm{M}_{\mathrm{T}}+\sum_{i=1}^{\mathrm{Nc}} \mathrm{M}_{\mathrm{c} i}\right) \cdot{ }^{\mathrm{G}} \dot{\mathbf{v}}_{\mathrm{R}}=\boldsymbol{\operatorname { R o t }}\left({ }^{\mathrm{G}} \boldsymbol{\theta}_{\mathrm{R}}\right) \cdot \sum_{i=1}^{\mathrm{N}} \operatorname{Rot}\left({ }^{\mathrm{R}} \boldsymbol{\theta}_{\mathrm{E} i}\right) \cdot \mathbf{F}_{\text {fric } i} \\
& \left(\mathrm{I}_{\mathrm{T}}+\sum_{i=1}^{\mathrm{Nc}} \mathrm{I}_{\mathrm{csi}}\right) \cdot \dot{\omega}+\sum_{i=1}^{\mathrm{Nc}} \mathrm{M}_{\mathrm{ci}} \cdot \mathbf{f}_{1 i}^{\mathrm{T}} \cdot{ }^{\mathrm{G}} \dot{\mathbf{v}}_{\mathrm{cm} i}+\sum_{i=1}^{\mathrm{Nc}} \mathrm{I}_{\mathrm{csi}} \cdot \ddot{\beta}_{\mathrm{c} i} \\
& +\sum_{i=1}^{\mathrm{No}} \mathrm{I}_{\mathrm{osi}} \cdot \ddot{\beta}_{\mathrm{o} i}=\sum_{i=1}^{\mathrm{N}}\left({ }^{\mathrm{R}} \mathbf{d}_{\mathrm{E} i} \times\left(\begin{array}{c}
\boldsymbol{\operatorname { R o t }}\left({ }^{\mathrm{R}} \theta_{\mathrm{E} i}\right) \cdot \mathbf{F}_{\text {fric } i} \\
0
\end{array}\right)\right) \cdot\left(\begin{array}{l}
0 \\
0 \\
1
\end{array}\right) \\
& \mathrm{I}_{\mathrm{csj}} \cdot\left(\dot{\omega}+\ddot{\beta}_{\mathrm{c} j}\right)+\mathrm{M}_{\mathrm{cj}} \cdot \mathbf{f}_{2 j}^{\mathrm{T}} \cdot{ }^{\mathrm{G}} \dot{\mathbf{v}}_{\mathrm{cmj}}=\tau_{\mathrm{csj}}-\left({ }^{\mathrm{E} j} \mathbf{d}_{\mathrm{s} j} \times\left(\begin{array}{c}
\mathbf{F}_{\text {fric } j} \\
0
\end{array}\right)\right) \cdot\left(\begin{array}{l}
0 \\
0 \\
1
\end{array}\right) \\
& \mathrm{I}_{\mathrm{osj}} \cdot\left(\ddot{\beta}_{\mathrm{oj}}+\dot{\omega}\right)=\tau_{\mathrm{os} j} \\
& \mathrm{I}_{\mathrm{rj}} \cdot \ddot{\varphi}_{j}=\tau_{\mathrm{r} j}+\mathrm{r}_{j} \cdot\left(\begin{array}{ll}
\mathrm{s} \gamma_{j} & \mathrm{c} \gamma_{j}
\end{array}\right) \cdot \mathbf{F}_{\text {fric } j}
\end{aligned}
$$




$$
\mathrm{I}_{\mathrm{rr} j} \cdot \ddot{\varphi}_{\mathrm{rj}}=\mathrm{r}_{\mathrm{r} j} \cdot\left(\begin{array}{ll}
0 & 1
\end{array}\right) \cdot \mathbf{F}_{\text {fric } j}
$$

con $j$ de 1 a $\mathrm{N}_{\mathrm{c}}$ (7.23), a $\mathrm{N}_{\mathrm{o}}$ (7.24), a $\mathrm{N}(7.25)$ ó a $\mathrm{N}_{\mathrm{s}}$ (7.26). 


\subsection{MODELOS DE TRACCIÓN (FUERZAS DE FRICCIÓN EN LAS RUEDAS)}

La componente $a$, tanto lateral $x$ como longitudinal $y$, de la fuerza de tracción en la rueda es:

$$
F_{\text {fric } a}=-\mu_{a} \cdot F_{\mathrm{N}}
$$

donde $F_{\mathrm{N}}$ es la fuerza normal o reacción en el punto de contacto entre la rueda y el suelo y $\mu_{a}$ el coeficiente de fricción o adhesión.

El coeficiente de adhesión es una función de la dinámica de la rueda y condiciones de tracción. Depende en cantidades tales como la velocidad de deslizamiento en la rueda, la rugosidad de la superficie, el material de la rueda, ...

En general para la fricción hay cuatro fenómenos estáticos y tres dinámicos:

+ Fenómenos estáticos (ilustrados en la Figura 7.1)

- Fricción estática

- Fricción de Coulomb ó fricción dinámica

- Fricción viscosa

- Efecto Stribeck

+ Fenómenos dinámicos

- Efecto Dahl

- Elevación de la fricción estática

- Retraso en la fricción

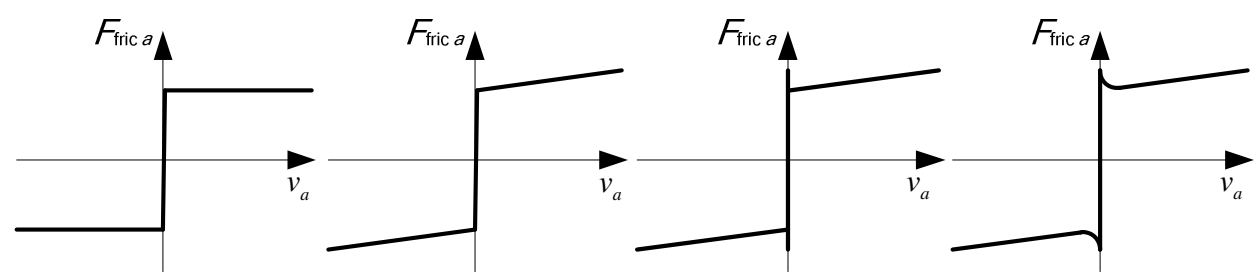
(a) Coulomb
(b) Coulomb + viscosa
(c) Ídem b. + estática
(d) todos

Figura 7.1- Fenómenos estáticos en la fricción

Los modelos estáticos de fricción, también denominados modelos de estadofijo (steady-state), tienen una dependencia lineal de la velocidad de deslizamiento a través de la llamada curva Stribeck [Stribeck 1902]. 
Mientras que los modelos dinámicos de fricción incorporan un comportamiento tipo muelle para pequeñas fuerzas. Ejemplos de modelos dinámicos son:

$\rightarrow$ Dahl [Dahl 68] [Dahl 75] [Dahl 76];

$\rightarrow$ Bristel and Reset Integrator [Haessig et al. 91];

$\rightarrow$ Bliman-Sorine [Bliman et al. 91] [Bliman et al. 93] [Bliman et al. 95];

$\rightarrow$ LuGre [Canudas et al. 95].

Por ejemplo, el modelo Dahl sólo considera la fricción de Coulomb dentro de los fenómenos estáticos y el desplazamiento pre-deslizamiento (efecto Dahl) dentro de los dinámicos.

Así mismo el modelo Bliman-Sorine puede verse como una conexión en paralelo de dos modelos Dahl de primer orden e incluye tres fenómenos estáticos (fricción estática, de Coulomb y viscosa) de la fricción y uno dinámico (efecto Dahl).

También, el modelo LuGre puede verse como un modelo Dahl de primer orden con un coeficiente de velocidad variable e incluye los cuatro fenómenos estáticos de la fricción y los tres dinámicos.

Tanto los modelos estáticos como dinámicos pueden asumir la rueda con fuerza de fricción puntual ó distribuida (ver Figura 7.2). Normalmente los modelos distribuidos se suelen aproximar a modelos puntuales realizando un promedio que depende de la distribución considerada para la fuerza de reacción normal en la rueda.
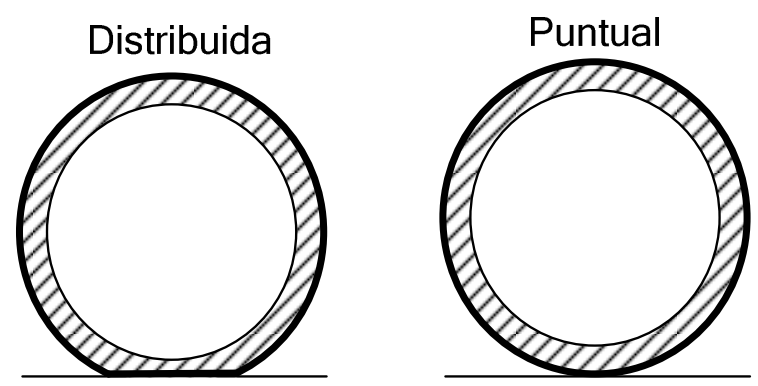

Figura 7.2- Rueda con fricción distribuida o puntual 
Debido al marco de movimiento quasi-estático del siguiente punto, en este estudio sólo se van a considerar modelos de fricción estáticos.

En [Alexander et al. 89] sólo se utiliza la Ley de Coulomb para modelar la fricción (fricción de Coulomb) y se asume el mismo coeficiente de adhesión en todas las direcciones, como se ha comentado en la introducción del capítulo.

No obstante, puesto que la fricción de Coulomb produce problemas algorítmicos en la simulación y diseño del control, se han propuesto algunas modificaciones. Por ejemplo [Karnopp 85] utiliza una zona muerta para un intervalo pequeño de velocidades, mientras que [Williams et al. 02] utiliza la siguiente función artificial tanto para el coeficiente de adhesión lateral como longitudinal:

$$
\mu_{a}=\mu_{a \max } \cdot(2 / \pi) \cdot \arctan \left(\mathrm{k}_{1} \cdot v_{\text {slip }}\right)
$$

donde $\mu_{a \max }$ es el coeficiente de fricción de Coulomb y $\mathrm{k}_{1}$ es una constante que establece la brusquedad del cambio entre $\pm \mu_{a \max }$ (ver Figura 7.2).

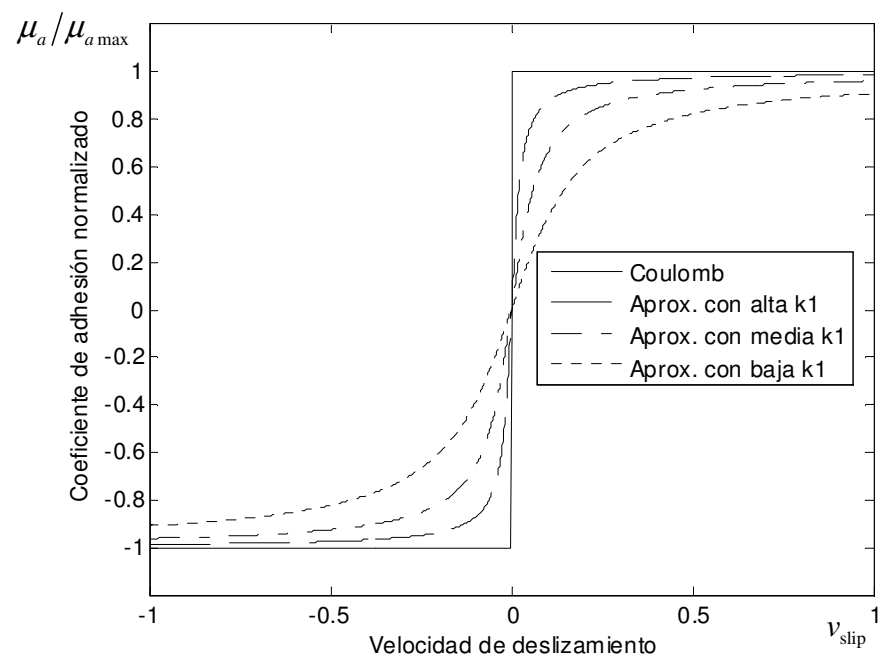

Figura 7.3- Coeficiente de adhesión para fricción de Coulomb y aproximada con (7.28)

Sin embargo, el tipo de planteamiento de [Alexander et al. 89] es inconsistente debido a la falta de acoplamiento entre los coeficientes de adhesión lateral y longitudinal. De hecho, en general produce una fuerza de fricción $\mathbf{F}_{\text {fric }}$ 
con una dirección y magnitud equivocada, además de la falta de unicidad de la fricción de Coulomb, es decir diferentes velocidades de deslizamiento producen la misma fuerza de fricción.

Para superar esta inconsistencia, se necesitan propiedades de acoplamiento obtenidas de las características de anisotropía de la fricción: conjunto de parámetros admisibles, maximización local del ratio de disipación, etc.

Por ejemplo, los coeficientes admisibles para el caso bidimensional de Coulomb vienen dados por (7.29) donde $\mu_{x 0} / \mu_{y 0}$ es el coeficiente de fricción lateral/longitudinal en un deslizamiento puramente lateral/longitudinal.

$$
\left(\mu_{x} / \mu_{x 0}\right)^{2}+\left(\mu_{y} / \mu_{y 0}\right)^{2} \leq 1
$$

El máximo ratio de disipación se obtiene para [Velenis et al. 05]:

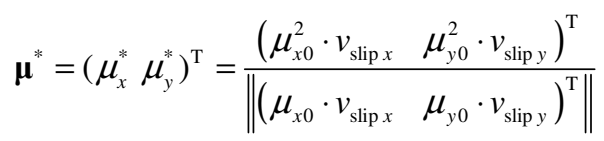

que está en el límite de la elipse de fricción dada por (7.29) (ver Figura 7.3).

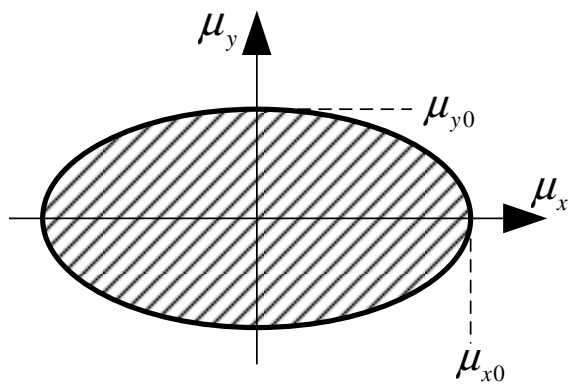

Figura 7.4- Coeficientes admisibles para el caso bidimensional de Coulomb

Muchos modelos se han desarrollado en la literatura para relacionar el coeficiente de fricción longitudinal de la rueda $\mu_{y}$ y el ratio de deslizamiento longitudinal $s$ (7.31) para deslizamiento puramente longitudinal.

$$
s=\frac{\left(\dot{\varphi} \cdot \mathrm{r}-v_{\text {wheel } y}\right)}{\max \left(|\dot{\varphi} \cdot \mathrm{r}|,\left|v_{\text {wheel } y}\right|\right)}=\frac{v_{\text {slip }}}{\max \left(|\dot{\varphi} \cdot \mathrm{r}|,\left|v_{\text {wheel } y}\right|\right)}
$$

donde $v_{\text {wheel } y}$ es la velocidad longitudinal del centro de la rueda. 
Por ejemplo, [Dugoff et al. 70] propuso la curva mostrada en la Figura 7.5 y verificó su ajuste cualitativo para diferentes superficies y velocidades de rueda $v_{\text {wheel } y}$. Este modelo ha sido ampliamente utilizado para análisis de estabilidad en la dinámica de vehículos [Matsumoto et al. 92] y para el control de tracción [Tan et al. 91]. En concreto, el método estándar de diseño de sistemas ABS consiste en una ley de control que fuerce el funcionamiento en (o cerca) el máximo del coeficiente de fricción $\mu_{y \max }$.

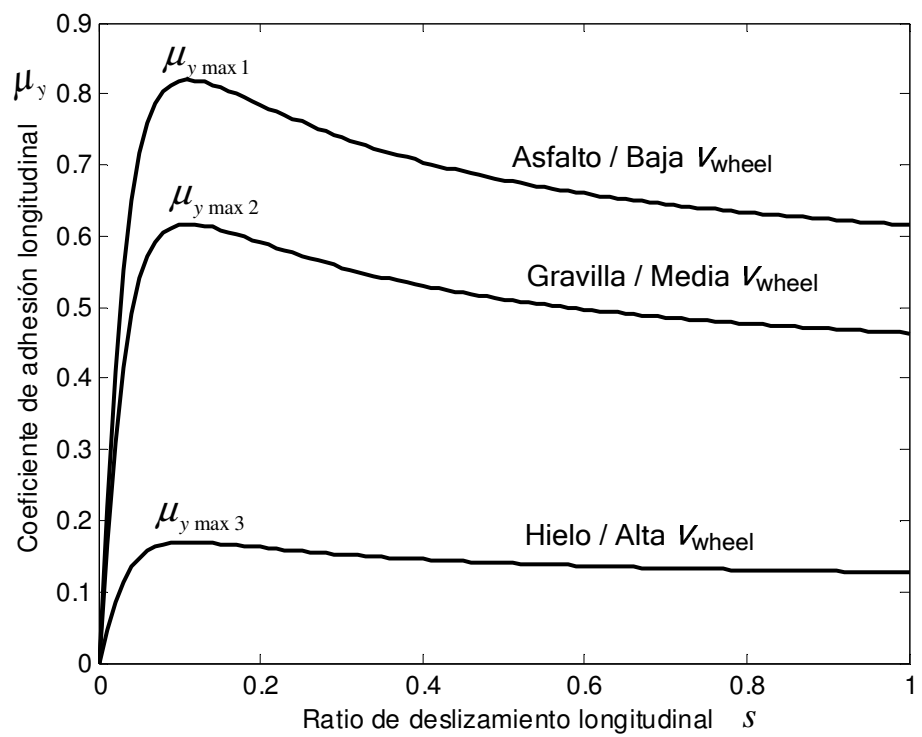

Figura 7.5- Curva propuesta en [Dugoff et al. 70] para el coeficiente de fricción en distintas condiciones de velocidad y adherencia

En [Bekker 60] [Bekker 62] se desarrollan complejos modelos basados en curvas con formas de sinusoides modificadas similares a la Figura 7.5. Ejemplos de la relación analítica $\mu_{y}-s$ son (7.32) [Okello et al. 94], (7.33) [Kiencke et al. 94], y (7.34) [Pacejka et al. 93], donde $k_{i}$ son parámetros de los modelos.

$$
\begin{gathered}
\mu_{y}=k_{2} \cdot\left(1-\left(k_{3} / s\right) \cdot\left(1-e^{-s / k_{3}}\right)\right) \\
\mu_{y}=k_{4} \cdot s /\left(k_{5} \cdot s^{2}+k_{6} \cdot s+1\right) \\
\mu_{y}=k_{7} \cdot \sin \left(k_{8} \cdot \arctan \left(k_{9} \cdot\left(1-k_{10}\right) \cdot s+k_{10} \cdot \arctan \left(k_{9} \cdot s\right)\right)\right)
\end{gathered}
$$

Estos modelos se utilizan también para relacionar (Figura 7.6) el coeficiente 
lateral de adhesión de la rueda $\mu_{x}$ y el ángulo de deslizamiento $\alpha_{s}$ (7.35) para deslizamiento puramente lateral.

$$
\alpha_{\mathrm{s}}=\operatorname{atan}\left(v_{\text {slip }} / v_{\text {wheel } y}\right)
$$

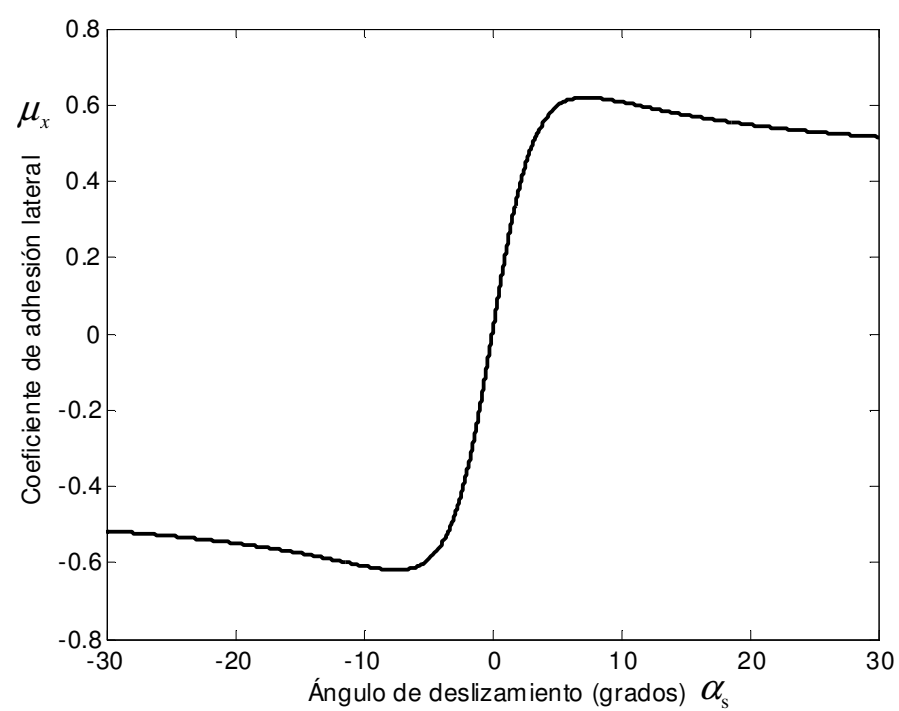

Figura 7.6- Curva estática para el coeficiente de fricción lateral vs. ángulo de deslizamiento

Notar que estos modelos tienen un comportamiento aproximado de línea recta (Figura 7.5 / Figura 7.6) para pequeño ratio / ángulo de deslizamiento.

Esta línea recta aproximada se caracteriza por la pendiente, comúnmente referida como rigidez o pendiente de deslizamiento. Por ejemplo, un neumático rígido da lugar a una gran pendiente de deslizamiento.

Como antes, se deben considerar las propiedades de acoplamiento de las componentes de la fricción para un deslizamiento combinado (longitudinal y lateral), obteniéndose un modelo más elaborado del de deslizamiento longitudinal/lateral puro [Pacejka et al. 97], [Velenis et al. 05], [Pacejka 02].

Todos los modelos de tracción anteriores, que relacionan fuerzas de tracción (fricciones) con las velocidades de deslizamiento, representan un trabajo disipativo. Esta pérdida de energía se justifica, para deslizamiento insignificante, por la deformación de la rueda (rodamiento no ideal). 


\subsection{MODELOS CON DESLIZAMIENTO}

\subsubsection{Modelo del movimiento quasi-estático}

Para explicar el planteamiento de este punto, considérese el modelo dinámico de segundo orden de (7.36) con $\mathbf{x}$ las incógnitas (posiciones) y $\mathbf{u}$ el vector de entradas (fuerzas/pares).

$$
\mathbf{f}(\ddot{\mathbf{x}}, \dot{\mathbf{x}}, \mathbf{x}, \mathbf{u})=0 \rightarrow \ddot{\mathbf{x}}
$$

La expresión anterior se utiliza de forma instantánea para calcular $\ddot{\mathbf{x}}$, y puede ser utilizada también para calcular el valor estacionario (si lo hubiere) $\dot{\mathbf{x}}_{\infty}$ de la situación quasi-estática (aceleraciones nulas):

$$
\mathbf{f}\left(\mathbf{0}, \dot{\mathbf{x}}_{\infty}, \mathbf{x}, \mathbf{u}\right)=0 \rightarrow \dot{\mathbf{x}}_{\infty}
$$

La situación (7.36) se corresponde con el modelo dinámico del punto 7.2 (junto con el punto 7.3), donde las variables calculadas son $\ddot{\mathbf{q}}$ y las entradas $\boldsymbol{\tau}$.

Mientras que la situación (7.37) se corresponde a un modelo quasi-estático con las variables calculadas $\dot{\mathbf{q}}_{\infty}$ y las entradas $\boldsymbol{\tau}$.

Esta segunda situación es la planteada en este punto.

Si se define el movimiento quasi-estático del vehículo como:

$$
{ }^{\mathrm{G}} \ddot{\mathbf{q}}=0 \rightarrow{ }^{\mathrm{G}} \dot{\mathrm{v}}_{\mathrm{R} x}={ }^{\mathrm{G}} \dot{v}_{\mathrm{R} y}=\dot{\omega}=\ddot{\beta}_{\mathrm{c} j}=\ddot{\beta}_{\mathrm{oj}}=\ddot{\varphi}_{j}=\ddot{\varphi}_{\mathrm{r} j}=0
$$

implica que el vehículo* tiene un movimiento rectilíneo uniforme ${ }^{\mathrm{G}} \dot{\mathbf{v}}_{\mathrm{R}}=\mathbf{0}$ con una velocidad angular constante $\dot{\omega}=0$.

Sin embargo, debido a que la orientación del vehículo* ${ }^{\mathrm{G}} \boldsymbol{\theta}_{\mathrm{R}}$ varía respecto al sistema $\mathrm{G}$, este movimiento no es posible ya que en general no hay un valor estacionario $\dot{\mathbf{x}}_{\infty}$ para $\left({ }^{\mathrm{G}} \mathbf{v}_{\mathrm{R}}, \omega, \dot{\varphi}_{j}, \ldots\right)$.

Por ejemplo, el vehículo basado en dos ruedas fijas alineadas (vehículo diferencial) no puede desarrollar un movimiento rectilíneo a velocidad angular constante. 
Por tanto, debe considerarse el movimiento quasi-estático del vehículo con respecto al sistema de coordinas coincidente $\overline{\mathrm{R}}$ :

$$
{ }^{\overline{\mathrm{R}}} \ddot{\mathbf{q}}=0 \rightarrow{ }^{\overline{\mathrm{R}}} \dot{v}_{\mathrm{R} x}={ }^{\overline{\mathrm{R}}} \dot{\mathrm{R}}_{\mathrm{R} y}=\dot{\omega}=\ddot{\beta}_{\mathrm{c} j}=\ddot{\beta}_{\mathrm{oj}}=\ddot{\varphi}_{j}=\ddot{\varphi}_{\mathrm{r} j}=0
$$

lo que implica que el vehículo* tiene un movimiento curvilíneo uniforme, caracterizado por ${ }^{\overline{\mathrm{R}}} \mathbf{v}_{\mathrm{R}}$ and $\omega$, con una velocidad angular constante $\omega$.

Para este movimiento la relación (7.40), obtenida de (7.14), se tiene que sustituir en (7.20) y (7.21).

$$
{ }^{\mathrm{G}} \dot{\mathbf{v}}_{\mathrm{R}}=\boldsymbol{\operatorname { R o t }}\left({ }^{\mathrm{G}} \boldsymbol{\theta}_{\mathrm{R}}\right) \cdot{ }^{\overline{\mathrm{R}}} \dot{\mathbf{v}}_{\mathrm{R}}+\omega \cdot\left(\partial \boldsymbol{\operatorname { R o t }}\left({ }^{\mathrm{G}} \boldsymbol{\theta}_{\mathrm{R}}\right) / \partial{ }^{\mathrm{G}} \boldsymbol{\theta}_{\mathrm{R}}\right) \cdot{ }^{\overline{\mathrm{R}}} \mathbf{v}_{\mathrm{R}}
$$

Bajo las condiciones de (7.39) todos los miembros de la izquierda de las ecuaciones dinámicas (7.21)-(7.26) son cero excepto por el término centrípeto $\left(\omega \cdot{ }^{\overline{\mathrm{R}}} \mathbf{v}_{\mathrm{R}}\right)$ de ${ }^{\mathrm{G}} \dot{\mathbf{v}}_{\mathrm{R}}$ en $(7.40)$ y los términos centrifugo $\left(\omega^{2}, \dot{\beta}_{i}^{2}\right)$ y Coriolis $\left(\omega \cdot \dot{\beta}_{i}\right)$ de ${ }^{\mathrm{G}} \dot{\mathbf{v}}_{\mathrm{m} c i}$ en (7.20).

Si los términos no nulos de las ruedas castor debidos a (7.20) se desprecian en (7.22) y (7.23), ej. la masa de las ruedas castor $\mathrm{M}_{c i}$ es muy pequeña, las ecuaciones dinámicas se convierten en las siguientes ecuaciones del movimiento quasi-estático:

$$
\begin{aligned}
& \left(\mathrm{M}_{\mathrm{T}}+\sum_{i=1}^{\mathrm{Nc}} \mathrm{M}_{\mathrm{c} i}\right) \cdot \omega \cdot\left(\begin{array}{cc}
0 & -1 \\
1 & 0
\end{array}\right) \cdot{ }^{\overline{\mathrm{R}}} \mathbf{v}_{\mathrm{R}}=\sum_{i=1}^{\mathrm{N}} \boldsymbol{\operatorname { R o t }}\left({ }^{\mathrm{R}} \theta_{\mathrm{E} i}\right) \cdot \mathbf{F}_{\text {fric } i} \\
& 0=\sum_{i=1}^{\mathrm{N}}\left({ }^{\mathrm{R}} \mathbf{d}_{\mathrm{E} i} \times\left(\begin{array}{c}
\boldsymbol{\operatorname { R o t }}\left({ }^{\mathrm{R}} \boldsymbol{\theta}_{\mathrm{E} i}\right) \cdot \mathbf{F}_{\text {fric } i} \\
0
\end{array}\right) \cdot\left(\begin{array}{lll}
0 & 0 & 1
\end{array}\right)^{\mathrm{T}}\right. \\
& 0=\tau_{\mathrm{csj}}-\left({ }^{\mathrm{Ej} j} \mathbf{d}_{\mathrm{S} j} \times\left(\begin{array}{c}
\mathbf{F}_{\text {fric } j} \\
0
\end{array}\right)\right) \cdot\left(\begin{array}{lll}
0 & 0 & 1
\end{array}\right)^{\mathrm{T}} \\
& 0=\tau_{\text {os } j} \\
& 0=\tau_{\mathrm{r} j}+\mathrm{r}_{j} \cdot\left(\begin{array}{ll}
\mathrm{s} \gamma_{j} & \mathrm{c} \gamma_{j}
\end{array}\right) \cdot \mathbf{F}_{\mathrm{fric} j} \\
& 0=\mathrm{r}_{\mathrm{r} j} \cdot\left(\begin{array}{ll}
0 & 1
\end{array}\right) \cdot \mathbf{F}_{\text {fric } j}
\end{aligned}
$$

con $j$ de 1 a $\mathrm{N}_{\mathrm{c}}(7.43)$, a $\mathrm{N}_{\mathrm{o}}(7.44)$, a $\mathrm{N}(7.45)$ ó a $\mathrm{N}_{\mathrm{s}}(7.46)$. 
El significado de cada ecuación del movimiento quasi-estático es: (7.41) la fuerza resultante producida por las fuerzas de fricción en el vehículo es igual a la fuerza centrípeta necesaria para producir el movimiento curvilíneo uniforme; (7.42) equilibrio de momentos en el vehículo; (7.43) el par de direccionamiento sobre la rueda castor $j$ es igual y contrario al momento de su fuerza de fricción respecto al eje $S_{z j}$; (7.44) par de direccionamiento nulo sobre la rueda orientable $j$; (7.45) el par de rotación sobre la rueda $j$ es igual y contrario al momento de su fuerza de fricción respecto al eje $\mathrm{L}_{x j}$; (7.46) fuerza de fricción nula sobre la rueda sueca $j$ en la dirección longitudinal del rodillo.

Para la rueda castor $j$ sin par de direccionamiento (direccionamiento libre) (7.43) resulta (7.47), con el significado de que su fuerza de fricción es cero en la dirección perpendicular al brazo de dirección.

$$
\left|\left(\begin{array}{lll}
\left({ }^{\mathrm{E} j} d_{\mathrm{S} j x}\right. & { }^{\mathrm{E} j} d_{\mathrm{S} j y} & 0
\end{array}\right)^{\mathrm{T}} \times\left(\mathbf{F}_{\text {fricj }}^{\mathrm{T}} \quad 0\right)^{\mathrm{T}}\right|=0
$$

De igual forma, para una rueda $j$ sin par de rotación (rotación libre) (7.45) resulta (7.48), con el significado de que no hay fuerza de fricción en la dirección definida por el plano de la rueda.

$$
\left(\begin{array}{ll}
\mathrm{s} \gamma_{j} & \mathrm{c} \gamma_{j}
\end{array}\right) \cdot \mathbf{F}_{\text {fric } j}=0
$$

Notar que, una rueda* castor/sueca completamente libre tiene fuerza de fricción nula (no-deslizamiento) en el modelo quasi-estático, debido a las ecuaciones (7.47)/(7.46) y (7.48). (*Asumiendo que no es singular: $\delta_{i} \neq 90^{\circ} / \gamma_{i} \neq 0^{\circ}$, ver Capítulo 6.)

\subsubsection{Modelo cinemático con deslizamiento}

En el modelo de movimiento quasi-estático previo las entradas al modelo $\mathbf{u}$ de (7.37) son los pares de rotación $\tau_{\text {raj }}$ de todas las ruedas de rotación no libre y los pares de dirección $\tau_{\text {csaj }}$ de todas las ruedas castor de dirección no libre.

Sin embargo, es posible directamente considerar las velocidades de rotación y direccionamiento $\left(\dot{\varphi}_{\text {raj }}, \dot{\beta}_{\text {caj }}\right)$ de esas ruedas no libres (asignadas) con las entradas $\mathbf{u}$ en (7.37), de modo que puede obviarse sus pares y ecuaciones asociadas, (7.43) y (7.45). 
Incluso es posible considerar las velocidades del vehículo como entradas (asignadas) en el modelo. Esto conduce a una solución inversa.

Una fuerza de fricción bidimensional genérica viene definida por:

$$
\mathbf{F}_{\text {fric } i}=-F_{\mathrm{N} i} \cdot \mathbf{f}_{\text {fric } i}\left(\mathbf{v}_{\text {slip } i}\right)
$$

donde $\mathbf{f}_{\text {fric } i}$ es una función bidimensional genérica que depende de la magnitud y dirección de la velocidad de deslizamiento.

Esta fuerza de fricción genérica se debe sustituir en (7.41), (7.42), (7.46), (7.47), y (7.48). Por ejemplo en (7.46) produce velocidad de deslizamiento nulo en la dirección longitudinal del rodillo.

Así pues, después de realizar esta sustitución múltiple, se tiene el modelo quasi-cinemático con deslizamiento dado por (7.41), (7.42), (7.46) (para todas las ruedas suecas), (7.47) (para todas las ruedas castor libres en dirección), y (7.48) (para todas las ruedas libres en rotación).

Debido a la fuerza centrípeta del miembro de la izquierda de (7.41), este modelo es quasi-cinemático con las velocidades asignadas como entradas.

Si se desprecia dicha fuerza centrípeta, lo que es posible para maniobras suaves (es decir cuando ${ }^{\bar{R}} \mathbf{v}_{\mathrm{R}}$ y $\omega$ no tienen valores grandes simultáneamente), el modelo resulta completamente cinemático: el modelo cinemático con deslizamiento.

Notar que cuando la fuerza centrípeta se desprecia en el movimiento curvilíneo uniforme (movimiento quasi-estático respecto a $\bar{R}$ ), éste es equivalente al movimiento rectilíneo uniforme (movimiento quasi-estático respecto a $\mathrm{G})$.

Por tanto, para el modelo cinemático con deslizamiento la reacción normal $F_{\mathrm{N} i}$ en las ruedas permanece constante, y el origen del sistema de coordenadas $\mathrm{R}$ se puede situar arbitrariamente (no necesariamente en el CM del vehículo*) puesto que hay equilibrio de fuerzas y momentos. 
Por otro lado, [Alexander et al. 89] define dos tipos de deslizamiento:

- Derrapaje (skidding): este tipo de deslizamiento está asociado con maniobras a alta velocidad y es debido a grandes fuerzas de inercia que saturan la fricción disponible.

- Resbalamiento ó Patinazo (scrubbing or slippage): este tipo de deslizamiento está producido por valores de entrada al modelo incompatibles con rodamiento ideal.

En el modelo dinámico (punto 7.2) solo el derrapaje está presente, ya que los pares en las ruedas (valores de entrada al modelo) no son incompatibles con el rodamiento ideal.

Por el contrario, en el modelo cinemático con deslizamiento previo (aceleraciones nulas) sólo el resbalamiento está presente, ya que las velocidades asignadas (valores de entrada al modelo) pueden ser incompatibles con rodamiento ideal. Si no lo son el modelo cinemático con deslizamiento indicará no resbalamiento.

Los valores estacionarios de las velocidades en el modelo cinemático con deslizamiento, ej. $\dot{\mathbf{x}}_{\infty}$ en (7.37), son más precisos desde el punto de vista del marco dinámico, para:

- Dinámica rápida: esto viene dado por valores de masas y momentos de inercia pequeños y grandes valores de coeficientes de adhesión.

- Entradas suaves: ya que cuando las entradas varían gradualmente la solución estacionaria está más próxima a la instantánea.

\subsubsection{Uso práctico del modelo cinemático con deslizamiento}

Básicamente hay tres opciones para utilizar el modelo cinemático con deslizamiento dependiendo de las velocidades asignadas:

\subsubsection{1) Las velocidades asignadas son velocidades de rueda sensorizadas}

En este caso el modelo es una solución directa utilizada para estimar el movimiento del vehículo con redundancia en la información de sensores. 
Asumiendo una actuación consistente en las ruedas tractoras, las velocidades de rueda sensorizadas no deben ser demasiado incompatibles con rodamiento ideal. Por tanto es posible considerar solo el comportamiento de línea recta (pendiente de deslizamiento) de la Figura 7.1 y Figura 7.2 para los coeficientes de adhesión de las fuerzas de fricción.

\subsubsection{2) Las velocidades asignadas son velocidades de rueda accionadas}

En este caso el modelo cinemático con deslizamiento es una solución directa utilizada para estimar el movimiento del vehículo con sobre-actuación independiente en las velocidades de rueda.

En general, los accionamientos independientes sobre las velocidades de rueda pueden producir mucha incompatibilidad con el rodamiento ideal. Así pues no es posible simplificar los coeficientes de adhesión a la pendiente de deslizamiento. Un sencillo ejemplo de este caso es un vehículo como movimiento lineal monodimensional con dos ruedas fijas accionadas de forma independiente.

\subsubsection{2) Las velocidades asignadas son velocidades del vehículo}

En este caso el modelo es una solución inversa utilizada para calcular las velocidades de rueda que producen un movimiento particular del vehículo.

Si la fuerza de fricción está bien modelada esta opción incrementa el grado de movilidad del vehículo. Por ejemplo, un vehículo triciclo (dos ruedas fijas alineadas y otra rueda orientable) podría tener una movilidad completa a través de la rotación de las tres ruedas, calculada a través de la solución inversa.

A diferencia de las soluciones directas, la solución inverse puede ser singular. En el ejemplo anterior, triciclo con movilidad completa, singularity la singularidad aparece cuando la rueda orientable es paralela a las ruedas fijas.

Las opciones 7.4.3.2) y 7.4.3.3) no son habituales en la práctica porque no se suele asumir el deslizamiento como herramienta de trabajo para incrementar la movilidad del vehículo, ... , ya que si fuera necesario se habría diseñado otro tipo de vehículo, con otro tipo de ruedas, características,...

Mientras que 7.4.3.1) es más habitual en la práctica. Por ejemplo, las ecuaciones cinemáticas con deslizamiento se podrían utiliza junto con el FKE para estimar la postura del vehículo [Tham et al. 98] [Scheding et al. 99]. 


\subsubsection{Solución de Mínimos Cuadrados ponderada del modelo cinemático}

Las restricciones cinemáticas de no deslizamiento del vehículo vienen dadas por (4.1). Aplicando una matriz de ponderación $\sqrt{\mu}$ a (4.1):

$$
\begin{aligned}
& \left(\begin{array}{cccc}
\sqrt{\boldsymbol{\mu}_{1}} & \mathbf{0} & \cdots & \mathbf{0} \\
\mathbf{0} & \sqrt{\boldsymbol{\mu}_{2}} & \cdots & \vdots \\
\vdots & \vdots & \ddots & \mathbf{0} \\
\mathbf{0} & \cdots & \mathbf{0} & \sqrt{\boldsymbol{\mu}_{\mathrm{N}}}
\end{array}\right) \cdot \mathbf{A} \cdot \dot{\mathbf{q}}=\sqrt{\boldsymbol{\mu}} \cdot \mathbf{A} \cdot \dot{\mathbf{q}}=\mathbf{0} \\
& \text { with } \sqrt{\boldsymbol{\mu}_{i}}=\left(\begin{array}{cc}
\sqrt{\mu_{x i}} & 0 \\
0 & \sqrt{\mu_{y i}}
\end{array}\right)
\end{aligned}
$$

Separando las velocidades asignadas y no asignadas:

$$
\sqrt{\boldsymbol{\mu}_{\mathrm{na}}} \cdot \mathbf{A}_{\mathrm{na}} \cdot \dot{\mathbf{q}}_{\mathrm{na}}=-\sqrt{\boldsymbol{\mu}_{\mathrm{a}}} \cdot \mathbf{A}_{\mathrm{a}} \cdot \dot{\mathbf{q}}_{\mathrm{a}}
$$

Calculando las velocidades no asignadas con la solución de Mínimos Cuadrados (Least Square LS):

$$
\dot{\mathbf{q}}_{\text {na }}=-\left(\left(\sqrt{\boldsymbol{\mu}_{\text {na }}} \cdot \mathbf{A}_{\text {na }}\right)^{\mathrm{T}} \cdot \sqrt{\boldsymbol{\mu}_{\text {na }}} \cdot \mathbf{A}_{\text {na }}\right)^{-1} \cdot\left(\sqrt{\boldsymbol{\mu}_{\text {na }}} \cdot \mathbf{A}_{\text {na }}\right)^{\mathrm{T}} \cdot \sqrt{\boldsymbol{\mu}_{\mathrm{a}}} \cdot \mathbf{A}_{\mathrm{a}} \cdot \dot{\mathbf{q}}_{\mathrm{a}}
$$

La singularidad de la solución de LS anterior está dada por $\mathbf{A}_{\text {na }}$ (Capítulo 6).

Por definición, el algoritmo de LS minimiza el índice $J_{\mathrm{LS}}$ de (7.53), suma del error cuadrático de las ecuaciones originales, respecto a las velocidades no asignadas.

$$
J_{\mathrm{LS}}=(\sqrt{\boldsymbol{\mu}} \cdot \mathbf{A} \cdot \dot{\mathbf{q}})^{\mathrm{T}} \cdot(\sqrt{\boldsymbol{\mu}} \cdot \mathbf{A} \cdot \dot{\mathbf{q}})=\dot{\mathbf{q}}^{\mathrm{T}} \cdot \mathbf{A}^{\mathrm{T}} \cdot \boldsymbol{\mu} \cdot \mathbf{A} \cdot \dot{\mathbf{q}}=\mathbf{v}_{\text {slip }}^{\mathrm{T}} \cdot \boldsymbol{\mu} \cdot \mathbf{v}_{\text {slip }}
$$

Las velocidades no asignadas de minimización pueden ser la velocidad lineal del vehículo ${ }^{\overline{\mathrm{R}}} \mathbf{v}_{\mathrm{R}}$, la velocidad angular del vehículo $\omega$, la velocidad de direccionamiento $\dot{\beta}_{\text {c } j}$ de la rueda castor $j$, la velocidad de rotación $\dot{\varphi}_{j}$ de la rueda $j$ y la velocidad de rotación del rodillo $\dot{\varphi}_{\mathrm{r} j}$ de la rueda sueca $j$.

La minimización de $J_{\mathrm{LS}}$ respecto a esas velocidades, teniendo en cuenta (3.6), (7.12) y (7.53), resulta: 


$$
\begin{aligned}
& \frac{\partial J_{\mathrm{LS}}}{\partial^{\overline{\mathrm{R}}} \mathbf{v}_{\mathrm{R}}}=\sum_{i=1}^{\mathrm{N}} \boldsymbol{\operatorname { R o t }}\left({ }^{\mathrm{R}} \boldsymbol{\theta}_{\mathrm{E} i}\right) \cdot \boldsymbol{\mu}_{i} \cdot \mathbf{v}_{\operatorname{sip} i}=0 \\
& \frac{\partial J_{\mathrm{LS}}}{\partial \omega}=\sum_{i=1}^{\mathrm{N}}\left({ }^{\mathrm{R}} \mathbf{d}_{\mathrm{E} i} \times\left(\begin{array}{c}
\boldsymbol{\operatorname { R o t }}\left({ }^{\mathrm{R}} \theta_{\mathrm{E} i}\right) \cdot \boldsymbol{\mu}_{i} \cdot \mathbf{v}_{\text {slip } i} \\
0
\end{array}\right)\right) \cdot\left(\begin{array}{l}
0 \\
0 \\
1
\end{array}\right)=0
\end{aligned}
$$

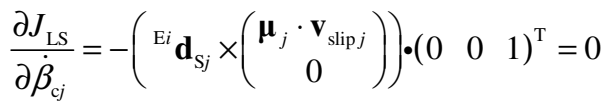

$$
\begin{aligned}
& \frac{\partial J_{\mathrm{LS}}}{\partial \dot{\varphi}_{j}}=\mathrm{r}_{j} \cdot\left(\begin{array}{ll}
\mathrm{s} \gamma_{j} & \mathrm{c} \gamma_{j}
\end{array}\right) \cdot \boldsymbol{\mu}_{j} \cdot \mathbf{v}_{\mathrm{sip} j}=0 \\
& \frac{\partial J_{\mathrm{LS}}}{\partial \dot{\varphi}_{\mathrm{r} j}}=\mathrm{r}_{\mathrm{r} j} \cdot\left(\begin{array}{ll}
0 & 1
\end{array}\right) \cdot \boldsymbol{\mu}_{j} \cdot \mathbf{v}_{\mathrm{slip} j}=0
\end{aligned}
$$

Para fuerzas de fricción (7.59) linealmente dependientes con las velocidades de deslizamiento, existen las siguientes equivalencias: $(7.55) \rightarrow(7.42)$, (7.56) $\rightarrow$ (7.47), (7.57) $\rightarrow$ (7.48), (7.58) $\rightarrow$ (7.46) y (7.54) $\rightarrow$ (7.41) si se desprecia la fuerza centrípeta en el miembro de la izquierda de (7.41).

$$
\mathbf{F}_{\text {fric } i}=-\left(\begin{array}{cc}
\mu_{x i} & 0 \\
0 & \mu_{y i}
\end{array}\right) \cdot\left(\begin{array}{l}
v_{\text {slip } x i} \\
v_{\text {slip } x i}
\end{array}\right)=-\boldsymbol{\mu}_{i} \cdot \mathbf{v}_{\text {slip } i}
$$

Notar que la fuerza de fricción previa (7.59) desprecia el acoplamiento entre los coeficientes longitudinal y lateral. Es posible acoplarlos de forma similar a (7.30), aunque produciría una solución no analítica para (7.52) ó (7.54)-(7.58).

Para un correcto significado físico la solución de En ese caso ponderada, las velocidades del vehículo deben ser consideradas no asignadas, de modo que el equilibrio de fuerzas de (7.54) y el equilibrio de momentos de (7.55) están siempre presentes. Las velocidades de rueda pueden ser asignadas o no.

Así pues, la solución de LS ponderada con las velocidades del vehículo como no asignadas es equivalente al modelo cinemático con deslizamiento para fuerzas de fricción linealmente dependientes con las velocidades de deslizamiento. 


\subsection{SIMULACIÓN Y RESULTADOS EXPERIMENTALES}

\subsubsection{Carretilla industrial (triciclo)}

Con objeto de probar los modelos con deslizamiento del punto anterior, se utilizará la carretilla industrial de la Figura 7.7. Este vehículo representa un vehículo próximo al mundo real.

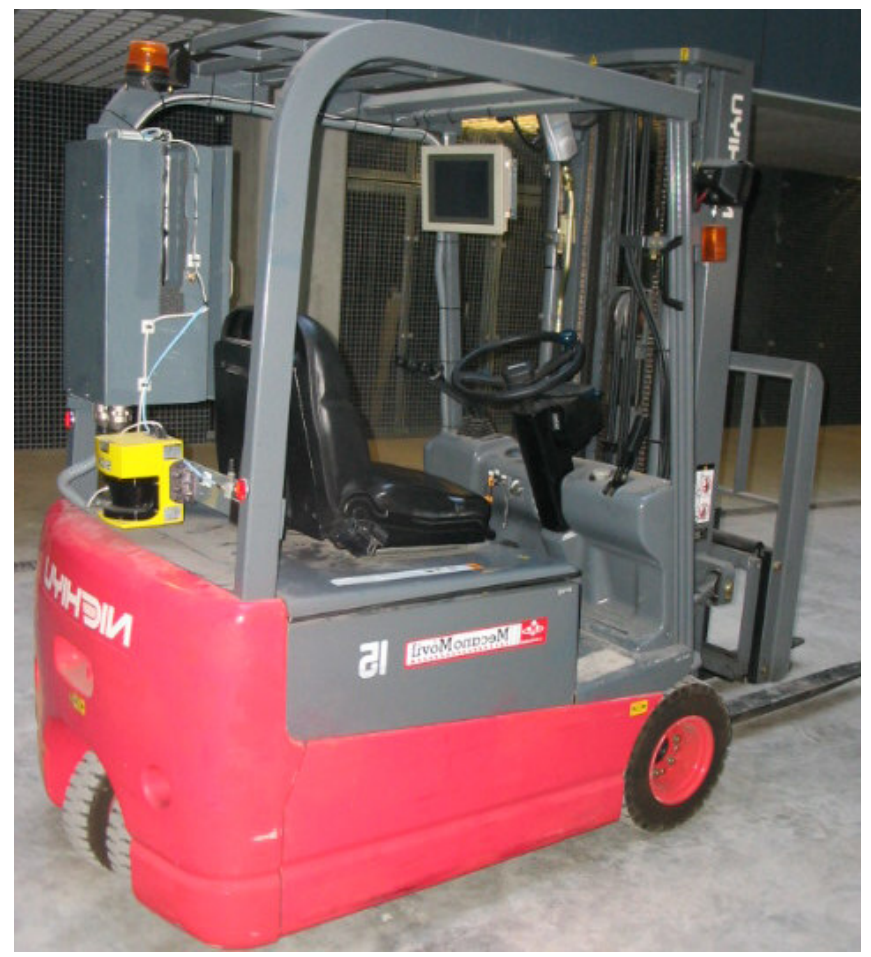

Figura 7.7- Carretilla industrial Nichiyu FBT15 serie 65

En la Figura 7.8 se muestra la representación equivalente del vehículo, con dos ruedas fijas alineadas y otra rueda orientable.

Los modelos con deslizamiento se utilizarán como una solución directa para estimar el movimiento del vehículo, con la información de sensores dada por tres encoders que miden la rotación de las tres ruedas fijas (encoders incrementales con 8290 pulsos por vuelta de la rueda) y la orientación de la rueda orientable (encoder absoluto de $1.41^{\circ}$ de resolución). 


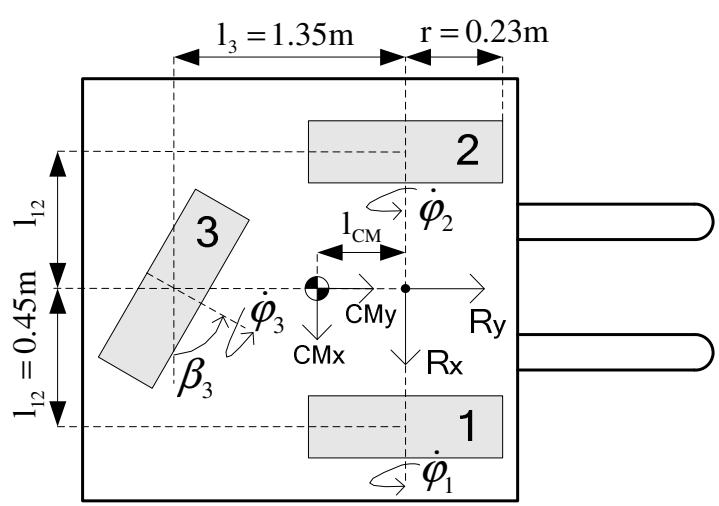

Figura 7.8- Vista de planta de la carretilla industrial con la representación esquemática tipo triciclo

Notar que el sistema de coordenadas $\mathrm{R}$ (ver Figura 7.8) no se ha situado en el CM del vehículo y que ${ }^{\mathrm{G}} \mathbf{v}_{\mathrm{CM}} \neq{ }^{\mathrm{G}} \mathbf{v}_{\mathrm{R}},{ }^{\overline{\mathrm{CM}}} \omega_{\mathrm{CM}}=\omega,{ }^{\mathrm{G}} \theta_{\mathrm{CM}}={ }^{\mathrm{G}} \theta_{\mathrm{R}}$.

De la definición gráfica de la Figura 7.8 y utilizando tres veces (3.11), la ecuación matricial cinemática con deslizamiento de (7.12) resulta:

$$
\mathbf{v}_{\text {slip }}=\left(\begin{array}{c}
\mathbf{v}_{\text {slip } 1} \\
\mathbf{v}_{\text {slip } 2} \\
\mathbf{v}_{\text {slip } 3}
\end{array}\right)=\left(\begin{array}{ccc|ccc}
1 & 0 & 0 & 0 & 0 & 0 \\
0 & 1 & 1_{12} & \mathrm{r} & 0 & 0 \\
\hline 1 & 0 & 0 & 0 & 0 & 0 \\
0 & 1 & -1_{12} & 0 & \mathrm{r} & 0 \\
\mathrm{c} \beta_{3} & \mathrm{~s} \beta_{3} & 1_{3} \cdot \mathrm{c} \beta_{3} & 0 & 0 & 0 \\
-\mathrm{s} \beta_{3} & \mathrm{c} \beta_{3} & -1_{3} \cdot \mathrm{s} \beta_{3} & 0 & 0 & \mathrm{r}
\end{array}\right) \cdot\left(\begin{array}{c}
\overline{\mathrm{R}} \dot{\mathbf{p}} \\
\dot{\varphi}_{1} \\
\dot{\varphi}_{2} \\
\dot{\varphi}_{3}
\end{array}\right)
$$

Las ecuaciones dinámicas del vehículo de (7.21)-(7.26), son:

$$
\begin{aligned}
& \mathrm{M}_{\mathrm{T}} \cdot{ }^{\mathrm{G}} \dot{\mathbf{v}}_{\mathrm{CM}}=\boldsymbol{\operatorname { R o t }}\left({ }^{\mathrm{G}} \theta_{\mathrm{R}}\right) \cdot\left(\mathbf{F}_{\text {fric } 1}+\mathbf{F}_{\text {fric } 2}+\operatorname{Rot}\left(\beta_{3}\right) \cdot \mathbf{F}_{\text {fric } 3}\right) \\
& \mathrm{I}_{\mathrm{T}} \cdot \dot{\omega}+\mathrm{I}_{\mathrm{s} 3} \cdot \ddot{\beta}_{3}=\left(1_{3}-1_{\mathrm{MC}}\right) \cdot\left(\mathrm{c} \beta_{3} \cdot F_{\text {fric } x 3}-\mathrm{s} \beta_{3} \cdot F_{\text {fric } y 3}\right) \\
& +1_{12} \cdot\left(F_{\text {fric } y 1}-F_{\text {fric } y 2}\right)-1_{\mathrm{CM}} \cdot\left(F_{\text {fric } x 1}+F_{\text {fric } x 2}\right) \\
& \mathrm{I}_{\mathrm{r} 1} \cdot \ddot{\varphi}_{1}=\tau_{\mathrm{r} 1}+\mathrm{r} \cdot\left(\begin{array}{ll}
0 & 1
\end{array}\right) \cdot \mathbf{F}_{\text {fric } 1} \quad \mathrm{I}_{\mathrm{r} 2} \cdot \ddot{\varphi}_{2}=\tau_{\mathrm{r} 2}+\mathrm{r} \cdot\left(\begin{array}{ll}
0 & 1
\end{array}\right) \cdot \mathbf{F}_{\text {fric } 2} \\
& \mathrm{I}_{\mathrm{r} 3} \cdot \ddot{\varphi}_{3}=\mathrm{r} \cdot\left(\begin{array}{ll}
0 & 1
\end{array}\right) \cdot \mathbf{F}_{\mathrm{fric} 3} \quad \mathrm{I}_{\mathrm{s} 3} \cdot\left(\ddot{\beta}_{3}+\dot{\omega}\right)=\tau_{\mathrm{s} 3}
\end{aligned}
$$

donde la rueda orientable es de rotación libre. 
Las ecuaciones del movimiento quasi-estático resultan:

$$
\begin{aligned}
& \mathrm{M}_{\mathrm{T}} \cdot \omega \cdot\left(\begin{array}{cc}
0 & -1 \\
1 & 0
\end{array}\right) \cdot{ }^{\overline{\mathrm{CM}}} \mathbf{v}_{\mathrm{CM}}=\left(\mathbf{F}_{\text {fric } 1}+\mathbf{F}_{\text {fric } 2}+\operatorname{Rot}\left(\beta_{3}\right) \cdot \mathbf{F}_{\text {fric } 3}\right) \\
& 0=1_{12} \cdot\left(F_{\text {fric } y 1}-F_{\text {fric } y 2}\right)-1_{\text {CM }} \cdot\left(F_{\text {fric } x 1}+F_{\text {fric } x 2}\right) \\
& +\left(1_{3}-1_{\mathrm{CM}}\right) \cdot\left(\mathrm{c} \beta_{3} \cdot F_{\text {fric } x 3}-\mathrm{s} \beta_{3} \cdot F_{\text {fric } y 3}\right) \\
& 0=\tau_{\mathrm{r} 1}+\mathrm{r} \cdot\left(\begin{array}{ll}
0 & 1
\end{array}\right) \cdot \mathbf{F}_{\text {fric } 1} \quad 0=\tau_{\mathrm{r} 2}+\mathrm{r} \cdot\left(\begin{array}{ll}
0 & 1
\end{array}\right) \cdot \mathbf{F}_{\text {fric } 2} \\
& 0=F_{\text {fric } y 3} \quad 0=\tau_{\mathrm{s} 3}
\end{aligned}
$$

Por tanto las ecuaciones del modelo con deslizamiento vienen dadas por:

$$
\begin{gathered}
0=F_{\text {fric } x 1}+F_{\text {fric } x 2}+\mathrm{c} \beta_{3} \cdot F_{\text {fric } x 3} \\
0=F_{\text {fric } y 1}+F_{\text {fric } y 2}+\mathrm{s} \beta_{3} \cdot F_{\text {fric } x 3} \\
0=\mathrm{l}_{12} \cdot\left(F_{\text {fric } y 1}-F_{\text {fric } y 2}\right)+1_{3} \cdot \mathrm{c} \beta_{3} \cdot F_{\text {fric } x 3}
\end{gathered}
$$

Además para todos los modelos anteriores $\{(7.61),(7.62),(7.63)\}$ se tiene que incluir (7.49) tres veces debido a las fuerzas de fricción en cada rueda.

Las reacciones normales en las ruedas se pueden calcula de:

$$
\begin{gathered}
F_{\mathrm{N} 1}+F_{\mathrm{N} 2}+F_{\mathrm{N} 3}=\mathrm{M}_{\mathrm{T}} \cdot \mathrm{g} \\
1_{12} \cdot\left(F_{\mathrm{N} 1}-F_{\mathrm{N} 2}\right)+\mathrm{h}_{\mathrm{CM}} \cdot \mathrm{M}_{\mathrm{T}} \cdot{ }^{\mathrm{CM}, \mathrm{G}} \dot{\mathrm{c}}_{\mathrm{CM} x}=0 \\
1_{\mathrm{CM}} \cdot\left(F_{\mathrm{N} 1}+F_{\mathrm{N} 2}\right)+\mathrm{h}_{\mathrm{CM}} \cdot \mathrm{M}_{\mathrm{T}} \cdot{ }^{\mathrm{CM}, \mathrm{G}} \dot{v}_{\mathrm{CM} y}=\left(1_{3}-1_{\mathrm{CM}}\right) \cdot F_{\mathrm{N} 3}+\tau_{\mathrm{r} 1}+\tau_{\mathrm{r} 2}
\end{gathered}
$$

donde $\mathrm{h}_{\mathrm{CM}}$ es la altura del CM respecto al suelo y la aceleración ${ }^{\mathrm{CM}, \mathrm{G}} \dot{\mathbf{v}}_{\mathrm{CM}}$ se particulariza para cada modelo.

Por otro lado (7.51) se puede particularizar en:

$$
\begin{aligned}
& \left(\begin{array}{ccc}
\sqrt{2 \cdot \mu_{x 12}} & 0 & 0 \\
0 & \sqrt{\mu_{y 12}} & \sqrt{\mu_{y 12}} \cdot 1_{12} \\
0 & \sqrt{\mu_{y 12}} & -\sqrt{\mu_{y 12}} \cdot 1_{12} \\
\sqrt{\mu_{x 3}} \cdot \mathrm{c} \beta_{3} & \sqrt{\mu_{x 3}} \cdot \mathrm{s} \beta_{3} & \sqrt{\mu_{x 3}} \cdot 1_{3} \cdot \mathrm{c} \beta_{3}
\end{array}\right) \cdot \overline{\mathrm{k}} \dot{\mathbf{p}}=-\left(\begin{array}{cc}
0 & 0 \\
\sqrt{\mu_{y 12}} \cdot \mathrm{r} & 0 \\
0 & \sqrt{\mu_{y 12}} \cdot \mathrm{r} \\
0 & 0
\end{array}\right) \cdot\left(\begin{array}{l}
\dot{\varphi}_{1} \\
\dot{\varphi}_{2}
\end{array}\right) \\
& \mathbf{A}_{\text {нпa }} \cdot \overline{\mathrm{R}} \dot{\mathbf{p}}=-\mathbf{A}_{\text {на }} \cdot\left(\begin{array}{l}
\dot{\varphi}_{1} \\
\dot{\varphi}_{2}
\end{array}\right)
\end{aligned}
$$

donde $\left(\mu_{x 12}, \mu_{y 12}\right)$ son los coeficientes de fricción de las identicas ruedas fijas y $\mu_{x 3}$ es el coeficiente de fricción de la rueda orientable en la dirección $\mathrm{L}_{x 3}$. 
Notar que (7.65) considera conjuntamente la primera y tercera ecuación de (7.60), a través de un coeficiente de fricción de doble valor, y se obvia la última ecuación utilizada para calcular $\dot{\varphi}_{3}$.

Por tanto la solución de LS ponderada (weighted least square: WLS) es:

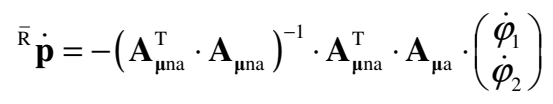

Para calcular la expresión anterior se tienen que conocer los tres coeficientes de fricción o dos relaciones entre ellos a través de las constantes $\mathrm{k}_{11}$ y $\mathrm{k}_{12}$ :

$$
\begin{aligned}
& \mu_{y 12}=\mathrm{k}_{11} \cdot \mu_{x 12} \\
& \mu_{x 3}=\mathrm{k}_{12} \cdot \mu_{x 12}
\end{aligned}
$$

\subsubsection{Estimación del vector de velocidad del vehículo con filtro de Kalman}

Las ecuaciones de estado y salida discretas son:

$$
\begin{gathered}
{ }^{\overline{\mathrm{R}}} \dot{\mathbf{p}}_{k+1}={ }^{\overline{\mathrm{R}}} \dot{\mathbf{p}}_{k}+\mathrm{T} \cdot{ }^{\overline{\mathrm{R}}} \ddot{\mathbf{p}}_{k} \\
\left(\begin{array}{c}
\dot{\varphi}_{1} \cdot \mathrm{r} \\
\dot{\varphi}_{2} \cdot \mathrm{r} \\
0
\end{array}\right)_{k}=\left(\begin{array}{ccc}
0 & -1 & -1_{12} \\
0 & -1 & 1_{12} \\
\mathrm{c} \beta_{3} & \mathrm{~s} \beta_{3} & 1_{3} \cdot \mathrm{c} \beta_{3}
\end{array}\right) \cdot{ }_{k}^{\overline{\mathrm{R}}} \dot{\mathbf{p}}_{k} \rightarrow \mathbf{y}_{1 k}=\mathbf{C}_{1 k} \cdot{ }^{\overline{\mathrm{R}}} \dot{\mathbf{p}}_{k}
\end{gathered}
$$

donde $k$ es el instante discreto de tiempo considerado y $\mathrm{T}$ el periodo.

La ecuación de salida (7.69) tiene tres elementos, uno para cada sensor, obtenidos de la cinemática sin deslizamiento de (7.60). Notar que no se ha despejado $\beta_{3}$ en la tercera ecuación escalar de (7.69) porque obligaría a trabajar con el filtro de Kalman extendido (FKE) que utiliza una aproximación de primer orden. Dicha aproximación no es necesaria con el planteamiento de (7.69).

Lo anterior, utilizar una ecuación escalar de salida por cada sensor, es lo habitual en el FK. Sin embargo se puede apreciar que (7.69) no incluye toda la cinemática de (7.65): faltaría su primer elemento (primero ó tercer elemento de (7.60)). Por ello alternativamente se puede plantear como ecuación de salida: 


$$
\left(\begin{array}{c}
0 \\
\dot{\varphi}_{1} \cdot \mathrm{r} \\
\dot{\varphi}_{2} \cdot \mathrm{r} \\
0
\end{array}\right)_{k}=\left(\begin{array}{ccc}
1 & 0 & 0 \\
0 & -1 & -1_{12} \\
0 & -1 & 1_{12} \\
\mathrm{c} \beta_{3} & \mathrm{~s} \beta_{3} & 1_{3} \cdot \mathrm{c} \beta_{3}
\end{array}\right)_{k} \cdot{ }^{\overline{\mathrm{p}}} \dot{\mathbf{p}}_{k} \rightarrow \mathbf{y}_{2 k}=\mathbf{C}_{2 k} \cdot{ }^{\overline{\mathrm{p}}} \dot{\mathbf{p}}_{k}
$$

Al FK que utiliza la ecuación de salida (7.69) se le denotará como FK1 y al que utiliza (7.70) como FK2.

El término aceleración ${ }^{\bar{R}} \ddot{\mathbf{p}}_{k}$ de (7.68) se considerará el ruido de proceso en la ecuación de estado, cuya matriz de estado sería la identidad.

Las ecuaciones recursivas del Filtro de Kalman (FK) resultan:

$$
\begin{gathered}
{ }^{\overline{\mathrm{R}}} \hat{\dot{\mathbf{p}}}_{k+1 / k}={ }^{\overline{\mathrm{R}}} \hat{\dot{\mathbf{p}}}_{k / k} \\
\mathbf{P}_{k+1 / k}=\mathbf{P}_{k / k}+\mathbf{Q}_{k} \\
\mathbf{K}_{k+1}=\mathbf{P}_{k+1 / k} \cdot \mathbf{C}_{k+1}^{\mathrm{T}} \cdot\left(\mathbf{C}_{k+1} \cdot \mathbf{P}_{k+1 / k} \cdot \mathbf{C}_{k+1}^{\mathrm{T}}+\mathbf{R}_{k+1}\right)^{-1} \\
{ }^{\overline{\mathrm{R}}} \hat{\mathbf{p}}_{k+1 / k+1}={ }^{\overline{\mathrm{R}}} \hat{\mathbf{p}}_{k+1 / k}+\mathbf{K}_{k+1} \cdot\left(\mathbf{y}_{k+1}-\mathbf{C}_{k+1} \cdot{ }^{\overline{\mathrm{R}}} \hat{\dot{\mathbf{p}}}_{k+1 / k}\right) \\
\mathbf{P}_{k+1 / k+1}=\left(\mathbf{I}-\mathbf{K}_{k+1} \cdot \mathbf{C}_{k+1}\right) \cdot \mathbf{P}_{k+1 / k}+\mathbf{Q}_{k}
\end{gathered}
$$

donde $\mathbf{C}_{k}=\mathbf{C}_{1 k}$ ó $\mathbf{C}_{2 k}, \mathbf{y}_{k}=\mathbf{y}_{1 k}$ ó $\mathbf{y}_{2 k},\left\{\mathbf{Q}_{k}, \mathbf{R}_{k}\right\}$ son las matrices de covarianza del ruido de proceso y la covarianza del ruido de medida, $\mathbf{K}_{k}$ es la matriz de corrección ó de ganancia del algoritmo y $\mathbf{P}_{k}$ es la matriz de covarianza del error. En cualquier caso, se asumirá que las matrices de covarianza del ruido en el proceso y en la medida son constantes, es decir que no cambian según el instante.

El FK propuesto es muy sencillo ya que la ecuación de estado (7.71) predice el valor de estado previo, el cual se corrige con las medidas en (7.74).

En particular, si las medidas se ponderan mucho más que el modelo del proceso $\mathbf{R}<<\mathbf{Q}$, es decir las medidas son más precisas que la ecuación de estado, la solución del FK1 se reduce a:

$$
{ }^{\overline{\mathrm{R}}} \dot{\mathbf{p}}_{k}=\mathbf{C}_{1 k}^{-1} \cdot \mathbf{y}_{k}
$$

donde no hay filtrado de los valores medidos. 
En general se consideraran las matrices de covarianza:

$$
\begin{gathered}
\mathbf{Q}=\mathrm{k}_{13}^{2} \cdot \mathbf{I} \quad \mathbf{R}=\mathbf{R}_{1} \sigma \mathbf{R}_{2} \\
\mathbf{R}_{1}=\left(\begin{array}{ccc}
\mathrm{k}_{14}^{2} & 0 & 0 \\
0 & \mathrm{k}_{14}^{2} & 0 \\
0 & 0 & \mathrm{k}_{15}^{2}
\end{array}\right) \quad \mathbf{R}_{2}=\left(\begin{array}{cccc}
\mathrm{k}_{16}^{2} & 0 & 0 & 0 \\
0 & \mathrm{k}_{17}^{2} & 0 & 0 \\
0 & 0 & \mathrm{k}_{17}^{2} & 0 \\
0 & 0 & 0 & \mathrm{k}_{18}^{2}
\end{array}\right)
\end{gathered}
$$

donde los encoders de las dos ruedas fijas y las ecuaciones escalares de estado se han supuesto igualmente fiables a través de las desviaciones típicas $\mathrm{k}_{14} / \mathrm{k}_{17} \mathrm{y} \mathrm{k}_{13}$, $\mathrm{y}$ los errores de las variables de estado y de salida se han asumido no correlados.

Para aplicar el FK1/FK2 se tienen que establecer dos/tres parámetros de (7.77), por ejemplo $\left\{\mathrm{k}_{14}, \mathrm{k}_{15}\right\} /\left\{\mathrm{k}_{16}, \mathrm{k}_{17}, \mathrm{k}_{18}\right\}$ si el otro se fija a un valor conocido, por ejemplo $\mathrm{k}_{13}=1$, sin pérdida de generalidad.

Notar que no es posible utilizar la solución estacionaria del FK, que vendría dada por la matriz de corrección estacionaria $\mathbf{K}_{\infty}$ y la ecuación (7.74), porque la matriz de salida $\mathbf{C}_{1 k} / \mathbf{C}_{2 k}$ es variable.

\subsubsection{Resultados de simulación}

En este apartado se comparará en simulación el modelo dinámico (MD) dado por (7.61) con los tres modelos con deslizamiento: el de movimiento quasi-estático (MQE) de (7.62), el cinemático con deslizamiento (MCD) de (7.63) y la solución de Mínimos Cuadrados ponderada (weighted least square: WLS) de (7.66).

Para $\mathbf{f}_{\text {fric } 1 / 2 / 3}$ se ha empleado la ampliamente utilizada "fórmula mágica" de Pacejka (7.34), con el procedimiento de acoplamiento presentado en [Pacejka et al. 97] y los parámetros (dependientes de la velocidad de la rueda) y coeficientes identificados en [Lu et al. 05] (ver punto 7.A1).

Los valores de las constantes utilizados en la simulación son: $\mathrm{M}_{\mathrm{T}}=2500 \mathrm{Kg}$; $\mathrm{I}_{\mathrm{T}}=1500 \mathrm{Kg} \cdot \mathrm{m}^{2} ; \mathrm{I}_{\mathrm{r} 1}=\mathrm{I}_{\mathrm{r} 2}=1 \mathrm{Kg} \cdot \mathrm{m}^{2} ; \mathrm{I}_{\mathrm{r} 3}=1.5 \mathrm{Kg} \cdot \mathrm{m}^{2} ; \mathrm{I}_{\mathrm{s} 3}=0.8 \mathrm{Kg} \cdot \mathrm{m}^{2} ; \mathrm{l}_{\mathrm{CM}}=0.45 \mathrm{~m}$; $\mathrm{h}_{\mathrm{CM}}=0.7 \mathrm{~m}$. Dichos valores son sólo orientativos, ya que no se ha aplicado ningún proceso de identificación.

Los valores de $\mathrm{k}_{11} \mathrm{y}_{12}$ se han calculado aplicando un proceso de optimización basado en minimizar la diferencia entre el vector de velocidad del vehículo ${ }^{\overline{\mathrm{R}}} \dot{\mathbf{p}}_{\mathrm{R}}$ calculado con MCD de (7.63) y con WLS de (7.66), ya que el 
segundo es la aproximación inmediatamente posterior del primero. En concreto se han considerado un número de puntos dados por un barrido en las velocidades de las ruedas fijas $\left(-\dot{\varphi}_{1} \cdot r=0: 0.5: 5,-\dot{\varphi}_{2} \cdot r=-5: 0.5: 5\right)$ y 5 valores de orientación $\beta_{3}$ para cada combinación: uno el teórico sin deslizamiento, redondeado con la resolución del encoder $\left(1.4118^{\circ}\right)$, y dos por arriba y dos por abajo con incrementos del doble de la resolución $\left(2.8236^{\circ}\right)$. Lo anterior es porque en condiciones normales el ángulo $\beta_{3}$ de la rueda orientable no debe ser "demasiado" incompatible con la velocidad de las ruedas fijas. En total el error se minimiza para un número de puntos igual a 11x21x5 = 1155 .

En la Figura 7.9 se indica la diferencia entre los elementos del vector de velocidad ${ }^{\overline{\mathrm{R}}} \dot{\mathbf{p}}_{\mathrm{R}}$ calculados con MCD y con WLS, una vez optimizadas $\mathrm{k}_{11}$ y $\mathrm{k}_{12}$, para las 1155 combinaciones de velocidades de las ruedas fijas y de orientación $\beta_{3}$. Se puede concluir que ambos modelos dan un resultado muy similar.
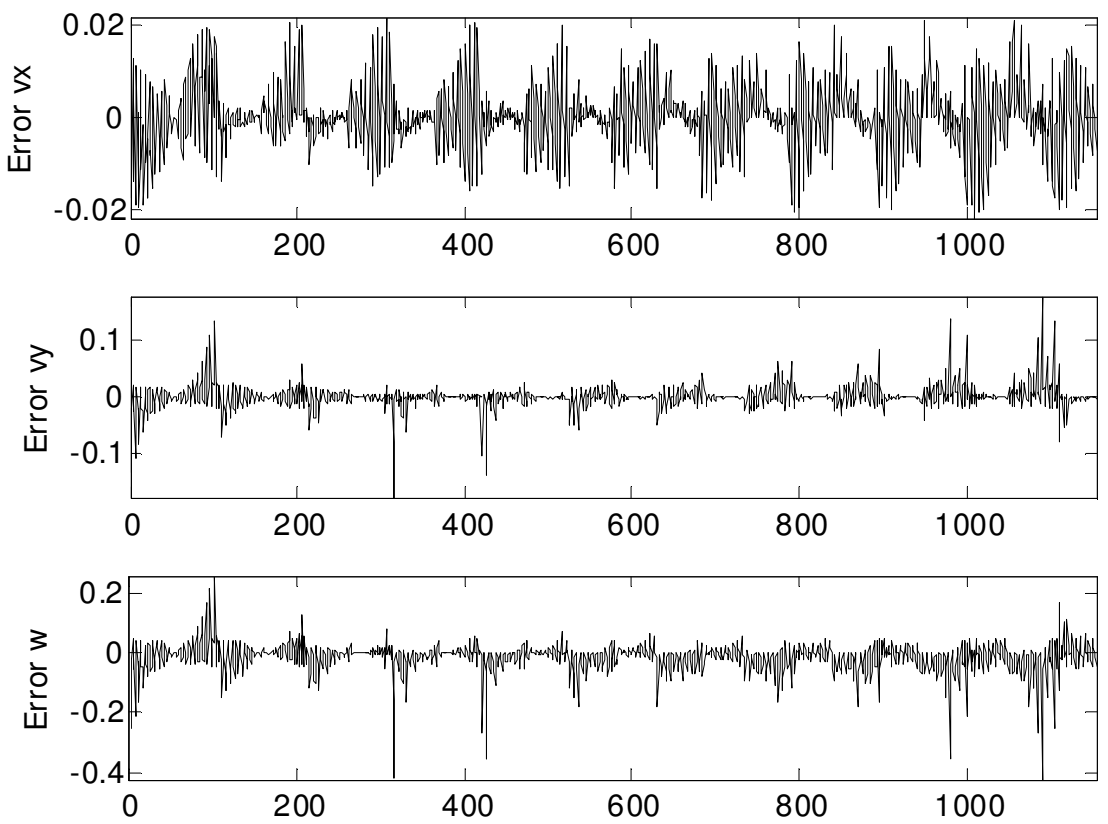

Figura 7.9- Diferencia en los elementos del vector de velocidad del vehículo ${ }^{\overline{\mathrm{R}}} \dot{\mathbf{p}}_{\mathrm{R}}$ al calcularlos con MCD y con WLS (para $\mathrm{k}_{11} \mathrm{y} \mathrm{k}_{12}$ optimizadas)

En las Figuras 7.10 y 7.11 se muestra el vector de velocidad ${ }^{\overline{\mathrm{R}}} \dot{\mathbf{p}}_{\mathrm{R}}$ calculado con MCD y el error medio producido en las tres ecuaciones de su modelo cinemático (7.63) al realizar el cálculo numérico de la solución. 

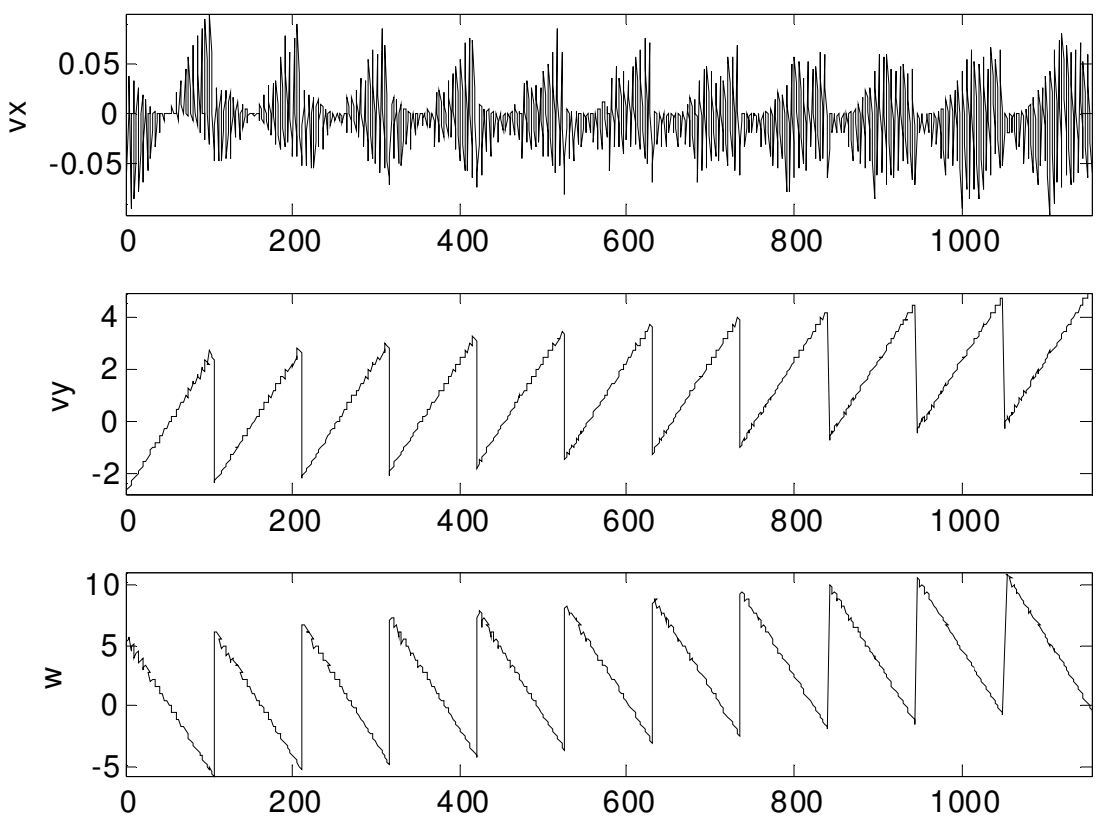

Figura 7.10- Vector de velocidad ${ }^{\overline{\mathrm{R}}} \dot{\mathbf{p}}_{\mathrm{R}}$ calculado con MCD para los 1155 puntos

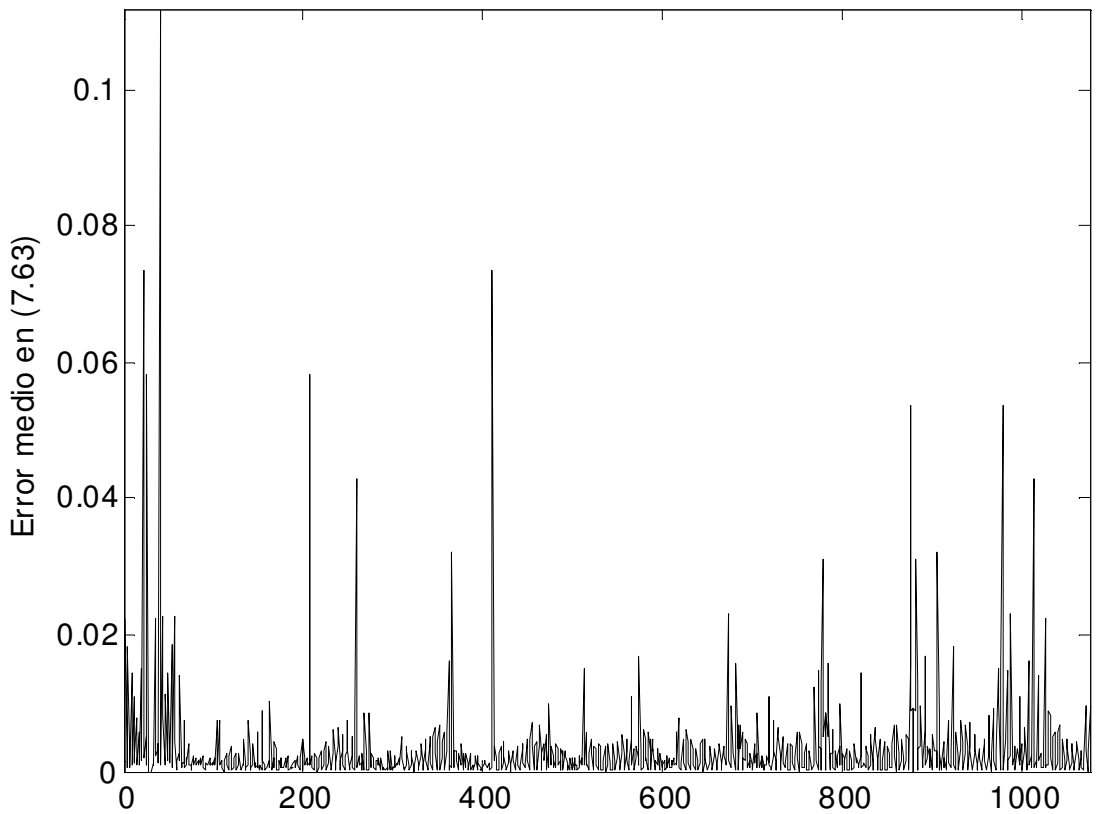

Figura 7.11- Error numérico medio cometido en (7.63) al calcular ${ }^{\overline{\mathrm{R}}} \dot{\mathbf{p}}_{\mathrm{R}}$ con MCD 
En la Figura 7.12 se muestra el camino obtenido en simulación con cada modelo, a través de la integración numérica de $\dot{\mathbf{p}}$, para pares nulos y condiciones iniciales no nulas: ${ }^{\overline{\mathrm{R}}} v_{\mathrm{R} y}(0)=2 \mathrm{~m} / \mathrm{s} ;{ }^{\overline{\mathrm{R}}} \omega_{\mathrm{R}}(0)=0.3 \mathrm{rad} / \mathrm{s}$ (los valores iniciales de las velocidades de rueda y el ángulo de la rueda orientable están en concordancia con esos valores).

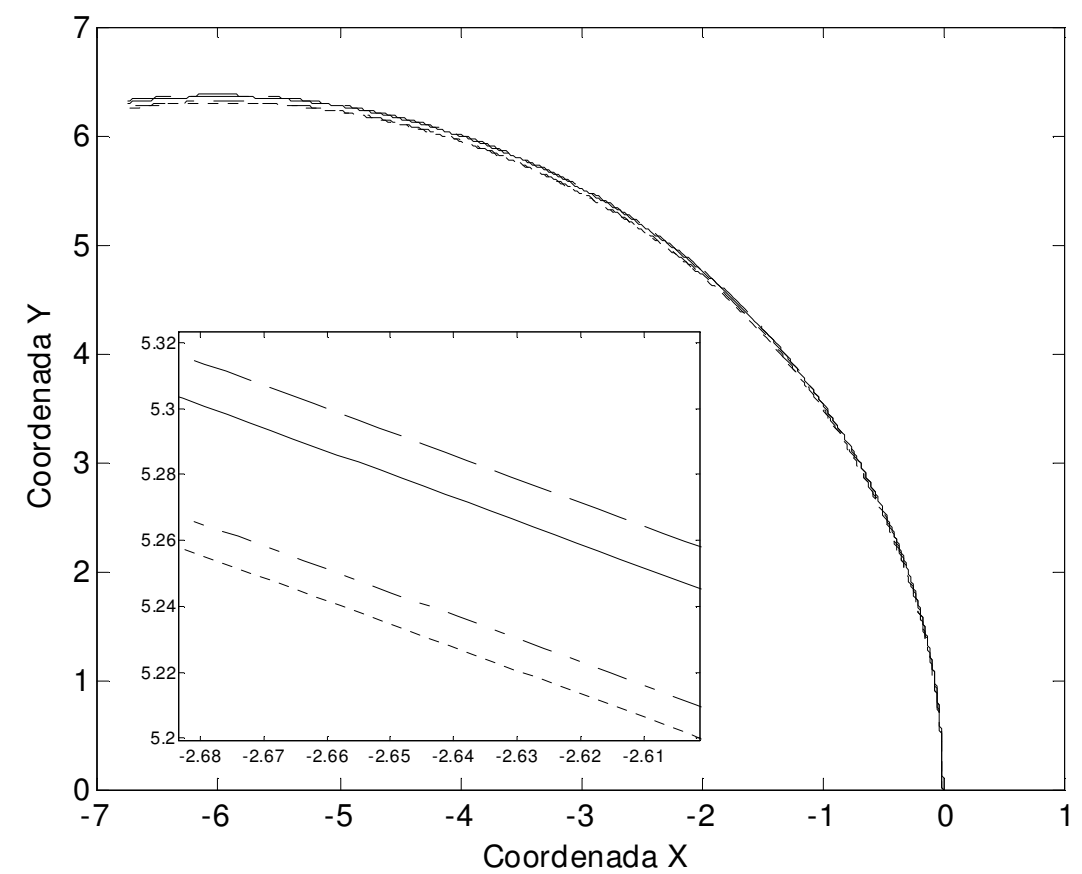

Figura 7.12- Caminos obtenidos con MD (-), MQE (--), MCD (- -) y WLS $(-\cdot-)$ para pares nulos y ${ }^{\overline{\mathrm{R}}} v_{\mathrm{R} y}(0)=2 \mathrm{~m} / \mathrm{s} \quad{ }^{\overline{\mathrm{R}}} \omega_{\mathrm{R}}(0)=0.3 \mathrm{rad} / \mathrm{s}$

Para evaluar la exactitud de los modelos con deslizamiento se utiliza el siguiente índice:

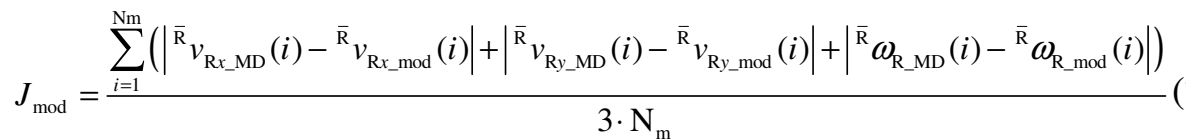

donde $\mathrm{N}_{\mathrm{m}}$ son el número de muestras de la simulación, el subíndice "MD" hace referencia al modelo dinámico y el subíndice "mod" al modelo con deslizamiento considerado (MQE, MCD ó WLS). 
Los índices para el ejemplo de simulación anterior resultan: $J_{\mathrm{MQE}}=0.24 \%$, $J_{\mathrm{MCD}}=0.88 \%, J_{\mathrm{WLS}}=0.92 \%$.

En la Figura 7.13 se muestra el camino obtenido con cada modelo con las condiciones iniciales de antes y pares de rotación sobre las ruedas fijas de 30 $\mathrm{N} \cdot \mathrm{m}$, aplicados durante 1 segundos. Para este ejemplo la orientación de la rueda orientable se ajusta en cada iteración, con el par de dirección, para evitar la incompatibilidad cinemática.

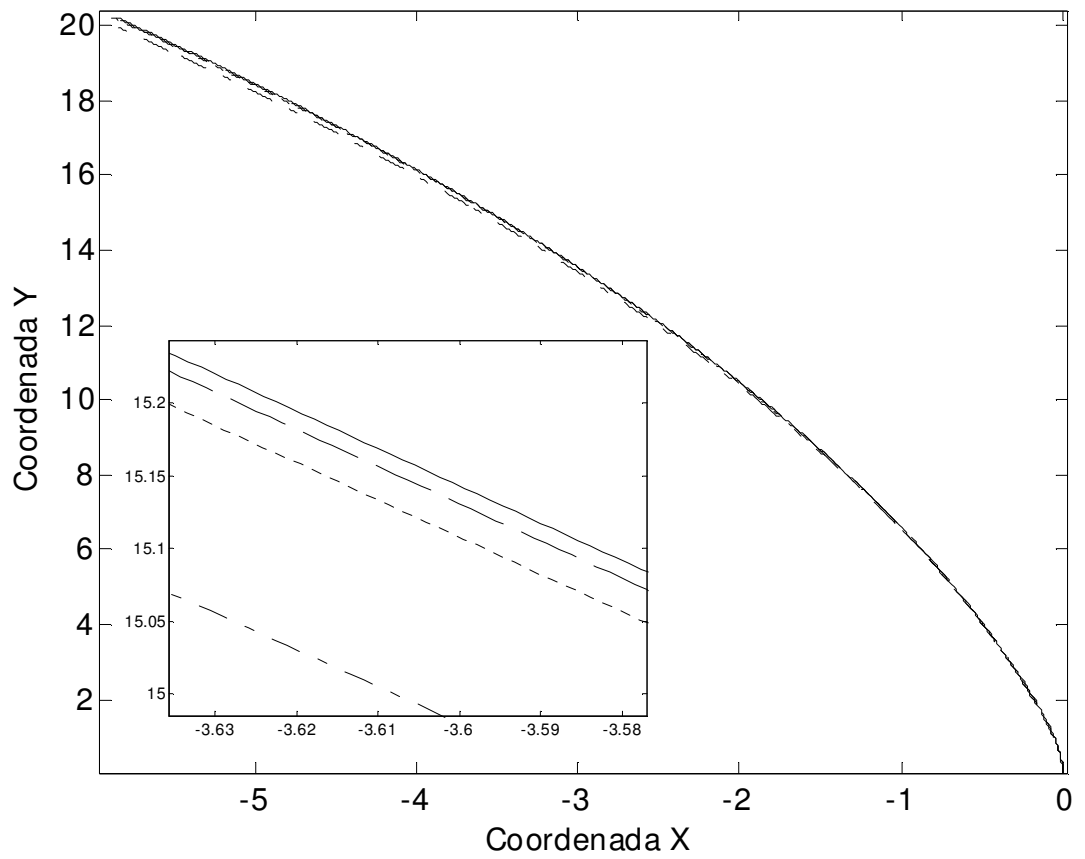

Figura 7.13- Caminos obtenidos con MD (-), MQE (--), MCD (- -) y WLS $(-\cdot-)$ con pares de rotación no nulos y ${ }^{\overline{\mathrm{R}}} v_{\mathrm{R} y}(0)=2 \mathrm{~m} / \mathrm{s}{ }^{\overline{\mathrm{R}}} \omega_{\mathrm{R}}(0)=0.3 \mathrm{rad} / \mathrm{s}$

Los índices para este segundo caso son: $J_{\mathrm{MQE}}=0.33 \%, J_{\mathrm{MCD}}=0.63 \%$, $J_{\mathrm{WLS}}=0.84 \%$.

En la Figura 7.14 se indica la evolución en la simulación de los elementos del vector de velocidad del vehículo ${ }^{\overline{\mathrm{R}}} \dot{\mathbf{p}}_{\mathrm{R}}$ según el MD.

En dicha figura se aprecia que, con los pares aplicados, se gana en velocidad de avance y se pierde en velocidad de rotación. También se observa que el deslizamiento en la dirección perpendicular a las ruedas fijas disminuye, lo cual es lógico porque se pasa de un movimiento circular inicial a otro más rectilíneo. 

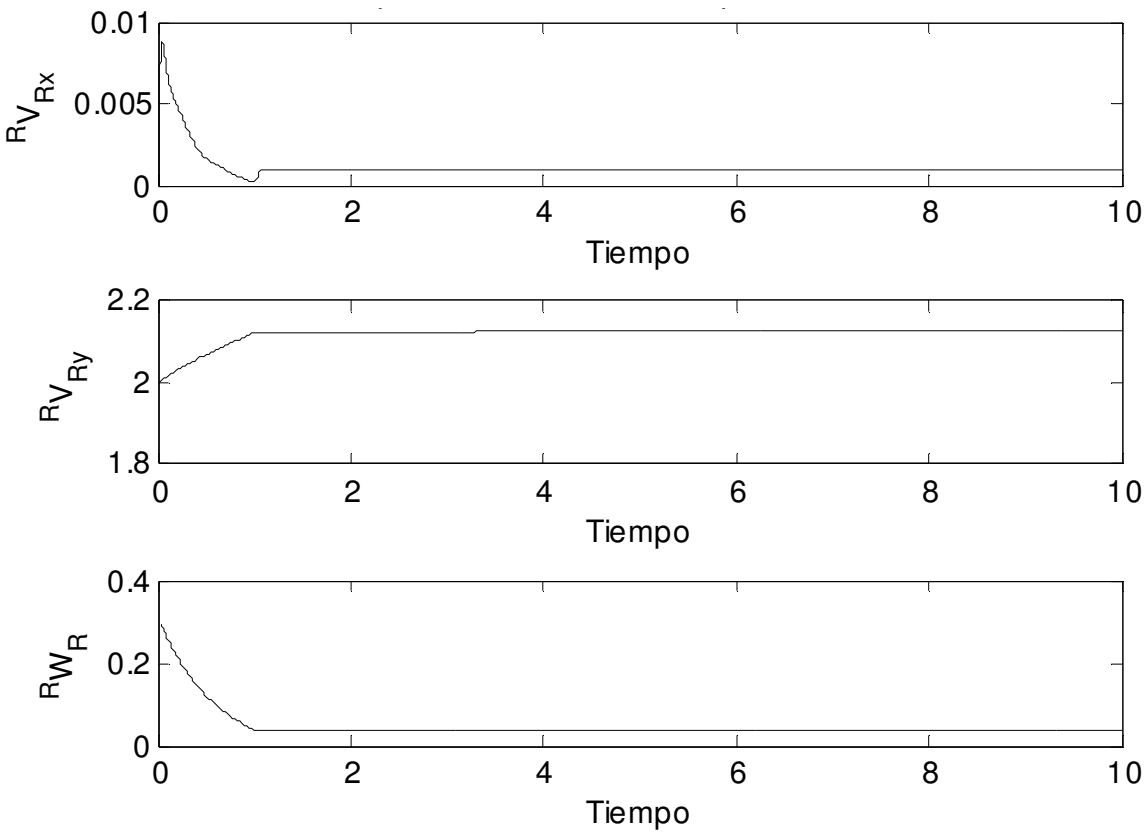

Figura 7.14- Evolución de los elementos del vector de velocidad del vehículo ${ }^{\bar{R}} \dot{\mathbf{p}}_{\mathrm{R}}$ según el MD para el segundo ejemplo de simulación

Los dos ejemplos de simulación anteriores evidencian que el MCD es más preciso que el WLS y menos que el MQE. 


\subsubsection{Resultados experimentales}

Aquí se considerará el tercer nivel de aproximación del modelo dinámico, solución de mínimos cuadrados ponderada (WLS), ya que se puede calcular analíticamente. Esta solución de WLS se comparará con los FK1 y FK2, presentados en un subapartado anterior, y con el clásico modelo odométrico (7.78) del vehículo diferencial (ver Capítulo 5).

$$
{ }^{\overline{\mathrm{R}}} \dot{\mathbf{p}}_{k}=\left(\begin{array}{cc}
0 & 0 \\
-\mathrm{r} / 2 & -\mathrm{r} / 2 \\
-\mathrm{r} /\left(2 \cdot \mathrm{l}_{12}\right) & \mathrm{r} /\left(2 \cdot \mathrm{l}_{12}\right)
\end{array}\right) \cdot\left(\begin{array}{l}
\dot{\varphi}_{1 k} \\
\dot{\varphi}_{2 k}
\end{array}\right)
$$

Dicha comparación se realizará en base a los caminos obtenidos con cada modelo, a través de la integración numérica de ${ }^{\overline{\mathrm{R}}} \dot{\mathbf{p}}$, para distintos experimentos. Estos experimentos serán realizados de forma que la postura final real del vehículo $\mathbf{p}(\infty)$ coincida exactamente con la postura inicial $\mathbf{p}(0)$.

Para establecer los valores de los parámetros de WLS $\left\{\mathrm{k}_{11}, \mathrm{k}_{12}\right\}$, del FK1 $\left\{\mathrm{k}_{14}, \mathrm{k}_{15}\right\}$ y del FK2 $\left\{\mathrm{k}_{16}, \mathrm{k}_{17}, \mathrm{k}_{18}\right\}$, se minimizará el siguiente índice de error $J_{\mathrm{k}}$ :

$$
J_{\mathrm{k}}=\frac{1}{2 \cdot \mathrm{N}_{\mathrm{e}}} \cdot \sum_{i=1}^{\mathrm{Ne}}\left(\frac{\sqrt{\left(x_{\infty}-x_{0}\right)^{2}+\left(y_{\infty}-y_{0}\right)^{2}}}{\text { distancia recorrida }}+\frac{\left|\theta_{\infty}-\theta_{0}\right|}{\text { ángulo recorrido }}\right)
$$

donde $\mathrm{N}_{\mathrm{e}}$ son el número de experimentos considerados.

Para la optimización de los parámetros de WLS/FK1/FK2 se han considerado 15 experimentos, realizados en un parking con una velocidad media entorno a $\operatorname{los} 2 \mathrm{~m} / \mathrm{s}$.

En el caso de WLS, después del proceso de optimización se obtiene $\mathrm{k}_{11}=$ $3.2 \cdot 10^{-3}, \mathrm{k}_{12}=19.2$ y $J_{\mathrm{k} \min }=2.02 \%$. Estos valores de parámetros evidencian que las ruedas fijas son menos fiables en la dirección longitudinal, es decir sus ecuaciones asociadas son menos precisas. Esto es lógico ya que las ruedas fijas son las ruedas tractoras y la tracción se produce principalmente, excepto para maniobras no suaves, en la dirección longitudinal. (Para pequeño deslizamiento el deslizamiento longitudinal se incrementa con la fuerza de tracción, Figura 7.5.)

Obviamente las fuerzas de tracción sobre las ruedas fijas las coordina la tarjeta de control de la carretilla con la orientación de la rueda orientable. 
La Figura 7.15 muestra el camino obtenido con WLS optimizado y no optimizado al recorrer aproximadamente un rectángulo. Mientras que la Figura 7.16 muestra los valores medidos por los sensores en este experimento.

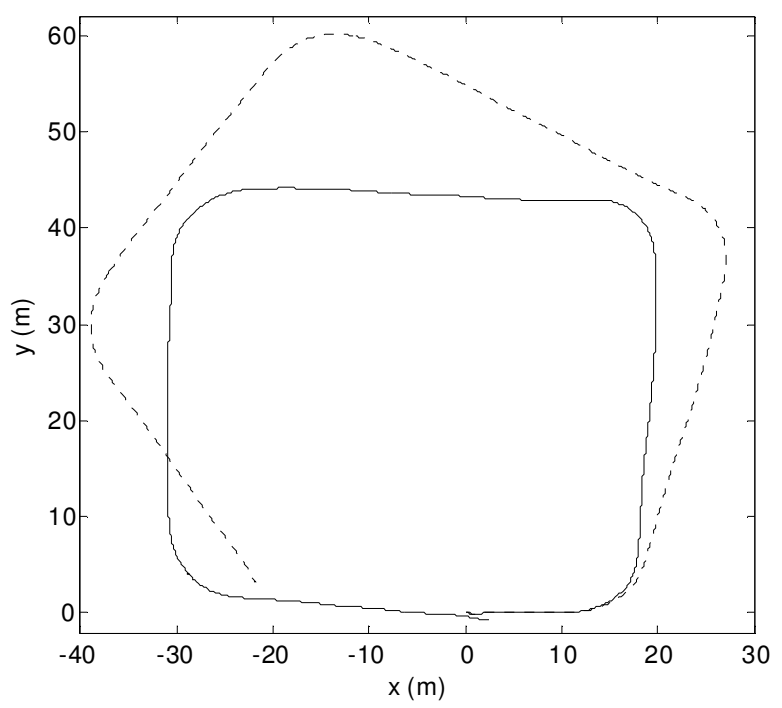

Figura 7.15- Camino obtenido con WLS optimizado (-) y no optimizado (- -) $\left(\mathrm{k}_{11}=0.32, \mathrm{k}_{12}=2, J_{\mathrm{k}}=11.55 \%\right)$ al recorrer aproximadamente un rectángulo
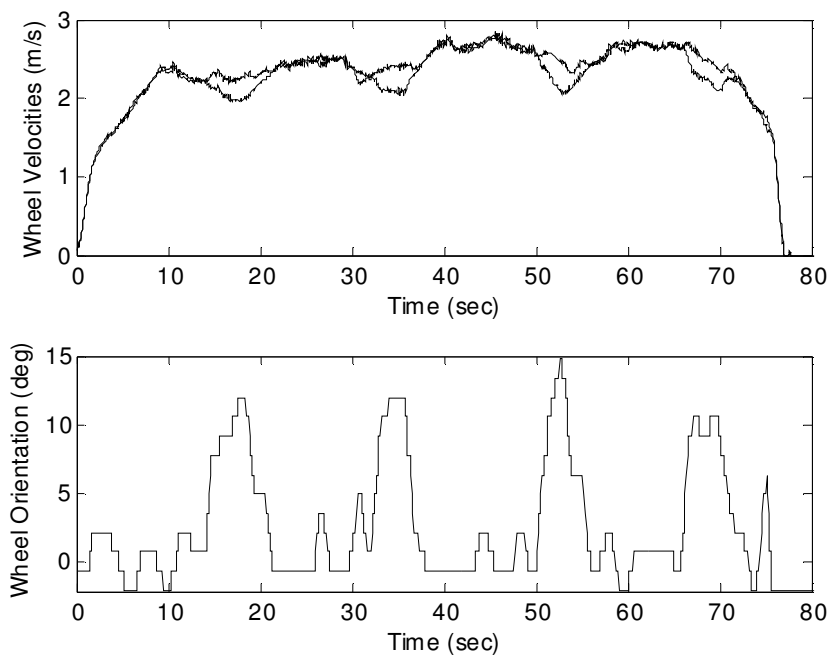

Figura 7.16- Medidas de velocidades en las ruedas fijas $\left(-\mathrm{r} \cdot \dot{\varphi}_{1},-\mathrm{r} \cdot \dot{\varphi}_{2}\right)$ y en la orientación $\beta_{3}$ de la rueda orientable en experimento del rectángulo (Fig. 7.15) 
Para el FK1, después del proceso de optimización se obtiene $\mathrm{k}_{14}=0.31$, $\mathrm{k}_{15}=1.09$, y $J_{\mathrm{k} \min }=7.11 \%$. Estos valores de parámetros implican que $\left\{\dot{\varphi}_{1}, \dot{\varphi}_{2}\right\}$ se filtran poco y $\beta_{3}$ un poco más.

La Figura 7.17 muestra el camino obtenido con el FK1 optimizado para el mismo experimento anterior del rectángulo (Figura 7.16).

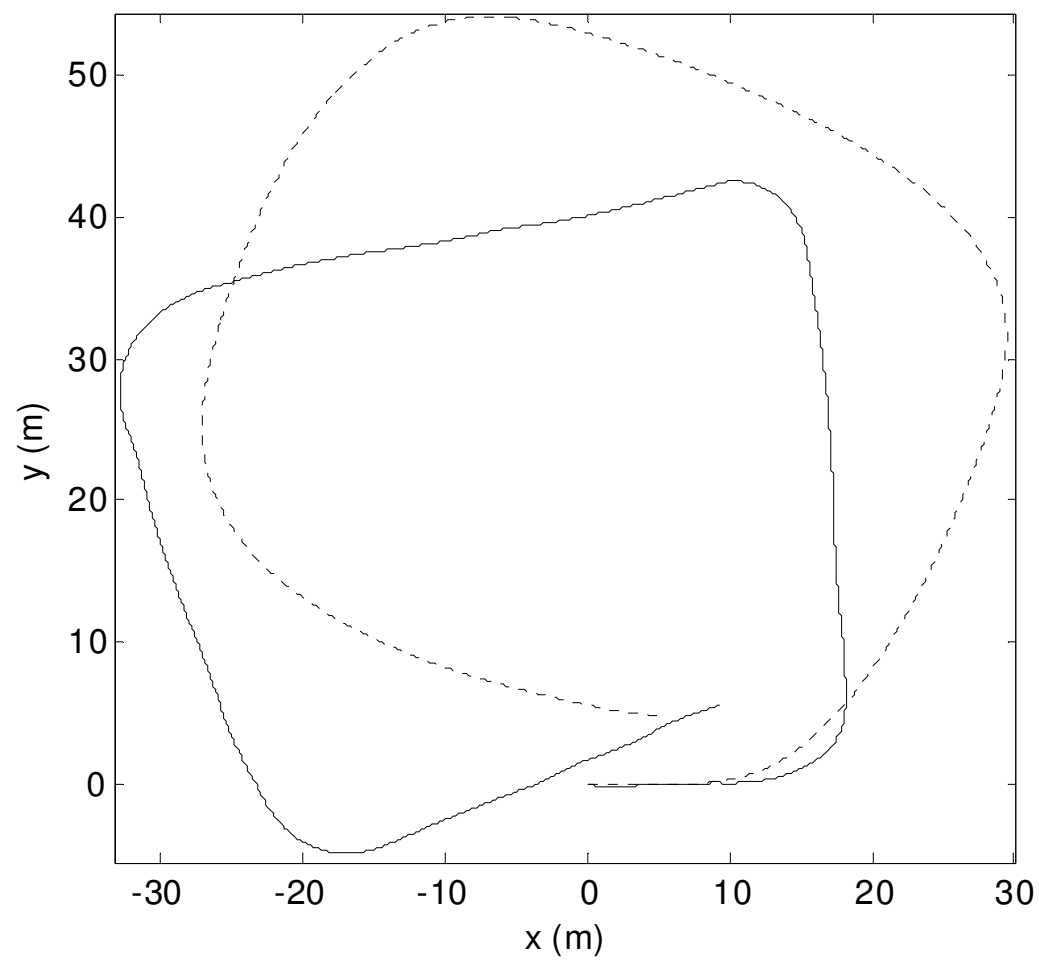

Figura 7.17- Camino obtenido con el FK1 optimizado (-) y no optimizado (- -) $\left(\mathrm{k}_{14}=\mathrm{k}_{15}=200, J_{\mathrm{k}}=12.16 \%\right)$ para los datos de la Figura 7.16

Para el FK2, después del proceso de optimización se obtiene $\mathrm{k}_{16}=0.28$, $\mathrm{k}_{17}=3.57, \mathrm{k}_{18}=0.28$ y $J_{\mathrm{k} \min }=2.1 \%$. Estos valores de parámetros implican que $\left\{\dot{\varphi}_{1}, \dot{\varphi}_{2}\right\}$ se filtran mucho y $\beta_{3}$ y la ecuación ${ }^{\overline{\mathrm{R}}} v_{\mathrm{R} x}=0$ poco. Esto concuerda cualitativamente con los valores de parámetros obtenidos para WLS.

La Figura 7.18 muestra el camino obtenido con el FK2 optimizado para el mismo experimento anterior del rectángulo (Figura 7.16). 


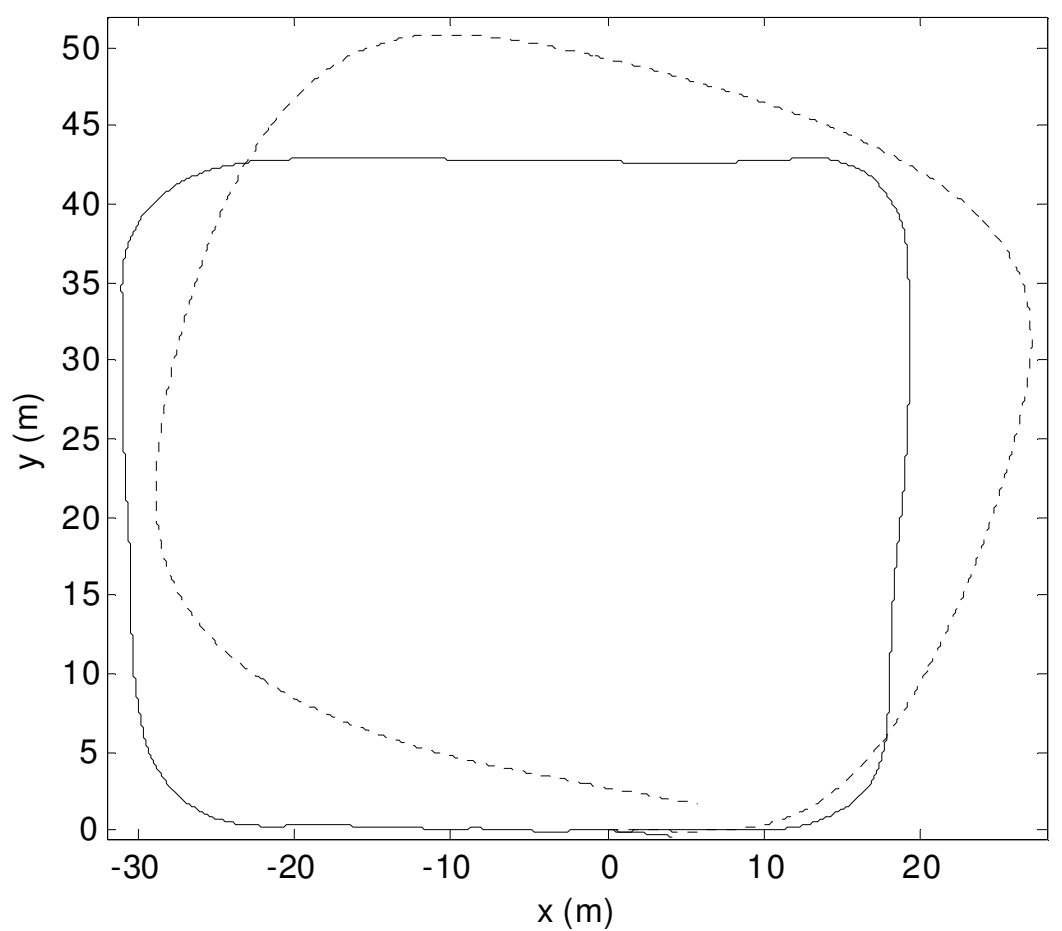

Figura 7.18- Camino obtenido con el FK2 optimizado (-) y no optimizado (- -) $\left(\mathrm{k}_{16}=\mathrm{k}_{17}=\mathrm{k}_{18}=200, J_{\mathrm{k}}=6.85 \%\right)$ para los datos de la Figura 7.16

La Figura 7.19 muestra los resultados de otros experimentos con mismo camino (rectángulo) para los modelos WLS, FK1, FK2 y diferencial (7.79). Se puede apreciar que el FK1 optimizado resulta similar al modelo del vehículo diferencial $\left(J_{\mathrm{k}}=7.14 \%\right)$ y el FK2 al WLS.

Las Figuras 7.20, 7.21, 7.22 y 7.23 muestran los caminos obtenidos con WLS, FK1, FK2 y diferencial con otros caminos seguidos. En concreto, el quinto camino seguido (triple bucle, ver la Figura 7.23) es el más crítico porque el ángulo recorrido (movimiento de rotación acumulado) es $3 \times 360^{\circ}$. De hecho el FK1 y el modelo diferencial fallan claramente en la estimación mientras que WLS y el FK2 la realizan bastante bien.

En general, para todos los experimentos WLS y el FK2 estiman mucho mejor que el FK1 y el modelo diferencial. 

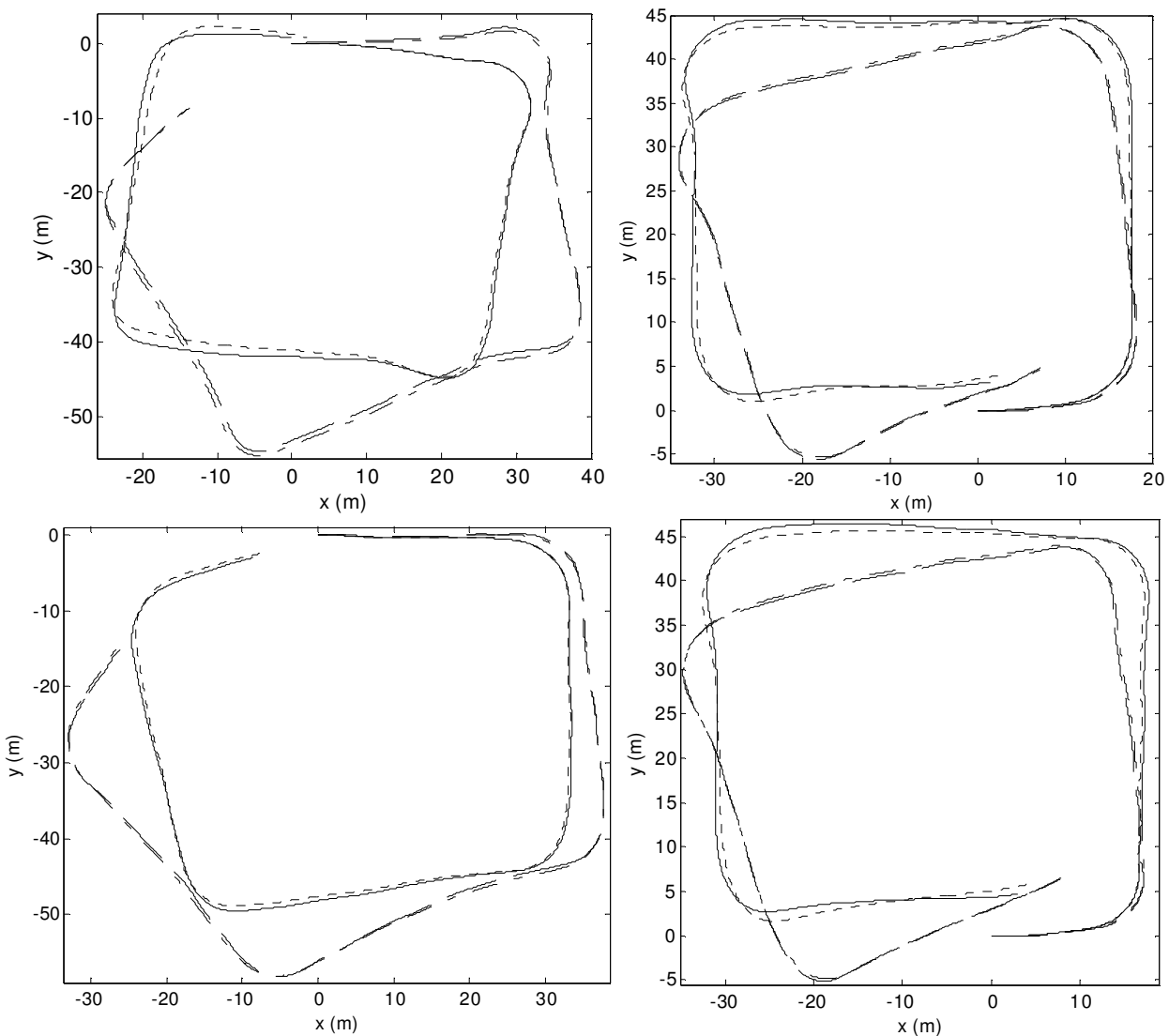

Figura 7.19- Caminos obtenidos con WLS (-), el FK1 (- -), el FK2 (- -) y el modelo diferencial $(-\cdot-)$ para distintos experimentos tipo rectángulo 


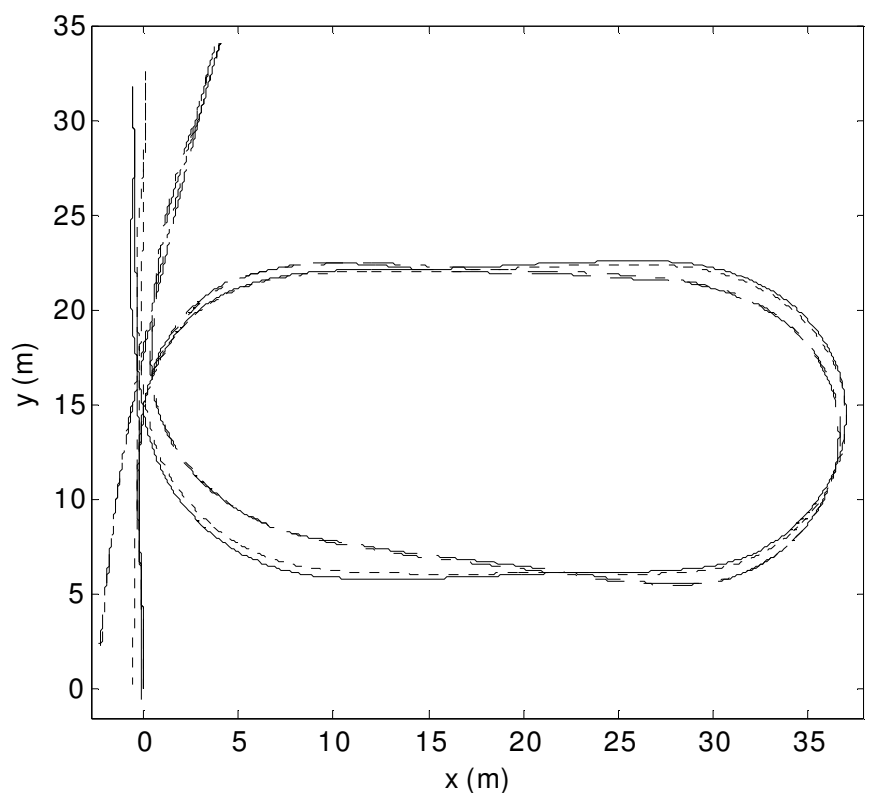

Figura 7.20- Caminos obtenidos con WLS (-), el FK1 (- -), el FK2 (- -) y el modelo diferencial $(-\cdot-)$ para el segundo tipo de camino en forma de tirabuzón

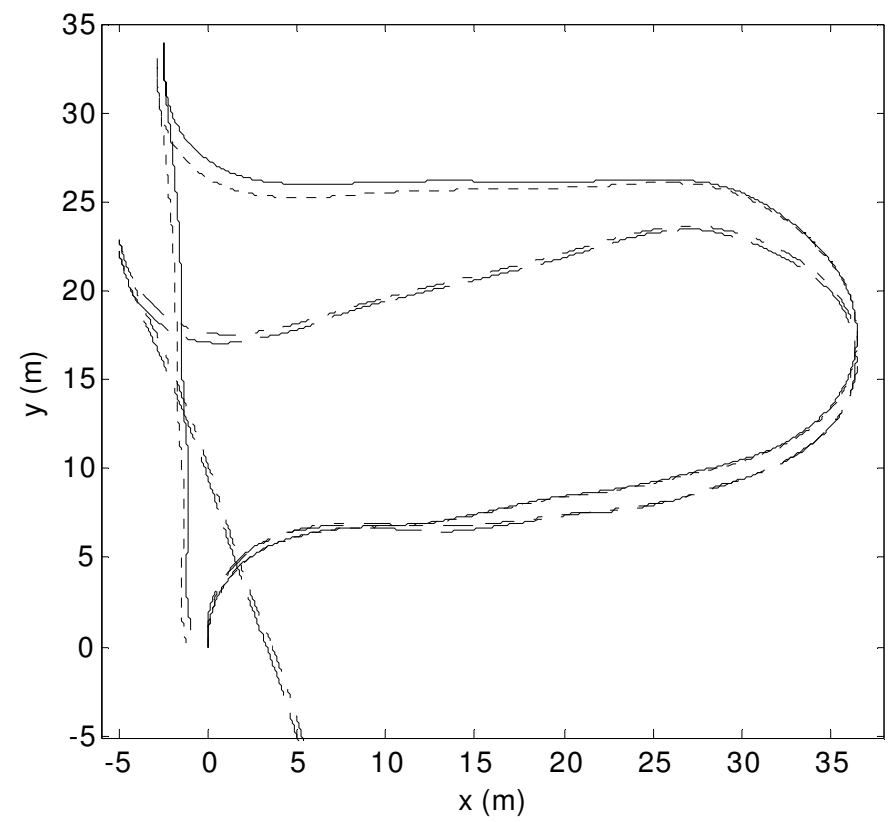

Figura 7.21- Caminos obtenidos con WLS (-), el FK1 (- -), el FK2 (- -) y el modelo diferencial $(-\cdot-)$ para el tercer tipo de camino en forma de $\mathrm{D}$ 


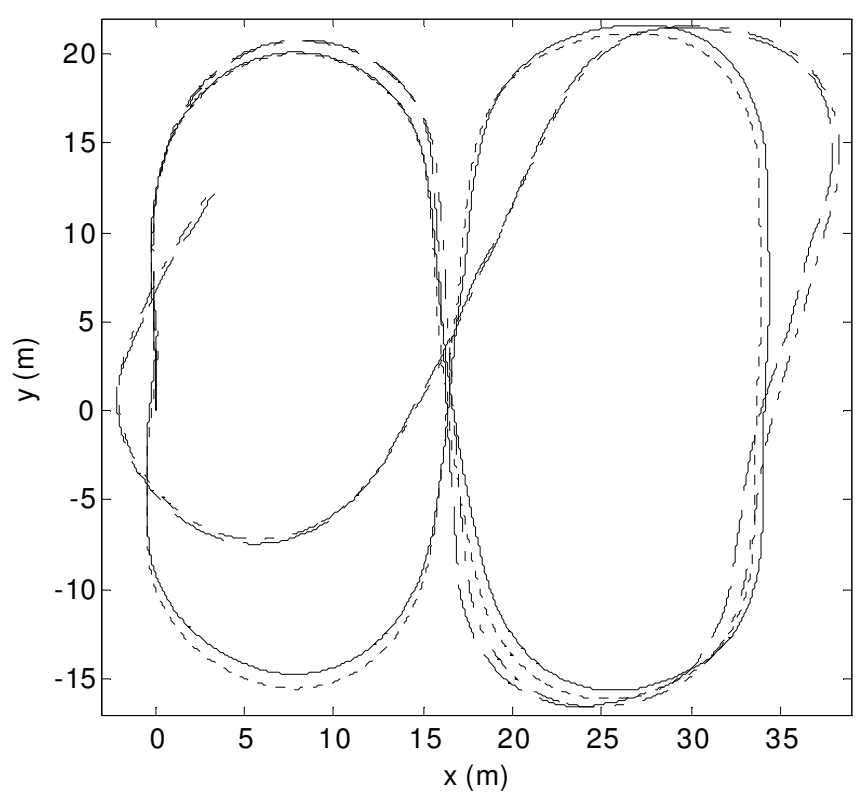

Figura 7.22- Caminos obtenidos con WLS (-), el FK1 (- -), el FK2 (- -) y el modelo diferencial (- - ) para el cuarto tipo de camino en forma de ocho

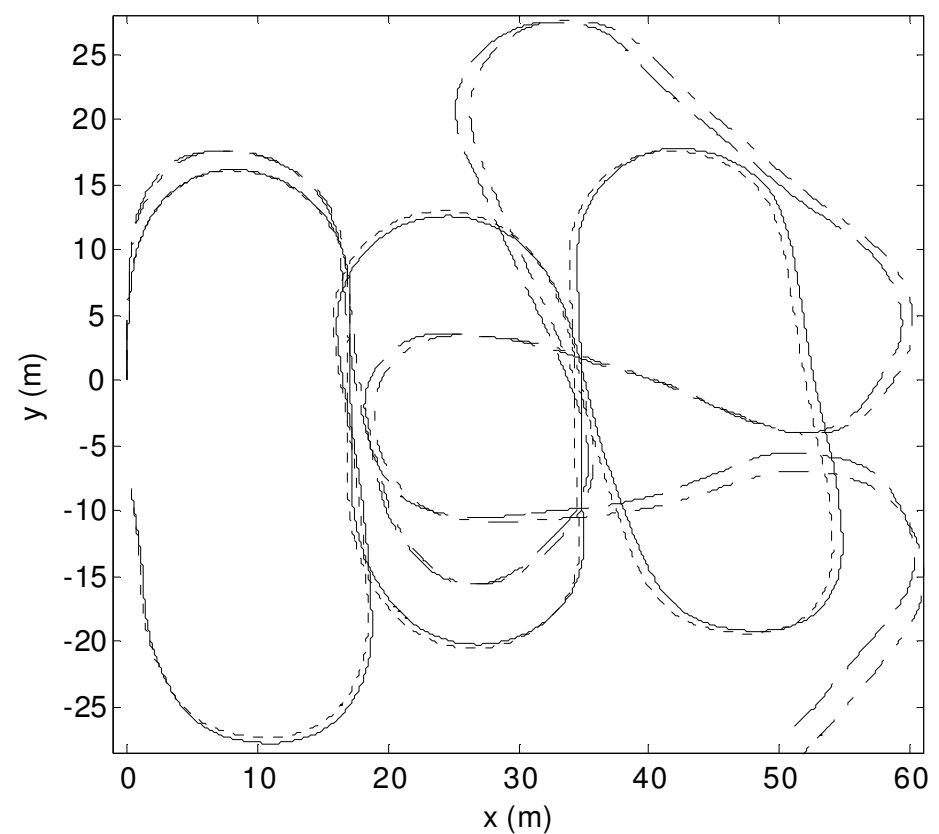

Figura 7.23- Caminos obtenido con WLS (-), el FK1 (- -), el FK2 (- -) y el modelo diferencial $(-\cdot-)$ para el quinto tipo de camino en forma de triple bucle 
La Figura 7.24 muestra el interfaz desarrollado (camino obtenido con WLS, indicadores, botones, etc.) con el software LabVIEW para el terminal táctil incorporado a la carretilla industrial.
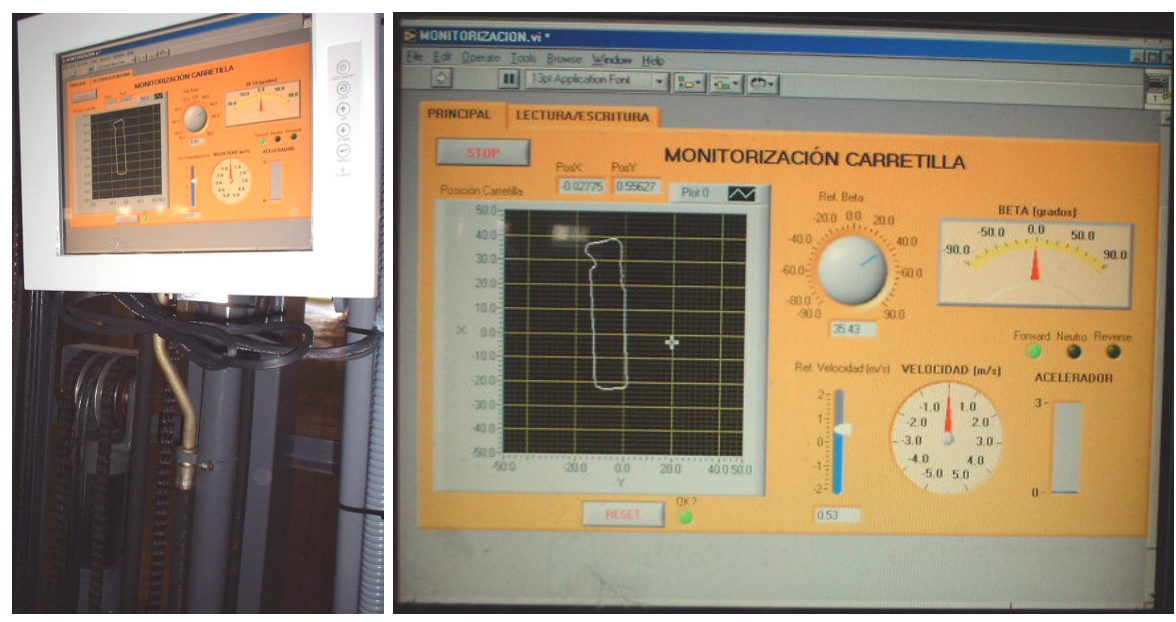

Figura 7.24- Interfaz del terminal táctil incorporado en la carretilla industrial

Como se ha comentado anteriormente, el FK2 resulta similar a WLS y el FK1 al modelo diferencial. Sin embargo, hay un aspecto que hace preferible WLS (7.66) frente al FK2 (7.71)-(7.75) y el modelo diferencial (7.79) frente al FK1 (7.71)-(7.75): el tiempo de cómputo. En la Tabla 7.3 se muestra una comparativa de las operaciones requeridas y tiempo de cómputo medido. En dicho tiempo de computo se aprecia que WLS cuesta un $16.5 \%$ menos que el FK2 y el modelo diferencial un $98.5 \%$ menos que el FK1.

Tabla 7.3- Comparativa de cómputo WLS-FK2 y modelo diferencial-FK1

\begin{tabular}{|c|c|c|c|c|}
\hline Parámetro & WLS & FK2 & Diferencial & FK1 \\
\hline Sumas & 30 & 131 & 3 & 102 \\
\hline Productos & 102 & 255 & 6 & 180 \\
\hline $\mathrm{N}^{\circ}$ Inversas & 1 de $3 \times 3$ & 1 de $4 \times 4$ & 0 & 1 de $3 \times 3$ \\
\hline $\begin{array}{c}\text { Tiempo Cómputo Iteración } \\
\text { (nanosegundos) }\end{array}$ & 46 & 55 & 0.8 & 51 \\
\hline
\end{tabular}




\subsection{RESULTADOS MÁS RELEVANTES Y CONCLUSIONES DEL CAPÍTULO}

A continuación, se indican los aspectos más relevantes que aporta el capítulo.

- La principal contribución de este capítulo son los tres niveles de modelos con deslizamiento presentados en el punto 7.4. A diferencia de otras investigaciones, estos modelos se han obtenido a partir de principios físicos, es decir de sucesivas aproximaciones de la dinámica del vehículo. Dicho modelo dinámico se ha obtenido considerando el caso general de vehículo con ruedas castor, no como en otros estudios, y teniendo en cuenta que el centro de masas de las ruedas centradas (orientables, fijas, suecas) permanece invariable respecto a la estructura del vehículo.

- El primer nivel de aproximación del planteamiento dinámico consiste en considerar un movimiento quasi-estático en el vehículo con respecto al sistema instantáneamente coincidente de la estructura del vehículo, que es equivalente a movimiento curvilíneo uniforme con una velocidad angular constante, además de desprecia la masa de las ruedas castor.

- El segundo nivel de aproximación desprecia la fuerza centrípeta requerida para el movimiento curvilíneo del vehículo, lo que es posible para maniobras suaves. El modelo obtenido es completamente cinemático: el modelo cinemático con deslizamiento.

- El tercer nivel de aproximación considera fuerzas de fricción linealmente dependientes de las velocidades de deslizamiento, lo que es posible para un movimiento de ruedas cercano al rodamiento puro, ej. en una solución directa con redundancia en la información de sensores. El modelo con deslizamiento resultante es equivalente al la solución de Mínimos Cuadrados ponderada de la cinemática sin deslizamiento del vehículo, donde las velocidades del vehículo deben considerarse no asignadas.

- La ventaja del tercer nivel de aproximación es que se calcula analíticamente, aunque es menos preciso debido a las tres aproximaciones. En cualquier caso, cualquiera de los tres modelos con deslizamiento se puede utilizar a través de cálculo numérico. 
- Los tres modelos con deslizamiento se han comparado con el modelo dinámico en un entorno de simulación para un vehículo triciclo. Dicha simulación ha evidenciando el grado de precisión de cada modelo.

- El tercer nivel de aproximación (solución de Mínimos Cuadrados ponderada) se compara, en una situación real para una carretilla industrial, con dos alternativas del Filtro de Kalman y con el modelo del vehículo diferencial. Para los datos experimentales el modelo con deslizamiento y uno de los dos filtros de Kalman dan claramente una mejor estimación del camino recorrido que el otro filtro y el modelo diferencial. Además se ha comprobado que el modelo con deslizamiento tiene un $16.5 \%$ menos de tiempo de computo por iteración que el filtro de Kalman equivalente. 


\section{A1 FÓRMULAS DE PACEJKA UTILIZADAS EN LA SIMULACIÓN}

La fórmula más utilizada para caracterizar la relación estática (curvas de estadofijo) entre los coeficientes de adhesión y el deslizamiento es la denominada fórmula mágica de Pacejka [Pacejka et al. 93].

La fórmula mágica de Pacejka es la indicada en (7.34):

$$
\mu_{a}=k_{7} \cdot \sin \left(k_{8} \cdot \arctan \left(k_{9} \cdot\left(1-k_{10}\right) \cdot a+k_{10} \cdot \arctan \left(k_{9} \cdot a\right)\right)\right)
$$

donde se tienen las variables y parámetros de la Tabla 7.4.

Tabla 7.4- Variables y parámetros de la fórmula mágica de Pacejka (7.81)

\begin{tabular}{|c|l|}
\hline Símbolo & \multicolumn{1}{c|}{ Descripción } \\
\hline$\mu_{a}=\mu_{y}$ ó $\mu_{x}$ & Coeficiente de adhesión longitudinal ó lateral \\
\hline$a=s$ ó $\alpha_{\mathrm{s}}$ & Ratio de deslizamiento longitudinal ó ángulo de deslizamiento \\
\hline$k_{7}$ & Factor de pico \\
\hline$k_{8}$ & Factor de forma \\
\hline$k_{9}$ & Factor de rigidez \\
\hline$k_{10}$ & Factor de curvatura \\
\hline
\end{tabular}

Los valores de los parámetros $k_{i}$ de la fórmula de Pacejka pueden ser constantes o función de otras variables: carga vertical, ángulo de peralte, etc.

Para el deslizamiento longitudinal puro de rueda en simulación se utilizarán las fórmulas y coeficientes identificados de [Lu et al. 05]:

$$
\begin{aligned}
& c_{s y}=\tan \left((\pi / 180) \cdot\left(84.21+5.16 \cdot \mathrm{e}^{-6.094 /\left(1+v_{\text {whecel }}, y\right.}\right)\right), \quad S=s+\left(0.0213+0.0086 \cdot v_{\text {wheel }}\right) / c_{s y} \\
& k_{8 y}=1.57,, k_{7 y}=0.8-0.0021 \cdot v_{\text {wheel } y}, k_{9 y}=c_{s y} /\left(k_{8 y} \cdot k_{7 y}\right), s_{m}=0.08+0.75 \cdot \mathrm{e}^{-v_{\text {whecl }} / / 8} \\
& k_{10 y}=\left(k_{9 y} \cdot s_{m}-\tan \left(0.5 \cdot \pi / k_{8 y}\right)\right) /\left(k_{9 y} \cdot s_{m}-\operatorname{atan}\left(k_{9 y} \cdot s_{m}\right)\right)-0.0162 \cdot v_{\text {wheel } y} \\
& \mu_{y}=k_{7 y} \cdot \sin \left(k_{8 y} \cdot \arctan \left(k_{9 y} \cdot\left(1-k_{10 y}\right) \cdot S+k_{10 y} \cdot \arctan \left(k_{9 y} \cdot S\right)\right)\right)
\end{aligned}
$$

La Figura 7.25 muestra el coeficiente longitudinal, con deslizamiento longitudinal puro, para una velocidad de rueda $v_{\text {wheel } y}$ entre 1 y $18 \mathrm{~m} / \mathrm{s}$. 


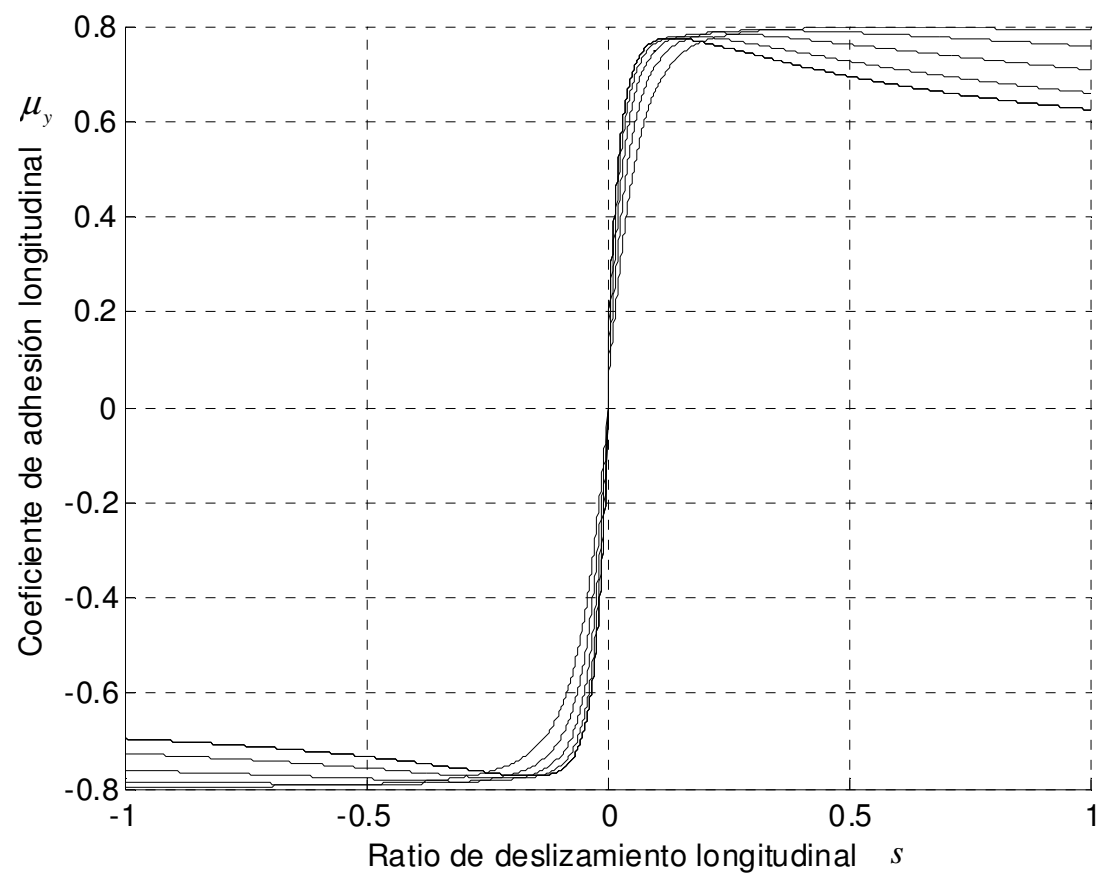

Figura 7.25- Coeficiente longitudinal en deslizamiento longitudinal puro y velocidad longitudinal de rueda $v_{\text {wheel } y}$ entre 1 y $18 \mathrm{~m} / \mathrm{s}$

Para el deslizamiento lateral puro de rueda en simulación se utilizarán las fórmulas y coeficientes identificados de [Lu et al. 05]:

$$
\begin{aligned}
& c_{s x}=0.3 \cdot \tan \left(\frac{\pi}{180} \cdot\left(84.21+5.16 \cdot \mathrm{e}^{\left.\frac{-6.094}{\left(1+5 \cdot v_{\text {whel }} y\right.}\right)}\right)\right), \quad \bar{\alpha}_{\mathrm{s}}=\alpha_{\mathrm{s}}+\frac{\left(0.00639+0.0026 \cdot v_{\text {wheel }}\right)}{c_{s x}} \\
& k_{8 x}=1.2, k_{7 x}=0.75+0.5 \cdot \mathrm{e}^{-v_{\text {whedel }} / 3}, k_{9 x}=c_{s x}\left(k_{8 x} \cdot k_{7 x}\right), \alpha_{m}=0.087+0.63 \cdot \mathrm{e}^{-v_{\text {wheal }} / 3} \\
& k_{10 x}=\left(k_{9 x} \cdot \alpha_{m}-\tan \left(0.5 \cdot \pi / k_{8 x}\right)\right) /\left(k_{9 x} \cdot \alpha_{m}-\operatorname{atan}\left(k_{9 x} \cdot \alpha_{m}\right)\right)-0.0162 \cdot v_{\text {wheel } y} \\
& \mu_{x}=k_{7 x} \cdot \sin \left(k_{8 x} \cdot \arctan \left(k_{9 x} \cdot\left(1-k_{10 x}\right) \cdot \bar{\alpha}_{\mathrm{s}}+k_{10 x} \cdot \arctan \left(k_{9 x} \cdot \bar{\alpha}_{\mathrm{s}}\right)\right)\right)
\end{aligned}
$$

La Figura 7.26 muestra el coeficiente lateral obtenido, con deslizamiento lateral puro, para una velocidad de rueda $v_{\text {wheel } y}$ entre 1 y $9 \mathrm{~m} / \mathrm{s}$. 


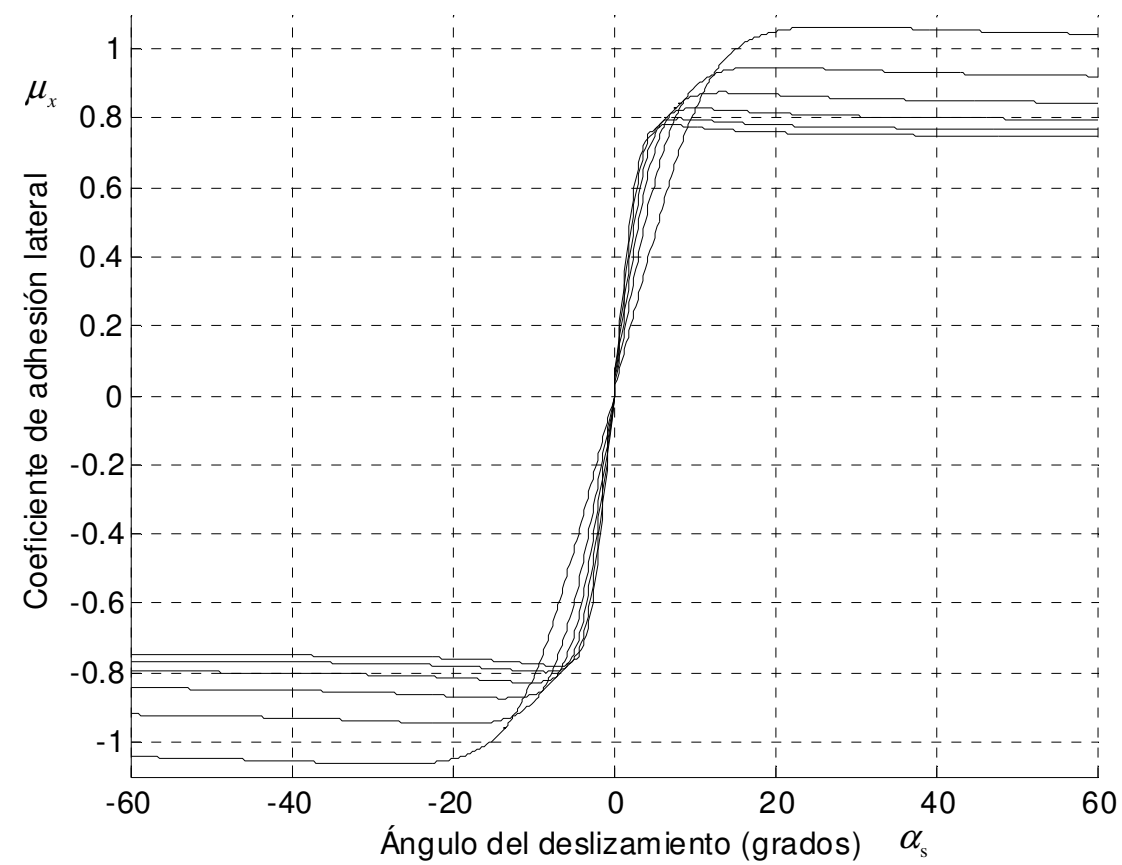

Figura 7.26- Coeficiente lateral en deslizamiento lateral puro y velocidad longitudinal de rueda $v_{\text {wheel } y}$ entre 1 y $9 \mathrm{~m} / \mathrm{s}$

Para el deslizamiento combinado hay que tener en cuenta el acoplamiento de los coeficientes longitudinal y lateral. Por ejemplo, un método sencillo de acoplamiento de coeficientes se presenta en [Pacejka 02].

Otro método de acoplamiento, que es el que aquí se utilizará, se describe en [Pacejka et al. 97] ("Delft tyre 97" parte steady-state). Sus fórmulas de acoplamiento con los coeficientes identificados en [Lu et al. 05] son:

$$
\begin{aligned}
& \mu_{y \text { comb }}=\mu_{y} \cdot \frac{\cos \left(1.43 \cdot \operatorname{atan}\left(1.25 \cdot \cos (\operatorname{atan}(0.513 \cdot s)) \cdot\left(0.0056+\alpha_{\mathrm{s}}\right)\right)\right)}{\cos (1.43 \cdot \operatorname{atan}(1.25 \cdot \cos (\operatorname{atan}(0.513 \cdot s)) \cdot 0.0056))} \\
& \mu_{x \text { comb }}=\mu_{x} \cdot \frac{\cos \left(1.01 \cdot \operatorname{atan}\left(20.18 \cdot \cos \left(\operatorname{atan}\left(0.137 \cdot\left(\alpha_{\mathrm{s}}-4.98\right)\right)\right) \cdot\left(0.204 \cdot \mu_{y}+s\right)\right)\right)}{\cos \left(1.01 \cdot \operatorname{atan}\left(20.18 \cdot \cos \left(\operatorname{atan}\left(0.137 \cdot\left(\alpha_{\mathrm{s}}-4.98\right)\right)\right) \cdot 0.204 \cdot \mu_{y}\right)\right)}
\end{aligned}
$$

En las Figuras 7.27, 7.28 7.29 y 7.30 se muestran los coeficientes de adhesión para deslizamiento combinado y velocidad longitudinal $v_{\text {wheel } y}$ de $2 \mathrm{~m} / \mathrm{s}$. 


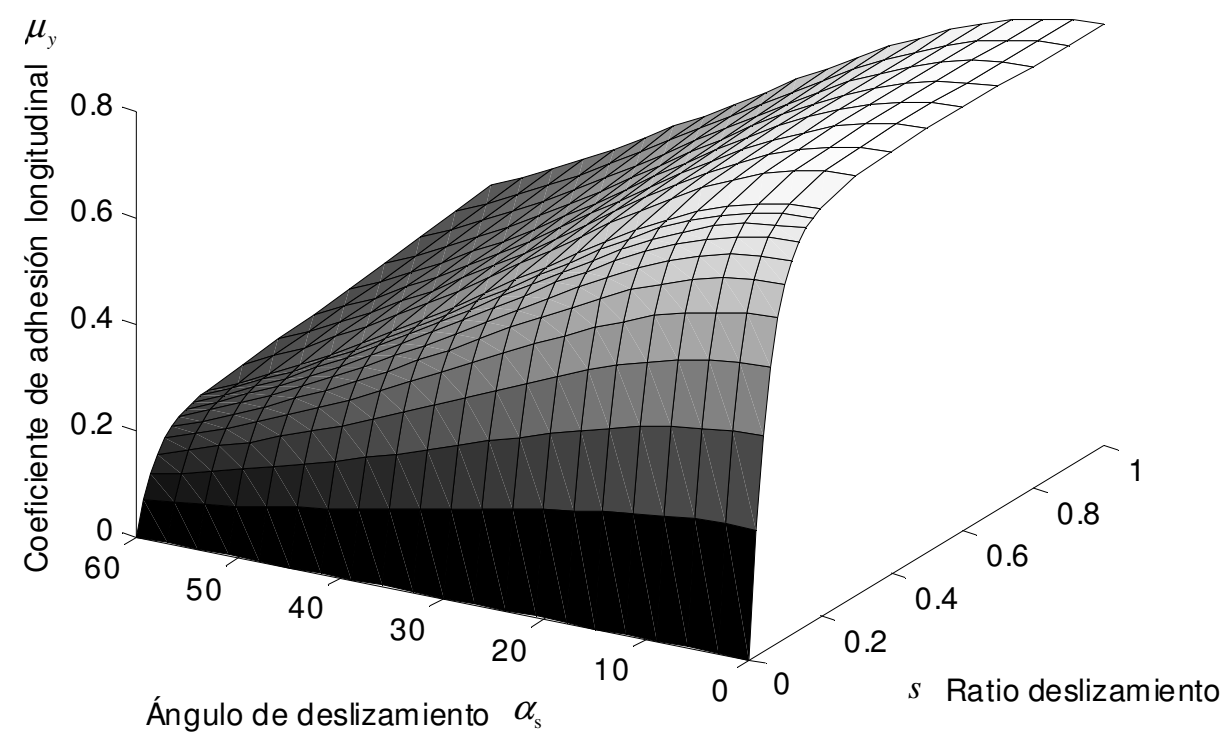

Figura 7.27- Coeficiente de adhesión longitudinal en deslizamiento combinado

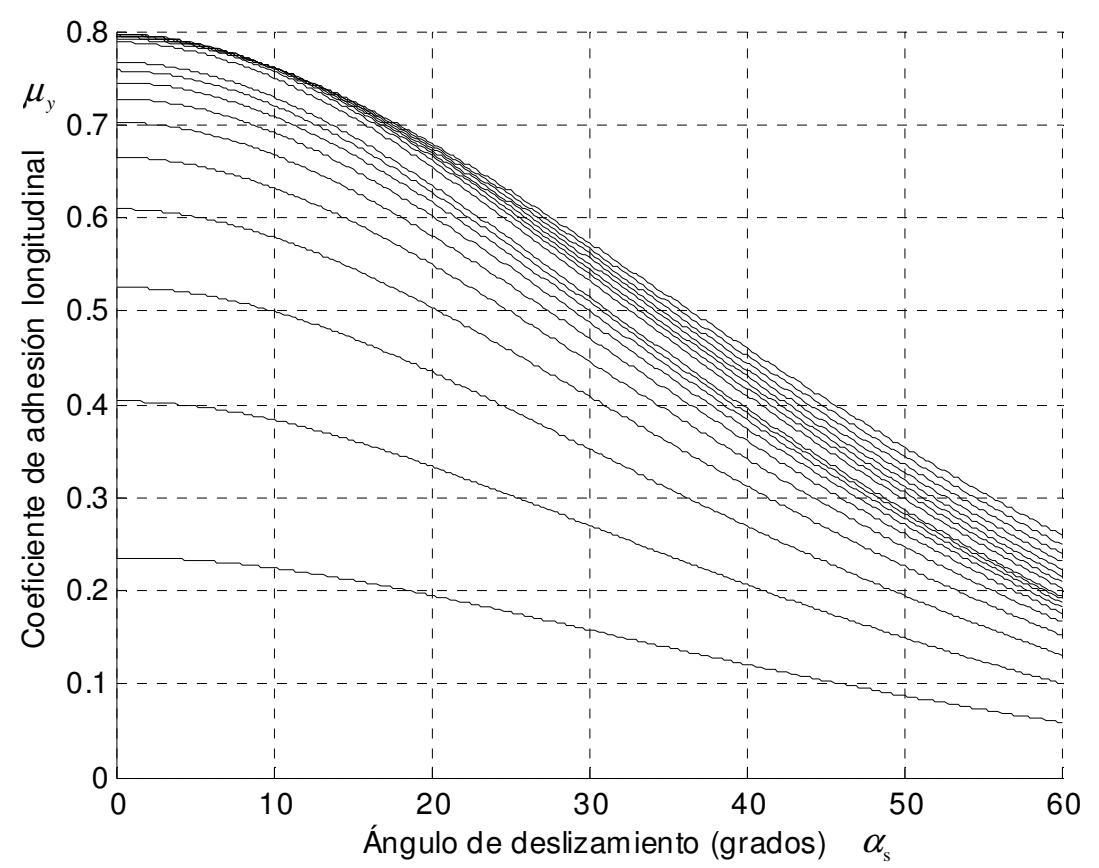

Figura 7.28- Proyección de las curvas de nivel del coeficiente de adhesión longitudinal en deslizamiento combinado respecto al ángulo de deslizamiento 


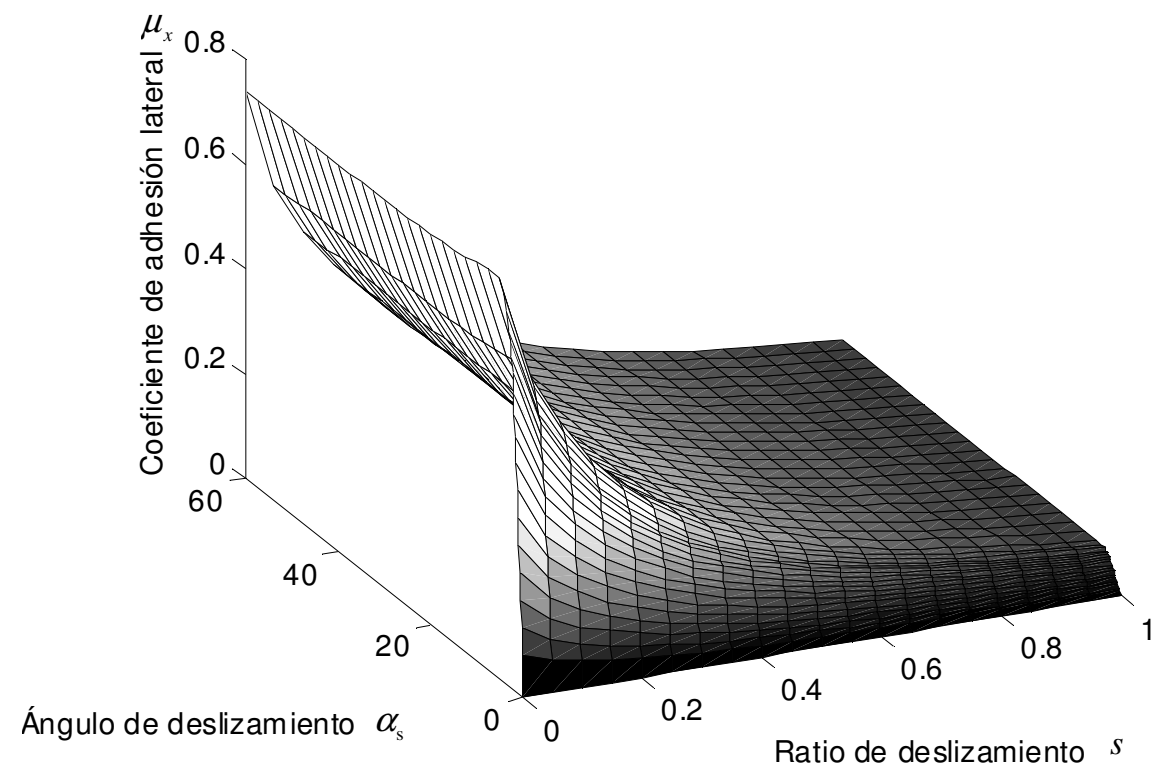

Figura 7.29- Coeficiente de adhesión lateral en deslizamiento combinado

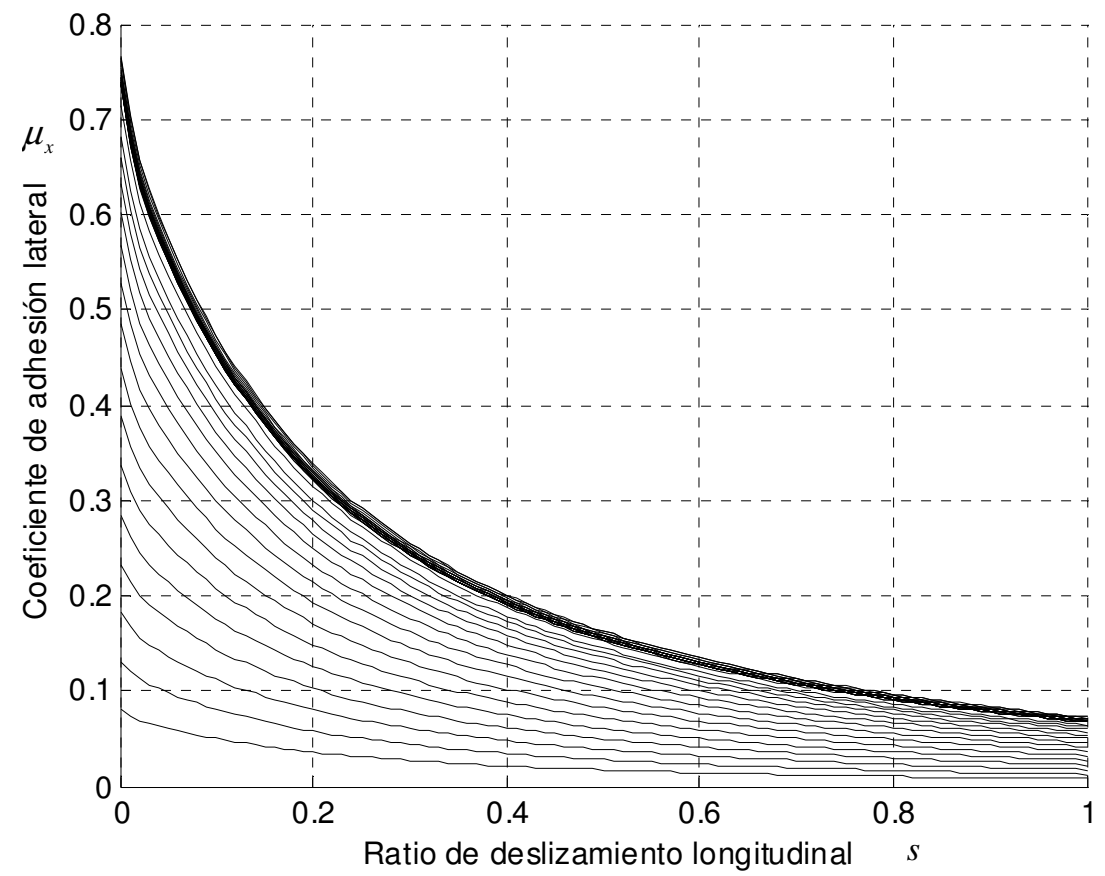

Figura 7.30- Proyección de las curvas de nivel del coeficiente de adhesión lateral en deslizamiento combinado respecto al ratio de deslizamiento 


\section{CAPÍTULO 8 \\ CONTROL CINEMÁTICO DE VEHÍCULOS CON RUEDAS}

\subsection{INTRODUCCIÓN}

Los vehículos autoguiados, utilizados en la automatización de procesos industriales u otros campos como la agricultura, se encuentran involucrados principalmente en el seguimiento de referencias, siendo imprescindible para ello un control adecuado del vehículo.

Para el diseño del control existen alternativas tan diversas como las desarrolladas en [Ollero et al. 94] [Shin 90] [O'Connor et al. 96] [Inoue et al. 97] [Lyshevski et al. 00] [Samson 95], entre muchas otras.

Se puede distinguir entre un control del vehículo de tipo cinemático o dinámico (dinámico global). En el cinemático se consideran dos bucles, uno principal denominado cinemático y otro de bajo nivel para los actuadores en las ruedas, evidentemente dinámico. De forma que hay un desacoplo entre la parte cinemática y la parte dinámica.

En el control dinámico global no existen los dos niveles/bucles anteriores, sino que la ley de control es única y considera todos los elementos del vehículo: relaciones cinemáticas, actuadores, masas, momentos de inercias, etc.

En general el control dinámico global presenta como inconvenientes: el análisis y cálculo adquieren una complejidad considerable; es muy sensible a las imprecisiones de los parámetros del modelo; a veces requiere sensores inerciales, 
que tienen alto coste, poca robustez y baja precisión, o estimadores de similares inconvenientes.

Mientras que el control cinemático es más sencillo y tiene validez siempre que el bucle dinámico de bajo nivel sea bastante más rápido que el cinemático. Este control es el más empleado en la bibliografía.

La referencia de entrada al control del vehículo, generadas normalmente por un planificador de alto nivel, puede tener 2 dimensiones (coordenadas $x$ e $y$ de posición) ó 3 (coordenadas $x$ e $y$ de posición y $\theta$ de orientación). Además las referencias pueden tener asociados instantes temporales (ej. trayectoria 2D) o no (ej. camino 2D), en cuyo caso se suele suponer una velocidad de avance en el movimiento constante.

Algunos autores han planteado el control del vehículo con métodos geométricos, aplicando acciones de control que hagan que el vehículo siga una curva que conecte su posición con una posición objetivo perteneciente a la trayectoria de referencia. Por ejemplo [Ollero et al. 94] utiliza como curvas arcos circulares mientras que [Shin 90] utiliza polinomios de quinto orden. Este método obliga a un seguimiento de la trayectoria punto a punto (persecución), siendo complicado garantizar la estabilidad. Además es difícil el ajuste óptimo, en función de la trayectoria, de algunos parámetros de la 'persecución' para conseguir un buen seguimiento de la misma.

Otros autores han utilizado herramientas de la teoría clásica de control:

- Control basado en aproximación lineal: Consiste en aproximar linealmente el modelo del vehículo entorno a un punto de funcionamiento y posteriormente diseñar un control lineal clásico. Incluso se puede discretizar el sistema aproximado lineal y hacer control discreto. El inconveniente de esta aproximación lineal es la perdida de validez del control diseñado a medida que el estado se aleja del punto de funcionamiento, pudiendo llegar a la inestabilidad. Para el control cinemático de vehículos el rango de validez suele ser muy pequeño. Por ejemplo en [O'Connor et al. 96] se realiza un control de cinemático de dirección basado en una aproximación lineal en torno $0^{\circ}$. Algunos controles similares se proponen en [Canudas et al. 97]. 
- Linealización exacta por realimentación del estado: Si existe, la linealización exacta del modelo del vehículo (continuo o discreto) permite aplicar un control lineal clásico (punto a punto, de trayectoria,...). Tiene el inconveniente de que las singularidades invalidan la linealización, aunque si no existen o no se alcanzan nunca es una opción muy adecuada. Esta herramienta se ha aplicado de forma cinemática en [d'Andréa-Novel et al. 95] [De Luca et al. 93] [Gracia2 et al. 03] [Park et al. 99] y dinámica global en [Tzafestas et al. 01].

- Controlabilidad: Algunos autores han estudiado la controlabilidad de distintos vehículos [Campion et al. 96] y sus condiciones para diseñar leyes de control [Samson 95] [Monaco et al. 91] [Murray et al. 93].

- Estabilidad por método de Lyapunov: Para el modelo no lineal del vehículo (continuo o discreto) es difícil encontrar una ley de control, en función de las magnitudes de error, que garantice la estabilidad del sistema. Una forma de garantizar la estabilidad es encontrar una función de Lyapunov que, para el algoritmo de control diseñado, cumpla el teorema de estabilidad de Lyapunov. Por ejemplo en [Lyshevski et al. 00] se realiza un control dinámico global continuo para el que existe dicha función. Igualmente, en [Canudas et al. 97] y [Dixon et al. 00] se presentan leyes de control demostrándose su estabilidad por este método.

- Control adaptativo: Por ejemplo en [Inoue et al. 97] se hace un control cinemático discreto adaptativo ajustando unos parámetros del algoritmo de control experimentalmente para que sea estable. Mientras que en [Dixon et al. 01] se realiza un control adaptativo para seguimiento con un sistema de visión sin calibrar. En [Fukao et al. 00] se propone una extensión, en forma de control adaptativo, para un controlador existente de vehículo no-holonómicos.

- Control predictivo: En [Ollero et al. 91] se presenta el seguimiento de caminos para el modelo bicicleta con control predictivo generalizado.

Así pues, el objetivo de este capítulo es proporcionar un método de control cinemático que anule el error en el seguimiento de referencias (con instante temporal asociado) para todo tipo de vehículos. 
En el punto 8.2 se plantea un esquema de control general que es similar conceptualmente a los empleados para robots manipuladores y que incluye tres bucles de control anidados: uno dinámico de bajo nivel, otro cinemático de nivel medio (en el que se profundiza posteriormente) y un último de planificación de alto nivel.

El bucle de control cinemático se desglosa en un control de posición (punto 8.3) y en la aplicación de la cinemática inversa de ruedas (punto 8.4), cuyo análisis permite caracterizar las referencias que puede seguir sin error cada tipo de vehículo (punto 8.5).

En el punto 8.6 se particulariza el control cinemático para el caso del vehículo triciclo y se comprueban a través de simulación sus restricciones y limitaciones. También se presentan algunos resultados experimentales obtenidos para una carretilla industrial sobre la cual se ha implementado el control anterior.

Finalmente en el punto de conclusiones se destacan los resultados más relevantes del capítulo. 


\subsection{ESQUEMA GLOBAL DEL CONTROL DEL VEHÍCULO}

En la Figura 8.1 se muestra el esquema general propuesto para el control del vehículo, donde se tienen las siguientes variables:

$\mathbf{p}=(x, y, \theta):$ Postura del vehículo;

$\dot{\mathbf{p}}=\left(v_{x}, v_{y}, \omega\right)$ : Vector de velocidad del vehículo;

$\mathbf{p}_{\text {ref: }}$ Postura de referencia establecida por el planificador;

$\dot{\mathbf{p}}_{\text {control }}:$ Vector de velocidad del vehículo a conseguir por el control dinámico;

$\dot{\varphi}_{i \text { ref }}:$ Velocidad de rotación a conseguir por control dinámico;

$\dot{\beta}_{i \text { ref }}:$ Velocidad de dirección a conseguir por control dinámico;

$\beta_{i \text { ref }}$ : Orientación de rueda orientable a conseguir por control dinámico;

$V_{i}$ : Tensión aplicada al actuador $i$ (calculadas por el control dinámico);

$\tau_{i}:$ Par aplicado por el actuadores $i$ (motores);

$\dot{\varphi}_{i \mathrm{~s}}:$ Velocidad de rotación sensorizada;

$\dot{\beta}_{i \mathrm{~s}}$ : Velocidad de dirección sensorizada;

$\beta_{i \mathrm{~s}}$ : Orientación sensorizada de rueda.

$\beta_{i \text { castor }}$ : Orientación de rueda castor con alguna velocidad de rueda accionada.

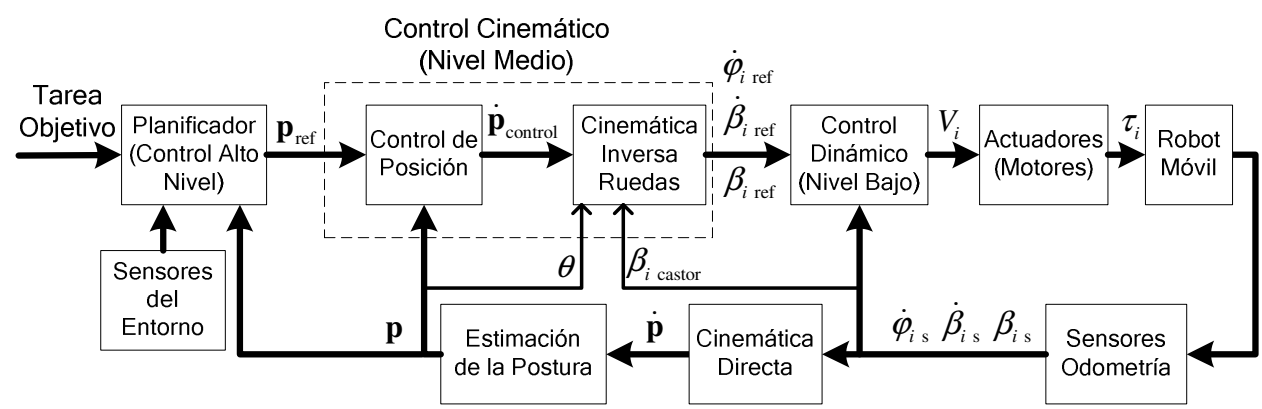

Figura 8.1: Esquema general de control del vehículo

El esquema anterior es similar conceptualmente a los empleados para robots manipuladores e incluye tres bucles de control anidados: uno dinámico de bajo nivel, otro cinemático de nivel medio (en el cual se profundiza en este capítulo) y un último de planificación de alto nivel. 
Para garantizar la estabilidad del conjunto, el bucle de control dinámico debería ser bastante más rápido que el cinemático y el cinemático que el de planificación.

El control dinámico de bajo nivel tendría en cuenta las masas, momentos de inercia, pares y fuerzas para generar los niveles de tensión de entrada a los actuadores (motores). Lo anterior podría hacerse de forma no acoplada: control individual de los actuadores sin tener en cuenta la influencia entre ellos (ideal); ó acoplada: control global del vehículo teniendo en cuenta interacción entre actuadores.

Tanto la postura de referencia del vehículo $\mathbf{p}_{\text {ref }}$ como el vector de velocidad de control $\dot{\mathbf{p}}_{\text {control }}$ pueden ser completos, asignándose las tres referencias de la postura, o limitado a dos componentes para vehículos de movimiento restringido.

El planificador de alto nivel debe especificar las referencias a seguir por el vehículo teniendo en cuenta: la tarea objetivo a realizar (postura destino o trayectoria deseada,...); las referencias posibles según tipo de vehículo (punto 8.5); la evitación de colisiones, utilizando para ello los sensores del entorno (globales y locales) y la postura estimada; las configuraciones isotrópicas y singulares de los Capítulos 5 y 6; etc.

La cinemática directa consiste en obtener el vector de velocidad del vehículo $\dot{\mathbf{p}}$ a partir de las velocidades de rueda sensorizada, pudiéndose utilizar los modelos cinemáticos con deslizamiento del Capítulo 7.

La estimación de la postura del vehículo p puede realizarse simplemente integrando el vector de velocidad del vehículo $\dot{\mathbf{p}}$ ó mediante el filtro extendido de Kalman, muy útil cuando hay otros sensores a parte de los odométricos (fusión sensorial) [Tham et al. 98] [Scheding et al. 99] [Lindgren et al. 02].

El número de actuadores a utilizar viene marcado por el criterio de actuación consistente (incompatibilidad imposible) del Capitulo 4 y por la necesidad de accionar la dirección de las ruedas orientables (las velocidades de rueda $\left\{\dot{\varphi}_{i}, \dot{\beta}_{i}, \dot{\varphi}_{\mathrm{r} i}\right\}$ se auto-ajustan, en caso de no estar actuadas, siempre que el rozamiento sea suficiente). En definitiva el número de actuadores coincidirá con la maniobrabilidad del vehículo (Capítulo 5) = grado de movilidad (GDLs instantáneos) + grado de direccionabilidad ( $\mathrm{n}^{\circ}$ de ruedas orientables). 


\subsection{CONTROL DE POSICIÓN}

El control de posición del esquema de la Figura 8.1 consiste en establecer el vector de velocidad del vehículo $\dot{\mathbf{p}}$ a conseguir por el control dinámico (vector de velocidad de control $\dot{\mathbf{p}}_{\text {control }}$ ) en función de las referencias de postura $\mathbf{p}$ suministradas por el planificador de alto nivel.

Se plantean los siguientes tipos de control:

$$
\begin{gathered}
\dot{\mathbf{p}}_{\text {control }}=\mathbf{A}_{\mathrm{c}} \cdot\left(\mathbf{p}_{\text {ref }}-\mathbf{p}\right) \\
\dot{\mathbf{p}}_{\text {control }}=\dot{\mathbf{p}}_{\text {ref }}+\mathbf{A}_{\mathrm{c}} \cdot\left(\mathbf{p}_{\text {ref }}-\mathbf{p}\right) \\
\dot{\mathbf{p}}_{\text {control }}=\dot{\mathbf{p}}_{\text {ref }}+\mathbf{A}_{\mathrm{c}} \cdot\left(\mathbf{p}_{\text {ref }}-\mathbf{p}\right)+\mathbf{B}_{\mathrm{c}} \cdot \int\left(\mathbf{p}_{\text {ref }}-\mathbf{p}\right)
\end{gathered}
$$

donde $\mathbf{A}_{\mathrm{c}}$ y $\mathbf{B}_{\mathrm{c}}$ son matrices diagonales de dimensión 3.

Suponiendo que el vector de velocidad $\dot{\mathbf{p}}$ converge al de velocidad de control $\dot{\mathbf{p}}_{\text {control }}$, la dinámica de cada control viene dada por:

$$
\begin{gathered}
\frac{\mathrm{d} \mathbf{p}}{\mathrm{d} t}+\mathbf{A}_{\mathrm{c}} \cdot\left(\mathbf{p}-\mathbf{p}_{\text {ref }}\right)=\mathbf{0} \\
\frac{\mathrm{d}\left(\mathbf{p}-\mathbf{p}_{\text {ref }}\right)}{\mathrm{d} t}+\mathbf{A}_{\mathrm{c}} \cdot\left(\mathbf{p}-\mathbf{p}_{\text {ref }}\right)=\mathbf{0} \\
\frac{\mathrm{d}^{2}\left(\mathbf{p}-\mathbf{p}_{\text {ref }}\right)}{\mathrm{d}^{2} t}+\mathbf{A}_{\mathrm{c}} \cdot \frac{\mathrm{d}\left(\mathbf{p}-\mathbf{p}_{\text {ref }}\right)}{\mathrm{d} t}+\mathbf{B}_{\mathrm{c}} \cdot\left(\mathbf{p}-\mathbf{p}_{\text {ref }}\right)=\mathbf{0}
\end{gathered}
$$

El control de (8.1) se denomina punto a punto y tiene error en régimen permanente no nulo (8.4) para referencias de tipo velocidad e infinito para las de tipo aceleración, por lo que no es conveniente en nuestro caso.

Los controles de (8.2) y (8.3) se denominan de trayectoria debido a la prealimentación derivativa* (primer sumando del segundo miembro) y tienen error en régimen permanente nulo (8.5) (8.6) para cualquier referencia continua.

*Nota: Una opción es no considerar la prealimentación derivativa hasta que el error entre dentro de un margen, ya que si el punto de seguimiento está muy alejado de la referencia la prealimentación pierde su sentido.

La diferencia entre ambos controles es que (8.2) aplica una realimentación proporcional y converge siempre de forma asintótica, mientras que (8.3) aplica una realimentación proporcional-integral y converge en general de forma 
oscilante. Además, a nivel práctico el de (8.3) permite corregir la posible zona muerta de los actuadores (motores) si la hubiere.

En principio en nuestro caso es suficiente con el control de (8.2), siempre y cuando la zona muerta de los actuadores sea pequeña. En ese caso los polos (dinámica del error) se asignan para cada coordenada con los elementos $a_{\mathrm{c}}$ de la diagonal de $\mathbf{A}_{\mathrm{c}}$.

En ocasiones el control debe realizarse de forma discreta, ej. en la aplicación de seguimiento de línea por visión del Capítulo 9 debido al procesamiento de imagen. En esos casos la dinámica asignada debe ser bastante más lenta que el periodo de control $\mathrm{T}$ para no tener problemas de estabilidad (Teorema de Shannon-Nyquist), por ejemplo $1 / a_{\mathrm{c}} \geq 10 \cdot \mathrm{T}$.

Para el resto del capítulo se considerará que el seguimiento de las referencias (posición y orientación) las realiza el sistema de coordenadas $\mathrm{R}$ del vehículo: en concreto su origen y orientación respecto al sistema global G.

Por tanto el modelado cinemático del vehículo, para aplicar después el control, tendrá en cuenta la elección de R con la consideración anterior. 


\subsection{MODELO CINEMÁTICO INVERSO DE RUEDA}

El modelo cinemático inverso de rueda es aquel que toma como entrada el vector de velocidad del vehículo $\dot{\mathbf{p}}$, respecto al sistema $\overline{\mathrm{R}}$ o $\mathrm{G}$, y obtiene las velocidades de la rueda $\dot{\mathbf{q}}_{\mathrm{wi}}$ y la orientación $\beta_{i}$ si la rueda es orientable.

Para el esquema de control de la Figura 8.1, dicho vector de velocidad de entrada será el de control $\dot{\mathbf{p}}_{\text {control }}$ generado por el control de posición.

Los valores de velocidades de rueda obtenidos $\dot{\mathbf{q}}_{\mathrm{wi}}$ con la cinemática inversa pueden ser utilizados o no para realizar el control dinámico de la Figura 8.1. Si no se utilizan se auto-ajustan siempre que el deslizamiento sea suficiente.

Sin embargo el valor de $\beta$, dado por la cinemática inversa, para el caso de rueda orientable, hay que utilizarlo en el control dinámico de la dirección ya que no se auto-ajusta.

\subsubsection{Rueda orientable sin ruedas fijas}

Se parte de la ecuación de rueda de (3.11) con deslizamiento nulo:

$$
\left(\begin{array}{cccc}
\mathrm{c} \beta_{i} & \mathrm{~s} \beta_{i} & 1_{i} \cdot \mathrm{s}\left(\beta_{i}-\alpha_{i}\right) & 0 \\
-\mathrm{s} \beta_{i} & \mathrm{c} \beta_{i} & 1_{i} \cdot \mathrm{c}\left(\beta_{i}-\alpha_{i}\right) & \mathrm{r}_{i}
\end{array}\right) \cdot\left(\begin{array}{c}
\overline{\mathrm{R}} \dot{\mathbf{p}} \\
\dot{\varphi}_{i}
\end{array}\right)=\mathbf{0}
$$

Despejando del primer elemento de la expresión anterior $\beta_{i}$ :

$$
\begin{gathered}
{ }^{\overline{\mathrm{R}}} v_{x} \cdot \mathrm{c} \beta_{i}+{ }^{\overline{\mathrm{R}}} v_{y} \cdot \mathrm{s} \beta_{i}+\omega \cdot 1_{i} \cdot \mathrm{s}\left(\beta_{i}-\alpha_{i}\right)=0 \\
{ }^{\overline{\mathrm{R}}} v_{x} \cdot \mathrm{c} \beta_{i}+{ }^{\overline{\mathrm{R}}} v_{y} \cdot \mathrm{s} \beta_{i}+\omega \cdot 1_{i} \cdot\left(\mathrm{s} \beta_{i} \cdot \mathrm{c} \alpha_{i}-\mathrm{c} \beta_{i} \cdot \mathrm{s} \alpha_{i}\right)=0 \\
{ }^{\overline{\mathrm{R}}} v_{x}+{ }^{\overline{\mathrm{R}}} v_{y} \cdot \tan \left(\beta_{i}\right)+\omega \cdot 1_{i} \cdot\left(\tan \left(\beta_{i}\right) \cdot \mathrm{c} \alpha_{i}-\mathrm{s} \alpha_{i}\right)=0 \\
\beta_{i}=\arctan \left(\frac{-{ }^{\overline{\mathrm{R}}} v_{x}+\omega \cdot 1_{i} \cdot \mathrm{s} \alpha_{i}}{{ }^{\overline{\mathrm{R}}} v_{y}+\omega \cdot 1_{i} \cdot \mathrm{c} \alpha_{i}}\right)
\end{gathered}
$$

Pasando las velocidades lineales del vehículo al sistema G con (3.15):

$$
\beta_{i}=\arctan \left(\frac{-{ }^{\mathrm{G}} v_{x} \cdot \mathrm{c} \theta-{ }^{\mathrm{G}} v_{y} \cdot \mathrm{s} \theta+\omega \cdot 1_{i} \cdot \mathrm{s} \alpha_{i}}{-{ }^{\mathrm{G}} v_{x} \cdot \mathrm{s} \theta+{ }^{\mathrm{G}} v_{y} \cdot \mathrm{c} \theta+\omega \cdot 1_{i} \cdot \mathrm{c} \alpha_{i}}\right)
$$

donde $\theta \equiv{ }^{\mathrm{G}} \theta_{\mathrm{R}}=\int \omega$. 
Por otra parte del segundo elemento de (8.7):

$$
\begin{aligned}
& \dot{\varphi}_{i}=-\left(1 / \mathrm{r}_{i}\right) \cdot\left(-\mathrm{s} \beta_{i} \quad \mathrm{c} \beta_{i} \quad 1_{i} \cdot \mathrm{c}\left(\beta_{i}-\alpha_{i}\right)\right) \cdot{ }^{\overline{\mathrm{p}}} \dot{\mathbf{p}} \\
& \dot{\varphi}_{i}=-\left(1 / \mathrm{r}_{i}\right) \cdot\left(-\mathrm{s} \beta_{i} \quad \mathrm{c} \beta_{i} \quad 1_{i} \cdot\left(\mathrm{c} \beta_{i} \cdot \mathrm{c} \alpha_{i}+\mathrm{s} \beta_{i} \cdot \mathrm{s} \alpha_{i}\right) \cdot{ }^{\overline{\mathrm{p}}} \dot{\mathbf{p}}\right.
\end{aligned}
$$

Sustituyendo $\beta_{i}$ de (8.9) en la expresión anterior y operando se obtiene:

$$
\dot{\varphi}_{i}=-\left(1 / \mathrm{r}_{i}\right) \cdot \sqrt{\left(-{ }^{\overline{\mathrm{R}}} v_{x}+\omega \cdot 1_{i} \cdot \mathrm{s} \alpha_{i}\right)^{2}+\left({ }^{\overline{\mathrm{R}}} v_{y}+\omega \cdot 1_{i} \cdot \mathrm{c} \alpha_{i}\right)^{2}}
$$

Pasando las velocidades lineales del vehículo al sistema G con (3.15):

$\dot{\varphi}_{i}=-\left(1 / \mathrm{r}_{i}\right) \cdot \sqrt{\left(-{ }^{\mathrm{G}} v_{x} \cdot \mathrm{c} \theta-{ }^{\mathrm{G}} v_{y} \cdot \mathrm{s} \theta+\omega \cdot 1_{i} \cdot \mathrm{s} \alpha_{i}\right)^{2}+\left(-{ }^{\mathrm{G}} v_{x} \cdot \mathrm{s} \theta+{ }^{\mathrm{G}} v_{y} \cdot \mathrm{c} \theta+\omega \cdot 1_{i} \cdot \mathrm{c} \alpha_{i}\right)^{2}}$

Por tanto la solución cinemática inversa de la rueda orientable viene dada por $\{(8.9),(8.12)\}$ ó $\{(8.10),(8.13)\}$. Esta cinemática inversa se particulariza en el apartado siguiente para cuando además hay una rueda fija independiente en el vehículo. (Según la clasificación del Capítulo 5 no puede haber más de una rueda fija independiente.)

Tener en cuenta que el cálculo de $\beta_{i}$ en (8.9)/(8.12) es en los 4 cuadrantes y que $\dot{\varphi}_{i}$ tiene siempre el mismo signo en $(8.10) /(8.13)$.

Alternativamente, se puede realizar el cálculo de $\beta_{i}$ en $(8.9) /(8.12)$ en 2 cuadrantes (primero y cuarto) y multiplicar en el segundo miembro de (8.10) /(8.13) por el signo de el segundo de los términos que hay elevado al cuadrado dentro de la raíz.

\subsubsection{Rueda fija y particularización de rueda orientable}

La cinemática inversa de la rueda fija viene dada por (8.10)/(8.13). No obstante, al ser $\beta_{i}$ constante hay una la ligadura entre los tres elementos del vector de velocidad $\dot{\mathbf{p}}$, la cual viene dada por el primer elemento de (8.7):

$$
\begin{aligned}
& \left(\begin{array}{lll}
\mathrm{c} \beta_{i} & \mathrm{~s} \beta_{i} & 1_{i} \cdot \mathrm{s}\left(\beta_{i}-\alpha_{i}\right)
\end{array}\right) \cdot{ }^{\overline{\mathrm{R}}} \dot{\mathbf{p}}=0 \\
& \left(\begin{array}{lll}
\mathrm{c}\left(\theta+\beta_{i}\right) & \mathrm{s}\left(\theta+\beta_{i}\right) & 1_{i} \cdot \mathrm{s}\left(\beta_{i}-\alpha_{i}\right)
\end{array}\right) \cdot{ }^{\mathrm{G}} \dot{\mathbf{p}}=0
\end{aligned}
$$

En caso de que el origen del sistema $R$ se encuentre en el eje de rotación de la rueda fija la ligadura afecta exclusivamente a las velocidades lineales de $\dot{\mathbf{p}}$ : 


$$
\beta_{\mathrm{f}}-\alpha_{\mathrm{f}}=0 \rightarrow\left\{\begin{array}{l}
{ }^{\overline{\mathrm{R}}} v_{x}={ }^{\overline{\mathrm{R}}} v_{y} \cdot \tan \beta_{i} \\
{ }^{\mathrm{G}} v_{x}={ }^{\mathrm{G}} v_{y} \cdot \tan \left(\theta+\beta_{i}\right)
\end{array}\right.
$$

En caso contrario, $R$ no está sobre eje de rotación de rueda fija, se puede obtener $\omega$ en función de las otras dos:

$$
\omega=\frac{{ }^{\overline{\mathrm{R}}} v_{x} \cdot \mathrm{c} \beta_{i}+{ }^{\overline{\mathrm{R}}} v_{y} \cdot \mathrm{s} \beta_{i}}{-\mathrm{l}_{i} \cdot \mathrm{s}\left(\beta_{i}-\alpha_{i}\right)}=\frac{{ }^{\mathrm{G}} v_{x} \cdot \mathrm{c}\left(\theta+\beta_{i}\right)+{ }^{\mathrm{G}} v_{y} \cdot \mathrm{s}\left(\theta+\beta_{i}\right)}{-\mathrm{l}_{i} \cdot \mathrm{s}\left(\beta_{i}-\alpha_{i}\right)}=f_{1}(\theta) \cdot{ }^{\mathrm{G}} v_{x}+f_{2}(\theta) \cdot{ }^{\mathrm{G}} v_{y}
$$

donde $f_{i}(\theta)$ es una función genérica que depende exclusivamente de la variable $\theta$.

Si se sustituye la ligadura (8.16) (para cuando el origen de R no esté en eje de rotación de la rueda fija) en (8.10) y (8.13) se obtiene:

$$
\begin{gathered}
\beta_{i}=\arctan \left(\frac{f_{3}(\theta) \cdot{ }^{\mathrm{G}} v_{x}+f_{4}(\theta) \cdot{ }^{\mathrm{G}} v_{y}}{f_{5}(\theta) \cdot{ }^{\mathrm{G}} v_{x}+f_{6}(\theta) \cdot{ }^{\mathrm{G}} v_{y}}\right)=\arctan \left(\frac{f_{3}(\theta)+f_{4}(\theta) \cdot\left({ }^{\mathrm{G}} v_{y} /{ }^{\mathrm{G}} v_{x}\right)}{f_{5}(\theta)+f_{6}(\theta) \cdot\left({ }^{\mathrm{G}} v_{y} /{ }^{\mathrm{G}} v_{x}\right)}\right) \\
\dot{\varphi}_{i}=-\sqrt{\left(f_{7}(\theta) \cdot{ }^{\mathrm{G}} v_{x}+f_{8}(\theta) \cdot{ }^{\mathrm{G}} v_{y}\right){ }^{2}+\left(f_{9}(\theta) \cdot{ }^{\mathrm{G}} v_{x}+f_{10}(\theta) \cdot{ }^{\mathrm{G}} v_{y}\right)^{2}}
\end{gathered}
$$

Si se sustituye la ligadura (8.15) (para cuando origen de R esté en eje de rotación de la rueda fija) en (8.10) y (8.13) se obtiene:

$$
\begin{gathered}
\beta_{i}=\arctan \left(\frac{f_{11}(\theta) \cdot{ }^{\mathrm{G}} v_{y}+f_{12}(\theta) \cdot \omega}{f_{13}(\theta) \cdot{ }^{\mathrm{G}} v_{y}+f_{14}(\theta) \cdot \omega}\right)=\arctan \left(\frac{f_{11}(\theta)+f_{12}(\theta) \cdot\left(\omega /{ }^{\mathrm{G}} v_{y}\right)}{f_{13}(\theta)+f_{14}(\theta) \cdot\left(\omega /{ }^{\mathrm{G}} v_{y}\right)}\right) \\
\dot{\varphi}_{i}=-\sqrt{\left(f_{15}(\theta) \cdot{ }^{\mathrm{G}} v_{y}+f_{16}(\theta) \cdot \omega\right)^{2}+\left(f_{17}(\theta) \cdot{ }^{\mathrm{G}} v_{y}+f_{18}(\theta) \cdot \omega\right)^{2}}
\end{gathered}
$$

Así pues, \{(8.17), (8.18)\} describen la cinemática inversa de ruedas orientables cuando además hay una rueda fija independiente y el punto de seguimiento no está sobre su eje de rotación, y $\{(8.21),(8.22)\}$ lo mismo pero para cuando el punto de seguimiento sí esta sobre dicho eje de rotación.

De igual forma (8.18) ó (8.22), dependiendo de la ubicación del punto de seguimiento (origen de $\mathrm{R}$ ), describen la cinemática inversa de las ruedas fijas. 


\subsubsection{Rueda castor}

Se parte de la ecuación de rueda de (3.12) con deslizamiento nulo:

$$
\left(\begin{array}{ccccc}
\mathrm{c}\left(\beta_{i}+\delta_{i}\right) & \mathrm{s}\left(\beta_{i}+\delta_{i}\right) & \mathrm{l}_{i} \cdot \mathrm{s}\left(\beta_{i}+\delta_{i}-\alpha_{i}\right)-\mathrm{d}_{i} \cdot \mathrm{c} \delta_{i} & -\mathrm{d}_{i} \cdot \mathrm{c} \delta_{i} & 0 \\
-\mathrm{s}\left(\beta_{i}+\delta_{i}\right) & \mathrm{c}\left(\beta_{i}+\delta_{i}\right) & 1_{i} \cdot \mathrm{c}\left(\beta_{i}+\delta_{i}-\alpha_{i}\right)+\mathrm{d}_{i} \cdot \mathrm{s} \delta_{i} & \mathrm{~d}_{i} \cdot \mathrm{s} \delta_{i} & \mathrm{r}_{i}
\end{array}\right) \cdot\left(\begin{array}{c}
\overline{\mathrm{R}} \dot{\mathbf{p}} \\
\dot{\beta}_{i} \\
\dot{\varphi}_{i}
\end{array}\right)=0
$$

Despejando $\dot{\beta}_{i}$ con el primer elemento de (8.23), se obtiene:

$$
\begin{gathered}
\dot{\beta}_{i}=\frac{1}{\mathrm{~d}_{i} \cdot \mathrm{c} \delta_{i}} \cdot\left(\mathrm{c}\left(\beta_{i}+\delta_{i}\right) \mathrm{s}\left(\beta_{i}+\delta_{i}\right) \quad 1_{i} \cdot \mathrm{s}\left(\beta_{i}+\delta_{i}-\alpha_{i}\right)-\mathrm{d}_{i} \cdot \mathrm{c} \delta_{i}\right) \cdot \overline{\mathrm{R}} \dot{\mathbf{p}} \\
\dot{\beta}_{i}=\frac{1}{\mathrm{~d}_{i} \cdot \mathrm{c} \delta_{i}} \cdot\left(\mathrm{c}\left(\theta+\beta_{i}+\delta_{i}\right) \mathrm{s}\left(\theta+\beta_{i}+\delta_{i}\right) \quad 1_{i} \cdot \mathrm{s}\left(\beta_{i}+\delta_{i}-\alpha_{i}\right)-\mathrm{d}_{i} \cdot \mathrm{c} \delta_{i}\right) \cdot{ }^{\mathrm{G}} \dot{\mathbf{p}}
\end{gathered}
$$

Premultiplicando (8.23) por $\left(\begin{array}{lll}\mathrm{s} \delta_{i} & \mathrm{c} \delta_{i}\end{array}\right)$ y despejando $\dot{\varphi}_{i}$ se obtiene:

$$
\begin{aligned}
& \dot{\varphi}_{i}=-\frac{1}{\mathrm{r}_{i} \cdot \mathrm{c} \delta_{i}} \cdot\left(-\mathrm{s} \beta_{i} \quad \mathrm{c} \beta_{i} \quad 1_{i} \cdot \mathrm{c}\left(\beta_{i}-\alpha_{i}\right)\right) \cdot{ }^{\overline{\mathrm{R}}} \dot{\mathbf{p}} \\
& \dot{\varphi}_{i}=-\frac{1}{\mathrm{r}_{i} \cdot \mathrm{c} \delta_{i}} \cdot\left(-\mathrm{s}\left(\theta+\beta_{i}\right) \quad \mathrm{c}\left(\theta+\beta_{i}\right) \quad \mathrm{l}_{i} \cdot \mathrm{c}\left(\beta_{i}-\alpha_{i}\right)\right) \cdot{ }^{\mathrm{G}} \dot{\mathbf{p}}
\end{aligned}
$$

Notar que la cinemática inversa de esta rueda, dada por (8.24) y (8.25), depende del ángulo del brazo de dirección, por lo que si se acciona alguna de las dos velocidades de la rueda castor hay que sensorizar el ángulo de la dirección $\beta_{i}$, como se indica en la Fig. 8.1.

\subsubsection{Rueda sueca}

Se parte de la ecuación de rueda (3.13) con deslizamiento nulo:

$$
\left(\begin{array}{ccccc}
\mathrm{c}\left(\beta_{i}+\gamma_{i}\right) & \mathrm{s}\left(\beta_{i}+\gamma_{i}\right) & 1_{i} \cdot \mathrm{s}\left(\beta_{i}+\gamma_{i}-\alpha_{i}\right) & \mathrm{r}_{i} \cdot \mathrm{s} \gamma_{i} & 0 \\
-\mathrm{s}\left(\beta_{i}+\gamma_{i}\right) & \mathrm{c}\left(\beta_{i}+\gamma_{i}\right) & 1_{i} \cdot \mathrm{c}\left(\beta_{i}+\gamma_{i}-\alpha_{i}\right) & \mathrm{r}_{i} \cdot \mathrm{c} \gamma_{i} & \mathrm{r}_{\mathrm{r} i}
\end{array}\right) \cdot\left(\begin{array}{c}
\overline{\mathrm{R}} \dot{\mathbf{p}} \\
\dot{\varphi}_{i} \\
\dot{\varphi}_{\mathrm{r} i}
\end{array}\right)=0
$$

Despejando $\dot{\varphi}_{i}$ con el primer elemento de (8.26), se obtiene: 


$$
\begin{aligned}
& \dot{\varphi}_{i}=-\frac{1}{\mathrm{r}_{i} \cdot \mathrm{s} \gamma_{i}} \cdot\left(\mathrm{c}\left(\beta_{i}+\gamma_{i}\right) \mathrm{s}\left(\beta_{i}+\gamma_{i}\right) 1_{i} \cdot \mathrm{s}\left(\beta_{i}+\gamma_{i}-\alpha_{i}\right)\right) \cdot{ }^{\bar{\alpha}} \dot{\mathbf{p}} \\
& \dot{\varphi}_{i}=-\frac{1}{\mathrm{r}_{i} \cdot \mathrm{s} \gamma_{i}} \cdot\left(\mathrm{c}\left(\theta+\beta_{i}+\gamma_{i}\right) \mathrm{s}\left(\theta+\beta_{i}+\gamma_{i}\right) \quad 1_{i} \cdot \mathrm{s}\left(\beta_{i}+\gamma_{i}-\alpha_{i}\right)\right) \cdot{ }^{\mathrm{G}} \dot{\mathbf{p}}
\end{aligned}
$$

Premultiplicando (8.26) por $\left(\mathrm{c} \gamma_{i}-\mathrm{s} \gamma_{i}\right)$ y despejando $\dot{\varphi}_{\mathrm{ri}}$ se obtiene:

$$
\begin{aligned}
& \dot{\varphi}_{\mathrm{ri} i}=\frac{1}{\mathrm{r}_{i} \cdot \mathrm{s} \gamma_{i}} \cdot\left(\mathrm{c} \beta_{i} \quad \mathrm{~s} \beta_{i} \quad 1_{i} \cdot \mathrm{s}\left(\beta_{i}-\alpha_{i}\right)\right) \cdot{ }^{\overline{\mathrm{R}}} \dot{\mathbf{p}} \\
& \dot{\varphi}_{\mathrm{r} i}=\frac{1}{\mathrm{r}_{i} \cdot \mathrm{s} \gamma_{i}} \cdot\left(\mathrm{c}\left(\theta+\beta_{i}\right) \mathrm{s}\left(\theta+\beta_{i}\right) \quad \mathrm{l}_{i} \cdot \mathrm{s}\left(\beta_{i}-\alpha_{i}\right)\right) \cdot{ }^{\mathrm{G}} \dot{\mathbf{p}}
\end{aligned}
$$

No obstante la velocidad de rueda anterior $\dot{\varphi}_{\mathrm{ri}}$ (rotación del rodillo) no se suele sensorizar ni actuar, como ya se indicó en el Capítulo 3, siendo el rodillo un elemento auxiliar de menor importancia. Por este motivo no se ha incluido en el diagrama de la Figura 8 a la salida del bloque "Cinemática Inversa Ruedas". 


\subsection{TIPOS DE REFERENCIAS POSIBLES PARA CADA TIPO DE VEHÍCULO}

\subsubsection{Introducción}

En primer lugar aclarar que el adjetivo posibles se refiere al hecho de que el vehículo siga las referencias sin ningún tipo de error, suponiendo que se parte de las condiciones iniciales adecuadas y que todos los elementos del esquema de control de la Figura 8.1 son ideales.

Por ejemplo, si se ha obtenido en la caracterización de referencias posibles la condición de continuidad de una variable, cuando la referencia seguida de lugar a una discontinuidad en dicha variable aparecería un error de forma instantánea, el cual se iría corrigiendo con el control de posición. Lo mismo ocurre si las condiciones iniciales (posiciones, orientaciones y velocidades) no son las adecuadas.

Por otra parte, si se considera un marco exclusivamente cinemático, es decir se obvia el control dinámico o es considerado instantáneo, las velocidades de rueda (es decir $\left\{\dot{\varphi}_{i}, \dot{\beta}_{i}, \dot{\varphi}_{\mathrm{ri}}\right\}$ ) accionadas pueden cambiar de forma instantánea.

Por el contrario si se considera un marco dinámico, y se desprecia la dinámica del actuador (ej. parte eléctrica del motor), son las derivadas de las velocidades de rueda (es decir las aceleraciones: $\left.\left\{\ddot{\varphi}_{i}, \ddot{\beta}_{i}, \ddot{\varphi}_{\mathrm{r} i}\right\}\right)$ ) accionadas las que cambian de forma instantánea.

Como se ha indicado en el punto 8.2 el número de actuadores a utilizar en el vehículo, para una actuación consistente (Capítulo 4), coincide con la maniobrabilidad del vehículo (Capítulo 5). Este valor también coincide con el número de referencias a considerar en la postura del vehículo $\mathbf{p}$.

Como se puede comprobar en la Tabla 5.1, todos los vehículos tienen una maniobrabilidad de 3 (tipo 1, tipo 3, tipo 5) ó de 2 (tipo 2, tipo 4). En los de maniobrabilidad completa (3) se generan referencias para los 3 elementos de la postura del vehículo p. 
Mientras que en los de maniobrabilidad reducida (2) se generan referencias sólo para dos elementos de la postura. Dada la connotación de los elementos de $\mathbf{p}$ (dos posiciones lineales y una angular) se asumirá que se generan referencias para las dos posiciones lineales, dando lugar a caminos de dos dimensiones (2D).

Se tienen las siguientes definiciones matemáticas de funciones monovariables:

$\rightarrow$ Una función es $\mathrm{C}^{0}$, o más comúnmente $\mathrm{C}$, si es continua (Figura 8.2)

$\rightarrow$ Una función es $\mathrm{C}^{1}$ si su derivada es continua (también llamada diferenciable de forma continua) (Figura 8.2)

$\rightarrow$ Una función es $\mathrm{C}^{\mathrm{n}}($ con $\mathrm{n}>0)$ si su derivada enésima es continua

$\rightarrow$ Una función es suave si pertenece a la clase $\mathrm{C}^{\mathrm{n}} \forall \mathrm{n}$ (también referidas como funciones $\mathrm{C}^{\infty}$ )

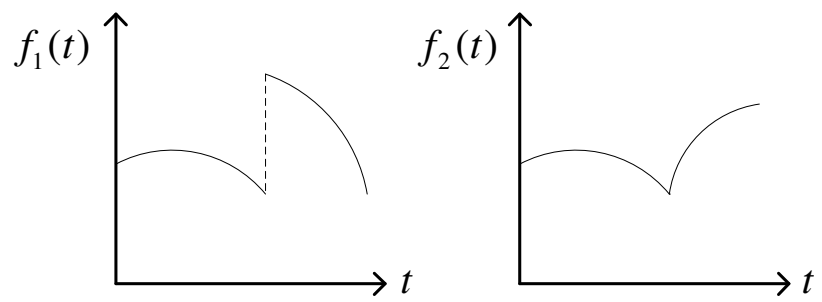

Figura 8.2: Ejemplos de función: $f_{1}$ no es $C^{0}$ (continua); $f_{2}$ es $C^{0}$ pero no $C^{1}$

A continuación se considerará el camino o curva (Fig.8.3) producida por dos coordenadas de posición lineal, $x(t)$ e $y(t)$, a lo largo tiempo (trayectoria en 2D).

Se denominará ángulo de la tangente al camino, denotado con $\chi$, al ángulo que forma el vector tangente al camino o curva con el eje $X$. Su valor es:

$$
\chi(t)=\arctan \left(\frac{d y(t)}{d x(t)}\right)=\arctan \left(\frac{d y(t) / d t}{d x(t) / d t}\right)=\arctan \left(\frac{v_{y}}{v_{x}}\right)
$$

Notar que un ángulo $\chi$ continuo $\left(\mathrm{C}^{0}\right)$ no implica que las posiciones lineales de $x(t)$ e $y(t)$ también lo sean (ej. tramos de línea recta en la dirección del eje $X$ o $Y$ ). No obstante, en lo que sigue se asumirá que las referencias lineales de posición generadas por el planificador de alto nivel son, lógicamente, continuas. 
Las velocidades de avance $v_{\mathrm{a}} \mathrm{y}$ giro $\omega_{\mathrm{g}}$ de la trayectoria $2 \mathrm{D}$ (Figura 8.3) son:

$$
\begin{gathered}
v_{\mathrm{a}}(t)=\sqrt{v_{x}^{2}+v_{y}^{2}} \\
\omega_{\mathrm{g}}(t)=\frac{d(\chi(t))}{d t}=\frac{\dot{v}_{y} \cdot v_{x}-v_{y} \cdot \dot{v}_{x}}{v_{x}^{2}+v_{y}^{2}}
\end{gathered}
$$

La siguiente coimplicación, entre la velocidad de giro $\omega_{\mathrm{g}}$ y el ángulo de la tangente a la curva $\chi$, es obvia:

$$
\omega_{\mathrm{g}} \text { es } \mathrm{C}^{0} \Leftrightarrow \chi \text { es } \mathrm{C}^{1}
$$

La curvatura del camino $\kappa_{\mathrm{c}}$ es la inversa del radio de curvatura $\rho_{\mathrm{c}}$, pudiéndose obtener a partir de las velocidades de avance y giro anteriores:

$$
\kappa_{\mathrm{c}}(t)=\frac{1}{\rho_{\mathrm{c}}(t)}=\frac{\omega_{\mathrm{g}}}{v_{\mathrm{a}}}=\frac{\dot{v}_{y} \cdot v_{x}-v_{y} \cdot \dot{v}_{x}}{\left(v_{x}^{2}+v_{y}^{2}\right)^{3 / 2}}
$$

Utilizando (8.30), (8.32) y (8.33) se obtiene la implicación:

$$
\text { Si } \chi \text { es } \mathrm{C}^{1} \Rightarrow\left\{\omega_{\mathrm{g}}, v_{\mathrm{a}}\right\} \text { son } \mathrm{C}^{0} \Rightarrow \kappa_{\mathrm{c}} \text { es } \mathrm{C}^{0}
$$

La implicación anterior al revés no es cierta, ya que puede mantenerse la relación $\kappa_{\mathrm{c}}$ entre $\omega_{\mathrm{g}}$ y $v_{\mathrm{a}}$ y cambiar ambas instantáneamente.

Si la curvatura $\kappa_{\mathrm{c}}$ está definida en un punto o en todo el camino, el ángulo de la tangente al camino es continuo en ese punto o en todo el camino:

$$
\mathrm{Si} \quad \kappa_{\mathrm{c}} \text { está definida } \Rightarrow \chi \text { es } \mathrm{C}^{0}
$$

El centro de curvatura del camino $\mathrm{C}_{\mathrm{c}}$ viene dado en cada instante por el radio de curvatura $\rho_{\mathrm{c}}$ y por el ángulo $\chi$ de la tangente al camino. Por tanto, si la curvatura del camino $\kappa_{\mathrm{c}}$ es continua (implica que $\rho_{\mathrm{c}}$ y $\chi$ son continuas) el lugar geométrico definido por el centro de curvatura es continuo.

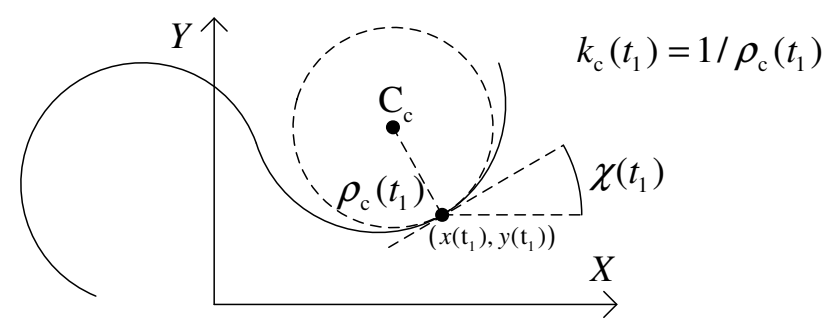

Figura 8.3: Variables del camino en un punto con ángulo de la tangente continuo 
Notar que se puede hablar de camino de curvatura $\kappa_{\mathrm{c}}$ (o ángulo $\chi$ ) continua, pero no de camino de curvatura $\kappa_{\mathrm{c}}$ (o ángulo $\chi$ ) de derivada continua. En ese caso hay que hablar de trayectoria 2D, ya que depende de la velocidad de avance.

\subsubsection{Vehículo tipo 1: Omnidireccional}

Este vehículo tiene maniobrabilidad completa por estar hecho con ruedas omnidireccionales, bien castor o suecas. Por tanto el planificador generará referencias para los tres elementos de la postura $\mathbf{p}$.

Considerando el marco exclusivamente cinemático (velocidades de rueda cambian de forma instantánea) y teniendo en cuenta la cinemática inversa dada por $\{(8.24),(8.25),(8.27),(8.28)\}$, resulta que este vehículo puede seguir cualquier tipo de referencia de la postura $\mathbf{p}\left(\mathbf{p}_{\text {ref }}\right)$ continua $\left(\mathrm{C}^{0}\right)$.

Para el marco dinámico (derivadas de velocidades de rueda cambian de forma instantánea) y con la cinemática inversa de antes, resulta que este vehículo puede seguir cualquier referencia de postura $\mathbf{p}$ de derivada continua $\left(\mathrm{C}^{1}\right)$.

\subsubsection{Vehículo tipo 2: Diferencial}

Este vehículo tiene maniobrabilidad reducida al tener una rueda fija independiente. Por tanto el planificador generará referencias sólo para las posiciones lineales $\{x(t), y(t)\}$ de la postura $\mathbf{p}$.

En caso de que el origen del sistema $\mathrm{R}$ (punto que hace el seguimiento de las referencias de posición) no esté sobre el eje de las ruedas fijas $\left(\beta_{\mathrm{f}}-\alpha_{\mathrm{f}} \neq 0\right)$, la cinemática inversa de las ruedas fijas es (8.18). Por lo que puede seguir cualquier trayectoria $2 \mathrm{D}$ con referencias de posición $\{x(t), y(t)\}$ continuas $\left(\mathrm{C}^{0}\right)$ para el marco cinemático y de derivadas continuas $\left(\mathrm{C}^{1}\right)$ para el marco dinámico.

En caso de que el origen del sistema $\mathrm{R}$ sí esté sobre el eje de las ruedas fijas $\left(\beta_{\mathrm{f}}-\alpha_{\mathrm{f}}=0\right)$ la cinemática inversa de las ruedas fijas viene dada por (8.20). Utilizando la ligadura de (8.15) debida a las ruedas fijas y (8.29), se obtiene la relación:

$$
\tan \left(\theta+\beta_{i}\right)=\frac{{ }^{\mathrm{G}} v_{x}}{{ }^{\mathrm{G}} v_{y}} \rightarrow \theta+\beta_{i}=\alpha_{\chi} \rightarrow \dot{\theta}=\dot{\alpha}_{\chi} \rightarrow \omega=\omega_{\mathrm{g}}
$$

es decir que la velocidad angular del vehículo $\omega$ coincide con la velocidad de giro $\omega_{\mathrm{g}}$ de la trayectoria $2 \mathrm{D}$. 
Teniendo en cuenta lo anterior, la cinemática inversa de (8.20) se reescribe:

$$
\dot{\varphi}_{i}=-\sqrt{\left(f_{15}(\theta) \cdot{ }^{\mathrm{G}} v_{y}+f_{16}(\theta) \cdot \omega_{\mathrm{g}}\right)^{2}+\left(f_{17}(\theta) \cdot{ }^{\mathrm{G}} v_{y}+f_{18}(\theta) \cdot \omega_{\mathrm{g}}\right)^{2}}
$$

Por tanto, dentro del marco cinemático se podrán seguir caminos con ángulo $\chi$ de la tangente continuo $\left(\mathrm{C}^{0}\right)$. No obstante, se podrán seguir caminos con ángulo discontinuo si se detiene el avance del vehículo en las discontinuidades para reorientarse. Desde el punto de vista dinámico se podrán seguir trayectorias 2D con ángulo $\chi$ de la tangente de derivada continua $\left(\mathrm{C}^{1}\right)$

\subsubsection{Vehículo tipo 4: Triciclo y bicicleta}

Este vehículo tiene maniobrabilidad reducida al tener una rueda fija independiente. Por tanto el planificador generará referencias sólo para las posiciones lineales $(x, y)$ de la postura $\mathbf{p}$.

En caso de que el origen del sistema $\mathrm{R}$ (punto que hace el seguimiento de las referencias de posición lineales) no esté sobre el eje de las ruedas fijas $\left(\beta_{\mathrm{f}}-\alpha_{\mathrm{f}} \neq 0\right)$, la cinemática inversa de la rueda orientable es $\{(8.17),(8.18)\}$ y la de las fijas (8.18). Usando (8.29), dichas expresiones resultan:

$$
\begin{gathered}
\beta_{i}=\arctan \left(\frac{f_{3}(\theta)+f_{4}(\theta) \cdot \chi}{f_{5}(\theta)+f_{6}(\theta) \cdot \chi}\right) \\
\dot{\varphi}_{i}=-{ }^{\mathrm{G}} v_{x} \cdot \sqrt{\left(f_{7}(\theta)+f_{8}(\theta) \cdot \chi\right)^{2}+\left(f_{9}(\theta)+f_{10}(\theta) \cdot \chi\right)^{2}}
\end{gathered}
$$

Considerando el marco cinemático y las ecuaciones anteriores, el vehículo podrá seguir caminos con ángulo $\chi$ de la tangente continuo $\left(\mathrm{C}^{0}\right)$. No obstante, podrá seguir caminos con ángulo discontinuo si se detiene el avance del vehículo en las discontinuidades para reorientar la rueda orientable. Para el marco dinámico, el vehículo podrá seguir trayectorias $2 \mathrm{D}$ con ángulo $\chi$ de la tangente de derivada continua $\left(\mathrm{C}^{1}\right)$.

En caso de que el origen del sistema $\mathrm{R}$ sí esté sobre el eje de las ruedas fijas $\left(\beta_{\mathrm{f}}-\alpha_{\mathrm{f}}=0\right)$ la cinemática inversa de la rueda orientable es $\{(8.19),(8.20)\}$ y la de las ruedas fijas (8.20). Puesto que (8.36) es válido también para este caso, (8.20) se convierte en (8.37) y (8.19) en se reescribe como: 


$$
\beta_{i}=\arctan \left(\frac{f_{11}(\theta)+f_{12}(\theta) \cdot\left(\omega_{\mathrm{g}} /{ }^{\mathrm{G}} v_{y}\right)}{f_{13}(\theta)+f_{14}(\theta) \cdot\left(\omega_{\mathrm{g}} /{ }^{\mathrm{G}} v_{y}\right)}\right)
$$

La curvatura del camino seguido por este vehículo, utilizando (8.15), es:

$$
\kappa_{c}=\frac{\omega_{\mathrm{g}}}{\sqrt{{ }^{\mathrm{G}} v_{x}^{2}+{ }^{\mathrm{G}} v_{y}^{2}}}=\frac{\omega_{\mathrm{g}}}{f_{19}(\theta) \cdot{ }^{\mathrm{G}} v_{y}}
$$

Por lo que (8.40) y (8.37) resultan:

$$
\begin{gathered}
\beta_{i}=\arctan \left(\frac{f_{11}(\theta)+f_{20}(\theta) \cdot \kappa_{c}}{f_{13}(\theta)+f_{21}(\theta) \cdot \kappa_{c}}\right) \\
\dot{\varphi}_{i}=-{ }^{\mathrm{G}} v_{y} \cdot \sqrt{\left(f_{15}(\theta)+f_{22}(\theta) \cdot \kappa_{c}\right)^{2}+\left(f_{17}(\theta)+f_{23}(\theta) \cdot \kappa_{c}\right)^{2}}
\end{gathered}
$$

Dentro del marco cinemático se podrán seguir caminos con curvatura $\kappa_{c}$ continua $\left(\mathrm{C}^{0}\right)$. No obstante, se podrán seguir caminos con curvatura $\kappa_{c}$ discontinua y ángulo $\chi$ de la tangente continuo (por (8.36)), deteniendo el vehículo en las discontinuidades para reorientar la rueda orientable. Si hay discontinuidad tanto en la curvatura $\kappa_{c}$ como en el ángulo $\chi$ de la tangente habría que reorientar el vehículo con doble reorientación de la rueda orientable.

Desde el punto de vista dinámico se podrán seguir trayectorias $2 \mathrm{D}$ con curvatura del camino $\kappa_{c}$ de derivada continua $\left(\mathrm{C}^{1}\right)$.

\subsubsection{Vehículo tipo 3 (una rueda orientable) y tipo 5 (dos ruedas orientables)}

Estos dos tipos de vehículo, tratados aquí de forma conjunta, tienen maniobrabilidad completa por no tener ruedas fijas. Por lo que el planificador generará referencias para los tres elementos de la postura $\mathbf{p}$.

En el caso de que haya más de una rueda orientable o haya una única rueda orientable pero el punto de seguimiento (origen del sistema $\mathrm{R}$ ) no esté en su centro, es decir existe alguna rueda orientable con el parámetro $l_{i}$ distinto de cero $\left(\exists 1_{o i} \neq 0\right)$, la cinemática inversa de la/s rueda/s orientable/s con $1_{o i} \neq 0$ viene dado por (8.10) y (8.13). Según estas fórmulas y considerando el marco cinemático, se podrán seguir referencias de los tres elementos de la postura $\mathbf{p}$ de derivadas continuas $\left(\mathrm{C}^{1}\right)$. Mientras que, según el punto de vista dinámico se podrán seguir referencias de $\mathbf{p}$ de derivadas segundas continuas $\left(\mathrm{C}^{2}\right)$. 
No obstante, para el marco cinemático se podrán seguir referencias de postura $\mathbf{p}$ con primera derivada discontinua si se detiene el vehículo en las discontinuidades para reorientar las ruedas orientables.

En caso de haber una única rueda orientable y el punto de seguimiento esté en el centro de dicha rueda $\left(\nexists 1_{\mathrm{o} i} \neq 0\right)$, las expresiones (8.10) y (8.13) se particularizan en:

$$
\begin{gathered}
\beta_{i}=\arctan \left(\frac{\mathrm{c} \theta+\chi \cdot \mathrm{s} \theta}{\mathrm{s} \theta-\chi \cdot \mathrm{c} \theta}\right) \\
\dot{\varphi}_{i}=-\frac{{ }^{\mathrm{G}} v_{x}}{\mathrm{r}_{i}} \cdot \sqrt{(\mathrm{c} \theta+\chi \cdot \mathrm{s} \theta)^{2}+(-\mathrm{s} \theta+\chi \cdot \mathrm{c} \theta)^{2}}
\end{gathered}
$$

Por lo que, dentro del marco cinemático se podrán seguir referencias con posición angular $\theta$ y ángulo $\chi$ de la tangente continuos $\left(\mathrm{C}^{0}\right)$. No obstante, se podrán seguir caminos con ángulo $\chi$ discontinuo si se detiene el vehículo en las discontinuidades para reorientar la rueda orientable.

Desde el punto de vista dinámico se podrán seguir referencias con posición angular $\theta$ y ángulo $\chi$ de la tangente de derivadas continuas $\left(\mathrm{C}^{1}\right)$.

\subsubsection{Resumen de tipos de referencias posibles}

En la Tabla 8.1 se muestra el resumen de tipos de referencias posibles según el tipo de vehículo. En ella se indican:

- Número de elementos de la postura $\mathbf{p}$ a considerar como referencias;

- Ecuaciones de cinemática inversa según el tipo de rueda ( $\{\mathrm{f}, \mathrm{o}, \mathrm{c}, \mathrm{s}\}$ para $\{$ fija, orientable, castor, sueca $\})$, donde para fijas y orientables ya se ha sustituido la posible ligadura dada por la rueda fija independiente;

- Condiciones de las referencias para el marco cinemático

- Condiciones de las referencias para el marco dinámico;

- Forma de evitar error de seguimiento si no se cumple una condición determinada. 
Tabla 8.1- Referencias posibles según el tipo de vehículo y marco considerados

\begin{tabular}{|c|c|c|c|c|c|c|c|}
\hline & \multirow{2}{*}{ Tipo 1} & \multicolumn{2}{|c|}{ Tipo 2} & \multicolumn{2}{|c|}{ Tipo 4} & \multicolumn{2}{|c|}{ Tipos 3 y 5} \\
\hline & & $\beta_{\mathrm{f}}-\alpha_{\mathrm{f}} \neq 0$ & $\beta_{\mathrm{f}}-\alpha_{\mathrm{f}}=0$ & $\beta_{\mathrm{f}}-\alpha_{\mathrm{f}} \neq 0$ & $\beta_{\mathrm{f}}-\alpha_{\mathrm{f}}=0$ & $\exists 1_{\mathrm{o} i} \neq 0$ & $\nexists \nexists 1_{\mathrm{o} i} \neq 0$ \\
\hline $\mathrm{N}^{\circ}$ refs. $\mathbf{p}$ & 3 & \multicolumn{2}{|c|}{2} & \multicolumn{2}{|c|}{2} & \multicolumn{2}{|c|}{3} \\
\hline \multirow[t]{2}{*}{$\begin{array}{l}\text { Cinemática } \\
\text { Inversa }\end{array}$} & \multirow{2}{*}{$\begin{array}{c}\text { c }\{(8.24), \\
(8.25)\} \\
\text { s }\{(8.27), \\
(8.28)\}\end{array}$} & f (8.18) & $\mathrm{f}(8.37)$ & $\begin{array}{c}\mathrm{f}(8.39) \\
\mathrm{o}\{(8.38) \\
(8.39)\}\end{array}$ & $\begin{array}{c}\mathrm{f}(8.43) \\
\mathrm{o}\{(8.42) \\
(8.43)\}\end{array}$ & $\begin{array}{c}\mathrm{o}\{(8.10) \\
(8.13)\}\end{array}$ & $\begin{array}{c}\mathrm{o}\{(8.44) \\
(8.45)\}\end{array}$ \\
\hline & & \multicolumn{2}{|c|}{$c, s$} ídem tipo 1 & \multicolumn{2}{|c|}{$c, s$} ídem tipo 1 & \multicolumn{2}{|c|}{$c, s$} ídem tipo 1 \\
\hline $\begin{array}{c}\text { Marco } \\
\text { cinemático }\end{array}$ & $\mathbf{p}_{\text {ref }}$ es $C^{0}$ & $\begin{array}{l}x_{\text {ref }} \text { es } C^{0} \\
y_{\text {ref }} \text { es C }^{0}\end{array}$ & $\chi \operatorname{es~}^{0 *}$ & $\chi \operatorname{es~C}^{0^{* *}}$ & $\kappa_{c}$ es $C^{0 * * *}$ & $\mathbf{p}_{\mathrm{ref}}$ es $\mathrm{C}^{1}$ & $\begin{array}{l}\theta_{\text {ref }} \text { es } C^{0} \\
\chi \text { es }^{0} C^{0 *}\end{array}$ \\
\hline $\begin{array}{c}\text { Marco } \\
\text { dinámico }\end{array}$ & $\mathbf{p}_{\text {ref }}$ es $C^{1}$ & $\begin{array}{l}x_{\text {ref }} \text { es } C^{1} \\
y_{\text {ref }} \text { es } C^{1}\end{array}$ & $\chi$ es $\mathrm{C}^{1}$ & $\chi$ es $\mathrm{C}^{1}$ & $\kappa_{c}$ es $\mathrm{C}^{1}$ & $\mathbf{p}_{\text {ref }}$ es $C^{2}$ & $\begin{array}{c}\theta_{\text {ref }} \text { es } C^{1} \\
\chi \text { es C }^{1}\end{array}$ \\
\hline
\end{tabular}

* Parar a reorientar el vehículo en las discontinuidades del ángulo de la tangente

** Parar a reorientar la rueda orientable en las discontinuidades del ángulo de la tangente

**** Parar a reorientar la rueda orientable en las discontinuidades de la curvatura y el vehículo en las discontinuidades de la curvatura y del ángulo de la tangente al camino

${ }^{* * * *}$ Parar a reorientar las ruedas orientables en las discontinuidades de la derivada de $\mathbf{p}_{\text {ref }}$ 


\subsection{APLICACIÓN DEL CONTROL AL CASO DEL TRICICLO}

\subsubsection{Particularización del control}

En este apartado se va a particularizar el control para el vehículo tipo triciclo correspondiente a la carretilla industrial que se presentó en el capítulo anterior. En la Figura 8.4 se puede apreciar la representación esquemática de este vehículo, donde el punto de seguimiento está situado en el extremo de las palas.

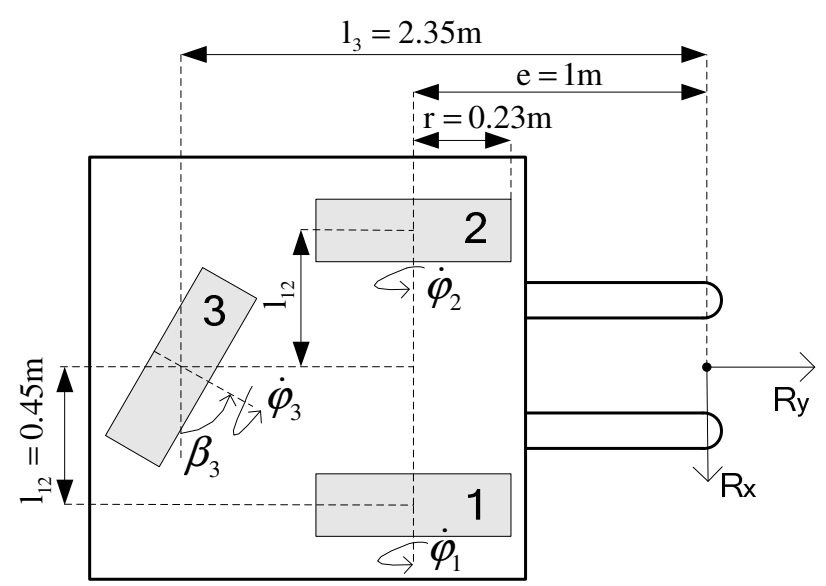

Figura 8.4: Variables y parámetros de la carretilla industrial (vehículo triciclo)

Los parámetros correspondientes a cada rueda son:

$$
\begin{array}{lll}
1_{1}=\sqrt{\mathrm{e}^{2}+\mathrm{l}_{12}^{2}} & \alpha_{1}=-\operatorname{atan}\left(\mathrm{e} / 1_{12}\right) & \beta_{1}=0 \\
1_{2}=\sqrt{\mathrm{e}^{2}+\mathrm{l}_{12}^{2}} & \alpha_{1}=\pi+\operatorname{atan}\left(\mathrm{e} / 1_{12}\right) & \beta_{2}=0 \\
1_{3}=1_{3} & \alpha_{3}=-\pi / 2 &
\end{array}
$$

La ligadura (8.16) dada por las ruedas fijas es:

$$
\omega=\frac{{ }^{\mathrm{G}} v_{x} \cdot \mathrm{c} \theta+{ }^{\mathrm{G}} v_{y} \cdot \mathrm{s} \theta}{\mathrm{l}_{1} \cdot \mathrm{s}\left(\alpha_{1}\right)}=-\frac{1}{\mathrm{e}} \cdot\left({ }^{\mathrm{G}} v_{x} \cdot \mathrm{c} \theta+{ }^{\mathrm{G}} v_{y} \cdot \mathrm{s} \theta\right)=-\frac{{ }^{\overline{\mathrm{R}}} v_{x}}{\mathrm{e}}
$$

La cinemática inversa de las ruedas fijas (8.18), dada por (8.12) y (8.47), es:

$$
\begin{aligned}
& \dot{\varphi}_{1}=-\frac{1}{\mathrm{r}} \cdot\left({ }^{\overline{\mathrm{R}}} v_{y}-\frac{1_{12}}{\mathrm{e}} \cdot{ }^{\overline{\mathrm{R}}} v_{x}\right)=-\frac{1}{\mathrm{r}} \cdot\left(-{ }^{\mathrm{G}} v_{x} \cdot \mathrm{s} \theta+{ }^{\mathrm{G}} v_{y} \cdot \mathrm{c} \theta-\frac{1_{12}}{\mathrm{e}} \cdot\left({ }^{\mathrm{G}} v_{x} \cdot \mathrm{c} \theta+{ }^{\mathrm{G}} v_{y} \cdot \mathrm{s} \theta\right)\right) \\
& \dot{\varphi}_{2}=-\frac{1}{\mathrm{r}} \cdot\left({ }^{\overline{\mathrm{R}}} v_{y}+\frac{1_{12}}{\mathrm{e}} \cdot{ }^{\overline{\mathrm{R}}} v_{x}\right)=-\frac{1}{\mathrm{r}} \cdot\left(-{ }^{\mathrm{G}} v_{x} \cdot \mathrm{s} \theta+{ }^{\mathrm{G}} v_{y} \cdot \mathrm{c} \theta+\frac{1_{12}}{\mathrm{e}} \cdot\left({ }^{\mathrm{G}} v_{x} \cdot \mathrm{c} \theta+{ }^{\mathrm{G}} v_{y} \cdot \mathrm{s} \theta\right)\right)
\end{aligned}
$$


La cinemática inversa de la rueda orientable $\{(8.17),(8.18)\}$, dada por $\{(8.9)$ ,(8.12)\} ó $\{(8.10),(8.13)\}$ y (8.47), es:

$$
\begin{aligned}
& \beta_{3}=\arctan \left(\frac{\left(l_{3}-\mathrm{e}\right)}{\mathrm{e}} \cdot \frac{{ }^{\overline{\mathrm{R}}} v_{x}}{{ }^{\overline{\mathrm{R}}} v_{y}}\right)=\arctan \left(\frac{\left(l_{3}-\mathrm{e}\right)}{\mathrm{e}} \cdot \frac{{ }^{\mathrm{G}} v_{x} \cdot \mathrm{c} \theta+{ }^{\mathrm{G}} v_{y} \cdot \mathrm{s} \theta}{-{ }^{\mathrm{G}} v_{x} \cdot \mathrm{s} \theta+{ }^{\mathrm{G}} v_{y} \cdot \mathrm{c} \theta}\right) \\
& \dot{\varphi}_{3}=-\frac{1}{\mathrm{r}} \cdot \sqrt{\frac{\left(\mathrm{l}_{3}-\mathrm{e}\right)^{2}}{\mathrm{e}^{2}} \cdot \overline{\mathrm{R}} v_{x}^{2}+{ }^{\overline{\mathrm{R}}} v_{y}^{2}} \\
& \dot{\varphi}_{3}=-\frac{1}{\mathrm{r}} \cdot \sqrt{\frac{\left(1_{3}-\mathrm{e}\right)^{2}}{\mathrm{e}^{2}} \cdot\left({ }^{\mathrm{G}} v_{x} \cdot \mathrm{c} \theta+{ }^{\mathrm{G}} v_{y} \cdot \mathrm{s} \theta\right)^{2}+\left(-{ }^{\mathrm{G}} v_{x} \cdot \mathrm{s} \theta+{ }^{\mathrm{G}} v_{y} \cdot \mathrm{c} \theta\right)^{2}}
\end{aligned}
$$

En caso de calcular $\beta_{3}$ en dos cuadrantes $\left(1^{\circ}\right.$ y $\left.4^{\circ}\right)$ se utilizaría para $\dot{\varphi}_{3}$ :

$$
\dot{\varphi}_{3}=-\frac{1}{\mathrm{r}} \cdot \operatorname{signo}\left({ }^{\overline{\mathrm{R}}} v_{y}\right) \cdot \sqrt{\frac{\left(1_{3}-\mathrm{e}\right)^{2}}{\mathrm{e}^{2}} \cdot{ }^{\overline{\mathrm{R}}} v_{x}^{2}+{ }^{\overline{\mathrm{R}}} v_{y}^{2}}
$$

El control de posición de (8.2) se particulariza para las coordenadas lineales:

$$
\begin{aligned}
& { }^{\mathrm{G}} v_{x \text { control }}={ }^{\mathrm{G}} \dot{x}_{\mathrm{R} \text { ref }}+a_{x} \cdot\left({ }^{\mathrm{G}} x_{\mathrm{R} \text { ref }}-{ }^{\mathrm{G}} x_{\mathrm{R}}\right) \\
& { }^{\mathrm{G}} v_{y \text { control }}={ }^{\mathrm{G}} \dot{y}_{\mathrm{R} \text { ref }}+a_{y} \cdot\left({ }^{\mathrm{G}} y_{\mathrm{R} \text { ref }}-{ }^{\mathrm{G}} y_{\mathrm{R}}\right)
\end{aligned}
$$

donde ${ }^{\mathrm{G}} x_{\mathrm{R} \text { ref }} \mathrm{e}^{\mathrm{G}} y_{\mathrm{R} \text { ref }}$ son las referencias en las coordenadas lineales del sistema global G y la dinámica en ambas coordenada viene dada por $a_{x}$ y $a_{y}$.

A la cinemática inversa dada por $\{(8.48),(8.49),(8.50) /(8.51)\}$ se le pasarían las velocidades de control del vehículo $\left\{{ }^{\mathrm{G}} v_{x \text { control }},{ }^{\mathrm{G}} v_{y \text { control }}\right\}$ de (8.52) y devolvería las referencias de las variables de rueda $\left\{\dot{\varphi}_{1 \text { ref }}, \dot{\varphi}_{2 \text { ref }}, \beta_{3 \text { ref }}, \dot{\varphi}_{3 \text { ref }}\right\}$ a conseguir por el control dinámico (ver Figura 8.1). Para una actuación consistente se actuaría sobre la orientación $\beta_{3}$ y sobre una de las velocidades de rotación de las ruedas.

\subsubsection{Resultados de simulación}

A continuación se presentan dos casos, a modo de ejemplo, en los que se ha aplicado el control cinemático anterior en un entorno de simulación. Éste se ha aplicado de forma continua y la parte dinámica de la Figura 8.1 se ha obviado.

Para los dos casos se ha tomado $a_{x}=a_{y}=2$ segundos $^{-1}$. Para el primero se ha tomado como postura inicial $\mathbf{p}(0)=\left(0,4 \mathrm{~m}, 180^{\circ}\right)^{\mathrm{T}}$ y $\mathbf{p}(0)=(0,0,0)^{\mathrm{T}}$ en el segundo. 


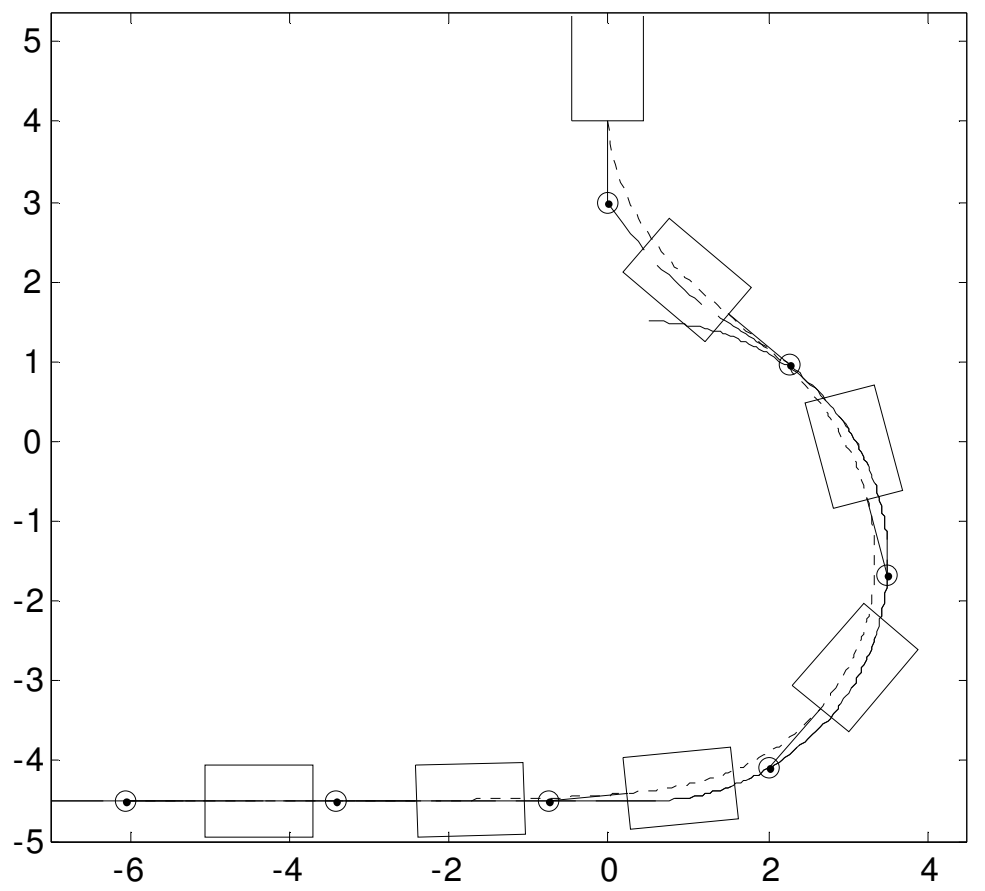

Figura 8.5: Ejemplo $1^{\circ}$ de seguimiento de referencia: camino seguido por la referencia (-), origen de $\mathrm{R}(--)$ y punto medio de las rueda fijas (- -)

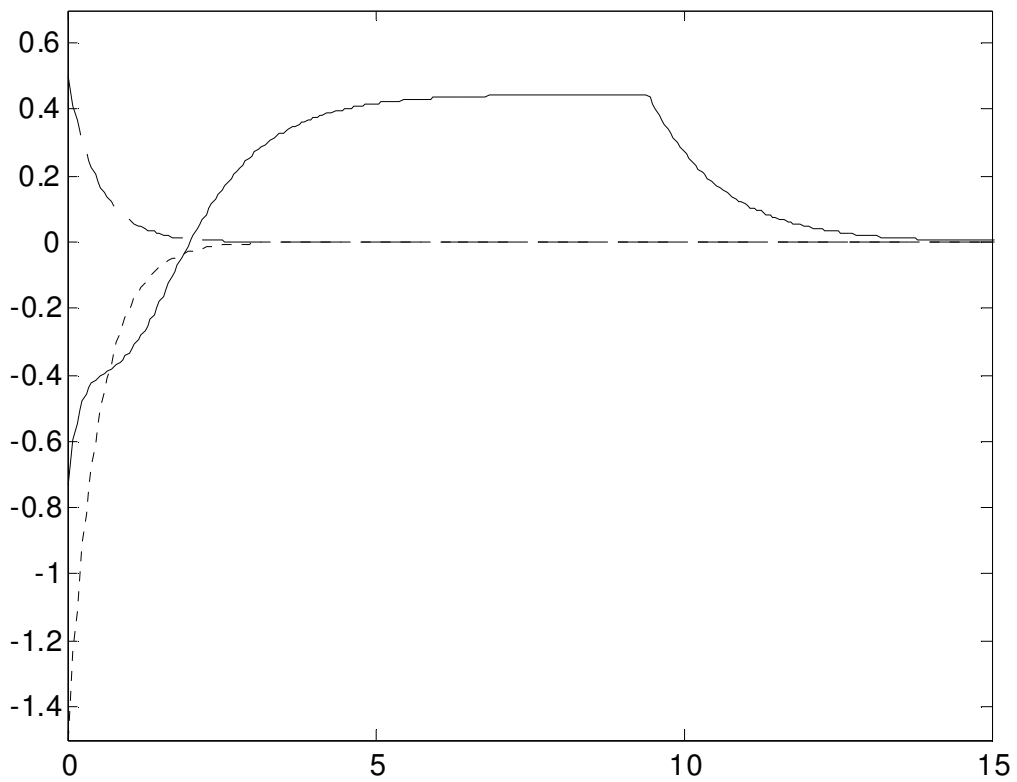

Figura 8.6: Orientación $\beta_{3}(-)$, error en $x(--)$ y error en $y(--)$ para ejemplo $1^{\circ}$ 


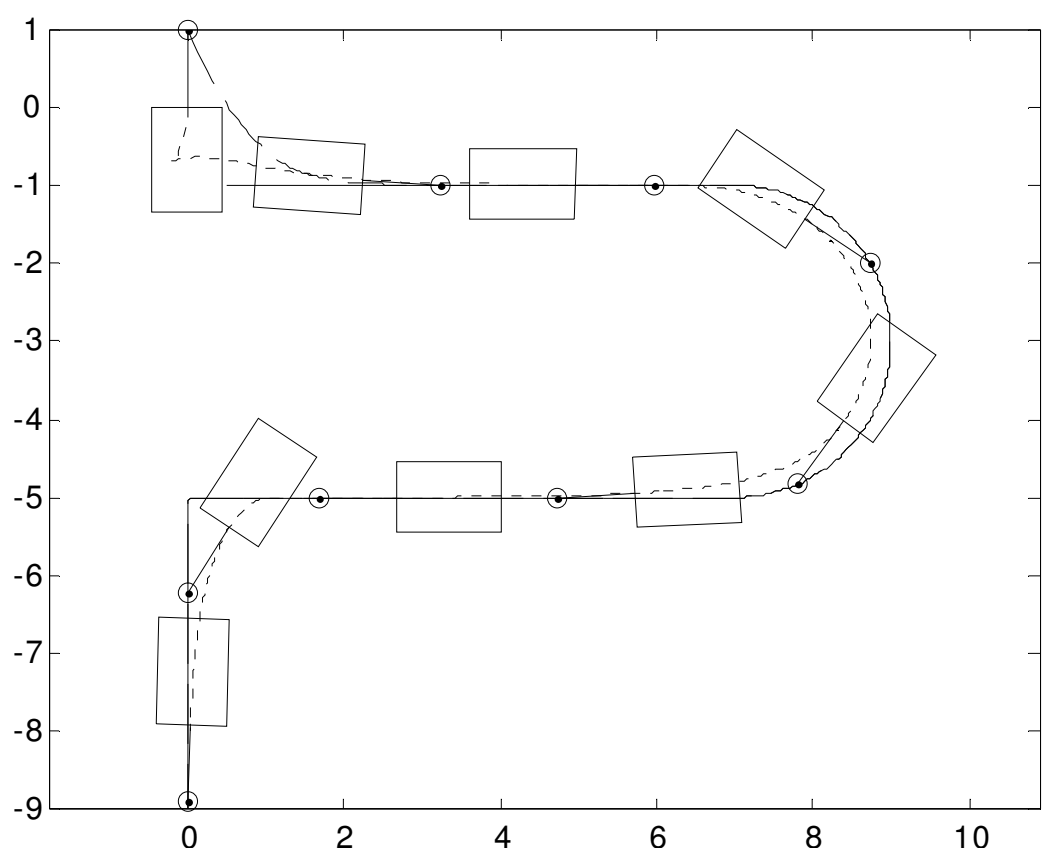

Figura 8.7: Ejemplo $2^{\circ}$ de seguimiento de referencia: camino seguido por la referencia (-), origen de $\mathrm{R}(--)$ y punto medio de las rueda fijas (- -)

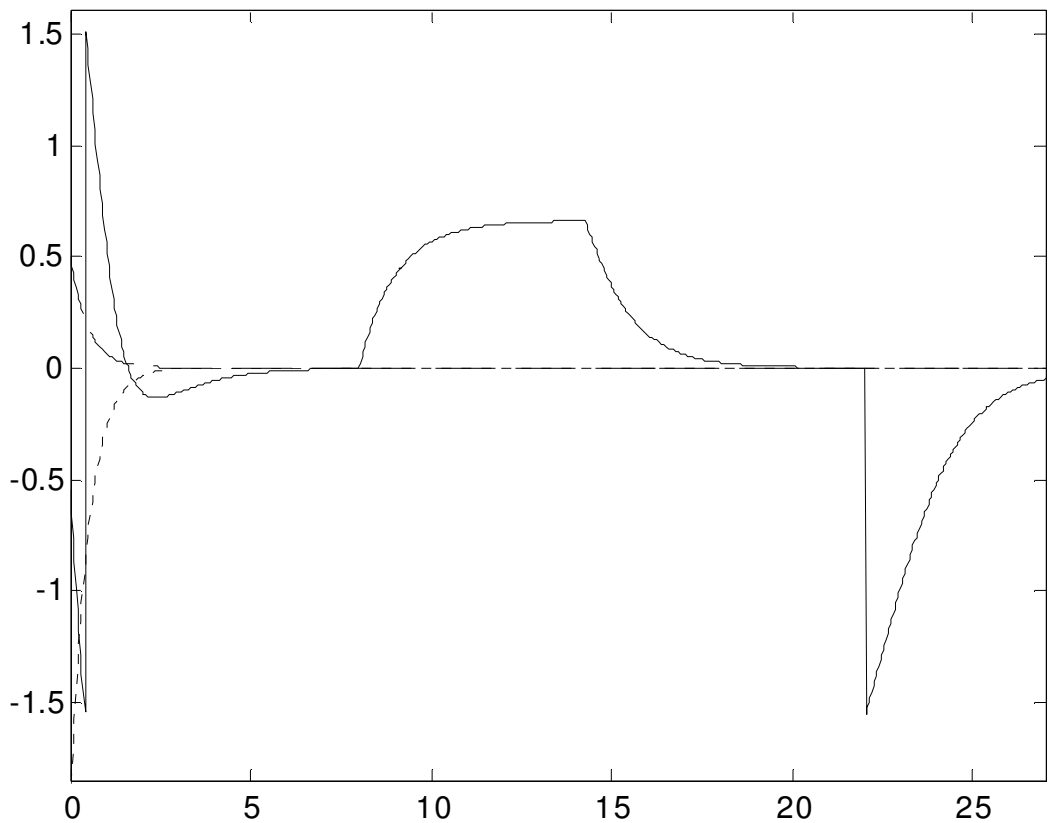

Figura 8.8: Orientación $\beta_{3}(-)$, error en $x(--)$ y error en $y(--)$ para ejemplo $2^{\circ}$ 
En el primer ejemplo la referencia consiste en un giro inicial de $180^{\circ}$ y una línea posterior. Se observa (Figuras 8.5 y 8.6) que el control funciona bien ya que el origen de $\mathrm{R}$ sigue la referencia $\mathrm{y}$ el error la dinámica asignada (exponencial de constante de tiempo 2 segundos).

También se aprecia (Figura 8.6) que la orientación $\beta_{3}$ de la rueda orientable es continua. Por lo que siempre existe una velocidad de control $\dot{\beta}_{3 \text { control }}$ no infinita, aunque cambia de forma instantánea en el punto de curvatura discontinua $\left(t_{\text {disc }} \cong 9.5 \mathrm{~s}\right)$ al pasar del arco a la recta. Por tanto dentro del marco cinemático la rueda orientable no supone ningún problema para seguir esta referencia, aunque inicialmente hay que dotarla del ángulo adecuado.

En el segundo ejemplo (Figuras 8.7 y 8.8 ) la referencia posee un punto de discontinuidad en el ángulo la tangente al camino. Para el control aplicado el origen de $\mathrm{R}$ sigue la referencia (Figura 8.7) con la dinámica asignada (Figura 8.8 ), sin embargo la orientación $\beta_{3}$ de la rueda orientable sufre dos discontinuidades.

La primera discontinuidad es de valor $\pi$ y evidencia que la orientación $\beta_{3}$ habría que calcularla en los cuatro cuadrantes para que sea continua. En caso de que la rueda sólo tenga $180^{\circ}$ de rango $\left( \pm 90^{\circ}\right)$, como es el caso de la carretilla industrial, obligaría a detener el vehículo para reorientarla.

La segunda discontinuidad es de valor $\pi / 2$ y se produce en el punto de discontinuidad del ángulo de la tangente al camino, implicando una acción de control irrealizable. Por tanto para poder seguir la referencia sin error habría que detener la marcha en ese punto y reorientarla.

En caso de que el vehículo fuera diferencial, es decir que no estuviera la rueda orientable, las discontinuidades anteriores no serían un problema (ver Tabla 8.1).

Un hecho curioso es que se produce una maniobra (cambio del sentido de movimiento) al principio del seguimiento de la referencia (Fig. 8.7). De esto se desprende que el control cinemático diseñado no distingue entre seguir la referencia con el vehículo de 'cara' o de 'espaldas'. Para evitar ir de 'espaldas' el vehículo debe estar ligeramente orientado hacia la referencia, o simplemente incorporar un trayecto previo que encare el vehículo con la referencia original.

Es interesante hacer notar que el camino seguido por el punto medio de las ruedas fijas (Fig. 8.7) tiene continuo el ángulo de la tangente, a pesar de que el ángulo de la tangente al camino seguido por la referencia es discontinuo. 


\subsubsection{Resultados con la carretilla industrial}

Se ha implementado sobre el autómata programable que controla la carretilla industrial la cinemática inversa de la rueda orientable, dada por (8.49) y (8.51), y el control de posición de (8.52).

No obstante, para el control de posición (8.52) se ha introducido la variante:

$$
\begin{aligned}
& { }^{\mathrm{G}} v_{x \text { control }}={ }^{\mathrm{G}} \dot{x}_{\mathrm{R} \text { ref }} \cdot f_{\text {aux }}+a_{x} \cdot\left({ }^{\mathrm{G}} x_{\mathrm{R} \text { ref }}-{ }^{\mathrm{G}} x_{\mathrm{R}}\right) \\
& { }^{\mathrm{G}} v_{y \text { control }}={ }^{\mathrm{G}} \dot{y}_{\mathrm{R} \text { ref }} \cdot f_{\text {aux }}+a_{y} \cdot\left({ }^{\mathrm{G}} y_{\mathrm{R} \text { ref }}-{ }^{\mathrm{G}} y_{\mathrm{R}}\right) \\
& \text { con } f_{\text {aux }}=\left\{\begin{array}{lll}
1 & \text { si } & \sqrt{\left({ }^{\mathrm{G}} x_{\mathrm{R} \text { ref }}-{ }^{\mathrm{G}} x_{\mathrm{R}}\right)^{2}+\left({ }^{\mathrm{G}} y_{\mathrm{R} \text { ref }}-{ }^{\mathrm{G}} y_{\mathrm{R}}\right)^{2}} \\
0 & \text { si } & \sqrt{\left({ }^{\mathrm{G}} x_{\mathrm{R} \text { ref }}-{ }^{\mathrm{G}} x_{\mathrm{R}}\right)^{2}+\left({ }^{\mathrm{G}} y_{\mathrm{R} \text { ref }}-{ }^{\mathrm{G}} y_{\mathrm{R}}\right)^{2}}>\mathrm{d}_{\text {max }}
\end{array}\right.
\end{aligned}
$$

donde $\mathrm{d}_{\max }$ es la distancia máxima para la que considerar la prealimentación derivativa, ya que si el error es grande (punto de seguimiento muy alejado de la referencia) la prealimentación pierde su sentido.

Notar que no es conveniente ponderar la presencia de la prealimentación derivativa en función del error porque produciría un error no nulo en régimen permanente.

Para el control de la carretilla se dispone de un motor de tracción, que actúa sobre el mecanismo diferencial de las ruedas fijas, y otro de dirección que actúa sobre la rueda orientable. El diseño del controlador dinámico de bajo nivel de cada motor se ha realizado de forma desacoplada.

Para el motor de dirección se ha conseguido un control desacoplado (con la carretilla parada) entorno a 1.5 segundos y sin sobreoscilación.

El caso del control del motor de tracción es un poco más complejo. El control se realiza sobre el valor de la velocidad de la rueda orientable. Esta velocidad se estima utilizando la información de los encoders de las ruedas fijas y la orientación de la orientable. En concreto se utiliza el modelo con deslizamiento del capítulo anterior junto con un pequeño filtrado.

Se ha conseguido un control del motor de tracción desacoplado (con rueda orientable a $0^{\circ}$ ) con un tiempo de establecimiento entorno a 3.5 segundos y una pequeña sobreoscilación entorno al 15\%. 
Se han realizado experiencias de dos trayectorias típicas, la línea recta y el círculo, ambas con una velocidad de seguimiento de $0.5 \mathrm{~m} / \mathrm{s}$.

En las Figuras 8.9 y 8.10 se muestra el resultado para el caso de trayectoria rectilínea con dos valores distintos de $\mathrm{a}_{x} \mathrm{y} \mathrm{a}_{y}$.

En el primer caso $\left(a_{x}=a_{y}=1 \mathrm{~s}^{-1}\right)$ el vehículo converge a la recta y el error se anula de forma ligeramente oscilante, mientras que en el segundo $\left(a_{x}=a_{y}=0.5 \mathrm{~s}^{-1}\right)$ se tiene una convergencia más lenta y no oscilante. Se ha podido comprobar empíricamente que asignar, al control cinemático, dinámicas más rápidas que las del primer caso produce inestabilidad. De hecho el tiempo de establecimiento del control cinemático sería de 5 segundos (al 99\%), que es ligeramente superior al tiempo dado por controlador dinámico de bajo nivel más lento.

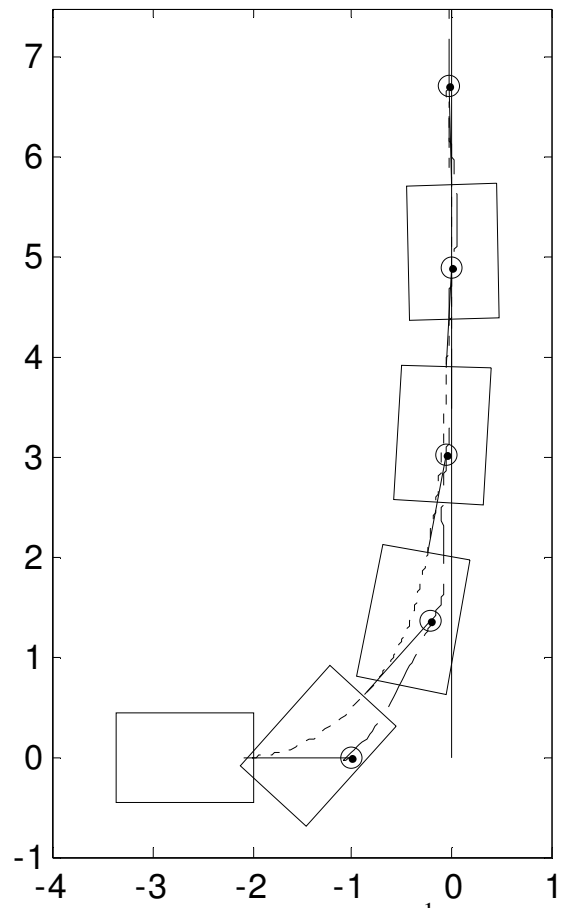

a) $a_{x}=a_{y}=1 \mathrm{~s}^{-1}$

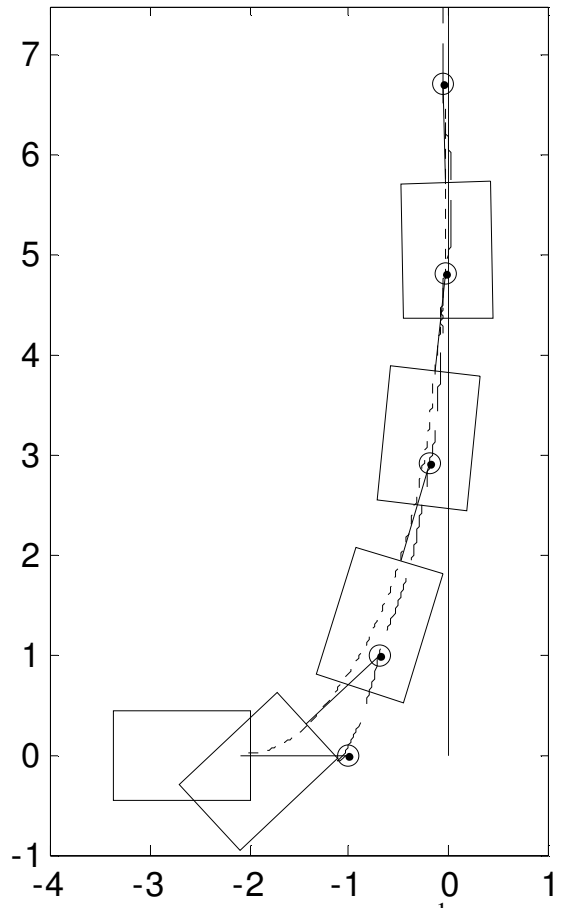

b) $a_{x}=a_{y}=0.5 \mathrm{~s}^{-1}$

Figura 8.9: Experiencias de seguimiento de trayectoria rectilínea: camino seguido por la referencia (-), origen de $\mathrm{R}(--)$ y punto medio de las rueda fijas (- -)

En las Figuras 8.11 y 8.12 se muestra el resultado para el caso de trayectoria circular con $a_{x}=a_{y}=1 \mathrm{~s}^{-1}$. Se puede comprobar que el seguimiento de la referencia es más o menos aceptable, aunque el valor de orientación de la rueda orientable se está ajustando constantemente durante todo el movimiento circular. 

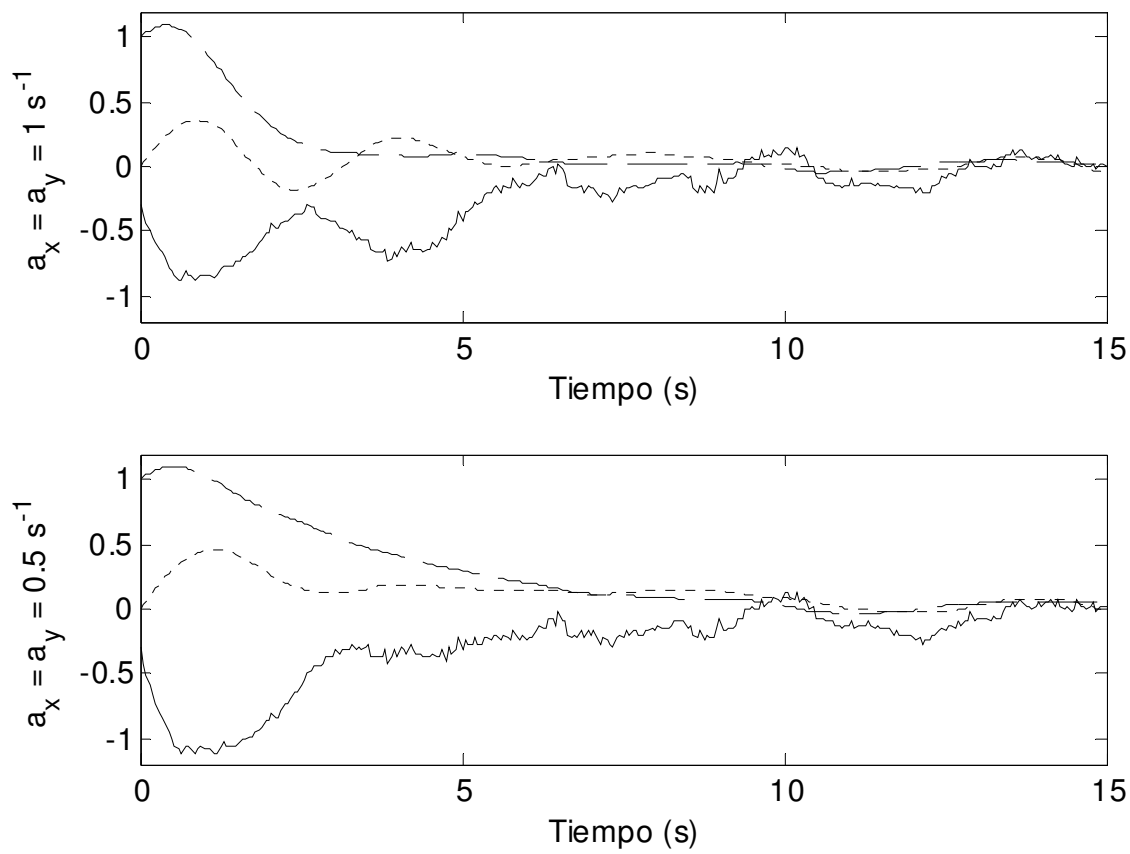

Figura 8.10: Orientación $\beta_{3}(-)$, error en $x(--)$ y error en $y(--)$ en caso de recta

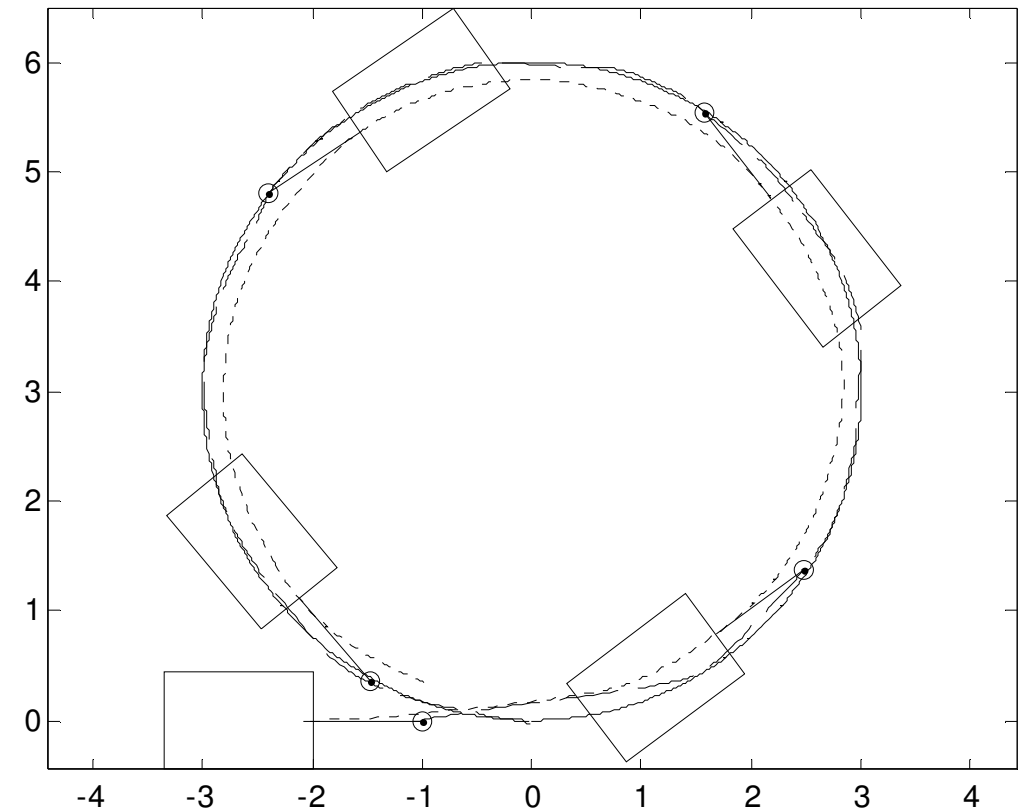

Figura 8.11: Experiencia de seguimiento de trayectoria circular: camino seguido por la referencia (-), origen de $\mathrm{R}(--)$ y punto medio de las rueda fijas (- -) 


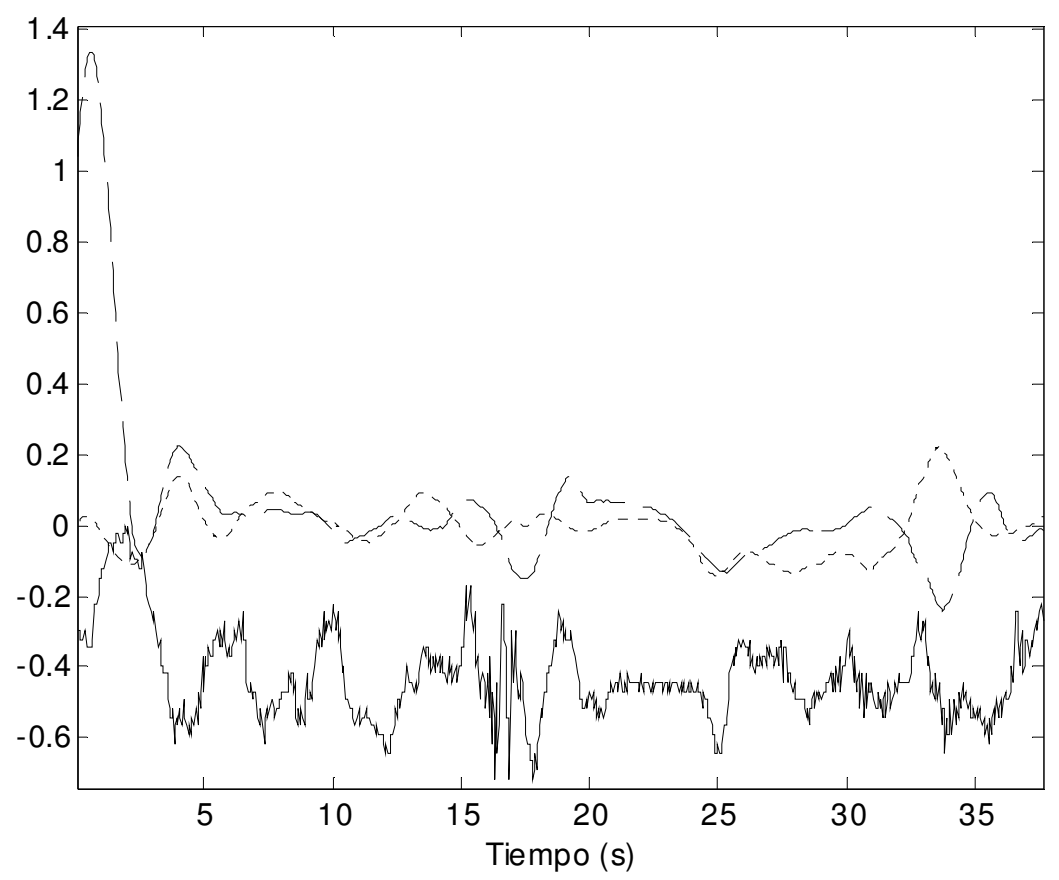

Figura 8.12: Orientación $\beta_{3}(-)$, error en $x(--)$ y error en $y(--)$ para el círculo

Cabe indicar que un control acoplado de los dos motores (bucle dinámico de bajo nivel) evitaría la perturbación que se infringe uno a otro, mejorando la respuesta conjunta.

Mientras que un control dinámico global evitaría la necesidad de un bucle dinámico de bajo nivel más rápido que el cinemático. 


\subsection{RESULTADOS MÁS RELEVANTES Y CONCLUSIONES DEL CAPÍTULO}

A continuación, se indican los aspectos más relevantes del capítulo.

- Se ha propuesto un control global válido para cualquier tipo de vehículo.

- El esquema de control es similar conceptualmente a los empleados para robots manipuladores y consiste en tres bucles de control anidados: uno dinámico de bajo nivel, otro cinemático de nivel medio y un último de planificación de alto nivel.

- En este capítulo se ha profundizado en el bucle de control cinemático e indirectamente en el de planificación. En concreto control cinemático propuesto incluye una parte de control de posición y otra parte de cinemática inversa de ruedas.

- El control de posición consiste en un regulador proporcional (o proporcional integral si hay zonas muertas) con prealimentación derivativa para cada una de las coordenadas sobre las que se realiza el seguimiento de referencia.

- La cinemática inversa se ha obtenido para los tipos de rueda habituales: fijas, orientable, castor y suecas.

- Para los cinco tipos de vehículos posibles, clasificados en el Capítulo 5, se han obtenido los tipos de referencias posibles (a tener en cuenta por el planificador) para que su seguimiento se realicen sin error. Lo anterior es la aportación teórica importante del capítulo, resumida en la Tabla 8.1, y se ha deducido analizando la cinemática inversa de las ruedas.

- Se ha particularizado el control cinemático para el caso del vehículo triciclo. $\mathrm{Su}$ posterior simulación ha permitido verificar las limitaciones así como obtener interesantes conclusiones de índole práctica.

- Se han presentado resultados experimentales del control cinemático implementado sobre una carretilla industrial, evidenciándose la importancia del control dinámico de bajo nivel para garantizar la estabilidad global. 

CAPÍTULO 9

\section{APLICACIONES PARA EL CONTROL CINEMÁTICO DE VEHÍCULOS CON RUEDAS}

\subsection{INTRODUCCIÓN}

En ese capítulo se presentan dos aplicaciones a las que poder aplicar el control cinemático presentado en el Capítulo 8.

Estas aplicaciones representan estudios parciales, donde la revisión bibliográfica no es exhaustiva, ya que más bien se ha querido dar soluciones prácticas a dos aplicaciones robóticas concretas. Un estudio más exhaustivo de de estas aplicaciones conduce a tesis doctorales en sí mismas, ej. [Gómez 01].

La primera aplicación es el seguimiento de línea por visión, que incluye un sistema de calibración y posicionamiento:

En los últimos años ha sido considerable el avance tecnológico para los sistemas de visión, por lo que cada vez más se utilizan como elementos sensores para gran variedad de aplicaciones.

Muchas de estas aplicaciones requieren conocer la situación de la unidad de visión o cámara respecto a elementos captados por ésta. Concretamente, conocer la situación de una cámara, incorporada como sensor a un vehículo, respecto a una línea permitiría al mismo, utilizando sus actuadores, seguir dicha línea (que representaría el eje de una 
calzada, una hilera de cultivo, etc.) de forma autoguiada como en [Gracia2 et al. 03]. Quedando justificado el interés por posicionar la cámara respecto a una línea de referencia.

La segunda aplicación es el aparcamiento en línea de un vehículo con maniobras a través de un planificador:

Los robots móviles con ruedas están usualmente involucrados en movimientos como el atraque y aparcamiento en paralelo, línea ó cordón con espacio limitado. Esta cuestión es compleja cuando se considera el vehículo clásico tipo coche, debido a sus restricciones no-holónomas [Laumond 92]. Muchos autores han intentado resolver estas situaciones con algoritmos eficientes de generación de movimiento de avance y retroceso [Barraquand et al. 89].

De hecho, el aparcamiento en línea o cordón es un problema interesante que justifica el diseño de planificadores potentes, algunos de ellos basados en razonamientos geométricos o criterios de optimización. 


\subsection{SEGUIMIENTO DE LÍNEA POR VISIÓN}

\subsubsection{Posicionamiento por visión}

\subsubsection{A) Introducción}

Existen varias posibilidades para posicionar un vehículo respecto a una línea, aunque las ventajas aportadas por un sistema de visión, comentadas en el subapartado posterior, hace preferible en ocasiones decantarse por esta opción.

Así pues, el problema se desglosará en dos partes: por un lado identificar la línea a partir de la imagen captada, utilizándose la transformada de Hough [Hough 59], ampliamente extendida [Luo 98]; y por otro calcular la posición del vehículo a través del modelo de cámara $[\mathrm{Fu}$ et al. 88] particularizado, alcanzándose una relación analítica que no requiere aproximaciones ni cálculo numérico como en [Marchant 95].

Dado que los parámetros del sistema de visión que intervienen en el cálculo (distancia focal, inclinación, etc.) son complicados de obtener se ha desarrollado un sistema de calibración especial sencillez.

\subsubsection{B) Alternativas para el posicionamiento de vehículos}

\section{- Basado en distancias a puntos de referencia}

Consiste en calcular las distancias a puntos de referencia conocidos para obtener la posición (local o global) del vehículo.

Un sistema muy utilizado para obtener la posición global del vehículo es el GPS ([Nagasaka et al 00]; [Rekow et al. 98], etc.), aunque existen otras alternativas para posicionar localmente el vehículo utilizando, por ejemplo, sensores de ultrasonidos, marcas en el suelo, sensores magnéticos, etc.

Este sistema de posicionamiento tiene, con objeto de autoguiar el vehículo, los siguientes inconvenientes:

1) Hay que tener almacenada previamente las características del espacio de movimientos del vehículo;

2) Para tener un buen conocimiento de éste, hay que obtener y almacenar mucha información, con los problemas que ello conlleva; 
3) Es poco robusto ante cambios sensibles en las características de dicho espacio.

- Basado en análisis de imagen

Consiste en utilizar sistemas, incorporados al vehículo, de visión por cámara (CCD por ejemplo) para caracterizar, a través de técnicas de tratamiento de imágenes, el entorno visualizado (líneas, curvas, etc.) que se utilizará como referencia. Posteriormente hay que realizar una análisis a través del modelo de cámara para posicionar el vehículo respecto a su entorno.

Este sistema de posicionamiento tiene como inconvenientes:

1) El tratamiento de imágenes puede ser lento;

2) El valor posicionamiento obtenido es local.

No obstante presenta la ventaja respecto a la opción anterior, de no depender (hasta cierto punto) de las características del espacio de movimientos del vehículo. Esta ventaja es definitiva para preferir esta opción, puesto que permite trabajar en un espectro más amplio de entornos utilizando el mismo sistema de posicionamiento.

\subsubsection{C) Obtención de las líneas del plano imagen}

Las líneas del plano imagen se pueden obtener por técnicas tradicionales basadas en el análisis de imagen. En este caso se aplican las siguientes:

$\left.1^{\circ}\right)$ Se identifican los píxeles de interés del plano imagen.

Los píxeles de interés pueden ser los del elemento que marque la referencia a seguir (raya en el suelo, cultivo, etc.), para lo que se aplicaría un filtrado, o los del contorno de dicha referencia, para lo que se aplicaría un operador gradiente. La segunda opción es más conveniente cuando el contorno de la referencia es rectilíneo y dicha referencia tiene una anchura apreciable. De forma que, se identificarían las dos líneas del contorno (computacionalmente más rápido) para después interpolarlas. 
$2^{\circ}$ ) Saturar la información anterior, es decir convertir a binario (blanco o negro) el color de cada píxel.

$3^{\circ}$ ) Calcular las líneas que se ajustan más a la ubicación de los píxeles de interés detectados.

La última fase es con diferencia, la más lenta del proceso de posicionamiento. Por ello es importante implementar un buen sistema de ajuste de línea. Por ejemplo, [Pinto et al. 99] implementan clasificadores basados en redes neuronales, mientras que en [Sánchez et al. 97] se utiliza la transformada de Hough, muy frecuente en aplicaciones de visión por su buena respuesta computacional comparativa. De hecho, esta segunda opción es la que los autores de este trabajo emplean.

La transformada de Hough se utiliza para identificar, en un espacio bidimensional, formas predefinidas caracterizadas por un número $n$ de parámetros. De tal forma que cada punto del espacio bidimensional da lugar a un conjunto de posibles valores de parámetros, en un espacio n-dimensional, tal que la forma predefinida, para esos valores de parámetros, pasa por ese punto. Para más información consúltese [Hough 59] [Luo 98].

En este caso la forma predefinida es la línea, caracterizada por dos parámetros. Con lo que, cada píxel de interés (identificado del plano imagen) da lugar a una curva (en el caso más general) en el plano de la transformada, cortándose en el mismo punto los puntos del plano imagen alineados.

Por tanto, una vez discretizado espacio de transformada se calcula el número de rectas que pasan por cada celda, y aquellas por las que, sin ser adyacentes o demasiado próximas, pasen más rectas dan lugar a los valores de parámetros de las líneas identificadas en el plano imagen.

Lo anterior es válido, sobre todo, para el caso de que los puntos de interés sean el contorno de la referencia. En el caso de que los puntos de interés sean la referencia, y ésta tenga una anchura apreciable, conviene calcular la celda media de entre todas las que, siendo adyacentes, sean atravesadas por un número de rectas elevado. De forma que, se evita abatir la línea identificada dentro de la referencia.

Además, para esta aplicación se utiliza la transformada de Hough normalizada, para evitar singularidades (Ej. pendientes infinitas). 


\subsubsection{D) Cálculo del posicionamiento}

A continuación se describe el modelo de cámara descrito en [Fu et al. 88] para después particularizar a las necesidades de este estudio.

\section{Modelo de cámara}

En la Figura 9.1 se muestra el modelo básico formación de imágenes. El significado de las variables utilizadas es:

$\left(X_{c}, Y_{c}, Z_{c}\right)$ : Sistema de coordenadas (en el objetivo) de la cámara;

$\left(x_{c}, y_{c}, z_{c}\right)$ : Posición de un punto respecto al sistema de la cámara;

$\left(X_{u}, Y_{u}\right)$ : Sistema de coordenadas del plano imagen;

$\left(x_{u}, y_{u}\right)$ : Posición en el plano imagen correspondiente al punto $\left(x_{c}, y_{c}, z_{c}\right)$;

$f$ : Distancia focal efectiva de la cámara, que se supone conocida.

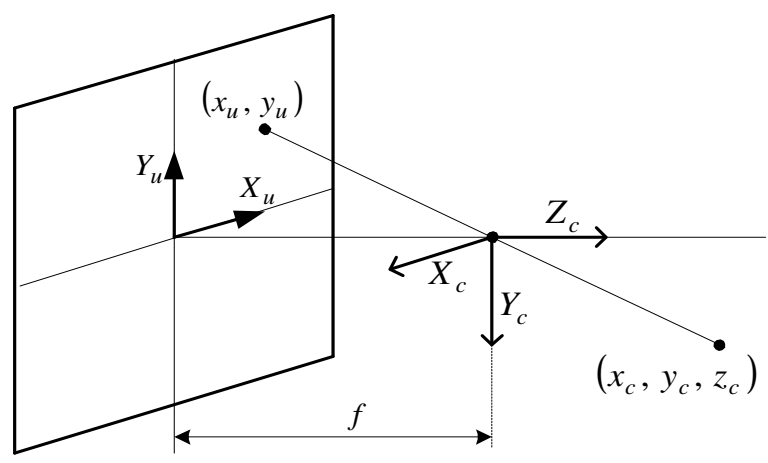

Figura 9.1- Modelo básico de formación de imagen

Teniendo en cuenta la Figura 9.1 se obtiene:

$$
x_{u}=\frac{f}{z_{c}} \cdot x_{c} \quad y_{u}=\frac{f}{z_{c}} \cdot y_{c}
$$

Relacionando la posición anterior en el plano imagen con sus correspondientes coordenadas en píxeles $\left(x_{p}, y_{p}\right)$, se obtiene:

$$
x_{u}=\left(x_{p}-\mathrm{C}_{x}\right) \cdot \mathrm{d}_{x} \quad y_{u}=\left(y_{p}-\mathrm{C}_{y}\right) \cdot \mathrm{d}_{y}
$$

donde $\left(\mathrm{C}_{x}, \mathrm{C}_{y}\right)$ son las coordenadas en píxeles del centro del plano imagen y $\left(\mathrm{d}_{x}\right.$, $\mathrm{d}_{y}$ ) el tamaño de los elementos sensores en cada eje. 


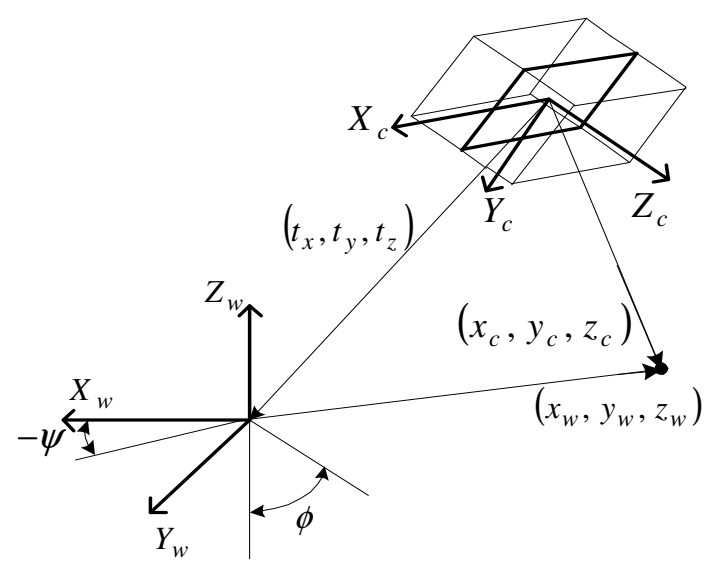

Figura 9.2- Relación entre el sistema de coordenadas del suelo y el de la cámara

El significado de las variables de la Figura 9.2 es:

$\left(X_{w}, Y_{w}, Z_{w}\right)$ : Sistema de coordenadas del suelo;

$\left(x_{w}, y_{w}, z_{w}\right)$ : Posición de un punto respecto al sistema del suelo;

$\left(t_{x}, t_{y}, t_{z}\right)$ : Vector de desplazamiento entre el sistema de coordenadas de la cámara y el del suelo;

$\phi$ : ángulo de inclinación (cabeceo) de la cámara respecto a la vertical;

$\psi$ : Ángulo de giro entre el sistema de la cámara y el del suelo.

Teniendo en cuenta la Figura 9.2 la relación entre los sistemas de coordenadas del suelo y de la cámara es:

$$
\left(\begin{array}{c}
x_{c} \\
y_{c} \\
z_{c} \\
1
\end{array}\right)=\left(\begin{array}{cccc}
\mathrm{c} \psi & \mathrm{s} \psi & 0 & t_{x} \\
\mathrm{~s} \psi \cdot \mathrm{c} \phi & -\mathrm{c} \psi \cdot \mathrm{c} \phi & -\mathrm{s} \phi & t_{y} \\
\mathrm{~s} \psi \cdot \mathrm{s} \phi & \mathrm{c} \psi \cdot \mathrm{s} \phi & -\mathrm{c} \phi & t_{z} \\
0 & 0 & 0 & 1
\end{array}\right) \cdot\left(\begin{array}{c}
x_{w} \\
y_{w} \\
z_{w} \\
1
\end{array}\right)
$$

Obsérvese que se ha hecho nulo el ángulo de vasculación entre la cámara y el suelo, ya que no aporta ninguna ventaja y se simplifican los cálculos.

Con (9.1), (9.2) y (9.3) se calcula la posición $\left(t_{x}, t_{y}, t_{z}\right)$ y orientación $(\phi, \psi)$ de la cámara respecto al suelo. En particular sustituyendo $\left(x_{c}, y_{c}, z_{c}\right)$ de $(9.3)$ en (9.1) se obtiene: 


$$
\begin{aligned}
& x_{u}=\frac{f \cdot\left(\mathrm{c} \psi \cdot x_{w}+\mathrm{s} \psi \cdot y_{w}+t_{x}\right)}{\mathrm{s} \phi \cdot\left(\mathrm{s} \psi \cdot x_{w}+\mathrm{c} \psi \cdot y_{w}\right)-\mathrm{c} \phi \cdot z_{w}+t_{z}} \\
& y_{u}=\frac{f \cdot\left(\mathrm{c} \phi \cdot\left(\mathrm{s} \psi \cdot x_{w}-\mathrm{c} \psi \cdot y_{w}\right)-\mathrm{s} \phi \cdot z_{w}+t_{y}\right)}{\mathrm{s} \phi \cdot\left(\mathrm{s} \psi \cdot x_{w}+\mathrm{c} \psi \cdot y_{w}\right)-\mathrm{c} \phi \cdot z_{w}+t_{z}}
\end{aligned}
$$

En general, en (9.4) hay 5 incógnitas $\left\{t_{x}, t_{y}, t_{z}, \phi, \psi\right\}$ (se ha hecho nulo el ángulo de vasculación), mientras que cada punto $\left(x_{u i}, y_{u i}\right)$ de la imagen, cuyas coordenadas $\left(x_{w i}, y_{w i}, z_{w i}\right)$ se conozcan, aporta 2 ecuaciones. Así pues, con tres puntos se obtiene el posicionamiento de la cámara respecto al sistema de coordenadas del suelo.

En caso de conocer alguna de las incógnitas $\left(t_{x}, t_{y}, t_{z}, \phi, \psi\right)$ se necesitarían menos puntos de referencia conocidos.

\section{Particularización del modelo de cámara}

Las Figuras 9.3 y 9.4 muestran la planta y perfil de la cámara, el vehículo, la línea a seguir y los sistemas de coordenadas.

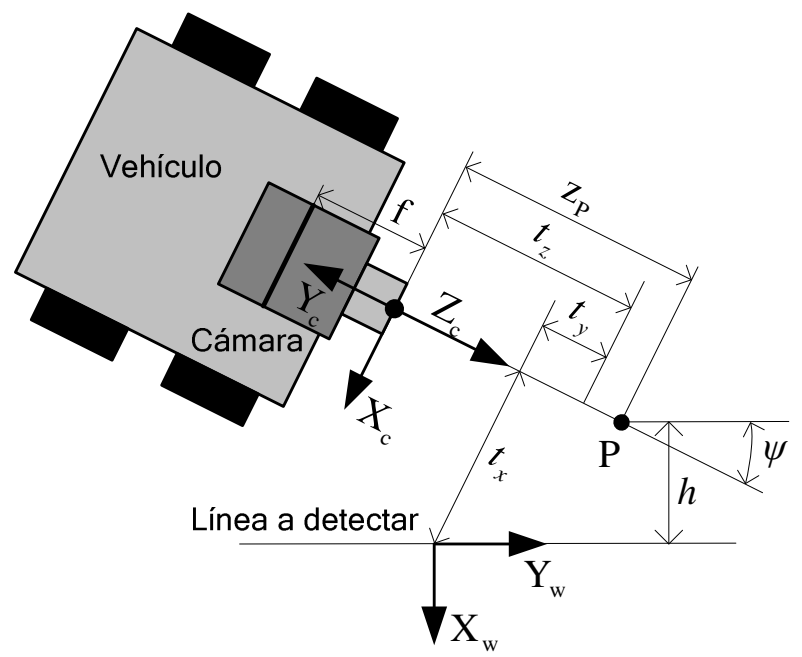

Figura 9.3- Vista de planta del vehículo, cámara, línea a seguir y sus sistemas de coordenadas 


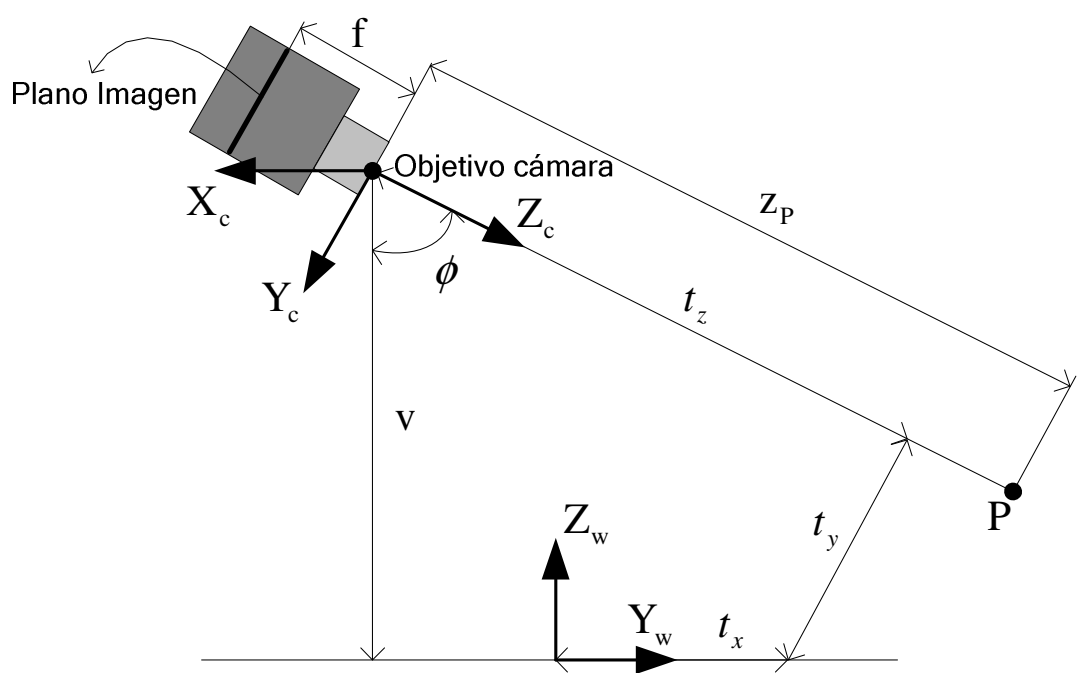

Figura 9.4- Vista de perfil de la cámara, línea a seguir y sus sistemas de coordenadas

Las nuevas variables y parámetros introducidos son:

v: altura (constante) a la que esta situada el objetivo de la cámara respecto a la línea a seguir;

P: Punto, solidario al vehículo y perteneciente al eje del objetivo de la cámara, que se quiere que haga el seguimiento;

Zp: Distancia desde el punto $\mathrm{P}$ al objetivo de la cámara;

$h$ : distancia entre P y la línea a seguir.

Al tener libertad para elegir el sistema de coordenadas del suelo, se fija con $Y_{w}$ sobre la línea a seguir (el sentido elegido establece el de $Y_{u}$ hacia abajo en la imagen captada).

Por lo que los puntos de la línea y de otras a la misma altura tienen:

$$
z_{w}=0
$$

De todos los orígenes posibles sobre la línea de seguimiento se elige aquel que hace:

$$
t_{y}=0
$$

Concretamente, éste origen se encuentra en el punto de corte de la línea a seguir con una línea paralela a $X_{c}$ que pase por el punto de corte del eje $Z_{c}$ con el plano $z_{w}=0$. No obstante, no será necesario calcular dicho origen. 
Ya que v y $\phi$ son constantes y supuestas conocidas se puede calcular:

$$
t_{z}=\frac{\mathrm{v}}{\cos (\phi)}
$$

Se considerará que los puntos de referencia utilizados están sobre la línea de seguimiento o paralela a ésta (ver Figura 9.5) a una distancia supuesta conocida $\pm d$, lo que se traduce en:

$$
x_{w}=\mathrm{n} \cdot \mathrm{d} \quad \text { con } \mathrm{n}=-1,0,1
$$

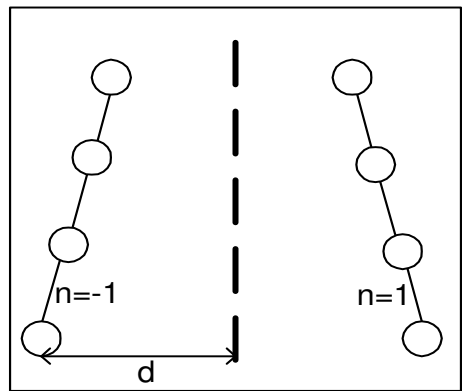

a) Ej. Calzada

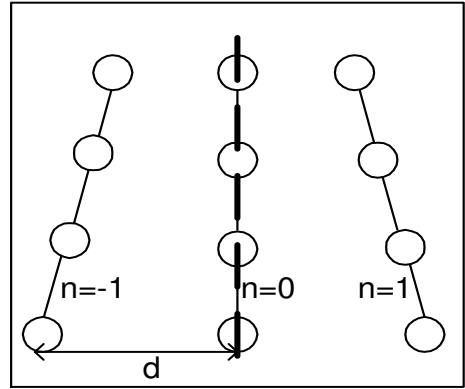

b) Ej. Línea de cultivo

Figura 9.5- Posibilidades de líneas de seguimiento

Por otro lado, no hay forma de conocer, a través de la imagen, el valor de $\mathrm{y}_{\mathrm{w}}$ de los puntos de referencia de la imagen. Por tanto, despejando $y_{w}$ de (9.4), y teniendo en cuenta (9.5), (9.6), (9.7) y (9.8), resulta:

$$
\mathrm{c} \psi \cdot\left(t_{x} \cdot\left(y_{u} \cdot \mathrm{s} \phi+f \cdot \mathrm{c} \phi\right)-\mathrm{v} \cdot x_{u}\right)+\mathrm{s} \psi \cdot y_{u} \cdot \mathrm{v} / \mathrm{c} \phi+\mathrm{n} \cdot \mathrm{d} \cdot\left(y_{u} \cdot \mathrm{s} \phi+f \cdot \mathrm{c} \phi\right)=0
$$

De (9.9) se obtienen los valores de $\left(t_{x}, \psi\right)$ para dos puntos del plano imagen, de los que hay que conocer si están sobre la línea a seguir, a su derecha ó a su izquierda. En el caso de utilizar dos puntos de la misma línea $\left(\mathrm{n}_{1}=\mathrm{n}_{2}=\mathrm{n}\right)$ se llega a la solución analítica:

$$
\begin{aligned}
& \psi=\arctan \left(\mathrm{c} \phi \cdot \frac{\left(x_{u 1}-x_{u 2}\right)}{\left(y_{u 1}-y_{u 2}\right)}+\frac{\mathrm{s} \phi}{f} \cdot \frac{\left(y_{u 2} \cdot x_{u 1}-y_{u 1} \cdot x_{u 2}\right)}{\left(y_{u 1}-y_{u 2}\right)}\right) \\
& t_{x}=\frac{1}{f} \cdot \frac{\mathrm{v}}{\mathrm{c} \phi} \cdot \frac{\left(y_{u 1} \cdot x_{u 2}-y_{u 2} \cdot x_{u 1}\right)}{\left(y_{u 1}-y_{u 2}\right)}-\frac{\mathrm{n} \cdot \mathrm{d}}{\mathrm{c} \psi}
\end{aligned}
$$


Siendo $h$, a partir de $\left(\psi, t_{x}\right)$ :

$$
h=\tan (\phi) \cdot\left(\mathrm{v}-z_{\mathrm{P}} \cdot \mathrm{c} \phi\right) \cdot \mathrm{s} \psi+t_{x} \cdot \mathrm{c} \psi
$$

Con lo cual, se tiene el posicionamiento $(\psi, h)$ a partir de los parámetros $(f$, $\left.\mathrm{v}, \phi, \mathrm{d}, z_{\mathrm{P}}\right)$ y de dos puntos $\left(x_{u i}, y_{u i}\right)$ de la imagen para los que se conoce la línea en la que están $\left(\mathrm{n}_{\mathrm{i}}\right)$.

El cálculo anterior no consigue situar el vehículo respecto a la dirección longitudinal de la línea a seguir. Esta limitación viene por el hecho de que, un sistema de visión no es capaz de determinar a qué altura de una "línea" se encuentran un punto de referencia. No obstante, esto no impide que el vehículo siga la línea de cultivo tal y como se muestra posteriormente.

\subsubsection{E) Sistema de calibración}

El problema de calibración consiste en el proceso inverso al posicionamiento, es decir se conoce la ubicación real de algunos puntos de la imagen y se establecen los parámetros del sistema de visión que producen esa correspondencia con el plano imagen.

\section{Situación para la calibración}

Con objeto de conseguir una solución analítica sencilla para dicho proceso inverso se fuerza:

$$
\psi=0 \quad t_{x}=0
$$

Para el caso de seguimiento de calzada de la Figura 9.5 (a) se tiene el plano imagen de la Figura 9.6 (a).

Para conseguir la situación anterior se desplazan los dos elementos paralelos de referencia (Ej.: rayas) respecto a la cámara (o al revés) hasta que la línea media, obtenida a partir de las dos identificadas para cada elemento (con el sistema descrito en una apartado anterior), divida el plano imagen en dos rectángulos iguales. 


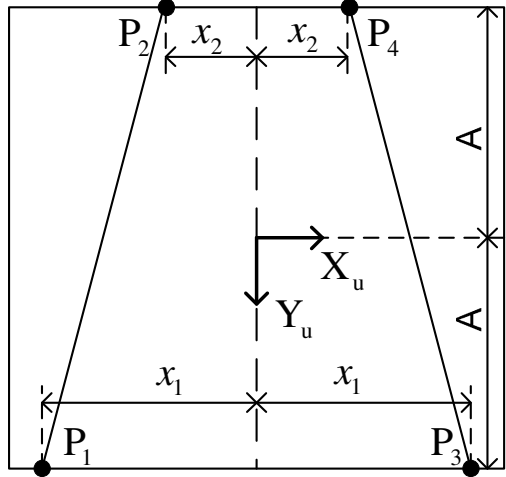

a) Plano imagen

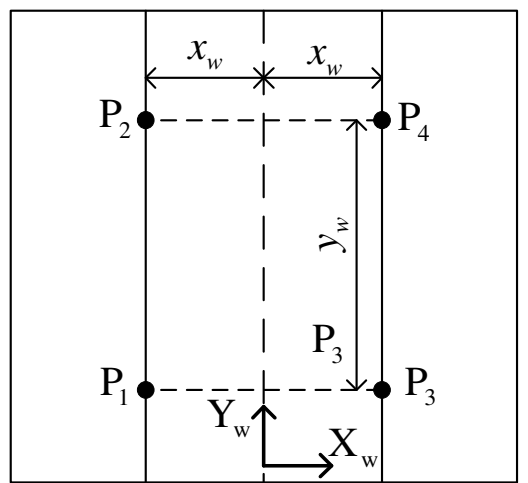

b) Plano real

Figura 9.6- Situación de calibración

\section{Cálculo de la inclinación de la cámara}

Una vez dada esta situación "especial" de calibración, se recurre a la fórmula (9.4) que, teniendo en cuenta (9.12) y (9.5), resulta:

$$
x_{u}=\frac{f \cdot x_{w}}{\mathrm{~s} \phi \cdot y_{w}+t_{z}} \quad y_{u}=\frac{f \cdot\left(t_{y}-\mathrm{c} \phi \cdot y_{w}\right)}{\mathrm{s} \phi \cdot y_{w}+t_{z}}
$$

Dividiendo las dos ecuaciones de (9.13):

$$
\frac{x_{u}}{y_{u}}=\frac{x_{w}}{\left(t_{y}-\mathrm{c} \phi \cdot y_{w}\right)}
$$

Tomando dos puntos $\mathrm{P}_{i}$ y $\mathrm{P}_{j}$ (de los cuatro de la imagen) se obtienen dos instancias de la expresión (9.14), de las cuales se puede eliminar $t_{y}$, resultando:

$$
\mathrm{c} \phi=\frac{1}{\left(y_{w j}-y_{w i}\right)} \cdot\left(\frac{x_{w i}}{x_{u i}} \cdot y_{u i}-\frac{x_{w j}}{x_{u j}} \cdot y_{u j}\right)
$$

Además, teniendo en cuenta la Figura 9.6:

$$
\begin{array}{cccc}
x_{w 1}=-x_{w} & \mathrm{y}_{w 1}=\mathrm{K} & x_{w 2}=-x_{w} & \mathrm{y}_{w 2}=\mathrm{K}+y_{w} \\
x_{w 3}=x_{w} & \mathrm{y}_{w 3}=\mathrm{K} & x_{w 4}=x_{w} & \mathrm{y}_{w 4}=\mathrm{K}+y_{w} \\
x_{u 1}=-x_{1} & \mathrm{y}_{u 1}=\mathrm{A} & x_{u 2}=-x_{2} & \mathrm{y}_{u 1}=-\mathrm{A} \\
x_{u 3}=x_{1} & \mathrm{y}_{u 3}=\mathrm{A} & x_{u 4}=x_{2} & \mathrm{y}_{u 4}=-\mathrm{A}
\end{array}
$$


Cualquier combinación de pares de puntos, salvo $\left\{\mathrm{P}_{1}, \mathrm{P}_{3}\right\}$ y $\left\{\mathrm{P}_{2}, \mathrm{P}_{4}\right\}$ que producen una indeterminación, hace que (9.15) resulte:

$$
\mathrm{c} \phi=\mathrm{A} \cdot \frac{x_{w}}{y_{w}} \cdot \frac{\left(x_{1}+x_{2}\right)}{x_{1} \cdot x_{2}}
$$

de donde se obtiene el parámetro $\phi$ (inclinación de la cámara) a partir de los valores $\left\{\mathrm{A}, x_{w}, y_{w}, x_{1}, x_{2}\right\}$, supuestamente conocidos.

\section{Cálculo de la distancia focal de la cámara}

Teniendo en cuenta (9.12), el primer elemento de (9.10) es:

$$
0=\mathrm{c} \phi \cdot \frac{\left(x_{u i}-x_{u j}\right)}{\left(y_{u i}-y_{u j}\right)}+\frac{\mathrm{s} \phi}{f} \cdot \frac{\left(y_{u j} \cdot x_{u i}-y_{u i} \cdot x_{u j}\right)}{\left(y_{u i}-y_{u j}\right)}
$$

donde $\mathrm{P}_{i}$ y $\mathrm{P}_{j}$ son puntos sobre la misma línea.

Despejando $f$ :

$$
f=-\tan (\phi) \cdot \frac{\left(y_{u j} \cdot x_{u i}-y_{u i} \cdot x_{u j}\right)}{\left(x_{u i}-x_{u j}\right)}
$$

Teniendo en cuenta (9.17), para cualquiera de las combinaciones $\left\{\mathrm{P}_{1}, \mathrm{P}_{2}\right\}$ ó $\left\{\mathrm{P}_{3}, \mathrm{P}_{4}\right\}$ se llega a:

$$
f=\tan (\phi) \cdot \mathrm{A} \cdot \frac{\left(x_{1}+x_{2}\right)}{\left(x_{1}-x_{2}\right)}
$$

de donde se obtiene el parámetro $f$ (distancia focal efectiva de la cámara) a partir de los valores conocidos $\left\{\mathrm{A}, x_{1}, x_{2}\right\}$ y $\{\phi\}$, que se calcula en (9.18).

Cálculo de la altura del objetivo de la cámara

A partir de (9.12) y del primer elemento de (9.10):

$$
0=\frac{1}{f} \cdot \frac{\mathrm{v}}{\mathrm{c} \phi} \cdot \frac{\left(y_{u i} \cdot x_{u j}-y_{u j} \cdot x_{u i}\right)}{\left(y_{u i}-y_{u j}\right)}-\mathrm{n} \cdot \mathrm{d}
$$

donde $\mathrm{P}_{i}$ y $\mathrm{P}_{j}$ son puntos sobre la misma línea y d equivale a $x_{w}$. 
Despejando v:

$$
\mathrm{v}=\mathrm{n} \cdot \mathrm{d} \cdot f \cdot \mathrm{c} \phi \cdot \frac{\left(y_{u i}-y_{u j}\right)}{\left(y_{u i} \cdot x_{u j}-y_{u j} \cdot x_{u i}\right)}
$$

Teniendo en cuenta (9.17) y (9.21), para cualquiera de las combinaciones $\left\{\mathrm{P}_{1}, \mathrm{P}_{2}\right\}$ ó $\left\{\mathrm{P}_{3}, \mathrm{P}_{4}\right\}$ se llega a:

$$
\mathrm{v}=\mathrm{s} \phi \cdot \mathrm{A} \cdot \frac{x_{w}}{\left(x_{1}-x_{2}\right)}
$$

de donde se obtiene el parámetro v (altura del objetivo de la cámara) a partir de los valores conocidos $\left\{\mathrm{A}, x_{w}, x_{1}, x_{2}\right\}$ y $\{\phi\}$ que se calcula en (9.18).

Por tanto, a partir de la información del plano imagen, caracterizada por $x_{l}$, $x_{2}$ y A, y de la ubicación real de los puntos, caracterizada por $x_{w}$ e $y_{w}$, se obtiene la calibración de los parámetros $\{\phi, f, v\}$, al aplicar (9.18), (9.21) y (9.24).

\subsubsection{F) Resultados del sistema de calibración y posicionamiento}

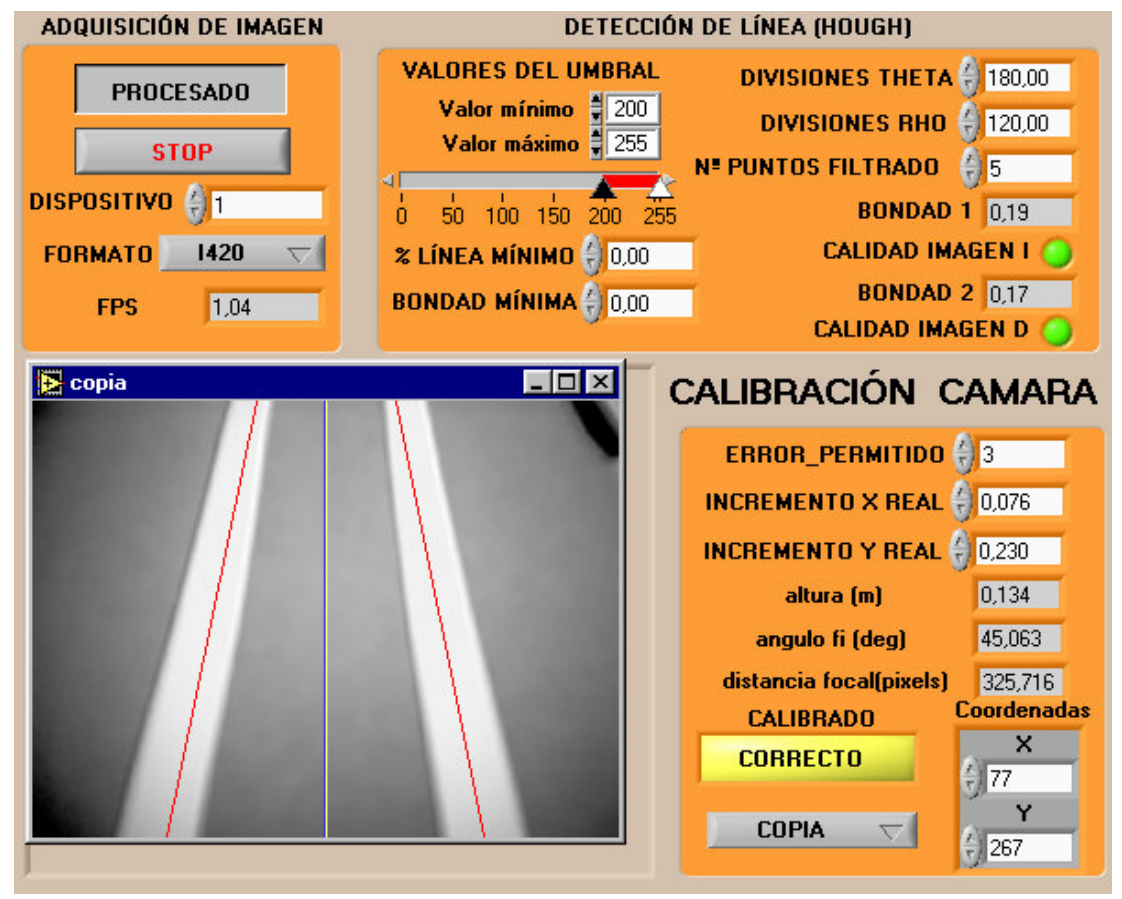

Figura 9.7- Ejemplo de Calibración 
En la Figura 9.7 se muestra un ejemplo de una aplicación basada en el sistema de calibración desarrollado. Obsérvese que las líneas se identifican bien a pesar de que la lente de la cámara deforma ligeramente la referencia (contornos de referencias un poco curvados).

Los valores de calibración obtenidos (Figura 9.7) son: altura $13.4 \mathrm{~cm}$, inclinación $45^{\circ}$, distancia focal 325.7 píxeles $\equiv 325.7$ x tamaño real del píxel.

Esta altura e inclinación se ajustan a las calculadas con herramientas métricas tradicionales $\left(13.5 \mathrm{~cm}\right.$ y $\left.45^{\circ}\right)$, lo que valida el sistema de calibración.

Una vez que se tiene el sistema de visión anterior calibrado, se ha utilizado para obtener el posicionamiento respecto a tres líneas (ver Figura 9.8) paralelas y equidistantes.

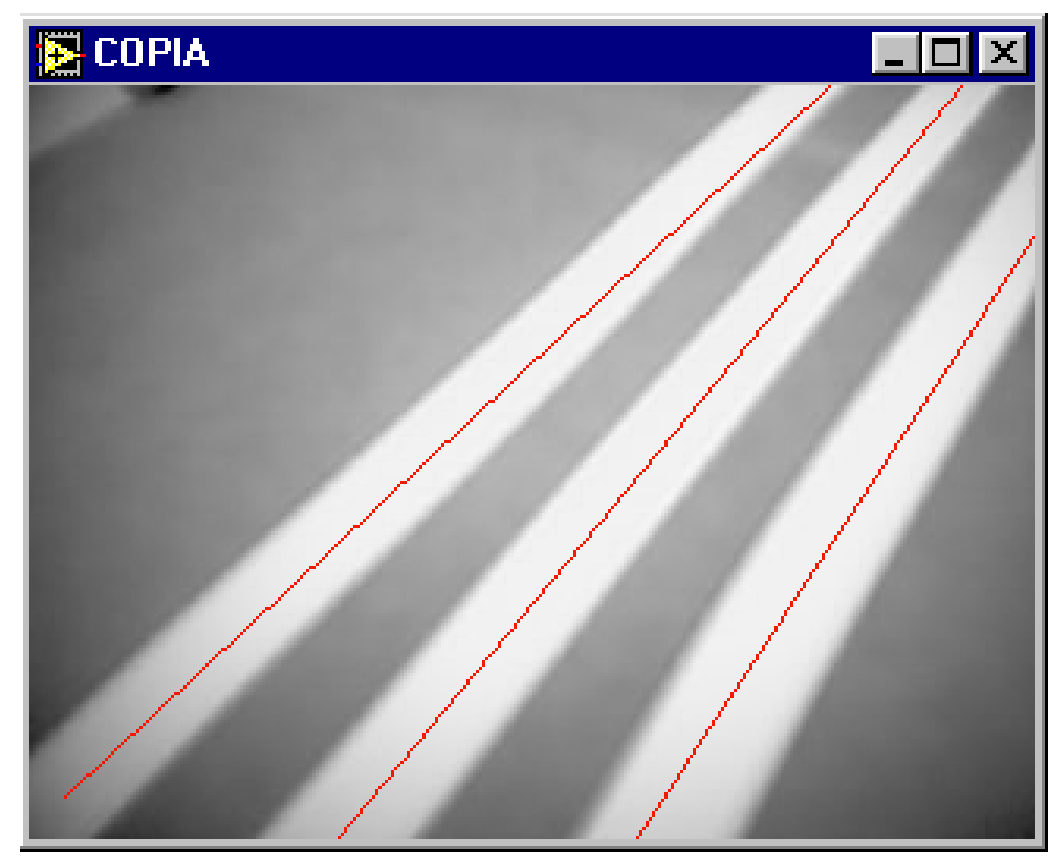

Figura 9.8- Líneas detectadas en ejemplo de posicionamiento con sistema calibrado

En la Figura 9.9 se muestran los resultados del posicionamiento del objetivo de la cámara $\left(z_{\mathrm{P}}=0\right)$ respecto a cada una de las líneas. Teniendo en cuenta que están separadas $3.5 \mathrm{~cm}$ el error de posicionamiento máximo (respecto a la media) es de $1.5^{\circ}$ y $2.3 \mathrm{~mm}$. 


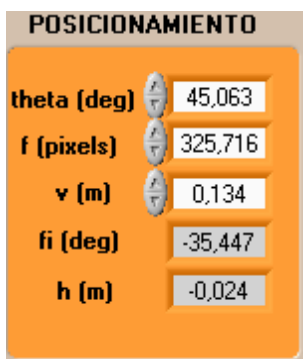

a) Izquierda

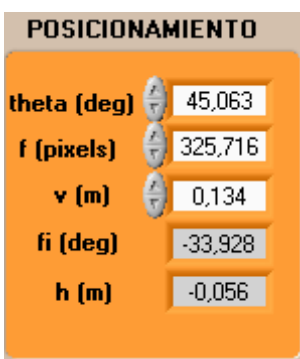

b) Centro

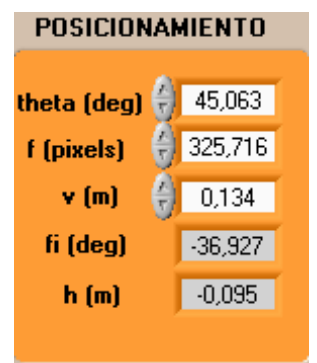

c) Derecha

Figura 9.9- Posicionamientos obtenidos

Con lo que la precisión lograda es bastante alta, por tanto queda también validado el sistema de posicionamiento desarrollado.

Finalmente, se aborda un ejemplo de posicionamiento en un campo de chufas. La Figura 9.10 (a) muestra la imagen original con las líneas detectadas superpuestas.

Para obtener las líneas se aplica el análisis de imagen indicado para referencias de contornos no rectilíneos, aunque el contorno del cultivo tampoco es totalmente abrupto (Figura 9.10 (b)).

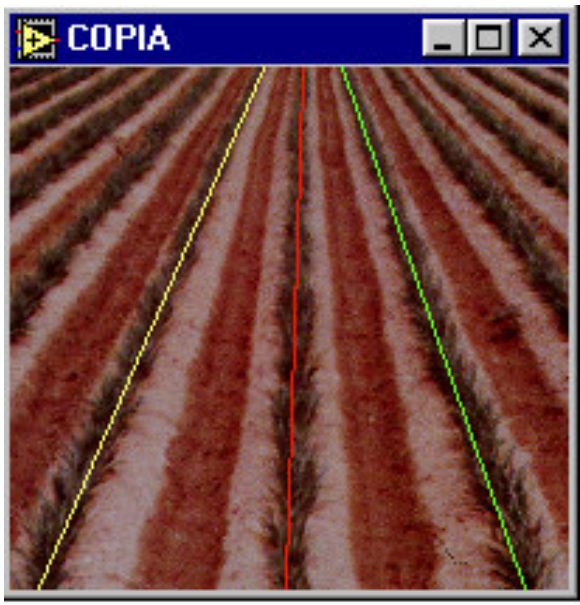

a) Imagen Original + líneas detectadas

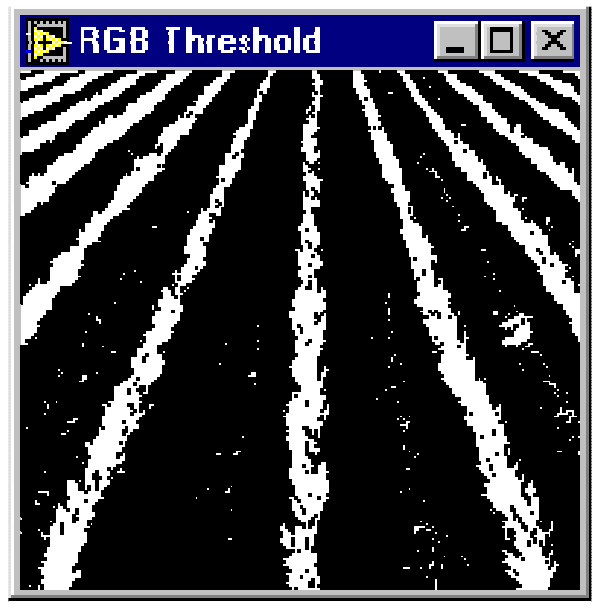

b) Imagen filtrada

Figura 9.10- Posicionamiento en campo de chufas

En la Figura 9.11 se muestra el resultado del plano de Hough de la imagen filtrada, donde se aprecian sobre todo los nodos de las 3 líneas centrales. 


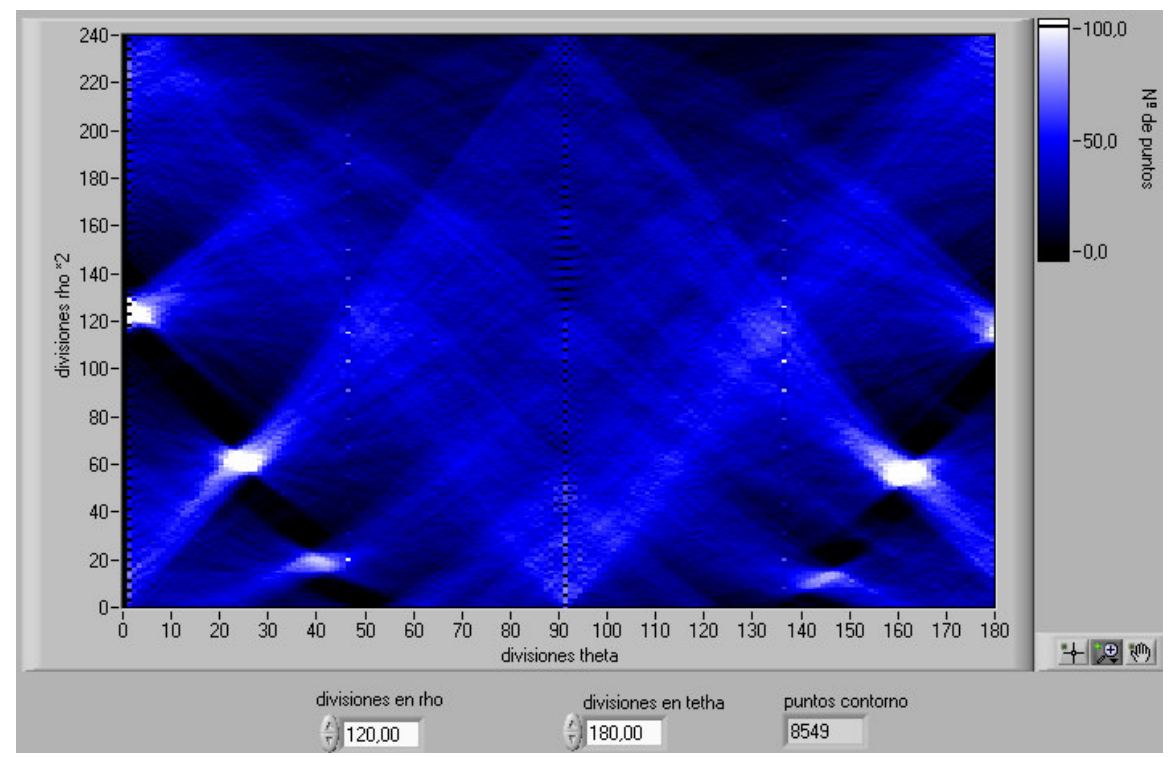

Figura 9.11- Transformada de Hough de la Figura 9.10 (b)

Las coordenadas de los puntos (en píxeles) para las líneas detectadas son:
a) $\quad\left\{n_{a}=1\right\} \quad P_{u 1 a}=(-10,-93) \quad P_{u 2 a}=(-90,93)$
b) $\quad\left\{n_{b}=-1\right\} \quad P_{u l b}=(4,-93) \quad P_{u 2 b}=(-2,93)$
c) $\quad\left\{n_{c}=0\right\} \quad P_{u l c}=(18,-93) \quad P_{u 2 c}=(83,93)$

El posicionamiento respecto a la línea central \{parámetros de visión: $f=545$ píxeles, v=1.42 $\left.\mathrm{m}, \mathrm{d}=60 \mathrm{~cm}, \phi=76^{\circ}\right\}$ según cada línea es:
a) $\psi_{\mathrm{a}}=-0.86^{\circ}$
$h_{a}=-2.4 \mathrm{~cm}$
b) $\psi_{\mathrm{b}}=-0.55^{\circ}$
$\mathrm{h}_{\mathrm{b}}=-4.2 \mathrm{~cm}$
c) $\psi_{\mathrm{c}}=-0.3^{\circ}$
$\mathrm{h}_{\mathrm{c}}=-8.5 \mathrm{~cm}$

Los tres posicionamientos son semejantes y de gran precisión (error máximo respecto a la media $0.3^{\circ}$ y $2.5 \mathrm{~cm}$ ), destacando la buena alineación de la cámara con la línea central. 


\subsubsection{Control cinemático para el seguimiento de línea por visión}

En este apartado se particulariza el control cinemático del vehículo triciclo (ver Capítulo 8) para poder aplicar al seguimiento de línea con la información del sistema de visión descrito en el apartado anterior.

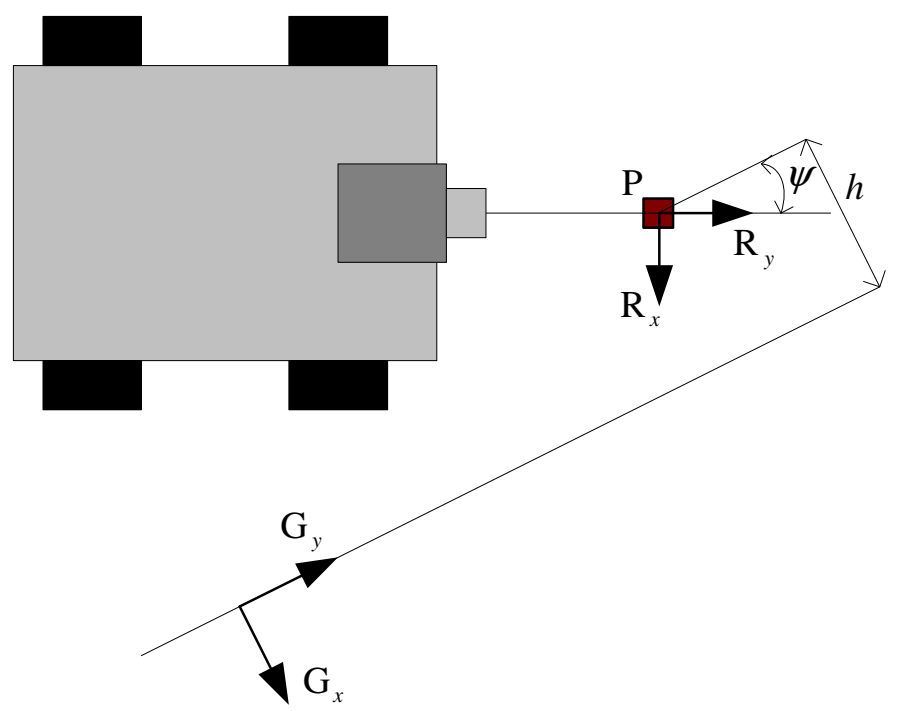

Figura 9.12- Posicionamiento obtenido con sistema de visión

El posicionamiento $\{\psi, h\}$ obtenido con el sistema de visión indicado anteriormente tiene la siguiente relación con la postura del vehículo (Fig. 9.12):

$$
\left(\begin{array}{c}
{ }^{\mathrm{G}} x_{\mathrm{R}} \\
{ }^{\mathrm{G}} y_{\mathrm{R}} \\
{ }^{\mathrm{G}} \theta_{\mathrm{R}}
\end{array}\right)=\left(\begin{array}{c}
-h \\
\text { Indeterminado } \\
-\psi
\end{array}\right)
$$

donde ${ }^{\mathrm{G}} x_{\mathrm{R}}$ y ${ }^{\mathrm{G}} \theta_{\mathrm{R}} \equiv \theta$ son observables con $(\psi, h)$ y ${ }^{\mathrm{G}} y_{\mathrm{R}}$ no lo es.

Por tanto el control de posición de (8.52) se concreta en:

$$
\begin{aligned}
& { }^{\mathrm{G}} v_{x \text { control }}={ }^{\mathrm{G}} \dot{x}_{\mathrm{R} \text { ref }}+a_{x} \cdot\left({ }^{\mathrm{G}} x_{\mathrm{R} \text { ref }}-{ }^{\mathrm{G}} x_{\mathrm{R}}\right)=-a_{x} \cdot h \\
& { }^{\mathrm{G}} v_{y \text { control }}={ }^{\mathrm{G}} \dot{y}_{\mathrm{R} \text { ref }}=v_{\text {seguimiento }}
\end{aligned}
$$

donde $v_{\text {seguimiento }}$ es la velocidad deseada para seguir la línea.

Notar que la imposibilidad de medir la posición en $y$ obliga a un control exclusivamente de velocidad en la coordenada longitudinal. 
La orientación del vehículo $\theta$ es necesaria, ver Figura 8.1, para poder calcular la cinemática inversa de las ruedas. Por ejemplo para la carretilla industrial de los capítulos 7 y 8 las cinemática inversa de las ruedas viene dada por $\{(8.48),(8.49),(8.50),(8.51)\}$.

Teniendo en cuenta la necesidad de procesar la señal del sensor de visión, la ecuación de control (9.26), y las dadas por la cinemática inversa, se implementa necesariamente de forma discreta. En general hay que asignar una dinámica con $a_{x}$ bastante más lenta que el periodo de muestreo (Teorema de Shannon-Nyquist).

\subsubsection{Resultados del seguimiento de línea por visión}

A continuación se muestran varios ejemplos de simulación del control cinemático para el seguimiento de línea del apartado anterior.

El vehículo considerado en este caso es de tipo diferencial (Figura 9.15), por lo que lo deducido en el Capítulo 8 para el vehículo triciclo es válido simplemente prescindiendo de las ecuaciones de la rueda orientable.

Los parámetros de este robot son: $\mathrm{r}=0.05 \mathrm{~m} ; \mathrm{l}_{12}=0.17 \mathrm{~m}$; e $=0.39 \mathrm{~m}$.

Para simular el seguimiento de línea se ha considerado un velocidad de seguimiento $v_{\text {seguimiento }}$ de $0.1 \mathrm{~m} / \mathrm{s}$, una postura inicial $\mathbf{p}(0)=(-0.3 \mathrm{~m}, 0,0)$ y un tiempo de 0.5 segundos para procesar la imagen del sistema de visión, haciéndolo coincidir con el periodo $\mathrm{T}$ de control.

En estas condiciones se ha simulado con tres valores distintos de $a_{x}$, que es la que establece la dinámica (polo) del error lateral.

Para el primer y segundo ejemplo de simulación se ha tomado $a_{x}=0.3 \mathrm{~s}^{-1}$, que supondría asignar una dinámica de seguimiento (constante de tiempo) aproximadamente 7 veces más lenta que el periodo del control. Lo cual se puede considerar aceptable.

El control del primer ejemplo es bueno: el punto de seguimiento $\mathrm{P}$ (origen de R) sigue la referencia asintóticamente (Figura 9.13 (a)) y las velocidades de control de las ruedas fijas (Figura 9.14 (a)) alcanza su régimen permanente (criterio del 95\%) a un valor un poco superior al previsto. 


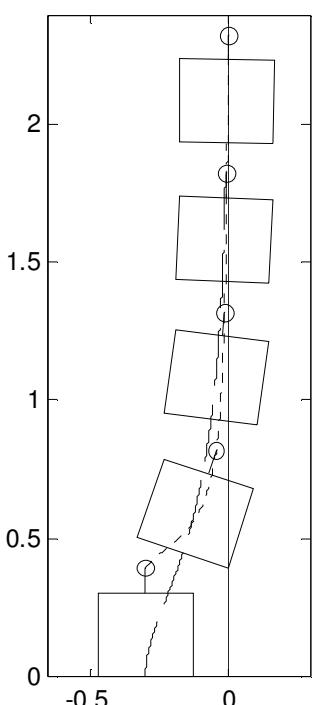

a) Ejemplo 1

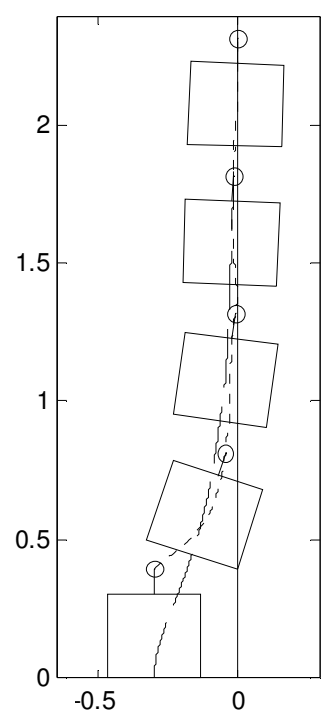

b) Ejemplo 2

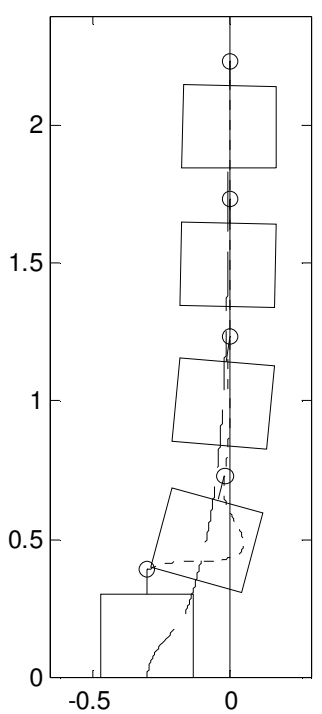

c) Ejemplo 3

Figura 9.13- Trazados en seguimiento de línea simulado: Referencia (-), P ó origen de R (- -) y punto medio de las ruedas fijas (--)

Con objeto de probar la robustez del control, en el segundo ejemplo se ha introducido un ruido aleatorio en los valores del posicionamiento, acotado a $2 \mathrm{~cm}$ en separación $(h)$ y $3^{\circ}$ en orientación $(\psi)$. Esto principalmente produce una pequeña inestabilidad en la velocidad de control (Figura 9.14 (b)), pero no impide que el seguimiento sea nuevamente aceptable (Figura 9.13 (b)).

En el tercer ejemplo se ha tomado $a_{x}=0.8 \mathrm{~s}^{-1}$, que supondría asignar una dinámica de seguimiento 2.5 veces más lenta que el periodo del control. Lo cual entra en el límite de lo marcado por el Teorema de Shannon-Nyquist. De hecho, el control ya no produce un acercamiento exponencial a la referencia (Figura 9.13 (c)), sino que hay una pequeña oscilación. Por lo que se empieza a atisbar una posible inestabilidad, no en vano las velocidades de control (Figura 9.14 (c)) toman valores más grandes.

Un problema práctico adicional al asignar dinámicas rápidas es la pérdida de la referencia en el plano imagen del sistema de visión, sobre todo si el punto $\mathrm{P}$ que realiza el seguimiento no coincide con el corte entre eje del objetivo de la cámara y el suelo.

De estas simulaciones se desprende que el control cinemático diseñado para seguir una línea con un sistema por visión funciona correctamente siempre que se respete una relación mínima entre la dinámica a asignar y el periodo de las acciones de control, y a pesar de no tener situado longitudinalmente el vehículo. 


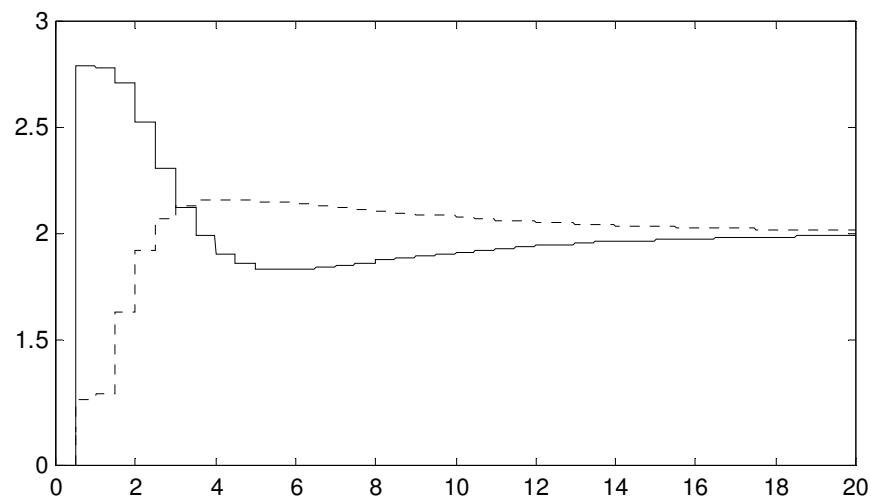

a) Ejemplo 1

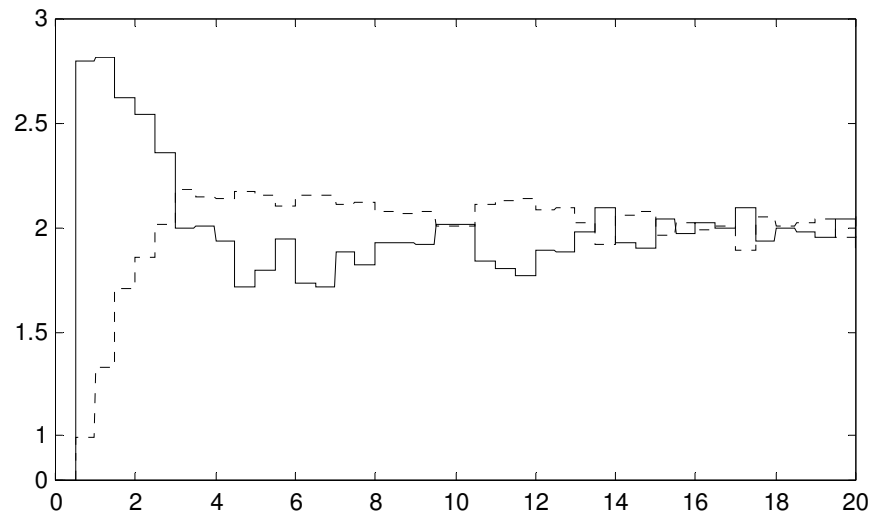

b) Ejemplo 2

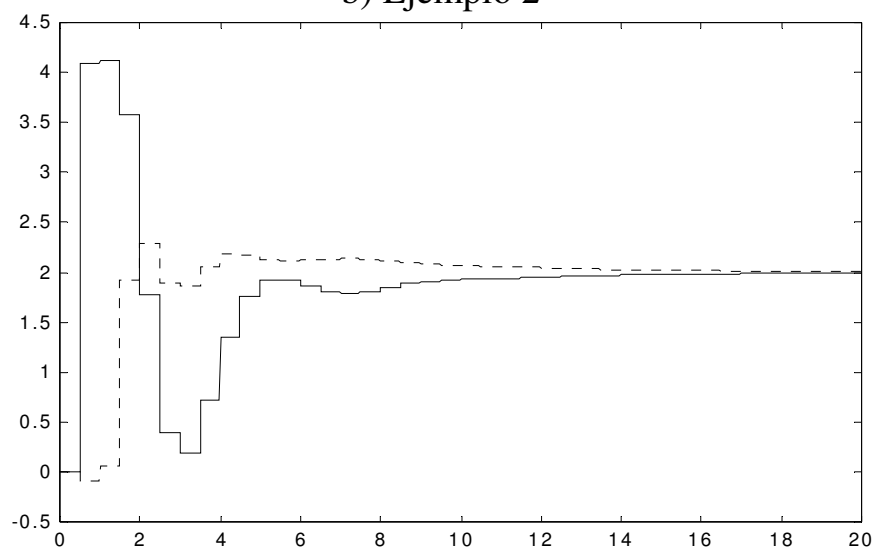

a) Ejemplo 3

Figura 9.14- Evolución de las velocidades de control de las ruedas fijas del vehículo diferencial en el seguimiento de línea simulado: izda (-), dcha (- -) 
En la Figura 9.15 se muestra el vehículo, de desarrollo propio, sobre el que se han realizado pruebas, con resultados medianamente satisfactorios (aunque no demasiado vistosos) de seguimiento de línea. En la Figura 9.16 se muestra la interfaz desarrollada y utilizada para las pruebas de seguimiento de línea.

Los tiempos de procesado de imagen para la obtención del posicionamiento han sido aceptables: en torno a los cuatro posicionamientos por segundo.

El mayor inconveniente que se ha encontrado al implementar dicho seguimiento de línea ha sido el control dinámico sobre las ruedas fijas. Dicho control, además de no ser instantáneo como idealmente supone el marco cinemático, se ha realizado sin considerar el acoplamiento entre ambas ruedas. A la postre esto a dado cierta inestabilidad en el seguimiento de línea.

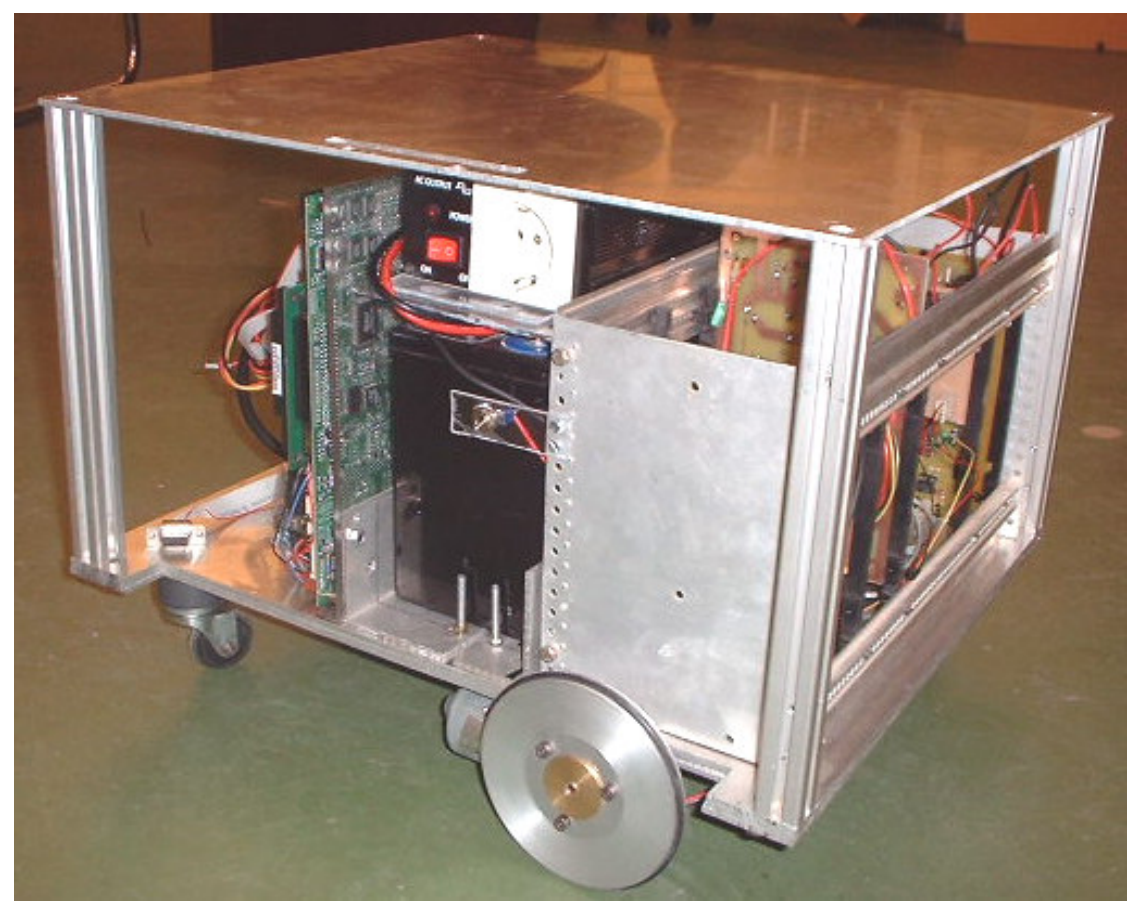

Figura 9.15- Vehículo de pruebas tipo diferencial 


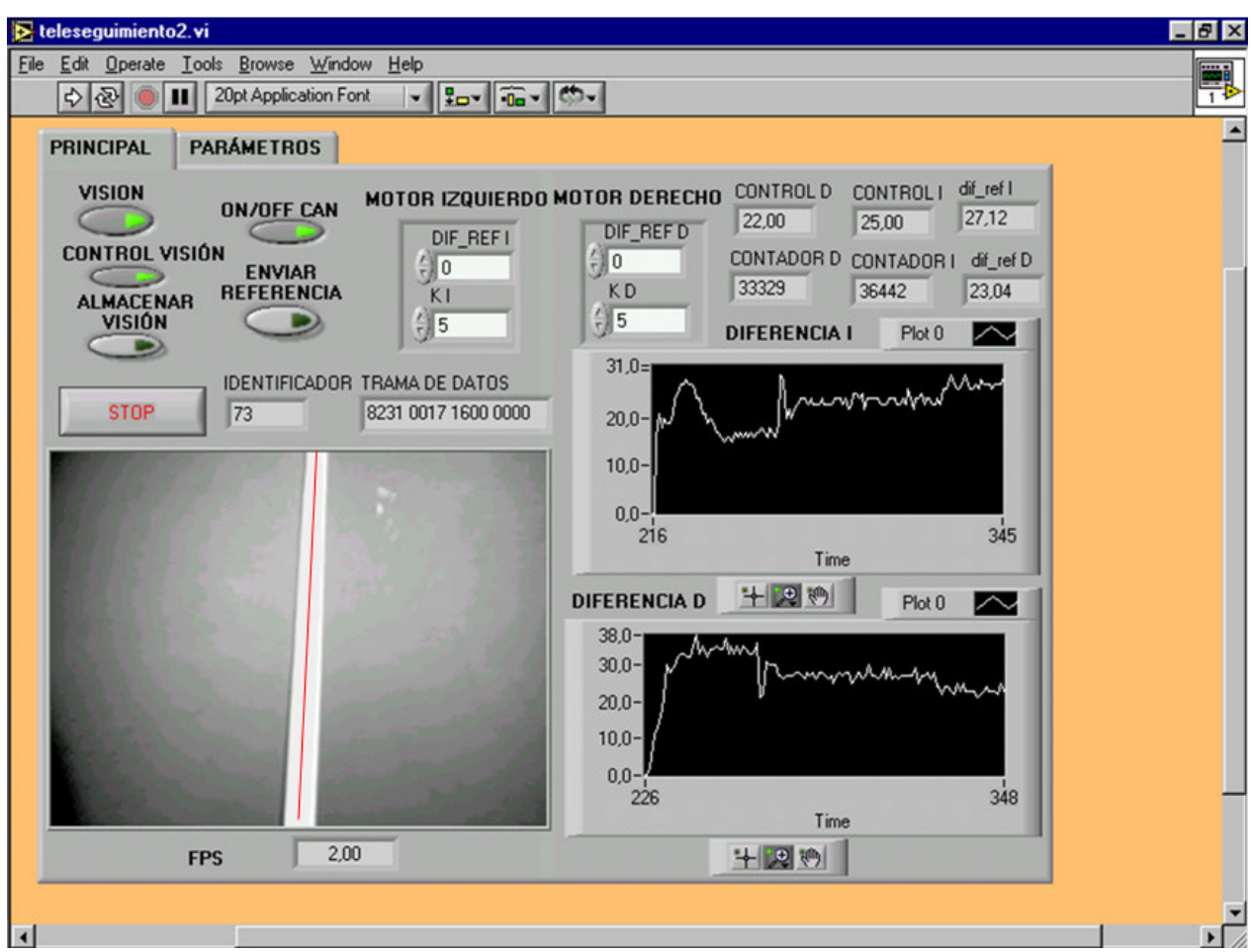

Figura 9.16- Interfaz desarrollada para el seguimiento de línea

Finalmente indicar que el seguimiento de línea aquí desarrollado también se ha implementado sobre la carretilla industrial de los Capítulos 7 y 8 y sobre una transpaleta automatizada, los resultados se describen en [Armesto 05]. Allí el tratamiento de imagen utilizado es distinto, ya que se utiliza la segmentación, y el control sobre las ruedas está planteado de forma multifrecuencia utilizando la información odométrica junto con la de la imagen [Armesto et al. 03]. 


\subsection{PLANIFICADOR DE APARCAMIENTO}

\subsubsection{Introducción}

Los planificadores de propósito general pueden utilizarse para el aparcamiento en línea. Algunos de ellos se basan en grafos en forma de árbol, donde las hojas son la postura (posición + orientación) del vehículo y los brazos los caminos de una postura a otra. El planificador comprueba durante la construcción del grafo si se ha alcanzado la meta. Ver por ejemplo [Latombe 91] [Laumond et al. 94] entre otras relevantes publicaciones. Su principal desventaja es el alto coste computacional requerido, que lo imposibilita para aplicaciones de tiempo real.

Otros planificadores utilizan métodos más particulares basados en herramientas como el fuzzy, la linealización por realimentación, las redes neuronales, la arquitectura reactiva, etc. Por ejemplo [Tilbury et al. 93] utiliza sinusoides para direccionar un vehículo autoguiado con n-trailers en un aparcamiento en línea, mientras que [Zhao et al. 05] emplea la lógica borrosa para aparcamientos en lugares pequeños. Otros ejemplos de planificadores específicos para el aparcamiento en línea se desarrollan en [Paromtchik et al. 96] [Jiang et al. 99] [Holve et al. 96] [Miyata et al. 96] [Baturone et al. 04] [Cuesta et al. 04].

De entre los planificadores anteriores, los presentados en [Paromtchik et al. 96] y [Jiang et al. 99] realizan el aparcamiento en las siguientes tres fases:

1) Detección con sensores de ultrasonidos para detectar el espacio (rectángulo) de aparcamiento;

2) Posicionamiento en el lateral del espacio de aparcamiento;

3) Maniobras para el aparcamiento con un patrón unificado.

En [Jiang et al. 99], las maniobras se calculan con una caracterización geométrica, mientras que [Paromtchik et al. 96] aplica unas acciones de control preestablecidas, que producirían las maniobras adecuadas. El algoritmo de [Jiang et al. 99] necesita detener el vehículo en el medio de la maniobra para reorientar las ruedas de dirección, mientras que en [Paromtchik et al. 96] la reorientación es progresiva. Finalmente [Paromtchik et al. 96] lleva más tiempo de cálculo, porque necesita integración numérica, mientras que en [Jiang et al. 99] todos los cálculos son analíticos. 
En este punto se presenta un planificador geométrico que mejora el planteamiento de [Jiang et al. 99] en dos aspectos:

1) Evitación de colisiones con los objetos delantero y trasero, no sólo con los laterales;

2) Optimización del punto de posicionamiento de la segunda fase.

\subsubsection{Planificador de aparcamiento en línea}

\subsubsection{A) Características generales del planificador}

El planificador desarrollado se basa en cálculos geométricos utilizando la estación de colisión con obstáculos en las tres fases tradicionales de la Fig. 9.17.

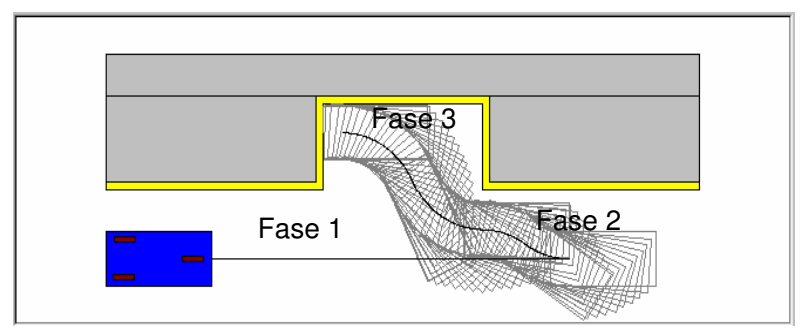

Figura 9.17- Fases del aparcamiento

Las tres fases son:

1) Detectar el espacio de aparcamiento (rectángulo) con una pasada.

2) Maniobrar hacia atrás hacia el punto de posicionamiento. Este punto se optimiza, en la prolongación del lateral del obstáculo delantero, para estar tan cerca como sea posible del obstáculo delantero pero evitando la colisión en la primera maniobra hacia atrás.

3) Maniobrar hacia delante y hacia atrás hasta que se alcanza la meta, evitando la colisión lateral, frontal y trasera con los obstáculos. Cada maniobra tiene dos tramos circulares, donde la rueda de dirección equivalente va el final de uno al otro. El vehículo está paralelo al espacio de aparcamiento al principio y final de cada maniobra. 


\subsubsection{B) Caracterización geométrica para el aparcamiento}

La Figura 9.18 muestra los parámetros geométricos del espacio de aparcamiento:

- J es la anchura del espacio de aparcamiento;

- H es la longitud del espacio de aparcamiento;

- MS es el margen de seguridad con los obstáculos;

- J' es el ancho efectivo del espacio de aparcamiento, equivalente a J;

- H' es la longitud efectiva del espacio de aparcamiento, equivalente a (H-2-MS).

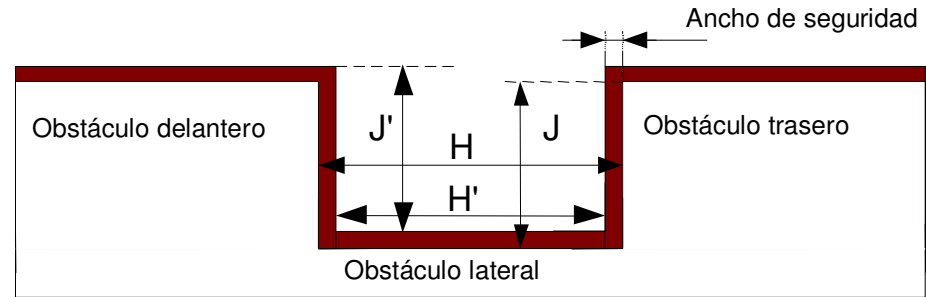

Figura 9.18- Parámetros geométricos del espacio de aparcamiento

La Figura 9.19 muestra los parámetros geométricos del vehículo tipo coche donde la rueda equivalente de dirección se ha representado. Si hay más de una rueda el mecanismo de Ackerman garantiza su equivalencia. Los parámetros son:

- $a_{1}$ es la distancia entre el eje trasero de ruedas y la parte delantera del vehículo;

- $b_{1}$ es la distancia entre eje trasero de ruedas y la parte de atrás del vehículo;

- $\mathrm{h}_{1}$ es la distancia entre el eje de ruedas trasero y el centro de la rueda orientable;

- $\mathrm{d}_{1}$ es la distancia entre los centros de las ruedas fijas del eje trasero;

- $\mathrm{c}_{1}$ es el ancho del vehículo;

- $r$ es el radio de las ruedas;

- $\phi_{\mathrm{d} \max }$ es la máxima orientación posible de la rueda de dirección equivalente;

- $\mathrm{P}_{\mathrm{f}}$ es el punto medio entre las ruedas fijas que sigue la trayectoria. 


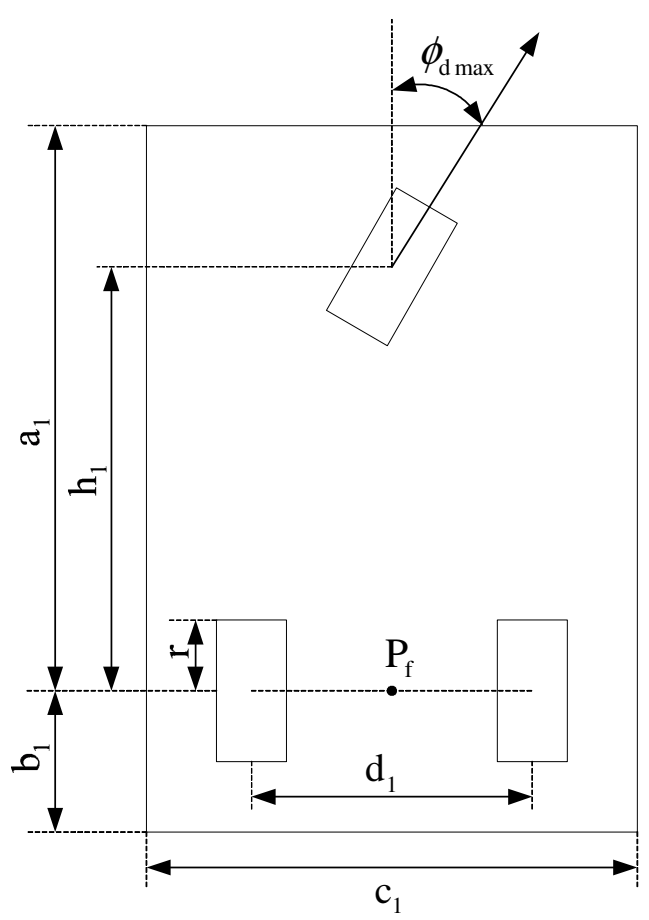

Figura 9.19- Parámetros geométricos del vehículo tipo coche

Relaciones geométricas útiles mostradas en las Figuras 9.20, 9.21 y 9.22:

$\rightarrow$ Relación entre el radio de giro $\rho$ (distancia de $\mathrm{P}_{\mathrm{f}}$ al Centro Instantáneo de Rotación, CIR) y la orientación $\phi_{\mathrm{d}}$ de la rueda equivalente de dirección:

$$
\rho=\frac{\mathrm{h}_{1}}{\tan \left(\phi_{\mathrm{d}}\right)}
$$

$\rightarrow$ Relación entre el radio de giro $\rho$ y la distancia $d_{\mathrm{i}}$ desde el CIR y la esquina $\mathrm{P}_{i}$ del vehículo:

$$
\begin{array}{ll}
d_{1}=\sqrt{\mathrm{b}_{1}{ }^{2}+\left(\rho+\mathrm{c}_{1} / 2\right)^{2}} & d_{2}=\sqrt{\mathrm{a}_{1}{ }^{2}+\left(\rho+\mathrm{c}_{1} / 2\right)^{2}} \\
d_{3}=\sqrt{\mathrm{b}_{1}{ }^{2}+\left(\rho-\mathrm{c}_{1} / 2\right)^{2}} & d_{4}=\sqrt{\mathrm{a}_{1}{ }^{2}+\left(\rho-\mathrm{c}_{1} / 2\right)^{2}}
\end{array}
$$




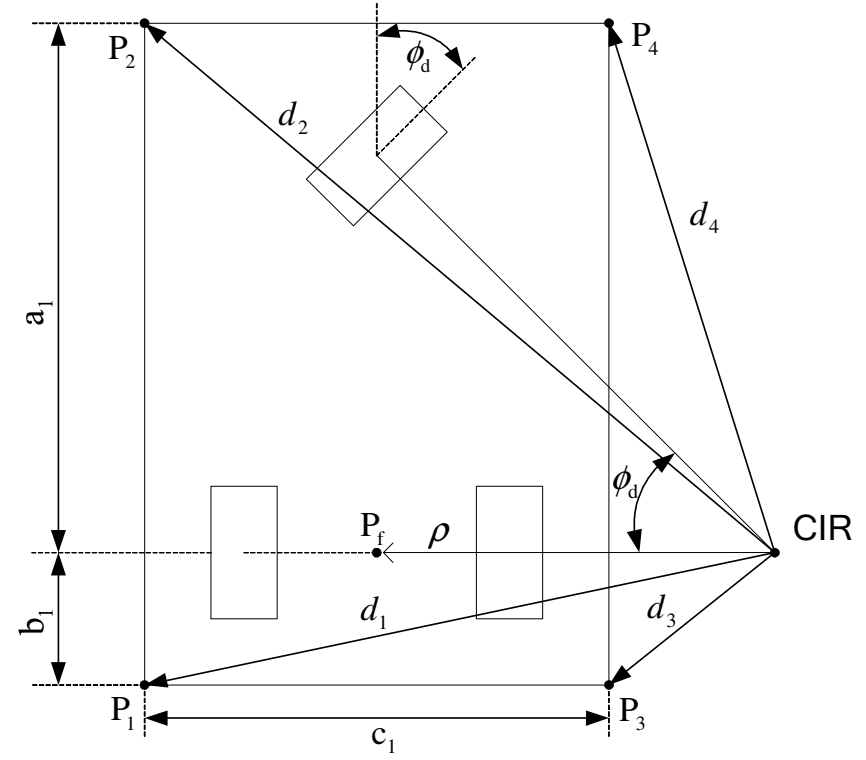

Figura 9.20- Descripción de CIR y distancias $d_{1}, d_{2}, d_{3}$ y $d_{4}$

$\rightarrow$ Relación entre la orientación $\theta$ del vehículo y la posición de la esquina $\mathrm{P}_{i}$ (respecto al sistema del CIR) en la Figura 9.21 es:

$$
\begin{aligned}
& \left(x_{\mathrm{P} i}, y_{\mathrm{P} i}\right)=\left(d_{i} \cdot \cos \left(\theta-\delta_{i}\right), d_{i} \cdot \sin \left(\theta-\delta_{i}\right)\right) \\
& \left(x_{\mathrm{P} j}, y_{\mathrm{P}_{j}}\right)=\left(d_{j} \cdot \cos \left(\theta+\delta_{j}\right), d_{j} \cdot \sin \left(\theta+\delta_{j}\right)\right)
\end{aligned}
$$

donde

$$
\begin{array}{ll}
i=1,2 & j=2,3 \\
\delta_{1}=\arctan \left(\frac{\mathrm{b}_{1}}{\rho+\mathrm{c}_{1} / 2}\right) & \delta_{2}=\arctan \left(\frac{\mathrm{a}_{1}}{\rho+\mathrm{cl} / 2}\right) \\
\delta_{3}=\arctan \left(\frac{\mathrm{b}_{1}}{\rho-\mathrm{c}_{1} / 2}\right) & \delta_{4}=\arctan \left(\frac{\mathrm{a}_{1}}{\rho-\mathrm{c}_{1} / 2}\right)
\end{array}
$$

$\rightarrow$ Desplazamientos longitudinal y transversal del camino consistente de dos arcos simétricos (Figura 9.22) del valor de $\xi$ :

$\mathrm{X}=2 \cdot \rho \cdot(1-\cos (\xi)) \quad \mathrm{Y}=2 \cdot \rho \cdot \operatorname{sen}(\xi) \quad \mathrm{Z}=\rho \cdot(1-2 \cdot \cos (\xi))$ 


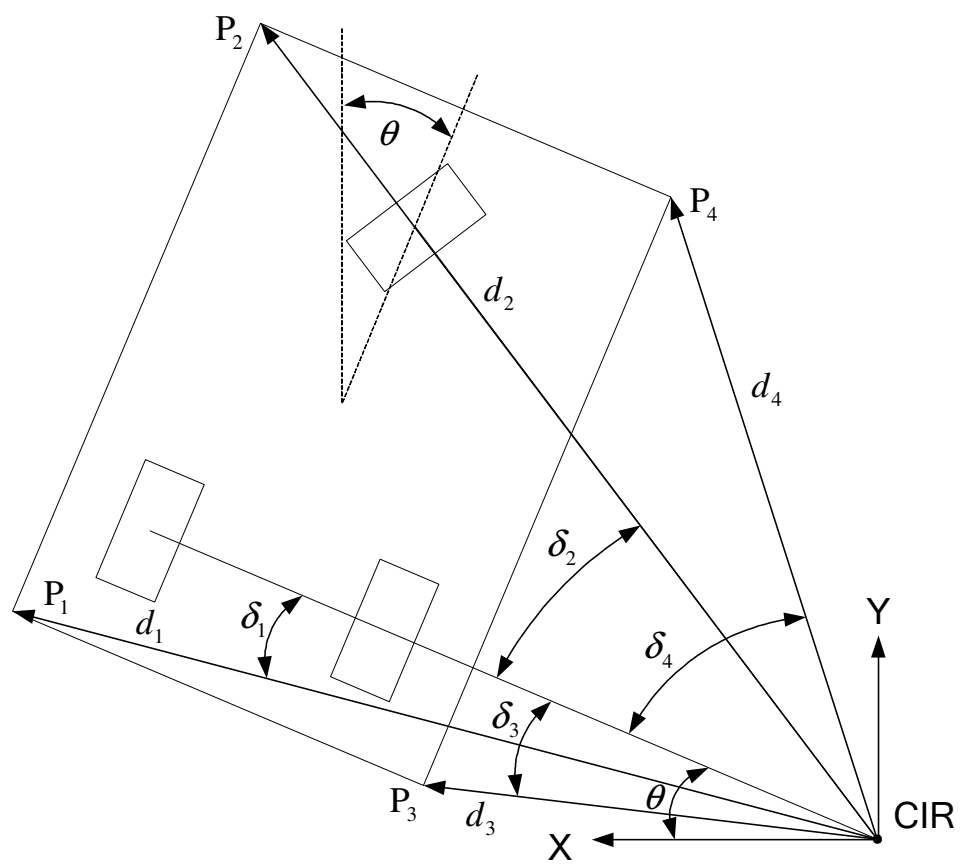

Figura 9.21- Relación entre el ángulo $\theta$ y la posición de $\mathrm{P}_{i}$

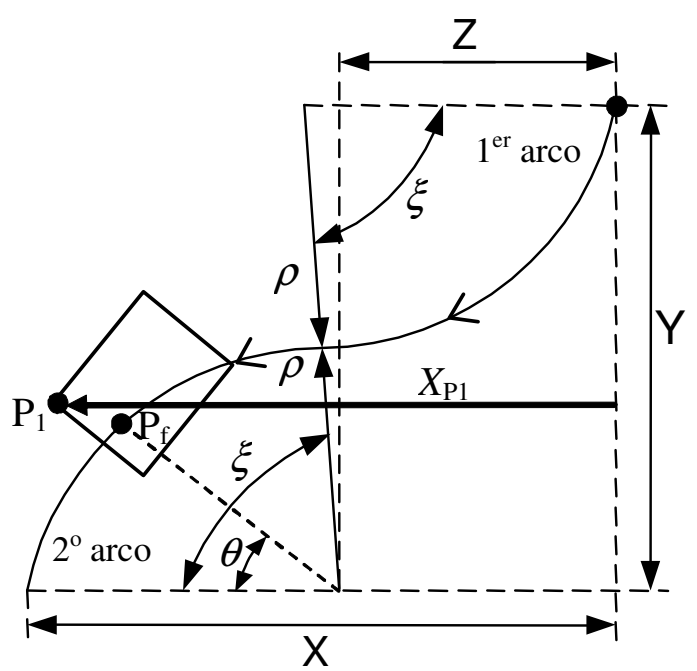

Figura 9.22- Desplazamiento entre dos arcos simétricos de valor $\xi$ 


\subsubsection{C) Caracterización de colisiones}

Las posibles colisiones son con los obstáculos lateral, delantero y trasero al maniobrar y con el delantero en la primera maniobra al abandonar del punto de posicionamiento.

La colisión lateral siempre se produce con la esquina más próxima del vehículo al obstáculo lateral en la dirección del movimiento (ej. según la Figura $9.20 \mathrm{P}_{1}$ puede colisionar en la maniobra hacia atrás o $\mathrm{P}_{2}$ en la maniobra hacia delante). Por tanto para evitar la colisión lateral en una maniobra, se debe garantizar la no-colisión de la esquina correspondiente.

En la Figura 9.22 se muestran dos arcos simétricos que conforman la maniobra planteada. Es suficiente con analizar el segundo arco, ya que está más cerca del obstáculo lateral. Por lo que la distancia lateral se debe calcular verificando el espacio lateral disponible.

De las ecuaciones (9.29) y (9.31) es posible calcular la distancia lateral entre la esquina $\mathrm{P}_{1}$ o $\mathrm{P}_{2}$ (movimiento hacia atrás o hacia delante) dependiendo del ángulo $\theta$ del segundo arco:

$$
X_{\mathrm{P} i}(\theta)=d_{i} \cdot \cos \left(\theta-\delta_{i}\right)+\rho \cdot(1-2 \cdot \cos (\xi)) \text { donde } i=1,2
$$

con el máximo:

$$
\hat{X}_{\mathrm{P} i}=d_{i}+\rho \cdot(1-2 \cdot \cos (\xi)) \text { para } \quad \hat{\theta}_{\mathrm{P} i}=\delta_{i}
$$

La detección de colisión lateral se comprueba comparando (9.33) con el espacio lateral disponible. Es decir, verificar la colisión implica verificar que la esquina crítica nunca colisiona.

La colisión delantera / trasera se produce con la esquina más próxima del vehículo en la dirección del movimiento. Es decir $\mathrm{P}_{2} / \mathrm{P}_{3}$ en una maniobra hacia atrás y $\mathrm{P}_{1} / \mathrm{P}_{4}$ en una maniobra hacia delante.

La colisión trasera en la maniobra hacia atrás, involucra a $\mathrm{P}_{3}$ y sólo en el segundo arco, y se produce si el mínimo local $y_{\mathrm{P} 3}$ en (9.29) tiene un valor $\hat{\theta}$ dentro del intervalo $] 0, \xi[$, donde:

$$
\hat{\theta}_{3}=\delta_{3}-\pi / 2 \quad \text { con } 0 \leq \theta \leq \xi \text { y } 0 \leq \delta_{3} \leq \pi
$$


Por tanto el punto singular (9.34) está fuera del rango de $\theta$ cuando:

$$
\rho \geq \mathrm{c}_{1} / 2 \quad \text { ó } \quad \rho \leq \mathrm{c}_{1} / 2-\mathrm{b}_{1} \cdot \tan (\xi)
$$

En colisiones traseras/frontales, otras esquinas pueden ser analizadas dando un resultado similar: para $\mathrm{P}_{2}$ y $\mathrm{P}_{4}$ el parámetro $b_{1}$ se sustituye con $\mathrm{a}_{1}$.

Se propone el siguiente algoritmo para detección de colisión frontal/trasera:

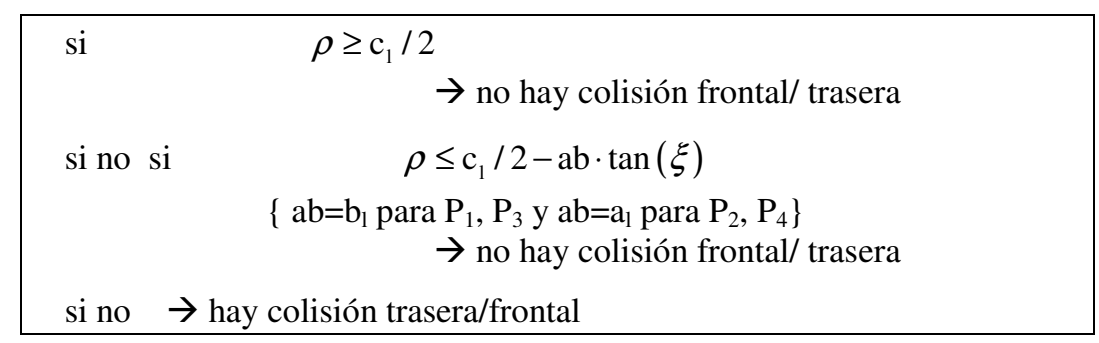

Algoritmo 1- Detección de colisión frontal/ trasera

Además las colisiones en la primera maniobra (entre el obstáculo delantero y la esquina $\mathrm{P}_{2}$ ) se producen sólo en el segundo arco.

Para evitar estas colisiones, el mínimo de $\mathrm{dx}_{1}$ en la Figura 9.23 se calcula con un algoritmo tal que $\mathrm{P}_{2}$ toque el origen del sistema $(X, Y)$. Para este caso se utiliza (9.36) obtenida de (9.29) y (9.31).

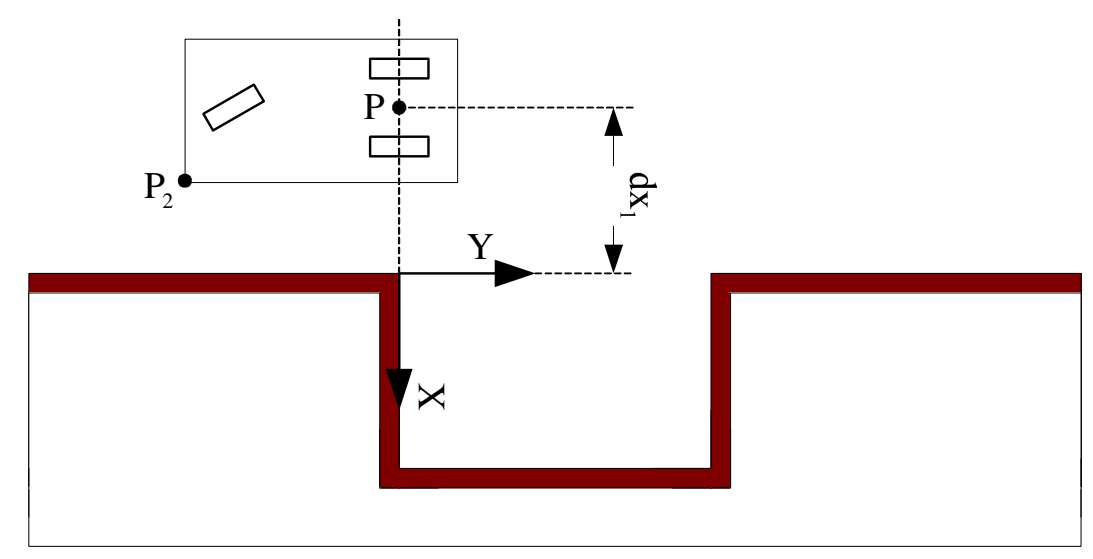

Figura 9.23- Características de la colisión en la primera maniobra 


$$
\begin{aligned}
& y_{\mathrm{P} 2}(\theta)=2 \cdot \rho \cdot \sin (\xi)-\left(\rho \cdot \sin (\theta)+\mathrm{c}_{1} / 2 \cdot \sin (\theta)+\mathrm{a}_{1} \cdot \cos (\theta)\right) \\
& x_{\mathrm{P} 2}(\theta)=\rho \cdot(1-2 \cdot \cos (\xi))+\rho \cdot \cos (\theta)+\mathrm{c}_{1} / 2 \cdot \cos (\theta)-\mathrm{a}_{1} \cdot \sin (\theta)-\mathrm{dx}_{1}
\end{aligned}
$$

Para establecer el valor definitivo de $\mathrm{dx}_{1}$ el algoritmo tiene en cuenta posibles colisiones laterales y traseras. Para calcular el valor de $\mathrm{dx}_{1}$ se han desarrollado los Algoritmos 2 y 3.

1- Se varía numéricamente $\theta$ empezando en $\theta=\xi$ y decrementado hacia cero.

2- Si $y_{\mathrm{P} 2}$ empiaza disminuyendo aux $=1$ si no aux $=0$.

3- Se detecta cuando $y_{\mathrm{P} 2}=0$. Si aux $=1$ se comprueba que en ese momento $y_{\mathrm{P} 2}$ aumenta si no se sigue hasta que $y_{\mathrm{P} 2}$ vuelva a ser cero.

4- Se sustituye el valor de $\theta$ que ha hecho $y_{\mathrm{P} 2}=0$ en $x_{\mathrm{P} 2}$

5- Se despeja $\mathrm{dx}_{1}$ de $x_{\mathrm{P} 2}=0$.

Algoritmo 2- Cálculo de $\mathrm{dx}_{1}$ de modo que se pase por $\left(x_{\mathrm{P} 2}, y_{\mathrm{P} 2}\right)=(0,0)$

1- Se calcula el espacio longitudinal disponible $\mathrm{D}_{\mathrm{y}}=\mathrm{H}-\mathrm{MS}-\mathrm{b}_{1}$

2- Se calcula el radio de giro mínimo de (9.27) $\operatorname{con} \phi_{\mathrm{d} \max }$.

3- Se calcula el $\xi$ de $(9.31)_{b}$ con los resultados de 1- y 2-.

4- Se comprueba que no hay colisión trasera con el Algoritmo 1 para los resultados de $\xi$ y $\rho$ obtenidos en 2- y 3-. En caso de colisión se hace $\rho=\mathrm{c}_{1} / 2$ y se calcula nuevamente $\xi$ con $(9.31)_{\mathrm{b}}$.

5- Se calcula la distancia máxima alcanzada por $\mathrm{P}_{1}$ a partir del punto inicial de la maniobra utilizando (9.33).

6- Se calcula $\mathrm{dx}_{1}$ con el Algoritmo 2.

7- Se utiliza 5- y 6- para calcular el valor definitivo de $\mathrm{dx}_{1}$ :

si $(5--\mathrm{J}>6-)$

si $(5--\mathrm{J}>$ Separación inicial $) \rightarrow$ Se decrementa $\phi_{\mathrm{d}}$ y se vuelve a 1-

si no $\rightarrow \mathrm{dx}_{1}=5--\mathrm{J}$

si no $\rightarrow \mathrm{dx}_{1}=6-$

8- Si $\mathrm{dx}_{1}<\mathrm{c}_{1} / 2 \rightarrow \mathrm{dx}_{1}=\mathrm{c}_{1} / 2$

Algoritmo 3- Cálculo del punto de aproximación

\section{Respecto al Algoritmo 3 comentar que:}

El hecho de que haya colisión trasera obliga indefectiblemente a reducir el ángulo de orientación de la rueda delantera, y en consecuencia el radio de giro, el cual se fija para que esta colisión no se produzca seguro. 
De los valores del barrido lateral de $\mathrm{P}_{1} \mathrm{y}$ del punto calculado por el Algoritmo 2 (para que no se produzca la colisión de punto de aproximación) se utiliza el más restrictivo para calcular $\mathrm{dx}_{1}$.

En caso de que el barrido de $\mathrm{P}_{1}$ sea mayor que la separación inicial y haga alejarse del obstáculo delantero, se disminuye el ángulo de orientación de la rueda delantera hasta que se avance tanto con la primera maniobra como distancia inicial al obstáculo lateral al vehículo.

En el caso de que el valor asignado a $\mathrm{dx}_{1}$ sea menor de la mitad del ancho del vehículo se hace que sea igual a la mitad del ancho del vehículo.

Los valores finales de $\xi$ y $\rho$ se utilizan para la maniobra backward que parte del punto de aproximación.

Una vez establecida la distancia de $\mathrm{dx}_{1}$, que caracteriza la posición del punto de posicionamiento, se calcula la mínima distancia $\mathrm{dy}_{3}$ de la Figura 9.24 para detener el primer movimiento en línea recta del vehículo (pasada de reconocimiento).

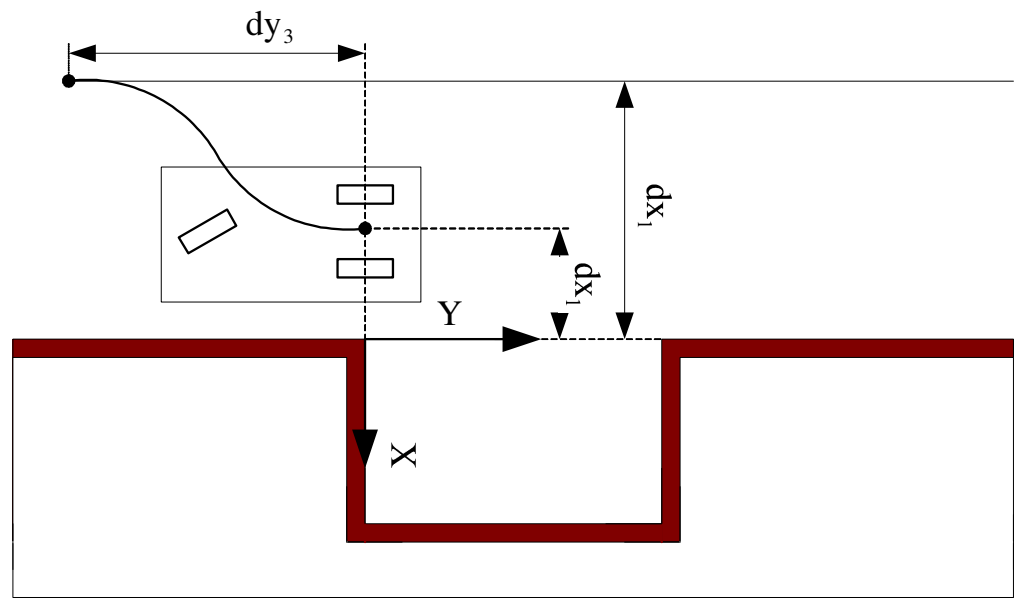

Figura 9.24- Maniobra previa para alcanzar el punto de posicionamiento

Este cálculo se realiza con un algoritmo con las entradas $\mathrm{dx}_{1} \mathrm{y} \mathrm{dx}_{2}$ (separación inicial del vehículo), que tiene en cuenta la evitación de colisión lateral, utilizando (9.33), y con el obstáculo delantero en la segunda fase. Para todo ello se ha desarrollado el Algoritmo 4. 
1- Se calcula el radio de giro mínimo de (9.27) con $\phi_{\mathrm{d} \max }$.

2- Se calcula el $\xi$ con $(9.31)_{a}$ teniendo en cuenta $X=\left|\mathrm{dx}_{1}-\mathrm{dx}_{2}\right|$.

3- Se calcula $\mathrm{dy}_{3}$ con $(9.31)_{b}$.

4- Se comprueba que no hay colisión lateral con el obstáculo delantero: si $\max (\mathrm{dx} 1, \mathrm{dx} 2) \geq(9.33) \rightarrow$ No hay colisión si no $\rightarrow$ SALIR

5- Se aumenta dy $\rightarrow \mathrm{dy}_{3}=\mathrm{dy}_{3}+\Delta \mathrm{dy}_{3}$.

6- Se calculan $\xi$ y $\rho$ con el sistema de ecuaciones dado por $(9.31)_{\mathrm{a}}$ y $(9.31)_{b}$ para $X=l d x_{1}-d x_{2} \mid$ y $Y=d_{3}$

7- Se vuelve a 4-

Algoritmo 4- Cálculo del punto previo al de aproximación

Para caracterizar cada maniobra necesaria en la tercera fase se ha desarrollado el Algoritmo 5 que utiliza la distancia actual disponible al obstáculo lateral $\left(\mathrm{W}_{\max }\right)$ como entrada y calcula los valores de $\xi$ y $\rho$ en esas maniobras. Para ello tiene en cuenta la evitación de colisiones lateral, frontal y trasera por medio de (9.33) y del Algoritmo 1. Las llamadas al algoritmo anterior se detienen cuando la distancia lateral, entre el vehículo y el espacio lateral, es menor que la separación final deseada.

1- Se calcula el radio de giro mínimo de (9.27) con $\phi_{\mathrm{d} \max }$.

2- Se comprueba que se cumple $\rho \geq \mathrm{c}_{1} / 2$ (no colisión frontal/trasera) si no se hace $\rho=\mathrm{c}_{1} / 2$

3- Se calcula $\xi$ con $(9.31)_{\mathrm{b}}$ teniendo en cuenta $\mathrm{Y}=\mathrm{H}-2 \cdot \mathrm{MS}=\mathrm{H}^{\prime}$.

4- Se comprueba que se cumple $\mathrm{W}_{\max } \geq(9.33)$, si no se calculan $\xi$ y $\rho$ para cumplir $(9.31)_{\mathrm{b}} \mathrm{y} \mathrm{W}_{\max }=(9.33)$

Algoritmo 5- Cálculo de maniobras en la fase 3

\subsubsection{Resultados del planificador en simulación}

Para validar el planificador desarrollado se ha implementado un entorno de simulación. También se ha creado una interfaz para introducir las características geométricas del vehículo (Figura 9.25) y el espacio de aparcamiento (Figura 9.26). Incluso los parámetros dinámicos del vehículo se pueden especificar para monitorizar un tracking (seguimiento) simulado.

La Figura 9.27 muestra un ejemplo de aparcamiento con los parámetros de la Tabla 9.1. 
Tabla 9.1- Valores de parámetros para el ejemplo $1^{\circ}$ de aparcamiento

\begin{tabular}{|c|l|c|l|c|l|}
\hline Parámetro & Valor & Parámetro & Valor & Parámetro & Valor \\
\hline $\mathrm{a}_{1}$ & $150 \mathrm{~cm}$ & $\mathrm{~b}_{1}$ & $50 \mathrm{~cm}$ & $\mathrm{c}_{1}$ & $140 \mathrm{~cm}$ \\
\hline $\mathrm{h}_{1}$ & $105 \mathrm{~cm}$ & $\mathrm{~d}_{1}$ & $50 \mathrm{~cm}$ & $\phi_{\mathrm{d} \max }$ & $46^{\circ}$ \\
\hline $\mathrm{H}$ & $335 \mathrm{~cm}$ & $\mathrm{~J}$ & $200 \mathrm{~cm}$ & MS & $20 \mathrm{~cm}$ \\
\hline $\mathrm{dx}_{2}$ & $70 \mathrm{~cm}$ & Separación Final & $7 \mathrm{~cm}$ & \multicolumn{1}{l}{} \\
\cline { 1 - 3 }
\end{tabular}

El tiempo de cómputo es 0.438 milisegundos y se requieren dos maniobras.

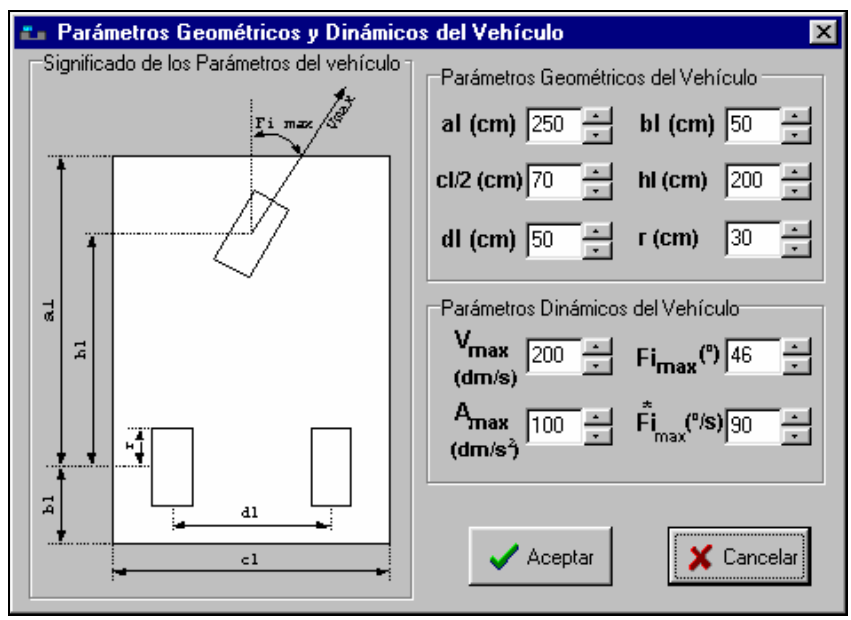

Figura 9.25- Interfaz de los parámetros del vehículo

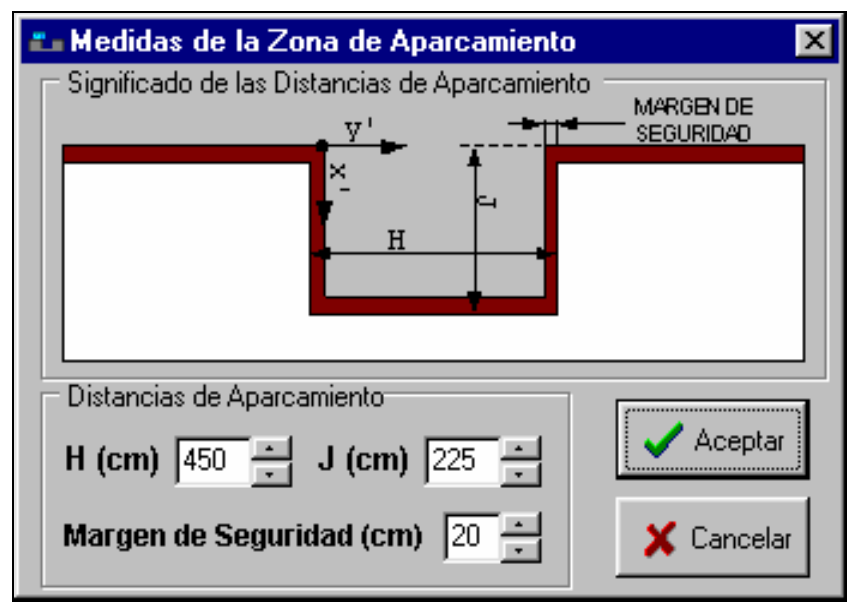

Figura 9.26- Interfaz para los parámetros del espacio de aparcamiento 


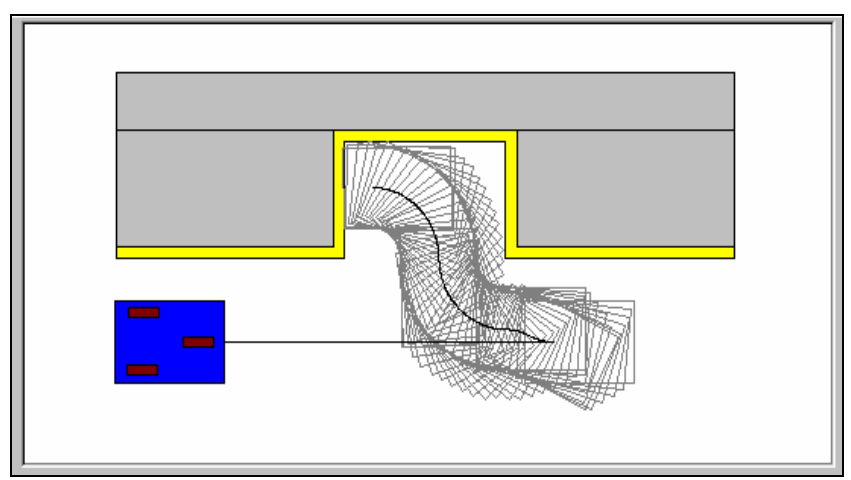

Figura 9.27- Ejemplo $1^{\circ}$ de aparcamiento con el planificador desarrollado

Las Figuras 9.28 y 9.29 muestran otro ejemplo con los parámetros de aparcamiento de la Tabla 9.2.

Tabla 9.2- Valores de parámetros para el ejemplo $2^{\circ}$ de aparcamiento

\begin{tabular}{|c|l|c|l|c|l|}
\hline Parámetro & Valor & Parámetro & Valor & Parámetro & Valor \\
\hline $\mathrm{a}_{1}$ & $75 \mathrm{~cm}$ & $\mathrm{~b}_{1}$ & $15 \mathrm{~cm}$ & $\mathrm{c}_{1}$ & $350 \mathrm{~cm}$ \\
\hline $\mathrm{h}_{1}$ & $60 \mathrm{~cm}$ & $\mathrm{~d}_{1}$ & $130 \mathrm{~cm}$ & $\phi_{\mathrm{d} \max }$ & $46^{\circ}$ \\
\hline $\mathrm{H}$ & $330 \mathrm{~cm}$ & $\mathrm{~J}$ & $360 \mathrm{~cm}$ & MS & $12 \mathrm{~cm}$ \\
\hline $\mathrm{dx}_{2}$ & $17 \mathrm{~cm}$ & Separación Final & $17 \mathrm{~cm}$ & \multicolumn{1}{l}{} \\
\cline { 1 - 4 }
\end{tabular}

En este caso el vehículo es mucho más ancho, que se correspondería por ejemplo a un vehículo agrícola. El tiempo de cómputo es de 1.3 milisegundos y se requieren 4 maniobras.

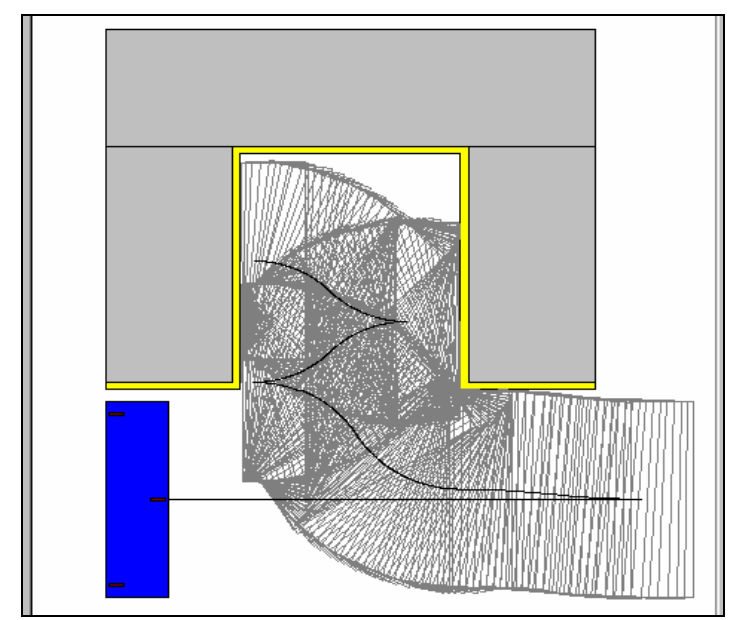

Figura 9.28- Ejemplo $2^{\circ}$ de aparcamiento con el planificador desarrollado 


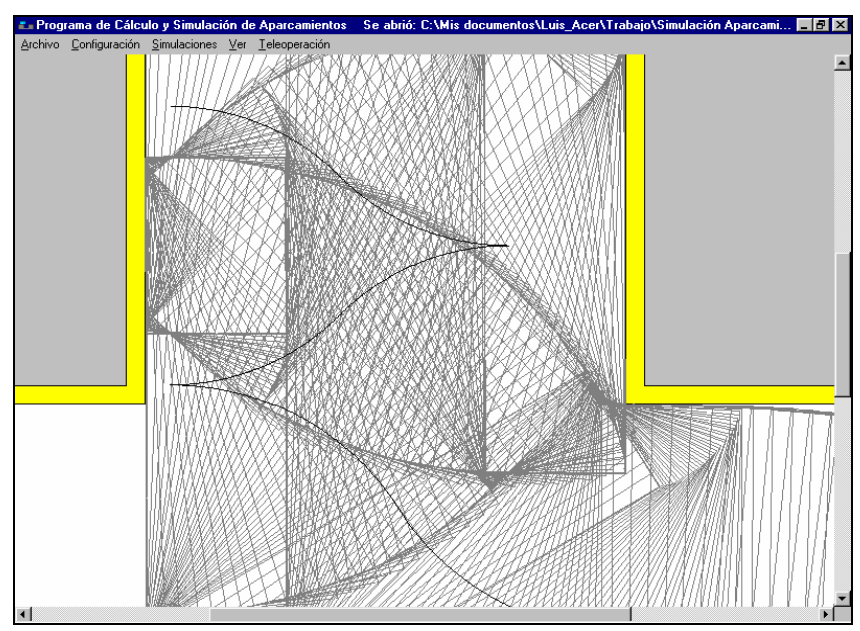

Figura 9.29- Zoom del Ejemplo $2^{\circ}$ de aparcamiento

La Figura 9.30 muestra el número de maniobras requeridas respecto al espacio longitudinal disponible en términos del ratio dado por (9.37). Notar que las maniobras requeridas crecen rápidamente cuando el ratio baja del $30 \%$.

ratio $(\%)=100 \cdot \frac{\text { esp. disponible }- \text { long. del vehículo }}{\text { esp. disponible }}=100 \cdot \frac{(\mathrm{H}-2 \cdot \mathrm{MS})-\left(\mathrm{a}_{1}+\mathrm{b}_{1}\right)}{(\mathrm{H}-2 \cdot \mathrm{MS})}(9.37)$

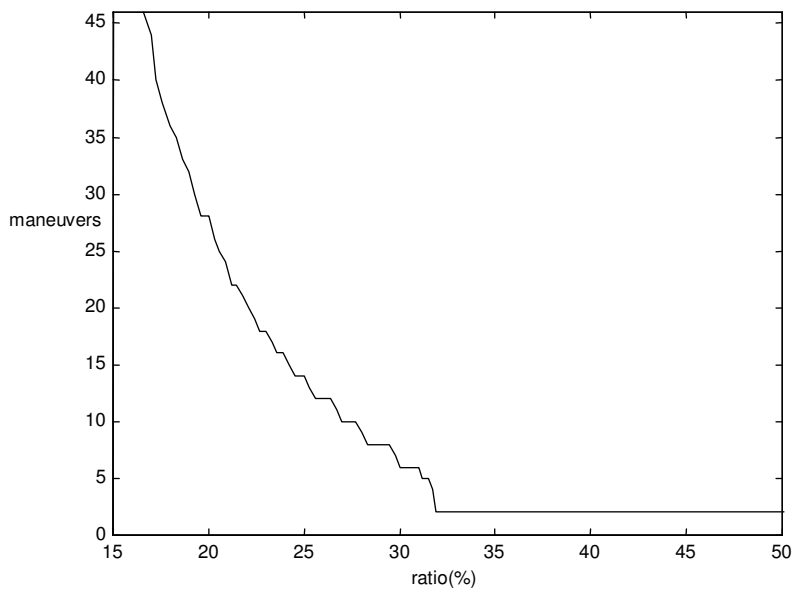

Figura 9.30- Relación maniobras-ratio (\%) en el ejemplo $1^{\circ}$ de aparcamiento 
En cuanto a la referencia de velocidad a generar para su posterior seguimiento, en el programa de simulación se han utilizado los valores de velocidad y aceleración máxima introducidos (ver Figura 9.25) de forma que para cada tramo se tenga un trapezoide. Estos tramos vienen marcados por la necesidad de detener el vehículo, bien por cambio en el sentido de la marcha o para reorientar la rueda directriz. De modo que hay un tramo en la primera fase de aparcamiento y dos por cada maniobra backward o forward.

Como trabajo futuro, el planificador probado en simulación se pretende implementar sobre el vehículo eléctrico de la Figura 9.31.

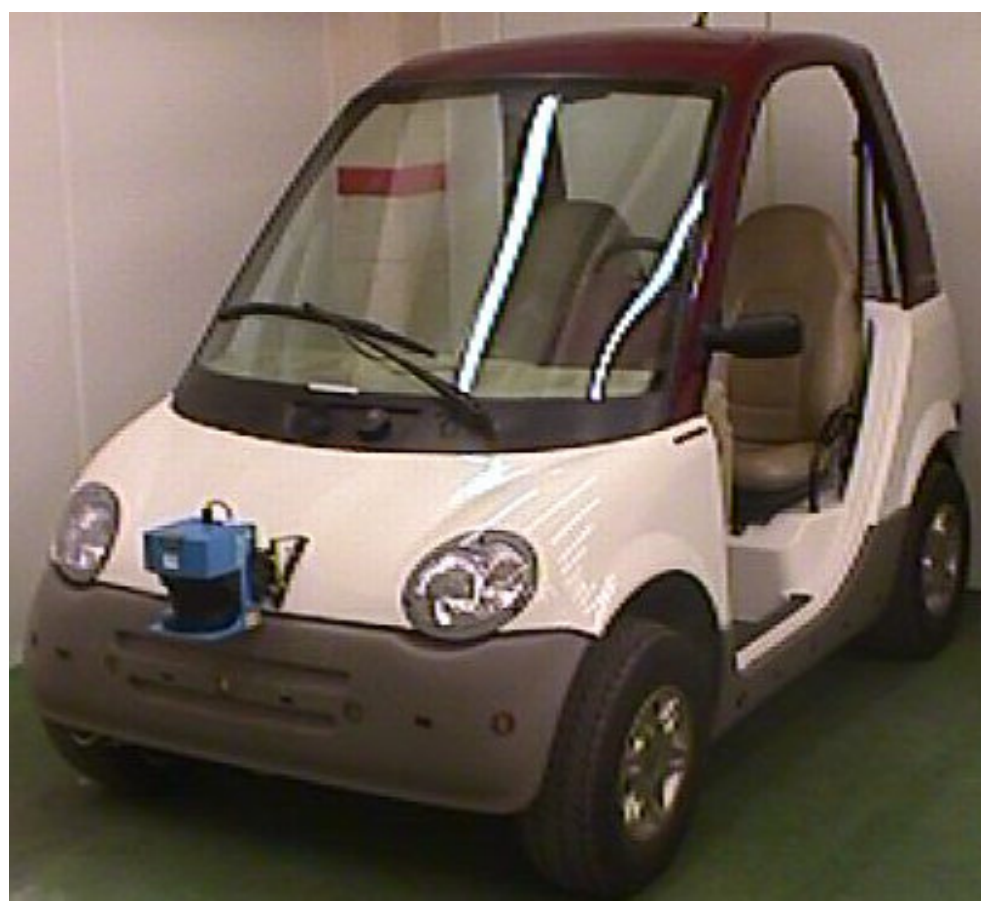

Figura 9.31- Vehículo eléctrico de pruebas sobre el que implementar el planificador de aparcamiento desarrollado 


\subsection{RESULTADOS MÁS RELEVANTES Y CONCLUSIONES DEL CAPÍTULO}

En cuanto al sistema de posicionamiento por visión:

- Se basa en la relación analítica que no requiere aproximaciones ni cálculo numérico como en otros autores [Marchant 95].

- Resulta útil en entornos tan variados como una carretera (tomando como referencia sus marcas longitudinales), una nave industrial (tomando como referencia una línea pintada al estilo filo guiado), un campo de cultivo (tomando como referencia las hileras de cultivo), etc., y sirve como entrada para realizar el seguimiento de línea por control cinemático.

- Se plantea también un sistema de calibración que utiliza el mismo análisis de imagen del sistema de posicionamiento, lo que simplifica su implementación, y tan sólo requiere de dos medidas de la situación real de los elementos captados.

- Para distintas aplicaciones se han probado los sistemas de posicionamiento y calibración desarrollados corroborándose su viabilidad y validez.

- La limitación de no disponer de información en la coordenada longitudinal, para la aplicación de seguimiento de línea por visión, se resuelve implementando un control de velocidad en esa dirección, lo que deja entrever una realidad práctica.

- El periodo (dado por la adquisición de la imagen y el cálculo del posicionamiento $\{\psi, \mathrm{h}\})$ en la aplicación de seguimiento de línea por visión, obliga a cumplir una mínima relación (teorema de Shannon-Nyquist) entre dicho periodo y la dinámica a asignar para garantizar la estabilidad.

En cuanto al planificador de aparcamiento:

- Un planificador de aparcamiento en línea, basado en cálculos geométricos de evitación de colisiones, se ha implementado. Mejoras importantes se han introducido respecto a otros planteamientos geométricos en el sentido de evitar las colisiones del vehículo con los obstáculos trasero y delantero y de un criterio de optimización. El bajo tiempo de cómputo requerido (cercano 
a unos pocos milisegundos) hace que el planificador sea adecuado para aplicaciones de tiempo real.

- Un entorno de simulación se ha diseñado para probar este planificador de aparcamiento bajo diferentes situaciones y vehículos. Varias simulaciones se han realizado para validar los planificadores y el software de simulación, indicando como el número de maniobras se incrementa en función del espacio disponible. 
CAPÍTULO 10

\section{CONCLUSIONES Y TRABAJOS FUTUROS}

\subsection{CONCLUSIONES}

A continuación se indican las conclusiones y aportaciones más relevantes de la presente tesis

- Se ha presentado un modelado cinemático basado en una rueda genérica (Capítulo 3) que incluye los tipos más comunes: fija, orientable, castor y sueca. Su modelado se ha obtenido con un método sistemático, sencillo, eficiente y novedoso, basado en una formulación cinemática recursiva.

- Se han desarrollado distintos métodos para la generación de modelos cinemáticos (Capítulo 4) de vehículo: el del espacio nulo, el del análisis de rangos y el de matrices Jacobianas de rueda de [Muir et al. 87] mejorado. Los tres métodos de modelado se han ilustrado con el ejemplo de vehículo tipo triciclo.

- Se han obtenido los modelos cinemáticos de los cinco tipos de vehículos (Capítulo 5) clasificados de acuerdo a [Campion et al. 96]. A dichos modelos se les ha aplicado un completo análisis de isotropía (transmisión de errores).

- Se ha establecido un planteamiento geométrico (Capítulo 6) que caracteriza la singularidad de cualquier modelo cinemático de cualquier vehículo con ruedas comunes (fijas, orientables, castor y suecas) y/o especiales (dobles, castor dobles, tipo bola y ortogonales). Dicho planteamiento se ha aplicado a los cinco tipos de vehículos existentes. 
- Los dos puntos anteriores representan una guía de la cinemática de vehículos útil para el diseño mecánico, desarrollo de aplicaciones de control, la planificación de caminos, etc.

- Se han deducido tres niveles de modelos con deslizamiento (Capítulo 7), obtenidos a partir de principios físicos (por aproximaciones de la dinámica del vehículo) a diferencia de otros estudios. En concreto el modelo correspondiente al tercer nivel de aproximación (solución de Mínimos Cuadrados ponderada) se calcula analíticamente. Los modelos presentados se comparan, para el caso del vehículo triciclo, con el modelo dinámico en simulación y con el Filtro de Kalman en una situación real. En concreto, con los datos experimentales el modelo con deslizamiento utilizado da mucho mejores resultados que el modelo del vehículo diferencial y una de las dos opciones planteadas de Filtro de Kalman. El otro filtro de Kalman planteado da resultado similares pero tiene un 20\% más de tiempo de cómputo por iteración que el modelo con deslizamiento.

- Se ha propuesto una estructura de control (Capítulo 8) valida para cualquier tipo de vehículo, la cual es similar a las empleadas en robots manipuladores y que consiste en tres bucles de control anidados (dinámico, cinemático y de planificación). En particular se ha profundizado en el bucle cinemático y se han caracterizado, a través del análisis de los modelos cinemáticos inversos de rueda, las referencias que puede seguir cada tipo de vehículo sin error. Además se ha particularizado el control cinemático para el caso del triciclo, el cual ha sido estudiado en simulación e implementado sobre una carretilla industrial.

- Se presenta un sistema de posicionamiento por visión respecto a una línea (Capítulo 9) basado en una relación analítica sin aproximaciones que no requiere cálculo numérico como en otros autores. Este posicionamiento es útil en diversos entornos (carretera, nave industrial, campos de de cultivos, etc.) y sirve como entrada para realizar el seguimiento de línea con el control desarrollado. Además se ha planteado un sistema de calibración con el mismo análisis de imagen que el de posicionamiento, lo que simplifica su implementación, que sólo requiere dos medidas de la situación real de los elementos captados.

- Se ha desarrollado un planificador de aparcamiento en línea basado en cálculos geométricos de evitación de colisiones. El bajo tiempo de cómputo requerido (pocos milisegundos) lo hace adecuado para aplicaciones de tiempo real. El planificador se ha probado en un entorno de simulación, donde se ha estudiado el incremento en el número de maniobras en función del espacio disponible. 


\subsection{TRABAJOS FUTUROS}

A continuación se indican los trabajos futuros en distintas líneas:

- Para la isotropía del Capítulo 5 se plantean dos trabajos futuros:

1) Desarrollar un planificador que tenga en cuenta las configuraciones isotrópicas de un vehículo con ruedas orientables o castor para la generación de caminos. Dado que el error acumulado se debe a la amplificación el error instantáneo (número de condición) y al camino seguido, un planificador podría minimizar el error acumulado al alcanzar una meta. (La isotropía solo minimiza el error instantáneo.)

2) Establecer un procedimiento para diseño mecánico basado en una isotropía global, para vehículos con ruedas orientables o castor, para optimizar los parámetros del vehículo: radio de ruedas, distancias entre ruedas, etc. Esto minimizaría el error medio de las condiciones de isotropía, o alternativamente la media del número de condición, a lo largo del espacio de configuración.

- Desde el punto de vista de la singularidad de la cinemática de vehículos (Capítulo 6) se pretende plantear un planificador y/o controlador de movimiento que tenga en cuenta las configuraciones singulares, de un vehículo con ruedas orientables o castor, para la generación de caminos y/o actuaciones. Este planificador y/o controlador de movimiento sería análogo a los ampliamente desarrollados para robots manipuladores.

- Para los modelos con deslizamiento del Capítulo 7 se pretende plantear un control que trabaje con estos modelos en lugar de los cinemáticos sin deslizamiento.

- Utilizando el modelado dinámico del Capítulo 7 se planteará un bucle de control dinámico de bajo nivel con actuadores acoplados y/o un control dinámico global (sin desacoplar cinemática y dinámica). Esto permitirá, tanto para el vehículo diferencial de investigación (Capítulo 9) como para la carretilla industrial (Capítulo 8), mejores prestaciones en el seguimiento de línea por visión y en el seguimiento de referencias respectivamente.

- El planificador de aparcamiento del Capítulo 9 se pretende implementar sobre el vehículo eléctrico mostrado en dicho capítulo. 
- Finalmente, en el marco de un proyecto interdisciplinar de restauración se pretende desarrollar el modelado cinemático, dinámico y control del conjunto formado por un vehículo (y/o estructura guiada en dos ejes con sistema de elevación) que transporta un brazo robot, destinado a la realización de tareas diversas: detección/corrección de errores de fachada, limpieza, etc. 


\section{BIBLIOGRAFÍA}

[Alexander et al. 89] J. C. Alexander, J. H. Maddocks, "On the Kinematics of Wheeled Mobile Robots", The International Journal of Robotics Research, vol. 8 no. 5, pp. 15-27, October 1989.

[An et al. 88] C. H. An, C. G. Atkenson, J. M. Hollerback, "Model-based control of a robot manipulator", Mit Press, Cambridge, Mass, 1988.

[Armesto et al. 03] L. Armesto, L. Gracia, J. Tornero, "Multi-rate line tracking for mobile robots based on artificial vision and odometry", Industrial Simulation Conference, 2003.

[Armesto 05] L. Armesto, "Técnicas de fusión sensorial y su aplicación a la robótica móvil”, Tesis Doctoral, Universidad Politécnica de Valencia, 2005.

[Balakrishna et al. 95] R. Balakrishna and A. Ghosal, "Modeling of slip for wheeled mobile robots", IEEE Transactions on Robotics and Automation, vol. 11 no. 1 , pp. 126-132, 1995.

[Barraquand et al. 89] J. Barraquand, J.-C. Latombe, "On non-holonomic mobile robots on optimal maneuvering", Revue d'Ingelligence Artificielle, vol. 3 no. 2, pp. 77-103, 1989.

[Baturone et al. 04] I. Baturone, F.J. Moreno-Velo, S. Sánchez-Solano, A. Ollero, "Automatic design of fuzzy controller for car-like autonomous robots", IEEE Transactions on Fuzzy Systems, vol. 12 no. 4, pp. 447-465, 2004.

[Bekker 60] M.G. Bekker, Off-The-Road Locomotion, Univ. of Michigan Press, 1960. 
[Bekker 62] M.G. Bekker, Theory of Land Locomotion, Univ. of Michigan Press, 1962.

[Bliman et al. 91] P.-A. Bliman and M. Sorine, "Friction modelling by hysteresis operators: application to Dahl, sticktion and Stribeck effects", In Proceedings of the Conference Models of Hysteresis, Trento, Italy, 1991.

[Bliman et al. 93] P.-A. Bliman and M. Sorine, "A system-theoretic approach of systems with hysteresis. Application to friction modeling and compensation", In Proceedings of the second European Control Conference, Groningen, Netherlands, pp. 1844-1849, 1993.

[Bliman et al. 95] P.-A. Bliman and M. Sorine, "Easy-to-use realistic dry friction models for automatic control", In Proceedings of the third European Control Conference, Rome, Italy, pp. 3788-3794, 1995.

[Borenstein et al. 94] J. Borenstein, L. Feng, "A method for measuring, comparing, and correcting dead-reckoning errors in mobile robots", University of Michigan, Technical Report UM-MEAM-94-22, 1994.

[Borenstein 95] J. Borenstein, "Control and kinematic design of multi-degree-offreedom mobile robots with compliant linkage", IEEE Transactions on Robotics and Automation, vol. 11 no. 1, 1995.

[Campion et al. 96] G. Campion, G. Bastin, B. D'Andrea-Novel, "Structural Properties and Classification of Kinematic and Dynamic Models of Wheeled Mobile Robots", IEEE Trans. on Robotics and Automation, vol. 12 no. 1, pp. 47-61, February 1996.

[Canudas et al. 95] C. Canudas de Wit, H. Olsson, K. J. Åström, P. Lischinsky, "A new model for control of systems with friction", IEEE Transactions on Automatic Control, vol. 40 no. 3, pp. 419-425, 1995.

[Canudas et al. 97] C. Canudas de Wit, B. Siciliano, G. Bastin, "Theory of Robot Control", Springer, 1997.

[Craig 88] J. J. Craig, "Adaptative control of mechanical manipulators", Addison Wesley, 1988. 
[Cuesta et al. 04] F. Cuesta, F. Gómez-Bravo, A. Ollero, "Parking maneuvers of industrial-like electrical vehicles with and without trailer", IEEE Transactions on Industrial Electronics, vol. 51 no. 2, pp. 257-263, 2004.

[d'Andréa-Novel et al. 95] B. d'Andréa-Novel, G. Campion, G. Bastin, "Control of nonholonomic wheeled mobile robots by state feedback linearization", The International Journal of Robotics Research, vol. 14 no. 6, pp. 543-559, 1995.

[Dahl 68] P. Dahl, “A solid friction model”, Technical Report TOR-0158H310718I-1, The Aerospace Corporation, El Segundo, CA, 1968.

[Dahl 75] P. Dahl, "Solid friction damping of spacecraft oscillations", AIAA paper no. 75-1104 at the AIAA Guidance and Control Conference, Boston Mass, 1975.

[Dahl 76] P. Dahl, "Solid friction damping of mechanical vibrations", AIAA Journal, vol. 14 no. 12, pp. 1675-1682, 1976.

[De Luca et al. 93] A. De Luca and M.D. Benedetto, "Control of nonholonomic systems via dynamic compensation", Kybernetica, vol. 29 no. 6, pp. 593608, 1993.

[Denavit et al. 55] J. Denavit and R. S. Hartenberg, "A kinematic notation for lower-pair mechanism based on matrices", Journal of Applied Mechanism, vol. 77 no. 2, pp. 215-221, 1955.

[Dinesh et al. 92] K. Dinesh and M.C. Leu, "Genericity and singularities of robot manipulators", IEEE Transactions on Robotics and Automation, vol. 8 no. 5 , pp. 545-559, 1992.

[Dixon et al. 00] W.E. Dixon, Z.P. Jiang, D.M. Dawson "Global exponential setpoint control of wheeled mobile robots: a Lyapunov approach", Automatica, vol. 36, pp. 1741-1746, 2000.

[Dixon et al. 01] W.E. Dixon, D.M. Dawson, E. Zergeroglu, A. Behal, "Adaptative tracking control of a wheeled mobile robot via an uncalibrated camera system", IEEE Transactions on Systems, Man, and Cybernetics-Part B: Cybernetics, vol. 31 no. 3, pp. 341-352, 2001. 
[Dugoff et al. 70] H. Dugoff, P. S.Fancher, L. Segel, "An analysis of tire traction properties and their influence on vehicle dynamic performance", SAE Transactions, 1970.

[Everett 79] H. R. Everett, "A second generation of autonomous sentry robot", Robotics age 7 (4): 29-32, 1979.

[Ferrière et al. 98] L. Ferrière and B. Raucent, "Rollmobs, a new universal wheel concept", Proceedings of the International Conference on Field and Service Robotics, Leuven, Belgium, pp. 1877-1882, 1998.

[Fortune et al. 88] F. Fortune and G. Wilfong, "Planning constrained motion", Proceedings of the $20^{\text {th }}$ annual ACM symposium on theory of computing, Chicago, pp. 445-459, 1988.

[Fu et al. 88] K. S. Fu, R. C. Gonzalez, C. S. G. Lee, "Robótica: Control, detección, visión e inteligencia”, McGraw-Hill, 1988.

[Fukao et al. 00] T. Fukao, H. Nakagawa, N. Adachi, "Adaptative tracking control of a nonholonomic mobile robot", IEEE Transactions on Robotics and Automation, vol. 16 no. 5, pp. 609-615, 2000.

[Golub et al. 83] G. H. Golub, C. F. Van Loan, Matrix Computations, The Johns Hopkins University Press, $3^{\text {rd }}$ ed. Baltimore, MD 1996.

[Gómez 01] F. Gómez Bravo, "Planificación de maniobras de sistemas robóticos no holónomos. Aplicación a robots móviles", Tesis Doctoral, Universidad de Huelva, 2001.

[Gracia et al. 01] L. Gracia, J. Tornero, "Análisis comparativo de metodologías para la obtención de modelos cinemáticos en robótica móvil”, Proceedings of ICSECIT'01, Punta Arenas - Chile, 2001.

[Gracia2 et al. 01] L. Gracia, J. Tornero, "Application of kinematic Modeling methods to wheeled mobile robots", Advances in Scientific Computing, Computational Intelligence and Applications, WSES PRESS, pp 141-146, 2001.

[Gracia3 et al. 01] L. Gracia, J. Tornero, C. Gracia, "Sistema de posicionamiento por visión en líneas de cultivo", AGROING’01, Valencia, 2001. 
[Gracia4 et al. 01] L. Gracia, J. Tornero, "Sistema de control para el seguimiento de líneas de cultivo”, AGROING’01, Valencia, 2001.

[Gracia et al. 02] L. Gracia, J. Tornero, "Vision-based system for self-positioning and calibration", Advances in Systems Engineering, Signal Processing and Communications, WSEAS PRESS, pp. 137-142, 2002.

[Gracia2 et al. 02] L. Gracia, J. Tornero, "Kinematic control system for car-like vehicles", Advances in Artificial Intelligence - Iberamia 2002, VerlagSpringer, pp. 882-892, 2002.

[Gracia et al. 03] L. Gracia, J. Tornero, C. Gracia, "Sistema de posicionamiento por visión y calibración", Información Tecnológica (ISSN 0716-8756), vol. 14 no. 1 , pp. 31-38, 2003

[Gracia2 et al. 03] L. Gracia, J. Tornero, "Sistema de control cinemático para vehículos", Información Tecnológica (ISSN 0716-8756), vol. 14 no. 1, pp. 39-48, 2003.

[Gracia3 et al. 03] L. Gracia, J. Tornero, "Geometric parallel parking planner for car-like vehicles", Industrial Simulation Conference, 2003.

[Gracia et al. 07] L. Gracia, J. Tornero, "Kinematic modeling and singularity of wheeled mobile robots kinematics", Advanced Robotics, vol. 21 no. 7, 2007.

[Helmers 85] C. Helmers, "Photo essay: A first glimpse at Gemini", Robotics age, vol. 7 no. 2, pp. 12-13, 1985.

[Haessig et al. 91] D.A. Haessig and B. Friedland, "On the modelling and simulation of friction", ASME Journal of Dynamic Systems, Measurement, and Control, vol. 113 no. 3, pp. 354-362, 1991.

[Higham 96] N. J. Higham, Accuracy and Stability of Numerical Algorithms, SIAM Publications, Philadelphia, 1996.

[Holve et al. 96] R. Holve, P. Protzel, "Reverse parking of a mobile car with fuzzy control", Proceedings of the $4^{\text {th }}$ European Congress on Intelligent Techniques and Soft Computing, pp. 2171-2175, 1996.

[Hough 59] P.V.C. Hough, "Machine analysis of bubble chamber pictures", Int. Conference on High Energy Accelerators and Instrumentation, 1959. 
[Iijima et al. 83] J. Iijima, S. Yuta, Y. Kanayama, "Elementary functions of a self-contained robot", Proc. of the $11^{\text {th }}$ Int. Symp. on Industrial Robots, Tokyo, pp. 211-218, 1983.

[Inoue et al. 97] K. Inoue, K. Otsuka, M. Sugimoto, N. Murakami, "Estimation of place of Tractor and Adaptative Control Method of Autonomous Tractor using INS and GPS”, In Proceedings of Biorobotics, Valencia - Spain, pp. 27-32, 1997.

[Jaynes 57] E. T. Jaynes, "Information theory and statistical mechanics", Physical Reviews, 620-630 (vol. 106), 171-190 (vol. 108), 1957.

[Jiang et al. 99] K. Jiang, D.Z. Zhang, L.D. Seneviratne, "A parallel parking system for a car-like robot with sensor guidance", Proc. IMechE, Part C, Jour. of Mechanical Engineering Science, vol. 213 no. 6, pp. 591-600, 1999.

[Kanayama et al. 86] Y. Kanayama and N. Miyaki, "Trajectory generation for mobile robots", Proc. of the $3^{\text {rd }}$ Int. Symp. on Robotics Research, France, pp. 333-340, 1985.

[Karnopp 85] D. Karnopp, "Computer simulation of slip-stick friction in mechanical dynamic systems." Journal of Dynamic Systems, Measurement, and Control, vol. 107 no. 1, pp. 100-103, 1985.

[Kiencke et al. 94] U. Kiencke, and A. Daiss, "Estimation of tyre friction for enhanced ABS-systems", In Proceedings of AVEG Congress, Tokyo, 1994.

[Killough et al. 92] S. M. Killough and F. G. Pin, "Design of an omni-directional and holonomic wheeled platform prototype", Proc. of the IEEE Int. Conf. on Robotics and Automation, Nice, pp. 84-90, 1992.

[Kim et al. 04] W. Kim, B-J Yi, D. J. Lim, "Kinematic modeling of mobile robots by transfer method of augmented generalized coordinates", Journal of Robotic Systems, vol. 21 no. 6, pp. 301-322, 2004.

[Kim2 et al. 04] S. Kim and H. Kim, "Isotropy analysis of caster wheeled omnidirectional mobile robots", Proc. of the Int. Conf. on Robotics and Automation, New Orleans, pp. 3093-3098, 2004. 
[Kim et al. 05] S. Kim, H. Kim, B. Moon, "Local and global isotropy of caster wheeled omnidirectional mobile robots", Proc. of the Int. Conf. on Robotics and Automation, Barcelona, pp. 3457-3462, 2005.

[Kleeman 95] L. Kleeman, "Odometry error covariance estimation for two wheel robot vehicles", Monash University, Technical Report MECSE-95-1, 1995.

[Lai et al. 90] J.Z.C. Lai, and D. Yang, "Efficient motion control algorithm for robots with wrist singularities", IEEE Transactions on Robotics and Automation, vol. 6 no. 1, pp. 113-117, 1990.

[Latombe 91] J.-C. Latombe, "Robot motion planning", Kluwer Academic Publishers, cap. 7, 1991.

[Laumond 92] J.-P. Laumond, "Singularities and topological aspects in nonholonomic motion planning", Eds. New York: The Kluwer International Series in Engineering and Computer Science, 1992.

[Laumond et al. 94] J.-P. Laumond, P.E. Jacobs, et al., "A motion planner for nonholonomic mobile robots", IEEE Transactions on Robotics and Automation, vol. 10 no. 5, 1994.

[Leow et al. 00] Y. P. Leow, J. Angeles, K. H. Low, "A comparative mobility study of three-wheeled mobile robots", Proc. of $6^{\text {th }}$ Int. Conf. on Control, Automation, Robotics and Vision, Singapore, 2000.

[Leow et al. 02] Y. P. Leow, K.H. Low, W.K. Loh, "Kinematic Modelling and Analysis of Mobile Robots with Omni-Directional Wheels", Proc. of $7^{\text {th }}$ Int. Conf. on Control, Automation, Robotics and Vision, Singapore, 2002.

[Lindgren et al. 02] D. R. Lindgren, T. Hague, P.J. Probert Smith, J. A. Marchant, "Relating torque and slip in an odometric model for autonomous agricultural vehicle", Autonomous Robots, vol. 13, pp. 73-86, 2002.

[Lipkin et al. 88] H. Lipkin and J. Duffy, "Hybrid twist and wrench control for a robotic manipulator", ASME Journal of Mechanisms, Transmissions, and Automation in Design, vol. 110, pp. 138-144, 1988.

[Lipkin et al. 91] H. Lipkin and E. Pohl, "Enumeration of Singular Configurations for Robotic Manipulators", ASME Journal of Mechanical Design, vol. 113, pp. 272-279, 1991. 
[Liu et al. 03] G. Liu, Y. Lou, Z. Li, "Singularities of parallel manipulators: A geometric treatment", IEEE Transactions on Robotics and Automation, vol. 19 no. 4, pp. 579-594, 2003.

[Lloyd et al. 01] J.E. Lloyd and V. Hayward, "Singularity-robust trajectory generation”, Int. Jour. of Robotics Research, vol. 20 no. 1, pp. 38-56, 2001.

[Loh et al. 03] W.K. Loh, K. H. Low, Y. P. Leow, "Mechatronics design and kinematic modeling of a singularityless omnidirectional wheeled mobile robot", Proc. of the Int. Conference of Robotics and Automation, Taiwan, pp. 3237-3242, 2003.

[Low et al. 05] K. H. Low and Y. P. Leow, "Kinematic modeling, mobility analysis and design of wheeled mobile robots", Advanced Robotics, Vol. 19, pp. 73-99, 2005.

[Lu et al. 05] C.Y. Lu and M.C. Shih, "An experimental study on the longitudinal and lateral adhesive coefficients between the tyre and the road for a light motorcycle", Vehicle System Dynamics Supplement, vol. 43, pp. 168-178, 2005.

[Luo 98] D. Luo, "Pattern recognition and image processing", Horwood Series in Engineering Science, Chichester: Horwood Publishing Ltd., cap. 6, 1998.

[Luo et al. 98] Z.-H. Luo, K. Machida, M. Funaki, "Design and control of a cartesian nonholonomic robot", Journal of Robotic Systems, vol. 15 no. 2, pp. 85-95, 1998.

[Lyshevski et al. 00] S. E. Lyshevski and A. Nazarov, "Lateral maneuvering of ground vehicles: Modeling and Control", Proc. of the American Control Conference, Chicago, Illinois, pp. 110-114, 2000.

[Ma et al. 91] O. Ma and J. Angeles, "Optimum architecture design of platform manipulators", Proc. IEEE Int. Conf. Adv. Robotics, pp. 1130-1135, 1991.

[Marchant 95] J. Marchant, "Tracking of row structure in three crops using image analasys", Computer and Electronic in Agriculture, 1995.

[Marrs 85] T. Marrs, "The personal robot book", Blue ridge summit, Pennsylvania: Tab Books, Inc, 1985. 
[Martinelli 02] A. Martinelli, "Evaluating the odometry error of a mobile robot", Proceedings of the IEEE/RSJ International Conference on Intelligent Robots and Systems, Lausanne (Switzerland), 2002.

[Matsumoto et al. 92] N. Matsumoto and M. Tomizuka, "Vehicle lateral velocity and yaw rate control with two independent control inputs", ASME Journal of Dynamic Systems, Measurement, and Control, Vol. 114, pp. 606 - 613, 1992.

[Miyata et al. 96] H. Miyata, M. Ohki, Y. Yokouchi, M. Ohkita, "Control of the autonomous mobile robot DREAM-1 for a parallel parking", Mathematics and Computers in Simulation, vol. 41 no. 1-2, pp. 129-138, 1996.

[Monaco et al. 91] S. Monaco and D. Normand-Cyrot, "Multirate sampling and zero dynamics: from linear to nonlinear, Nonlinear Synthesis", C. I. Byrnes and A. Kurzhansky Eds., Birkauser, pp. 200-213, 1991.

[Muir et al. 87] P. F. Muir and C. P. Neuman., "Kinematic modeling of wheeled mobile robots", J. Robotic Syst., vol. 4 no. 2, pp. 281-329, 1987.

[Murray et al. 93] R. M. Murray and S. Sastry, "Nonholonomic motion planning: Steering using sinusoids", IEEE Transactions on Automatic Control, vol. 38 no. 5, pp. 700-716, 1993.

[Nagasaka et al 00] Y. Nagaska, K. Taniwaki, R. Otani, K. Shigeta, "A study about an automated rice transplanter with GPS and FOG", ASAE paper no. 001066, 2000.

[Nenchev et al. 97] D.N. Nenchev and M. Uchiyama, "Singularity-Consistent Path Planning and Motion Control Through Instantaneous Self-Motion Singularities of Parallel-Link Manipulators”, Journal of Robotic Systems, vol. 14 no. 1 , pp. 27-36, 1997.

[O'Connor et al. 96] M. O'Connor, T. Bell, G. Elkaim, B. Parkinson, "Automatic steering of farm vehicles using GPS", Proc. of $3^{\text {rd }}$ Int. Conference on Precision Agriculture pp. 767-778, 1996.

[Okello et al. 94] J. Okello, M.J. Dwyer, F.B. Cottrell, "The tractive performance of rubber tracks and a tractor driving wheel tyre as influenced by design parameters", J. Agricultural Eng. Research, Vol. 59 no. 1, pp. 33-43, 1994. 
[Ollero et al. 91] A. Ollero and O. Amidi, "Predictive path-tracking of mobile robots. Applications to the CMU Navlab", Proceedings of 5th International Conference on Advanced Robotics, Robots in Unstructured Environments (ICAR '91), vol. 2, pp. 1081-1086, 1991.

[Ollero et al. 94] A. Ollero, A. García-Cerezo, J. Martínez, "Fuzzy supervisory path tracking of mobile robots", Control Engineering Practice 2 (2) pp. 313319, Pergamon Press, 1994.

[Ollero 01] A. Ollero, "Robótica: Manipuladores y robots móviles", Marcombo, 2001.

[Onsager 31] L. Onsager, "Reciprocal relations in irreversible processes", Physical Reviews, 405-426 (vol. 37), 2265-2279 (vol. 38), 1931.

[Ortega et al. 89] R. Ortega and M. W. Spong, "Adaptative motion control of rigid robots: a tutorial”, Automatica, vol. 25, pp. 887-888, 1989.

[Ostrovskaya et al. 00] S. Ostrovskaya, J. Angeles, R. Spiteri, "Dynamics of a mobile robot with three ball-wheels", The International Journal of Robotic Research, vol. 19 no. 4, pp. 383-393, 2000.

[Pacejka et al. 93] H.B. Pacejka and E. Bakker, "The 'magic formula' tyre model”, Vehicle System Dynamics, vol. 21, pp. 1-18, 1993.

[Pacejka et al. 97] H.B. Pacejka and I.J.M. Besselink, "Magic formula tyre model with transient properties", Vehicle System Dynamics Supplement, vol. 27, pp. 234-249, 1997.

[Pacejka 02] H.B. Pacejka, Tyre and Vehicle Dynamics, Butterworth Heinemann, 2002.

[Paromtchik et al. 96] I.E. Paromtchik, C. Laugier, "Motion generation and control for parking an autonomous vehicle", Proceedings of the IEEE Int. Conf. on Robotics and Aut., Minneapolis (USA), pp. 3117-3122, 1996.

[Park et al. 99] K. Park, H. Chung, J.G. Lee, "Point stabilization of mobile robots via state space exact feedback linearization", Proc of the IEEE International Conference on Robotics and Automation, Detroit, pp. 2626-2631, 1999. 
[Pin et al. 94] F. Pin and S. Killough, "A new family of omnidirectional and holonomic wheeled platforms for mobile robots", IEEE Transactions on Robotics and Automation, vol. 10 no. 4, pp. 480-489, 1994.

[Pinto et al. 99] F.A.C. Pinto, J.F. Reid, Q. Zhang, N. Noguchi, "Guidance parameter determination using artificial neural network classifier", ASAE paper no. 993004, 1999.

[Rajagopalan 97] R. Rajagopalan, "A generic kinematic formulation for wheeled mobile robots”, Journal of Robotic Systems, vol. 14, pp. 77-91, 1997.

[Rayleigh 1896] J. W. S. Rayleigh, "The Theory of Sound", $2^{\text {nd }}$ ed. London: Macmillan. Reprinted by Dover, New York, 1945.

[Rekow et al. 98] A. Rekow, T. Bell, M. O'Connor, B. Parkinson, "CDGPS based identification of farm tractor steering effectiveness", Standford U. 07803-4330-1/98 IEEE, 1998.

[Rogers 84] M. Rogers, "Birth of the killer robots", Newsweek CIII (26):51, 1984.

[Routh 1905] E. Routh, "Dynamics of a System of Rigid Bodies", Part 1. London 7th ed, New-York: The Macmillan Co., 1905.

[Saha et al. 95] S. K. Saha, J. Ángeles, J. Darcovich, "The design of kinematically isotropic rolling robots with omnidirectional wheels", Mech. Machine Theory, vol. 30 no. 8, pp. 1127-137, 1995.

[Samson et al. 91] C. Samson and K. Ait-Abderrahim, "Feedback control of a nonholonomic wheeled cart in Cartesian space", Proc of the 1991 IEEE International Conference on Robotics and Automation, Sacramento, CA, pp. 1136-1141, 1991.

[Samson 95] C. Samson, "Control of chained systems. Application to path following and time-varying point-stabilization of mobile robots", IEEE Transactions on Robotics and Automation, vol. 40 no. 1, pp. 64-77, 1995.

[Sánchez et al. 97] A. Sánchez, J. Marchant, "Fast and robust method for tracking crop rows using a two point Hough transform", Biorobotics'97, Valencia, , pp. 33-281, 1997. 
[Scheding et al. 99] S. Scheding, G. Dissanayake, E. M. Nebot, H. DurrantWhyte, "An experiment in autonomous navigation of an underground mining vehicle", IEEE Transactions on Robotics and Automation, Vol. 15 no. 1, pp. 85-95, 1999.

[Scheuer et al. 98] A. Scheuer, Y Ch. Laugier, "Planning Sub-Optimal and Continuous-Curvature Paths for Car-Like Robots", International Conference on Intelligent Robots and Systems, 1998.

[Sheth et al. 71] P. N. Sheth and J. J. Uicker Jr., "A generalized symbolic notation for mechanisms", Journal of Engineering for Industry, vol. 93 no. 70, pp. 102-112, 1971.

[Shin 90] D. Shin, "High Performance Tracking of Explicit Paths by Roadworthy Mobile Robots", Ph. D. Dissertation, Carnegie Mellon University, 1990.

[Shin et al. 01] D. H. Shin and K. H. Park, "Velocity kinematic modeling for wheeled mobile robots", Proc of the 2001 IEEE International Conference on Robotics and Automation, Seoul, Korea, pp. 3516-3522, 2001.

[Smith 73] M. H. Smith, "Design of a low cost, general purpose robot", Proc. Of $3^{\text {rd }}$ Int. Joint Conf. on Artificial Intelligence, Stanford (California), pp. 324335, 1973.

[Stanisic et al. 90] M.M Stanisic and O. Duta, "Symmetrically actuated double pointing systems: the basis of singularity-free robot wrists", IEEE Transactions on Robotics and Automation, vol. 6 no. 5, pp. 562-569, 1990.

[Stocco et al. 98] L. Stocco, S. E. Salcudean, F. Sassani, "Matrix normalization for optimal robot design", IEEE Int. Conf. on Robotics and Automation, Leuven (Belgium), vol. 2, pp. 1346-1351, 1998.

[Stocco2 et al. 98] L. Stocco, S. E. Salcudean, F. Sassani, "Fast constrained global minimax optimization of robot parameters", Robotica, vol. 16, pp. 595-605, 1998.

[Stocco et al. 99] L.J. Stocco, S.E. Salcudean, F. Sassani, "On the use of scaling matrices for task-specific robot design", IEEE Trans. Robotics and Automation, vol. 15 no. 5, pp. 958-965, 1999. 
[Stribeck 1902] R. Stribeck, "Die wesentlichen Eigenschaften der Gleit- und Rollenlager -The key qualities of sliding and roller bearings", Zeitschrift des Vereines Seutscher Ingenieure, 46 (38,39), pp. 1342-48,1432-37, 1902.

[Tan et al. 91] H . S . Tan and Y.-K. Chin, "Vehicle traction control: variable structure control approach", ASME Journal of Dynamic Systems, Measurement, and Control, Vol. 113, pp. 223-230, 1991.

[Tandirci et al. 92] M. Tandirci, J. Angeles, F. Ranjbaran, "The characteristic point and the characteristic length of robotic manipulators", Proc. ASME 22nd Biennial Mechanisms Conf., Arizona, USA, vol. 45, pp. 203-208, 1992.

[Tham et al. 98] Y. K. Tham, H. Wang, E. K. Teoh, "Adaptative State Estimation for 4-Wheel Steerable Industrial Vehicles", Proceedings of the 37th IEEE Conference on Decision and Control, Florida USA, pp. 4509-4514, 1998.

[Tilbury et al. 93] D. Tilbury, R. Murray, S. Sastry, "Trajectory generation for the n-trailer problem using Goursat normal form", Proceedings of the IEEE Conference on Decision and Control, San Antonio, 1993.

[Tourassis et al. 92] V.D. Tourassis and H. Ang Jr., "Identification and analysis of robot manipulator singularities", International Journal of Robotics Research, vol. 11 no. 3, pp. 248-259, 1992.

[Tzafestas et al. 01] C.S. Tzafestas and S.G. Tzafestas, "Full-state modeling, motion planning and control of mobile manipulators", Studies in Informatics and Control, vol. 10 no. 2, pp 109-128, 2001.

[Velenis et al. 05] E. Velenis, P. Tsiotras, C. Canudas-de-Wit, M. Sorine, "Dynamic tyre friction models for combined longitudinal and lateral vehicle motion”, Vehicle System Dynamics, Vol. 43 no. 1, pp. 3-29, 2005.

[Wada et al. 00] M. Wada, A. Takagi, S. Mori, "Caster drive mechanisms for holonomic and omni-directional mobile platforms with no over constraint", Proceedings of the IEEE International Conference on Robotics and Automation, San Francisco, USA, pp. 1531-1538, 2000.

[West et al. 92] M. West and H. Asada, "Design of a holonomic omnidirectional vehicle", Proc. of the IEEE International Conference on Robotics and Automation, Nice, France, pp. 153-161, 1992. 
[West et al. 95] M. West and H. Asada, "Design and control of ball wheel omnidirectional vehicles", Proc. of the IEEE International Conference on Robotics and Automation, Nagoya, Japan, Vol. 2, pp. 1931-1938, 1995.

[Whittaker 62] E. T. Whittaker, "Stair climbing device", U.S. Patent No. $3,058,754,1962$.

[Wilfong 88] G. T. Wilfong, " Motion planning for an autonomous vehicle”, Proc of the IEEE International Conference on Robotics and Automation, Philadelphia, pp. 529-533, 1988.

[Williams et al. 02] R. L. Williams, B. E. Carter, P. Gallina, G. Rosati, "Dynamic model with slip for wheeled omnidirectional robots", IEEE Transactions on Robotics and Automation, Vol. 18 no. 3, pp.285-293, 2002.

[Yi et al. 02] B.-J. Yi and W. K. Kim, "The kinematics for redundantly actuated omnidirectional mobile robots", Journal of Robotic Systems, vol. 19 no. 6, pp. 255-267, 2002.

[Zhao et al. 05] Y. Zhao, E.G. Collins Jr., "Robust automatic parallel parking in tight spaces via fuzzy logic", Robotics and Autonomous Systems, vol. 51 no. 2, pp. 111-127, 2005. 